\title{
The Second Spectrum of Ytterbium (Yb II)
}

\author{
William F. Meggers
}

\author{
(Edited by Charlotte E. Moore)
}

\begin{abstract}
(May 26, 1967)
The analysis of $\mathrm{Yb}$ II provides the most complete interpretation of a complex rare-earth spectrum known to date. The total number of energy levels is $315 ; 141$ even levels and 174 odd levels. Three coupling schemes are represented: $L S$-coupling for the terms $4 f^{14} n s^{2} S(n=6,7,8)$; $4 f^{14} n d^{2} \mathrm{D}(n=5,6,7)$ and $4 f^{13} 6 s^{2}{ }^{2} \mathrm{~F}^{\circ}: J_{1} J_{\mathrm{II}^{-}}$coupling for the levels from the configurations $4 f^{13} 6 s 6 p$ and $4 f^{13} 5 d 6 p$; and $J_{1} L_{11}$-coupling for those from the $4 f^{13} 5 d 6 s$ and $4 f^{13} 5 d^{2}$ configurations.

More than $5000 \mathrm{Yb}$ II lines are listed, of which about 80 percent have been classified, and 95 percent of the total intensity is accounted for. Observed Zeeman patterns for over 1000 lines have yielded $g$-values for 258 levels. The agreement between observation and theory is excellent as regards both the positions of the energy levels and the g-values. The theoretical work was done in collaboration with Racah and his associates over more than a decade.

A limit, $98150 \mathrm{~K}$, giving an ionization potential of $12.17 \mathrm{eV}$, has been derived from the three member ${ }^{2} \mathrm{~S}$ series.
\end{abstract}

Key Words: Analysis of Yb II. Atomic energy levels of Yb II. Classified lines of Yb II. Spectrum, $\mathrm{Yb}$ II. Ytterbium, second spectrum. Zeeman effect in Yb II.

\section{Introduction}

In November 1966 death claimed the eminent spectroscopist, William F. Meggers, the author of the present paper. It was characteristic of this author to leave a rich heritage for his colleagues. Part of this heritage is the analysis of $\mathrm{Yb}$ II. His interest in this rare-earth spectrum dates back at least to 1932 when the first regularities were known. In 1937 he and Scribner "provided the first useful description of conventional arc and spark spectra of this element" [1]. ${ }^{1}$ Some $1250 \mathrm{Yb}$ II lines in the range 2000 to $11000 \AA$ were reported. The resonance lines of $\mathrm{Yb}$ II were interpreted "with considerable certainty" as due to the transition from the ground state $4 f^{14} 6 s^{2} \mathrm{~S}$ to $4 f^{14} 6 p{ }^{2} \mathrm{P}^{\circ}$, giving rise to the strong lines at 3694.190 and $3289.370 \AA$. The second series member $4 f^{14} 7 s^{2} \mathrm{~S}$ was also reported at this time.

Although this work was handicapped by the use of impure materials and conventional light sources, Meggers succeeded, in 1939, in photographing the first Zeeman patterns of ytterbium lines with the Bitter magnet and concave gratings of the Massachusetts Institute of Technology. With these Zeeman data and further observations of spark spectra he was able to extend the quantum interpretation of the spectrum. In 1947 he had measured 400 Zeeman patterns, reobserved and measured the spectrum from 2000 to $9000 . \AA$. and classified about 700 Yb II lines "as combinations between 38 odd and 76 even levels." Additional configurations were detected. The configurations $4 f^{13} 5 d 6 s, 4 f^{13} 5 d^{2}$, and $4 f^{13} 6 s 6 d$ give rise to many odd levels whose combinations with larger families of even levels from $4 f^{13} 6 s 6 p$ and $4 f^{13} 5 d 6 p$ give $\mathrm{Yb}$ II "its rare earth characteristics" [2].

\footnotetext{
${ }^{1}$ Figures in brackets indicate the literature references at the end of this paper.
}

"When pure ytterbium metal and new light sources became available in 1950, Meggers and Corliss decided to make a new description of ytterbium spectra" [3]. In the new list some $5100 \mathrm{Yb}$ II lines are recorded together with the Zeeman Effect Type for more than 1000 lines. "The spectra of microwave and ring discharges of ytterbium were photographed side-by-side together with short images of superimposed standards from iron arcs or from thorium-iodide tubes. To disperse and focus the spectra, four concave gratings of $6.5 \mathrm{~m}$ radius, in Wadsworth-type mountings, were used." The "region from $2000 \AA$ to $2500 \AA$ was photographed in the first order of two $(30,000$ lines per inch) gratings with a plate factor of $2.4 \AA / \mathrm{mm}$." From " $2500 \AA$ to $4500 \AA$, the spectra were recorded in the second order of either grating with a plate factor of $0.87 \AA / \mathrm{mm}$, and the same setting served to record the range $5000 \AA$ to $9000 \AA$ in the first order with a plate factor of $1.74 \AA / \mathrm{mm}$." From 7000 to $10000 \AA$ a third grating having 15,000 lines per inch was used, the plate factor being $5 \AA / \mathrm{mm}$.

In 1962 Sugar developed a light source "for producing self-reversed lines in both first and second spectra of rare-earth elements" [4]. He made the first observations with this source with ytterbium electrodes, covering the range 2400 to $7000 \AA$. "These spectro-rams were evaluated by Meggers . . . who found that 50 lines of $\mathrm{Yb}$ II were absorbed or self-reversed."

In 1963 N. Spector photographed ytterbium spectra from 6600 to $11600 \AA$ by means of a sliding spark, a source described in a paper by Sugar [5]. Longer than $9000 \AA$ Meggers found several hundred additional lines on these spectrograms, "many of which have been explained as transitions between previously established Yb II energy levels." 
There are four classified $\mathrm{Yb}$ II lines longer than $11600 \mathrm{~A}$ : these were observed by Humphreys and Paul with a lead sulfide cell as detector, and electrodeless lamps as source [6].

In the 1966 reference, Meggers gives a clearly written history of other observations and work on ytterbium spectra, which need not be repeated here. It was his intention to prepare a Monograph on this spectrum that would serve as an inspiration to others who were engaged in the difficult task of interpreting complex rare-earth spectra. Although the material was not in final form for publication, the analysis was essentially completed at the time of his death. The details of publication have been carried out with his general idea in mind, insofar as it could be anticipated.

\section{Interpretation of the Spectrum}

The first recollection of the writer (C. E. M.) regarding $\mathrm{Yb}$ II is connected with the concern of both Meggers and $H$. N. Russell over the failure to find the elusive $4 f^{14} 5 d^{2} \mathrm{D}$ term in spite of an exhaustive search made with the earlier line lists. The late G. Racah became interested in this problem and in December 1952 pointed out that this term was not a rare-earth term, and could, therefore, be extrapolated from the spectra Hf II to W II. His predicted position placed the two respective levels surprisingly high i.e., near 23500 and $25000 \mathrm{~K}$, thus explaining why the earlier searches had been fruitless. Earlier, however (1951) with the aid of Meggers' data, Racah had detected an unusual kind of coupling $\left(J_{\mathrm{I}} J_{\mathrm{II}}\right)$ in the configuration $4 f^{13} 6 s 6 p$ of $\mathrm{Yb}$ II, which is described below. Thus started the long and fruitful collaboration between these eminent spectroscopists, which has resulted in the present analysis. From this date to the present time, with the aid of digital computers in both the experimental and theoretical aspects of the work, this complex spectrum has been unraveled slowly, by using the observed levels to obtain parameters for predicting missing levels, and repeating the process. The early correspondence reveals the painstaking care used in this step-by-step method to push the search for new levels to its present state. The collaboration for nearly 15 years has contributed results of far-reaching importance in the realm of complex rare-earth spectra.

To date there are 141 known even energy levels of Yb II. These are tabulated in table 1: the odd levels, 174 in all, are given in table 2. The level values are accurate "to about $\pm 0.02 \mathrm{~K}$." The tables are arranged similarly. Each table contains eight columns, headed, respectively: Configuration, Designation, J, Level, Interval, Observed $g$, Calculated $g$, and the number of observed combinations on which the level is based.

Three types of coupling-schemes are needed to describe the energy levels of $\mathrm{Yb}$ II. ${ }^{2}$ The only entries in the "Interval" column are those for the regular terms

\footnotetext{
${ }^{2}$ A summary of coupling schemes for one-electron spectra may be found in a paper by Gutman and Levinson [7], which has been translated by J. Tech. This may serve, also, as a useful general guide for the interpretation of spectra having more than one electron outside the core.
}

having LS-coupling. These terms result from the configurations having the completed shell of $f$-electrons: $4 f^{14} n s^{2} \mathrm{~S}(n=6,7,8), 4 f^{14} n d^{2} \mathrm{D}(n=5,6,7), 4 f^{14} 6 p^{2} \mathrm{P}^{\circ}$, and from the configuration $4 f^{13} 6 s^{2}{ }^{2} \mathrm{~F}^{\circ}$. All other assigned levels belong to the configurations $4 f^{13} 6 s 6 p$, $4 f^{13} 5 d 6 p, 4 f^{13} 5 d 6 s$, and $4 f^{13} 5 d^{2}$.

The key to the interpretation of the spectrum was given by the configuration $4 f^{13} 6 s 6 p$ [8]. The coupling is a $\mathrm{J}_{\mathrm{I}} \mathrm{J}_{\mathrm{II}^{-}}$-coupling, a type first noted by Shenstone in the $3 d^{9} 4 s 4 p$ configuration of $\mathrm{Cu} \mathrm{I}$, who described it as $3 d^{9}{ }^{2} \mathrm{D}+4 s 4 p^{3} \mathrm{P},{ }^{1} \mathrm{P}$ [9]. In $\mathrm{Yb}$ II "the low levels are given by the three states of $s p^{3} \mathrm{P}_{\mathrm{J}_{\mathrm{II}}}$; each of these states may assume different orientations in the quadrupole field of $f^{13}{ }^{2} \mathrm{~F}_{3 \frac{1}{2}}$, and thus splits into 1,3 , or 5 levels depending on the $J_{I I}$ value. The three levels of ${ }^{1} \mathrm{P}_{1}$ lie somewhat higher, and the whole distribution is repeated $10000 \mathrm{~cm}^{-1}$ higher, in the field of $f^{13}{ }^{2} \mathbf{F}_{2 \frac{1}{2}}$." The interval of the ground term of the grandparent, 4. $f^{13}{ }^{2} \mathrm{~F}^{\circ}$ in $\mathrm{Yb} \mathrm{IV}, 10090 \mathrm{~K}[10]$ is clearly reflected in $\mathrm{Yb}$ II and "the J-value of the grandparent is very well defined" [8]. The complete $\mathrm{J}_{\mathrm{I}} \mathrm{J}_{\mathrm{II}}$-coupling notation is given in column one of table 1 , and the corresponding groups of levels are given in column four. All of the 24 predicted levels of the $4 f^{13} 6 s 6 p$ configuration have been found.

Similar notation is used for the levels from the $4 f^{13} 5 d 6 p$ configuration (table 1$)$. Of the 113 predicted levels, 108 are known.

Except for the regular terms mentioned above, the identified odd levels (table 2) have the configurations $4 f^{13} 5 d 6 s$ or $4 f^{13} 5 d^{2}$. Racah [8] has noted that "the orbital momentum of $s d^{3} \mathrm{D}$ in the former group is orientated in the electrostatic field of the core before it is coupled with its own spin. The resulting coupling scheme is a $\mathrm{J}_{\mathrm{I}} \mathrm{L}_{\mathrm{II}^{-}}$-coupling." This "is the natural generalization of the $j l$ coupling for configurations with two electrons outside the core." Instead of pairs, there are mostly groups of three levels or isolated levels according to the resultant spin of the outer electrons, and the multiplicity is indicated by the superscript at the left of the brackets in column two. The complete $\mathrm{J}_{\mathrm{I}} \mathrm{L}_{\mathrm{II}}$-coupling notation is given in column one of table 2. All 39 predicted levels from $4 f^{13} 5 d 6 s$ are known. Of the 81 predicted levels from $4 f^{13} 5 d^{2}, 73$ have been found.

The two coupling schemes described above are illustrated in figures 1 and 2 , which are self-explanatory [8]. Racah noted that the present work on Yb II provides the first example of $\mathrm{J}_{\mathrm{I}} \mathrm{L}_{\mathrm{II}}$-coupling in a complex spectrum. It has already served as a "model" for Tm II, as reported by Spector [11]. Both types of coupling may well be expected in other complex rare-earth spectra.

After tables 1 and 2 had been completed, a final machine search was made by J. Tech for the five missing levels from the $4 f^{13} 5 d 6 p$ configuration and for the nine missing levels from $4 f^{13} 5 d^{2}$. The range covered in this search was $\pm 300 \mathrm{~K}$ from the predicted values. He succeeded in finding the level $59632.21_{6 \frac{1}{2}}^{\circ}$, which has been included in the tables with a question, since the total number of combinations on which it is based, is five, and one of these may be a blend. 


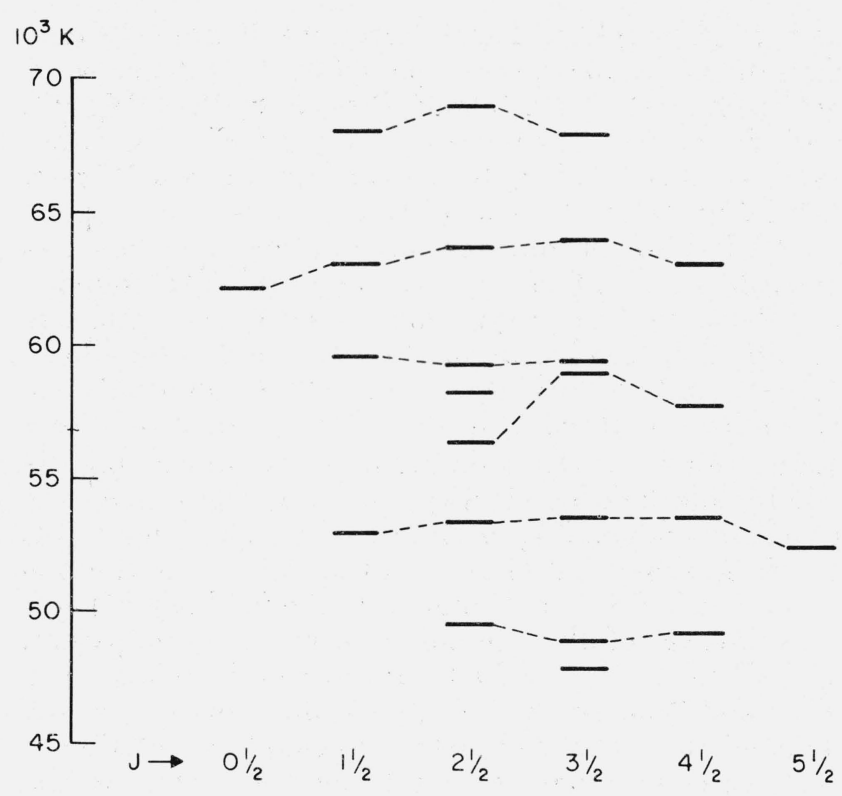

FIGURE 1. The configuration $4 \mathrm{f}{ }^{13}\left({ }^{2} \mathrm{~F}_{31 / 2,21 / 2}^{\circ}\right) 6 \mathrm{~s} 6 \mathrm{p}$ in $\mathrm{Yb}$ II: $\mathrm{J}_{\mathrm{I}} \mathrm{J}_{\mathrm{II}}$-coupling.

The arrays of observed levels as described in the various coupling schemes are presented in table 3 , which shows the relation of the various groups to the atomic structure.

In addition there are 58 miscellaneous high odd levels, above $84000 \mathrm{~K}$, in table 2, which have not yet been assigned configurations.

The calculations of the energy matrices for $\mathrm{Yb}$ II have been carried out at The Hebrew University in Jerusalem. New diagonalizations including interaction were reported by Racah in August 1965 [12]. After his untimely death a few weeks later, the work was continued by Z. Goldschmidt and others. In fact, the $4 f^{13} 5 d 6 p\left({ }^{1} \mathrm{P}_{1}\right)$ configuration was interpreted by Mrs. Goldschmidt after introducing configuration interaction. She has kindly furnished the final data on the coupling schemes, and on the calculated positions and $g$-values for the individual levels. The observed and calculated values for both levels and $g$-values are in remarkably good agreement. The mean error (o-c) in the level values is $103 \mathrm{~K}$ for the even levels, and $144 \mathrm{~K}$ for the odd levels. A separate publication on the theoretical interpretation of the spectrum, the exact composition of the levels, and the agreement between observation and theory is in the hands of Mrs. Goldschmidt, who is familiar with the details of the work on Yb II carried on by Racah and his associates over the years.

\section{Observations of Zeeman Effect}

In a letter to Racah dated July 16, 1965, Meggers writes "I have spent many months of dreary drudgery deriving $g$-factors and classified lines from 98 pages of square array." As stated above, the first Zeeman observations were made at the Massachusetts Institute

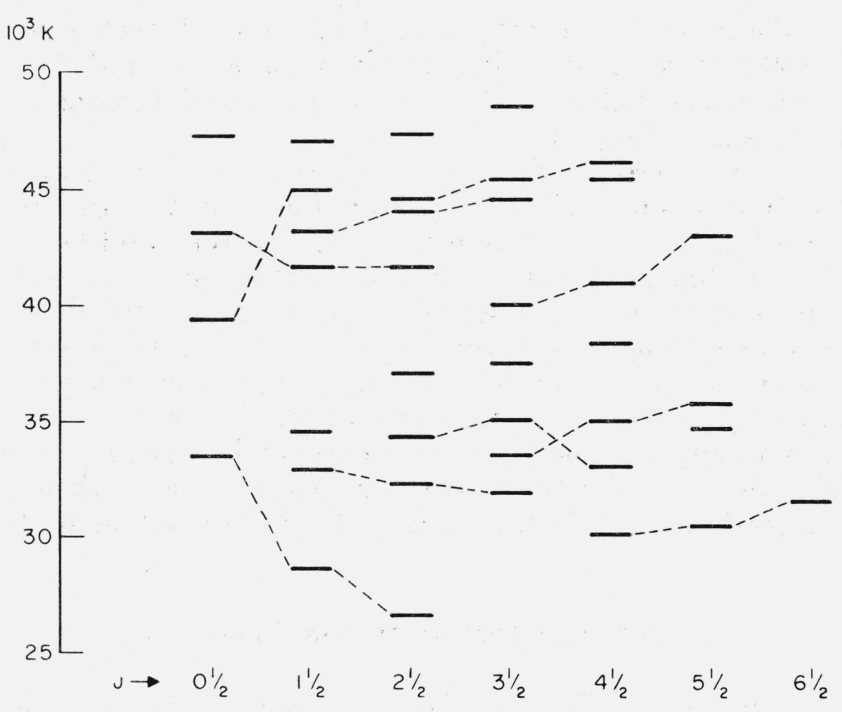

FIGURE 2. The configuration $4 \mathrm{f}^{13}\left({ }^{2} \mathrm{~F}_{31 / 2,21 / 2}^{\circ}\right) 5 \mathrm{~d} 6 \mathrm{~s}$ in $\mathrm{Yb}$ II; $\mathrm{J}_{\mathrm{I}} \mathrm{L}_{\mathrm{II}}$-coupling.

of Technology in 1939. At this time, Zeeman patterns for $400 \mathrm{Yb}$ lines were obtained by accident, when they appeared as impurities in Tm spectra. "In favorable cases the resolution reached 0.05 Lorentz unit." Further Zeeman observations in the range 2000 to $9000 \AA$ were made in 1959 at the National Bureau of Standards, where a Weiss magnet was used. "The highest resolution achieved was about 0.1 Lorentz unit.'

"These investigations extending intermittently over a quarter century" have resulted in observed Zeeman patterns for more than 1000 lines of $\mathrm{Yb}$ II. Observed $g$-values have been derived for 136 even levels (column six, table 1) and for 122 odd levels (column six, table 2).

Details of the Zeeman observations are included in the last five columns of table 6 . The standard Zeeman Types 4, 5, 6 for even multiplicity, and Type 7, which has "a single undisplaced parallel $(p)$ component and two symmetrically displaced normal $(n)$ components," are indicated in column seven, labeled "Type." Excellent illustrations of these types may be found in the paper by Meggers and Corliss on an "Improved Description of Hafnium Spectra" [13].

The next two columns, eight and nine, contain the $g$-values of the respective levels of the transition giving rise to the line. The "lst" $g$-value is that of the low level involved, i.e., the first entry in column six of table 6. The "2nd" $g$-value is that of the high level, i.e., the second entry in column six of this table. For example, $8781.96 \AA$ is designated as $37516_{31 / 2}^{\circ}$ $48900_{31 / 2}$. The $g$-value for $37516^{\circ}$ is 1.114 ; that for 48900 is 1.034 . The last two columns of table 6 , ten and eleven, give the strongest $p$-component, and the strongest $n$-component, respectively.

All entries in parentheses have been assumed in making the calculations to derive observed $g$-values. For some lines the strongest $p$-component is in parentheses. If $\Delta g$ is small, it has been assumed that the measurement refers to the weakest $p$-component. The 
entry in parentheses is, therefore, the appropriate fraction of the observed value.

The observed $g$-values in tables 1 and 2 were obtained by averaging the separate values. Many are based on resolved patterns. The average value can generally be used as an assumed value for one level, to derive the $g$-value for the other level in cases of unresolved patterns. When this has been done, the assumed $g$-value is in parentheses.

For resolved patterns, an excellent value of $\Delta g$ can often be determined, which may differ slightly from the one indicated by the strongest $p$-component. The best value has been used in the calculations, which explains small differences between the value of $\Delta g$ from the observed $g$-values and that from column ten.

Most of the final $g$-values were assigned by Meggers, who had completed the reductions of all Zeeman observations and interpreted practically all of the resolved patterns. A number of $g$-values from unresolved patterns have been derived by C. E. M. as described above. They have been added in table 6 , and used only to supplement the averages adopted by Meggers.

Most of the lines for which the Zeeman Effect has been observed, are classified. The remaining unclassified lines having a known Zeeman Effect, are listed in table 4, which gives in successive columns the wavelength, spark intensity, wave number Type, strongest $p$-, and strongest $n$-components. As in table 5 which contains the strongest unclassified lines, the entries are from table 6.

The overall consistency of the individual $g$-values reveals the painstaking skill and care of the author in making the observations, in reducing them, and in interpreting them.

TABLE 1. Even levels of $\mathrm{Yb}$ II

\begin{tabular}{|c|c|c|c|c|c|c|c|}
\hline Configuration & Desig. & $J$ & Level & Interval & Obs. $g$ & Calc. $g$ & No. Comb. \\
\hline $4 f^{1+}(1 S) 6 s$ & $6 s \div \mathrm{S}$ & $0^{1 / 2}$ & 0.00 & & 1.998 & 2.000 & 14 \\
\hline $4 f^{1+1}(' S) .5 d$ & $5 d l^{\prime} \mathrm{D}$ & $\begin{array}{l}11 / 2 \\
21 / 2\end{array}$ & $\begin{array}{l}22960.80 \\
24332.69\end{array}$ & 1371.89 & $\begin{array}{l}1.802 \\
1.202\end{array}$ & $\begin{array}{l}0.800 \\
1.200\end{array}$ & $\begin{array}{l}37 \\
39\end{array}$ \\
\hline $4 f^{13}\left(-\mathrm{F}_{3 !}^{\circ}\right) 6 s 6 p\left({ }^{3} \mathrm{P}_{01}\right)$ & & $31 / 2$ & 47912.31 & & 1.280 & 1.284 & 26 \\
\hline $4 f^{13}\left({ }^{2} \mathrm{~F}_{3}^{\circ}\right) 6 s 6 p\left({ }^{3} \mathrm{P}_{\mathrm{i}}\right)$ & & $\begin{array}{l}11 / 2 \\
31 / 2 \\
21 / 2\end{array}$ & $\begin{array}{l}49301.16 \\
48900.41 \\
49498.04\end{array}$ & & $\begin{array}{l}1.187 \\
1.044 \\
1.145\end{array}$ & $\begin{array}{l}1.196 \\
1.051 \\
1.140\end{array}$ & $\begin{array}{l}27 \\
26 \\
24\end{array}$ \\
\hline 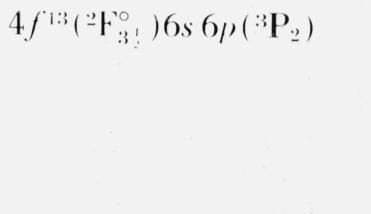 & & $\begin{array}{l}51 / 2 \\
4^{1 / 2} \\
3^{1 / 2} \\
2^{1 / 2} \\
1^{1 / 2}\end{array}$ & $\begin{array}{l}52517.35 \\
53720.71 \\
5: 371.5 .26 \\
53404.96 \\
52938.01\end{array}$ & & $\begin{array}{l}1.27 \\
1.26 \\
1.181 \\
1.033 \\
0.877\end{array}$ & $\begin{array}{l}1.272 \\
1.270 \\
1.190 \\
1.043 \\
0.879\end{array}$ & $\begin{array}{l}19 \\
30 \\
26 \\
35 \\
17\end{array}$ \\
\hline $4 f^{1+}\left({ }^{\prime} S\right) 7 s$ & $7 s \div \mathrm{S}$ & $0^{1 / 2}$ & 54304.30 & & 2.001 & 2.000 & 12 \\
\hline $4 f^{1: 3}\left({ }^{2} \mathrm{r}_{3 !}^{\circ}\right) 6 s 6 p\left({ }^{1} \mathrm{P}_{1}\right)$ & & $\begin{array}{l}21 / 2 \\
33^{1 / 2} \\
4^{1 / 2}\end{array}$ & $\begin{array}{l}56375.91 \\
59090.13 \\
57765.32\end{array}$ & & $\begin{array}{l}1.226 \\
1.122 \\
1.12\end{array}$ & $\begin{array}{l}1.233 \\
1.112 \\
1.112\end{array}$ & $\begin{array}{l}34 \\
39 \\
36\end{array}$ \\
\hline 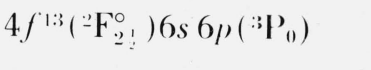 & & $2^{1 / 2}$ & 58283.91 & & 0.77 & 0.761 & 25 \\
\hline $4 f^{13}\left(\cdot{ }^{\prime} \mathrm{F}_{2 !}^{\circ}\right) 6 s 6 p\left(: 3 \mathrm{P}_{1}\right)$ & & $\begin{array}{l}31 / 2 \\
21 / 2 \\
11 / 2\end{array}$ & $\begin{array}{l}59618.90 \\
59259.24 \\
59710.65\end{array}$ & & $\begin{array}{l}1.00 \\
1.105 \\
0.82\end{array}$ & $\begin{array}{l}1.020 \\
1.114 \\
0.777\end{array}$ & $\begin{array}{l}23 \\
31 \\
22\end{array}$ \\
\hline $4 f^{13}\left({ }^{2} \mathrm{~F}_{2 !}^{\circ}\right) 6 s 6 p\left(: \mathrm{P}_{2}^{\circ}\right)$ & & $\begin{array}{l}0^{1 / 2} \\
11 / 2 \\
21 / 2 \\
3^{1 / 2} \\
41 / 2\end{array}$ & $\begin{array}{l}62136.94 \\
63011.85 \\
63702.32 \\
63957.80 \\
63163.79\end{array}$ & & $\begin{array}{l}0.08 \\
0.79 \\
1.07 \\
1.148 \\
1.12\end{array}$ & $\begin{array}{l}0.113 \\
0.812 \\
1.040 \\
1.144 \\
1.138\end{array}$ & $\begin{array}{l}11 \\
21 \\
29 \\
23 \\
22\end{array}$ \\
\hline
\end{tabular}


TABLE 1. Even levels of $\mathrm{Yb} \mathrm{II}-$ Continued

\begin{tabular}{|c|c|c|c|c|c|c|c|}
\hline Configuration & Desig. & $J$ & Level & Interval & Obs. $g$ & Calc. $g$ & No. Comb. \\
\hline $4 f^{13}\left({ }^{2} \mathrm{~F}_{2 !}^{\circ}\right) 6 s 6 p\left({ }^{1} \mathrm{P}_{1}\right)$ & & $\begin{array}{l}31 / 2 \\
21 / 2 \\
11 / 2\end{array}$ & $\begin{array}{l}67938.68 \\
69079.88 \\
68150.57\end{array}$ & & $\begin{array}{l}0.960 \\
0.89 \\
0.928\end{array}$ & $\begin{array}{l}0.954 \\
0.907 \\
0.927\end{array}$ & $\begin{array}{l}42 \\
29 \\
42\end{array}$ \\
\hline $4 f^{13}\left({ }^{2} \mathrm{~F}_{3^{\frac{1}{2}}}^{\circ}\right) 5 d 6 p\left({ }^{3} \mathrm{~F}_{2}\right)$ & & $\begin{array}{l}11 / 2 \\
21 / 2 \\
31 / 2 \\
41 / 2 \\
51 / 2\end{array}$ & $\begin{array}{l}55702.07 \\
58823.58 \\
61051.06 \\
61214.66 \\
58961.37\end{array}$ & & $\begin{array}{l}1.693 \\
1.266 \\
1.18 \\
1.07 \\
0.974\end{array}$ & $\begin{array}{l}1.700 \\
1.278 \\
1.176 \\
1.054 \\
0.985\end{array}$ & $\begin{array}{l}17 \\
39 \\
35 \\
44 \\
21\end{array}$ \\
\hline $4 f^{13}\left({ }^{2} \mathrm{~F}_{3 !}^{\circ}\right) 5 d 6 p\left({ }^{3} \mathrm{~F}_{3}\right)$ & & $\begin{array}{l}01 / 2 \\
11 / 2 \\
21 / 2 \\
31 / 2 \\
41 / 2 \\
5^{1 / 2} \\
61 / 2\end{array}$ & $\begin{array}{l}61120.49 \\
60586.46 \\
64055.82 \\
65093.14 \\
64923.11 \\
66082.25 \\
61873.40\end{array}$ & & $\begin{array}{l}1.661 \\
1.323 \\
1.108 \\
0.973 \\
1.10 \\
1.141 \\
1.10\end{array}$ & $\begin{array}{l}1.661 \\
1.339 \\
1.062 \\
1.006 \\
1.125 \\
1.147 \\
1.114\end{array}$ & $\begin{array}{l}13 \\
26 \\
42 \\
37 \\
49 \\
29 \\
16\end{array}$ \\
\hline $4 f^{13}\left({ }^{2} \mathrm{~F}_{3 !}^{\circ}\right) 5 d 6 p\left({ }^{3} \mathrm{~F}_{4}\right)$ & & $\begin{array}{l}71 / 2 \\
6^{1 / 2} \\
5^{1 / 2} \\
4^{1 / 2} \\
3^{1 / 2} \\
2^{1 / 2} \\
1^{1 / 2} \\
0^{1 / 2}\end{array}$ & $\begin{array}{l}64891.30 \\
68720.44 \\
68148.88 \\
68549.23 \\
68450.11 \\
66395.72 \\
65888.46 \\
66462.86\end{array}$ & & $\begin{array}{l}1.19 \\
1.157 \\
1.20 \\
1.157 \\
1.08 \\
1.235 \\
1.206 \\
1.11\end{array}$ & $\begin{array}{l}1.200 \\
1.155 \\
1.207 \\
1.147 \\
1.100 \\
1.249 \\
1.212 \\
1.084\end{array}$ & $\begin{array}{r}8 \\
23 \\
29 \\
49 \\
39 \\
37 \\
30 \\
16\end{array}$ \\
\hline $4 f^{1: 3}\left({ }^{2} \mathrm{~F}_{3 !}^{\circ}\right) 5 d 6 p\left({ }^{\circ} \mathrm{D}_{1}\right)$ & & $\begin{array}{l}2^{1 / 2} \\
31 / 2 \\
4^{1 / 2}\end{array}$ & $\begin{array}{l}61374.49 \\
64598.28 \\
63726.83\end{array}$ & & $\begin{array}{l}1.299 \\
1.223 \\
1.005\end{array}$ & $\begin{array}{l}1.298 \\
1.217 \\
1.009\end{array}$ & $\begin{array}{l}34 \\
48 \\
47\end{array}$ \\
\hline $4 f^{13}\left({ }^{2} \mathrm{~F}_{3 !}^{\circ}\right) 5 d 6 p\left({ }^{3} \mathrm{D}_{2}\right)$ & & $\begin{array}{l}51 / 2 \\
41 / 2 \\
31 / 2 \\
2^{1 / 2} \\
1^{1 / 2}\end{array}$ & $\begin{array}{l}63944.18 \\
65950.95 \\
66558.00 \\
66351.21 \\
64461.08\end{array}$ & & $\begin{array}{l}1.115 \\
1.09 \\
1.100 \\
1.125 \\
1.176\end{array}$ & $\begin{array}{l}1.102 \\
1.074 \\
1.105 \\
1.106 \\
1.164\end{array}$ & $\begin{array}{l}33 \\
46 \\
47 \\
41 \\
25\end{array}$ \\
\hline $4 f^{13}\left({ }^{\prime} \mathrm{F}_{3 !}^{\circ}\right) 5 d 6 p\left({ }^{\circ} \mathrm{D}_{33}\right)$ & & $\begin{array}{l}6^{1 / 2} \\
5^{1 / 2} \\
4^{1 / 2} \\
3^{1 / 2} \\
2^{1 / 2} \\
1^{1 / 2} \\
0^{1 / 2}\end{array}$ & $\begin{array}{l}65875.77 \\
66571.58 \\
67416.03 \\
68756.04 \\
68135.00 \\
67204.97 \\
67955.23\end{array}$ & & $\begin{array}{l}1.205 \\
1.189 \\
1.26 \\
1.32 \\
1.087 \\
1.04 \\
0.895\end{array}$ & $\begin{array}{l}1.198 \\
1.189 \\
1.262 \\
1.343 \\
1.087 \\
1.064 \\
0.880\end{array}$ & $\begin{array}{l}26 \\
44 \\
50 \\
48 \\
39 \\
29 \\
18\end{array}$ \\
\hline $4 f^{13}\left({ }^{2} \mathrm{~F}_{3 \frac{1}{2}}^{\circ}\right) 5 d 6 p\left({ }^{3} \mathrm{P}_{0}\right)$ & & $3 \frac{1 / 2}{2}$ & 69395.20 & & 0.94 & 0.944 & 43 \\
\hline $4 f^{13}\left({ }^{2} \mathrm{~F}_{3 !}^{\circ}\right) 5 d 6 p\left({ }^{3} \mathrm{P}_{1}\right)$ & & $\begin{array}{l}21 / 2 \\
31 / 2 \\
41 / 2\end{array}$ & $\begin{array}{l}69607.54 \\
61822.58 \\
69957.49\end{array}$ & & $\begin{array}{l}1.210 \\
1.327 \\
1.112\end{array}$ & $\begin{array}{l}1.202 \\
1.338 \\
1.115\end{array}$ & $\begin{array}{l}45 \\
39 \\
53\end{array}$ \\
\hline $4 f^{13}\left({ }^{2} \mathrm{~F}_{3 !}^{\circ}\right) 5 d 6 p\left({ }^{3} \mathrm{P}_{2}\right)$ & & $\begin{array}{l}51 / 2 \\
41 / 2 \\
31 / 2 \\
21 / 2 \\
11 / 2\end{array}$ & $\begin{array}{l}70793.96 \\
70858.18 \\
71222.36 \\
70954.27 \\
71113.84\end{array}$ & & $\begin{array}{l}1.20 \\
1.28 \\
1.15 \\
1.014 \\
0.967\end{array}$ & $\begin{array}{l}1.214 \\
1.284 \\
1.149 \\
1.017 \\
0.955\end{array}$ & $\begin{array}{l}39 \\
46 \\
43 \\
43 \\
33\end{array}$ \\
\hline
\end{tabular}


TABLE 1. Even levels of $\mathrm{Yb} \mathrm{II}-$ Continued

\begin{tabular}{|c|c|c|c|c|c|c|c|}
\hline Configuration & Desig. & $J$ & Level & Interval & Obs. $g$ & Calc. $g$ & No. Comb. \\
\hline $4 f^{14}\left({ }^{1} \mathrm{~S}\right) 6 d$ & $6 d^{2} \mathrm{D}$ & $\begin{array}{l}1^{1 / 2} \\
21 / 2\end{array}$ & $\begin{array}{l}62174.10 \\
62559.02\end{array}$ & 384.92 & $\begin{array}{l}0.80 \\
1.22\end{array}$ & $\begin{array}{l}0.800 \\
1.200\end{array}$ & $\begin{array}{l}17 \\
19\end{array}$ \\
\hline $4 f^{13}\left({ }^{2} \mathrm{~F}_{3 !}^{\circ}\right) 5 d 6 p\left({ }^{1} \mathrm{D}_{2}\right)$ & & $\begin{array}{l}51 / 2 \\
41 / 2 \\
31 / 2 \\
21 / 2 \\
11 / 2\end{array}$ & $\begin{array}{l}62861.27 \\
65199.54 \\
65577.11 \\
63234.11 \\
63647.73\end{array}$ & & $\begin{array}{l}1.140 \\
1.08 \\
1.055 \\
1.208 \\
1.149\end{array}$ & $\begin{array}{l}1.139 \\
1.078 \\
1.050 \\
1.282 \\
1.133\end{array}$ & $\begin{array}{l}30 \\
42 \\
44 \\
37 \\
31\end{array}$ \\
\hline $4 f^{13}\left({ }^{2} \mathrm{~F}_{3 !}^{\circ}\right) 5 d 6 p\left({ }^{1} \mathrm{~F}_{3}\right)$ & & $\begin{array}{l}01 / 2 \\
11 / 2 \\
21 / 2 \\
31 / 2 \\
41 / 2 \\
51 / 2 \\
61 / 2\end{array}$ & $\begin{array}{l}65566.72 \\
69443.52 \\
71468.70 \\
72779.99 \\
73283.35 \\
73291.95 \\
70136.24\end{array}$ & & $\begin{array}{l}1.43 \\
1.15 \\
1.02 \\
1.10 \\
1.04 \\
1.09 \\
1.12\end{array}$ & $\begin{array}{l}1.460 \\
1.175 \\
0.996 \\
1.105 \\
1.011 \\
1.084 \\
1.150\end{array}$ & $\begin{array}{l}20 \\
35 \\
34 \\
41 \\
43 \\
38 \\
26\end{array}$ \\
\hline $4 f^{13}\left({ }^{2} \mathrm{~F}_{3 !}^{\circ}\right) 5 d 6 p\left({ }^{1} \mathrm{P}_{1}\right)$ & & $\begin{array}{l}21 / 2 \\
31 / 2 \\
41 / 2\end{array}$ & $\begin{array}{l}77606.58 \\
79628.71 \\
78070.19\end{array}$ & & $\begin{array}{l}1.14 \\
1.11 \\
1.10\end{array}$ & $\begin{array}{l}1.169 \\
1.126 \\
1.114\end{array}$ & $\begin{array}{l}30 \\
29 \\
33\end{array}$ \\
\hline $4 f^{13}\left({ }^{2} \mathrm{~F}_{2 !}^{\circ}\right) 5 d 6 p\left({ }^{3} \mathrm{~F}_{2}\right)$ & & $\begin{array}{l}4^{1 / 2} \\
31 / 2 \\
2^{1 / 2} \\
11 / 2 \\
01 / 2\end{array}$ & $\begin{array}{l}68943.65 \\
72202.03 \\
71270.01 \\
71763.67 \\
75905.77\end{array}$ & & $\begin{array}{l}0.89 \\
0.86 \\
0.91 \\
1.11 \\
1.28\end{array}$ & $\begin{array}{l}0.881 \\
0.864 \\
0.912 \\
1.101 \\
1.308\end{array}$ & $\begin{array}{l}23 \\
29 \\
39 \\
31 \\
15\end{array}$ \\
\hline $4 f^{13}\left({ }^{2} \mathrm{~F}_{2 \frac{1}{2}}^{\circ}\right) 5 d 6 p\left({ }^{3} \mathrm{~F}_{3}\right)$ & & $\begin{array}{l}51 / 2 \\
41 / 2 \\
31 / 2 \\
21 / 2 \\
11 / 2 \\
01 / 2\end{array}$ & $\begin{array}{l}72615.74 \\
76516.11 \\
75640.06 \\
74270.48 \\
73657.29 \\
73636.83\end{array}$ & & $\begin{array}{l}0.99 \\
1.01 \\
0.81 \\
0.92 \\
1.04 \\
0.70\end{array}$ & $\begin{array}{l}0.996 \\
0.999 \\
0.830 \\
0.910 \\
1.071 \\
0.692\end{array}$ & $\begin{array}{l}24 \\
24 \\
29 \\
32 \\
22 \\
17\end{array}$ \\
\hline $4 f^{13}\left({ }^{2} \mathrm{~F}_{2 !}^{\circ}\right) 5 d 6 p\left({ }^{3} \mathrm{~F}_{4}\right)$ & & $\begin{array}{l}6^{1 / 2} \\
51 / 2 \\
4^{1 / 2} \\
3^{1 / 2} \\
2^{1 / 2} \\
1^{1 / 2}\end{array}$ & $\begin{array}{l}76233.60 \\
79126.83 \\
77882.04 \\
76170.25 \\
76517.21\end{array}$ & & $\begin{array}{l}1.07 \\
1.15 ? \\
1.13 \\
1.12 \\
0.85\end{array}$ & $\begin{array}{l}1.108 \\
\\
1.140 \\
1.142 \\
1.093 \\
0.792\end{array}$ & $\begin{array}{l}10 \\
\\
17 \\
28 \\
31 \\
14\end{array}$ \\
\hline $4 f^{13}\left({ }^{2} \mathrm{~F}_{2 !}^{\circ}\right) 5 d 6 p\left({ }^{1} \mathrm{D}_{2}\right)$ & & $\begin{array}{l}01 / 2 \\
11 / 2 \\
21 / 2 \\
31 / 2 \\
41 / 2\end{array}$ & $\begin{array}{l}70029.04 \\
73116.71 \\
75550.94 \\
74973.41 \\
73750.90\end{array}$ & & $\begin{array}{l}0.68 \\
0.863 \\
0.91 \\
1.07 \\
1.07\end{array}$ & $\begin{array}{l}0.670 \\
0.797 \\
0.945 \\
1.040 \\
1.007\end{array}$ & $\begin{array}{l}16 \\
21 \\
38 \\
26 \\
37\end{array}$ \\
\hline $4 f^{14} 8 s$ & $8 s^{2} \mathrm{~S}$ & $0^{1 / 2}$ & 73039.61 & & & 2.000 & 7 \\
\hline $4 f^{13}\left({ }^{2} \mathrm{~F}_{2 !}^{\circ}\right) 5 d 6 p\left({ }^{3} \mathrm{D}_{1}\right)$ & & $\begin{array}{l}31 / 2 \\
21 / 2 \\
11 / 2\end{array}$ & $\begin{array}{l}73966.81 \\
76676.31 \\
77284.02\end{array}$ & & $\begin{array}{l}0.763 \\
1.10 \\
0.90\end{array}$ & $\begin{array}{l}0.765 \\
1.048 \\
0.964\end{array}$ & $\begin{array}{l}23 \\
39 \\
23\end{array}$ \\
\hline
\end{tabular}


TABIE 1. Even levels of $\mathrm{Yb} \mathrm{II}-$ Continued

\begin{tabular}{|c|c|c|c|c|c|c|c|}
\hline Configuration & Desig. & $J$ & Level & Interval & Obs. $g$ & Calc. $g$ & No. Comb. \\
\hline $4 f^{13}\left({ }^{2} \mathrm{~F}_{2 !}^{\circ}\right) 5 d 6 p\left({ }^{3} \mathrm{D}_{2}\right)$ & & $\begin{array}{l}41 / 2 \\
31 / 2 \\
21 / 2 \\
11 / 2 \\
01 / 2\end{array}$ & $\begin{array}{l}74568.58 \\
76323.49 \\
79583.11 \\
74991.36 \\
74989.49\end{array}$ & & $\begin{array}{l}1.04 \\
1.00 \\
0.68 \\
1.05 \\
0.52\end{array}$ & $\begin{array}{l}1.032 \\
0.960 \\
0.671 \\
1.060 \\
0.511\end{array}$ & $\begin{array}{l}22 \\
37 \\
15 \\
30 \\
15\end{array}$ \\
\hline $4 f^{13}\left({ }^{2} \mathrm{~F}_{2 !}^{\circ}\right) 5 d 6 p\left({ }^{3} \mathrm{D}_{3}\right)$ & & $\begin{array}{l}5^{1 / 2} \\
4^{1 / 2} \\
3^{1 / 2} \\
2^{1 / 2} \\
1^{1 / 2} \\
01 / 2\end{array}$ & $\begin{array}{l}76649.80 \\
77747.40 \\
77449.32 \\
78814.86 \\
79605.95 \\
77887.30\end{array}$ & & $\begin{array}{l}1.08 \\
1.07 \\
1.16 \\
1.22 \\
1.16 \\
2.20\end{array}$ & $\begin{array}{l}1.083 \\
1.087 \\
1.161 \\
1.210 \\
1.129 \\
2.170\end{array}$ & $\begin{array}{l}14 \\
25 \\
29 \\
22 \\
24 \\
12\end{array}$ \\
\hline $4 f^{14}\left({ }^{1} \mathrm{~S}\right) 7 d$ & $7 d^{2} \mathrm{D}$ & $\begin{array}{l}11 / 2 \\
21 / 2\end{array}$ & $\begin{array}{l}76799.07 \\
76839.00\end{array}$ & 39.93 & $\begin{array}{l}0.84 \\
1.11\end{array}$ & $\begin{array}{l}0.800 \\
1.200\end{array}$ & $\begin{array}{l}15 \\
35\end{array}$ \\
\hline $4 f^{13}\left({ }^{2} \mathrm{~F}_{2 !}^{\circ}\right) 5 d 6 p\left({ }^{3} \mathrm{P}_{0}\right)$ & & $21 / 2$ & 81657.13 & & & 1.051 & 26 \\
\hline $4 f^{13}\left({ }^{2} \mathrm{~F}_{2 !}^{\circ}\right) 5 d 6 p\left({ }^{3} \mathrm{P}_{1}\right)$ & & $\begin{array}{l}31 / 2 \\
21 / 2 \\
11 / 2\end{array}$ & $\begin{array}{l}80457.71 \\
75058.11 \\
81512.62\end{array}$ & & $\begin{array}{l}0.99 \\
0.76 \\
0.48\end{array}$ & $\begin{array}{l}0.984 \\
0.732 \\
0.502\end{array}$ & $\begin{array}{l}23 \\
29 \\
12\end{array}$ \\
\hline $4 f^{13}\left({ }^{2} \mathrm{~F}_{2 !}^{\circ}\right) 5 d 6 p\left({ }^{3} \mathrm{P}_{2}\right)$ & & $\begin{array}{l}01 / 2 \\
11 / 2 \\
21 / 2 \\
31 / 2 \\
41 / 2\end{array}$ & $\begin{array}{l}78464.76 \\
81312.65 \\
81692.92 \\
81205.27\end{array}$ & & $\begin{array}{l}1.18 \\
1.11 ? \\
1.16 \\
1.09\end{array}$ & $\begin{array}{l}1.193 \\
1.049 \\
1.164 \\
1.115\end{array}$ & $\begin{array}{l}22 \\
29 \\
18 \\
17\end{array}$ \\
\hline $4 f^{13}\left({ }^{2} \mathrm{~F}_{2 !}^{\circ}\right) 5 d 6 p\left({ }^{1} \mathrm{~F}_{3}\right)$ & & $\begin{array}{l}51 / 2 \\
41 / 2 \\
31 / 2 \\
21 / 2 \\
11 / 2 \\
01 / 2\end{array}$ & $\begin{array}{l}80679.18 \\
\\
83363.88 \\
82891.80 \\
82398.98 \\
82091.32\end{array}$ & & $\begin{array}{l}1.00 ? \\
0.97 \\
1.03 ? \\
1.042\end{array}$ & $\begin{array}{l}1.019 \\
\\
0.956 \\
1.066 \\
1.061 \\
1.223\end{array}$ & $\begin{array}{r}13 \\
\\
21 \\
27 \\
18 \\
7\end{array}$ \\
\hline $4 f^{13}\left({ }^{2} \mathrm{~F}_{2 !}^{\circ}\right) 5 d 6 p\left({ }^{1} \mathrm{P}_{1}\right)$ & & $\begin{array}{l}11 / 2 \\
2^{1 / 2} \\
31 / 2\end{array}$ & 89713.17 & & & 0.869 & 14 \\
\hline
\end{tabular}

TABLE 2. Odd levels of $\mathrm{Yb} \mathrm{II}$

\begin{tabular}{|c|c|c|c|c|c|c|c|}
\hline Configuration & Desig. & $J$ & Level & Interval & Obs. $g$ & Calc. $g$ & No. Comb. \\
\hline $4 f^{13} 6 s^{2}$ & $6 s^{2}{ }^{2} \mathrm{~F}^{\circ}$ & $\begin{array}{l}31 / 2 \\
21 / 2\end{array}$ & $\begin{array}{l}21418.75 \\
31568.08\end{array}$ & -10149.33 & $\begin{array}{l}1.145 \\
0.862\end{array}$ & $\begin{array}{l}1.143 \\
0.866\end{array}$ & $\begin{array}{l}30 \\
50\end{array}$ \\
\hline $4 f^{14}\left({ }^{1} \mathrm{~S}\right) 6 p$ & $6 p^{2} \mathrm{P}^{\circ}$ & $\begin{array}{l}01 / 2 \\
11 / 2\end{array}$ & $\begin{array}{l}27061.82 \\
30392.23\end{array}$ & 3330.41 & $\begin{array}{l}0.667 \\
1.333\end{array}$ & $\begin{array}{l}0.667 \\
1.333\end{array}$ & $\begin{array}{l}13 \\
28\end{array}$ \\
\hline $4 f^{13}\left({ }^{2} \mathrm{~F}_{3 !}^{\circ}\right) 5 d 6 s\left({ }^{3} \mathrm{D}^{\circ}\right)$ & $3[1 / 2]^{\circ}$ & $\begin{array}{l}2^{1 / 2} \\
1^{1 / 2} \\
0^{1 / 2}\end{array}$ & $\begin{array}{l}26759.02 \\
28757.98 \\
33653.86\end{array}$ & & $\begin{array}{l}1.570 \\
1.440 \\
1.320\end{array}$ & $\begin{array}{l}1.576 \\
1.447 \\
1.318\end{array}$ & $\begin{array}{l}27 \\
30 \\
22\end{array}$ \\
\hline
\end{tabular}


TABLE 2. Odd levels of $\mathrm{Yb}$ II - Continued

\begin{tabular}{|c|c|c|c|c|c|c|c|}
\hline Configuration & Desig. & $J$ & Level & Interval & Obs. $g$ & Calc. $g$ & No. Comb. \\
\hline \multirow[t]{4}{*}{$\begin{array}{l}4 f^{13}\left({ }^{2} \mathrm{~F}_{3 \frac{1}{2}}^{\circ}\right) 5 d 6 s\left({ }^{3} \mathrm{D}^{\circ}\right) \\
\text { Continued }\end{array}$} & $3[51 / 2]^{0}$ & $\begin{array}{l}41 / 2 \\
51 / 2 \\
61 / 2\end{array}$ & $\begin{array}{l}30224.33 \\
30562.79 \\
31631.59\end{array}$ & & $\begin{array}{l}0.935 \\
1.112 \\
1.230\end{array}$ & $\begin{array}{l}0.936 \\
1.124 \\
1.231\end{array}$ & $\begin{array}{l}31 \\
25 \\
12\end{array}$ \\
\hline & $3[21 / 2]^{\circ}$ & $\begin{array}{l}31 / 2 \\
21 / 2 \\
11 / 2\end{array}$ & $\begin{array}{l}31979.90 \\
32371.10 \\
32981.59\end{array}$ & & $\begin{array}{l}1.331 \\
1.170 \\
0.866\end{array}$ & $\begin{array}{l}1.336 \\
1.157 \\
0.908\end{array}$ & $\begin{array}{l}43 \\
44 \\
40\end{array}$ \\
\hline & $3[31 / 2]^{\circ}$ & $\begin{array}{l}4^{1 / 2} \\
3^{1 / 2} \\
2^{1 / 2}\end{array}$ & $\begin{array}{l}33052.29 \\
35059.00 \\
34389.76\end{array}$ & & $\begin{array}{l}1.264 \\
1.124 \\
1.007\end{array}$ & $\begin{array}{l}1.267 \\
1.112 \\
1.017\end{array}$ & $\begin{array}{l}36 \\
51 \\
47\end{array}$ \\
\hline & $3\left[4^{1 / 2}\right]^{\circ}$ & $\begin{array}{l}31 / 2 \\
41 / 2 \\
51 / 2\end{array}$ & $\begin{array}{l}33494.68 \\
35019.13 \\
35831.68\end{array}$ & & $\begin{array}{l}0.991 \\
1.158 \\
1.214\end{array}$ & $\begin{array}{l}1.003 \\
1.159 \\
1.219\end{array}$ & $\begin{array}{l}48 \\
38 \\
29\end{array}$ \\
\hline \multirow[t]{5}{*}{$4 f^{13}\left({ }^{2} \mathrm{~F}_{3 \frac{1}{2}}^{\circ}\right) 5 d 6 s\left({ }^{1} \mathrm{D}^{\circ}\right)$} & ${ }^{1}[1 / 2]^{\circ}$ & $11 / 2$ & 34575.37 & & 1.434 & 1.395 & 41 \\
\hline & $1\left[5^{1 / 2}\right]^{\circ}$ & $5^{1 / 2}$ & 34784.95 & & 1.119 & 1.120 & 29 \\
\hline & ${ }^{1}\left[2^{1 / 2}\right]^{\circ}$ & $21 / 2$ & 37077.59 & & 1.113 & 1.113 & 52 \\
\hline & ${ }^{1}\left[3^{1 / 2} \mathbf{2}\right]^{\circ}$ & $31 / 2$ & 37516.59 & & 1.119 & 1.126 & 59 \\
\hline & ${ }^{1}[4,1 / 2]^{\circ}$ & $4^{1 / 2}$ & 38342.02 & & 1.093 & 1.090 & 42 \\
\hline \multirow[t]{5}{*}{$4 f^{13}\left({ }^{2} \mathrm{~F}_{2 \frac{1}{2}}^{\circ}\right) 5 d 6 s\left({ }^{3} \mathrm{D}^{\circ}\right)$} & ${ }^{3}\left[0^{1 / 2}\right]^{\circ}$ & $\begin{array}{l}0^{1 / 2} / 2 \\
11 / 2\end{array}$ & $\begin{array}{l}39378.89 \\
44940.61\end{array}$ & & $\begin{array}{l}1.850 \\
1.244\end{array}$ & $\begin{array}{l}1.859 \\
1.248\end{array}$ & $\begin{array}{l}21 \\
53\end{array}$ \\
\hline & $3\left[4^{1 / 2}\right]^{\circ}$ & $\begin{array}{l}31 / 2 \\
4^{1 / 2} \\
51 / 2\end{array}$ & $\begin{array}{l}40035.82 \\
40917.91 \\
42915.13\end{array}$ & & $\begin{array}{l}0.720 \\
0.967 \\
1.115\end{array}$ & $\begin{array}{l}0.714 \\
0.978 \\
1.125\end{array}$ & $\begin{array}{l}53 \\
40 \\
20\end{array}$ \\
\hline & $3[1 / 2]^{\circ}$ & $\begin{array}{l}2^{1 / 2} \\
1^{1 / 2} \\
0^{1 / 2}\end{array}$ & $\begin{array}{l}41678.73 \\
41688.45 \\
43007.79\end{array}$ & & $\begin{array}{l}1.193 \\
0.784 \\
0.15\end{array}$ & $\begin{array}{l}1.182 \\
0.784 \\
0.148\end{array}$ & $\begin{array}{l}43 \\
39 \\
21\end{array}$ \\
\hline & $3[21 / 2]^{\circ}$ & $\begin{array}{l}1^{1 / 2} \\
2^{1 / 2} \\
31 / 2\end{array}$ & $\begin{array}{l}43075.09 \\
43956.41 \\
44438.03\end{array}$ & & $\begin{array}{l}0.96 \\
0.99 \\
1.10\end{array}$ & $\begin{array}{l}0.928 \\
0.967 \\
1.091\end{array}$ & $\begin{array}{l}49 \\
47 \\
55\end{array}$ \\
\hline & $3\left[3^{1 / 2}\right]^{\circ}$ & $\begin{array}{l}2^{1 / 2} \\
3^{1 / 2} \\
4^{1 / 2}\end{array}$ & $\begin{array}{l}44497.51 \\
45429.82 \\
46169.78\end{array}$ & & $\begin{array}{l}0.734 \\
1.150 \\
1.10\end{array}$ & $\begin{array}{l}0.770 \\
1.128 \\
1.104\end{array}$ & $\begin{array}{l}45 \\
51 \\
45\end{array}$ \\
\hline \multirow[t]{5}{*}{$4 f^{13}\left({ }^{2} \mathbf{F}_{2 \frac{1}{2}}^{\circ}\right) 5 d 6 s\left({ }^{1} \mathrm{D}^{\circ}\right)$} & ${ }^{1}\left[4^{1} / 2\right]^{\circ}$ & $4 \frac{1}{2}$ & 45335.03 & & 1.01 & 0.981 & 39 \\
\hline & ${ }^{1}\left[1^{1 / 2}\right]^{\circ}$ & $1^{1 / 2}$ & 47005.46 & & 0.990 & 0.971 & 45 \\
\hline & ${ }^{1}\left[0^{1 / 2}\right]^{\circ}$ & $01 / 2$ & 47228.96 & & 0.74 & 0.806 & 26 \\
\hline & ${ }^{1}\left[2^{1 / 2}\right]^{\circ}$ & $2 \frac{1 / 2}{2}$ & 47329.44 & & 0.990 & 0.974 & 56 \\
\hline & $1\left[3^{1} / 2\right]^{\circ}$ & $3 \frac{1}{2}$ & 48556.57 & & 0.941 & 0.921 & 50 \\
\hline
\end{tabular}


TABLE 2. Odd levels of $\mathrm{Yb} \mathrm{II}$ - Continued

\begin{tabular}{|c|c|c|c|c|c|c|c|}
\hline Configuration & Desig. & $J$ & Level & Interval & Obs. $g$ & Calc. $g$ & No. Comb. \\
\hline \multirow[t]{7}{*}{$4 f^{13}\left({ }^{2} \mathrm{~F}_{3 \frac{1}{2}}^{\circ}\right) 5 d^{2}\left({ }^{3} \mathrm{~F}^{\circ}\right)$} & $3\left[2^{1 / 2}\right]^{\circ}$ & $\begin{array}{l}31 / 2 \\
21 / 2 \\
11 / 2\end{array}$ & $\begin{array}{l}47758.54 \\
45012.79 \\
50832.65\end{array}$ & & $\begin{array}{l}1.08 \\
1.281 \\
0.956\end{array}$ & $\begin{array}{l}1.123 \\
1.298 \\
0.977\end{array}$ & $\begin{array}{l}43 \\
38 \\
22\end{array}$ \\
\hline & ${ }^{3}\left[3^{1 / 2}\right]^{\circ}$ & $\begin{array}{l}41 / 2 \\
31 / 2 \\
21 / 2\end{array}$ & $\begin{array}{l}46354.55 \\
45273.02 \\
51248.86\end{array}$ & & $\begin{array}{l}1.29 \\
1.18 \\
1.034\end{array}$ & $\begin{array}{l}1.284 \\
1.209 \\
1.052\end{array}$ & $\begin{array}{l}32 \\
56 \\
44\end{array}$ \\
\hline & $3\left[1^{1 / 2}\right]^{\circ}$ & $\begin{array}{l}01 / 2 \\
11 / 2 \\
21 / 2\end{array}$ & $\begin{array}{l}46902.71 \\
45737.29 \\
48272.77\end{array}$ & & $\begin{array}{l}1.30 \\
1.333 \\
1.14\end{array}$ & $\begin{array}{l}1.201 \\
1.384 \\
1.147\end{array}$ & $\begin{array}{l}20 \\
50 \\
55\end{array}$ \\
\hline & ${ }^{3}\left[6^{1 / 2}\right]^{\circ}$ & $\begin{array}{l}5^{1 / 2} \\
61 / 2 \\
71 / 2\end{array}$ & $\begin{array}{l}46547.72 \\
47680.40 \\
48923.30\end{array}$ & & $\begin{array}{l}0.97 \\
1.12 \\
1.20\end{array}$ & $\begin{array}{l}0.971 \\
1.118 \\
1.195\end{array}$ & $\begin{array}{r}21 \\
13 \\
6\end{array}$ \\
\hline & $3\left[5^{1 / 2}\right]^{\circ}$ & $\begin{array}{l}41 / 2 \\
5^{1 / 2} \\
6^{1 / 2}\end{array}$ & $\begin{array}{l}47663.52 \\
48503.82 \\
49727.19\end{array}$ & & $\begin{array}{l}0.98 \\
1.10 \\
1.20\end{array}$ & $\begin{array}{l}1.011 \\
1.129 \\
1.200\end{array}$ & $\begin{array}{l}38 \\
25 \\
12\end{array}$ \\
\hline & $3[01 / 2]^{\circ}$ & $\begin{array}{l}1 / 2 \\
0^{1 / 2}\end{array}$ & $\begin{array}{l}48024.70 \\
49419.13\end{array}$ & & $\begin{array}{l}1.38 \\
1.41\end{array}$ & $\begin{array}{l}1.354 \\
1.432\end{array}$ & $\begin{array}{l}30 \\
16\end{array}$ \\
\hline & $3[41 / 2]^{\circ}$ & $\begin{array}{l}31 / 2 \\
41 / 2 \\
5^{1 / 2}\end{array}$ & $\begin{array}{l}49008.93 \\
49916.50 \\
50468.05\end{array}$ & & $\begin{array}{l}1.03 \\
1.090 \\
1.213\end{array}$ & $\begin{array}{l}1.046 \\
1.062 \\
1.198\end{array}$ & $\begin{array}{l}43 \\
27 \\
22\end{array}$ \\
\hline \multirow[t]{8}{*}{$4 f^{13}\left({ }^{2} \mathrm{~F}_{3 !}^{\circ}\right) 5 d^{2}\left({ }^{1} \mathrm{G}^{\circ}\right)$} & $1\left[0^{1 / 2}\right]^{\circ}$ & $0^{1 / 2}$ & 52067.87 & & 0.54 & 0.530 & 21 \\
\hline & ${ }^{1}\left[1^{1 / 2}\right]^{\circ}$ & $1 \frac{1 / 2}{2}$ & 52987.76 & & 1.131 & 1.116 & 28 \\
\hline & ${ }^{1}\left[7^{1} / 2\right]^{\circ}$ & $71 / 2$ & 53322.89 & & 1.04 & 1.072 & 5 \\
\hline & $1\left[2^{1 / 2}\right]^{\circ}$ & $2^{1 / 2}$ & 53716.38 & & 1.183 & 1.154 & 42 \\
\hline & $1\left[3 \frac{1}{2}\right]^{\circ}$ & $3 \frac{1 / 2}{2}$ & 57103.02 & & 1.10 & 1.078 & 25 \\
\hline & ${ }^{\mathrm{r}}\left[6^{1 / 2} /\right]^{\circ}$ & $6^{1 / 2}$ & 57561.62 & & 1.04 & 1.042 & 7 \\
\hline & ${ }^{1}\left[4^{1} / 2\right]^{\circ}$ & $41 / 2$ & 58484.54 & & 1.02 & 1.061 & 17 \\
\hline & ${ }^{1}\left[5 \frac{1}{2}\right]^{\circ}$ & $5 \frac{1}{2}$ & 59753.84 & & 1.11 & 1.082 & 11 \\
\hline \multirow[t]{3}{*}{$4 f^{13}\left({ }^{2} \mathrm{~F}_{3 !}^{\circ}\right) 5 d^{2}\left({ }^{3} \mathrm{P}^{\circ}\right)$} & $3[31 / 2]^{\circ}$ & $\begin{array}{l}41 / 2 \\
31 / 2 \\
2^{1 / 2}\end{array}$ & $\begin{array}{l}52880.75 \\
52921.62 \\
53120.58\end{array}$ & & $\begin{array}{l}1.26 \\
1.15 \\
0.944\end{array}$ & $\begin{array}{l}1.268 \\
1.173 \\
0.950\end{array}$ & $\begin{array}{l}26 \\
35 \\
34\end{array}$ \\
\hline & $3[41 / 2]^{\circ}$ & $\begin{array}{l}31 / 2 \\
41 / 2 \\
51 / 2\end{array}$ & $\begin{array}{l}55462.68 \\
56088.39 \\
56621.15\end{array}$ & & $\begin{array}{l}0.878 \\
1.11 \\
1.16\end{array}$ & $\begin{array}{l}0.874 \\
1.100 \\
1.215\end{array}$ & $\begin{array}{l}28 \\
20 \\
15\end{array}$ \\
\hline & $3\left[2^{1 / 2}\right]^{\circ}$ & $\begin{array}{l}31 / 2 \\
21 / 2 \\
11 / 2\end{array}$ & $\begin{array}{l}56500.64 \\
57798.51 \\
58162.75\end{array}$ & & $\begin{array}{l}1.352 \\
1.151 \\
0.94\end{array}$ & $\begin{array}{l}1.360 \\
1.152 \\
0.930\end{array}$ & $\begin{array}{l}20 \\
30 \\
17\end{array}$ \\
\hline
\end{tabular}


TABLE 2. Odd levels of $\mathrm{Yb} \mathrm{II} \ldots$ ontinued

\begin{tabular}{|c|c|c|c|c|c|c|c|}
\hline Configuration & Desig. & $J$ & Level & Interval & Obs. $g$ & Calc. $g$ & No. Comb. \\
\hline \multirow[t]{5}{*}{$4 f^{13}\left({ }^{2} \mathrm{~F}_{3 !}^{\circ}\right) 5 d^{2}\left({ }^{1} \mathrm{D}^{\circ}\right)$} & $1\left[3^{1 / 2}\right]^{\circ}$ & $3^{1 / 2}$ & 53644.89 & & 1.18 & 1.181 & 38 \\
\hline & ${ }^{1}\left[5^{1 / 2}\right]^{\circ}$ & $5 \frac{1}{2}$ & 54192.51 & & 1.11 & 1.110 & 19 \\
\hline & $1\left[4^{1 / 2}\right]^{\circ}$ & $4^{1 / 2}$ & 54640.82 & & 1.13 & 1.128 & 30 \\
\hline & ${ }^{1}[1 / 1 / 2]^{\circ}$ & $1 \frac{1 / 2}{2}$ & 55221.46 & & 1.11 & 1.069 & 27 \\
\hline & ${ }^{1}\left[2^{1} / 2\right]^{\circ}$ & $2^{1 / 2}$ & 56056.91 & & 1.12 & 1.131 & 22 \\
\hline $4 f^{13}\left({ }^{2} \mathrm{~F}_{3 !}^{\circ}\right) 5 d^{\circ}\left({ }^{1} \mathrm{~S}^{\circ}\right)$ & ${ }^{1}\left[3^{1 / 2}\right]^{\circ}$ & $3^{1 / 2}$ & & & & & \\
\hline \multirow[t]{6}{*}{$4 f^{13}\left({ }^{2} \mathrm{~F}_{2 !}^{\circ}\right) 5 d^{2}\left({ }^{3} \mathrm{~F}^{\circ}\right)$} & ${ }^{3}\left[5^{1 / 2}\right]^{0}$ & $\begin{array}{l}41 / 2 \\
51 / 2 \\
61 / 2\end{array}$ & $\begin{array}{l}56480.77 \\
58051.51 \\
59632.21 ?\end{array}$ & & $\begin{array}{l}0.76 \\
0.95\end{array}$ & $\begin{array}{l}0.767 \\
0.966 \\
1.087\end{array}$ & $\begin{array}{r}23 \\
10 \\
5\end{array}$ \\
\hline & ${ }^{3}\left[2^{1 / 2}\right]^{0}$ & $\begin{array}{l}11 / 2 \\
2^{1 / 2} \\
3^{1 / 2}\end{array}$ & $\begin{array}{l}56840.04 \\
62163.92 \\
62046.07\end{array}$ & & $\begin{array}{l}0.90 \\
1.14\end{array}$ & $\begin{array}{l}0.930 \\
1.082 \\
1.134\end{array}$ & $\begin{array}{l}20 \\
24 \\
17\end{array}$ \\
\hline & $3\left[0^{1 / 2}\right]^{0}$ & $\begin{array}{l}0^{1 / 2} \\
1^{1 / 2}\end{array}$ & $\begin{array}{l}56977.71 \\
59777.07\end{array}$ & & $\begin{array}{l}2.19 \\
1.63\end{array}$ & $\begin{array}{l}2.174 \\
1.623\end{array}$ & $\begin{array}{l}11 \\
12\end{array}$ \\
\hline & $3[4,1 / 2]^{\circ}$ & $\begin{array}{l}31 / 2 \\
41 / 2 \\
51 / 2\end{array}$ & $\begin{array}{l}57534.45 \\
59046.89 \\
61442.82\end{array}$ & & $\begin{array}{l}0.87 \\
1.08 \\
1.11\end{array}$ & $\begin{array}{l}0.837 \\
1.051 \\
1.134\end{array}$ & $\begin{array}{l}27 \\
14 \\
12\end{array}$ \\
\hline & ${ }^{3}\left[3^{1 / 2}\right]^{0}$ & $\begin{array}{l}21 / 2 \\
31 / 2 \\
41 / 2\end{array}$ & $\begin{array}{l}58661.13 \\
59624.66 \\
60649.86\end{array}$ & & $\begin{array}{l}0.86 \\
1.10\end{array}$ & $\begin{array}{l}0.821 \\
0.964 \\
1.123\end{array}$ & $\begin{array}{l}25 \\
19 \\
13\end{array}$ \\
\hline & $3\left[1 \frac{1}{2}\right]^{0}$ & $\begin{array}{l}01 / 2 \\
11 / 2 \\
21 / 2\end{array}$ & $\begin{array}{l}58672.53 \\
60910.14 \\
59439.08\end{array}$ & & $\begin{array}{l}0.538 \\
1.00 \\
0.994\end{array}$ & $\begin{array}{l}0.522 \\
1.095 \\
1.054\end{array}$ & $\begin{array}{l}13 \\
17 \\
22\end{array}$ \\
\hline \multirow[t]{3}{*}{$4 f^{13}\left({ }^{2} \mathrm{~F}_{2 !}^{\circ}\right) 5 d^{2}\left({ }^{3} \mathrm{P}^{\circ}\right)$} & $3[21 / \mathbf{2}]^{\circ}$ & $\begin{array}{l}1^{1 / 2} \\
2^{1 / 2} \\
3^{1 / 2}\end{array}$ & $\begin{array}{l}63028.26 \\
63417.03 \\
64169.39\end{array}$ & & $\begin{array}{l}0.51 \\
1.01\end{array}$ & $\begin{array}{l}0.531 \\
1.019 \\
1.078\end{array}$ & $\begin{array}{l}11 \\
16 \\
17\end{array}$ \\
\hline & ${ }^{3}\left[3^{1 / 2}\right]^{\circ}$ & $\begin{array}{l}21 / 2 ? \\
31 / 2 \\
41 / 2\end{array}$ & $\begin{array}{l}65594.06 \\
66796.03\end{array}$ & & $1.19 ?$ & $\begin{array}{l}0.809 \\
0.970\end{array}$ & $\begin{array}{l}8 \\
7\end{array}$ \\
\hline & ${ }^{3}[1 / 2]^{\circ}$ & $\begin{array}{l}21 / 2 \\
11 / 2 \\
01 / 2\end{array}$ & $\begin{array}{l}67933.51 \\
67789.60 ?\end{array}$ & & 1.17 & $\begin{array}{l}1.203 \\
1.051\end{array}$ & $\begin{array}{l}8 \\
5\end{array}$ \\
\hline \multirow[t]{3}{*}{$4 f^{13}\left({ }^{2} \mathrm{~F}_{2 \frac{1}{2}}^{\circ}\right) 5 d^{2}\left({ }^{1} \mathrm{G}^{\circ}\right)$} & ${ }^{1}\left[6^{1 / 2}\right]^{\circ}$ & $61 / 2$ & & & & & \\
\hline & ${ }^{1}\left[1^{1 / 2}\right]^{0}$ & $11 / 2$ & 64191.97 & & 1.193 & 1.105 & 8 \\
\hline & $1\left[2 \frac{1}{2}\right]^{\circ}$ & $21 / 2$ & 65739.47 & & 0.879 & 0.896 & 11 \\
\hline
\end{tabular}


TABLE 2. Odd levels of $\mathrm{Yb} \mathrm{II}$ - Continued

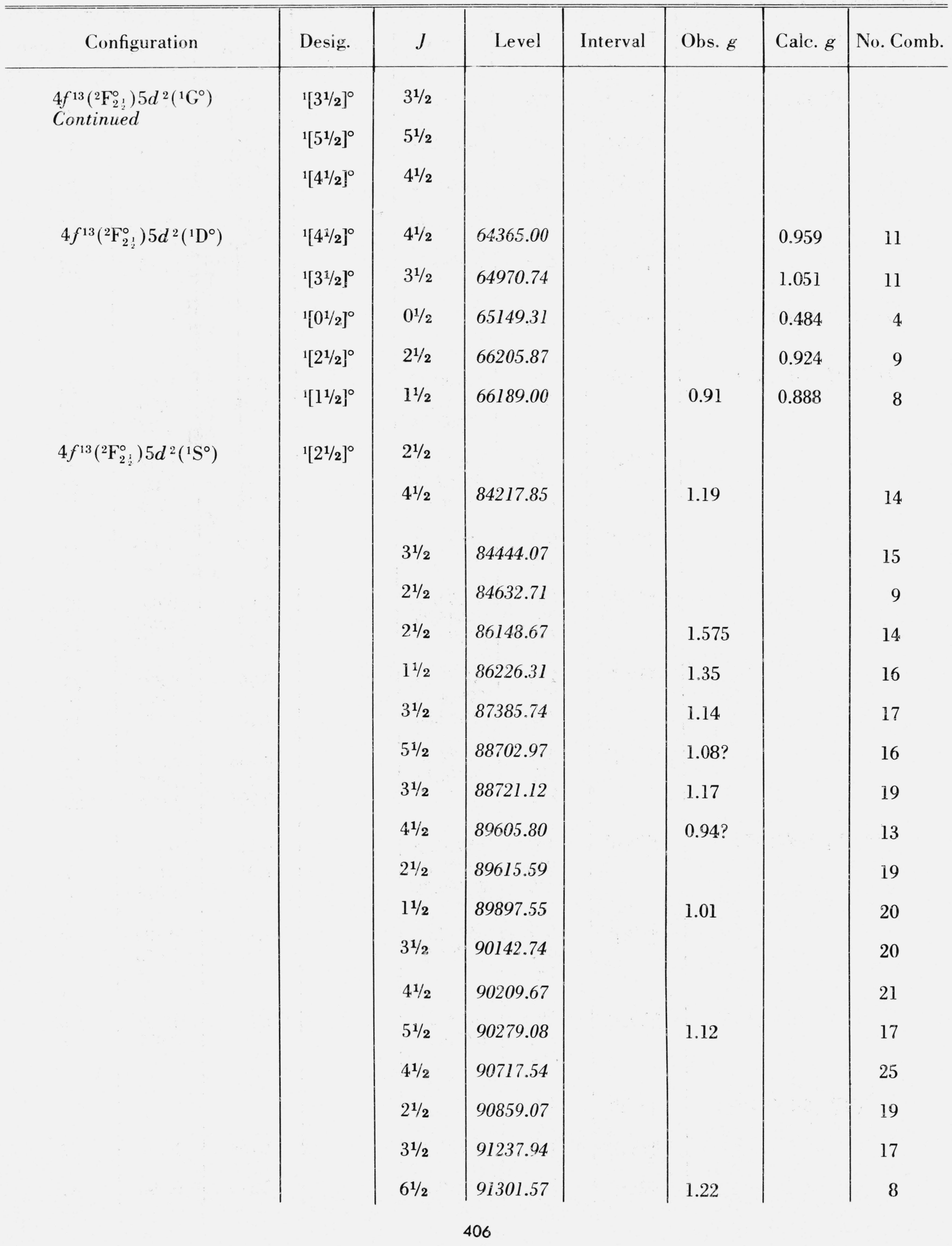


TABle 2. Odd levels of $\mathrm{Yb}$ in - Continued

\begin{tabular}{|c|c|c|c|c|c|c|c|}
\hline \multirow[t]{29}{*}{ Configuration } & Desig. & $J$ & Level & Interval & Obs. $g$ & Calc. $g$ & No. Comb. \\
\hline & & $31 / 2$ & 91536.40 & & $1.13 ?$ & & 16 \\
\hline & & $21 / 2$ & 91602.54 & & 1.17 & & 16 \\
\hline & & $41 / 2$ & 92015.76 & & & & 20 \\
\hline & & $11 / 2$ & 92278.27 & & $1.49 ?$ & & 10 \\
\hline & & $31 / 2$ & 92303.24 & & & & 17 \\
\hline & & $5 \frac{1}{2}$ & 92647.34 & & & & 14 \\
\hline & & $4^{1 / 2}$ & 92914.15 & & & & 15 \\
\hline & & $31 / 2$ & 92919.10 & & & & 17 \\
\hline & & $3 \frac{1 / 2}{2}$ & 92976.50 & & & & 13 \\
\hline & & $5^{1 / 2}$ & 93096.30 & & 1.23 & & 14 \\
\hline & & $3^{1 / 2}$ & 93195.03 & & & & 13 \\
\hline & & $51 / 2$ & 93242.39 & & & & 15 \\
\hline & & $6^{1 / 2}$ & 93246.19 & - & $1.21 ?$ & & 8 \\
\hline & & $3 \frac{1 / 2}{2}$ & 93463.01 & & & & 15 \\
\hline & & $4 \frac{1 / 2}{2}$ & 93503.89 & & & & 16 \\
\hline & & $2^{1 / 2}$ & 93546.00 & & & & 16 \\
\hline & & $2^{1 / 2}$ & 93864.57 & & & & 11 \\
\hline & & $4,1 / 2$ & 93867.22 & & & & 16 \\
\hline & & $1 \frac{1 / 2}{2}$ & 93998.52 & & & & 17 \\
\hline & & $3^{1 / 2}$ & 94008.96 & & & & 22 \\
\hline & & $31 / 2$ & 94303.58 & & 1.12 & & 23 \\
\hline & & $51 / 2$ & 94335.70 & & & & 18 \\
\hline & & $41 / 2$ & 94360.00 & & & & 12 \\
\hline & & $1^{1 / 2}$ & 94428.80 & & & & 6 \\
\hline & & $1 \frac{1 / 2}{2}$ & 94823.18 & & & & 11 \\
\hline & & $41 / 2$ & 95550.55 & & & & 14 \\
\hline & & $3 \frac{1 / 2}{2}$ & 95691.32 & & & $:$ & 19 \\
\hline & & $51 / 2$ & 95754.30 & & $0.89 ?$ & & 8 \\
\hline
\end{tabular}


TABLE 2. Odd levels of $\mathrm{Yb} \mathrm{II}-$ Continued

\begin{tabular}{|c|c|c|c|c|c|c|c|}
\hline Configuration & Desig. & $J$ & Level & Interval & Obs. $g$ & Calc. $g$ & No. Comb. \\
\hline & & $\begin{array}{l}5 \frac{1 / 2}{} \\
41 / 2 \\
51 / 2 \\
61 / 2 \\
41 / 2 \\
41 / 2 \\
61 / 2 \\
51 / 2 \\
61 / 2 \\
51 / 2 \\
41 / 2 \\
51 / 2\end{array}$ & $\begin{array}{l}96382.06 \\
96482.44 \\
96898.03 \\
97043.28 \\
97086.51 \\
98521.73 \\
98620.02 \\
98802.66 \\
98829.49 \\
99246.37 \\
99414.42 \\
100025.57\end{array}$ & & 1.23 & & $\begin{array}{r}10 \\
13 \\
12 \\
7 \\
14 \\
15 \\
8 \\
15 \\
6 \\
7 \\
12 \\
11\end{array}$ \\
\hline
\end{tabular}

\section{Ionization Potential}

In the 1937 paper [1] Meggers refers to the collaboration with $\mathrm{H}$. N. Russell on the interpretation of $\mathrm{Yb}$ spectra. As early as 1932 [14], Russell derived an ionization potential of $12.1 \mathrm{eV}$ for $\mathrm{Yb}$ II from the threemember series $n s^{2} \mathrm{~S}(n=6,7,8)$, reported in 1947 by Meggers [2]. Since then the third member of the series has been corrected. From a modified Ritz formula the revised limit 98150 has been determined, giving an ionization potential $12.17 \mathrm{eV}$ [15] from multiplication by the present conversion factor $\left(\mathrm{cm}^{-1}\right.$ to $\left.\mathrm{eV}\right)$ 0.000123981 .

A semi-empirical method of deriving ionization potentials by a smooth run of $\Delta n^{*}$ was suggested by Russell and Meggers in their analyses of La spectra [16]. Sugar and Reader [15] discuss the details of the method and the general run of ionization potentials for second spectra of the lanthanon group of rare earths.

\section{Classified Lines}

All observed lines of $\mathrm{Yb}$ II in the 1966 description of the spectrum [3] are listed in table 6. Column one contains the wavelength. With the exception of the first four entries, which are from Humphreys and Paul [6], practically all wavelengths are from [3], although a few additions and corrections to the published list noted by Meggers, have been included.
Columns two and three contain, respectively, estimated intensities of the lines as observed with the Meggers lamp and with the Thomson lamp. These sources are described in detail in [3]. "Literal symbols following intensity numbers have the following meanings:

$A=$ nearly all absorbed in the pulsed arc.

$R=$ wide reversal in the pulsed arc.

$r=$ narrow reversal in the pulsed arc.

$d=$ double but not resolved.

$e=$ enhanced near wall of Thomson tube.

$h=$ hazy as distinguished from sharp.

$H=$ very hazy.

$l=$ shaded or displaced to longer waves.

$s=$ shaded or displaced to shorter waves."

The wave number is in column four. The next column, "o-c," gives the difference between the observed wave number and that calculated from the energy levels listed in tables 1 and 2 . The levels giving rise to the transitions producing the lines are designated numerically in column six. They are the whole numbers of the levels tabulated in tables 1 and 2 . The respective $J$-values are entered as subscripts. Odd levels are printed in italics. The last five columns contain Zeeman data, as explained in detail in section 3 .

The total number of known Yb II lines is 5129. Of these, 3907 have been classified. There are 145 lines entered as blends, i.e., having more than one classifi- 
TABLE 3. Yb II Observed Levels

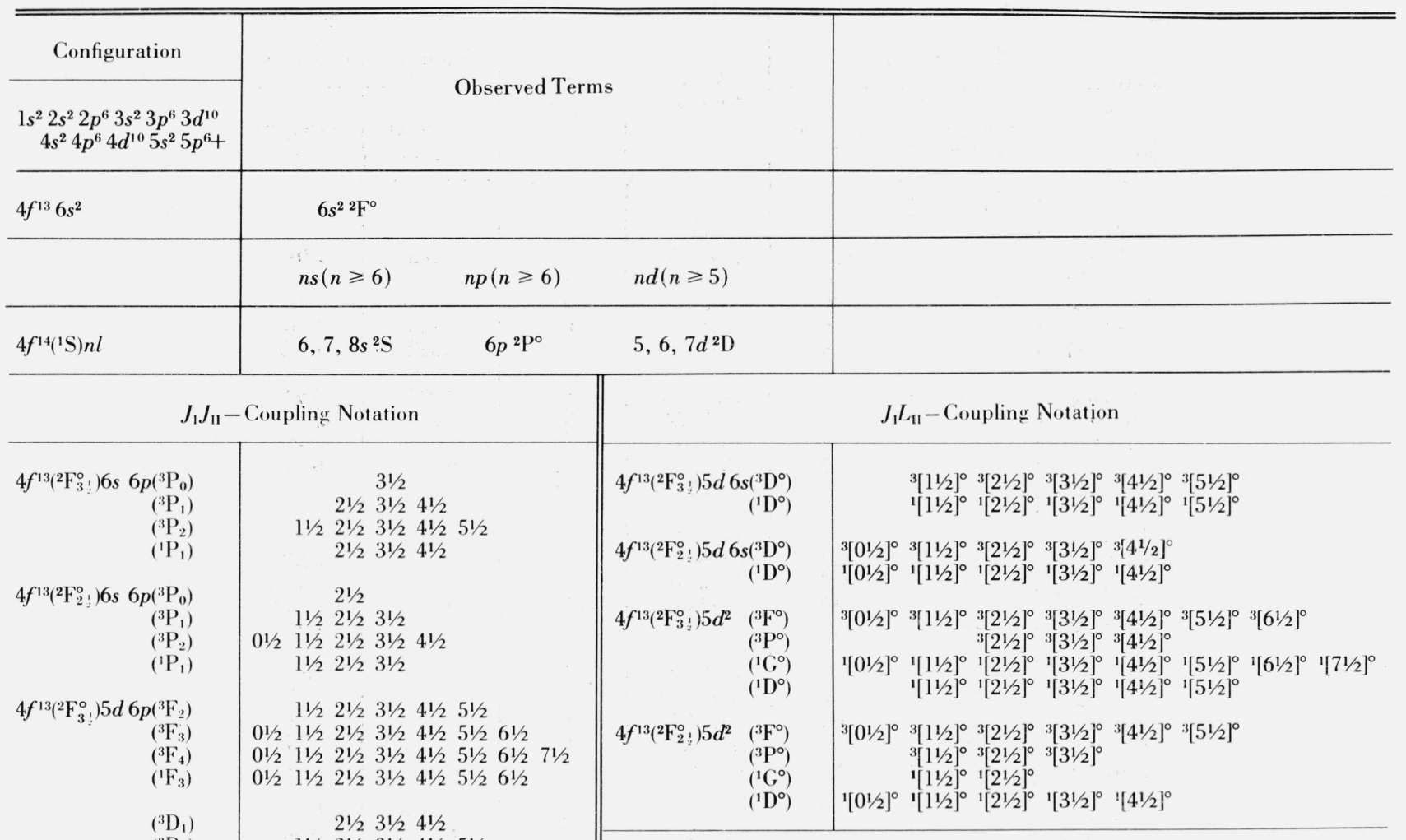

$4 f^{13}\left({ }^{2} \mathrm{~F}_{2}^{\circ}\right) 5 d 6 p\left({ }^{3} \mathrm{~F}_{2}\right) \quad 01 / 2 \quad 11 / 2 \quad 21 / 2 \quad 31 / 2 \quad 41 / 2$

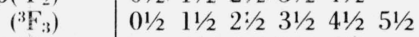

$\left(3 F_{4}\right) \quad 11 / 2 \quad 21 / 231 / 2 \quad 41 \frac{1}{2} \quad 61 / 2$

$\begin{array}{llllll}\left(\mathrm{F}_{3}\right) & 01 / 2 & 11 / 2 & 21 / 2 & 31 / 2 & 51 / 2\end{array}$

$\left({ }^{3} D_{1}\right) \quad 11 / 22^{1 / 2} 3^{1 / 2}$

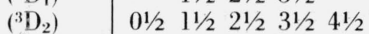

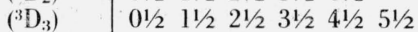

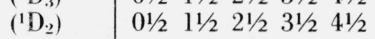

$4 f^{13}\left({ }^{2} \mathrm{~F}_{2 !}^{\circ}\right) 5 d 6 p\left({ }^{3} \mathrm{P}_{0}\right)$

$\left({ }^{3} \mathrm{P}_{1}\right)$
$\left({ }^{3} \mathrm{P}, 2\right)$

$\left({ }^{3} P_{2}\right)$
$\left(1 P_{1}\right)$

$21 / 2$

$11 / 22^{1 / 2} \cdot 31 / 2$

$11 / 2 \quad 21 / 2 \quad 31 / 2 \quad 41 / 2$

$21 / 2$

cation, thus bringing the total number of classifications to 4054 . In addition, 49 classifications which are considered to be masked, are entered in parentheses in table 6; these have not been included in the counts. In most cases the Zeeman pattern has been used as the deciding factor regarding masked lines. The number of computed combinations in the square array totals 10360. These numbers are, however, somewhat misleading. Nearly all of the strong lines are classified and "95 percent of the total intensity is accounted for." The strongest unclassified lines are listed in table 5 , which includes all remaining lines having an estimated spark intensity of 15 or greater. The wavelengths and intensities are from table 6.

The present Monograph brings to a conclusion an accomplishment that is a lasting tribute to W. F. Meggers. In this work an ambition of more than 30 years of his distinguished career is realized. It is characteristic that he collaborated with the late Professor Racah and his associates for over 15 years on the interpretation of this complex rare-earth spectrum. The writer is deeply indebted to these eminent spectroscopists for their unfailing and expert work. The success of that part of the project of the "Atomic Energy Levels" program concerned with rare-earth spectra depends entirely on such contributions. Difficult ground-breaking research is required for this success. 
TABLE 4. Unclassified lines of $\mathrm{Yb}$ II having an observed Zeeman effect

\begin{tabular}{|c|c|c|c|c|c|c|}
\hline \multirow{2}{*}{$\begin{array}{l}\text { Wavelength } \\
\text { Air }\end{array}$} & \multicolumn{2}{|c|}{ Intensity } & \multirow{2}{*}{$\begin{array}{l}\text { Wave } \\
\text { Number }\end{array}$} & \multirow{2}{*}{$\begin{array}{c}\text { Zeeman } \\
\text { Type }\end{array}$} & \multicolumn{2}{|c|}{ Zeeman Components } \\
\hline & $\begin{array}{l}\text { Meggers } \\
\text { lamp }\end{array}$ & $\begin{array}{l}\text { Thomson } \\
\text { lamp }\end{array}$ & & & $\begin{array}{c}\text { Strongest } \\
p\end{array}$ & $\begin{array}{c}\text { Strongest } \\
n\end{array}$ \\
\hline$\AA$ & & & K & & & \\
\hline 8263.640 & 4 & 150 & 12097.88 & 4 & $0.16 w$ & 1.03 \\
\hline 8192.400 & & 30 & 12203.08 & 4 & $w$ & 1.03 \\
\hline 8171.820 & 1 & 30 & 12233.81 & 5 & $w$ & 1.40 \\
\hline 8149.080 & & 15 & 12267.95 & 6 & 1.150 & 1.20 \\
\hline 8093.030 & & 8 & 12352.91 & 6 & 0.512 & 1.478 \\
\hline 7803.650 & 20 & 300 & 12810.99 & $6 ?$ & 0.938 & 0.938 \\
\hline 7362.920 & 2 & 15 & 13577.83 & $5 ?$ & 0.11 & 0.99 \\
\hline 7339.800 & & 60 & 13620.60 & $7 ?$ & 0 & 1.12 \\
\hline 7317.740 & & 30 & 13661.66 & $7 ?$ & 0 & 0.963 \\
\hline 7306.630 & 1 & 100 & 13682.43 & $5 ?$ & & \\
\hline 7086.080 & & 20 & 14108.29 & 4 & $w$ & 0.96 \\
\hline 6582.790 & 2 & 250 & 15186.93 & 7 & 0 & 0.97 \\
\hline 5042.982 & 3 & 20 & 19824.01 & 7 & 0 & 1.20 \\
\hline 3573.750 & 1 & 10 & 27973.82 & 7 & 0 & 1.013 \\
\hline 3443.587 & 30 & 20 & 29031.17 & $?$ & 0 & 1.18 \\
\hline 3337.171 & 30 & 100 & 29956.89 & 7 & 0 & 1.019 \\
\hline 3014.526 & 10 & 60 & 33163.05 & 7 & 0 & 1.12 \\
\hline 2963.460 & 40 & 300 & 33734.49 & 6 & 0.16 & 1.08 \\
\hline 2940.517 & 25 & 150 & 33997.68 & 5 & $w$ & 1.44 \\
\hline 2881.930 & 7 & 60 & 34688.79 & 5 & $w$ & 1.24 \\
\hline 2864.725 & 4 & 40 & 34897.12 & 4 & $w$ & 0.94 \\
\hline 2830.880 & 4 & 30 & 35314.32 & 7 & & 0.99 \\
\hline 2594.200 & 3 & 15 & 38536.01 & 6 & 0.22 & \\
\hline 2516.820 & 50 & 300 & 39720.72 & 7 & 0 & 1.348 \\
\hline 2515.600 & 2 & 7 & 39739.99 & 7 & 0 & 0.91 \\
\hline
\end{tabular}

In Israel, warmest thanks are due Mrs. Z. B. Goldschmidt and her associates for their collaboration in providing the theoretical calculations of the configurations and matrices. The theses by S. Aberbach and D. Sperber in 1953 were useful contributions to earlier calculations for Yb II. At this Bureau, the cooperation of the Spectroscopy Section is appreciated. Special thanks are due W. C. Martin, J. Sugar, C. H. Corliss, N. Spector, and J. Tech.

The writer is deeply indebted to Mrs. Isabel D. Murray for her extreme competence and painstaking care in preparing for publication the enormous amount of tabular data on $\mathrm{Yb}$ II.

It is most regrettable that the author did not live to see his Monograph on $\mathrm{Yb}$ II thru publication, after his long years of labor. The writer has attempted to present the material as accurately as possible, with the aid of his beautifully kept records of work. In spite of her inability to do justice to the description of his accomplishment, she hopes that this monumental contribution will serve as the model he had in mind over the years.

Washington, D.C.

May 26, 1967

\section{References}

[1] W. F. Meggers and B. F. Scribner, J. Res. NBS 19, 651 to 664 (1937) RP1053.

[2] W. F. Meggers, J. Opt. Soc. Am. 37, 988, 989(A) (1947).

[3] W. F. Meggers and C. H. Corliss, J. Res. NBS 70A (Phys. and Chem.) No. 1, 63 to 106 (1966).

[4] J. Sugar, J. Res. NBS 66A (Phys, and Chem.) No. 4, 321 to 324 (1962).

[5] J. Sugar, J. Opt. Soc. Am. 53, 831 to 839 (1963).

[0] C. J. Humphreys and E. Paul, Jr., Quarterly Report Foundational Research Projects, NAVORD Report 5970, NOLC Report 473, 57 to 67 (1959).

[7] A. M. Gutman and I. B. Levinson; Translated by J. Tech., Smithsonian Inst. Astroph. Obs., Astron. Translations Ser. No. 8, 20 to 25 (1967).

[8] G. Racah, Proc. Rydberg Centennial Conference on Atomic Spectroscopy, Lunds Univ. Årsskrift. N. F. Avd. 2, 50, No. 21; Kungl. Fys. Sällsk. Handl. N.F. 65, No. 21, 31 to 42 (1954). See also, J. Opt. Soc. Am. 50, 408 to 411 (1960).

[9] A. G. Shenstone, Phil. Trans. Roy. Soc. London A241, No. 832 , 301 (1948).

[10] B. W. Bryant, J. Opt. Soc. Am. 55, 771 to 779 (1965).

[11] N. Spector, J. Opt. Soc. Am. 57, 312 to 317 (1967).

[12] (;. Racah, letter, Augusț 15 (1965).

[13] C. H. Corliss and W. F. Meggers, J. Res. NBS 61, 270, 271 (1958) RP2904.

[14] H. N. Russell, private communication, February (1932).

[15] J. Sugar and J. Reader, J. Opt. Soc. Am. 55, 1286 to 1290 (1965).

[16] H. N. Russell and W. F. Meggers, J. Res. NBS 9, 625 to 668 (1932) RP497. 
TABLE 5. Strongest unclassified tines of $\mathrm{Yb}$ II

\begin{tabular}{|c|c|c|c|c|c|c|c|}
\hline \multirow{2}{*}{$\begin{array}{c}\text { Wavelength } \\
\text { Air }\end{array}$} & \multicolumn{2}{|c|}{ Intensity } & \multirow{2}{*}{ Wave number } & \multirow{2}{*}{$\begin{array}{c}\text { Wavelength } \\
\text { Air }\end{array}$} & \multicolumn{2}{|c|}{ Intensity } & \multirow{2}{*}{ Wave number } \\
\hline & $\begin{array}{c}\text { Meggers } \\
\text { Lamp }\end{array}$ & $\begin{array}{c}\text { Thomson } \\
\text { Lamp }\end{array}$ & & & $\begin{array}{l}\text { Meggers } \\
\text { Lamp }\end{array}$ & $\begin{array}{c}\text { Thomson } \\
\text { Lamp }\end{array}$ & \\
\hline $\begin{array}{c}\AA \\
10830.36 \\
10829.11 \\
10711.60 \\
10516.65\end{array}$ & & $\begin{array}{c}100 \\
40 \\
80 h \\
40\end{array}$ & $\begin{array}{c}K \\
9230.77 \\
9231.84 \\
9333.12 \\
9506.13\end{array}$ & \begin{tabular}{c}
\multicolumn{1}{c}{$\AA$} \\
5042.982 \\
4965.43 \\
4837.045 \\
4350.801
\end{tabular} & $\begin{array}{r}3 \\
3 \\
500\end{array}$ & $\begin{array}{r}20 \\
15 \\
1000 \\
15\end{array}$ & $\begin{array}{c}K \\
19824.01 \\
20133.62 \\
20668.00 \\
22977.81\end{array}$ \\
\hline $\begin{array}{l}9893.90 \\
9693.72 \\
9664.23 \\
9482.44 \\
9428.10\end{array}$ & & $\begin{array}{c}100 H \\
50 \\
20 \\
20 \\
30\end{array}$ & $\begin{array}{l}10104.47 \\
10313.13 \\
10344.60 \\
10542.92 \\
10603.68\end{array}$ & $\begin{array}{l}4281.972 \\
4215.519 \\
4202.44 \\
4177.40 \\
4119.25\end{array}$ & $\begin{array}{r}20 \\
6 \\
15 \\
2 \\
20\end{array}$ & $\begin{array}{l}50 \\
20 \\
30 h l \\
50 \\
30\end{array}$ & $\begin{array}{l}23347.16 \\
23715.19 \\
23789.00 \\
23931.59 \\
24269.42\end{array}$ \\
\hline $\begin{array}{l}9380.18 \\
9325.68 \\
9314.88 \\
9272.68 \\
9253.57\end{array}$ & $\begin{array}{l}2 \\
3 \\
7\end{array}$ & $\begin{array}{l}15 \\
20 h \\
40 \\
30 \\
70\end{array}$ & $\begin{array}{l}10657.85 \\
10720.14 \\
10732.57 \\
10781.41 \\
10803.68\end{array}$ & $\begin{array}{l}4077.713 \\
3857.586 \\
3807.574 \\
3779.184 \\
3743.138\end{array}$ & $\begin{array}{r}10 \\
10 \\
50 \\
1\end{array}$ & $\begin{array}{c}20 \\
20 \\
500 \\
15 \\
15 h\end{array}$ & $\begin{array}{l}24516.63 \\
25915.60 \\
26255.99 \\
26453.22 \\
26707.96\end{array}$ \\
\hline $\begin{array}{l}9196.50 \\
9167.06 \\
9163.69 \\
9105.95 \\
9096.88\end{array}$ & 3 & $\begin{array}{l}20 H \\
40 \\
15 \\
15 h \\
20\end{array}$ & $\begin{array}{l}10870.72 \\
10905.63 \\
10909.64 \\
10978.82 \\
10989.76\end{array}$ & $\begin{array}{l}3716.139 \\
3574.58 \\
3482.564 \\
3477.022 \\
3472.324\end{array}$ & $\begin{array}{r}10 \\
5 \\
20 \\
5\end{array}$ & $\begin{array}{l}40 \\
30 h \\
30 h \\
30 \\
20\end{array}$ & $\begin{array}{l}26902.00 \\
27967.33 \\
28706.26 \\
28752.01 \\
28790.9 !\end{array}$ \\
\hline $\begin{array}{l}9080.35 \\
8985.71 \\
8980.74 \\
8855.12 \\
8291.01\end{array}$ & $\begin{array}{r}1 \\
8 \\
50\end{array}$ & $\begin{array}{r}60 \\
60 \\
20 \\
300 \\
20\end{array}$ & $\begin{array}{l}11009.77 \\
11125.73 \\
11131.88 \\
11289.80 \\
12057.94\end{array}$ & $\begin{array}{l}3390.421 \\
3390.246 \\
3376.623 \\
3363.645 \\
3362.438\end{array}$ & $\begin{array}{l}1 h \\
1 h \\
3 \\
10 \\
10\end{array}$ & $\begin{array}{l}30 h \\
40 h \\
30 \\
20 \\
70\end{array}$ & $\begin{array}{l}29486.40 \\
29487.92 \\
29606.89 \\
29721.11 \\
29731.78\end{array}$ \\
\hline $\begin{array}{l}8263.64 \\
8192.40 \\
8171.82 \\
8149.08 \\
8124.50\end{array}$ & $\begin{array}{l}4 \\
1\end{array}$ & $\begin{array}{r}150 \\
30 \\
30 \\
15 \\
15\end{array}$ & $\begin{array}{l}12097.88 \\
12203.08 \\
12233.81 \\
12267.95 \\
12305.07\end{array}$ & $\begin{array}{l}3352.491 \\
3342.93 \\
3337.171 \\
3315.101 \\
3179.344\end{array}$ & $\begin{array}{l}10 \\
20 h \\
30 \\
1 h \\
30\end{array}$ & $\begin{array}{l}30 \\
100 h \\
100 \\
15 h \\
70\end{array}$ & $\begin{array}{l}29820.00 \\
29905.28 \\
29956.89 \\
30156.31 \\
31443.93\end{array}$ \\
\hline $\begin{array}{l}8023.46 \\
7803.65 \\
7362.92 \\
7339.80 \\
7317.74\end{array}$ & $\begin{array}{r}1 \\
20 \\
2\end{array}$ & $\begin{array}{r}20 \\
300 \\
15 \\
60 \\
30\end{array}$ & $\begin{array}{l}12460.02 \\
12810.99 \\
13577.83 \\
13620.60 \\
13661.66\end{array}$ & $\begin{array}{l}3166.727 \\
3114.905 \\
3014.526 \\
3007.923 \\
3004.642\end{array}$ & $\begin{array}{r}2 \\
3 \\
10 \\
5 \\
1\end{array}$ & $\begin{array}{l}20 \\
20 \\
60 \\
30 \\
15\end{array}$ & $\begin{array}{l}31569.21 \\
32094.40 \\
33163.05 \\
33235.85 \\
33272.14\end{array}$ \\
\hline $\begin{array}{l}7306.63 \\
7086.08 \\
6829.80 \\
6670.18 \\
6616.40\end{array}$ & $\begin{array}{l}1 \\
1 \\
2\end{array}$ & $\begin{array}{r}100 \\
20 \\
70 \\
50 \\
40\end{array}$ & $\begin{array}{l}13682.43 \\
14108.29 \\
14637.68 \\
14987.96 \\
15109.79\end{array}$ & $\begin{array}{l}2963.46 \\
2950.52 \\
2940.517 \\
2881.93 \\
2864.725\end{array}$ & $\begin{array}{r}40 \\
2 \\
25 \\
7 \\
4\end{array}$ & $\begin{array}{r}300 \\
20 \\
150 \\
60 \\
40\end{array}$ & $\begin{array}{l}33734.49 \\
33882.43 \\
33997.68 \\
34688.79 \\
34897.12\end{array}$ \\
\hline $\begin{array}{l}6582.79 \\
6485.55 \\
6453.11 \\
6150.62 \\
6120.30\end{array}$ & $\begin{array}{r}2 \\
10 \\
10 \\
5\end{array}$ & $\begin{array}{l}250 \\
200 h \\
150 \\
70 h \\
250\end{array}$ & $\begin{array}{l}15186.93 \\
15414.63 \\
15492.12 \\
16254.03 \\
16334.55\end{array}$ & $\begin{array}{l}2830.880 \\
2594.20 \\
2516.82 \\
2369.42 \\
2284.99\end{array}$ & $\begin{array}{r}4 \\
3 \\
50\end{array}$ & $\begin{array}{r}30 \\
15 \\
300 \\
50 \\
30\end{array}$ & $\begin{array}{l}35314.32 \\
38536.01 \\
39720.72 \\
42191.53 \\
43750.37\end{array}$ \\
\hline $\begin{array}{l}5833.99 \\
5581.05 \\
5331.536 \\
5256.85 \\
5074.609\end{array}$ & $\begin{array}{l}5 h \\
2\end{array}$ & $\begin{array}{c}200 h \\
40 \\
20 \\
20 \\
80\end{array}$ & $\begin{array}{l}17136.18 \\
17912.80 \\
18751.10 \\
19017.51 \\
19700.46\end{array}$ & $\begin{array}{l}2161.60 \\
2155.50 \\
2102.73\end{array}$ & $\begin{array}{r}10 \\
5\end{array}$ & $\begin{array}{l}40 \\
30 \\
20\end{array}$ & $\begin{array}{l}46247.50 \\
46378,37 \\
47542.14\end{array}$ \\
\hline
\end{tabular}


TABLE 6. Yb II-Observed and classified lines

\begin{tabular}{|c|c|c|c|c|c|c|c|c|c|c|}
\hline 1 & 2 & 3 & 4 & 5 & 6 & 7 & 8 & 9 & 10 & 11 \\
\hline \multirow{2}{*}{$\begin{array}{l}\text { Wave- } \\
\text { length } \\
\text { air }\end{array}$} & \multicolumn{2}{|c|}{ Intensity } & \multirow{2}{*}{$\begin{array}{l}\text { Wave } \\
\text { number }\end{array}$} & \multirow[b]{2}{*}{$\mathrm{o}-\mathrm{c}$} & \multirow{2}{*}{ Classification } & \multirow{2}{*}{ Zee- } & \multirow[b]{2}{*}{ lst $g$} & \multirow[b]{2}{*}{ 2nd $g$} & \multicolumn{2}{|c|}{$\begin{array}{l}\text { Zeeman } \\
\text { components }\end{array}$} \\
\hline & $\begin{array}{l}\text { Meggers } \\
\text { lamp }\end{array}$ & $\begin{array}{c}\text { Thomson } \\
\text { lamp }\end{array}$ & & & & & & & Strong $p$ & Strong $n$ \\
\hline $\begin{array}{c}\AA \\
24377.26 \\
16498.42 \\
13452.62 \\
12692.48 \\
11559.05\end{array}$ & $\begin{array}{r}250 \\
1500 \\
300 \\
450\end{array}$ & 5 & $\begin{array}{c}K \\
4101.06 \\
6059.53 \\
7431.46 \\
7876.53 \\
8648.86\end{array}$ & $\begin{aligned} & K \\
+ & 0.04 \\
- & 0.01 \\
+ & 0.03 \\
+ & 0.04 \\
- & 0.04\end{aligned}$ & $\begin{array}{l}22960_{1 \frac{1}{2}}-27061_{0 \frac{1}{2}}^{\circ} \\
24332_{2 \frac{1}{2}}-30392_{1 \frac{1}{2}}^{\circ} \\
22960_{1 \frac{1}{2}}-30392_{1 \frac{1}{2}}^{\circ}-47912_{3 \frac{1}{2}}^{\circ} \\
40035_{3 \frac{1}{2}}^{\circ}-472981_{1 \frac{1}{2}}^{\circ} \\
24332_{2 \frac{1}{2}}-32\end{array}$ & & & & & $\therefore$ \\
\hline $\begin{array}{l}11442.70 \\
11299.78 \\
11277.83 \\
11060.25 \\
11051.05\end{array}$ & & $\begin{array}{l}1 \\
5 h \\
1 \\
2 h \\
2\end{array}$ & $\begin{array}{l}8736.80 \\
8847.31 \\
8864.53 \\
9038.91 \\
9046.44\end{array}$ & $\begin{array}{l}-0.06 \\
-0.03\end{array}$ & $\begin{array}{l}40035_{3 \frac{1}{2}}^{\circ}-48900_{3 \frac{1}{2}} \\
47329_{2 \frac{1}{2}}^{\circ}-56375_{2 \frac{1}{2}}\end{array}$ & & & & & \\
\hline $\begin{array}{l}11035.15 \\
11023.37 \\
11002.95 \\
10980.47 \\
10959.94\end{array}$ & & $\begin{array}{l}1 h \\
1 h \\
4 \\
3 h \\
1 h\end{array}$ & $\begin{array}{l}9059.47 \\
9069.15 \\
9085.98 \\
9104.58 \\
9121.64\end{array}$ & -0.03 & $54640_{4 \frac{1}{2}}^{\circ}-63726_{4 \frac{1}{2}}$ & & & & & \\
\hline $\begin{array}{l}10943.17 \\
10882.92 \\
10856.25 \\
10845.53 \\
10830.36\end{array}$ & & $\begin{array}{c}1 \\
1 h \\
1 \\
3 h \\
100\end{array}$ & $\begin{array}{l}9135.62 \\
9186.19 \\
9208.76 \\
9217.86 \\
9230.77\end{array}$ & $\begin{array}{l}+0.02 \\
+0.01\end{array}$ & $\begin{array}{l}55462_{3 \frac{1}{2}}^{\circ}-64598_{3 \frac{1}{2}} \\
48556_{3 \frac{1}{2}}^{\circ}-57765_{4 \frac{1}{2}}\end{array}$ & & & & & \\
\hline $\begin{array}{l}10829.11 \\
10810.82 \\
10802.47 \\
10776.11 \\
10774.38\end{array}$ & & $\begin{array}{l}40 \\
5 h \\
4 \\
1 \\
1\end{array}$ & $\begin{array}{l}9231.84 \\
9247.46 \\
9254.61 \\
9277.25 \\
9278.73\end{array}$ & $\begin{array}{l}-0.01 \\
+0.02\end{array}$ & $\begin{array}{l}56621_{5 \frac{1}{2}}^{\circ}-65875_{6 \frac{1}{2}} \\
44438_{3 \frac{1}{2}}^{\circ}-53715_{3 \frac{1}{2}}\end{array}$ & & & & & \\
\hline $\begin{array}{l}10754.99 \\
10745.87 \\
10731.94 \\
10721.68 \\
10717.07\end{array}$ & & $\begin{array}{l}1 \\
3 \\
3 \\
3 h \\
2\end{array}$ & $\begin{array}{l}9295.46 \\
9303.35 \\
9315.43 \\
9324.34 \\
9328.35\end{array}$ & $\begin{array}{l}-0.01 \\
-0.01\end{array}$ & $\begin{array}{l}53716_{2 \frac{1}{2}}^{\circ}-63011_{1 \frac{1}{2}}^{\circ} \\
54640_{4 \frac{1}{2}}^{\circ}-63944_{5 \frac{1}{2}}\end{array}$ & & & & & \\
\hline $\begin{array}{l}10711.60 \\
10691.02 \\
10689.20 \\
10678.64\end{array}$ & & $\begin{array}{c}80 h \\
1 h \\
2 h \\
1\end{array}$ & $\begin{array}{l}9333.12 \\
9351.08 \\
9352.67 \\
9361.92\end{array}$ & $\begin{array}{l}-0.06 \\
-0.02\end{array}$ & $\begin{array}{l}61442_{5 \frac{1}{2}}^{\circ}-70793_{5 \frac{1}{2}} \\
66189_{1 \frac{1}{2}}^{\circ}-75550_{2 \frac{1}{2}}\end{array}$ & & & & & \\
\hline 10676.73 & & 30 & 9363.60 & $\left\{\begin{array}{l}-0.09 \\
+0.04\end{array}\right.$ & $\begin{array}{l}44940_{1 \frac{1}{2}}^{\circ}-54304_{0 \frac{1}{2}}^{\circ} \\
56375_{\frac{1}{2}}-65739_{2 \frac{1}{2}}^{\circ}\end{array}$ & & & & & \\
\hline 10666.30 & & $2 h$ & 9372.75 & & & & & & & \\
\hline 10651.36 & & 5 & 9385.90 & $\left\{\begin{array}{r}0.00 \\
-0.06\end{array}\right.$ & $\begin{array}{l}64365_{4 \frac{1}{2}}^{\circ}-73750^{\frac{1}{2}} \\
75058\end{array}$ & & & & & \\
\hline $\begin{array}{r}10638.35 \\
\text { See } \mathrm{f}\end{array}$ & otnotes a & $\begin{array}{l}1 \\
\text { at end of } t\end{array}$ & $\begin{array}{l}9397.38 \\
\text { able. }\end{array}$ & +0.08 & $65594_{2 \frac{1}{2}}^{\circ}-74991_{1 \frac{1}{2}}^{\circ}$ & & & & & \\
\hline
\end{tabular}


TABLE 6. Yb II-Observed and classified lines-Continued

\begin{tabular}{|c|c|c|c|c|c|c|c|c|c|c|}
\hline 1 & 2 & 3 & 4 & 5 & 6 & 7 & 8 & 9 & 10 & 11 \\
\hline \multirow{2}{*}{$\begin{array}{l}\text { Wave- } \\
\text { length } \\
\text { air }\end{array}$} & \multicolumn{2}{|c|}{ Intensity } & \multirow{2}{*}{$\begin{array}{c}\text { Wave } \\
\text { number }\end{array}$} & \multirow[b]{2}{*}{$\mathrm{o}-\mathrm{c}$} & \multirow[b]{2}{*}{ Classification } & \multirow{2}{*}{$\mid \begin{array}{l}\text { Zee- } \\
\text { man } \\
\text { type }\end{array}$} & \multirow[b]{2}{*}{ lst $g$} & \multirow[b]{2}{*}{ 2nd $g$} & \multicolumn{2}{|c|}{$\begin{array}{l}\text { Zeeman } \\
\text { component's }\end{array}$} \\
\hline & $\begin{array}{c}\text { Meggers } \\
\text { lamp }\end{array}$ & $\begin{array}{c}\text { Thomson } \\
\text { lamp }\end{array}$ & & & & & & & Strong $p$ & Strong $n$ \\
\hline A & & & $K$ & $K$ & & & & & & \\
\hline $\begin{array}{l}10628.11 \\
10602.75\end{array}$ & & $\begin{array}{l}4 \\
2 h\end{array}$ & $\begin{array}{l}9406.43 \\
9428.93\end{array}$ & -0.03 & $57798_{2 \frac{1}{2}}^{\circ}-67204_{1 \frac{1}{2}}$ & & & & & \\
\hline 10584.87 & & 1 & 9444.86 & 0.00 & $64191_{1 \frac{1}{2}}^{\circ}-73636_{0 \frac{1}{2}}$ & & & & & $\therefore$ \\
\hline 10580.74 & & 1 & 9448.55 & 0.00 & $43956_{2 \frac{1}{2}}^{\circ}-534044_{2 \frac{1}{2}}$ & & & & & \\
\hline 10574.50 & & $2 h$ & 9454.12 & -0.02 & $58484_{4 \frac{1}{2}}^{\circ}-67938_{3 \frac{1}{2}}$ & & & & & \\
\hline 10570.51 & & 1 & 9457.69 & & & & & & & \\
\hline 10567.42 & & 3 & 9460.46 & +0.03 & $55462_{3 \frac{1}{2}}^{\circ}-64923_{4 \frac{1}{2}}$ & & & & & \\
\hline 10565.46 & & 4 & 9462.21 & -0.01 & $40035_{3 \frac{1}{2}}^{\circ}-49498_{2 \frac{1}{2}}$ & & & & & \\
\hline 10556.48 & & $2 h$ & 9470.26 & +0.08 & $56480_{4 \frac{1}{2}}^{\circ}-65950_{4 \frac{1}{2}}$ & & & & & \\
\hline 10547.78 & & 4 & 9478.07 & +0.03 & $58672_{0 \frac{1}{2}}^{\circ}-68150_{1 \frac{1}{2}}$ & & & & & \\
\hline 10546.24 & & 1 & 9479.46 & & & & & & & \\
\hline 10516.65 & & 40 & 9506.13 & & & & & & & \\
\hline 10502.56 & & 1 & 9518.88 & -0.02 & $53644_{3 \frac{1}{2}}^{\circ}-63163_{4 \frac{1}{2}}$ & & & & & \\
\hline 10501.08 & & 4 & 9520.22 & +0.02 & $56056_{2 \frac{1}{2}}^{\circ}-65577_{3 \frac{1}{2}}$ & & & & & \\
\hline 10490.03 & & 1 & 9530.25 & & 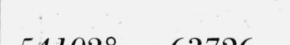 & & & & 1 & \\
\hline 10485.56 & & 2 & 9534.31 & -0.01 & $54192_{5 \frac{1}{2}}^{\circ}-63726_{4 \frac{1}{2}}$ & & & & & \\
\hline 10477.15 & & $1 h$ & 9541.97 & & & & & & & \\
\hline 10456.88 & & 1 & 9560.46 & & & & & & & \\
\hline 10455.88 & & 2 & 9561.38 & & & & & & & \\
\hline 10446.15 & & 1 & 9570.28 & -0.01 & $38342_{4 \frac{1}{2}}^{\circ}-47912_{3 \frac{1}{2}}$ & & & & & \\
\hline $\begin{array}{l}10445.27 \\
10425.42\end{array}$ & & $4 h$ & $\begin{array}{l}9571.09 \\
9589.31\end{array}$ & +0.09 & $53644^{\circ}-63234$ & & & & & \\
\hline 1042.42 & & & 9589.31 & +0.09 & $500+73 \frac{1}{2}$ & & & & & \\
\hline 10416.16 & & 5 & 9597.84 & 0.00 & $64970_{3 \frac{1}{2}}^{\circ}-74568_{4 \frac{1}{2}}$ & & & & & \\
\hline 10414.15 & & 1 & 9599.69 & -0.06 & $62163_{2 \frac{1}{2}}^{\circ}-71763_{1 \frac{1}{2}}$ & & & & & \\
\hline 10411.40 & & 10 & 9602.23 & +0.01 & $42915_{5 \frac{1}{2}}^{\circ}-52517_{5 \frac{1}{2}}$ & & & & & \\
\hline 10397.98 & & 3 & 9614.62 & & & & & & & \\
\hline 10391.64 & & $1 h$ & 9620.48 & & $\therefore$ & & & & & \\
\hline 10388.85 & & $2 h$ & 9623.07 & & & & & & & \\
\hline 10363.89 & & 3 & 9646.24 & & & & $\therefore$ & & & \\
\hline 10345.62 & & $1 h$ & 9663.28 & & ' & & & & & \\
\hline 10343.99 & & $4 h$ & 9664.80 & & & & & & & \\
\hline 10339.49 & & $2 h$ & 9669.01 & & $\because$ & & & & & \\
\hline 10337.35 & & $1 h$ & 9671.01 & & & & & & & \\
\hline 10328.74 & & $2 h$ & 9679.07 & & & & & & & \\
\hline 10327.33 & & 1 & 9680.39 & & & & & & & \\
\hline 10293.40 & & $2 h$ & 9712.30 & $\because$ & & & & & & \\
\hline 10283.38 & & 2 & 9721.76 & & & & & & & \\
\hline 10275.82 & & 1 & 9728.92 & & & & & & & \\
\hline 10259.93 & & $3 h$ & 9743.98 & & & & & & & \\
\hline 10257.97 & & $1 h$ & 9745.85 & & & & & & & \\
\hline
\end{tabular}


TABLE 6. Y Y II-Observed and classified lines-Continued

\begin{tabular}{|c|c|c|c|c|c|c|c|c|c|c|}
\hline 1 & 2 & 3 & 4 & 5 & 6 & 7 & 8 & 9 & 10 & 11 \\
\hline \multirow{2}{*}{$\begin{array}{l}\text { Wave- } \\
\text { length } \\
\text { air }\end{array}$} & \multicolumn{2}{|c|}{ Intensity } & \multirow{2}{*}{$\begin{array}{c}\text { Wave } \\
\text { number }\end{array}$} & \multirow[b]{2}{*}{$0-c$} & \multirow{2}{*}{ Classification } & \multirow{2}{*}{$\begin{array}{c}\text { Zee- } \\
\text { man } \\
\text { type }\end{array}$} & \multirow[b]{2}{*}{ lst $g$} & \multirow{2}{*}{ 2nd $g$} & \multicolumn{2}{|c|}{$\begin{array}{c}\text { Zeeman } \\
\text { components }\end{array}$} \\
\hline & $\begin{array}{l}\text { Meggers } \\
\text { lamp }\end{array}$ & $\begin{array}{c}\text { Thomson } \\
\text { lamp }\end{array}$ & & & & & & & Strong $p$ & Strong $n$ \\
\hline$\AA$ & & & $K$ & $K$ & & & & & & \\
\hline $\begin{array}{l}10251.85 \\
10242.30\end{array}$ & & $\begin{array}{l}10 \\
1 h\end{array}$ & $\begin{array}{l}9751.66 \\
9760.76\end{array}$ & $\begin{array}{l}-0.01 \\
+0.04\end{array}$ & $\begin{array}{l}54192_{5 \frac{1}{2}}^{\circ}-63944_{5 \frac{1}{2}} \\
48900_{3 \frac{1}{2}}-58661_{2 \frac{1}{2}}^{\circ}\end{array}$ & & & & & \\
\hline 10222.98 & & $2 h$ & 9779.20 & +0.04 & $77606_{2 \frac{1}{2}}-87385_{3 \frac{1}{2}}^{\circ}$ & & & & & \\
\hline 10212.34 & & $1 h$ & 9789.39 & & & & & & & \\
\hline 10198.92 & & $1 h$ & 9802.27 & +0.07 & $51248_{2 \frac{1}{2}}^{\circ}-61051_{3 \frac{1}{2}}$ & & & & & \\
\hline 10189.53 & & $10 h$ & 9811.31 & & & & & & & \\
\hline 10186.03 & & 5 & 9814.68 & +0.03 & $49008_{3 \frac{1}{2}}^{\circ}-58823_{2 \frac{1}{2}}$ & & & & & \\
\hline 10172.39 & & 2 & 9827.84 & & & & & & & \\
\hline 10168.71 & & $1 h$ & 9831.39 & & & & & & & \\
\hline 10167.42 & & $1 h$ & 9832.64 & & & & & & & \\
\hline 10157.63 & & 1 & 9842.12 & +0.07 & $65149_{0 \frac{1}{2}}^{\circ}-74991_{1 \frac{1}{2}}^{\circ}$ & & : & & & \\
\hline 10148.91 & & 3 & 9850.57 & 0.00 & $56500_{3 \frac{1}{2}}^{\circ}-66351_{2 \frac{1}{2}}$ & & & & & \\
\hline 10145.30 & & $4 h$ & 9854.08 & $\because$ & & & & & & \\
\hline 10136.34 & & $3 h$ & 9862.79 & & & & & & & \\
\hline 10107.09 & & 1 & 9891.33 & +0.06 & $53120_{2 \frac{1}{2}}^{\circ}-63011_{1 \frac{1}{2}}$ & & & & & \\
\hline 10103.24 & & 3 & 9895.10 & +0.02 & $56500_{3 \frac{1}{2}}^{\circ}-66395_{2 \frac{1}{2}}^{\circ}$ & & & & & \\
\hline 10100.39 & & $1 h$ & 9897.89 & & & & & & & \\
\hline 10086.67 & & 1 & 9911.36 & & & & & & & \\
\hline 10068.79 & & $2 h$ & 9928.96 & & & & & & & \\
\hline 10067.47 & & 1 & 9930.26 & +0.04 & $43007_{0 \frac{1}{2}}^{\circ}-52938_{1 \frac{1}{2}}$ & & & & & \\
\hline 10066.30 & & 5 & 9931.41 & +0.06 & $53716_{2 \frac{1}{2}}^{\circ}-63647_{1 \frac{1}{2}}$ & & & & & \\
\hline 10063.22 & & $2 h$ & 9934.45 & & & & & & & \\
\hline 10046.95 & & 2 & 9950.54 & & & & & & & . \\
\hline 10039.90 & & 1 & 9957.53 & +0.07 & $54640_{4 \frac{1}{2}}^{\circ}-64598_{3 \frac{1}{2}}$ & & & & & \\
\hline 10032.55 & & 50 & 9964.82 & +0.04 & $45737_{1 \frac{1}{2}}^{\circ}-55702_{1 \frac{1}{2}}$ & & & & & \\
\hline 10020.70 & & 1 & 9976.61 & & & & & & & \\
\hline 10016.78 & & 15 & 9980.51 & -0.01 & $52880_{4 \frac{1}{2}}^{\circ}-62861_{5 \frac{1}{2}}$ & & & & & \\
\hline 10007.96 & & 3 & 9989.31 & & & & & & & \\
\hline 10003.38 & & 4 & 9993.88 & +0.02 & $56088_{4 \frac{1}{2}}^{\circ}-66082_{5 \frac{1}{2}}$ & & $\cdots$ & & & \\
\hline 9994.64 & & 1 & 10002.62 & -0.05 & $64970_{3 \frac{1}{2}}^{\circ}-74973_{3 \frac{1}{2}}$ & & ..... & & & \\
\hline 9992.75 & & 1 & 10004.51 & +0.07 & $59439_{2 \frac{1}{2}}^{\circ}-69443_{1 \frac{1}{2}}$ & & & & & \\
\hline 9990.47 & & 6 & 10006.80 & +0.02 & $47758_{3 \frac{1}{2}}^{\circ}-57765_{4 \frac{1}{2}}$ & & 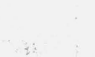 & & & \\
\hline 9984.66 & & 1 & 10012.62 & & & & & & & \\
\hline 9976.50 & 2 & 30 & 10020.81 & +0.02 & $22960_{\frac{1}{2}}-32981_{1 \frac{1}{2}}^{\circ}$ & & & & & \\
\hline 9970.10 & & $200 H$ & 10027.24 & -0.06 & $82891_{2 \frac{1}{2}}-92919_{3 \frac{1}{2}}^{\circ}$ & & & & & \\
\hline 9959.25 & & 2 & 10038.16 & +0.05 & $62163_{2 \frac{1}{2}}^{\circ}-72202_{3 \frac{1}{2}}$ & & & & & \\
\hline 9953.32 & & 4 & 10044.15 & +0.02 & $60910_{1 \frac{1}{2}}^{\circ}-70954_{2 \frac{1}{2}}$ & & & & & \\
\hline 9945.98 & & 4 & 10051.56 & & & & & & & \\
\hline
\end{tabular}


TABLE 6. Yb II-Observed and classified lines-Continued

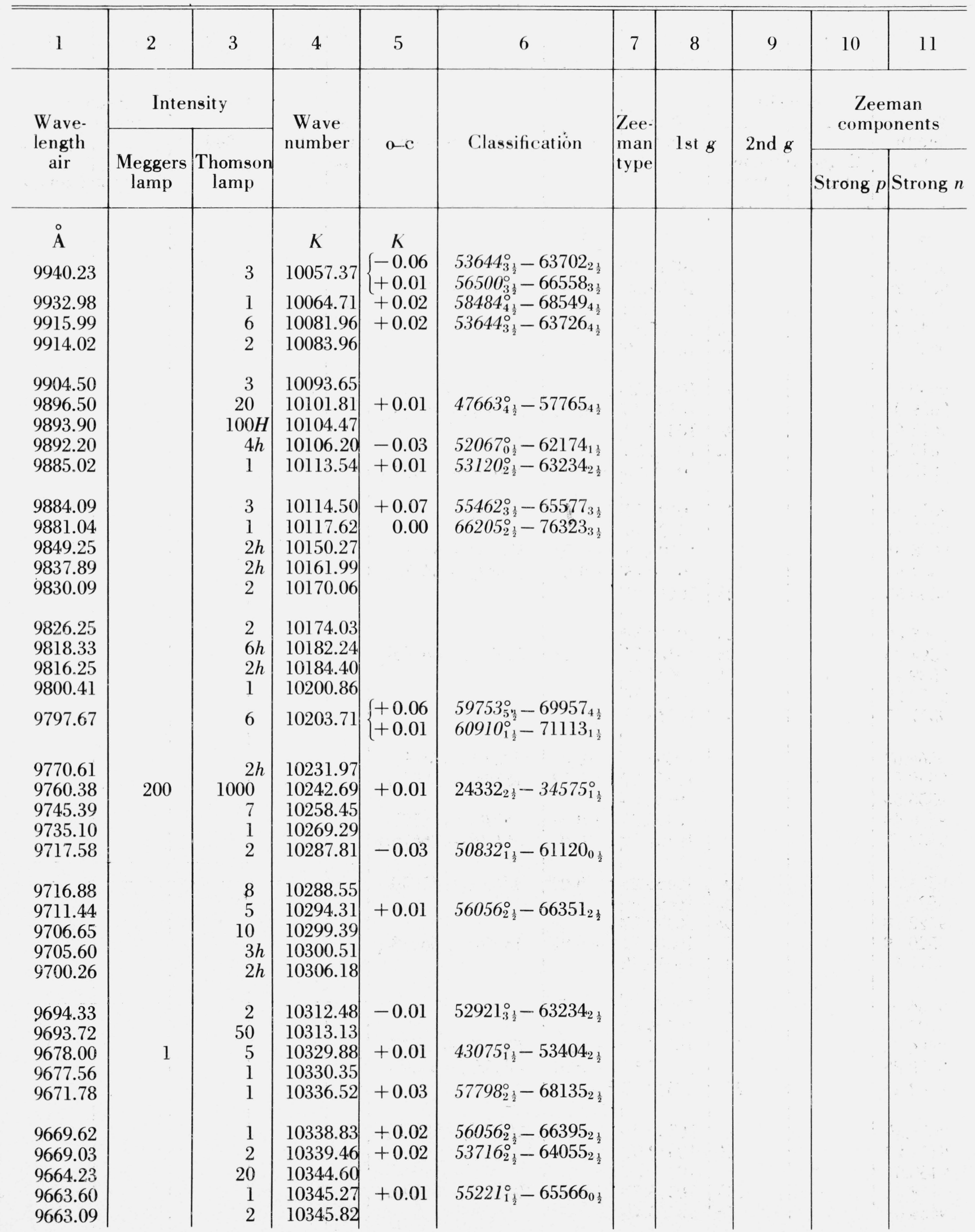


TABLE 6. Yb II-Observed and classified lines-Continued

\begin{tabular}{|c|c|c|c|c|c|c|c|c|c|c|}
\hline 1 & 2 & 3 & 4 & 5 & 6 & 7 & 8 & 9 & 10 & 11 \\
\hline \multirow{2}{*}{$\begin{array}{l}\text { Wave- } \\
\text { length } \\
\text { air }\end{array}$} & \multicolumn{2}{|c|}{ Intensity } & \multirow{2}{*}{$\begin{array}{c}\text { Wave } \\
\text { number }\end{array}$} & \multirow[b]{2}{*}{$\mathrm{o}-\mathrm{c}$} & \multirow{2}{*}{ Classification } & \multirow{2}{*}{$\left|\begin{array}{c}\text { Zee- } \\
\text { man } \\
\text { type }\end{array}\right|$} & \multirow[b]{2}{*}{ lst $g$} & \multirow{2}{*}{ 2nd $g$} & \multicolumn{2}{|c|}{$\begin{array}{c}\text { Zeeman } \\
\text { components }\end{array}$} \\
\hline & $\begin{array}{c}\text { Meggers } \\
\text { lamp }\end{array}$ & $\begin{array}{c}\text { Thomson } \\
\text { lamp }\end{array}$ & & & & & & & Strong $p$ & Strong $n$ \\
\hline$\AA$ & & & $K$ & $K$ & & & & & & \\
\hline 9661.01 & & $6 h$ & 10348.05 & & & & & & & \\
\hline 9657.25 & & 3 & 10352.08 & +0.02 & $57798_{2 \frac{1}{2}}^{\circ}-68150_{1 \frac{1}{2}}$ & & & & & \\
\hline $\begin{array}{l}9648.86 \\
9644.92\end{array}$ & & $\begin{array}{l}3 \\
2 h\end{array}$ & 10361.08 & & & & & & & \\
\hline 9637.59 & & $\begin{array}{l}2 n \\
3 h\end{array}$ & 10373.19 & & & & & & & \\
\hline 9630.97 & & 5 & 10380.32 & & & & & & & \\
\hline 9629.04 & & 2 & 10382.40 & 0.00 & $59753_{5 \frac{1}{2}}^{\circ}-70136_{6 \frac{1}{2}}$ & & & & & \\
\hline 9623.60 & & $9 h$ & 10388.27 & & & & & & & \\
\hline 9619.82 & & $3 h$ & 10392.35 & & & & & & & \\
\hline 9613.49 & & 1 & 10399.20 & +0.01 & $64169_{3 \frac{1}{2}}^{\circ}-74568_{4 \frac{1}{2}}$ & & & & & \\
\hline 9611.30 & & 2 & 10401.57 & & & & & & & \\
\hline 9610.77 & & 2 & 10402.14 & & & & & & & \\
\hline 9608.81 & & 2 & 10404.26 & +0.03 & $57534_{3 \frac{1}{2}}^{\circ}-67938_{3 \frac{1}{2}}$ & & & & & \\
\hline 9602.64 & & 20 & 10410.95 & +0.02 & $53644_{3 \frac{1}{2}}^{\circ}-64055_{2 \frac{1}{2}}$ & & & & & \\
\hline 9600.55 & & $2 h$ & 10413.21 & & & & & & & \\
\hline 9595.60 & & $2 h$ & 10418.59 & & & & & & & \\
\hline 9594.26 & & $2 h$ & 10420.04 & -0.08 & $56375_{2 \frac{1}{2}}-66796_{3 \frac{1}{2}}^{\circ}$ & & & & & \\
\hline 9589.14 & & $7 h$ & 10425.60 & & & & & & & \\
\hline 9578.21 & & 1 & 10437.50 & & & & & & & \\
\hline 9577.45 & & 3 & 10438.33 & & & & & & & \\
\hline 9564.64 & & 3 & 10452.31 & -0.01 & $54640_{4 \frac{1}{2}}^{\circ}-65093_{3 \frac{1}{2}}$ & & & & & \\
\hline 9559.83 & & 5 & 10457.57 & +0.02 & $48503_{5 \frac{1}{2}}^{\circ}-58961_{5 \frac{1}{2}}^{\circ}$ & & & & & \\
\hline 9555.55 & & 5 & 10462.25 & & & & & & & \\
\hline 9536.43 & & 3 & 10483.23 & +0.04 & $56088_{4 \frac{1}{2}}^{\circ}-66571_{5 \frac{1}{2}}$ & & & & & \\
\hline 9532.71 & & $8 h$ & 10487.32 & +0.01 & $66189_{1 \frac{1}{2}}^{\circ}-76676_{2 \frac{1}{2}}$ & & & & & \\
\hline 9531.83 & & 20 & 10488.29 & +0.02 & $55462_{3 \frac{1}{2}}^{\circ}-65950_{4 \frac{1}{2}}$ & & & & & \\
\hline 9527.91 & & $3 h$ & 10492.60 & & & & & & & \\
\hline 9522.68 & & $4 h$ & 10498.37 & & & & & & & \\
\hline 9520.19 & & 3 & 10501.11 & +0.02 & $56056_{2 \frac{1}{2}}^{\circ}-66558_{3 \frac{1}{2}}$ & & & & & \\
\hline 9511.61 & & 3 & 10510.58 & & & & & & & \\
\hline 9504.03 & & 1 & 10518.97 & & & & & & & \\
\hline 9498.90 & & 3 & 10524.65 & & & & & & & \\
\hline 9496.63 & & 10 & 10527.16 & +0.01 & $53120_{2 \frac{1}{2}}^{\circ}-63647_{1 \frac{1}{2}}$ & & & & & \\
\hline 9490.85 & & 2 & 10533.57 & +0.01 & $48556_{3 \frac{1}{2}}^{\circ}-59090_{3 \frac{1}{2}}$ & & & & & \\
\hline 9482.44 & & 20 & 10542.92 & & & & & & & \\
\hline 9475.35 & & 1 & 10550.81 & 0.00 & $48272_{2 \frac{1}{2}}^{\circ}-58823_{2 \frac{1}{2}}$ & & & & & \\
\hline 9474.20 & & $2 h$ & 10552.09 & & & & & & & \\
\hline $9468.54^{\mathrm{a}}$ & 2 & 3 & 10558.39 & & & & & & & \\
\hline 9468.20 & & $10 h$ & 10558.77 & +0.05 & $54640_{4 \frac{1}{2}}^{\circ}-65199_{4 \frac{1}{2}}$ & & & & & \\
\hline 9464.03 & & $4 h$ & 10563.43 & & & & & & & \\
\hline
\end{tabular}


TABLE 6. Yb II-Observed and classified lines-Continued

\begin{tabular}{|c|c|c|c|c|c|c|c|c|c|c|}
\hline 1 & 2 & 3 & 4 & 5 & 6 & 7 & 8 & 9 & 10 & 11 \\
\hline \multirow{2}{*}{$\begin{array}{l}\text { Wave- } \\
\text { length } \\
\text { air }\end{array}$} & \multicolumn{2}{|c|}{ Intensity } & \multirow{2}{*}{$\begin{array}{c}\text { Wave } \\
\text { number }\end{array}$} & \multirow[b]{2}{*}{$o-c$} & \multirow{2}{*}{ Classification } & \multirow{2}{*}{$\left|\begin{array}{l}\text { Zee- } \\
\text { man } \\
\text { type }\end{array}\right|$} & \multirow[b]{2}{*}{ lst $g$} & \multirow[b]{2}{*}{ 2nd $g$} & \multicolumn{2}{|c|}{$\begin{array}{c}\text { Zeeman } \\
\text { components }\end{array}$} \\
\hline & $\begin{array}{l}\text { Meggers } \\
\text { lamp }\end{array}$ & $\begin{array}{c}\text { Thomson } \\
\text { lamp }\end{array}$ & & & & & & & Strong $p$ & Strong $n$ \\
\hline A & & & $K$ & $K$ & & & & & & \\
\hline 9457.07 & & $6 h$ & 10571.20 & -0.01 & $82891_{2 \frac{1}{2}}-93463_{3 \frac{1}{2}}^{\circ}$ & & & & & \\
\hline 9445.45 & & $6 h$ & 10584.20 & & & & & & & \\
\hline 9442.75 & & $\begin{array}{r}7 \\
20\end{array}$ & $\begin{array}{l}10587.23 \\
10603.68\end{array}$ & -0.03 & $57561_{6 \frac{1}{2}}^{\circ}-68148_{5 \frac{1}{2}}^{\circ}$ & & & & & \\
\hline $\begin{array}{l}9428.10 \\
9423.79\end{array}$ & & $\begin{array}{l}30 \\
3 d\end{array}$ & $\begin{array}{l}10603.68 \\
10608.53\end{array}$ & -0.04 & $63028_{1 \frac{1}{2}}^{\circ}-73636_{0 \frac{1}{2}}$ & & & & & \\
\hline 9417.09 & & 5 & 10616.08 & +0.01 & $62163_{2 \frac{1}{2}}^{\circ}-72779_{3 \frac{1}{2}}$ & & & & & \\
\hline 9408.11 & & 4 & 10626.21 & & & & & & & \\
\hline 9405.75 & & 3 & 10628.88 & & & & & & & \\
\hline 9404.73 & & 10 & 10630.03 & & & & & & & \\
\hline 9397.79 & & $2 h$ & 10637.88 & & & & & & & \\
\hline 9390.61 & & $8 h$ & 10646.01 & & & & & & & \\
\hline 9388.32 & & 2 & 10648.61 & & & & & & & \\
\hline 9380.18 & & 15 & 10657.85 & & & & & & & \\
\hline 9378.29 & & 3 & 10660.00 & +0.03 & $52987_{1 \frac{1}{2}}^{\circ}-63647_{1 \frac{1}{2}}$ & & & & & \\
\hline 9372.12 & & 3 & 10667.02 & +0.02 & $55221_{1 \frac{1}{2}}^{\circ^{2}}-65888_{1 \frac{1}{2}}$ & & & & & \\
\hline 9367.86 & & 1 & 10671.87 & & & & & & & \\
\hline 9367.21 & & 2 & 10672.61 & & & & & & & \\
\hline 9352.58 & 8 & 80 & 10689.30 & +0.02 & $45012_{2 \frac{1}{2}}^{\circ}-55702_{1 \frac{1}{2}}^{\circ}$ & & & & & \\
\hline 9349.25 & 80 & 300 & 10693.11 & +0.05 & $22960_{\frac{1}{2}}-33653_{0 \frac{1}{2}}^{\circ}$ & & & & & \\
\hline 9341.89 & & $4 h$ & 10701.54 & & & & & & & \\
\hline 9334.67 & & 3 & 10709.81 & & & & & & & \\
\hline 9328.56 & & $3 h$ & 10716.83 & & & & & & & \\
\hline 9325.68 & & $20 h$ & 10720.14 & & & & $\because$ & & & \\
\hline 9324.60 & & $2 h$ & 10721.38 & & & & & & & \\
\hline 9321.14 & & $2 h$ & 10725.36 & & & & & & & \\
\hline 9320.30 & & 1 & 10726.33 & +0.02 & $24332_{2_{\frac{1}{2}}}-35059_{3 \frac{1}{2}}^{\circ}$ & & & & & \\
\hline 9316.58 & & 1 & 10730.61 & +0.01 & $54192_{5 \frac{1}{2}}^{\circ}-64923_{4 \frac{1}{2}}$ & & & & & \\
\hline 9314.88 & 2 & 40 & 10732.57 & & & & & & & \\
\hline 9302.74 & & 2 & 10746.57 & -0.04 & $50468_{5 \frac{1}{2}}^{\circ}-61214_{4 \frac{1}{2}}^{\circ}$ & & & & & \\
\hline 9301.33 & & 3 & 10748.20 & & & & & & & \\
\hline 9299.67 & & 1 & 10750.12 & & & & & & & \\
\hline 9298.74 & & 6 & 10751.20 & & & & & & & \\
\hline 9289.87 & 4 & 100 & 10761.46 & 0.00 & $44940_{1 \frac{1}{2}}^{\circ}-55702_{1 \frac{1}{2}}$ & & & & & \\
\hline 9287.36 & & 1 & 10764.37 & -0.06 & $53404_{2_{\frac{1}{2}}}-64169_{\frac{1}{2}}^{\circ}$ & & & & & \\
\hline 9272.68 & 3 & 30 & 10781.41 & & & & & & & \\
\hline 9271.84 & & 2 & 10782.39 & 0.00 & $58661_{2 \frac{1}{2}}^{\circ}-69443_{1 \frac{1}{2}}$ & & & & & \\
\hline 9263.73 & & $4 h$ & 10791.83 & & & & & & & \\
\hline 9261.11 & & $4 h$ & 10794.88 & 0.00 & $56621_{5 \frac{1}{2}}^{\circ}-67416_{4 \frac{1}{2}}$ & & & & & \\
\hline 9257.70 & & 8 & 10798.86 & -0.02 & $48024_{1 \frac{1}{2}}^{\circ}-58823_{2 \frac{1}{2}}$ & & & & & \\
\hline 9253.57 & 7 & 70 & 10803.68 & & & & & & & \\
\hline
\end{tabular}


TABLE 6. Yb II-Observed and classified lines-Continued

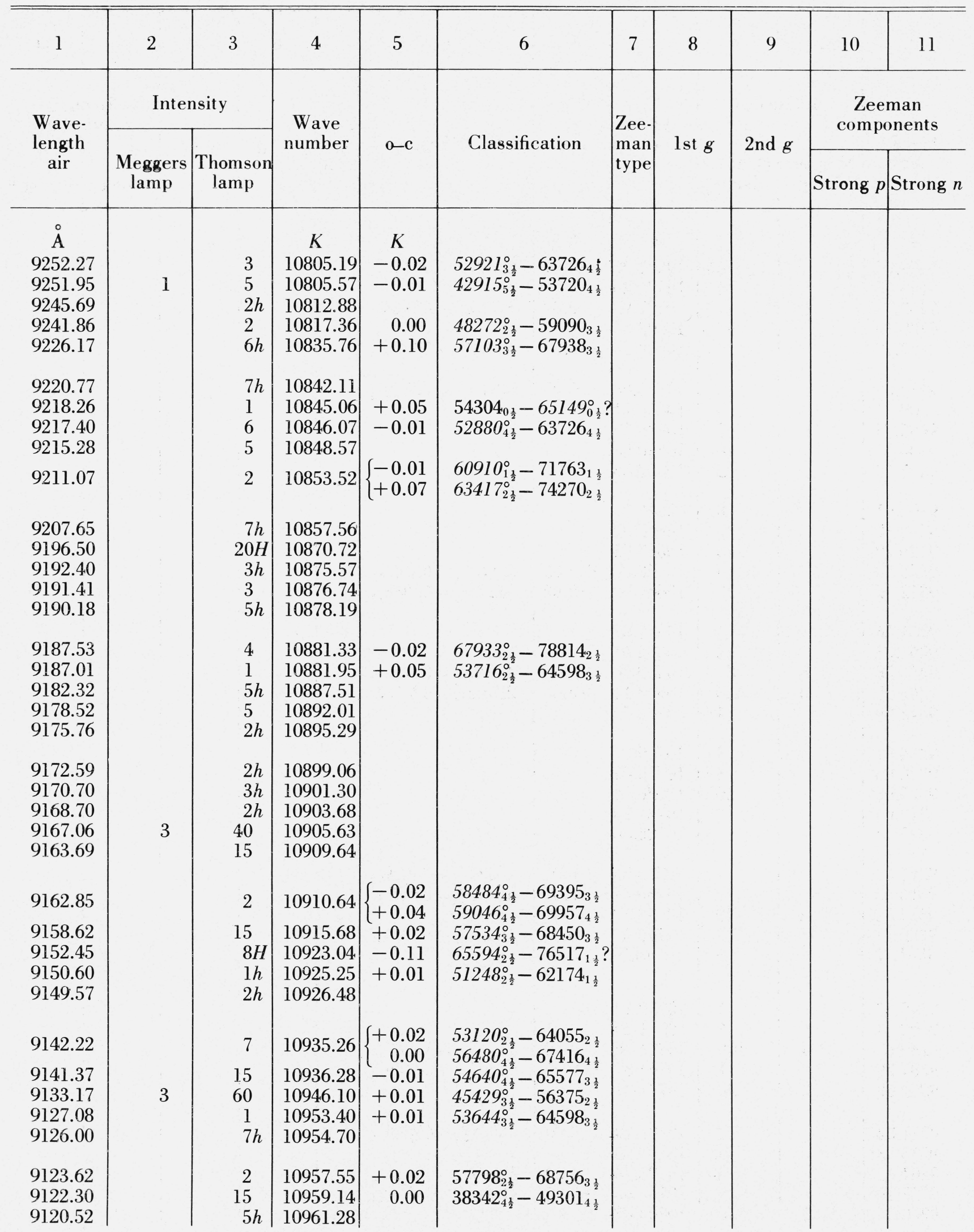


TABLE 6. Yb II-Observed and classified lines-Continued

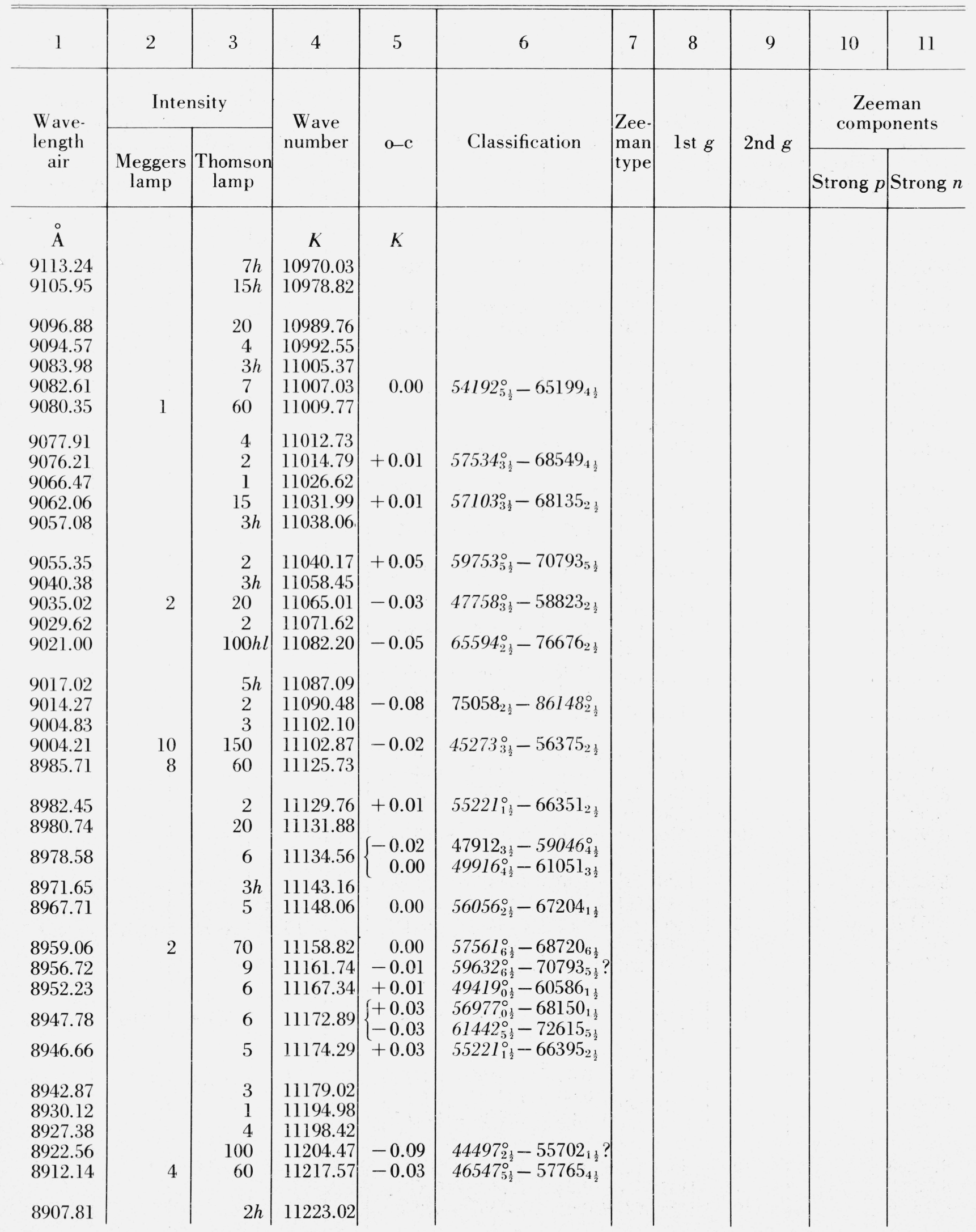


TABLE 6. Yb II-Observed and classified lines-Continued

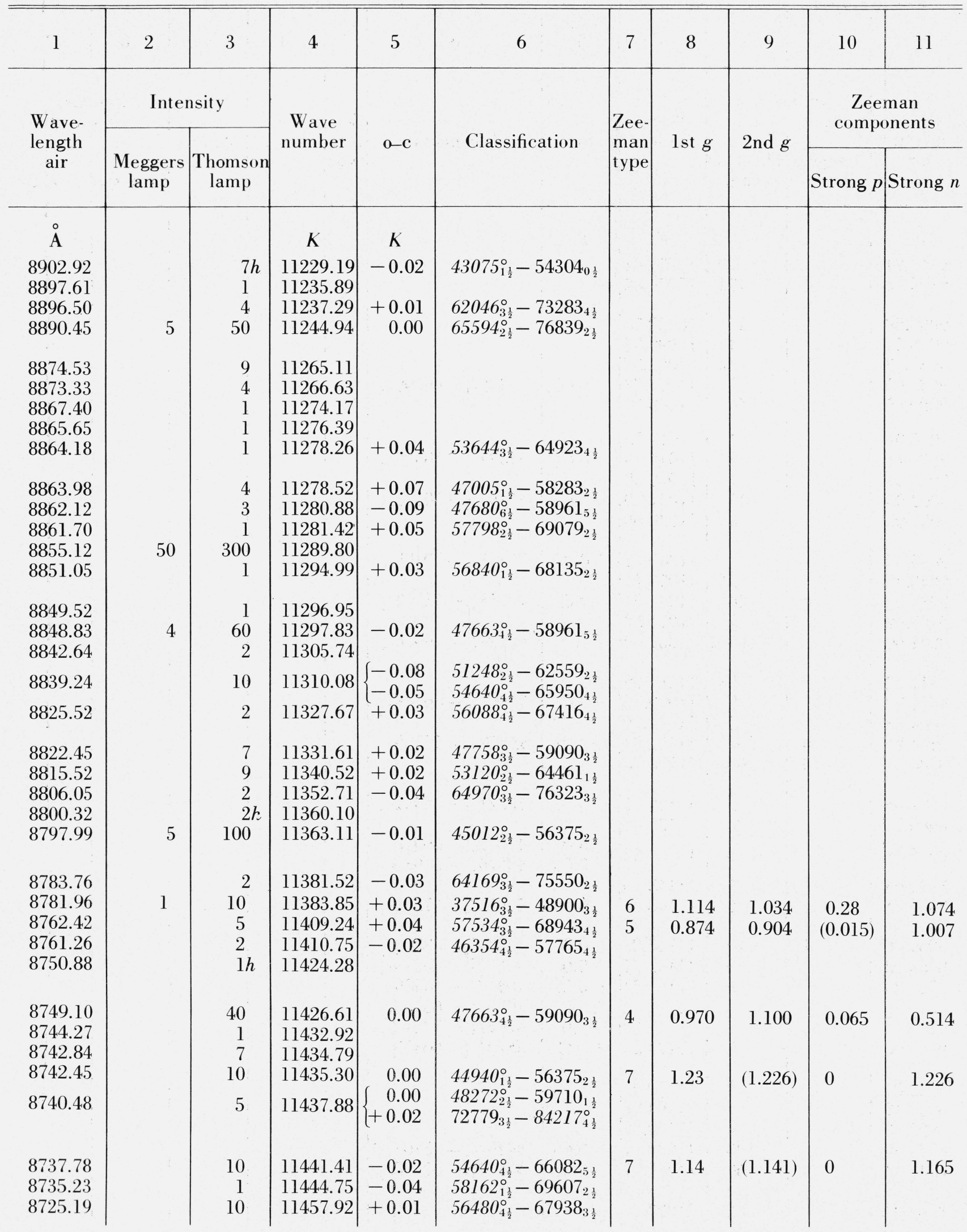


TABLE 6. Yb II-Observed and classified lines-Continued

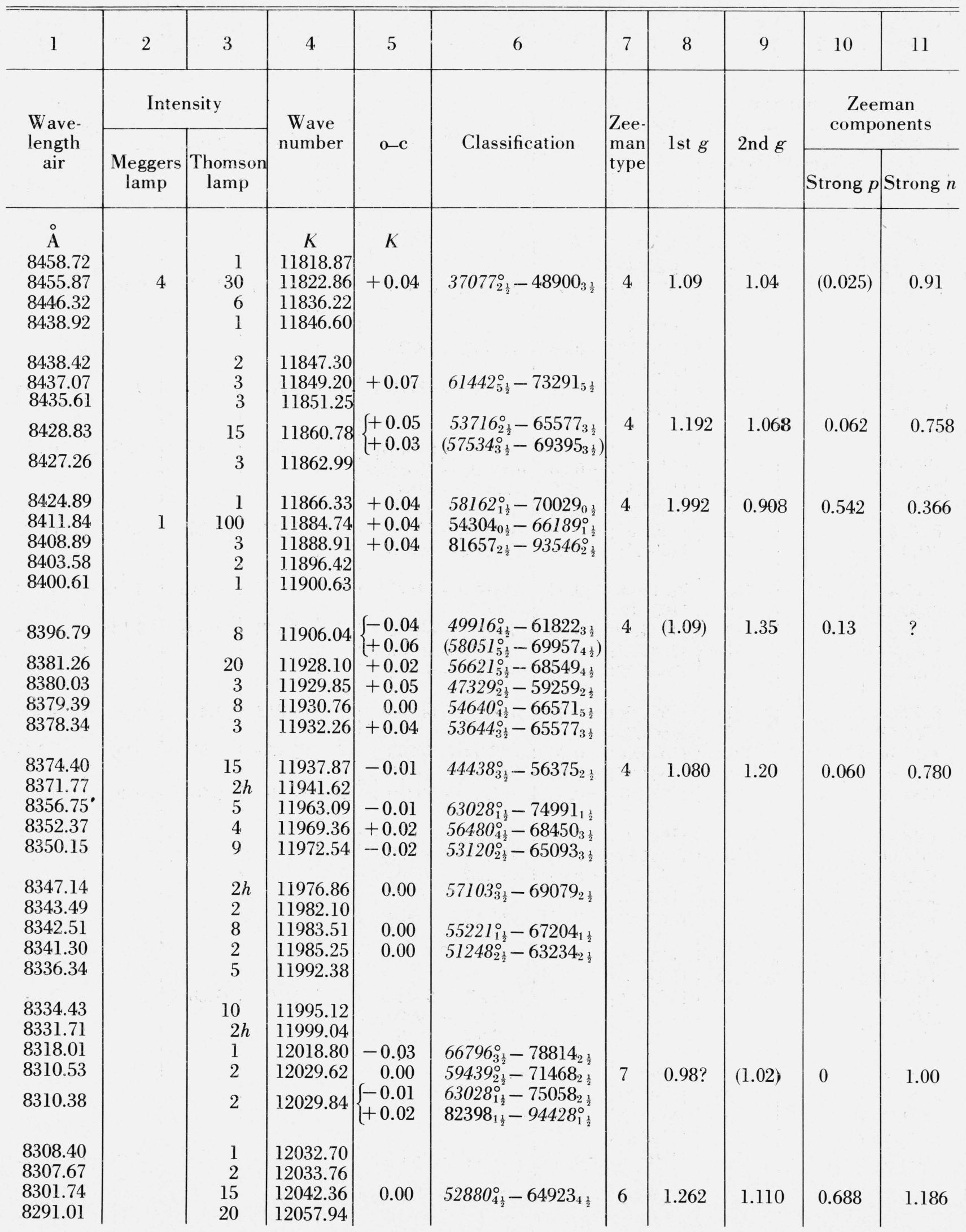


TABLE 6. Yb II-Observed and classified lines - Continued

\begin{tabular}{|c|c|c|c|c|c|c|c|c|c|c|}
\hline 1 & 2 & 3 & 4 & 5 & 6 & 7 & 8 & 9 & 10 & 11 \\
\hline \multirow{2}{*}{$\begin{array}{l}\text { Wave- } \\
\text { length } \\
\text { air }\end{array}$} & \multicolumn{2}{|c|}{ Intensity } & \multirow{2}{*}{$\begin{array}{l}\text { Wave } \\
\text { number }\end{array}$} & \multirow{2}{*}{$o-c$} & \multirow{2}{*}{ Classification } & \multirow{2}{*}{$\begin{array}{l}\text { Zee- } \\
\text { man } \\
\text { type }\end{array}$} & \multirow{2}{*}{ lst $g$} & \multirow{2}{*}{2 nd $g$} & \multicolumn{2}{|c|}{$\begin{array}{c}\text { Zeeman } \\
\text { components }\end{array}$} \\
\hline & $\begin{array}{c}\text { Meggers } \\
\text { lamp }\end{array}$ & $\begin{array}{c}\text { Thomson } \\
\text { lamp }\end{array}$ & & & & & & & Strong $p$ & Strong $n$ \\
\hline A & & & K & $K$ & & & & & & \\
\hline 8289.25 & & 3 & 12060.50 & +0.01 & $56088_{4 \frac{1}{2}}^{\circ}-68148_{5 \frac{1}{2}}$ & & & & & \\
\hline $\begin{array}{l}8283.78 \\
8282.14 \\
8280.60 \\
8276.29 \\
8267.90\end{array}$ & & $\begin{array}{l}15 \\
2 \\
2 \\
2 h \\
2\end{array}$ & $\begin{array}{l}12068.47 \\
12070.86 \\
12073.10 \\
12079.39 \\
12091.65\end{array}$ & $\begin{array}{l}+0.01 \\
+0.01\end{array}$ & $\begin{array}{l}56480_{4 \frac{1}{2}}^{\circ}-68549_{4 \frac{1}{2}} \\
57534_{3 \frac{1}{2}}^{\circ}-69607_{2 \frac{1}{2}}\end{array}$ & 6 & $0.758 ?$ & 1.160 & 1.815 & $(0.959 ?)$ \\
\hline $\begin{array}{l}8266.52 \\
8263.64 \\
8261.70 \\
8246.48 \\
8241.66\end{array}$ & 4 & $\begin{array}{c}7 \\
150 \\
1 \\
1 h \\
5\end{array}$ & $\begin{array}{l}12093.66 \\
12097.88 \\
12100.72 \\
12123.05 \\
12130.14\end{array}$ & $\begin{array}{r}0.00 \\
+0.01\end{array}$ & $\begin{array}{l}56056_{2 \frac{1}{2}}^{\circ}-68150_{1 \frac{1}{2}} \\
60649_{4 \frac{1}{2}}^{\circ}-72779_{3 \frac{1}{2}}^{\circ}\end{array}$ & 4 & & & $0.16 w$ & 1.03 \\
\hline $\begin{array}{l}8240.01 \\
8237.20 \\
8230.78 \\
8227.41 \\
8225.41\end{array}$ & & $\begin{array}{l}1 \\
2 \\
1 \\
1 \\
5\end{array}$ & $\begin{array}{l}12132.57 \\
12136.71 \\
12146.18 \\
12151.15 \\
12154.11\end{array}$ & $\begin{array}{r} \\
-0.03 \\
+0.04 \\
+0.01\end{array}$ & $\begin{array}{l}49727_{6 \frac{1}{2}}^{\circ}-61873_{6 \frac{1}{2}} \\
64365_{4 \frac{1}{2}}^{\circ}-76516_{4 \frac{1}{2}} \\
64169_{3 \frac{1}{2}}^{\circ}-76323_{3 \frac{1}{2}}\end{array}$ & 6 & & & 0.35 & \\
\hline $\begin{array}{l}8213.64 \\
8213.24 \\
8206.55 \\
8199.08 \\
8194.83\end{array}$ & & $\begin{array}{r}30 \\
10 \\
3 \\
1 \\
2\end{array}$ & $\begin{array}{l}12171.52 \\
12172.12 \\
12182.04 \\
12193.14 \\
12199.46\end{array}$ & $\begin{array}{r}0.00 \\
+0.04 \\
\end{array}$ & $\begin{array}{l}52921_{\frac{1}{2}}^{\circ}-65093_{3 \frac{1}{2}} \\
53716_{2 \frac{1}{2}}^{\circ}-65888_{1 \frac{1}{2}}\end{array}$ & $\begin{array}{l}6 \\
6 ?\end{array}$ & $\begin{array}{l}1.150 \\
1.16\end{array}$ & $\begin{array}{c}0.972 \\
(1.206)\end{array}$ & $\begin{array}{l}0.625 \\
d ?\end{array}$ & $\begin{array}{l}1.060 \\
1.10\end{array}$ \\
\hline $\begin{array}{l}8192.40 \\
8190.61 \\
8189.53 \\
8183.28 \\
8180.34\end{array}$ & & $\begin{array}{r}30 \\
10 \\
2 \\
1 \\
2 h\end{array}$ & $\begin{array}{l}12203.08 \\
12205.75 \\
12207.36 \\
12216.68 \\
12221.07\end{array}$ & +0.02 & $49008_{3 \frac{1}{2}}^{\circ}-61214_{4 \frac{1}{2}}$ & $\begin{array}{l}4 \\
7\end{array}$ & & & $\begin{array}{l}w \\
0\end{array}$ & $\begin{array}{l}1.03 \\
1.050\end{array}$ \\
\hline $\begin{array}{l}8179.01 \\
8178.46 \\
8178.14 \\
8173.60 \\
8171.82\end{array}$ & 1 & $\begin{array}{l}4 \\
4 \\
3 \\
3 h \\
30\end{array}$ & $\begin{array}{l}12223.06 \\
12223.88 \\
12224.36 \\
12231.15 \\
12233.81\end{array}$ & $\begin{array}{l}+0.03 \\
-0.05\end{array}$ & $\begin{array}{l}63417_{2 \frac{1}{2}}^{\circ}-75640_{3 \frac{1}{2}} \\
62046_{3 \frac{1}{2}}^{\circ}-74270_{2 \frac{1}{2}}\end{array}$ & 5 & & & $w$ & 1.40 \\
\hline $\begin{array}{l}8169.69 \\
8169.29 \\
8166.73 \\
8166.05 \\
8162.00\end{array}$ & & $\begin{array}{l}1 h \\
2 h \\
4 h \\
1 \\
2 h\end{array}$ & $\begin{array}{l}12237.00 \\
12237.60 \\
12241.44 \\
12242.46 \\
12248.53\end{array}$ & & & & & & & \\
\hline $\begin{array}{l}8158.49 \\
8157.40 \\
8155.12 \\
8149.08\end{array}$ & 5 & $\begin{array}{r}80 \\
150 \\
3 \\
15\end{array}$ & $\begin{array}{l}12253.80 \\
12255.44 \\
12258.86 \\
12267.95\end{array}$ & $\begin{array}{l}+0.02 \\
+0.04 \\
-0.03\end{array}$ & $\begin{array}{l}47005_{\frac{1}{2}}^{\circ}-59259_{2 \frac{1}{2}} \\
56500_{3 \frac{1}{2}}^{\circ}-68756_{3 \frac{1}{2}}^{\circ} \\
66205_{\frac{1}{2}}^{\circ}-78464_{1 \frac{1}{2}}^{\circ}\end{array}$ & $\begin{array}{l}5 \\
6 \\
6\end{array}$ & $\begin{array}{c}0.982 \\
(1.352)\end{array}$ & $\begin{array}{l}1.100 \\
1.316\end{array}$ & $\begin{array}{l}0.059 \\
w \\
0.150\end{array}$ & $\begin{array}{l}1.276 \\
1.334 \\
\\
1.20\end{array}$ \\
\hline
\end{tabular}


TABLE 6. Yb II-Observed and classified lines-Continued

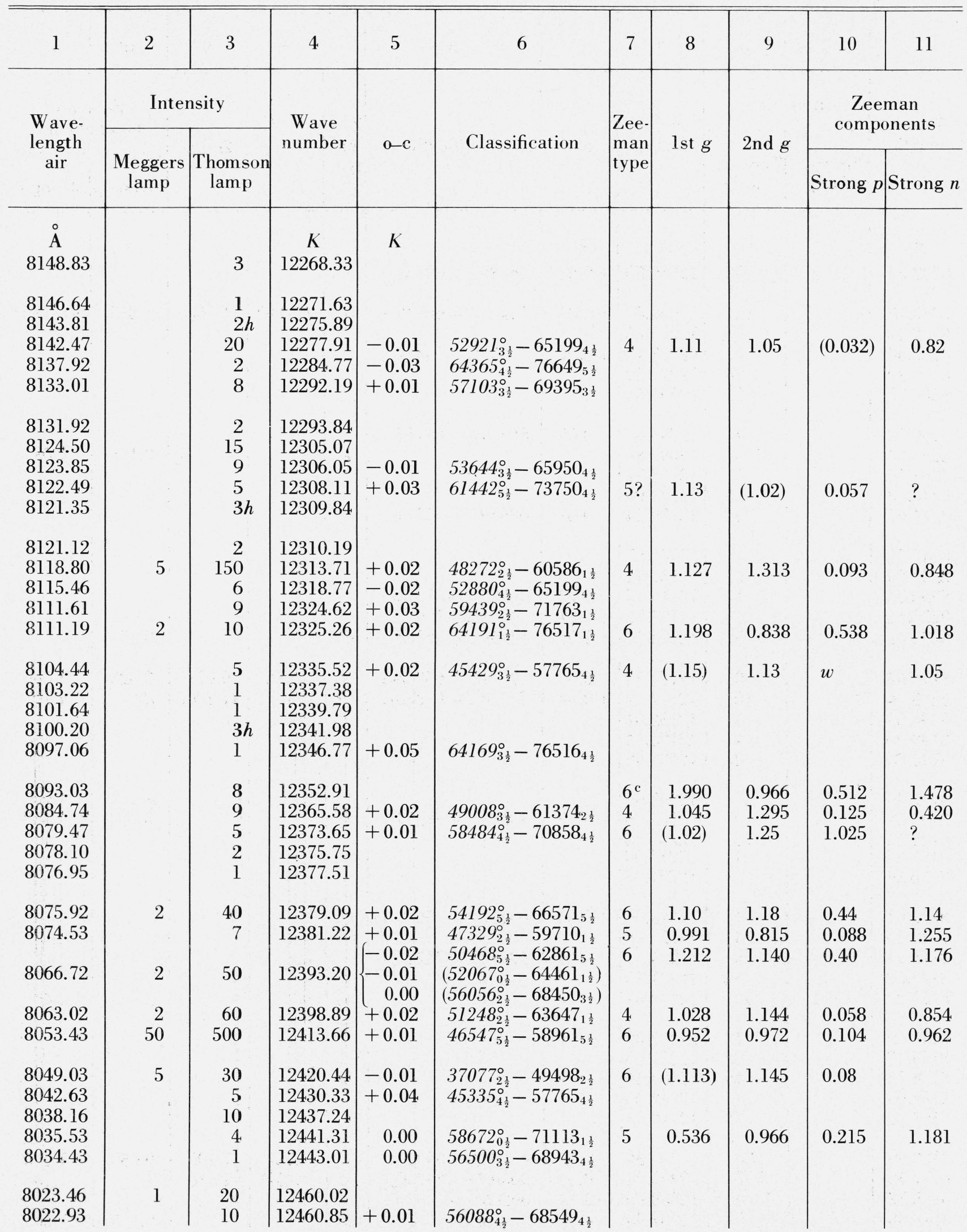


TABLE 6. Yb II-Observed and classified lines-Continued

\begin{tabular}{|c|c|c|c|c|c|c|c|c|c|c|}
\hline 1 & 2 & 3 & 4 & 5 & 6 & 7 & 8 & 9 & 10 & 11 \\
\hline \multirow{2}{*}{$\begin{array}{l}\text { Wave- } \\
\text { length } \\
\text { air }\end{array}$} & \multicolumn{2}{|c|}{ Intensity } & \multirow{2}{*}{$\begin{array}{c}\text { Wave } \\
\text { number }\end{array}$} & \multirow[b]{2}{*}{$o-c$} & \multirow[b]{2}{*}{ Classification } & \multirow{2}{*}{$\begin{array}{l}\text { Zee- } \\
\text { man } \\
\text { type }\end{array}$} & \multirow[b]{2}{*}{ lst $g$} & \multirow[b]{2}{*}{ 2nd $g$} & \multicolumn{2}{|c|}{$\begin{array}{c}\text { Zeeman } \\
\text { components }\end{array}$} \\
\hline & $\begin{array}{l}\text { Meggers } \\
\text { lamp }\end{array}$ & $\begin{array}{c}\text { Thomson } \\
\text { lamp }\end{array}$ & & & & & & & Strong $p$ & Strong $n$ \\
\hline А & & & $K$ & $K$ & & & & & & \\
\hline 8021.62 & 3 & 150 & 12462.88 & 0.00 & $56480_{4 \frac{1}{2}}^{\circ}-68943_{4 \frac{1}{2}}$ & 6 & 0.747 & 0.859 & 0.508 & 0.803 \\
\hline 8013.17 & & 15 & 12476.02 & +0.02 & $55462_{3 \frac{1}{2}}^{\circ}-67938_{3 \frac{1}{2}}$ & 6 & 0.878 & 0.954 & 0.263 & 0.916 \\
\hline 8009.53 & 4 & 50 & 12481.69 & 0.00 & $47228_{0 \frac{1}{2}}^{\circ}-59710_{1 \frac{1}{2}}^{\circ}$ & 4 & 1.035 & 0.917 & 0.059 & $0.858 ?$ \\
\hline 8007.83 & 15 & 300 & 12484.34 & 0.00 & $64191_{1 \frac{1}{2}}^{\circ}-76676_{2 \frac{1}{2}}$ & 4 & 1.190 & 1.088 & 0.051 & 0.935 \\
\hline 8002.73 & 1 & 20 & 12492.30 & 0.00 & $45273_{3 \frac{1}{2}}^{\circ}-57765_{4 \frac{1}{2}}$ & 4 & $1.053 ?$ & $1.001 ?$ & $(0.026)$ & $0.818 ?$ \\
\hline 8001.30 & & $2 h$ & 12494.53 & +0.04 & $48556_{3 \frac{1}{2}}^{\circ}-61051_{3 \frac{1}{2}}$ & & & & & \\
\hline 7994.90 & & 30 & 12504.53 & +0.01 & $57103_{3 \frac{1}{2}}^{\circ}-69607_{2 \frac{1}{2}}^{\circ}$ & 4 & 1.084 & 1.208 & 0.062 & 0.774 \\
\hline 7984.31 & & 4 & 12521.12 & & & & & & & \\
\hline 7968.09 & 1 & 6 & 12546.61 & -0.01 & $45737_{1 \frac{1}{2}}^{\circ}-58283_{2 \frac{1}{2}}$ & & & & & \\
\hline 7964.12 & 2 & 80 & 12552.86 & -0.02 & $53322_{7 \frac{1}{2}}^{\circ}-65875_{6 \frac{1}{2}}$ & 4 & 1.090 & 1.222 & 0.066 & 0.232 \\
\hline 7958.80 & & 3 & 12561.25 & +0.02 & $58661_{2 \frac{1}{2}}^{\circ}-71222_{3 \frac{1}{2}}^{\circ}$ & & & & & \\
\hline 7958.48 & 2 & 40 & 12561.76 & 0.00 & $48024_{1 \frac{1}{2}}^{\circ}-60586_{1 \frac{1}{2}}^{\circ}$ & 6 & 1.35 & 1.31 & 0.070 & 1.33 \\
\hline 7950.35 & 2 & 60 & 12574.60 & -0.02 & $57561_{6 \frac{1}{2}}^{\circ}-70136_{6 \frac{1}{2}}$ & 6 & & & & \\
\hline 7950.01 & & 2 & 12575.14 & & & & & & & \\
\hline 7947.60 & 1 & 40 & 12578.95 & -0.01 & $52987_{1^{\frac{1}{2}}}^{\circ}-65566_{0 \frac{1}{2}}$ & 4 & 1.129 & 1.423 & 0.147 & 0.982 \\
\hline 7930.04 & & 8 & 12606.81 & -0.01 & $46354_{4 \frac{1}{2}}^{\circ}-58961_{5 \frac{1}{2}}$ & 4 & 1.28 & 0.98 & 0.146 & 0.37 \\
\hline 7928.71 & & 7 & 12608.92 & $\left\{\begin{array}{l}+0.04 \\
-0.07\end{array}\right.$ & $\begin{array}{c}58661_{2 \frac{1}{2}}^{\circ}-71270_{2 \frac{1}{2}} \\
\left(66205^{\circ}-78814^{2}\right.\end{array}$ & & & & & \\
\hline 7913.30 & & 6 & 12633.48 & -0.01 & $60649_{4 \frac{1}{2}}^{\circ}-73283_{4 \frac{1}{2}}^{\circ}$ & & & & & \\
\hline 7912.46 & & 10 & 12634.82 & -0.01 & $53716_{2 \frac{1}{2}}^{\circ}-66351_{2 \frac{1}{2}}$ & & & & & \\
\hline 7906.25 & & $2 h$ & 12644.74 & & & & & & & \\
\hline 7904.82 & 10 & 200 & 12647.03 & 0.00 & $64191_{1 \frac{1}{2}}^{\circ}-76839_{2 \frac{1}{2}}$ & 4 & 1.200 & 1.108 & 0.046 & 0.970 \\
\hline 7899.54 & & 5 & 12655.48 & -0.01 & $52921_{3 \frac{1}{2}}^{\circ}-65577_{3 \frac{1}{2}}$ & 6 & 1.17 & (1.055) & 0.407 & $?$ \\
\hline 7897.90 & & 2 & 12658.11 & +0.02 & $48556_{3 \frac{1}{2}}^{\circ}-61214_{4 \frac{1}{2}}$ & & & & & \\
\hline 7891.93 & & 1 & 12667.69 & +0.04 & $56088_{4 \frac{1}{2}}^{\circ}-68756_{3 \frac{1}{2}}$ & & & & & \\
\hline 7874.10 & 1 & 20 & 12696.37 & +0.01 & $52880_{4 \frac{1}{2}}^{\circ}-65577_{3 \frac{1}{2}}^{\circ}$ & 5 & 1.261 & 1.049 & 0.106 & 2.003 \\
\hline 7865.13 & & 7 & 12710.85 & +0.01 & $48503_{5 \frac{1}{2}}^{\circ}-61214_{4 \frac{1}{2}}$ & 5 & 1.151 & 1.093 & $(0.029)$ & 1.414 \\
\hline 7856.20 & & 4 & 12725.30 & +0.01 & $65739_{2 \frac{1}{2}}^{\circ}-78464_{1 \frac{1}{2}}^{\circ}$ & 4 & 0.880 & 1.176 & 0.148 & 0.436 \\
\hline 7849.85 & & 6 & 12735.59 & +0.01 & $46354_{4 \frac{1}{2}}^{\circ}-59090_{3 \frac{1}{2}}$ & & & & & \\
\hline 7848.49 & & 15 & 12737.80 & -0.02 & $58484_{4 \frac{1}{2}}^{\circ}-71222_{3 \frac{1}{2}}$ & 4 & 1.040 & 1.122 & $(0.041)$ & 0.751 \\
\hline 7840.45 & 1 & 40 & 12750.86 & +0.03 & $53644_{3 \frac{1}{2}}^{\circ}-66395_{2 \frac{1}{2}}$ & 4 & 1.179 & 1.231 & $(0.026)$ & 1.048 \\
\hline 7839.17 & & $1 h$ & 12752.94 & & & & & & & \\
\hline 7833.02 & & 9 & 12762.96 & +0.01 & $59439_{2 \frac{1}{2}}^{\circ}-72202_{3 \frac{1}{2}}$ & 4 & 0.991 & 0.855 & 0.068 & 0.515 \\
\hline 7825.52 & 1 & 50 & 12775.19 & -0.02 & $54640_{4 \frac{1}{2}}^{\circ}-67416_{4 \frac{1}{2}}$ & 6 & 1.132 & 1.260 & 0.582 & 1.195 \\
\hline 7824.64 & & 3 & 12776.63 & $\left\{\begin{array}{l}-0.03 \\
+0.04\end{array}\right.$ & $\begin{array}{l}64970_{\frac{11}{2}}^{\circ}-77747_{4 \frac{1}{2}}^{\circ} \\
76839_{2 \frac{1}{2}}^{\circ}-89615_{2 \frac{1}{2}}^{\circ}\end{array}$ & & & & & \\
\hline 7815.52 & & 5 & 12791.53 & $\left\{\begin{array}{l}+0.01 \\
-0.06\end{array}\right.$ & $\begin{array}{c}58162_{\frac{1}{1 \frac{1}{2}}}^{\circ}-70954_{2 \frac{1}{2}} \\
\left(46169_{4 \frac{1}{2}}^{\circ}-58961_{\left.5 \frac{1}{2}\right)}\right.\end{array}$ & 5 & 0.95 & (1.014) & $w$ & 1.107 \\
\hline 7811.96 & 1 & 8 & 12797.36 & +0.01 & $40917_{4 \frac{1}{2}}^{\circ}-53715_{3 \frac{1}{2}}$ & 4 & 0.965 & 1.175 & 0.105 & 0.230 \\
\hline 7808.64 & & 4 & 12802.80 & 0.00 & $40917_{4 \frac{1}{2}}^{\circ}-53720_{4 \frac{1}{2}}$ & 6 & $(0.967)$ & 1.265 & 1.338 & $?$ \\
\hline
\end{tabular}


TABLE 6. Yb II-Observed and classified lines - Continued

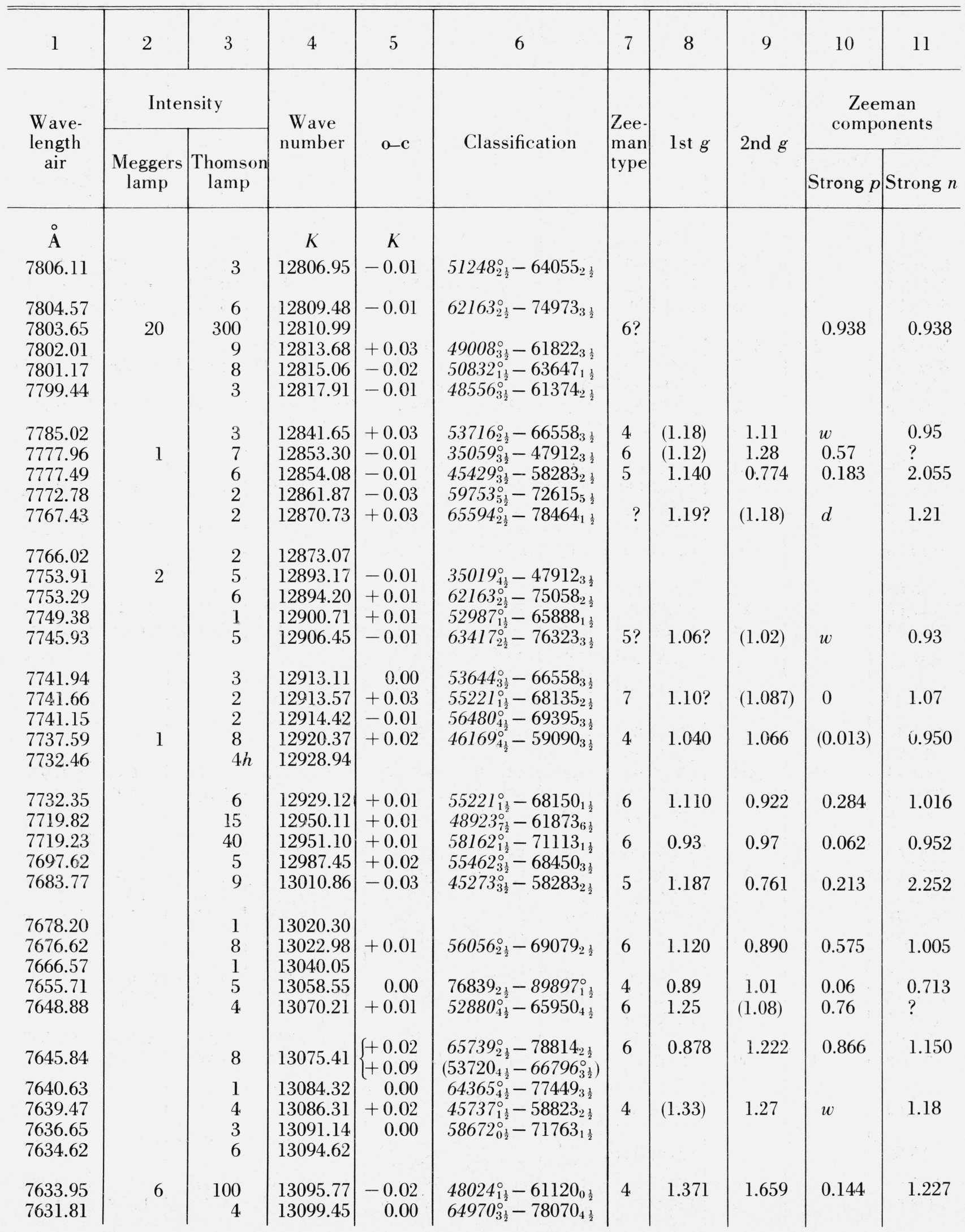


TABLE 6. Yb II-Observed and classified lines-Continued

\begin{tabular}{|c|c|c|c|c|c|c|c|c|c|c|}
\hline 1 & 2 & 3 & 4 & 5 & 6 & 7 & 8 & 9 & 10 & 11 \\
\hline \multirow{3}{*}{$\begin{array}{l}\text { Wave- } \\
\text { length } \\
\text { air }\end{array}$} & \multicolumn{2}{|c|}{ Intensity } & \multirow{3}{*}{$\begin{array}{c}\text { Wave } \\
\text { number }\end{array}$} & \multirow{3}{*}{$o-c$} & \multirow{3}{*}{ Classification } & \multirow{3}{*}{$\mid \begin{array}{l}Z e e- \\
\text { man } \\
\text { type }\end{array}$} & \multirow{3}{*}{ lst $g$} & \multirow{3}{*}{2 nd $g$} & \multirow{2}{*}{\multicolumn{2}{|c|}{$\begin{array}{c}\text { Zeeman } \\
\text { components }\end{array}$}} \\
\hline & \multirow[b]{2}{*}{$\begin{array}{c}\text { Meggers } \\
\text { lamp }\end{array}$} & \multirow[b]{2}{*}{$\begin{array}{c}\text { Thomson } \\
\text { lamp }\end{array}$} & & & & & & & & \\
\hline & & & & & & & & & Strong $p$ & Strong $n$ \\
\hline$\AA$ & & & $K$ & K & & & & & & \\
\hline 7630.87 & & 1 & 13101.06 & +0.02 & $60649_{4 \frac{1}{2}}^{\circ}-73750_{4 \frac{1}{2}}$ & & & & & \\
\hline 7630.48 & & 9 & 13101.73 & +0.01 & $48272_{2 \frac{1}{2}}^{\circ}-61374_{2 \frac{1}{2}}$ & & & & & \\
\hline 7630.00 & & 8 & 13102.55 & +0.01 & $58661_{2 \frac{1}{2}}^{\circ}-71763_{1 \frac{1}{2}}$ & & & & & \\
\hline 7627.45 & 1 & 50 & 13106.93 & +0.03 & $56500_{3 \frac{1}{2}}^{\circ}-69607_{2 \frac{1}{2}}$ & 5 & 1.355 & 1.221 & 0.067 & 1.690 \\
\hline 7627.24 & 1 & 70 & 13107.29 & +0.03 & $58162_{1 \frac{1}{2}}^{\circ}-71270_{2 \frac{1}{2}}$ & 7 & 0.93 & $(0.91)$ & & 0.88 \\
\hline 7616.50 & & 2 & 13125.78 & +0.02 & $61442_{5 \frac{1}{2}}^{\circ}-74568_{4 \frac{1}{2}}$ & & & & & \\
\hline 7611.69 & & 15 & 13134.07 & -0.01 & $49727_{6 \frac{1}{2}}^{\circ}-62861_{5 \frac{1}{2}}$ & 5 & 1.200 & $(1.140)$ & $?$ & 1.530 \\
\hline 7607.09 & & 2 & 13142.01 & +0.02 & $63028^{\circ} \frac{1}{2}-76170_{2 \frac{1}{2}}$ & & & & & \\
\hline 7599.13 & & 20 & 13155.78 & +0.02 & $57798_{2 \frac{1}{2}}^{\circ}-70954_{2 \frac{1}{2}}$ & 6 & 1.148 & 1.000 & 0.368 & 1.074 \\
\hline 7582.93 & & 40 & 13183.88 & -0.02 & $24332_{2 \frac{1}{2}}-37516_{3 \frac{1}{2}}^{\circ}$ & 4 & 1.184 & 1.120 & $(0.032)$ & 0.960 \\
\hline 7579.97 & 1 & 60 & 13189.03 & +0.03 & $56840_{1 \frac{1}{2}}^{\circ}-70029_{0 \frac{1}{2}}$ & 5 & 0.910 & 0.682 & 0.115 & 1.024 \\
\hline 7572.81 & & 5 & 13201.50 & 0.00 & $52880_{4 \frac{1}{2}}^{\circ}-66082_{5 \frac{1}{2}}$ & & & & & \\
\hline 7566.65 & & 15 & 13212.25 & +0.03 & $51248_{2 \frac{1}{2}}^{\circ}-64461_{1 \frac{1}{2}}$ & 4 & 1.034 & 1.178 & 0.072 & 0.818 \\
\hline 7560.18 & & 1 & 13223.56 & +0.04 & $54192_{5 \frac{1}{2}}^{\circ}-67416_{4 \frac{1}{2}}$ & & & & & \\
\hline 7556.13 & 1 & 40 & 13230.65 & +0.02 & $53120^{\circ}{ }_{2 \frac{1}{2}}^{2}-66351_{2 \frac{1}{2}}$ & 6 & $0.819 ?$ & $0.645 ?$ & 0.438 & $0.732 ?$ \\
\hline 7555.14 & 1 & 60 & 13232.38 & +0.04 & $57561_{6 \frac{1}{2}}^{-2}-70793_{5 \frac{1}{2}}^{-2}$ & 4 & 1.032 & 1.204 & 0.086 & $0.086 ?$ \\
\hline 7541.10 & 5 & 80 & 13257.01 & -0.01 & $47329_{2 \frac{1}{2}}^{\circ}-60586_{1 \frac{1}{2}}^{\circ}$ & 4 & 0.980 & 1.314 & 0.167 & 0.480 \\
\hline 7537.40 & & 1 & 13263.52 & +0.01 & $48900_{3 \frac{1}{2}}-62163_{2 \frac{1}{2}}^{\circ}$ & & & & & \\
\hline 7533.09 & & 3 & 13271.11 & -0.01 & $45012_{2 \frac{1}{2}}^{\circ}-58283_{2 \frac{1}{2}}$ & & & & & \\
\hline 7530.78 & & 3 & 13275.18 & +0.04 & $53120_{2 \frac{1}{2}}^{\circ}-66395_{2 \frac{1}{2}}^{\circ}$ & & & & & \\
\hline 7528.07 & & 8 & 13279.96 & +0.03 & $64169_{3 \frac{1}{2}}^{\circ}-77449_{3 \frac{1}{2}}^{\circ}$ & & & & & \\
\hline 7520.94 & & 4 & 13292.55 & +0.03 & $47758_{3 \frac{1}{2}}^{\circ}-61051_{3 \frac{1}{2}}$ & & & & & \\
\hline 7520.46 & & 15 & 13293.40 & +0.04 & $55462_{3 \frac{1}{2}}^{\circ}-68756_{3 \frac{1}{2}}^{\circ}$ & 6 & 0.88 & 1.32 & 1.560 & 1.10 \\
\hline 7517.94 & & 2 & 13297.85 & -0.01 & $54640_{4 \frac{1}{2}}^{\circ}-67938_{3 \frac{1}{2}}$ & & & & & \\
\hline 7516.24 & & 2 & 13300.86 & +0.04 & $43075_{1 \frac{1}{2}}^{\circ}-56375_{2 \frac{1}{2}}$ & & & & & \\
\hline 7512.86 & & 15 & 13306.85 & +0.04 & $56088_{4 \frac{1}{2}}^{\circ}-69395_{3 \frac{1}{2}}$ & & & & & \\
\hline 7508.07 & & 8 & 13315.34 & +0.01 & $57798_{2 \frac{1}{2}}^{\circ}-71113_{1 \frac{1}{2}}$ & 5 & 1.150 & 0.970 & 0.090 & 1.420 \\
\hline 7503.32 & & $1 h$ & 13323.76 & +0.03 & $57534_{3 \frac{1}{2}}^{\circ}-70858_{4 \frac{1}{2}}$ & & & & & \\
\hline 7501.33 & & $1 h$ & 13327.30 & +0.01 & $44438_{3 \frac{1}{2}}^{\circ}-57765_{4 \frac{1}{2}}$ & & & & & \\
\hline 7500.09 & & $3 h$ & 13329.50 & & & & & & & \\
\hline 7496.24 & & 20 & 13336.35 & +0.01 & $56621_{5 \frac{1}{2}}^{\circ}-69957_{4 \frac{1}{2}}$ & & & & & \\
\hline 7495.15 & & 2 & 13338.29 & 0.00 & $56056_{2 \frac{1}{2}}^{\circ}-69395_{3 \frac{1}{2}}^{\circ}$ & & & & & 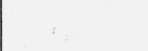 \\
\hline 7494.37 & & 3 & 13339.68 & +0.04 & $59777_{1 \frac{1}{2}}^{\circ}-73116_{1 \frac{1}{2}}$ & & & & & : \\
\hline 7492.34 & 1 & 6 & 13343.29 & -0.01 & $44940_{1 \frac{1}{2}}^{\circ}-58283_{2 \frac{1}{2}}$ & 4 & 1.237 & 0.767 & 0.235 & 0.062 \\
\hline 7483.19 & & 2 & 13359.61 & -0.06 & $70858_{4 \frac{1}{2}}-84217_{4 \frac{1}{2}}^{\circ}$ & & & & & \\
\hline 7482.80 & & 1 & 13360.30 & -0.04 & $60910_{1 \frac{1}{2}}^{\circ}-74270_{2 \frac{1}{2}}$ & & & & & \\
\hline 7481.04 & & 6 & 13363.45 & 0.00 & $52987_{1 \frac{1}{2}}^{\circ}-66351_{2 \frac{1}{2}}$ & 7 & $1.13 ?$ & $(1.125)$ & 0 & 1.12 \\
\hline 7477.86 & 1 & 5 & 13369.13 & -0.01 & $40035_{3 \frac{1}{2}}^{\circ}-53404_{2 \frac{1}{2}}^{2}$ & & & & & \\
\hline 7477.61 & 7 & 200 & 13369.58 & 0.00 & $48503_{5 \frac{1}{2}}^{\circ}-61873_{6 \frac{1}{2}}$ & 7 & $(1.10)$ & 1.10 & 0 & 1.08 \\
\hline
\end{tabular}


TABLE 6. Y Y II-Observed and classified lines-Continued

\begin{tabular}{|c|c|c|c|c|c|c|c|c|c|c|}
\hline 1 & 2 & 3 & 4 & 5 & 6 & 7 & 8 & 9 & 10 & 11 \\
\hline \multirow{2}{*}{$\begin{array}{l}\text { Wave- } \\
\text { length } \\
\text { air }\end{array}$} & \multicolumn{2}{|c|}{ Intensity } & \multirow{2}{*}{$\begin{array}{l}\text { Wave } \\
\text { number }\end{array}$} & \multirow[b]{2}{*}{$o-c$} & \multirow{2}{*}{ Classification } & \multirow{2}{*}{$\begin{array}{l}\text { Zee- } \\
\text { man } \\
\text { type }\end{array}$} & \multirow{2}{*}{ lst $g$} & \multirow{2}{*}{ 2nd $g$} & \multicolumn{2}{|c|}{$\begin{array}{c}\text { Zeeman } \\
\text { components }\end{array}$} \\
\hline & $\begin{array}{l}\text { Meggers } \\
\text { lamp }\end{array}$ & $\begin{array}{c}\text { Thomson } \\
\text { lamp }\end{array}$ & & & & & & & Strong $p$ & Strong $n$ \\
\hline $\begin{array}{c}\text { A } \\
7475.24 \\
7473.32 \\
7468.09 \\
7467.58^{\mathrm{a}}\end{array}$ & 40 & $\begin{array}{r}2 \\
5 \\
8 \\
30\end{array}$ & $\begin{array}{c}K \\
13373.81 \\
13377.25 \\
13386.62 \\
13387.53\end{array}$ & $\begin{array}{l}+0.01 \\
+0.01\end{array}$ & $\begin{array}{l}66205_{2 \frac{1}{2}}^{\circ}-79583_{2 \frac{1}{2}} \\
56056_{2 \frac{1}{2}}^{\circ}-69443_{1 \frac{1}{2}}^{\circ}\end{array}$ & & & & & \\
\hline $\begin{array}{l}7464.10 \\
7456.20 \\
7451.21 \\
7449.60\end{array}$ & 5 & $\begin{array}{r}60 \\
2 \\
1 \\
1\end{array}$ & $\begin{array}{l}13393.77 \\
13407.96 \\
13416.94 \\
13419.84\end{array}$ & $\begin{array}{r}+0.01 \\
0.00 \\
-0.01 \\
+0.02\end{array}$ & $\begin{array}{l}45429_{3 \frac{1}{2}}^{\circ}-58823_{2 \frac{1}{2}}^{\circ} \\
52987_{1 \frac{1}{2}}^{\circ}-66395_{2 \frac{1}{2}} \\
66189_{1 \frac{1}{2}}^{\circ}-79605_{1 \frac{1}{2}} \\
57534_{3 \frac{1}{2}}^{\circ}-70954_{2 \frac{1}{2}}^{\circ}\end{array}$ & 4 & 1.149 & 1.275 & 0.063 & 0.834 \\
\hline 7447.37 & 1 & 60 & 13423.86 & $\left\{\begin{array}{l}+0.01 \\
-0.03\end{array}\right.$ & $\begin{array}{c}57798_{2 \frac{1}{2}}^{\circ}-71222_{3 \frac{1}{2}}^{2} \\
\left(70793_{5 \frac{1}{2}}^{2}-84217_{4 \frac{1}{2}}^{\circ}\right)\end{array}$ & 7 & 1.16 & (1.15) & 0 & 1.136 \\
\hline $\begin{array}{l}7444.20 \\
7439.84 \\
7433.39 \\
7429.51 \\
7429.10\end{array}$ & $\begin{array}{l}1 \\
4 \\
2\end{array}$ & $\begin{array}{r}2 \\
80 \\
30 \\
40 \\
2\end{array}$ & $\begin{array}{l}13429.58 \\
13437.45 \\
13449.11 \\
13456.13 \\
13456.87\end{array}$ & $\begin{array}{l}-0.01 \\
+0.03 \\
-0.01 \\
+0.01 \\
+0.02\end{array}$ & $\begin{array}{l}52921_{3 \frac{1}{2}}^{\circ}-66351_{2 \frac{1}{2}} \\
53120_{2 \frac{1}{2}}^{\circ}-66558_{3 \frac{1}{2}} \\
46169_{4 \frac{1}{2}}^{\circ}-59618_{3 \frac{1}{2}}^{\circ} \\
47758_{3 \frac{1}{2}}^{\circ}-61214_{4 \frac{1}{2}}^{\circ} \\
56500_{3 \frac{1}{2}}^{\circ}-69957_{4 \frac{1}{2}}^{\circ}\end{array}$ & $\begin{array}{l}5 \\
5 \\
4\end{array}$ & $\begin{array}{l}0.943 \\
1.115 \\
1.067\end{array}$ & $\begin{array}{l}1.098 \\
1.057 \\
1.029\end{array}$ & $\begin{array}{c}0.078 \\
(0.028) \\
(0.019)\end{array}$ & $\begin{array}{l}1.485 \\
1.315 \\
0.896\end{array}$ \\
\hline $\begin{array}{l}7422.15 \\
7421.04\end{array}$ & 40 & $\begin{array}{r}200 \\
2\end{array}$ & $\begin{array}{l}13469.48 \\
13471.49\end{array}$ & $\begin{array}{r}0.00 \\
-0.01\end{array}$ & $\begin{array}{l}35831_{5 \frac{1}{2}}^{\circ}-49301_{4 \frac{1}{2}} \\
57798_{2 \frac{1}{2}}^{\circ}-71270_{2 \frac{1}{2}}\end{array}$ & 5 & $(1.214)$ & 1.20 & $w$ & 1.295 \\
\hline $\begin{array}{l}7419.60 \\
7419.06\end{array}$ & 1 & $\begin{array}{r}40 \\
8\end{array}$ & $\begin{array}{l}13474.10 \\
13475.09\end{array}$ & $\begin{array}{r}0.00 \\
-0.01\end{array}$ & $\begin{array}{l}52921_{3 \frac{1}{2}}^{\circ}-66395_{2 \frac{1}{2}} \\
52987_{1 \frac{1}{2}}^{\circ}-66462_{0 \frac{1}{2}}\end{array}$ & 4 & 1.155 & 1.233 & $(0.039)$ & 0.96 \\
\hline 7418.49 & & 4 & 13476.12 & $\left\{\begin{array}{l}-0.01 \\
-0.02\end{array}\right.$ & $\begin{array}{l}50468_{5 \frac{1}{2}}^{\circ}-63944_{5 \frac{1}{2}}^{\circ} \\
62163_{2 \frac{1}{2}}^{\circ}-75640_{3 \frac{1}{2}}\end{array}$ & & & & & \\
\hline 7418.15 & & 3 & 13476.74 & +0.02 & $56480_{4 \frac{1}{2}}^{\circ}-69957_{4 \frac{1}{2}}$ & & & & & \\
\hline 7411.63 & & 4 & 13488.59 & 0.00 & $53716_{2 \frac{1}{2}}^{\circ}-67204_{1 \frac{1}{2}}^{2}$ & 5 & 1.17 & 1.05 & 0.061 & 1.35 \\
\hline $\begin{array}{l}7406.00 \\
7402.70\end{array}$ & 10 & $\begin{array}{r}150 \\
2\end{array}$ & $\begin{array}{l}13498.85 \\
13504.86\end{array}$ & $\begin{array}{r}0.00 \\
-0.01\end{array}$ & $\begin{array}{l}52067_{0 \frac{1}{2}}^{\circ}-65566_{0 \frac{1}{2}}^{\circ} \\
62046^{\circ}-73550 .\end{array}$ & 6 & 0.51 & 1.41 & 0.45 & 0.96 \\
\hline 7402.45 & & 1 & 13505.32 & & $020403 \frac{1}{2}-155300_{2 \frac{1}{2}}$ & & & & & \\
\hline 7400.97 & & 5 & 13508.02 & -0.04 & $54640_{4 \frac{1}{2}}^{\circ}-68148_{5 \frac{1}{2}}$ & & & & & \\
\hline $\begin{array}{l}7397.10 \\
7396.04\end{array}$ & 1 & $\begin{array}{r}40 \\
1\end{array}$ & $\begin{array}{l}13515.09 \\
13517.03\end{array}$ & $\begin{array}{r}0.00 \\
-0.01\end{array}$ & $\begin{array}{l}56621_{5 \frac{1}{2}}^{\circ}-70136_{6 \frac{1}{2}} \\
64365_{4 \frac{1}{2}}^{\circ}-77882_{3 \frac{1}{2}}^{\circ}\end{array}$ & 4 & 1.126 & 1.076 & $(0.025)$ & 0.800 \\
\hline $\begin{array}{l}7393.34 \\
7393.03\end{array}$ & 4 & $\begin{array}{r}30 \\
1\end{array}$ & $\begin{array}{l}13521.96 \\
13522.53\end{array}$ & $\begin{array}{l}+0.01 \\
-0.02\end{array}$ & $\begin{array}{l}45737_{\frac{1}{2}}^{0^{2}}-59259_{2 \frac{1}{2}} \\
34389_{2 \frac{1}{2}}^{\circ}-47912_{3 \frac{1}{2}}\end{array}$ & 4 & 1.333 & 1.099 & 0.117 & 0.748 \\
\hline 7389.22 & 6 & 150 & 13529.50 & -0.01 & $59753_{5 \frac{1}{2}}^{\circ}-73283_{4 \frac{1}{2}}$ & 5 & 1.112 & 1.050 & $(0.031)$ & 1.395 \\
\hline $\begin{array}{l}7384.50 \\
7383.00\end{array}$ & 7 & $\begin{array}{r}150 \\
2\end{array}$ & $\begin{array}{l}13538.15 \\
13540.90\end{array}$ & $\begin{array}{r}+0.04 \\
0.00\end{array}$ & $\begin{array}{l}59753_{5 \frac{1}{2}}^{\circ}-73291_{5 \frac{1}{2}} \\
58661_{2 \frac{1}{2}}^{\circ}-72202_{3 \frac{1}{2}}\end{array}$ & 6 & $(1.08)$ & $1.08 ?$ & 0 & 1.08 \\
\hline 7377.73 & 20 & 400 & 13550.57 & $\left\{\begin{array}{l}+0.01 \\
-0.06\end{array}\right.$ & $\begin{array}{l}45273_{3 \frac{1}{2}}^{\circ}-58823_{2 \frac{1}{2}} \\
56056^{\circ}-69607_{2 \frac{1}{2}}^{\circ}\end{array}$ & & & & & \\
\hline 7377.43 & 10 & 300 & 13551.12 & -0.02 & $47663_{4 \frac{1}{2}}^{\circ}-61214_{4 \frac{1}{2}}$ & & & & & \\
\hline 7373.06 & 3 & 15 & 13559.15 & +0.03 & $39378_{0 \frac{1}{2}}^{\circ}-52938_{1 \frac{1}{2}}$ & 4 & 1.833 & 0.873 & 0.48 & 0.393 \\
\hline 7367.78 & & 20 & 13568.87 & +0.02 & $59046_{4 \frac{1}{2}}^{\circ}-72615_{5 \frac{1}{2}}^{\circ}$ & 4 & (1.08) & $1.01 ?$ & $?$ & 0.70 \\
\hline 7362.92 & 2 & 15 & 13577.83 & & & 5 & $0.88^{\mathrm{e}}$ & $0.66^{\mathrm{e}}$ & 0.11 & 0.99 \\
\hline
\end{tabular}


TABLE 6. Yb II-Observed and classified lines-Continued

\begin{tabular}{|c|c|c|c|c|c|c|c|c|c|c|}
\hline 1 & 2 & 3 & 4 & 5 & 6 & 7 & 8 & 9 & 10 & 11 \\
\hline \multirow{2}{*}{$\begin{array}{l}\text { Wave- } \\
\text { length } \\
\text { air }\end{array}$} & \multicolumn{2}{|c|}{ Intensity } & \multirow{2}{*}{$\begin{array}{c}\text { Wave } \\
\text { number }\end{array}$} & \multirow[b]{2}{*}{$\mathrm{o}-\mathrm{c}$} & \multirow[b]{2}{*}{ Classification } & \multirow{2}{*}{$\begin{array}{l}\text { Zee- } \\
\text { man } \\
\text { type }\end{array}$} & \multirow[b]{2}{*}{ lst $g$} & \multirow[b]{2}{*}{ 2nd $g$} & \multicolumn{2}{|c|}{$\begin{array}{c}\text { Zeeman } \\
\text { components }\end{array}$} \\
\hline & $\begin{array}{l}\text { Meggers } \\
\text { lamp }\end{array}$ & $\begin{array}{c}\text { Thomson } \\
\text { lamp }\end{array}$ & & & & & & & Strong $p$ & Strong $n$ \\
\hline A & \multirow{7}{*}{10} & & $K$ & K & & & & & & $\therefore$ \\
\hline 7362.83 & & 60 & 13577.99 & -0.02 & $64169_{3 \frac{1}{2}}^{\circ}-77747_{4 \frac{1}{2}}$ & & & & & \\
\hline 7354.17 & & 4 & 13593.98 & -0.01 & $62046_{3 \frac{1}{2}}^{\circ}-75640_{3 \frac{1}{2}}$ & 6 & 1.14 & $(0.81)$ & 1.16 & $?$ \\
\hline 7350.42 & & 15 & 13600.92 & 0.00 & $58162_{1 \frac{1}{2}}^{\circ}-71763_{1 \frac{1}{2}}$ & & & & & \\
\hline 7342.30 & & 400 & 13615.96 & +0.01 & $47758_{3 \frac{1}{2}}^{\circ}-61374_{2 \frac{1}{2}}$ & 4 & 1.098 & 1.294 & 0.098 & 0.608 \\
\hline 7341.64 & & 4 & 13617.18 & -0.02 & $55462_{3 \frac{1}{2}}^{\circ}-69079_{2 \frac{1}{2}}^{\circ}$ & & & & & \\
\hline 7339.80 & & 60 & 13620.60 & & & 7 & & & 0 & 1.12 \\
\hline 7331.31 & \multirow[t]{6}{*}{1} & 80 & 13636.37 & -0.01 & $52921_{3 \frac{1}{2}}^{\circ}-66558_{3 \frac{1}{2}}$ & 6 & 1.15 & 1.09 & 0.21 & 1.12 \\
\hline 7329.29 & & 2 & 13640.13 & & & & & & & \\
\hline 7323.05 & & 2 & 13651.75 & & & & & & & \\
\hline 7318.77 & & 1 & 13659.73 & -0.01 & $59632_{6 \frac{1}{2}}^{\circ}-73291_{5 \frac{1}{2}}$ & & & & & \\
\hline 7317.74 & & 30 & 13661.66 & & & 7 & & & 0 & 0.963 \\
\hline 7313.18 & & 15 & 13670.18 & -0.01 & $57798_{2 \frac{1}{2}}^{\circ}-71468_{2 \frac{1}{2}}$ & & & & & \\
\hline 7309.19 & \multirow{15}{*}{1} & 40 & 13677.64 & +0.01 & $59439_{2 \frac{1}{2}}^{\circ}-73116_{1 \frac{1}{2}}$ & \multirow{3}{*}{$\begin{array}{l}5 \\
5 ?\end{array}$} & \multirow[t]{2}{*}{0.998} & \multirow[t]{2}{*}{0.862} & \multirow[t]{2}{*}{0.068} & \multirow[t]{3}{*}{1.202} \\
\hline 7306.63 & & 100 & 13682.43 & & & & & & & \\
\hline 7305.92 & & 8 & 13683.76 & +0.01 & $46902_{0 \frac{1}{2}}^{\circ}-60586_{1 \frac{1}{2}}$ & & \multirow{8}{*}{1.244} & \multirow{7}{*}{1.188} & & \\
\hline 7302.15 & & 15 & 13690.82 & -0.01 & $52880_{4 \frac{1}{2}}^{\circ}-66571_{5 \frac{1}{2}}^{2}$ & \multirow[t]{7}{*}{4} & & & \multirow[t]{6}{*}{$(0.028)$} & \multirow[t]{7}{*}{0.934} \\
\hline 7294.50 & & 1 & 13705.18 & -0.01 & $64365_{4 \frac{1}{2}}^{\circ}-78070_{4 \frac{1}{2}}$ & & & & & \\
\hline 7290.56 & & 3 & 13712.59 & -0.06 & $64169_{3 \frac{1}{2}}^{\circ}-77882_{3 \frac{1}{2}}$ & & & & & \\
\hline 7287.96 & & 2 & 13717.48 & -0.01 & $58484_{4 \frac{1}{2}}^{\circ}-72202_{3 \frac{1}{2}}^{\circ}$ & & & & & \\
\hline 7285.02 & & 1 & 13723.02 & 0.00 & $67789_{1 \frac{1}{2}}^{\circ}-81512_{1 \frac{1}{2}} ?$ & & & & & \\
\hline $\begin{array}{l}7284.70 \\
7279.67\end{array}$ & & $\begin{array}{l}1 \\
5\end{array}$ & $\begin{array}{l}13723.62 \\
13733.10\end{array}$ & $\begin{array}{l}0.00 \\
0.00\end{array}$ & $\begin{array}{l}67933_{2 \frac{1}{2}}^{\circ}-81657_{2 \frac{1}{2}} \\
59046^{\circ}-72779^{2}\end{array}$ & & & & & \\
\hline & & & & & $020704 \frac{1}{2}-12613 \frac{1}{2}$ & & & & & \\
\hline 7278.37 & & 30 & 13735.56 & 0.00 & $57534_{3 \frac{1}{2}}^{\circ}-71270_{2 \frac{1}{2}}$ & 4 & $0.86 ?$ & $(0.91)$ & $(0.027)$ & $0.638 ?$ \\
\hline 7268.03 & & 4 & 13755.10 & $\left\{\begin{array}{r}0.00 \\
-0.06\end{array}\right.$ & $\begin{array}{l}45335_{4 \frac{1}{2}}^{\circ}-59090_{3 \frac{1}{2}} \\
57103^{\circ}-70858^{2}\end{array}$ & $\therefore$ & & & & \\
\hline 7265.76 & & 10 & 13759.39 & -0.02 & $67933_{2 \frac{1}{2}}^{\circ}-81692_{3 \frac{1}{2}}$ & 7 & 1.16 & (1.16) & 0 & 1.15 \\
\hline 7259.76 & & 6 & 13770.77 & -0.04 & $63028_{1 \frac{1}{2}}^{\circ}-76799_{1 \frac{1}{2}}^{\circ}$ & & & & & \\
\hline 7259.56 & & 3 & 13771.14 & 0.00 & $53644_{3 \frac{1}{2}}^{0^{2}}-67416_{4 \frac{1}{2}}^{2}$ & & & & & \\
\hline 7251.55 & 30 & 200 & 13786.36 & -0.04 & $44497_{2 \frac{1}{2}}^{\circ}-58283_{2 \frac{1}{2}}$ & 6 & 0.730 & 0.764 & 0.084 & 0.747 \\
\hline 7238.95 & 1 & 80 & 13810.35 & +0.02 & $49916_{4 \frac{1}{2}}^{\circ}-63726_{4 \frac{1}{2}}^{\circ}$ & & & & & \\
\hline 7238.73 & 4 & 80 & 13810.77 & $\left\{\begin{array}{l}-0.02 \\
+0.03\end{array}\right.$ & $45012_{2 \frac{1}{2}}^{\circ}-58823_{2 \frac{1}{2}}$ & & & & & \\
\hline 7235.42 & 1 & 40 & 13817.09 & $\begin{array}{r}1+0.03 \\
-0.02\end{array}$ & $\begin{array}{l}63028_{1 \frac{1}{2}}^{\circ}-76839_{2 \frac{1}{2}} \\
45273_{3 \frac{1}{2}}^{\circ}-59090_{3 \frac{1}{2}}\end{array}$ & 6 & 1.19 & 1.13 & 0.218 & 1.16 \\
\hline 7228.98 & & 2 & 13829.40 & -0.02 & $45429_{3 \frac{1}{2}}^{\circ}-59259_{2 \frac{1}{2}}^{\circ}$ & 5 & 1.14 & (1.105) & $w$ & 1.22 \\
\hline 7222.72 & 20 & 150 & 13841.39 & -0.02 & $35059_{3 \frac{1}{2}}^{\circ}-48900_{3 \frac{1}{2}}$ & 6 & 1.10 & 1.03 & 0.245 & 1.065 \\
\hline 7221.50 & & 10 & 13843.72 & +0.08 & $65739_{2 \frac{1}{2}}^{\circ}-79583_{2 \frac{1}{2}}^{\circ}$ & & & & & \\
\hline 7221.21 & 1 & 60 & 13844.28 & 0.00 & $51248_{2 \frac{1}{2}}^{\circ}-65093_{3 \frac{1}{2}}^{2}$ & 7 & $(1.034)$ & 0.99 & 0 & 0.90 \\
\hline 7220.38 & 2 & 50 & 13845.87 & $\left\{\begin{array}{r}-0.01 \\
0.00\end{array}\right.$ & $\begin{array}{r}44438_{3 \frac{1}{2}}^{\circ}-58283_{2 \frac{1}{2}} \\
\left(80457^{2}-94303^{\circ}\right)\end{array}$ & 5 & 1.10 & 0.76 & 0.168 & 1.94 \\
\hline 7217.57 & & 60 & 13851.26 & +0.01 & $57103_{3 \frac{1}{2}}^{\circ}-70954^{\frac{1}{2}}$ & 5 & 1.11 & 1.05 & $(0.03)$ & 1.265 \\
\hline
\end{tabular}


TABLE 6. Yb II-Observed and classified lines-Continued

\begin{tabular}{|c|c|c|c|c|c|c|c|c|c|c|}
\hline 1 & 2 & 3 & 4 & 5 & 6 & 7 & 8 & 9 & 10 & 11 \\
\hline \multirow{2}{*}{$\begin{array}{l}\text { Wave- } \\
\text { length } \\
\text { air }\end{array}$} & \multicolumn{2}{|c|}{ Intensity } & \multirow{2}{*}{$\begin{array}{c}\text { Wave } \\
\text { number }\end{array}$} & \multirow[b]{2}{*}{$o-c$} & \multirow[b]{2}{*}{ Classification } & \multirow{2}{*}{$\begin{array}{l}\text { Zee- } \\
\text { man } \\
\text { type }\end{array}$} & \multirow[b]{2}{*}{ lst $g$} & \multirow[b]{2}{*}{ 2nd $g$} & \multicolumn{2}{|c|}{$\begin{array}{c}\text { Zeeman } \\
\text { components }\end{array}$} \\
\hline & $\begin{array}{c}\text { Meggers } \\
\text { lamp }\end{array}$ & $\begin{array}{c}\text { Thomson } \\
\text { lamp }\end{array}$ & & & & & & & Strong $p$ & Strong $n$ \\
\hline A & \multirow{11}{*}{4} & & $K$ & $K$ & & & & & & \\
\hline 7209.65 & & 1 & 13866.48 & 0.00 & $65739_{2 \frac{1}{2}}^{\circ}-79605_{1 \frac{1}{2}}^{\circ}$ & & & & & \\
\hline 7209.38 & & 2 & 13867.00 & +0.01 & $63417_{2 \frac{1}{2}}^{\circ}-77284_{1 \frac{1}{2}}^{2}$ & & & & & \\
\hline 7209.10 & & 8 & 13867.54 & +0.01 & $67789_{1 \frac{1}{2}}^{\circ}-81657_{2 \frac{1}{2}}^{\circ} ?$ & & & & & \\
\hline 7208.29 & & 200 & 13869.09 & -0.01 & $56088_{4 \frac{1}{2}}^{\circ}-69957_{4 \frac{1}{2}}$ & 7 & $1.09 ?$ & (1.11) & 0 & 1.10 \\
\hline 7201.09 & & 20 & 13882.96 & -0.01 & $44940_{1 \frac{1}{2}}^{\circ}-58823_{2 \frac{1}{2}}$ & 7 & (1.244) & 1.260 & 0 & 1.285 \\
\hline 7191.87 & & 1 & 13900.76 & -0.04 & $64169_{3 \frac{1}{2}}^{\circ}-78070_{4 \frac{1}{2}}$ & & & & & \\
\hline 7182.60 & & 2 & 13918.70 & -0.02 & $60649_{4 \frac{1}{2}}^{\circ}-74568_{4 \frac{1}{2}}^{\circ}$ & & & & & \\
\hline 7175.48 & & 300 & 13932.51 & -0.01 & $55462_{3 \frac{1}{2}}^{\circ}-69395_{3 \frac{1}{2}}$ & 6 & 0.878 & 0.924 & 0.165 & 0.90 \\
\hline 7174.60 & & 8 & 13934.22 & -0.03 & $57534_{3 \frac{1}{2}}^{\circ}-71468_{2 \frac{1}{2}}^{\circ}$ & & & & & \\
\hline 7154.52 & & 5 & 13973.33 & -0.03 & $45737_{1 \frac{1}{2}}^{\circ}-59710_{1 \frac{1}{2}}$ & 6 & 1.32 & 0.80 & 0.800 & 1.060 \\
\hline 7148.16 & \multirow{10}{*}{1} & 2 & 13985.76 & & & \multirow{5}{*}{$\begin{array}{l}7 \\
5\end{array}$} & \multirow{5}{*}{1.145} & \multirow{5}{*}{1.077} & \multirow{5}{*}{$\begin{array}{l}0 \\
(0.034)\end{array}$} & \multirow{5}{*}{$\begin{array}{l}0.86 \\
1.450\end{array}$} \\
\hline 7147.94 & & 8 & 13986.19 & -0.03 & $45273_{3 \frac{1}{2}}^{\circ}-59259_{2 \frac{1}{2}}$ & & & & & \\
\hline 7145.13 & & 4 & 13991.69 & -0.09 & $75905_{0 \frac{1}{2}}-89897_{1 \frac{1}{2}}^{\circ}$ & & & & & \\
\hline 7142.39 & & 120 & 13997.06 & 0.00 & $59753_{5 \frac{1}{2}}^{\circ}-73750_{4 \frac{1}{2}}^{\circ}$ & & & & & \\
\hline 7137.67 & & 2 & 14006.31 & -0.02 & $62163_{2 \frac{1}{2}}^{\circ}-76170_{2 \frac{1}{2}}$ & & & & & \\
\hline 7134.83 & & 2 & 14011.89 & 0.00 & $65594_{2 \frac{1}{2}}^{\circ}-79605_{1 \frac{1}{2}}$ & & & & & \\
\hline 7128.94 & & 2 & 14023.47 & +0.13 & $41678_{2 \frac{1}{2}}^{\circ}-55702_{1 \frac{1}{2}}$ & & & & & \\
\hline 7126.81 & & 100 & 14027.66 & -0.02 & $49916_{4 \frac{1}{2}}^{0}-63944_{5 \frac{1}{2}}$ & 5 & 1.087 & 1.113 & $(0.013)$ & 1.230 \\
\hline 7124.46 & & 5 & 14032.28 & -0.01 & $63417_{2 \frac{1}{2}}^{\circ}-77449_{3 \frac{1}{2}}$ & 5 & 1.011 & 1.161 & 0.075 & 1.536 \\
\hline 7117.99 & & 10 & 14045.04 & -0.01 & $47329_{2 \frac{1}{2}}^{\circ}-61374_{2 \frac{1}{2}}$ & 6 & 0.986 & 1.298 & 0.780 & 1.142 \\
\hline 7108.37 & & 6 & 14064.05 & +0.01 & $47758_{3 \frac{1}{2}}^{\circ}-61822_{3 \frac{1}{2}}$ & 6 & 1.104 & 1.326 & 0.774 & 1.215 \\
\hline 7101.66 & & 10 & 14077.33 & -0.01 & $45012_{2 \frac{1}{2}}^{\circ}-59090_{3 \frac{1}{2}}^{\circ}$ & 4 & 1.279 & 1.102 & 0.088 & 0.662 \\
\hline 7099.70 & & 2 & 14081.22 & 0.00 & $60910_{1 \frac{1}{2}}^{\circ}-74991_{1 \frac{1}{2}}^{\circ}$ & & & & & \\
\hline 7098.11 & & 6 & 14084.37 & -0.02 & $53120_{2 \frac{1}{2}}^{\circ}-67204_{1 \frac{1}{2}}^{\circ}$ & 4 & 0.95 & 1.04 & $(0.045)$ & 0.815 \\
\hline 7089.40 & & 1 & 14101.68 & & & & & & & \\
\hline 7086.08 & & 20 & 14108.29 & & & 4 & & & $w$ & 0.96 \\
\hline 7082.61 & & 80 & 14115.20 & -0.02 & $54640_{4 \frac{1}{2}}^{\circ}-68756_{3 \frac{1}{2}}$ & 4 & 1.129 & 1.325 & 0.098 & 0.443 \\
\hline 7081.81 & & 4 & 14116.79 & 0.00 & $22960_{1 \frac{1}{2}}-37077_{2 \frac{1}{2}}^{\circ}$ & & & & & \\
\hline 7080.77 & & 1 & 14118.87 & +0.01 & $58661_{2 \frac{1}{2}}^{\circ}-72779_{3 \frac{1}{2}}^{\circ}$ & & & & & \\
\hline 7080.53 & & 40 & 14119.34 & 0.00 & $57103_{\frac{1}{2}}^{\rho^{2}}-71222_{3 \frac{1}{2}}$ & 6 & 1.10 & 1.16 & 0.212 & 1.127 \\
\hline 7078.11 & & 5 & 14124.17 & -0.01 & $62046_{3 \frac{1}{2}}^{\circ}-76170_{2 \frac{1}{2}}$ & 7 & (1.14) & $1.13 ?$ & 0 & 1.170 \\
\hline 7077.07 & & 1 & 14126.25 & +0.01 & $59624_{3 \frac{1}{2}}^{\circ}-73750_{4 \frac{1}{2}}^{\circ}$ & & & & & \\
\hline 7074.60 & & 2 & 14131.18 & -0.02 & $58484_{4 \frac{1}{2}}^{\circ}-72615_{5 \frac{1}{2}}^{\circ}$ & & & & & \\
\hline 7072.12 & & 4 & 14136.13 & 0.00 & $56977_{0 \frac{1}{2}}^{\circ}-71113_{1 \frac{1}{2}}$ & 4 & 2.188 & 0.968 & 0.611 & 0.358 \\
\hline 7066.20 & & 10 & 14147.98 & +0.01 & $60910_{1 \frac{1}{2}}^{\circ}-75058_{2 \frac{1}{2}}^{\circ}$ & & & & & \\
\hline 7060.67 & & 60 & 14159.06 & 0.00 & $47663_{4 \frac{1}{2}}^{\circ}-61822_{3 \frac{1}{2}}$ & 4 & 0.99 & 1.32 & 0.165 & $-0.165 ?$ \\
\hline 7060.41 & & 2 & 14159.58 & +0.01 & $62163_{2 \frac{1}{2}}^{\circ}-76323_{3 \frac{1}{2}}^{\circ}$ & & & & & \\
\hline 7056.72 & & 4 & 14166.98 & -0.01 & $57103_{3 \frac{1}{2}}^{\circ}-71270_{2 \frac{1}{2}}^{\circ}$ & 5 & 1.115 & 0.937 & 0.089 & 1.560 \\
\hline 7053.80 & 1 & 200 & 14172.85 & +0.04 & $56621_{5 \frac{1}{2}}^{\circ}-70793_{5 \frac{1}{2}}^{\circ}$ & 7 & 1.20 & 1.20 & & 1.203 \\
\hline 7052.58 & & 10 & 14175.30 & -0.03 & $38342_{4 \frac{1}{2}}^{\circ}-52517_{5 \frac{1}{2}}^{\circ}$ & 5 & 1.088 & 1.273 & 0.093 & 2.110 \\
\hline
\end{tabular}


TABLE 6. Yb II-Observed and classified lines-Continued

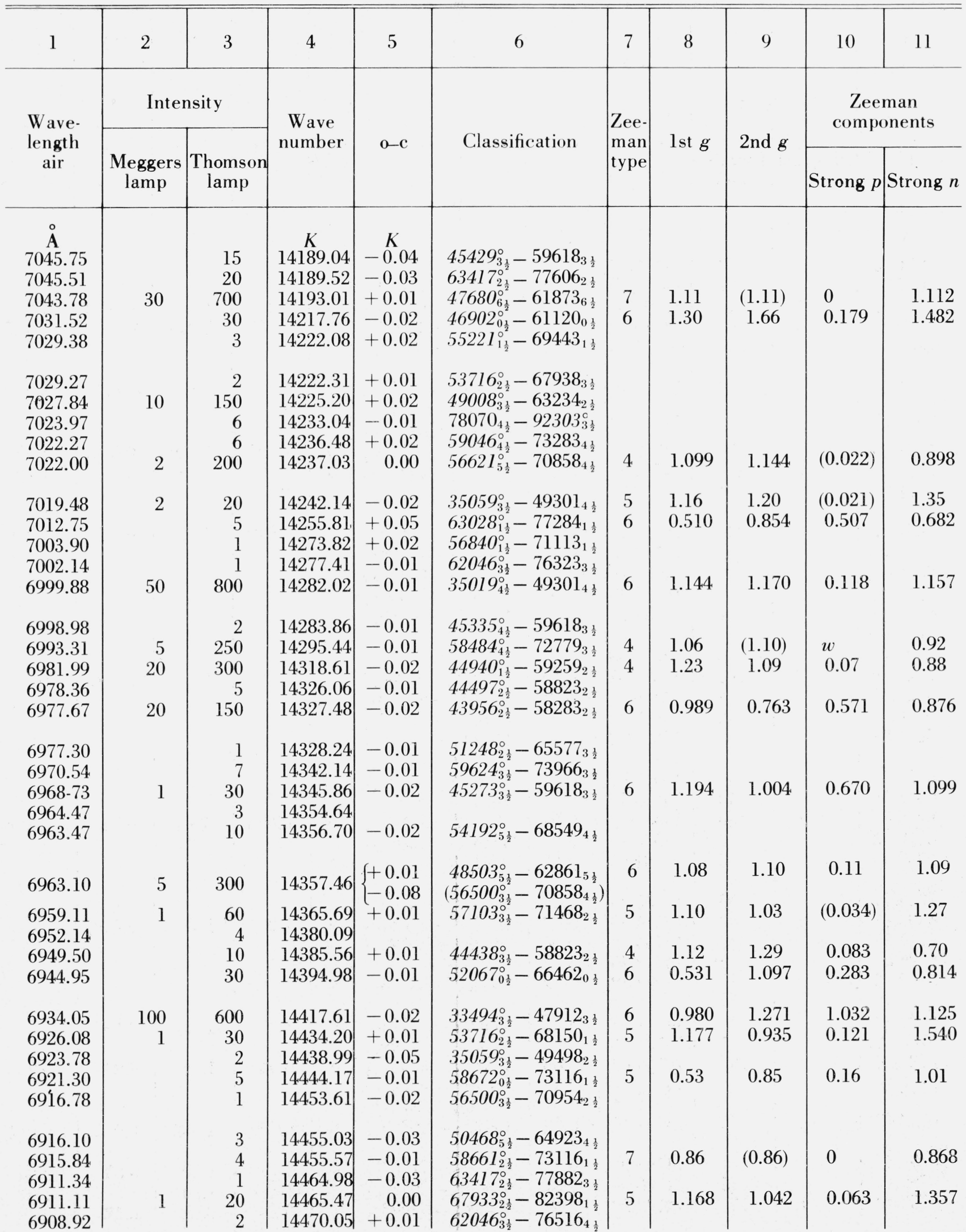


TABLE 6. Yb II-Observed and classified lines-Continued

\begin{tabular}{|c|c|c|c|c|c|c|c|c|c|c|}
\hline 1 & 2 & 3 & 4 & 5 & 6 & 7 & 8 & 9 & 10 & 11 \\
\hline \multirow{2}{*}{$\begin{array}{l}\text { Wave- } \\
\text { length } \\
\text { air }\end{array}$} & \multicolumn{2}{|c|}{ Intensity } & \multirow{2}{*}{$\begin{array}{c}\text { Wave } \\
\text { number }\end{array}$} & \multirow{2}{*}{$0-c$} & \multirow{2}{*}{ Classification } & \multirow{2}{*}{$\begin{array}{l}\text { Zee- } \\
\text { man } \\
\text { type }\end{array}$} & \multirow{2}{*}{ lst $g$} & \multirow{2}{*}{2 nd $g$} & \multicolumn{2}{|c|}{$\begin{array}{c}\text { Zeeman } \\
\text { components }\end{array}$} \\
\hline & $\begin{array}{c}\text { Meggers } \\
\text { lamp }\end{array}$ & $\begin{array}{c}\text { Thomson } \\
\text { lamp }\end{array}$ & & & & & & & Strong $p$ & Strong $n$ \\
\hline A & \multirow{8}{*}{$\begin{array}{r}1 \\
10\end{array}$} & & $K$ & $K$ & \multirow{6}{*}{$\begin{array}{l}69957_{4 \frac{1}{2}}-84444_{3 \frac{1}{2}}^{\circ} \\
59777_{1 \frac{1}{2}}^{\circ}-74270_{2 \frac{1}{2}} \\
52921_{3 \frac{1}{2}}^{\circ}-67416_{4 \frac{1}{2}} \\
34389_{2 \frac{1}{2}}^{\circ}-48900_{3 \frac{1}{2}}^{\circ} \\
62163_{2 \frac{1}{2}}^{\circ}-76676_{2 \frac{1}{2}}^{\circ}\end{array}$} & \multirow{6}{*}{$\begin{array}{l}5 \\
5\end{array}$} & \multirow{6}{*}{$\begin{array}{l}1.227 \\
(1.007)\end{array}$} & \multirow{6}{*}{$\begin{array}{l}1.305 \\
1.038\end{array}$} & \multirow{6}{*}{$\begin{array}{l}(0.039) \\
w\end{array}$} & \multirow{6}{*}{$\begin{array}{l}1.577 \\
1.117\end{array}$} \\
\hline 6901.06 & & 2 & 14486.53 & -0.05 & & & & & & \\
\hline 6897.78 & & 1 & 14493.42 & +0.01 & & & & & & \\
\hline 6897.31 & & 40 & 14494.41 & 0.00 & & & & & & \\
\hline 6889.59 & & 100 & 14510.65 & 0.00 & & & & & & \\
\hline 6888.76 & & & 14512.40 & +0.01 & & & & & & \\
\hline 6886.95 & & 8 & 14516.21 & 0.00 & $34784_{5 \frac{1}{2}}^{\circ}-49301_{4 \frac{1}{2}}^{\circ}$ & 4 & 1.11 & 1.16 & $(0.026)$ & 0.87 \\
\hline 6886.77 & & 5 & 14516.59 & $\left\{\begin{array}{l}-0.03 \\
-0.03\end{array}\right.$ & $\begin{array}{r}-48900_{3 \frac{1}{2}}-63417_{2 \frac{1}{2}}^{\circ} \\
66796_{3 \frac{1}{2}}^{\circ}-81312_{2 \frac{1}{2}}^{\circ}\end{array}$ & & & & & \\
\hline 6881.40 & 1 & 100 & 14527.92 & -0.01 & $54192_{5 \frac{1}{2}}^{\circ}-68720_{6 \frac{1}{2}}^{\circ}$ & 5 & 1.123 & 1.159 & $(0.018)$ & 1.357 \\
\hline $\begin{array}{l}6877.93 \\
6871.51\end{array}$ & 2 & $\begin{array}{r}250 \\
3\end{array}$ & $\begin{array}{l}14535.25 \\
14548.83\end{array}$ & -0.03 & $52880_{4 \frac{1}{2}}^{\circ}-67416_{4 \frac{1}{2}}$ & 6 & 1.26 & 1.26 & 0 & 1.26 \\
\hline 6864.27 & \multirow[t]{5}{*}{2} & 300 & 14564.17 & -0.06 & $58051_{5 \frac{1}{2}}^{\circ}-72615_{5 \frac{1}{2}}$ & \multirow[t]{5}{*}{6} & \multirow[t]{5}{*}{0.949} & \multirow[t]{5}{*}{0.971} & \multirow[t]{5}{*}{0.117} & \multirow[t]{5}{*}{0.960} \\
\hline 6857.64 & & 2 & 14578.25 & -0.07 & $63028_{1 \frac{1}{2}}^{\circ}-77606_{2 \frac{1}{2}}^{\circ}$ & & & & & \\
\hline 6844.05 & & 1 & 14607.20 & -0.02 & $48556_{3 \frac{1}{2}}^{\circ}-63163_{4 \frac{1}{2}}^{\circ}$ & & & & & \\
\hline 6843.02 & & 2 & 14609.40 & +0.02 & $67789_{1 \frac{1}{2}}^{\circ}-82398_{1 \frac{1}{2}}^{\circ} ?$ & & & & & \\
\hline 6830.99 & & 2 & 14635.13 & -0.02 & $62163_{2 \frac{1}{2}}^{\circ}-76799_{1 \frac{1}{2}}^{2}$ & & & & & \\
\hline 6829.80 & 1 & 70 & 14637.68 & & & & & & & \\
\hline 6828.90 & 2 & 200 & 14639.61 & +0.01 & $51248_{2 \frac{1}{2}}^{\circ}-65888_{1 \frac{1}{2}}$ & 4 & 1.03 & 1.20 & 0.090 & 0.75 \\
\hline 6828.35 & & 2 & 14640.79 & -0.01 & $60910_{1 \frac{1}{2}}^{\circ}-75550_{2 \frac{1}{2}}^{\circ}$ & & & & & \\
\hline 6826.18 & & 5 & 14645.44 & -0.03 & $64169_{3 \frac{1}{2}}^{\circ}-78814_{2 \frac{1}{2}}^{\circ}$ & & & & & \\
\hline 6826.02 & & 10 & 14645.78 & -0.04 & $59624_{3 \frac{1}{2}}^{\circ}-74270_{2 \frac{1}{2}}$ & & & & & \\
\hline 6819.41 & & 2 & 14659.98 & +0.01 & $48503_{5 \frac{1}{2}}^{\circ}-63163_{4 \frac{1}{2}}$ & & & & & \\
\hline 6816.18 & & 40 & 14666.93 & -0.01 & $46547_{5 \frac{1}{2}}^{\circ}-61214_{4 \frac{1}{2}}^{\circ}$ & & & & & \\
\hline 6815.88 & 1 & 100 & 14667.57 & -0.01 & $57534_{3 \frac{1}{2}}^{\circ}-72202_{3 \frac{1}{2}}^{\circ}$ & 6 & 0.865 & 0.831 & 0.12 & 0.848 \\
\hline 6812.42 & & 2 & 14675.02 & -0.06 & $62163_{2 \frac{1}{2}}^{\circ}-76839_{2 \frac{1}{2}}^{2}$ & & & & & \\
\hline 6809.30 & & 5 & 14681.75 & -0.03 & $49916_{4 \frac{1}{2}}^{\circ}-64598_{3 \frac{1}{2}}^{\circ}$ & 4 & & & & \\
\hline 6803.91 & & 20 & 14693.38 & -0.01 & $49008_{3 \frac{1}{2}}^{\circ}-63702_{2 \frac{1}{2}}$ & & & & & \\
\hline 6802.47 & 2 & 400 & 14696.49 & -0.02 & $46354_{4 \frac{1}{2}}^{\circ}-61051_{3 \frac{1}{2}}^{\circ}$ & 5 & 1.28 & 1.18 & $(0.050)$ & 1.63 \\
\hline 6798.29 & & $2 h$ & 14705.52 & -0.05 & $56088_{4 \frac{1}{2}}^{\circ}-70793_{5 \frac{1}{2}}$ & & & & & \\
\hline 6796.80 & & $2 h$ & 14708.75 & & & & & & & \\
\hline 6792.58 & & 30 & 14717.88 & -0.02 & $49008_{3 \frac{1}{2}}^{\circ}-63726_{4 \frac{1}{2}}$ & & & & & \\
\hline 6790.82 & & 20 & 14721.70 & -0.02 & $56500_{3 \frac{1}{2}}^{\circ}-71222_{3 \frac{1}{2}}$ & 6 & 1.35 & (1.15) & 0.69 & \\
\hline 6786.32 & & 1 & 14731.46 & -0.03 & $50468_{5 \frac{1}{2}}^{\circ}-65199_{4 \frac{1}{2}}$ & & & & & \\
\hline 6785.14 & 1 & 150 & 14734.02 & -0.05 & $50832_{1 \frac{1}{2}}^{\circ}-65566_{0 \frac{1}{2}}^{\circ}$ & 4 & 0.94 & 1.42 & 0.24 & 0.70 \\
\hline 6775.79 & & 2 & 14754.35 & $\left\{\begin{array}{l}-0.03 \\
+0.04\end{array}\right.$ & $\begin{array}{l}54640_{4 \frac{1}{2}}^{\circ}-69395_{3 \frac{1}{2}}^{2} \\
73966^{2}-88721^{\mathrm{c}^{2}}\end{array}$ & & & & & \\
\hline 6772.42 & 1 & 50 & 14761.70 & $\begin{array}{l}++0.04 \\
-0.03\end{array}$ & $\begin{array}{l}44000_{3 \frac{1}{2}}-88121_{3 \frac{1}{2}}^{\circ} \\
44497_{2 \frac{1}{2}}^{\circ}-59259_{2 \frac{1}{2}}\end{array}$ & 6 & 0.729 & 1.095 & 0.920 & 0.912 \\
\hline 6761.31 & & 4 & 14785.95 & -0.01 & $56977_{0 \frac{1}{2}}^{\circ}-71763_{1 \frac{1}{2}}$ & 4 & 2.20 & 1.10 & 0.55 & 0.55 \\
\hline 6759.10 & & 15 & 14790.79 & +0.01 & $61442_{5 \frac{1}{2}}^{\circ}-76233_{6 \frac{1}{2}}^{\circ}$ & 4 & (1.11) & $1.10 ?$ & $w$ & 1.01 \\
\hline 6755.44 & 1 & 150 & 14798.80 & -0.01 & $58484_{4 \frac{1}{2}}^{\sigma^{2}}-73283_{4 \frac{1}{2}}^{d^{2}}$ & 6 & 0.58 & 0.62 & 0.18 & $0.60 ?$ \\
\hline
\end{tabular}


TABlE 6. Yb II-Observed and classified lines-Continued

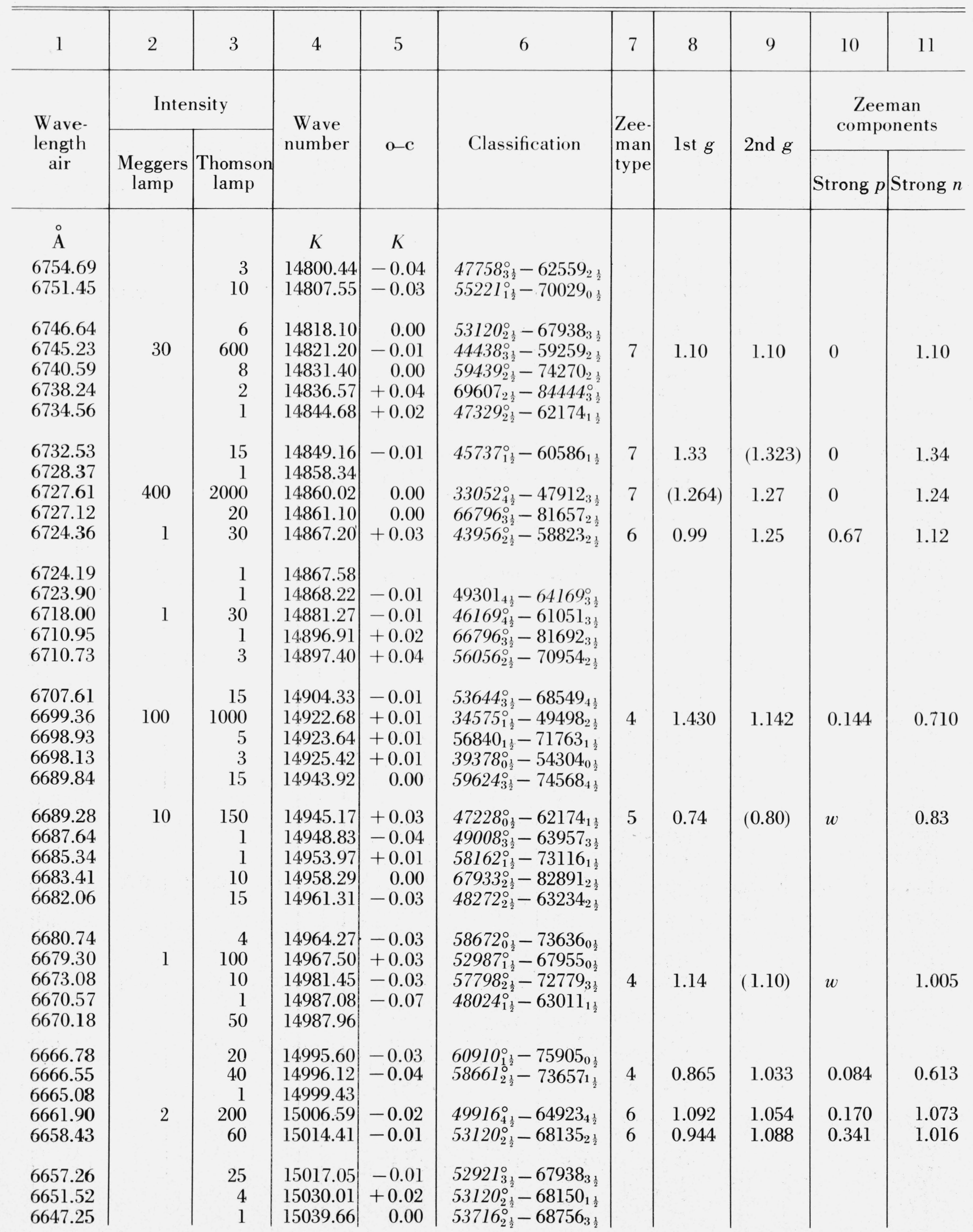


TABLE 6. Yb II-Observed and classified lines-Continued

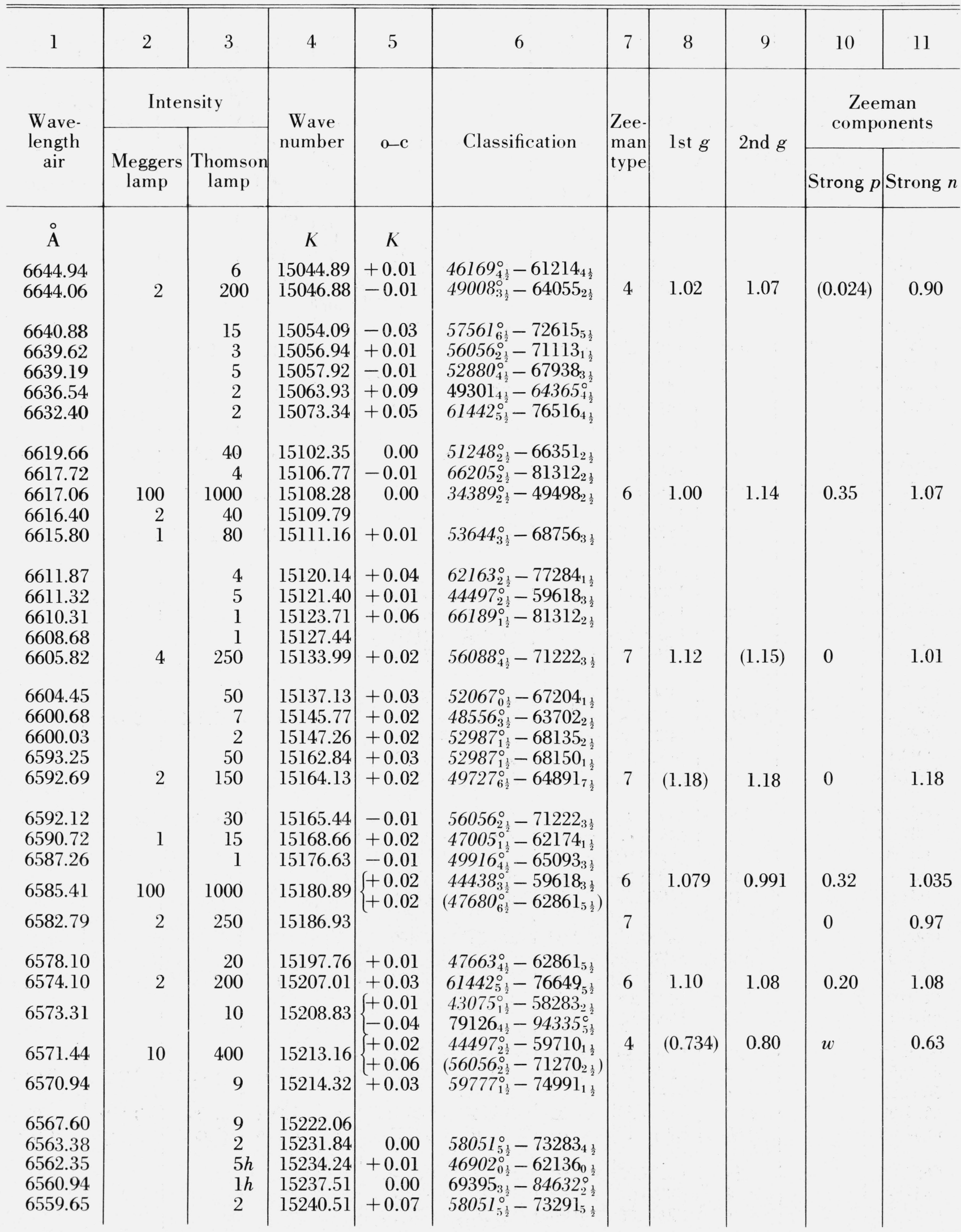


TABLE 6. YbII-Observed and classified lines-Continued

\begin{tabular}{|c|c|c|c|c|c|c|c|c|c|c|}
\hline 1 & 2 & 3 & 4 & 5 & 6 & 7 & 8 & 9 & 10 & 11 \\
\hline \multirow{2}{*}{$\begin{array}{l}\text { Wave- } \\
\text { length } \\
\text { air }\end{array}$} & \multicolumn{2}{|c|}{ Intensity } & \multirow{2}{*}{$\begin{array}{l}\text { Wave } \\
\text { number }\end{array}$} & \multirow{2}{*}{$o-c$} & \multirow{2}{*}{ Classification } & \multirow{2}{*}{$\left|\begin{array}{l}\text { Zee- } \\
\text { man } \\
\text { type }\end{array}\right|$} & \multirow{2}{*}{ lst $g$} & \multirow{2}{*}{ 2nd $g$} & \multicolumn{2}{|c|}{$\begin{array}{c}\text { Zeeman } \\
\text { components }\end{array}$} \\
\hline & $\begin{array}{l}\text { Meggers } \\
\text { lamp }\end{array}$ & $\begin{array}{c}\text { Thomson } \\
\text { lamp }\end{array}$ & & & & & & & Strong $p$ & Strong $n$ \\
\hline $\begin{array}{c}\AA \\
6557.46 \\
6552.61 \\
6548.53 \\
6547.77 \\
6546.35\end{array}$ & 2 & $\begin{array}{r}4 \\
2 \\
200 \\
9 \\
2\end{array}$ & $\begin{array}{c}K \\
15245.60 \\
15256.88 \\
15266.39 \\
15268.16 \\
15271.47\end{array}$ & $\begin{aligned} & K \\
+ & 0.06 \\
& +0.03 \\
+ & 0.03 \\
+ & 0.08\end{aligned}$ & $\begin{array}{l}57534_{3 \frac{1}{2}}^{\circ}-72779_{3 \frac{1}{2}} \\
58484_{4 \frac{1}{2}}^{\circ}-73750_{4 \frac{1}{2}} \\
52880_{4 \frac{1}{2}}^{\circ}-68148_{5 \frac{1}{2}} \\
46902_{0 \frac{1}{2}}^{\circ}-62174_{1 \frac{1}{2}}\end{array}$ & 6 & 0.96 & 1.01 & 0.22 & 0.99 \\
\hline $\begin{array}{l}6541.38 \\
6540.36 \\
6531.71 \\
6531.26 \\
6527.01\end{array}$ & 3 & $\begin{array}{r}400 \\
1 \\
5 \\
4 \\
25\end{array}$ & $\begin{array}{l}15283.07 \\
15285.46 \\
15305.70 \\
15306.75 \\
15316.72\end{array}$ & $\begin{array}{r}+0.03 \\
+0.06 \\
+0.02 \\
0.00 \\
+0.05\end{array}$ & $\begin{array}{l}49916_{3_{\frac{1}{2}}}^{\circ}-65199_{4 \frac{1}{2}} \\
62163_{2 \frac{1}{2}}^{\circ}-77449_{3 \frac{1}{2}} \\
58661_{2 \frac{1}{2}}^{\circ}-73966_{3 \frac{1}{2}} \\
66205_{2 \frac{1}{2}}^{\circ}-81512_{1 \frac{1}{2}} \\
54640_{4 \frac{1}{2}}^{\circ}-69957_{4 \frac{1}{2}}^{\circ}\end{array}$ & 6 & (1.090) & $1.05 ?$ & $d$ & 1.07 \\
\hline $\begin{array}{l}6526.36 \\
6523.19 \\
6521.52 \\
6507.15 \\
6503.01\end{array}$ & 50 & $\begin{array}{r}3 \\
250 \\
25 \\
1 \\
500\end{array}$ & $\begin{array}{l}15318.24 \\
15325.69 \\
15329.61 \\
15363.47 \\
15373.25\end{array}$ & $\begin{array}{l}+0.04 \\
+0.01 \\
+0.08 \\
-0.03 \\
+0.01\end{array}$ & $\begin{array}{l}57798_{2 \frac{1}{2}}^{\circ}-73116_{1 \frac{1}{2}} \\
46547_{5 \frac{1}{2}}^{\circ}-61873_{6 \frac{1}{2}} \\
53120_{2 \frac{1}{2}}^{\circ}-68450_{3 \frac{1}{2}}^{\circ} \\
53716_{2 \frac{1}{2}}^{\circ}-69079_{2 \frac{1}{2}} \\
38342_{4 \frac{1}{2}}^{\circ}-53715_{3 \frac{1}{2}}^{\circ}\end{array}$ & 4 & 1.00 & 1.08 & $(0.038)$ & 0.734 \\
\hline $\begin{array}{l}6502.28 \\
6500.70 \\
6498.79 \\
6493.58\end{array}$ & $\begin{array}{l}2 \\
1\end{array}$ & $\begin{array}{r}20 \\
30 \\
20 \\
5\end{array}$ & $\begin{array}{l}15374.97 \\
15378.71 \\
15383.23 \\
15395.57\end{array}$ & $\begin{array}{l}+0.01 \\
+0.02 \\
+0.03 \\
+0.07\end{array}$ & $\begin{array}{l}48272_{2 \frac{1}{2}}^{\circ}-63647_{1 \frac{1}{2}} \\
38342_{4 \frac{1}{2}}^{\circ}-53720_{4 \frac{1}{2}} \\
45737_{1 \frac{1}{2}}^{\circ}-61120_{0 \frac{1}{2}}^{\circ} \\
55462_{3 \frac{1}{2}}^{\circ}-70858_{4 \frac{1}{2}}^{\circ}\end{array}$ & & & & & \\
\hline 6492.73 & 10 & 700 & 15397.59 & +0.04 & $53322_{7 \frac{1}{2}}^{\circ}-68720_{6 \frac{1}{2}}$ & 4 & 0.98 & 1.04 & $(0.032)$ & 0.57 \\
\hline $\begin{array}{l}6491.18 \\
6490.32 \\
6488.47 \\
6487.08 \\
6486.74\end{array}$ & & $\begin{array}{r}5 \\
20 \\
200 \\
10 \\
50\end{array}$ & $\begin{array}{l}15401.26 \\
15403.30 \\
15407.70 \\
15411.00 \\
15411.81\end{array}$ & $\begin{array}{l}+0.03 \\
+0.05 \\
-0.02 \\
-0.02 \\
+0.02\end{array}$ & $\begin{array}{l}48556_{3 \frac{1}{2}}^{\circ}-63957_{3 \frac{1}{2}} \\
62046_{3 \frac{1}{2}}^{\circ}-77449_{3 \frac{1}{2}} \\
50468_{5 \frac{1}{2}}^{\circ}-65875_{6 \frac{1}{2}} \\
73291_{5 \frac{1}{2}}-88702_{5 \frac{1}{2}}^{\circ} \\
56056_{2 \frac{1}{2}}^{\circ}-71468_{2 \frac{1}{2}}^{\circ}\end{array}$ & & & & & \\
\hline $\begin{array}{l}6485.55 \\
6477.63 \\
6476.36\end{array}$ & 10 & $\begin{array}{c}200 h \\
6 \\
1\end{array}$ & $\begin{array}{l}15414.63 \\
15433.48 \\
15436.51\end{array}$ & $\begin{array}{l}+0.03 \\
+0.01\end{array}$ & $\begin{array}{l}59624_{3 \frac{1}{2}}^{\circ}-75058_{2 \frac{1}{2}}^{\circ} \\
63028^{\circ \frac{1}{2}}-78464_{1 \frac{1}{2}}\end{array}$ & & & & & \\
\hline $\begin{array}{l}6474.74 \\
6470.17\end{array}$ & 30 & $\begin{array}{r}1500 \\
1\end{array}$ & $\begin{array}{l}15440.37 \\
15451.27\end{array}$ & $\begin{array}{l}+0.01 \\
+0.01\end{array}$ & $\begin{array}{l}48503_{5 \frac{1}{2}}^{\circ}-63944_{5 \frac{1}{2}}^{2} \\
66205_{2 \frac{1}{2}}^{\circ}-81657_{2 \frac{1}{2}}\end{array}$ & 6 & 1.10 & 1.12 & 0.09 & 1.11 \\
\hline $\begin{array}{l}6466.79 \\
6465.78\end{array}$ & & $\begin{array}{l}7 \\
3 h\end{array}$ & $\begin{array}{l}15459.35 \\
15461.77\end{array}$ & $\begin{array}{l}+0.03 \\
-0.04\end{array}$ & $\begin{array}{l}64169_{3 \frac{1}{2}}^{\circ}-79628_{3 \frac{1}{2}}^{\circ} \\
68756_{3 \frac{1}{2}}-84217_{4 \frac{1}{2}}^{\circ}\end{array}$ & & & & & \\
\hline $\begin{array}{l}6463.15 \\
6456.95\end{array}$ & $\begin{array}{r}50 \\
2\end{array}$ & $\begin{array}{r}2000 \\
150\end{array}$ & $\begin{array}{l}15468.06 \\
15482.91\end{array}$ & $\begin{array}{l}+0.03 \\
+0.01\end{array}$ & $\begin{array}{l}46354_{4 \frac{1}{2}}^{\circ}-61822_{3 \frac{1}{2}}^{\circ} \\
50468_{5 \frac{1}{2}}^{\circ}-65950_{4 \frac{1}{2}}\end{array}$ & 7 & 1.30 & (1.327) & 0 & 1.21 \\
\hline 6455.23 & & 4 & 15487.03 & $\left\{\begin{array}{l}+0.06 \\
-0.02\end{array}\right.$ & $\begin{array}{l}64970_{3 \frac{1}{2}}^{\circ}-80457_{3 \frac{1}{2}}^{\circ} \\
66205_{2 \frac{1}{2}}^{\circ}-81692_{3 \frac{1}{2}}\end{array}$ & & & & & \\
\hline $\begin{array}{l}6453.30 \\
6453.11 \\
6452.10 \\
6442.10\end{array}$ & $\begin{array}{r}1 \\
10\end{array}$ & $\begin{array}{r}150 \\
150 \\
15 \\
9\end{array}$ & $\begin{array}{l}15491.67 \\
15492.12 \\
15494.55 \\
15518.60\end{array}$ & $\begin{array}{l}+0.08 \\
+0.01 \\
+0.04\end{array}$ & $\begin{array}{l}55462_{3 \frac{1}{2}}^{\circ}-70954_{2 \frac{1}{2}} \\
58162_{1 \frac{1}{2}}^{\circ}-73657_{1 \frac{1}{2}}^{\circ} \\
50832_{1^{\frac{1}{2}}}^{\circ}-66351_{2 \frac{1}{2}}\end{array}$ & & & & & \\
\hline
\end{tabular}


TABLE 6. Yb II-Observed and classified lines-Continued

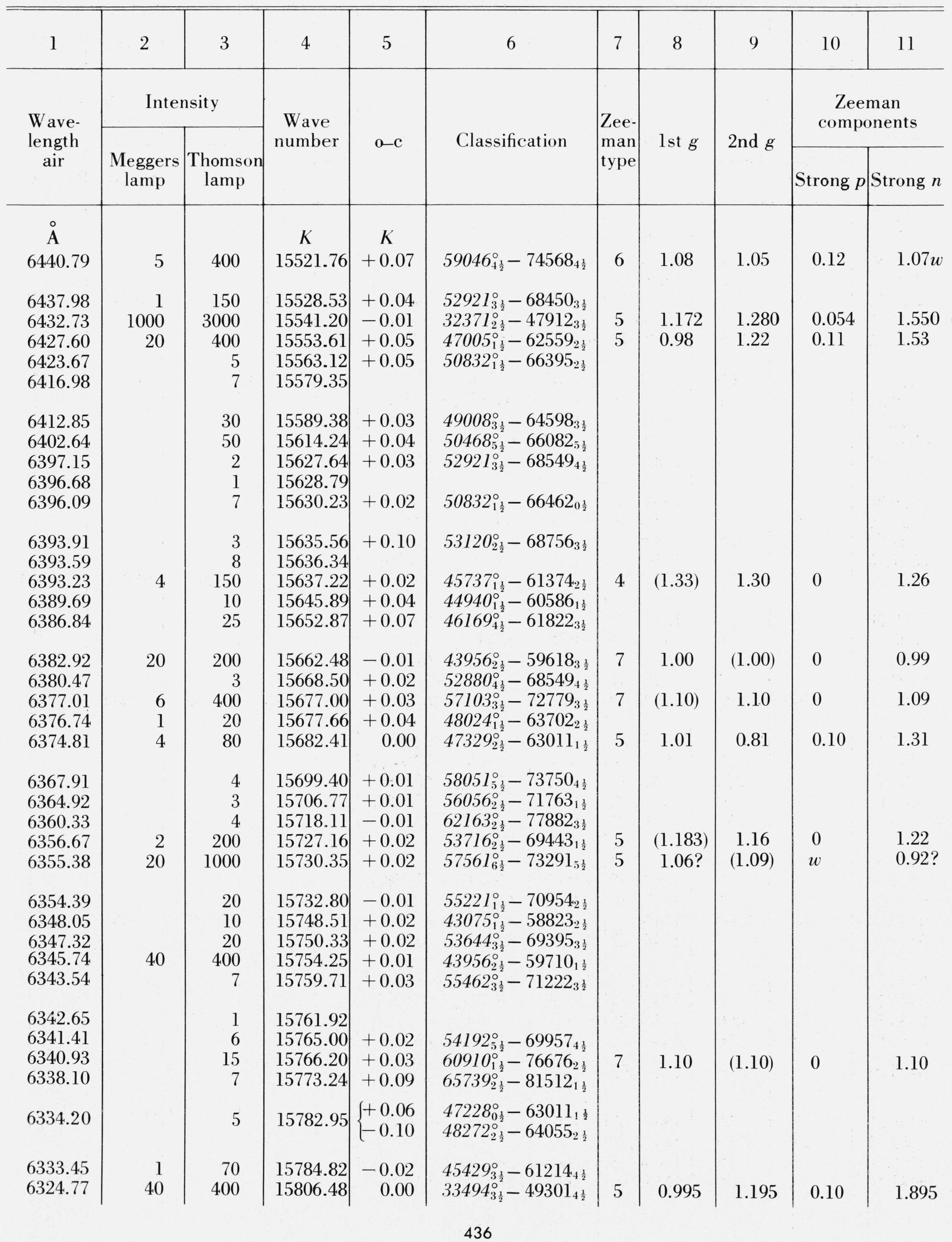


TABLE 6. Yb II-Observed and classified lines-Continued

\begin{tabular}{|c|c|c|c|c|c|c|c|c|c|c|}
\hline 1 & 2 & 3 & 4 & 5 & 6 & 7 & 8 & 9 & 10 & 11 \\
\hline \multirow{2}{*}{$\begin{array}{l}\text { Wave- } \\
\text { length } \\
\text { air }\end{array}$} & \multicolumn{2}{|c|}{ Intensity } & \multirow{2}{*}{$\begin{array}{c}\text { Wave } \\
\text { number }\end{array}$} & \multirow[b]{2}{*}{$0-c$} & \multirow{2}{*}{ Classification } & \multirow{2}{*}{$\begin{array}{l}\text { Zee- } \\
\text { man } \\
\text { type }\end{array}$} & \multirow{2}{*}{ lst $g$} & \multirow{2}{*}{ 2nd $g$} & \multicolumn{2}{|c|}{$\begin{array}{c}\text { Zeeman } \\
\text { components }\end{array}$} \\
\hline & $\begin{array}{l}\text { Meggers } \\
\text { lamp }\end{array}$ & $\begin{array}{c}\text { Thomson } \\
\text { lamp }\end{array}$ & & & & & & & Strong $p$ & Strong $n$ \\
\hline $\begin{array}{c}\AA \\
6324.43 \\
6313.61 \\
6312.99\end{array}$ & 1 & $\begin{array}{r}90 \\
1 \\
20\end{array}$ & \begin{tabular}{c|}
$K$ \\
15807.33 \\
15834.42 \\
15835.98
\end{tabular} & $\begin{aligned} & K \\
& 0.00 \\
& 0.00 \\
&+ 0.01\end{aligned}$ & $\begin{array}{l}55462_{3 \frac{1}{2}}^{\circ}-71270_{2 \frac{1}{2}} \\
52921_{3 \frac{1}{2}}^{\circ}-68756_{3 \frac{1}{2}} \\
62046_{3 \frac{1}{2}}^{\circ}-77882_{3 \frac{1}{2}}\end{array}$ & $\begin{array}{l}4 \\
7\end{array}$ & $\begin{array}{l}(0.878) \\
1.14\end{array}$ & $\begin{array}{l}0.89 \\
(1.12)\end{array}$ & $\begin{array}{l}0 \\
0\end{array}$ & $\begin{array}{l}0.85 \\
1.13\end{array}$ \\
\hline $\begin{array}{l}6308.15 \\
6303.91 \\
6303.27 \\
6300.94 \\
6297.35\end{array}$ & $\begin{array}{r}50 \\
5\end{array}$ & $\begin{array}{r}2000 \\
5 \\
400 \\
8 \\
400\end{array}$ & $\begin{array}{l}15848.13 \\
15858.79 \\
15860.40 \\
15866.26 \\
15875.31\end{array}$ & $\begin{array}{l}+0.01 \\
+0.01 \\
-0.02 \\
+0.01 \\
+0.02\end{array}$ & $\begin{array}{l}33052_{4 \frac{1}{2}}^{\circ}-48900_{3 \frac{1}{2}} \\
57798_{2 \frac{1}{2}}^{\circ}-73657_{1 \frac{1}{2}} \\
37077_{2 \frac{1}{2}}^{\circ}-52938_{1 \frac{1}{2}} \\
60649_{4 \frac{1}{2}}^{\circ}-76516_{4 \frac{1}{2}} \\
52880_{4 \frac{1}{2}}^{\circ}-68756_{3 \frac{1}{2}}^{\circ}\end{array}$ & 5 & 1.260 & 1.030 & 0.115 & 1.45 \\
\hline $\begin{array}{l}6292.56 \\
6292.17 \\
6291.06 \\
6285.72 \\
6281.96\end{array}$ & 2 & $\begin{array}{r}2 \\
20 \\
5 \\
3 \\
4\end{array}$ & $\begin{array}{l}15887.39 \\
15888.37 \\
15891.18 \\
15904.68 \\
15914.20\end{array}$ & $\begin{array}{r}+0.03 \\
0.00 \\
+0.02 \\
+0.01 \\
+0.02\end{array}$ & $\begin{array}{l}52067_{0 \frac{1}{2}}^{\circ}-67955_{0 \frac{1}{2}} \\
37516_{3 \frac{1}{2}}^{\circ}-53404_{2 \frac{1}{2}} \\
53716_{2 \frac{1}{2}}^{\circ}-69607_{2 \frac{1}{2}} \\
47329_{2 \frac{1}{2}}^{\circ}-63234_{2 \frac{1}{2}}^{\circ} \\
49008_{3 \frac{1}{2}}^{\circ}-64923_{4 \frac{1}{2}}\end{array}$ & & & & & \\
\hline $\begin{array}{l}6280.60 \\
6277.08 \\
6274.78 \\
6271.15\end{array}$ & $\begin{array}{r}2 \\
2000 \\
6\end{array}$ & $\begin{array}{r}2 \\
150 \\
10000 \\
250\end{array}$ & $\begin{array}{l}15917.64 \\
15926.57 \\
15932.41 \\
15941.63\end{array}$ & $\begin{array}{r}-0.02 \\
+0.05 \\
0.00 \\
-0.01\end{array}$ & $\begin{array}{l}65739_{2 \frac{1}{2}}^{\circ}-81657_{2 \frac{1}{2}} \\
59046_{4 \frac{1}{2}}^{\circ}-74973_{3 \frac{1}{2}} \\
31979_{3 \frac{1}{2}}^{\circ}-47912_{3 \frac{1}{2}} \\
45273_{3 \frac{1}{2}}^{\circ}-61214_{4 \frac{1}{2}}^{\circ}\end{array}$ & $\begin{array}{l}7 \\
6 \\
4\end{array}$ & $\begin{array}{l}1.08 \\
1.326 \\
(1.18)\end{array}$ & $\begin{array}{l}1.07 \\
1.280 \\
1.04\end{array}$ & $\begin{array}{l}0 \\
0.16 \\
w\end{array}$ & $\begin{array}{l}1.07 \\
1.303 \\
0.57\end{array}$ \\
\hline 6270.31 & 3 & 100 & 15943.77 & $\left\{\begin{array}{l}-0.01 \\
+0.04\end{array}\right.$ & $\begin{array}{c}47758_{3 \frac{1}{2}}^{\circ}-63702_{2 \frac{1}{2}}^{\circ} \\
\left(54192_{5 \frac{1}{2}}^{\circ}-70136_{6 \frac{1}{2}}\right)\end{array}$ & 5 & (1.08) & 1.01 & $w$ & 1.26 \\
\hline $\begin{array}{l}6269.95 \\
6265.45 \\
6262.89\end{array}$ & $\begin{array}{l}1 \\
1\end{array}$ & $\begin{array}{l}40 \\
80 \\
15\end{array}$ & $\begin{array}{l}15944.68 \\
15956.13 \\
15962.65\end{array}$ & $\begin{array}{r}+0.01 \\
+0.02 \\
0.00\end{array}$ & $\begin{array}{l}45429_{3 \frac{1}{2}}^{\circ}-61374_{2 \frac{1}{2}} \\
51248_{2 \frac{1}{2}}^{\circ}-67204_{1 \frac{1}{2}} \\
53644_{3 \frac{1}{2}}^{\circ}-69607_{2 \frac{1}{2}}\end{array}$ & 7 & (1.034) & 1.04 & 0 & 1.02 \\
\hline $\begin{array}{l}6260.79 \\
6250.39\end{array}$ & 30 & $\begin{array}{r}1500 \\
1\end{array}$ & $\begin{array}{l}15968.01 \\
15994.58\end{array}$ & $\begin{array}{l}+0.01 \\
-0.01\end{array}$ & $\begin{array}{l}48923_{\frac{1}{2}}^{\circ}-64891_{7 \frac{1}{2}}^{\circ} \\
56621_{5 \frac{1}{2}}^{\circ}-72615_{5 \frac{1}{2}}^{\circ}\end{array}$ & $6 ?$ & 1.20 & 1.20 & 0 & 1.20 \\
\hline $\begin{array}{l}6249.54 \\
6248.29 \\
6246.97 \\
6245.93 \\
6244.39\end{array}$ & 500 & $\begin{array}{r}1 \\
20 \\
4000 \\
6 \\
1\end{array}$ & $\begin{array}{l}15996.75 \\
15999.95 \\
16003.33 \\
16006.00 \\
16009.95\end{array}$ & $\begin{array}{r}+0.01 \\
-0.03 \\
-0.02\end{array}$ & $\begin{array}{l}60649_{4 \frac{1}{2}}^{\circ}-76649_{5 \frac{1}{2}} \\
33494_{3 \frac{1}{2}}^{\circ}-49498_{2 \frac{1}{2}} \\
55462_{3 \frac{1}{2}}^{\circ}-71468_{2 \frac{1}{2}}\end{array}$ & 4 & 0.990 & 1.140 & $(0.08)$ & 0.60 \\
\hline $\begin{array}{l}6242.25 \\
6239.67\end{array}$ & 1 & $\begin{array}{r}30 \\
3\end{array}$ & $\begin{array}{l}16015.43 \\
16022.06\end{array}$ & $\begin{array}{l}+0.03 \\
+0.03\end{array}$ & $\begin{array}{l}59624_{3 \frac{1}{2}}^{\circ}-75640_{\frac{1}{2}}^{\circ} \\
52921_{3 \frac{1}{2}}^{\circ}-68943_{4^{\frac{1}{2}}}\end{array}$ & & & & & \\
\hline 6236.13 & 3 & 150 & 16031.15 & +0.03 & $48024_{1 \frac{1}{2}}^{\circ}-64055_{2 \frac{1}{2}}$ & 4 & 1.36 & 1.12 & 0.12 & 0.76 \\
\hline 6234.85 & 3 & 250 & 16034.44 & -0.01 & $49916_{4 \frac{1}{2}}^{\mathrm{o}}-65950_{4 \frac{1}{2}}$ & 6 & 1.08 & 1.06 & 0.11 & 1.07 \\
\hline 6233.37 & 5 & 250 & 16038.25 & -0.02 & $45012_{2 \frac{1}{2}}^{\circ}-61051_{3 \frac{1}{2}}^{\circ}$ & 4 & 1.20 & 1.12 & $(0.042)$ & 0.91 \\
\hline $\begin{array}{l}6232.02 \\
6229.36\end{array}$ & & $\begin{array}{r}2 \\
10\end{array}$ & $\begin{array}{l}16041.72 \\
16048.57\end{array}$ & $\begin{array}{l}+0.01 \\
+0.02\end{array}$ & $\begin{array}{l}48556_{3 \frac{1}{2}}^{\circ}-64598_{3 \frac{1}{2}} \\
55221_{1 \frac{1}{2}}^{\circ}-71270_{2 \frac{1}{2}}\end{array}$ & & & & & \\
\hline $\begin{array}{l}6223.63 \\
6216.14\end{array}$ & 15 & $\begin{array}{r}500 \\
9\end{array}$ & $\begin{array}{l}16063.35 \\
16082.70\end{array}$ & $\begin{array}{r}+0.04 \\
0.00\end{array}$ & $\begin{array}{l}47663_{4 \frac{1}{2}}^{\circ}-63726_{4 \frac{1}{2}} \\
52067_{0 \frac{1}{2}}^{\circ}-68150_{1 \frac{1}{2}}\end{array}$ & 7 & 1.00 & (1.005) & 0 & 1.000 \\
\hline 6215.56 & 6 & 200 & 16084.20 & -0.01 & $49008_{3 \frac{1}{2}}^{\circ}-65093_{3 \frac{1}{2}}^{\circ}$ & 6 & 1.01 & 0.96 & 0.19 & 0.99 \\
\hline 6213.74 & & 3 & 16088.92 & -0.03 & $44497_{2 \frac{1}{2}}^{\circ}-60586_{1 \frac{1}{2}}$ & & & & & \\
\hline
\end{tabular}


TABLE 6. Yb II-Observed and classified lines-Continued

\begin{tabular}{|c|c|c|c|c|c|c|c|c|c|c|}
\hline 1 & 2 & 3 & 4 & 5 & 6 & 7 & 8 & 9 & 10 & 11 \\
\hline \multirow{2}{*}{$\begin{array}{l}\text { Wave- } \\
\text { length } \\
\text { air }\end{array}$} & \multicolumn{2}{|c|}{ Intensity } & \multirow{2}{*}{$\begin{array}{c}\text { Wave } \\
\text { number }\end{array}$} & \multirow[b]{2}{*}{$o-c$} & \multirow{2}{*}{ Classification } & \multirow{2}{*}{$\begin{array}{l}\text { Zee- } \\
\text { man } \\
\text { type }\end{array}$} & \multirow{2}{*}{ lst $g$} & \multirow{2}{*}{ 2nd $g$} & \multicolumn{2}{|c|}{$\begin{array}{c}\text { Zeeman } \\
\text { components }\end{array}$} \\
\hline & $\begin{array}{l}\text { Meggers } \\
\text { lamp }\end{array}$ & $\begin{array}{c}\text { Thomson } \\
\text { lamp }\end{array}$ & & & & & & & Strong $p$ & Strong $n$ \\
\hline A & & & $K$ & K & & & & & & \\
\hline 6212.28 & & 2 & 16092.70 & -0.01 & $64365_{4 \frac{1}{2}}^{\circ}-80457_{3 \frac{1}{2}}$ & & & & & \\
\hline 6211.10 & & 1 & 16095.75 & -0.02 & $66796_{3 \frac{1}{2}}^{\circ}-82891_{2 \frac{1}{2}}$ & & & & & \\
\hline 6208.89 & 3 & 70 & 16101.48 & +0.01 & $45273_{3 \frac{1}{2}}^{\circ}-61374_{2 \frac{1}{2}}^{2}$ & & & & & \\
\hline 6208.10 & 10 & 500 & 16103.53 & 0.00 & $50468_{5 \frac{1}{2}}^{\circ}-66571_{5 \frac{1}{2}}^{\circ}$ & 6 & 1.22 & 1.20 & 0.09 & 1.21 \\
\hline 6206.49 & & 3 & 16107.71 & -0.02 & $58162_{1 \frac{1}{2}}^{\circ}-74270_{2 \frac{1}{2}}$ & & & & & \\
\hline 6204.88 & & 4 & 16111.89 & +0.03 & $59439_{2 \frac{1}{2}}^{\circ}-75550_{2 \frac{1}{2}}^{\circ}$ & & & & & \\
\hline $\begin{array}{l}6204.20 \\
619601\end{array}$ & & 4 & 16113.66 & +0.02 & $56088_{4 \frac{1}{2}}^{\circ}-72202_{3 \frac{1}{2}}$ & & & & & \\
\hline 6191.14 & & $\begin{array}{l}0 \\
7 h\end{array}$ & $\begin{array}{l}16134.95 \\
16147.65\end{array}$ & $\begin{array}{l}-0.02 \\
+0.06\end{array}$ & $\begin{array}{l}56480_{4 \frac{1}{2}}-72615_{5 \frac{1}{2}} \\
49419_{0 \frac{1}{2}}^{0}-65566_{0 \frac{1}{2}}\end{array}$ & & & & & \\
\hline 6190.78 & 15 & 1000 & 16148.59 & +0.01 & $49727_{6 \frac{1}{2}}^{\circ}-65875_{6 \frac{1}{2}}$ & 7 & 1.21 & (1.205) & 0 & 1.206 \\
\hline 6189.03 & 1 & 90 & 16153.15 & +0.01 & $54640_{4 \frac{1}{2}}^{\circ}-70793_{5 \frac{1}{2}}^{\circ}$ & & & & & \\
\hline 6187.08 & & 20 & 16158.24 & -0.02 & $52921_{3 \frac{1}{2}}^{\circ}-69079_{2 \frac{1}{2}}^{\circ}$ & & & & & \\
\hline $\begin{array}{l}6185.43 \\
6184.05\end{array}$ & & $\begin{array}{l}2 \\
1\end{array}$ & $\begin{array}{l}16162.55 \\
16166.16\end{array}$ & +0.08 & $63417^{\circ}-79583$ & & & & & \\
\hline & & & & 0.00 & $034162 \frac{1}{2}-195033_{2 \frac{1}{2}}$ & & & & & \\
\hline 6178.81 & 1 & 20 & 16179.87 & $\left\{\begin{array}{l}-0.01 \\
+0.04\end{array}\right.$ & $\begin{array}{l}44940_{1 \frac{1}{2}}^{\circ}-61120_{0 \frac{1}{2}} \\
75058^{2}-91237^{\circ}\end{array}$ & & & & & \\
\hline 6178.63 & & 3 & 16180.34 & +0.01 & $57103_{3 \frac{1}{2}}^{\circ \frac{1}{2}}-73283_{4 \frac{1}{2}}$ & & & & & \\
\hline 6175.58 & & 90 & 16188.33 & +0.02 & $48272_{2 \frac{1}{2}}^{\circ}-64461_{1 \frac{1}{2}}^{\circ}$ & 4 & 1.15 & $(1.176)$ & 0 & 1.11 \\
\hline 6175.36 & & 9 & 16188.91 & -0.01 & $63417_{2 \frac{1}{2}}^{\circ}-79605_{1 \frac{1}{2}}$ & & & & & \\
\hline 6174.70 & 1 & 60 & 16190.64 & +0.03 & $49008_{3 \frac{1}{2}}^{\circ}-65199_{4 \frac{1}{2}}$ & & & & & \\
\hline 6173.76 & & 7 & 16193.10 & -0.01 & $66205_{2 \frac{1}{2}}^{\circ}-82398_{1 \frac{1}{2}}$ & & & & & \\
\hline 6171.63 & 30 & 300 & 16198.69 & +0.02 & $37516_{3 \frac{1}{2}}^{\circ}-53715_{3 \frac{1}{2}}^{\circ}$ & 6 & 1.13 & 1.18 & 0.17 & 1.16 \\
\hline 6169.55 & 100 & 200 & 16204.15 & +0.03 & $37516_{3 \frac{1}{2}}^{\circ}-53720_{4 \frac{1}{2}}^{\circ}$ & & & & & \\
\hline 6164.53 & & 2 & 16217.35 & -0.01 & $54640_{4 \frac{1}{2}}^{\circ}-70858_{4 \frac{1}{2}}^{\circ}$ & & & & & \\
\hline 6152.57 & 800 & 4000 & 16248.87 & 0.00 & $33052_{4 \frac{1}{2}}^{\circ}-49301_{4 \frac{1}{2}}$ & 6 & 1.262 & 1.190 & 0.336 & 1.23 \\
\hline 6150.62 & & $70 h$ & 16254.03 & & & & & & & :-... \\
\hline 6149.44 & & 1 & 16257.14 & +0.06 & $47912_{3 \frac{1}{2}}-64169_{3 \frac{1}{2}}^{\circ}$ & & & & & \\
\hline 6146.91 & 10 & 400 & 16263.84 & +0.06 & $47680_{6 \frac{1}{2}}^{\circ}-63944_{5 \frac{1}{2}}^{\circ}$ & 7 & 1.12 & (1.115) & 0 & 1.14 \\
\hline 6146.29 & & 3 & 16265.48 & -0.03 & $78070_{4 \frac{1}{2}}-94335_{5 \frac{1}{2}}^{\circ}$ & & & & & \\
\hline 6142.83 & 1 & 60 & 16274.64 & +0.02 & $53120_{2 \frac{1}{2}}^{\circ}-69395_{3 \frac{1}{2}}^{\circ}$ & 7 & $(0.944)$ & 0.94 & 0 & 0.95 \\
\hline 6141.70 & & 1 & 16277.63 & & & & & & & \\
\hline 6141.05 & & 3 & 16279.35 & 0.00 & $56500_{3 \frac{1}{2}}^{\circ}-72779_{3 \frac{1}{2}}$ & & & & & \\
\hline 6140.55 & & 2 & 16280.68 & +0.02 & $47663_{4 \frac{1}{2}}^{\circ}-63944_{5 \frac{1}{2}}^{\circ}$ & & & & & \\
\hline 6135.41 & & 2 & 16294.32 & +0.04 & $47663_{4 \frac{1}{2}}^{\circ}-63957_{3 \frac{1}{2}}$ & & & & & \\
\hline 6134.28 & 7 & 200 & 16297.32 & +0.04 & $47758_{3 \frac{1}{2}}^{\circ}-64055_{2 \frac{1}{2}}^{2}$ & 7 & 1.10 & (1.108) & 0 & 1.09 \\
\hline 6133.55 & & 3 & 16299.26 & +0.04 & $56480_{4 \frac{1}{2}}^{\circ}-72779_{3 \frac{1}{2}}$ & & & & & \\
\hline 6132.94 & & 25 & 16300.88 & +0.04 & $62163_{2 \frac{1}{2}}^{\circ}-78464_{1 \frac{1}{2}}^{\circ}$ & & & & & \\
\hline 6131.53 & 1 & 80 & 16304.63 & +0.05 & $61442_{5 \frac{1}{2}}^{2}-77747_{4 \frac{1}{2}}^{2}$ & 5 & 1.11 & (1.07) & $w$ & 1.30 \\
\hline 6128.51 & & 5 & 16312.67 & +0.07 & $53644_{3 \frac{1}{2}}^{\circ}-69957_{4 \frac{1}{2}}$ & & & & & \\
\hline 6128.18 & 10 & 300 & 16313.54 & -0.01 & $46547_{5 \frac{1}{2}}^{\circ}-62861_{5 \frac{1}{2}}^{\circ}$ & & & & & \\
\hline
\end{tabular}


TABLE 6. Yb II-Observed and classified lines-Continued

\begin{tabular}{|c|c|c|c|c|c|c|c|c|c|c|}
\hline 1 & 2 & 3 & 4 & 5 & 6 & 7 & 8 & 9 & 10 & 11 \\
\hline \multirow{2}{*}{$\begin{array}{l}\text { Wave- } \\
\text { length } \\
\text { air }\end{array}$} & \multicolumn{2}{|c|}{ Intensity } & \multirow{2}{*}{$\begin{array}{l}\text { Wave } \\
\text { number }\end{array}$} & \multirow{2}{*}{$\mathrm{o}-\mathrm{c}$} & \multirow{2}{*}{ Classification } & \multirow{2}{*}{ 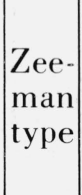 } & \multirow{2}{*}{ lst $g$} & \multirow{2}{*}{2 nd $g$} & \multicolumn{2}{|c|}{$\begin{array}{c}\text { Zeeman } \\
\text { components }\end{array}$} \\
\hline & $\begin{array}{l}\text { Meggers } \\
\text { lamp }\end{array}$ & $\begin{array}{c}\text { Thomson } \\
\text { lamp }\end{array}$ & & & & & & & Strong $p$ & Strong $n$ \\
\hline $\begin{array}{c}\AA \\
6127.92 \\
6126.89 \\
6126.39 \\
6124.63 \\
6123.67\end{array}$ & 1 & $\begin{array}{r}10 \\
1 \\
100 \\
3 \\
30\end{array}$ & \begin{tabular}{c|}
$K$ \\
16314.24 \\
16316.98 \\
16318.31 \\
16323.00 \\
16325.56
\end{tabular} & $\begin{array}{c}K \\
+0.06 \\
+0.02 \\
+0.02 \\
+0.06 \\
+0.05\end{array}$ & $\begin{array}{l}64365_{4 \frac{1}{2}}^{\circ}-80679_{5 \frac{1}{2}} \\
58672_{0 \frac{1}{2}}^{\circ}-74989_{0 \frac{1}{2}} \\
47329_{2 \frac{1}{2}}^{\circ}-63647_{1 \frac{1}{2}} \\
53120_{2 \frac{1}{2}}^{\circ}-69443_{1 \frac{1}{2}} \\
48272_{2 \frac{1}{2}}^{\circ}-64598_{3 \frac{1}{2}}^{\circ}\end{array}$ & & & & & \\
\hline $\begin{array}{l}6122.98 \\
6121.91 \\
6120.30 \\
6117.54 \\
6116.67\end{array}$ & $\begin{array}{r}40 \\
\\
5 \\
2 \\
10\end{array}$ & $\begin{array}{r}250 \\
9 \\
250 \\
10 \\
70\end{array}$ & $\begin{array}{l}16327.40 \\
16330.25 \\
16334.55 \\
16341.92 \\
16344.24\end{array}$ & $\begin{array}{l}+0.03 \\
+0.02 \\
+0.01 \\
+0.01\end{array}$ & $\begin{array}{l}37077_{2 \frac{1}{2}}^{\circ}-53404_{2 \frac{1}{2}} \\
58661_{2 \frac{1}{2}}^{\circ}-74991_{1 \frac{1}{2}} \\
64970_{3 \frac{1}{2}}^{\circ}-81312_{2 \frac{1}{2}} \\
31568_{2 \frac{1}{2}}^{\circ}-47912_{3 \frac{1}{2}}\end{array}$ & 6 & 1.118 & 1.052 & 0.164 & 1.085 \\
\hline $\begin{array}{l}6110.13 \\
6108.32\end{array}$ & 6 & $\begin{array}{r}200 \\
2\end{array}$ & $\begin{array}{l}16361.74 \\
16366.58\end{array}$ & $\begin{array}{l}+0.04 \\
+0.04\end{array}$ & $\begin{array}{l}45012_{2 \frac{1}{2}}^{\circ}-61374_{2 \frac{1}{2}} \\
48556_{3 \frac{1}{2}}^{\circ}-64923_{4 \frac{1}{2}}\end{array}$ & 7 & 1.30 & (1.299) & 0 & 1.30 \\
\hline $\begin{array}{l}6106.18 \\
6105.58\end{array}$ & $\begin{array}{l}5 \\
1\end{array}$ & $\begin{array}{r}200 \\
10\end{array}$ & $\begin{array}{l}16372.32 \\
16373.93\end{array}$ & $\begin{array}{r}0.00 \\
+0.05\end{array}$ & $\begin{array}{l}50832_{1 \frac{1}{2}}^{\circ}-67204_{1 \frac{1}{2}}^{\circ} \\
60910_{1 \frac{1}{2}}^{\circ}-77284_{1 \frac{1}{2}}\end{array}$ & 6 & 0.957 & 1.043 & 0.129 & 1.000 \\
\hline 6098.89 & & 7 & 16391.89 & $\left\{\begin{array}{l}+0.05 \\
-0.05\end{array}\right.$ & $\begin{array}{l}73750_{4 \frac{1}{2}}-90142_{3 \frac{1}{2}}^{\circ} \\
77606_{2 \frac{1}{2}}-93998_{1 \frac{1}{2}}^{\circ}\end{array}$ & & & & & \\
\hline $\begin{array}{l}6098.54 \\
6096.98 \\
6095.98 \\
6089.14\end{array}$ & $\begin{array}{l}3 \\
1\end{array}$ & $\begin{array}{r}250 \\
30 \\
3 \\
100\end{array}$ & $\begin{array}{l}16392.83 \\
16397.02 \\
16399.71 \\
16418.14 \\
16410.2\end{array}$ & $\begin{array}{l}+0.07 \\
+0.04 \\
+0.06 \\
+0.05\end{array}$ & $\begin{array}{l}45429_{3 \frac{1}{2}}^{\circ}-61822_{3 \frac{1}{2}} \\
58661_{2 \frac{1}{2}}^{\circ}-75058_{2 \frac{1}{2}} \\
45737_{1 \frac{1}{2}}^{\circ}-62136_{0 \frac{1}{2}}^{\circ} \\
22960_{1 \frac{1}{2}}-39378_{0 \frac{1}{2}}^{\circ}\end{array}$ & & & & & \\
\hline 6088.70 & 3 & 200 & 16419.32 & +0.03 & $48503_{5 \frac{1}{2}}^{\circ}-64923_{4 \frac{1}{2}}^{\circ}$ & 7 & 1.11 & $(1.10)$ & 0 & 1.16 \\
\hline 6083.86 & 1 & 30 & 16432.38 & +0.02 & $57534_{3 \frac{1}{2}}^{\circ}-73966_{3 \frac{1}{2}}$ & & & & & \\
\hline 6083.29 & 5 & 200 & 16433.92 & +0.04 & $44940_{1 \frac{1}{2}}^{\circ}-61374_{2 \frac{1}{2}}^{\circ}$ & 5 & 1.26 & (1.299) & $w$ & 1.36 \\
\hline $\begin{array}{l}6082.37 \\
6082.21\end{array}$ & 6 & $\begin{array}{r}500 \\
10\end{array}$ & $\begin{array}{l}16436.41 \\
16436.84\end{array}$ & $\begin{array}{l}+0.03 \\
+0.03\end{array}$ & $\begin{array}{l}48024_{1 \frac{1}{2}}^{\circ}-64461_{1 \frac{1}{2}} \\
45737_{1 \frac{1}{3}}^{\circ}-62174_{1 \frac{1}{2}}\end{array}$ & 6 & 1.39 & 1.19 & 0.30 & 1.28 \\
\hline 6075.21 & 3 & 200 & 16455.78 & +0.02 & $52987_{1 \frac{1}{2}}^{\circ}-69443_{1 \frac{1}{2}}$ & 7 & 1.14 & (1.145) & 0 & 1.14 \\
\hline $\begin{array}{l}6074.10 \\
6070.19 \\
6068.64 \\
6063.01 \\
6059.93\end{array}$ & 1 & $\begin{array}{r}1 \\
20 \\
60 \\
4 \\
3\end{array}$ & $\begin{array}{l}16458.79 \\
16469.39 \\
16473.60 \\
16488.89 \\
16497.27\end{array}$ & $\begin{array}{l}+0.02 \\
+0.06 \\
+0.02 \\
+0.02\end{array}$ & $\begin{array}{l}73750_{4 \frac{1}{2}}-90209_{4 \frac{1}{2}}^{\circ} \\
49419_{0 \frac{1}{2}}^{\circ}-65888_{1 \frac{1}{2}} \\
52921_{3 \frac{1}{2}}^{\circ}-69395_{3 \frac{1}{2}} \\
58484_{4 \frac{1}{2}}^{\circ}-74973_{3 \frac{1}{2}}\end{array}$ & & & & & \\
\hline $\begin{array}{l}6056.46 \\
6053.61\end{array}$ & 10 & $\begin{array}{r}500 \\
3\end{array}$ & $\begin{array}{l}16506.72 \\
16514.50\end{array}$ & $\begin{array}{r}0.00 \\
+0.05\end{array}$ & $\begin{array}{l}46354_{4 \frac{1}{2}}^{\circ}-62861_{5 \frac{1}{2}} \\
52880_{4 \frac{1}{2}}^{\circ}-69395_{3 \frac{1}{2}}\end{array}$ & 4 & 1.288 & (1.140) & $w$ & 0.475 \\
\hline $\begin{array}{l}6052.88 \\
6052.66 \\
6048.87\end{array}$ & 100 & $\begin{array}{r}900 \\
250 \\
2\end{array}$ & $\begin{array}{l}16516.49 \\
16517.09 \\
16527.44\end{array}$ & $\begin{array}{l}+0.04 \\
+0.02 \\
+0.09\end{array}$ & $\begin{array}{l}32981_{1 \frac{1}{2}}^{\circ}-49498_{2 \frac{1}{2}}^{\circ} \\
58051_{5 \frac{1}{2}}^{\circ}-74568_{4 \frac{1}{2}} \\
56088_{4 \frac{1}{2}}^{\circ}-72615_{5 \frac{1}{2}}^{\circ}\end{array}$ & 5 & 0.87 & 1.15 & 0.14 & 1.57 \\
\hline $\begin{array}{l}6048.18 \\
6045.52 \\
6043.45 \\
6042.22 \\
6040.77\end{array}$ & $\begin{array}{r}2 \\
15\end{array}$ & $\begin{array}{r}50 \\
6 \\
1 \\
100 \\
500\end{array}$ & $\begin{array}{l}16529.32 \\
16536.60 \\
16542.26 \\
16545.63 \\
16549.60\end{array}$ & $\begin{array}{l}+0.01 \\
+0.03 \\
+0.05 \\
+0.04 \\
+0.04\end{array}$ & $\begin{array}{l}32371_{2 \frac{1}{2}}^{\circ}-48900_{3 \frac{1}{2}} \\
48556_{3 \frac{1}{2}}^{\circ}-65093_{3 \frac{1}{2}} \\
55221_{1 \frac{1}{2}}^{\circ}-71763_{1 \frac{1}{2}} \\
59624_{3 \frac{1}{2}}^{\circ}-76170_{2 \frac{1}{2}} \\
45273^{\circ}-61822^{\circ}\end{array}$ & & & & & 25 \\
\hline & & & 16549.60 & +0.04 & $45273_{3 \frac{1}{2}}^{\circ}-61822_{3 \frac{1}{2}}$ & 6 & 1.19 & 1.31 & 0.43 & 1.25 \\
\hline
\end{tabular}


TABLE 6. Yb II-Observed and classified lines-Continued

\begin{tabular}{|c|c|c|c|c|c|c|c|c|c|c|}
\hline 1 & 2 & 3 & 4 & 5 & 6 & 7 & 8 & 9 & 10 & 11 \\
\hline \multirow{2}{*}{$\begin{array}{l}\text { Wave- } \\
\text { length } \\
\text { air }\end{array}$} & \multicolumn{2}{|c|}{ Intensity } & \multirow{2}{*}{$\begin{array}{l}\text { Wave } \\
\text { number }\end{array}$} & \multirow{2}{*}{$o-c$} & \multirow{2}{*}{ Classification } & \multirow{2}{*}{$\left|\begin{array}{l}Z \text { ee } \\
\text { man } \\
\text { type }\end{array}\right|$} & \multirow{2}{*}{ lst $g$} & \multirow{2}{*}{ 2nd $g$} & \multicolumn{2}{|c|}{$\begin{array}{c}\text { Zeeman } \\
\text { components }\end{array}$} \\
\hline & $\begin{array}{l}\text { Meggers } \\
\text { lamp }\end{array}$ & $\begin{array}{c}\text { Thomson } \\
\text { lamp }\end{array}$ & & & & & & & Strong $p$ & Strong $n$ \\
\hline A & & & $K$ & K & & & & & & \\
\hline 6038.82 & & 9 & 16554.94 & +0.09 & $63028^{\circ}{ }_{1 \frac{1}{2}}-79583_{2 \frac{1}{2}}$ & & & & & \\
\hline $\begin{array}{l}6029.76 \\
6024.08\end{array}$ & 40 & $\begin{array}{r}1 \\
400\end{array}$ & $\begin{array}{l}16579.82 \\
16595.45\end{array}$ & -0.01 & $41688_{1}^{\circ}-58283$ & 7 & $(0784)$ & 0.77 & 0 & 076 \\
\hline 0024.08 & 40 & & 10595.45 & $(-0.01$ & $\begin{array}{l}41088_{1 \frac{1}{2}}-58283_{2 \frac{1}{2}} \\
54192_{5 \frac{1}{2}}^{\circ}-70793_{5 \frac{1}{2}}\end{array}$ & 8 & $(0.184)$ & 0.77 & 0 & 0.16 \\
\hline 6021.92 & 10 & 400 & 16601.40 & $\{+0.01$ & $59632_{6_{\frac{1}{2}}^{2}}^{\circ}-76233_{6^{\frac{1}{2}}}$ & 6 & 1.08 & 1.10 & 0.11 & 1.09 \\
\hline 6020.55 & 40 & 400 & 16605.18 & 0.00 & $41678_{2 \frac{1}{2}}^{\circ}-58283_{2 \frac{1}{2}}$ & & & & & \\
\hline 6017.70 & & 2 & 16613.04 & +0.01 & $44438_{3 \frac{1}{2}}^{\circ}-61051_{3 \frac{1}{2}}$ & & & & & \\
\hline 6016.62 & 5 & 50 & 16616.03 & -0.04 & $46547_{5 \frac{1}{2}}^{\circ}-63163_{4 \frac{1}{2}}^{\circ}$ & & & & & \\
\hline 6012.51 & & 9 & 16627.38 & +0.01 & $61442_{5 \frac{1}{2}}^{\circ}-78070_{4 \frac{1}{2}}^{\circ}$ & & & & & \\
\hline 6012.16 & 1 & $\begin{array}{r}15 \\
8\end{array}$ & 16628.35 & -0.01 & $47329_{2 \frac{1}{2}}^{\circ}-63957_{3 \frac{1}{2}}^{\circ}$ & & & & & \\
\hline & & & 16630.04 & -0.01 & $43956_{2 \frac{1}{2}}^{\circ}-60586_{1 \frac{1}{2}}^{\circ}$ & & & & & \\
\hline 6009.56 & 1 & 10 & 16635.55 & -0.01 & $43075_{1 \frac{1}{2}}^{\circ}-59710_{1 \frac{1}{2}}$ & & & & & \\
\hline 6008.80 & 5 & 50 & 16637.65 & -0.02 & $37077_{2 \frac{1}{2}}^{\circ}-53715_{3 \frac{1}{2}}^{\frac{1}{2}}$ & & & & & \\
\hline 6007.41 & 20 & 1000 & 16641.50 & 0.00 & $49916_{4 \frac{1}{2}}^{\circ}-66558_{3 \frac{1}{2}}^{2}$ & 4 & 1.08 & (1.100) & $w$ & 0.99 \\
\hline 6004.015 & & 10 & 16650.91 & -0.03 & $62163_{2 \frac{1}{2}}^{\circ}-78814_{2 \frac{1}{2}}^{2}$ & & & & & \\
\hline 6002.52 & 3 & 90 & 16655.06 & -0.02 & $49916_{4 \frac{1}{2}}^{0}-66571_{5 \frac{1}{2}}^{2}$ & & & & & \\
\hline 6001.05 & 1 & 30 & 16659.14 & +0.02 & $56977_{0 \frac{1}{2}}^{\circ}-73636_{0 \frac{1}{2}}$ & 6 & 2.20 & 0.71 & 0.745 & 1.45 \\
\hline 6000.91 & & 8 & 16659.53 & +0.02 & $65739_{2 \frac{1}{2}}^{\circ}-82398_{1 \frac{1}{2}}^{\circ}$ & & & & & \\
\hline 5999.95 & 1 & 30 & 16662.19 & -0.01 & $56621_{5 \frac{1}{2}}^{\circ}-73283_{4 \frac{1}{2}}^{\circ}$ & & & & & \\
\hline 5998.71 & & 25 & 16665.64 & -0.03 & $54192_{5 \frac{1}{2}}^{\circ}-70858_{4 \frac{1}{2}}$ & & & & & \\
\hline 5996.84 & & 1 & 16670.83 & +0.03 & $56621_{5 \frac{1}{2}}^{\circ}-73291_{5 \frac{1}{2}}^{\circ}$ & & & & & \\
\hline 5993.95 & & 1 & 16678.87 & -0.05 & $76516_{4 \frac{1}{2}}-93195_{3 \frac{1}{2}}^{\circ}$ & & & & & \\
\hline 5993.704 & & 15 & 16679.55 & -0.03 & $56977_{0 \frac{1}{2}}^{\circ}-73657_{1 \frac{1}{2}}^{\circ}$ & & & & & \\
\hline 5991.51 & 2000 & 6000 & 16685.66 & -0.01 & $35831_{5 \frac{1}{2}}^{\circ}-52517_{5 \frac{1}{2}}^{\circ}$ & 6 & 1.213 & 1.260 & 0.26 & 1.236 \\
\hline 5990.01 & & 10 & 16689.84 & +0.02 & $51248_{2 \frac{1}{2}}^{\circ}-67938_{3 \frac{1}{2}}$ & & & & & \\
\hline 5987.91 & 15 & 500 & 16695.69 & -0.03 & $48503_{5 \frac{1}{2}}^{\circ}-65199_{4 \frac{1}{2}}^{\circ}$ & & & & & \\
\hline 5986.78 & 1 & 40 & 16698.84 & +0.01 & $59624_{3 \frac{1}{2}}^{\circ}-76323_{3 \frac{1}{2}}$ & & & & & \\
\hline 5985.35 & 20 & 300 & 16702.83 & $\left\{\begin{array}{l}-0.03 \\
+0.03\end{array}\right.$ & $43007_{0 \frac{1}{2}}^{\circ}-59710_{1 \frac{1}{2}}$ & 5 & 0.16 & 0.82 & 0.32 & 1.15 \\
\hline 5978.11 & & 10 & 16723.06 & $\begin{array}{r}{[+0.03} \\
-0.02\end{array}$ & $\begin{array}{c}\left(60189_{1 \frac{1}{2}}-82891_{2 \frac{1}{2}}\right) \\
56056_{2 \frac{1}{2}}^{\circ}-72779_{3 \frac{1}{2}}\end{array}$ & & & & & \\
\hline 5976.93 & & 2 & 16726.36 & -0.02 & $47329_{2 \frac{1}{2}}^{\circ}-64055_{2 \frac{1}{2}}^{\circ}$ & & - & & & \\
\hline 5973.49 & 2 & 20 & 16736.00 & -0.03 & $57534_{3 \frac{1}{2}}^{\circ}-74270_{2 \frac{1}{2}}^{2}$ & & & & & \\
\hline 5972.29 & & 1 & 16739.36 & +0.01 & $55462_{3 \frac{1}{2}}^{\circ}-72202_{3 \frac{1}{2}}$ & & . & & & \\
\hline 5972.02 & & 2 & 16740.12 & -0.02 & $59777_{1 \frac{1}{2}}^{\circ}-76517_{1 \frac{1}{2}}^{\circ}$ & & & & & \\
\hline 5970.26 & & 5 & 16745.05 & +0.03 & $46902_{0 \frac{1}{2}}^{\circ}-63647_{1 \frac{1}{2}}^{\circ}$ & & & & & \\
\hline 5964.12 & & 1 & 16762.29 & +0.02 & $59753_{\frac{1}{2}}^{\circ}-76516_{4 \frac{1}{2}}$ & & & & & \\
\hline 5961.83 & 1 & 60 & 16768.73 & -0.06 & $62046_{3 \frac{1}{2}}^{\circ}-78814_{2 \frac{1}{2}}$ & 4 & 1.15 & $(1.22)$ & $w$ & 0.99 \\
\hline 5959.03 & & 7 & 16776.61 & -0.02 & $44438_{3 \frac{1}{2}}^{\circ}-61214_{4 \frac{1}{2}}$ & & & & & \\
\hline 5956.86 & & 7 & 16782.72 & $\left\{\begin{array}{l}-0.07 \\
+0.01\end{array}\right.$ & $\begin{array}{l}69443_{1 \frac{1}{2}}-86226_{1 \frac{1}{2}}^{\circ} \\
56500_{3 \frac{1}{2}}^{\circ}-73283_{4 \frac{1}{2}}^{\circ}\end{array}$ & & & & & \\
\hline
\end{tabular}


TABLE 6. Yb II-Observed and classified lines-Continued

\begin{tabular}{|c|c|c|c|c|c|c|c|c|c|c|}
\hline 1 & 2 & 3 & 4 & 5 & 6 & 7 & 8 & 9 & 10 & 11 \\
\hline \multirow{2}{*}{$\begin{array}{l}\text { Wave- } \\
\text { length } \\
\text { air }\end{array}$} & \multicolumn{2}{|c|}{ Intensity } & \multirow{2}{*}{$\begin{array}{l}\text { Wave } \\
\text { number }\end{array}$} & \multirow[b]{2}{*}{$\mathrm{o}-\mathrm{c}$} & \multirow{2}{*}{ Classification } & \multirow{2}{*}{$\begin{array}{l}\text { Zee- } \\
\text { man } \\
\text { type }\end{array}$} & \multirow[b]{2}{*}{ lst $g$} & \multirow[b]{2}{*}{2 nd $g$} & \multicolumn{2}{|c|}{$\begin{array}{c}\text { Zeeman } \\
\text { components }\end{array}$} \\
\hline & $\begin{array}{c}\text { Meggers } \\
\text { lamp }\end{array}$ & $\begin{array}{c}\text { Thomson } \\
\text { lamp }\end{array}$ & & & & & & & Strong $p$ & Strong $n$ \\
\hline$\stackrel{\circ}{\AA}$ & & & $K$ & $K$ & & & & & & \\
\hline $\begin{array}{l}5950.93 \\
5949.83 \\
5948.98\end{array}$ & 5 & $\begin{array}{r}250 \\
20 \\
5\end{array}$ & $\begin{array}{l}16799.44 \\
16802.55 \\
16804.95\end{array}$ & $\begin{array}{l}-0.02 \\
-0.03 \\
+0.03\end{array}$ & $\begin{array}{l}60649_{\frac{1}{2}}^{\circ}-77449_{3 \frac{1}{2}}^{\circ} \\
56480_{4 \frac{1}{2}}^{\circ}-73283_{4 \frac{1}{2}} \\
65594_{2 \frac{1}{2}}^{\circ}-82398_{1 \frac{1}{2}}^{\circ}\end{array}$ & $7 ?$ & 1.13 & (1.16) & $w$ & 1.03 \\
\hline $\begin{array}{l}5947.26 \\
5946.00 \\
5944.63 \\
5943.55 \\
5943.05\end{array}$ & $\begin{array}{r}15 \\
40 \\
1 \\
5 \\
20\end{array}$ & $\begin{array}{r}500 \\
2000 \\
10 \\
200 \\
250\end{array}$ & $\begin{array}{l}16809.81 \\
16813.37 \\
16817.25 \\
16820.30 \\
16821.72\end{array}$ & $\begin{array}{r}+0.02 \\
+0.02 \\
0.00 \\
-0.07 \\
-0.01\end{array}$ & $\begin{array}{l}45012_{2 \frac{1}{2}}^{\circ}-61822_{3 \frac{1}{2}} \\
53322_{7 \frac{1}{2}}^{\circ}-70136_{6 \frac{1}{2}}^{\circ} \\
56840_{1 \frac{1}{2}}^{\circ}-73657_{1 \frac{1}{2}} \\
48272_{2 \frac{1}{2}}^{\circ}-65093_{3 \frac{1}{2}}^{\circ} \\
45737_{1 \frac{1}{2}}^{\circ}-62559_{2 \frac{1}{2}}^{\circ}\end{array}$ & $\begin{array}{l}5 \\
4\end{array}$ & $\begin{array}{l}1.32 \\
1.01\end{array}$ & $\begin{array}{l}(1.327) \\
1.07\end{array}$ & $\begin{array}{l}0 \\
(0.03)\end{array}$ & $\begin{array}{l}1.34 \\
0.61\end{array}$ \\
\hline $\begin{array}{l}5941.29 \\
5940.63 \\
5939.35 \\
5938.15 \\
5936.68\end{array}$ & $\begin{array}{l}2 \\
1\end{array}$ & $\begin{array}{r}2 \\
1 \\
8 \\
10 \\
20\end{array}$ & $\begin{array}{l}16826.70 \\
16828.57 \\
16832.20 \\
16835.60 \\
16839.77\end{array}$ & $\begin{array}{r}-0.04 \\
-0.04 \\
0.00 \\
+0.03\end{array}$ & $\begin{array}{l}58162_{1 \frac{1}{2}}^{\circ}-74989_{0 \frac{1}{2}} \\
58162_{1 \frac{1}{2}}^{\circ}-74991_{1_{\frac{1}{2}}}^{\circ} \\
72779_{3 \frac{1}{2}}-89615_{2 \frac{1}{2}}^{\circ} \\
47758_{3 \frac{1}{2}}^{\circ}-64598_{3 \frac{1}{2}}^{\circ}\end{array}$ & & & & & \\
\hline $\begin{array}{l}5935.05 \\
5934.01 \\
5929.77 \\
5928.24 \\
5920.98\end{array}$ & 20 & $\begin{array}{r}1000 \\
1 \\
10 \\
2 \\
2\end{array}$ & $\begin{array}{l}16844.39 \\
16847.34 \\
16859.39 \\
16863.74 \\
16884.42\end{array}$ & $\begin{array}{r}0.00 \\
-0.07 \\
0.00 \\
-0.05 \\
+0.01\end{array}$ & $\begin{array}{l}49727_{6 \frac{1}{2}}^{\circ}-66571_{5 \frac{1}{2}} \\
40917_{4 \frac{1}{2}}^{\circ}-57765_{4 \frac{1}{2}} \\
73283_{4 \frac{1}{2}}-90142_{3 \frac{1}{2}}^{\circ} \\
57103_{3 \frac{1}{2}}^{\circ}-73966_{3 \frac{1}{2}}^{\circ} \\
59439_{2 \frac{1}{2}}^{\circ}-76323_{3 \frac{1}{2}}^{\circ}\end{array}$ & 5 & 1.20 & (1.19) & 0 & 1.24 \\
\hline $\begin{array}{l}5920.38 \\
5919.10 \\
5916.95 \\
5914.912 \\
5909.33\end{array}$ & $\begin{array}{l}2 \\
2\end{array}$ & $\begin{array}{r}300 \\
7 \\
2 \\
20 \\
10\end{array}$ & $\begin{array}{l}16886.13 \\
16889.78 \\
16895.92 \\
16901.74 \\
16917.70\end{array}$ & $\begin{array}{l}-0.01 \\
-0.03 \\
-0.04 \\
+0.03 \\
-0.02\end{array}$ & $\begin{array}{l}51248_{2 \frac{1}{2}}^{\circ}-68135_{2 \frac{1}{2}} \\
58661_{2 \frac{1}{2}}^{\circ}-75550_{2 \frac{1}{2}} \\
59753_{5 \frac{1}{2}}^{\circ}-76649_{5 \frac{1}{2}} \\
51248_{2 \frac{1}{2}}^{\circ}-68150_{\frac{1}{2}}^{\circ} \\
73291_{5 \frac{1}{2}}-90209_{4 \frac{1}{2}}^{\circ}\end{array}$ & 6 & 1.029 & 1.081 & $(0.128)$ & 1.06 \\
\hline $\begin{array}{l}5908.36 \\
5906.33 \\
5903.38 \\
5902.80 \\
5900.85\end{array}$ & 400 & $\begin{array}{r}1500 \\
2 \\
90 \\
3 \\
20\end{array}$ & $\begin{array}{l}16920.48 \\
16926.30 \\
16934.76 \\
16936.42 \\
16942.02\end{array}$ & $\begin{array}{r}-0.03 \\
-0.02 \\
0.00 \\
-0.04 \\
\left\{\begin{array}{r}0.00 \\
+0.01\end{array}\right.\end{array}$ & $\begin{array}{l}31979_{3 \frac{1}{2}}^{\circ}-48900_{3 \frac{1}{2}} \\
73283_{\frac{1}{2}}^{\circ}-90209_{4 \frac{1}{2}}^{\circ} \\
47663_{4 \frac{1}{2}}^{\circ}-64598_{3 \frac{1}{2}} \\
44438_{3 \frac{1}{2}}^{\circ}-61374_{2 \frac{1}{2}} \\
49008_{3 \frac{1}{2}}^{\circ}-65950_{4 \frac{1}{2}} \\
65149_{0 \frac{1}{2}}^{\circ}-82091_{0 \frac{1}{2}}^{\circ}\end{array}$ & 6 & 1.335 & 1.045 & 1.015 & 1.190 \\
\hline $\begin{array}{l}5898.77 \\
5897.21 \\
5896.63 \\
5892.30 \\
5888.02\end{array}$ & $\begin{array}{l}15 \\
50\end{array}$ & $\begin{array}{c}800 \\
2500 \\
6 d \\
2 \\
4\end{array}$ & $\begin{array}{l}16947.99 \\
16952.47 \\
16954.14 \\
16966.60 \\
16978.93\end{array}$ & $\begin{array}{r}+0.01 \\
0.00 \\
-0.04 \\
0.00\end{array}$ & $\begin{array}{l}50468_{5 \frac{1}{2}}^{\circ}-67416_{4 \frac{1}{2}} \\
48923_{7 \frac{1}{2}}^{\circ}-65875_{6 \frac{1}{2}} \\
73750_{4 \frac{1}{2}}-90717_{4 \frac{1}{2}}^{\circ} \\
58661_{2 \frac{1}{2}}^{\circ}-75640_{3 \frac{1}{2}}\end{array}$ & $\begin{array}{l}4 \\
7\end{array}$ & $\begin{array}{l}1.21 \\
1.20\end{array}$ & $\begin{array}{l}1.25 \\
(1.205)\end{array}$ & $\begin{array}{l}(0.021) \\
0\end{array}$ & $\begin{array}{l}1.02 \\
1.174\end{array}$ \\
\hline $\begin{array}{l}5882.80 \\
5874.67 \\
5873.12 \\
5872.15 \\
5868.94\end{array}$ & $\begin{array}{r}100 \\
2\end{array}$ & $\begin{array}{r}600 \\
200 \\
10 \\
1 \\
7\end{array}$ & $\begin{array}{l}16994.00 \\
17017.52 \\
17022.01 \\
17024.82 \\
17034.13\end{array}$ & $\begin{array}{r}-0.01 \\
-0.07 \\
+0.01 \\
+0.04 \\
0.00\end{array}$ & $\begin{array}{l}46169_{4 \frac{1}{2}}^{\circ}-63163_{4 \frac{1}{2}} \\
59632_{6 \frac{1}{2}}^{\circ}-76649_{5 \frac{1}{2}}^{\circ} \\
59777_{\frac{1}{2}}^{\circ}-76799_{1 \frac{1}{2}} \\
76170_{2 \frac{1}{2}}^{\circ}-93195_{3 \frac{1}{2}}^{\circ} \\
57534_{3 \frac{1}{2}}^{\circ}-74568_{4 \frac{1}{2}}^{\circ}\end{array}$ & 7 & & & 0 & 1.08 \\
\hline
\end{tabular}


TABLE 6. Yb II-Observed and classified lines-Continued

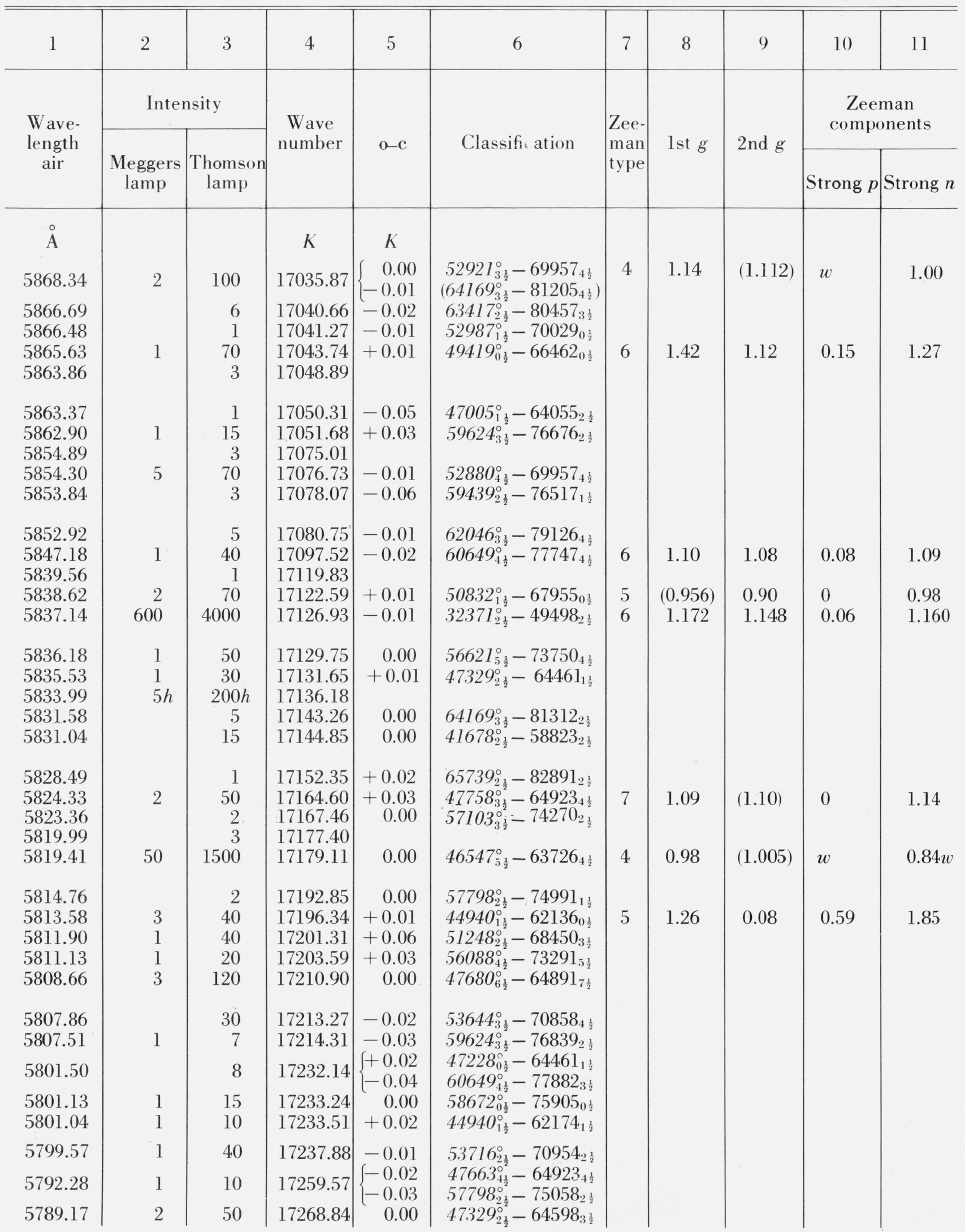


TABLE 6. Yb II-Observed and classified lines-Continued

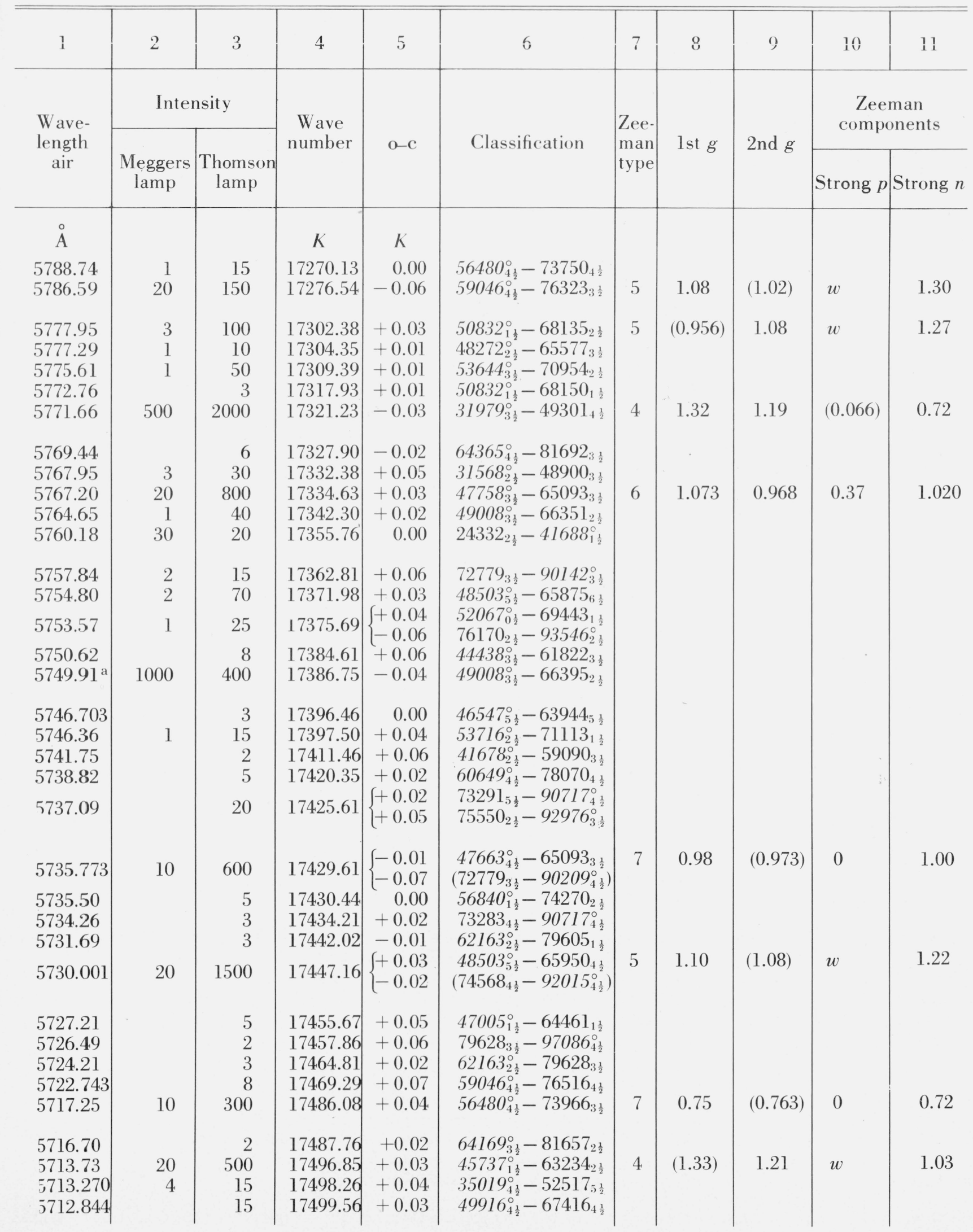


TABLE 6. Yb II-Observed and classified lines-Continued

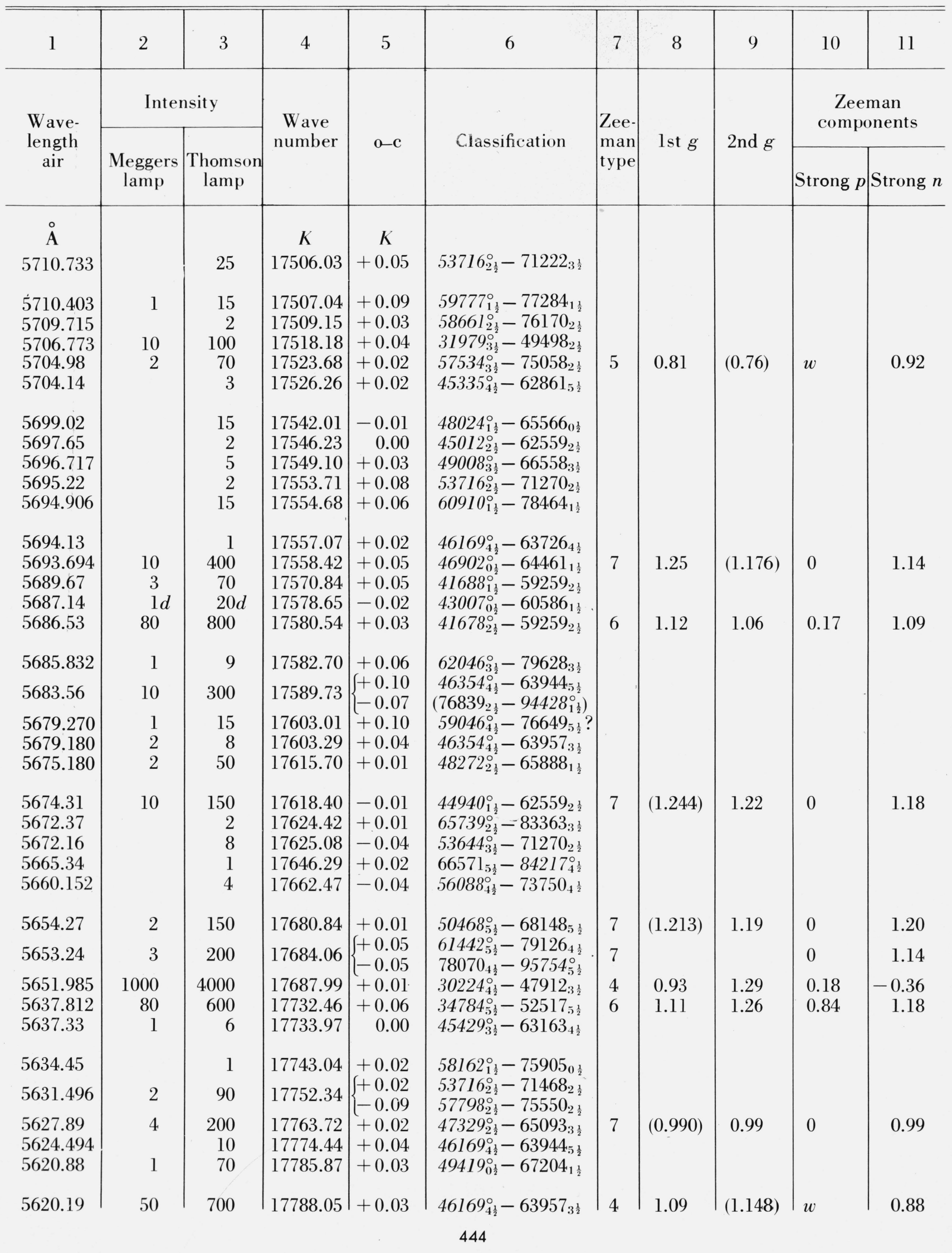


TABLE 6. Y Y II-Observed and classified lines-Continued

\begin{tabular}{|c|c|c|c|c|c|c|c|c|c|c|}
\hline 1 & 2 & 3 & 4 & 5 & 6 & 7 & 8 & 9 & 10 & 11 \\
\hline \multirow{2}{*}{$\begin{array}{l}\text { Wave- } \\
\text { length } \\
\text { air }\end{array}$} & \multicolumn{2}{|c|}{ Intensity } & \multirow{2}{*}{$\begin{array}{l}\text { Wave } \\
\text { number }\end{array}$} & \multirow{2}{*}{$o-c$} & \multirow{2}{*}{ Classification } & \multirow{2}{*}{ 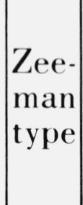 } & \multirow{2}{*}{ lst $g$} & \multirow{2}{*}{ 2nd $g$} & \multicolumn{2}{|c|}{$\begin{array}{c}\text { Zeeman } \\
\text { components }\end{array}$} \\
\hline & $\begin{array}{c}\text { Meggers } \\
\text { lamp }\end{array}$ & $\begin{array}{c}\text { Thomson } \\
\text { lamp }\end{array}$ & & & & & & & Strong $p$ & Strong $n$ \\
\hline A & \multirow{9}{*}{10} & & $K$ & $K$ & \multirow{5}{*}{$\begin{array}{l}55462_{3 \frac{1}{2}}^{\circ}-73283_{4 \frac{1}{2}} \\
53644_{3 \frac{1}{2}}^{\circ}-71468_{2 \frac{1}{2}} \\
59624_{3 \frac{1}{2}}^{\circ}-77449_{3 \frac{1}{2}}\end{array}$} & & & & & \\
\hline 5618.55 & & 1 & 17793.25 & & & & & & & \\
\hline 5609.89 & & 1 & 17820.71 & +0.04 & & & & & & \\
\hline $\begin{array}{l}5608.914 \\
5608.64\end{array}$ & & $\begin{array}{r}50 \\
2\end{array}$ & $\begin{array}{l}17823.82 \\
17824.69\end{array}$ & $\begin{array}{l}+0.01 \\
+0.03\end{array}$ & & & & & & \\
\hline 5607.91 & & 1 & 17827.01 & & & & & & & \\
\hline 5607.345 & & 150 & 17828.80 & +0.04 & $45335_{4 \frac{1}{2}}^{\circ}-63163_{4 \frac{1}{2}}$ & & & & & \\
\hline 5607.124 & & 4 & 17829.51 & 0.00 & $59777_{1 \frac{1}{2}}^{\circ}-77606_{2 \frac{1}{2}}^{2}$ & & & & & \\
\hline 5606.64 & & 1 & 17831.04 & +0.02 & $51248_{2 \frac{1}{2}}^{\circ}-69079_{2 \frac{1}{2}}$ & & & & & \\
\hline 5604.145 & & 9 & 17838.98 & +0.03 & $58484_{4 \frac{1}{2}}^{\circ}-76323_{3 \frac{1}{2}}$ & & & & & \\
\hline 5602.35 & 2 & 20 & 17844.70 & +0.02 & $58672_{0 \frac{1}{2}}^{\circ}-76517_{1 \frac{1}{2}}$ & & & & & \\
\hline 5602.264 & 3 & 40 & 17844.97 & +0.03 & $59439_{2 \frac{1}{2}}^{\circ}-77284_{1 \frac{1}{2}}^{\circ}$ & & & & & \\
\hline 5588.452 & 500 & 4000 & 17889.08 & +0.05 & $35831_{5 \frac{1}{2}}^{\circ}-53720_{4 \frac{1}{2}}^{\circ}$ & & & & & \\
\hline 5585.248 & & 20 & 17899.34 & -0.01 & $64191_{1 \frac{1}{2}}^{\circ}-82091_{0 \frac{1}{2}}$ & & & & & \\
\hline 5583.57 & & 1 & 17904.72 & 0.00 & $60910_{1 \frac{1}{2}}^{\circ}-78814_{2 \frac{1}{2}}^{\circ}$ & & & & & \\
\hline 5582.236 & & 2 & 17909.00 & -0.01 & $70793_{5 \frac{1}{2}}-88702_{5 \frac{1}{2}}^{\circ}$ & & & & & \\
\hline 5581.778 & & 8 & 17910.47 & +0.03 & $45737_{1 \frac{1}{2}}^{\circ}-63467_{1 \frac{1}{2}}$ & & & & & \\
\hline 5581.05 & 2 & 40 & 17912.80 & & & & & & & \\
\hline 5580.79 & 20 & 700 & 17913.64 & +0.05 & $47663_{4 \frac{1}{2}}^{\circ}-65577_{3 \frac{1}{2}}$ & & & & & \\
\hline 5578.483 & & 4 & 17921.04 & -0.02 & $64970_{3 \frac{1}{2}}^{\circ}-82891_{2 \frac{1}{2}}$ & & & & & \\
\hline 5575.705 & 10 & 100 & 17929.97 & +0.01 & $31568_{2 \frac{1}{2}}^{\circ}-49498_{2 \frac{1}{2}}$ & & & & & \\
\hline 5573.64 & & 1 & 17936.62 & +0.06 & $52921_{3 \frac{1}{2}}^{\circ}-70858_{4 \frac{1}{2}}$ & & & & & \\
\hline 5572.528 & 30 & 300 & 17940.20 & +0.03 & $41678_{2 \frac{1}{2}}^{\circ}-59618_{3 \frac{1}{2}}$ & & & & & \\
\hline 5566.04 & & 3 & 17961.11 & $\left\{\begin{array}{l}+0.02 \\
-0.06\end{array}\right.$ & $\begin{array}{l}45273_{3 \frac{1}{2}}^{\circ}-63234_{2 \frac{1}{2}} \\
52067_{0 \frac{1}{2}}^{\circ}-70029_{0 \frac{1}{2}}\end{array}$ & & & & & \\
\hline 5564.352 & & 3 & 17966.56 & +0.05 & $52987_{1 \frac{1}{2}}^{\circ}-70954_{2 \frac{1}{2}}^{0}$ & & & & & \\
\hline 5559.58 & & 1 & 17981.98 & +0.06 & $59624_{3 \frac{1}{2}}^{\circ}-77606_{2 \frac{1}{2}}$ & & & & & \\
\hline 5554.30 & & 4 & 17999.07 & +0.01 & $45012_{2 \frac{1}{2}}^{\circ}-63011_{1 \frac{1}{2}}^{\circ}$ & & & & & \\
\hline 5551.69 & & 2 & 18007.53 & +0.03 & $58162_{1 \frac{1}{2}}^{\circ}-76170_{2 \frac{1}{2}}^{2}$ & & & & & \\
\hline 5550.37 & & 7 & 18011.81 & +0.03 & $56977_{0 \frac{1}{2}}^{\circ}-74989_{0 \frac{1}{2}}$ & & & & & \\
\hline 5549.79 & & 20 & 18013.70 & $\left\{\begin{array}{l}+0.03 \\
+0.05\end{array}\right.$ & $\begin{array}{l}68135_{2 \frac{1}{2}}-86148_{2 \frac{1}{2}}^{\mathrm{c}} \\
56977_{0 \frac{1}{2}}^{\mathrm{o}}-74991_{1 \frac{1}{2}}\end{array}$ & & & & & \\
\hline 5549.32 & & 1 & 18015.22 & +0.04 & $58661_{2 \frac{1}{2}}^{\circ}-76676_{2 \frac{1}{2}}$ & & & & & \\
\hline 5548.918 & & 50 & 18016.53 & +0.04 & $57534_{3 \frac{1}{2}}^{\circ}-75550_{2 \frac{1}{2}}$ & & & & & \\
\hline 5547.16 & 50 & 500 & 18022.24 & $\left\{\begin{array}{l}+0.04 \\
+0.06\end{array}\right.$ & $\begin{array}{l}41688_{1 \frac{1}{2}}^{\circ} 59710_{1 \frac{1}{2}} \\
49916^{\circ}-67938_{3 \frac{1}{1}}\end{array}$ & 7 & & & 0 & 0.75 \\
\hline $\begin{array}{l}5544.17 \\
5543.96\end{array}$ & & $\begin{array}{l}1 h \\
8\end{array}$ & $\begin{array}{l}18031.96 \\
18032.64\end{array}$ & $\begin{array}{l}+0.04 \\
-0.01\end{array}$ & $\begin{array}{l}41678_{2 \frac{1}{2}}^{\circ}-59710_{1 \frac{1}{2}} \\
52921_{3 \frac{1}{2}}^{\circ}-70954_{2 \frac{1}{2}}^{\circ}\end{array}$ & & & & & \\
\hline 5540.03 & & 10 & 18045.43 & +0.03 & $43075_{\frac{1}{2}}^{\circ}-61120_{0 \frac{1}{3}}$ & & & & & \\
\hline 5533.21 & & 7 & 18067.67 & -0.09 & $48503_{5 \frac{1}{2}}^{\circ}-66571_{5 \frac{1}{2}}$ & & & & & \\
\hline 5533.14 & & 10 & 18067.90 & -0.04 & $56500_{3 \frac{1}{2}}^{\circ}-74568_{4 \frac{1}{2}}^{\circ}$ & & & & & \\
\hline 5532.111 & & 3 & 18071.26 & +0.02 & $44940_{1 \frac{1}{2}}^{\circ}-63011_{1 \frac{1}{2}}$ & & & & & \\
\hline
\end{tabular}


TABLE 6. Yb II-Observed and classified lines-Continued

\begin{tabular}{|c|c|c|c|c|c|c|c|c|c|c|}
\hline 1 & 2 & 3 & 4 & 5 & 6 & 7 & 8 & 9 & 10 & 11 \\
\hline \multirow{2}{*}{$\begin{array}{l}\text { Wave- } \\
\text { length } \\
\text { air }\end{array}$} & \multicolumn{2}{|c|}{ Intensity } & \multirow{2}{*}{$\begin{array}{l}\text { Wave } \\
\text { number }\end{array}$} & \multirow{2}{*}{$a-c$} & \multirow{2}{*}{ Classification } & \multirow{2}{*}{$\begin{array}{l}\text { Zee- } \\
\text { man } \\
\text { type }\end{array}$} & \multirow{2}{*}{ lst $g$} & \multirow{2}{*}{2 nd $g$} & \multicolumn{2}{|c|}{$\begin{array}{c}\text { Zeeman } \\
\text { components }\end{array}$} \\
\hline & $\begin{array}{l}\text { Meggers } \\
\text { lamp }\end{array}$ & $\begin{array}{c}\text { Thomson } \\
\text { lamp }\end{array}$ & & & & & & & Strong $p$ & Strong $n$ \\
\hline А & & & $K$ & K & & & & & & \\
\hline 5530.73 & 1 & 1 & 18075.78 & +0.04 & $68150_{1 \frac{1}{2}}-86226_{1 \frac{1}{2}}^{\circ}$ & & & & & \\
\hline 5529.92 & 4 & 150 & 18078.42 & -0.02 & $48272_{2 \frac{1}{2}}^{\circ}-66351_{2 \frac{1}{2}}$ & 7 & 1.12 & (1.125) & 0 & 1.12 \\
\hline $\begin{array}{l}5529.08 \\
5521.61\end{array}$ & 5 & $\begin{array}{r}250 \\
3\end{array}$ & $\begin{array}{l}18081.17 \\
18105.63\end{array}$ & $\begin{array}{l}-0.01 \\
+0.02\end{array}$ & $\begin{array}{l}50468_{5 \frac{1}{2}}^{\circ}-68549_{4 \frac{1}{2}} \\
57534_{3 \frac{1}{2}}^{\circ}-75640_{3 \frac{1}{3}}\end{array}$ & 5 & 1.21 & (1.157) & $w$ & 1.44 \\
\hline $\begin{array}{l}5520.22 \\
5519.83\end{array}$ & 1 & $\begin{array}{r}50 \\
2\end{array}$ & $\begin{array}{l}18110.19 \\
18111.47\end{array}$ & -0.04 & $59777_{\frac{1}{2}}^{2}-77887_{0 \frac{1}{2}}^{\frac{1}{2}}$ & 4 & 1.63 & 2.21 & 0.29 & 1.34 \\
\hline $\begin{array}{l}5516.334 \\
5515.38\end{array}$ & & $\begin{array}{r}10 \\
3\end{array}$ & $\begin{array}{l}18122.95 \\
18126.08\end{array}$ & $\begin{array}{l}0.00 \\
0.00\end{array}$ & $\begin{array}{l}48272_{2 \frac{1}{2}}^{\circ}-66395_{2 \frac{1}{2}} \\
52987_{1 \frac{1}{2}}^{\circ}-71113_{1 \frac{1}{1}}\end{array}$ & & & & & \\
\hline 5511.764 & 1 & 30 & 18137.97 & +0.03 & $58661_{2 \frac{1}{2}}^{\circ}-76799_{1 \frac{1}{2}}^{2}$ & 7 & $(0.86)$ & 0.83 & 0 & 0.90 \\
\hline $\begin{array}{l}5511.401 \\
5509.198\end{array}$ & 2 & $\begin{array}{l}70 \\
20\end{array}$ & $\begin{array}{l}18139.17 \\
18146.42\end{array}$ & $\begin{array}{r}0.00 \\
+0.08\end{array}$ & $\begin{array}{l}54640_{4 \frac{1}{2}}^{\circ}-72779_{3 \frac{1}{2}}^{\circ} \\
51248_{2 \frac{1}{2}}^{\circ}-69395_{3 \frac{1}{2}}^{\circ}\end{array}$ & & & & 0 & 1.20 \\
\hline 5509.046 & & 3 & 18146.92 & $\left\{\begin{array}{l}+0.03 \\
+0.05\end{array}\right.$ & $\begin{array}{l}71468_{2 \frac{1}{2}}-89615_{2 \frac{1}{2}}^{\circ} \\
76676_{2 \frac{1}{2}}^{\circ}-94823_{1 \frac{1}{2}}^{\circ}\end{array}$ & & & & & \\
\hline 5508.266 & & 20 & 18149.49 & $\left\{\begin{array}{l}+0.04 \\
+0.06\end{array}\right.$ & $\begin{array}{c}56840_{\frac{1}{2}}^{\circ}-74989_{0 \frac{1}{2}}^{\circ} \\
\left(53120_{2 \frac{1}{2}}^{\circ}-71270_{2 \frac{1}{2}}^{\circ}\right)\end{array}$ & 5 & 0.83 & 0.27 & $0.28 ?$ & 1.11 \\
\hline $\begin{array}{l}5507.70 \\
5502.802\end{array}$ & 1 & $\begin{array}{r}1 \\
10\end{array}$ & $\begin{array}{l}18151.36 \\
18167.51\end{array}$ & $\begin{array}{l}+0.04 \\
+0.01\end{array}$ & $\begin{array}{l}56840_{1 \frac{1}{2}}^{\circ}-74991_{1 \frac{1}{2}} \\
59439_{2 \frac{1}{3}}^{\circ}-77606_{2 \frac{1}{2}}\end{array}$ & & & & & \\
\hline 5501.37 & 2 & 20 & 18172.24 & +0.02 & $40917_{4 \frac{1}{2}}^{0}-59090_{3 \frac{1}{2}}^{2 \frac{1}{2}}$ & & & & & \\
\hline 5499.65 & & 2 & 18177.93 & +0.06 & $58661_{2 \frac{1}{2}}^{\circ}-76839_{2 \frac{1}{2}}$ & & & & & \\
\hline 5498.390 & & 3 & 18182.09 & 0.00 & $58051_{5 \frac{1}{2}}^{\circ}-76233_{6 \frac{1}{2}}$ & & & & & \\
\hline $\begin{array}{l}5494.58 \\
5494.364\end{array}$ & 1 & $\begin{array}{r}1 \\
10\end{array}$ & $\begin{array}{l}18194.70 \\
18195.41\end{array}$ & $\begin{array}{l}+0.04 \\
+0.04\end{array}$ & $\begin{array}{l}51248_{2 \frac{1}{2}}^{\circ}-69443_{1 \frac{1}{2}} \\
47680^{\circ}-65875^{\circ}\end{array}$ & & & & & \\
\hline 5490.847 & & 3 & 18207.07 & +0.06 & $64191_{1 \frac{1}{2}}^{\circ}-82398_{1 \frac{1}{2}}^{\circ}$ & & & & & \\
\hline 5488.883 & & 8 & 18213.58 & +0.01 & $56056_{2 \frac{1}{2}}^{\circ}-74270_{2 \frac{1}{2}}$ & & & & & \\
\hline 5487.512 & 1 & 9 & 18218.13 & +0.06 & $56840_{1 \frac{1}{2}}^{-2}-75058_{2 \frac{1}{2}}$ & & & & & \\
\hline 5486.535 & 7 & 150 & 18221.38 & +0.06 & $45012_{2 \frac{1}{2}}^{\circ}-63234_{2 \frac{1}{2}}^{2}$ & 6 & 1.286 & 1.214 & 0.18 & 1.25 \\
\hline 5483.212 & & 3 & 18232.42 & +0.04 & $49916_{\frac{1}{2}}^{\circ}-68148_{5 \frac{1}{2}}$ & & & & & \\
\hline 5479.806 & 1 & 50 & 18243.75 & +0.02 & $46354_{4 \frac{1}{2}}^{\circ}-64598_{3 \frac{1}{2}}$ & & & & & \\
\hline $\begin{array}{l}5479.122 \\
5478.50\end{array}$ & 100 & $2 d$ & 18246.03 & & & 4 & ( 2 ( & 077 & $\mu$ & 060 \\
\hline $\begin{array}{l}5478.50 \\
5477.206\end{array}$ & 100 & $\begin{array}{r}1500 \\
9\end{array}$ & $\begin{array}{l}18248.10 \\
18252.41\end{array}$ & $\begin{array}{l}+0.01 \\
+0.02\end{array}$ & $\begin{array}{l}40035_{3 \frac{1}{2}}^{\circ} 58283_{2 \frac{1}{2}}^{\circ} \\
50468_{5 \frac{1}{2}}^{\circ}-68720_{6 \frac{1}{2}}\end{array}$ & 4 & $(0.12)$ & 0.76 & $w$ & 0.00 \\
\hline $\begin{array}{l}5475.706 \\
5475.132\end{array}$ & & 10 & 18257.41 & +0.03 & $59624_{3 \frac{1}{2}}^{\circ}-77882_{3 \frac{1}{2}}^{\circ}$ & & & & & \\
\hline & & & & & & & & & & \\
\hline 5471.17 & 30 & 400 & 18272.55 & +0.05 & $45429_{3 \frac{1}{2}}^{\circ}-63702_{2 \frac{1}{2}}^{\circ}$ & 5 & 1.15 & 1.08 & $(0.036)$ & 1.33 \\
\hline $\begin{array}{l}5468.260 \\
5467.495\end{array}$ & 1 & $\begin{array}{l}3 \\
9\end{array}$ & $\begin{array}{l}18282.27 \\
18284.83\end{array}$ & +0.02 & $52987_{1 \frac{1}{2}}^{\circ}-71270_{2 \frac{1}{2}}$ & & & & & \\
\hline 5466.714 & 1 & 25 & 18287.44 & +0.01 & $47663_{4 \frac{1}{2}}^{\circ}-65950_{4 \frac{1}{2}}$ & & & & & \\
\hline 5464.894 & 6 & 200 & 18293.53 & $\left\{\begin{array}{l}+0.03 \\
-0.03\end{array}\right.$ & $\begin{array}{c}44940_{\frac{1}{2}}^{\circ}-63234_{2 \frac{1}{2}}^{\circ} \\
\left(47912_{3 \frac{1}{2}}-66205_{2 \frac{1}{2}}^{\circ}\right)\end{array}$ & 7 & 1.21 & (1.208) & 0 & 1.21 \\
\hline
\end{tabular}


TABLE 6. Yb II-Observed and classified lines-Continued

\begin{tabular}{|c|c|c|c|c|c|c|c|c|c|c|}
\hline 1 & 2 & 3 & 4 & 5 & 6 & 7 & 8 & 9 & 10 & 11 \\
\hline \multirow{2}{*}{$\begin{array}{l}\text { W ave- } \\
\text { length } \\
\text { air }\end{array}$} & \multicolumn{2}{|c|}{ Intensity } & \multirow{2}{*}{$\begin{array}{l}\text { Wave } \\
\text { number }\end{array}$} & \multirow{2}{*}{$\mathrm{o}-\mathrm{c}$} & \multirow{2}{*}{ Classification } & \multirow{2}{*}{$\begin{array}{l}\text { Zee- } \\
\text { man } \\
\text { type }\end{array}$} & \multirow{2}{*}{ lst $g$} & \multirow{2}{*}{ 2nd $g$} & \multicolumn{2}{|c|}{$\begin{array}{c}\text { Zeeman } \\
\text { components }\end{array}$} \\
\hline & $\begin{array}{c}\text { Meggers } \\
\text { lamp }\end{array}$ & $\begin{array}{c}\text { Thomsom } \\
\text { lamp }\end{array}$ & & & & & & & Strong $p$ & Strong $n$ \\
\hline$\stackrel{\circ}{\mathrm{A}}$ & & & K & K & & & & & & \\
\hline 5463.850 & & 3 & 18297.03 & +0.02 & $45429^{\circ}{ }_{3 \frac{1}{2}}-63726_{4 \frac{1}{2}}$ & & & & & \\
\hline 5463.12 & & 10 & 18299.47 & $\left\{\begin{array}{l}-0.06 \\
+0.07\end{array}\right.$ & $\begin{array}{l}42915_{5 \frac{1}{2}}^{\circ}-61214_{4 \frac{1}{2}} \\
43075^{\circ}-61374^{\circ}\end{array}$ & & & & & \\
\hline 5458.088 & 2 & 60 & 18316.34 & -0.01 & $59753_{\frac{1}{2}}^{\circ}-78070_{4 \frac{1}{2}}$ & & & & & \\
\hline 5457.427 & 4 & 150 & 18318.56 & +0.03 & $45737_{1 \frac{1}{2}}^{\circ}-64055_{2 \frac{1}{2}}^{\circ}$ & & & & & \\
\hline 5455.046 & 5 & 250 & 18326.56 & +0.05 & $48024_{1 \frac{1}{2}}^{2}-66351_{2 \frac{1}{2}}$ & 4 & 1.365 & 1.119 & 0.123 & 0.75 \\
\hline $\begin{array}{l}5449.84 \\
5449.270\end{array}$ & 500 & $\begin{array}{r}1 \\
2500\end{array}$ & $\begin{array}{l}18344.06 \\
18345.98\end{array}$ & +0.02 & $35059_{31}^{\circ}-53404_{2 \frac{1}{1}}$ & 5 & (1.124) & 1.04 & $w$ & 1.33 \\
\hline 5448.565 & & 4 & 18348.36 & -0.03 & $52921_{3 \frac{1}{2}}^{\circ}-71270_{2 \frac{1}{2}}$ & & & & & \\
\hline 5445.492 & 1 & 40 & 18358.71 & +0.03 & $51248_{2 \frac{1}{2}}^{\circ}-69607_{2 \frac{1}{2}}^{\circ}$ & & - & & & \\
\hline 5441.615 & 2 & 5 & 18371.79 & +0.05 & $57798_{2 \frac{1}{2}}^{\circ}-76170_{2 \frac{1}{2}}$ & & & & & \\
\hline 5440.548 & 10 & 200 & 18375.39 & 0.00 & $46547_{5 \frac{1}{2}}^{\circ}-64923_{4 \frac{1}{2}}$ & & & & & \\
\hline 5435.27 & & 6 & 18393.24 & $\left\{\begin{array}{l}+0.10 \\
+0.01\end{array}\right.$ & $\begin{array}{l}64970_{3 \frac{1}{2}}^{\circ}-83363_{3 \frac{1}{2}} \\
71222_{31}-89615^{\circ}\end{array}$ & & & & & \\
\hline 5432.71 & 60 & 1500 & 18401.91 & +0.06 & $47680_{6 \frac{1}{2}}^{\circ}-66082_{5 \frac{1}{2}}^{\circ}$ & 4 & 1.12 & (1.141) & 0 & 0.985 \\
\hline 5431.167 & 2 & 80 & 18407.13 & +0.03 & $49008_{3 \frac{1}{2}}^{\circ}-67416_{4 \frac{1}{2}}^{\circ}$ & & & & & \\
\hline 5429.838 & & 8 & 18411.64 & 0.00 & $62046_{3 \frac{1}{2}}^{\circ}-80457_{3 \frac{1}{2}}^{2}$ & & & & & \\
\hline 5426.867 & 30 & 900 & 18421.72 & +0.03 & $49727_{6 \frac{1}{2}}^{\circ}-68148_{5 \frac{1}{2}}$ & 7 & (1.20) & 1.20 & 0 & 1.175 \\
\hline 5424.627 & 30 & 300 & 18429.32 & +0.02 & $45273_{3 \frac{1}{2}}^{\circ}-63702_{2 \frac{1}{2}}^{2}$ & 5 & 1.20 & 1.08 & $(0.06)$ & 1.51 \\
\hline 5422.715 & & 1 & 18435.82 & -0.01 & $55221_{1 \frac{1}{2}}^{\circ}-73657_{1 \frac{1}{2}}^{\circ}$ & & & & & \\
\hline 5420.608 & & 7 & 18442.99 & +0.03 & $59439_{2 \frac{1}{2}}^{\circ}-77882_{3 \frac{1}{2}}^{\circ}$ & & & & & \\
\hline 5417.421 & & 9 & 18453.84 & +0.03 & $45273_{3 \frac{1}{2}}^{\circ}-63726_{4 \frac{1}{2}}$ & & & & & \\
\hline 5414.258 & 6 & 200 & 18464.62 & +0.02 & $58051_{5 \frac{1}{2}}^{\circ}-76516_{4 \frac{1}{2}}$ & 7 & 0.98 & (1.01) & 0 & 0.87 \\
\hline 5411.038 & 1 & 40 & 18475.61 & +0.01 & $50468_{5 \frac{1}{2}}^{\circ}-68943_{4 \frac{1}{2}}$ & & & & & \\
\hline 5410.635 & & 15 & 18476.98 & +0.01 & $60649_{4 \frac{1}{2}}^{\circ}-79126_{4 \frac{1}{2}}^{\circ}$ & & & & & \\
\hline 5409.473 & 2 & 60 & 18480.95 & +0.01 & $52987_{\frac{1}{2}}^{\circ}-71468_{2 \frac{1}{2}}^{2}$ & 4 & (1.13) & 1.02 & $w$ & 0.86 \\
\hline 5408.092 & & 4 & 18485.67 & +0.02 & $53716_{2 \frac{1}{2}}^{\circ}-72202_{3 \frac{1}{2}}^{2}$ & & & & & \\
\hline 5402.706 & & 3 & 18504.10 & -0.03 & $55462_{3 \frac{1}{2}}^{\circ}-73966_{3 \frac{1}{2}}$ & & & & & \\
\hline 5399.71 & 50 & 500 & 18514.37 & +0.03 & $44497_{2 \frac{1}{2}}^{\circ}-63011_{1 \frac{1}{2}}^{\circ}$ & 7 & $(0.734)$ & 0.78 & 0 & 0.67 \\
\hline 5397.23 & & 1 & 18522.87 & & & & & & & \\
\hline 5395.73 & 20 & 300 & 18528.02 & +0.04 & $45429_{3 \frac{1}{2}}^{\circ}-63957_{3 \frac{1}{2}}$ & 7 & $(1.150)$ & 1.17 & 0 & 1.16 \\
\hline 5394.084 & & 3 & 18533.68 & +0.07 & $49916_{4 \frac{1}{2}}^{\circ}-68450_{3 \frac{1}{2}}^{\circ}$ & & & & & \\
\hline 5393.37 & 5 & 150 & 18536.13 & +0.03 & $49419_{0 \frac{1}{2}}^{\circ}-67955_{0 \frac{1}{2}}$ & 6 & 1.40 & 0.90 & 0.25 & 1.15 \\
\hline 5390.18 & & 6 & 18547.10 & +0.02 & $52921_{3 \frac{1}{2}}^{\circ}-71468_{2 \frac{1}{2}}^{2}$ & & & & & \\
\hline 5389.84 & 200 & 800 & 18548.27 & +0.02 & $34389_{2 \frac{1}{2}}^{\circ}-52938_{1 \frac{1}{2}}^{2}$ & 5 & (1.007) & 0.90 & $w$ & 1.17 \\
\hline 5386.72 & 1 & 50 & 18559.01 & -0.01 & $47329_{2 \frac{1}{2}}^{\circ-2}-65888_{1 \frac{1}{2}}^{2}$ & & & & & \\
\hline 5386.07 & & 10 & 18561.25 & -0.01 & $47005_{1 \frac{1}{2}}^{\circ}-65566_{0 \frac{1}{2}}$ & & & & & \\
\hline 5384.48 & 5 & 50 & 18566.73 & 0.00 & $70136_{6 \frac{1}{2}}-88702_{5 \frac{1}{2}}^{\circ}$ & 5 & (1.07) & 1.03 & $w$ & 1.32 \\
\hline 5383.95 & 3 & 60 & 18568.56 & 0.00 & $46354_{4 \frac{1}{2}}^{\circ}-64923_{4 \frac{1}{2}}$ & & & & & \\
\hline 5376.97 & 5 & 250 & 18592.66 & -0.01 & $47758_{3 \frac{1}{2}}^{\circ}-66351_{2 \frac{1}{2}}$ & 7 & 1.11 & (1.125) & 0 & 1.07 \\
\hline
\end{tabular}


TABLE 6. Yb II-Observed and classified lines-Continued

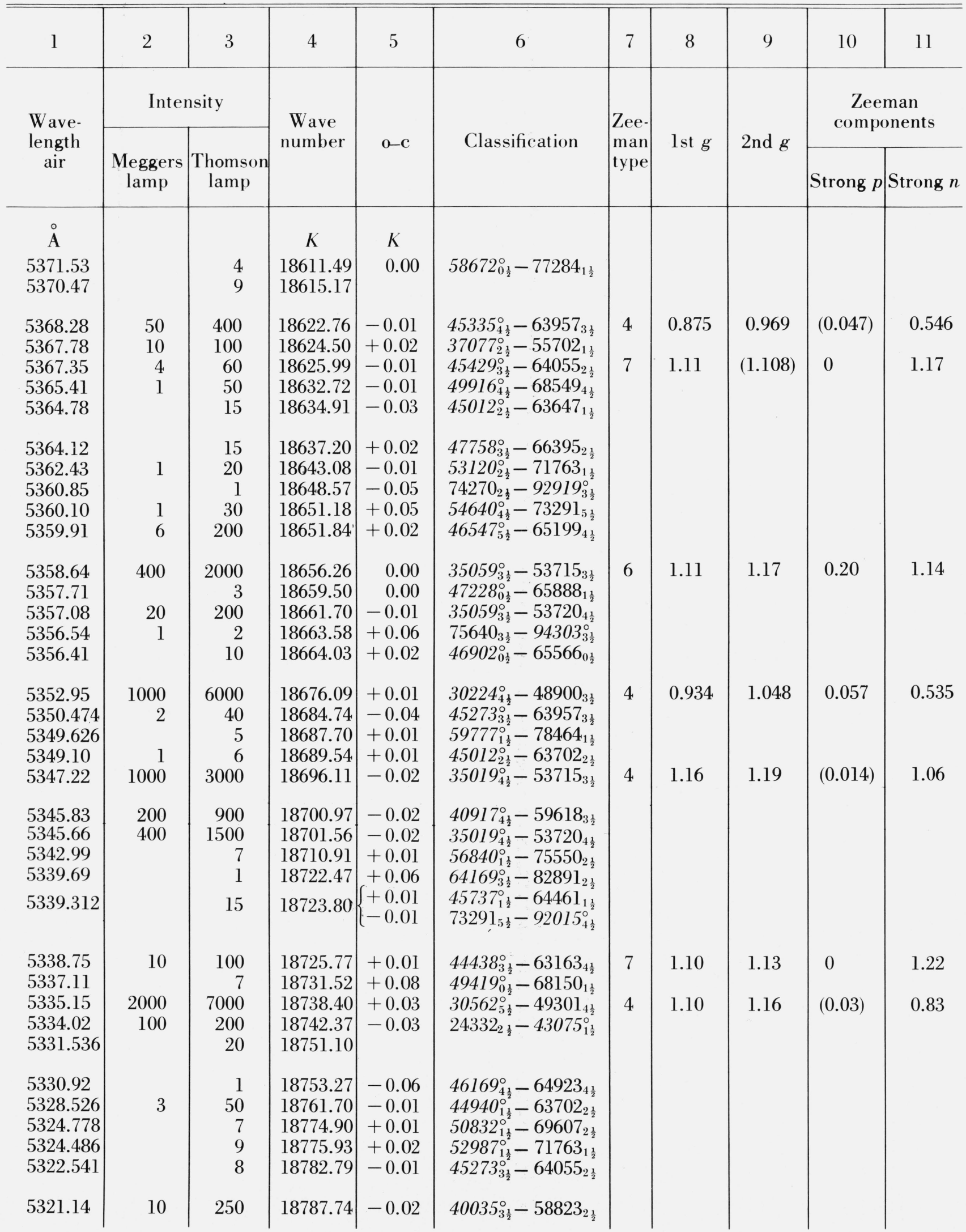


TABle 6. Yb II-Observed and classified lines-Continued

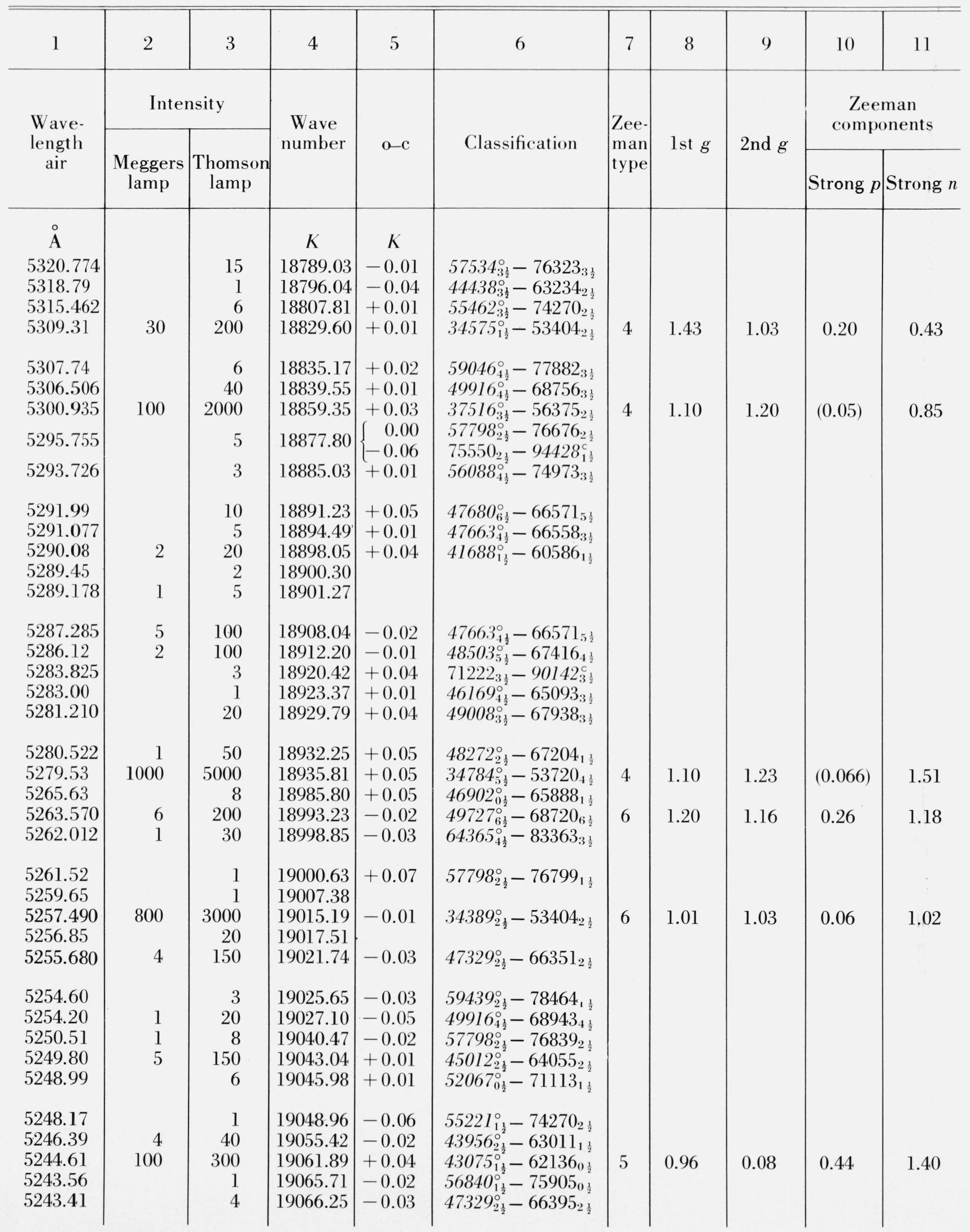


TABle 6. Yb II-Observed and classified lines-Continued

\begin{tabular}{|c|c|c|c|c|c|c|c|c|c|c|}
\hline 1 & 2 & 3 & 4 & 5 & 6 & 7 & 8 & 9 & 10 & 11 \\
\hline \multirow{2}{*}{$\begin{array}{l}\text { Wave- } \\
\text { length } \\
\text { air }\end{array}$} & \multicolumn{2}{|c|}{ Intensity } & \multirow{2}{*}{$\begin{array}{c}\text { Wave } \\
\text { number }\end{array}$} & \multirow[b]{2}{*}{$\mathrm{o}-\mathrm{c}$} & \multirow[b]{2}{*}{ Classification } & \multirow{2}{*}{$\begin{array}{l}\text { Zee- } \\
\text { man } \\
\text { type }\end{array}$} & \multirow[b]{2}{*}{ lst $g$} & \multirow{2}{*}{2 nd $g$} & \multicolumn{2}{|c|}{$\begin{array}{c}\text { Zeeman } \\
\text { components }\end{array}$} \\
\hline & $\begin{array}{l}\text { Meggers } \\
\text { lamp }\end{array}$ & $\begin{array}{c}\text { Thomson } \\
\text { lamp }\end{array}$ & & & & & & & Strong $p$ & Strong $r$ \\
\hline$\AA$ & & & $K$ & $\dot{K}$ & & & & & & \\
\hline $\begin{array}{l}5243.15 \\
5240.51\end{array}$ & & $\begin{array}{r}2 \\
1000\end{array}$ & $\begin{array}{l}19067.20 \\
19076.80\end{array}$ & $\begin{array}{l}-0.03 \\
-0.03\end{array}$ & $\begin{array}{l}57103_{3 \frac{1}{2}}^{\circ}-76170_{2 \frac{1}{2}} \\
30224_{4 \frac{1}{2}}^{\circ}-49301_{4 \frac{1}{12}}\end{array}$ & 6 & 0.94 & 1.20 & 1.15 & $1.07 ?$ \\
\hline $\begin{array}{l}5240.51 \\
5239.25\end{array}$ & 300 & $\begin{array}{r}1000 \\
2\end{array}$ & $\begin{array}{l}19080.80 \\
19081.39\end{array}$ & $\begin{array}{l}-0.03 \\
-0.06\end{array}$ & $\begin{array}{l}30224_{4 \frac{1}{2}}-495011_{\frac{1}{2}} \\
53120_{2 \frac{1}{2}}-72202_{3 \frac{1}{2}}\end{array}$ & 0 & 0.94 & 1.20 & 1.15 & $1.06 \mathrm{e}$ \\
\hline 5236.66 & 3 & 150 & 19090.83 & -0.01 & $54192_{5 \frac{1}{2}}^{\circ}-73283_{4 \frac{1}{2}}$ & & & & & \\
\hline 5234.28 & & 2 & 19099.51 & +0.07 & $54192_{5 \frac{1}{2}}^{\circ}-73291_{5 \frac{1}{2}}$ & & & & & \\
\hline 5232.537 & 6 & 40 & 19105.87 & $\left\{\begin{array}{l}+0.06 \\
-0.03\end{array}\right.$ & $\begin{array}{l}30392_{1 \frac{1}{2}}^{\circ}-49498_{2 \frac{1}{2}} \\
55462^{\circ}-74568_{4 \frac{1}{1}}\end{array}$ & & & & & \\
\hline 5229.963 & 1 & 60 & 19115.27 & +0.06 & $44940_{1 \frac{1}{2}}^{\circ}-64055_{2 \frac{1}{2}}^{\circ}$ & & & & & \\
\hline 5227.01 & 1 & 70 & 19126.07 & 0.00 & $49008_{3 \frac{1}{2}}^{\circ}-68135_{2 \frac{1}{2}}$ & & & & & \\
\hline 5226.18 & 20 & 150 & 19129.11 & -0.04 & $43007_{0 \frac{1}{2}}^{\circ}-62136_{0 \frac{1}{2}}$ & & & & & \\
\hline 5222.68 & & 3 & 19141.93 & +0.07 & $57534_{3 \frac{1}{2}}^{\circ}-76676_{2 \frac{1}{2}}$ & & & & & \\
\hline 5220.82 & & 5 & 19148.75 & +0.02 & $62163_{2 \frac{1}{2}}^{\circ}-81312_{2 \frac{1}{2}}$ & & & & & \\
\hline 5220.41 & & 10 & 19150.25 & +0.03 & $44497_{2 \frac{1}{2}}^{0}-63647_{1 \frac{1}{2}}^{2}$ & & & & & \\
\hline 5217.936 & 3 & 70 & 19159.33 & +0.04 & $56480_{4 \frac{1}{2}}^{\circ}-75640_{3 \frac{1}{2}}^{\circ}$ & 7 & 0.77 & $(0.81)$ & 0 & $0.63 h$ \\
\hline 5215.44 & 4 & 100 & 19168.50 & +0.04 & $45429_{3 \frac{1}{2}}^{\circ}-64598_{3 \frac{1}{2}}^{\circ}$ & & & & & \\
\hline 5212.232 & & 25 & 19180.30 & +0.03 & $48024_{1 \frac{1}{2}}^{\circ}-67204_{1 \frac{1}{2}}^{2}$ & & & & & \\
\hline 5209.54 & & 1 & 19190.21 & +0.01 & $59624_{3 \frac{1}{2}}^{\circ}-78814_{2 \frac{1}{2}}$ & & & & & \\
\hline 5205.574 & 4 & 30 & 19204.83 & +0.02 & $44497_{2 \frac{1}{2}}^{\circ}-63702_{2 \frac{1}{2}}^{2}$ & & & & & \\
\hline 5204.57 & & 1 & 19208.53 & & & & & & & \\
\hline 5202.87 & & 1 & 19214.81 & +0.04 & $58672_{0 \frac{1}{2}}^{\circ}-77887_{0 \frac{1}{2}}$ & & & & & \\
\hline 5201.32 & & 1 & 19220.54 & +0.07 & $57103_{3 \frac{1}{2}}^{\circ}-76323_{3 \frac{1}{2}}$ & & & & & \\
\hline 5201.21 & & 2 & 19220.94 & +0.03 & $58661_{2_{2 \frac{1}{2}}}^{\circ}-77882_{3 \frac{1}{2}}$ & & & & & \\
\hline 5200.75 & & 4 & 19222.64 & +0.08 & $46354_{4 \frac{1}{2}}^{\circ}-65577_{3 \frac{1}{2}}$ & & & & & \\
\hline 5200.54 & 50 & 500 & 19223.42 & 0.00 & $40035_{3 \frac{1}{2}}^{\circ}-59259_{2 \frac{1}{2}}^{\circ}$ & 4 & $(0.720)$ & 1.110 & 0.195 & $?$ \\
\hline 5199.14 & & 1 & 19228.60 & +0.04 & $47329_{2 \frac{1}{2}}^{\circ}-66558_{3 \frac{1}{2}}$ & & & & & \\
\hline 5189.89 & & 7 & 19262.87 & +0.01 & $58484_{4 \frac{1}{2}}^{\circ-2}-77747_{4 \frac{1}{2}}^{2}$ & & & & & \\
\hline 5189.50 & & 8 & 19264.31 & +0.02 & $44438_{3 \frac{1}{2}}^{\circ}-63702_{2 \frac{1}{2}}$ & & & & & \\
\hline 5188.89 & & 9 & 19266.58 & 0.00 & $62046_{3 \frac{1}{2}}^{\circ}-81312_{2 \frac{1}{2}}^{\circ}$ & & & & & \\
\hline 5187.33 & & 3 & 19272.37 & & & & & & & \\
\hline 5185.15 & & 1 & 19280.48 & +0.07 & $52921_{3 \frac{1}{2}}^{\circ}-72202_{3 \frac{1}{2}}$ & & & & & \\
\hline 5184.66 & & 1 & 19282.30 & & & & & & & \\
\hline 5184.15 & 150 & 1000 & 19284.19 & +0.04 & $33653_{0 \frac{1}{2}}^{\circ}-52938_{1 \frac{1}{2}}$ & 4 & 1.34 & 0.90 & 0.22 & 0.68 \\
\hline 5180.355 & 15 & 150 & 19298.32 & 0.00 & $37077_{2 \frac{1}{2}}^{\circ}-56375_{2 \frac{1}{2}}$ & 6 & 1.116 & 1.224 & 0.27 & 1.17 \\
\hline 5173.11 & 2 & 200 & 19325.35 & +0.09 & $45273_{3 \frac{1}{2}}^{\circ}-64598_{3 \frac{1}{2}}$ & & & & & \\
\hline 5173.064 & 10 & 100 & 19325.52 & +0.02 & $34389_{2 \frac{1}{2}}^{\circ}-53715_{3 \frac{1}{2}}^{\circ}$ & & & & & \\
\hline 5172.40 & & 1 & 19328.00 & -0.05 & $46547_{5 \frac{1}{2}}^{\circ}-65875_{6 \frac{1}{2}}$ & & & & & \\
\hline 5167.660 & & 3 & 19345.73 & -0.02 & $47005_{\frac{1}{2}}^{\circ}-66351_{2 \frac{1}{2}}$ & & & & & \\
\hline 5160.99 & & 1 & 19370.73 & $\left\{\begin{array}{l}+0.01 \\
+0.04\end{array}\right.$ & $\begin{array}{l}63028_{1 \frac{1}{2}}^{\circ}-82398_{1 \frac{1}{2}} \\
75058_{2 \frac{1}{2}}-9448^{c_{11}}\end{array}$ & & & & & \\
\hline 5159.655 & & 3 & 19375.74 & -0.04 & $59439_{2 \frac{1}{2}}^{\circ}-78814_{2 \frac{1}{2}}^{\circ}$ & & & & & \\
\hline
\end{tabular}


TABLE 6. Yb II-Observed and classified lines-Continued

\begin{tabular}{|c|c|c|c|c|c|c|c|c|c|c|}
\hline 1 & 2 & 3 & 4 & 5 & 6 & 7 & 8 & 9 & 10 & 11 \\
\hline \multirow{2}{*}{$\begin{array}{l}\text { Wave- } \\
\text { length } \\
\text { air }\end{array}$} & \multicolumn{2}{|c|}{ Intensity } & \multirow{2}{*}{$\begin{array}{c}\text { Wave } \\
\text { number }\end{array}$} & \multirow{2}{*}{$\mathrm{o}-\mathrm{c}$} & \multirow{2}{*}{ Classification } & \multirow{2}{*}{ Zee- } & \multirow{2}{*}{ lst $g$} & \multirow{2}{*}{ 2nd $g$} & \multicolumn{2}{|c|}{$\begin{array}{l}\text { Zeeman } \\
\text { components }\end{array}$} \\
\hline & $\begin{array}{l}\text { Meggers } \\
\text { lamp }\end{array}$ & $\begin{array}{c}\text { Thomson } \\
\text { lamp }\end{array}$ & & & & & & & Strong $p$ & Strong $n$ \\
\hline A & & & $K$ & $K$ & & & & & & \\
\hline 5157.962 & 3 & 100 & 19382.10 & -0.01 & $48556_{3 \frac{1}{2}}^{\circ}-67938_{3 \frac{1}{2}}$ & & & & & \\
\hline 5152.34 & 7 & 200 & 19403.25 & +0.02 & $46547_{5 \frac{1}{2}}^{\circ}-65950_{4 \frac{1}{2}}$ & & & & & \\
\hline 5151.25 & & 4 & 19407.36 & +0.03 & $46169_{4 \frac{1}{2}}^{\circ}-65577_{3 \frac{1}{2}}$ & & & & & \\
\hline 5149.04 & & 1 & 19415.69 & -0.02 & $70793_{5 \frac{1}{2}}-90209_{4 \frac{1}{2}}^{\circ}$ & & & & & \\
\hline 5147.02 & 50 & 700 & 19423.31 & +0.01 & $38342_{4 \frac{1}{2}}^{\circ}-57765_{4 \frac{1}{2}}^{\circ}$ & 6 & (1.093) & 1.13 & $w$ & 1.11 \\
\hline 5142.28 & 3 & 100 & 19441.21 & +0.03 & $49008_{3 \frac{1}{2}}-68450_{3 \frac{1}{2}}$ & & & & & \\
\hline 5141.61 & & 3 & 19443.74 & -0.09 & $58162_{1 \frac{1}{2}}^{\circ}-77606_{2 \frac{1}{2}}^{2}$ & & & & & \\
\hline 5138.01 & & 1 & 19457.37 & -0.03 & $47005^{\circ \frac{1}{2}}-66462_{0 \frac{1}{2}}$ & & & & & \\
\hline 5137.43 & 2 & 4 & 19459.56 & & & & & & & \\
\hline 5137.23 & & 1 & 19460.32 & +0.03 & $44497_{2 \frac{1}{2}}^{\circ}-63957_{3 \frac{1}{2}}$ & & & & & \\
\hline $\begin{array}{l}5136.49 \\
5135.98\end{array}$ & 200 & $\begin{array}{r}1 \\
1500\end{array}$ & $\begin{array}{l}19463.12 \\
19465.06\end{array}$ & 0.00 & & 7 & $(1.264)$ & 1.25 & 0 & 1.29 \\
\hline & & & 19400.00 & 0.00 & $50004 \frac{1}{2}-520165 \frac{1}{2}$ & 8 & & 1.25 & 0 & 1.29 \\
\hline 5133.42 & & 1 & 19474.76 & -0.01 & $63417_{2 \frac{1}{2}}^{\circ}-82891_{2 \frac{1}{2}}$ & & & & & \\
\hline 5132.40 & 1 & 60 & 19478.64 & -0.06 & $49916_{4 \frac{1}{2}}^{\circ}-69395_{3 \frac{1}{2}}^{2}$ & & & & & \\
\hline 5131.01 & 1 & 10 & 19483.91 & -0.02 & $43075_{1 \frac{1}{2}}^{\circ}-62559_{2 \frac{1}{2}}$ & & & & & \\
\hline 5130.711 & & 2 & 19485.05 & -0.07 & $70793_{5 \frac{1}{2}}-90279_{5 \frac{1}{2}}^{c}$ & & & & & \\
\hline 5130.56 & & 1 & 19485.62 & +0.11 & $57798_{2 \frac{1}{2}}^{\circ}-77284_{1 \frac{1}{2}}$ & & & & & \\
\hline 5129.56 & & 60 & 19489.42 & -0.02 & $50468_{5 \frac{1}{2}}^{\circ}-69957_{4 \frac{1}{2}}$ & & & & & \\
\hline 5128.55 & 3 & 100 & 19493.26 & $\left\{\begin{array}{l}-0.03 \\
+0.05\end{array}\right.$ & $\begin{array}{l}45429_{3 \frac{1}{2}}^{\circ}-64923_{4 \frac{1}{2}} \\
62163_{2 \frac{1}{2}}^{\circ}-81657_{2 \frac{1}{2}}\end{array}$ & & & & & \\
\hline 5121.58 & 30 & 300 & 19519.79 & +0.02 & $44438_{3 \frac{1}{2}}^{\circ}-63957_{3 \frac{1}{2}}$ & 6 & 1.11 & 1.15 & 0.13 & 1.13 \\
\hline 5117.72 & 1 & 25 & 19534.51 & -0.02 & $46547_{5 \frac{1}{2}}^{\circ}-66082_{5 \frac{1}{2}}^{\circ}$ & & & & & \\
\hline 5116.200 & & 10 & 19540.31 & +0.01 & $49008_{3 \frac{1}{2}}^{\circ}-68549_{4 \frac{1}{2}}^{2}$ & & & & & \\
\hline 5111.47 & 1 & 60 & 19558.39 & $\left\{\begin{array}{r}+0.08 \\
0.00\end{array}\right.$ & $44497_{2 \frac{1}{2}}^{\circ}-64055_{2 \frac{1}{2}}$ & & & & & \\
\hline 5107.58 & & 1 & 19573.29 & $\begin{array}{l}0.00 \\
0.00\end{array}$ & $\begin{array}{l}54192_{5 \frac{1}{2}} \\
57103_{3 \frac{1}{2}}^{\circ}-76676_{2 \frac{1}{2}}\end{array}$ & & & & & \\
\hline 5106.12 & & 1 & 19578.89 & & & & & & & \\
\hline 5105.028 & 10 & 150 & 19583.07 & $\left\{\begin{array}{l}-0.01 \\
-0.08\end{array}\right.$ & $\begin{array}{l}40035_{3 \frac{1}{2}}^{\circ}-59618_{3 \frac{1}{2}} \\
56056_{2 \frac{1}{\circ}}^{\circ}-75640_{3}\end{array}$ & & & & & \\
\hline 5104.42 & 20 & 400 & 19585.41 & -0.08 & $45012_{2 \frac{1}{2}}^{\circ}-64598_{3 \frac{1}{2}}^{\circ}$ & $?$ & $(1.28)$ & 1.23 & $w$ & $1.12 h$ \\
\hline 5101.810 & & 3 & 19595.43 & 0.00 & $55462_{3 \frac{1}{2}}^{\circ}-75058_{2 \frac{1}{2}}$ & & & & & \\
\hline 5101.57 & 3 & 80 & 19596.35 & -0.05 & $46354_{4 \frac{1}{2}}^{\circ}-65950_{4 \frac{1}{2}}^{2}$ & & & & & \\
\hline 5099.11 & & 3 & 19605.80 & & & & & & & \\
\hline $\begin{array}{l}5097.75 \\
5095.99\end{array}$ & & $\begin{array}{l}2 \\
3\end{array}$ & 19611.03 & -0.03 & $62046_{3 \frac{1}{2}}^{\circ}-81657_{2 \frac{1}{2}}$ & & & & & \\
\hline 5095.99 & & 3 & 19617.80 & +0.01 & $44438_{3 \frac{1}{2}}-64055_{2 \frac{1}{2}}$ & & & & & \\
\hline 5090.628 & & 15 & 19638.47 & +0.01 & $53644_{3 \frac{1}{2}}^{\circ}-73283_{4 \frac{1}{2}}$ & & & & & \\
\hline 5088.92 & 1 & 30 & 19645.06 & 0.00 & $48503_{5 \frac{1}{2}}^{\circ}-68148_{5 \frac{1}{2}}^{\circ}$ & & & & & \\
\hline 5087.61 & 10 & 150 & 19650.12 & +0.03 & $45273_{3 \frac{1}{2}}^{\circ}-64923_{\frac{1}{2}}^{\circ}$ & 4 & 1.17 & $(1.10)$ & $w$ & 0.86 \\
\hline 5085.70 & 2 & 80 & 19657.50 & +0.01 & $47758_{3 \frac{1}{2}}^{\circ}-67416_{4 \frac{1}{2}}$ & & & & & \\
\hline 5085.22 & & 1 & 19659.35 & -0.06 & $53120_{2 \frac{1}{2}}^{\circ}-72779_{3 \frac{1}{2}}^{2}$ & & & & & \\
\hline
\end{tabular}


TABLE 6. Yb II-Observed and classified lines-Continued

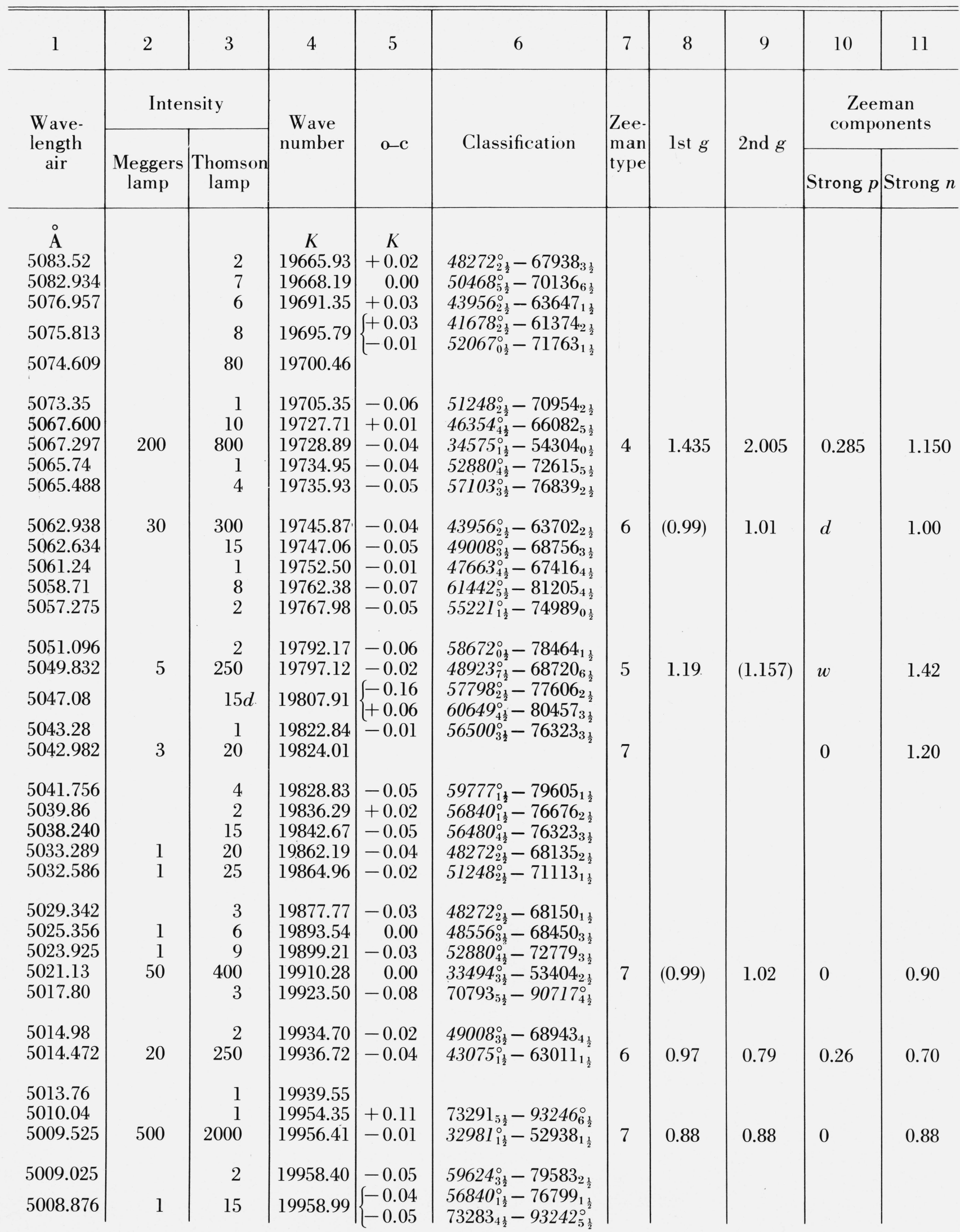


TABLE 6. Yb II-Observed and classified lines-Continued

\begin{tabular}{|c|c|c|c|c|c|c|c|c|c|c|}
\hline 1 & 2 & 3 & 4 & 5 & 6 & 7 & 8 & 9 & 10 & 11 \\
\hline \multirow{2}{*}{$\begin{array}{l}\text { W ave- } \\
\text { length } \\
\text { air }\end{array}$} & \multicolumn{2}{|c|}{ Intensity } & \multirow{2}{*}{$\begin{array}{l}\text { Wave } \\
\text { number }\end{array}$} & \multirow{2}{*}{$\mathrm{o}-\mathrm{c}$} & \multirow{2}{*}{ Classification } & \multirow{2}{*}{$\begin{array}{l}\text { Zee- } \\
\text { man } \\
\text { type }\end{array}$} & \multirow{2}{*}{ lst $g$} & \multirow{2}{*}{ 2nd $g$} & \multicolumn{2}{|c|}{$\begin{array}{c}\text { Zeeman } \\
\text { components }\end{array}$} \\
\hline & $\begin{array}{l}\text { Meggers } \\
\text { lamp }\end{array}$ & $\begin{array}{c}\text { Thomson } \\
\text { lamp }\end{array}$ & & & & & & & Strong $p$ & Strong $n$ \\
\hline $\begin{array}{c}\AA \\
\text { A } \\
5007.75 \\
5005.25 \\
5004.63\end{array}$ & & $\begin{array}{l}1 \\
1 \\
1\end{array}$ & $\begin{array}{c}K \\
19963.48 \\
19973.45 \\
19975.93\end{array}$ & $\begin{array}{c}K \\
-0.09 \\
-0.05 \\
-0.08\end{array}$ & $\begin{array}{l}44497_{2 \frac{1}{2}}^{\circ} 64461_{1 \frac{1}{2}} \\
51248_{2 \frac{1}{2}}^{\circ}-71222_{3 \frac{1}{2}} \\
47228_{0 \frac{1}{2}}^{\circ}-67204_{1 \frac{1}{2}}\end{array}$ & & & & & \\
\hline $\begin{array}{l}5002.98 \\
5000.452 \\
4998.87 \\
4998.266 \\
4996.604\end{array}$ & 3 & $\begin{array}{r}70 \\
2 \\
3 \\
15 \\
4\end{array}$ & $\begin{array}{l}19982.51 \\
19992.62 \\
19998.94 \\
20001.36 \\
20008.01\end{array}$ & $\begin{array}{l}-0.02 \\
-0.04 \\
-0.02 \\
-0.03 \\
-0.04\end{array}$ & $\begin{array}{l}68720_{6 \frac{1}{2}}-88702_{5 \frac{1}{2}}^{\circ} \\
48556_{3 \frac{1}{2}}^{\circ}-68549_{4 \frac{1}{2}}^{\circ} \\
56840_{1 \frac{1}{2}}^{\circ}-76839_{2 \frac{1}{2}}^{\circ} \\
43956_{2 \frac{1}{2}}^{\circ}-63957_{3 \frac{1}{2}}^{\circ} \\
69607_{2 \frac{1}{2}}^{\circ}-89615_{2 \frac{1}{2}}^{\circ}\end{array}$ & 5 & (1.157) & 1.12 & $w$ & 1.38 \\
\hline $\begin{array}{l}4994.74 \\
4993.32 \\
4992.654 \\
4992.520 \\
4989.794\end{array}$ & & $\begin{array}{l}1 \\
6 \\
7 \\
4\end{array}$ & $\begin{array}{l}20015.48 \\
20021.17 \\
20023.84 \\
20024.38 \\
20035.32\end{array}$ & $\begin{array}{r}+0.01 \\
\left\{\begin{array}{l}-0.03 \\
+0.02\end{array}\right. \\
-0.02 \\
-0.01 \\
-0.02\end{array}$ & $\begin{array}{l}56500_{3 \frac{1}{2}}^{\circ}-76516_{4 \frac{1}{2}} \\
47912_{3 \frac{1}{2}}^{\circ}-67933_{2 \frac{1}{2}}^{\circ} \\
51248_{2 \frac{1}{2}}^{\circ}-71270_{2 \frac{1}{2}} \\
46547_{5 \frac{1}{2}}^{\circ}-66571_{5 \frac{1}{2}} \\
49419_{0 \frac{1}{2}}^{\circ}-69443_{1 \frac{1}{2}} \\
56480_{4 \frac{1}{2}}^{\circ}-76516_{4 \frac{1}{2}}^{\circ}\end{array}$ & & & & & \\
\hline $\begin{array}{l}4988.383 \\
4988.022 \\
4987.292 \\
4986.88^{\mathrm{a}} \\
4978.62\end{array}$ & $\begin{array}{l}1 \\
6 \\
1\end{array}$ & $\begin{array}{r}15 \\
1 \\
15 \\
3 \\
9\end{array}$ & $\begin{array}{l}20040.99 \\
20042.44 \\
20045.37 \\
20047.02 \\
20080.28\end{array}$ & $\begin{array}{r}0.00 \\
-0.04 \\
+0.03 \\
-0.07\end{array}$ & $\begin{array}{l}49916_{4 \frac{1}{2}}^{\circ}-69957_{4 \frac{1}{2}} \\
48503_{5 \frac{1}{2}}^{\circ}-68549_{4 \frac{1}{2}} \\
22960_{1 \frac{1}{2}}^{\circ}-43007_{0 \frac{1}{2}}^{\circ} \\
45012_{2 \frac{1}{2}}^{\circ}-65093_{3 \frac{1}{2}}^{\circ}\end{array}$ & & & & & \\
\hline $\begin{array}{l}4976.65 \\
4973.90 \\
4972.26 \\
4971.20 \\
4970.203\end{array}$ & 3 & $\begin{array}{r}2 \\
15 \\
20 \\
8 \\
4\end{array}$ & $\begin{array}{l}20088.23 \\
20099.34 \\
20105.97 \\
20110.26 \\
20114.29\end{array}$ & $\begin{array}{r}-0.03 \\
-0.07 \\
-0.04 \\
-0.04 \\
0.00\end{array}$ & $\begin{array}{l}55462_{3 \frac{1}{2}}^{\circ}-75550_{2 \frac{1}{2}}^{\circ} \\
43956_{2 \frac{1}{2}}^{\circ}-64055_{2 \frac{1}{2}}^{\circ} \\
53644_{3 \frac{1}{2}}^{\circ}-73750_{4 \frac{1}{2}}^{\circ} \\
48024_{1 \frac{1}{2}}^{\circ}-68135_{2 \frac{1}{2}}^{\circ} \\
22960_{1 \frac{1}{2}}^{\circ}-43075_{\frac{1}{2}}^{\circ}\end{array}$ & & & & & \\
\hline $\begin{array}{l}4967.35 \\
4965.43 \\
4963.18 \\
4961.124 \\
4958.895\end{array}$ & 3 & $\begin{array}{r}2 \\
15 \\
2 \\
3 \\
2\end{array}$ & $\begin{array}{l}20125.84 \\
20133.62 \\
20142.75 \\
20151.10 \\
20160.16\end{array}$ & $\begin{array}{l}-0.03 \\
-0.09 \\
-0.07 \\
-0.09\end{array}$ & $\begin{array}{l}48024_{1 \frac{1}{2}}^{\circ}-68150_{1 \frac{1}{2}} \\
70136_{6 \frac{1}{2}}-90279_{5 \frac{1}{2}}^{\circ} \\
45737_{\frac{1}{2}}^{\circ}-65888_{\frac{1}{2}}^{\circ} \\
44438_{3 \frac{1}{2}}^{\circ}-64598_{\frac{1}{2}}^{\circ}\end{array}$ & & & & & \\
\hline $\begin{array}{l}4957.262 \\
4955.98\end{array}$ & & $\begin{array}{l}7 \\
1\end{array}$ & $\begin{array}{l}20166.80 \\
20172.01\end{array}$ & $\begin{array}{l}-0.07 \\
-0.06\end{array}$ & $\begin{array}{l}59439_{2 \frac{1}{2}}^{\circ}-79605_{1 \frac{1}{2}}^{\circ} \\
69443_{1 \frac{1}{2}}-89615_{2 \frac{1}{2}}^{\circ}\end{array}$ & & & & & \\
\hline 4954.68 & 3 & 50 & 20177.31 & $\left\{\begin{array}{l}-0.03 \\
-0.07\end{array}\right.$ & $\left.\begin{array}{l}48272_{2 \frac{1}{2}}^{\circ}-68450_{3 \frac{1}{2}}^{\circ} \\
55462_{3 \frac{1}{2}}^{\circ}-75640_{3 \frac{1}{2}}\end{array}\right\}$ & 7 & 0.98 & 0.98 & 0 & 0.98 \\
\hline $\begin{array}{l}4953.622 \\
4952.740\end{array}$ & & $\begin{array}{l}3 \\
2\end{array}$ & $\begin{array}{l}20181.62 \\
20185.21\end{array}$ & -0.04 & $69957_{4 \frac{1}{2}}-90142_{3 \frac{1}{2}}^{\circ}$ & & & & & \\
\hline 4949.24 & & 2 & 20199.48 & $\left\{\begin{array}{l}-0.03 \\
+0.01\end{array}\right.$ & $\begin{array}{l}47005_{\frac{11}{2}}^{\circ}-67204_{1 \frac{1}{2}} \\
48556_{3 \frac{1}{2}}^{\circ}-68756_{3 \frac{1}{2}}^{\circ}\end{array}$ & & & & & \\
\hline $\begin{array}{l}4946.524 \\
4946.19 \\
4945.944 \\
4944.956\end{array}$ & 5 & $\begin{array}{r}1 \\
1 \\
3 \\
150\end{array}$ & $\begin{array}{l}20210.58 \\
20211.94 \\
20212.95 \\
20216.98\end{array}$ & $\begin{array}{r}-0.02 \\
0.00 \\
0.00 \\
-0.05\end{array}$ & $\begin{array}{l}69395_{3 \frac{1}{2}}-89605_{4 \frac{1}{2}}^{\circ} \\
73291_{5 \frac{1}{2}}^{\circ}-93503_{4 \frac{1}{2}}^{\circ} \\
57534_{3 \frac{1}{2}}^{\circ}-77747_{4 \frac{1}{2}}^{\circ} \\
46354_{4 \frac{1}{2}}^{\circ}-66571_{5 \frac{1}{2}}^{\circ}\end{array}$ & & & & & \\
\hline
\end{tabular}


TABLE 6. Yb II-Observed and classified lines-Continued

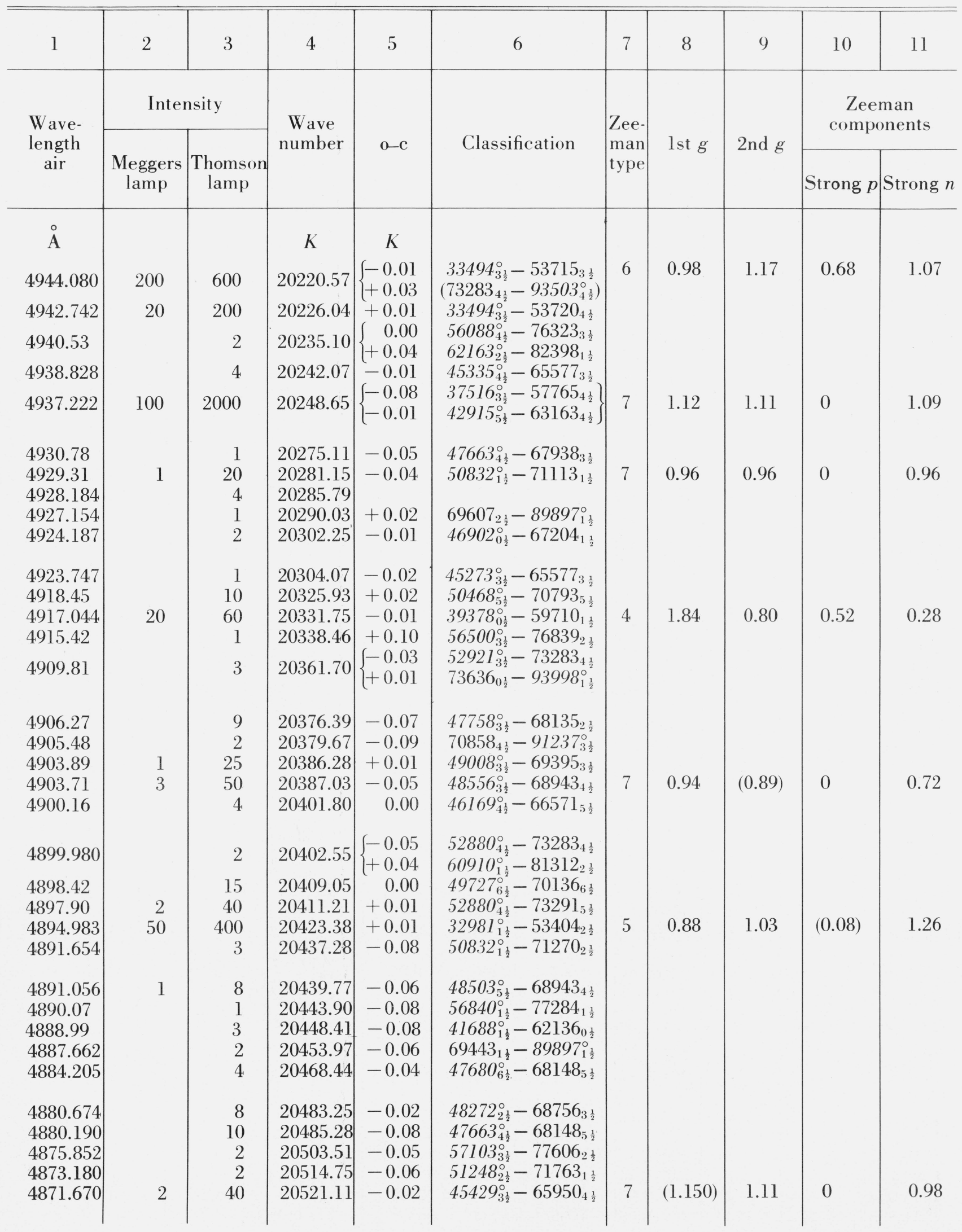


TABLE 6. Yb II-Observed and classified lines-Continued

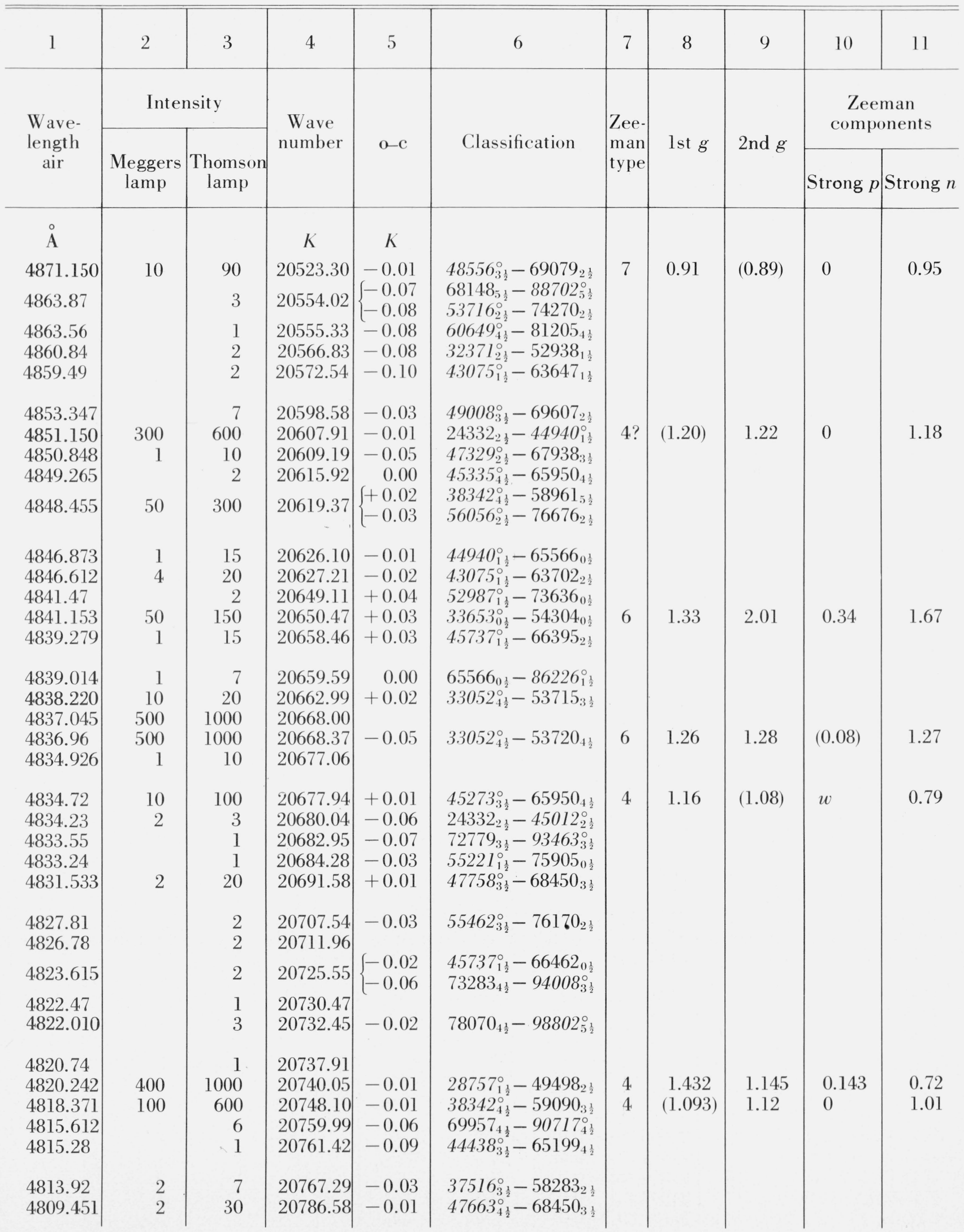


TABLE 6. Yb II-Observed and classified lines-Continued

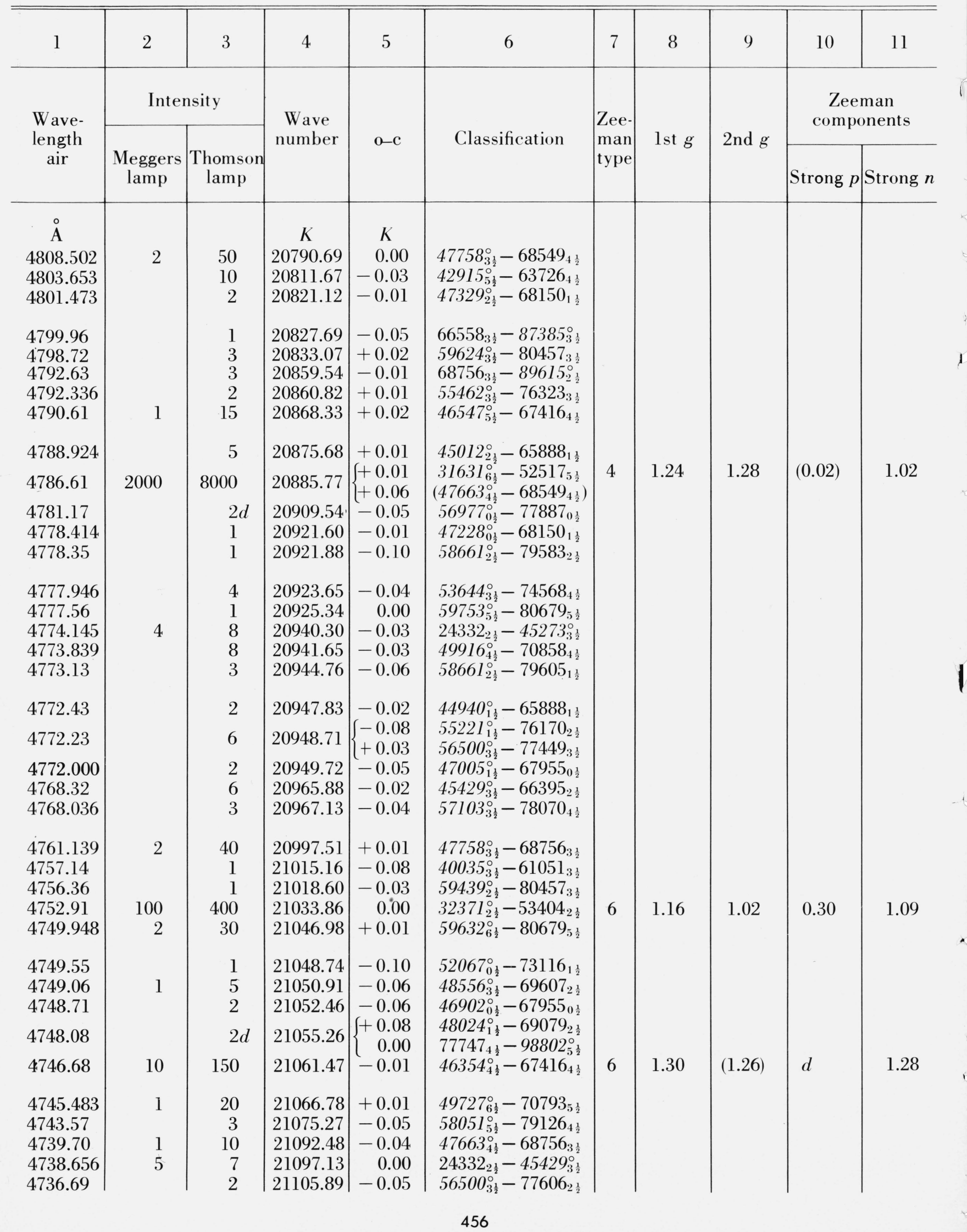


TABLE 6. Yb II-Observed and classified lines-Continued

\begin{tabular}{|c|c|c|c|c|c|c|c|c|c|c|}
\hline 1 & 2 & 3 & 4 & 5 & 6 & 7 & 8 & 9 & 10 & 11 \\
\hline \multirow{2}{*}{$\begin{array}{l}\text { Wave- } \\
\text { length } \\
\text { air }\end{array}$} & \multicolumn{2}{|c|}{ Intensity } & \multirow{2}{*}{$\begin{array}{c}\text { Wave } \\
\text { number }\end{array}$} & \multirow{2}{*}{$o-c$} & \multirow{2}{*}{ Classification } & \multirow{2}{*}{$\begin{array}{l}\text { Zee- } \\
\text { man } \\
\text { type }\end{array}$} & \multirow{2}{*}{ lst $g$} & \multirow{2}{*}{2 nd $g$} & \multicolumn{2}{|c|}{$\begin{array}{c}\text { Zeeman } \\
\text { components }\end{array}$} \\
\hline & $\begin{array}{c}\text { Meggers } \\
\text { lamp }\end{array}$ & $\begin{array}{c}\text { Thomson } \\
\text { lamp }\end{array}$ & & & & & & & Strong $p$ & Strong $n$ \\
\hline ̊ & & & K & $K$ & & & & & & \\
\hline $\begin{array}{l}4732.935 \\
4732.025 \\
4731.70 \\
4729.25 \\
4728.109\end{array}$ & $\begin{array}{r}1 \\
30\end{array}$ & $\begin{array}{r}25 \\
200 \\
2 \\
3 \\
8\end{array}$ & $\begin{array}{l}21122.63 \\
21126.69 \\
21128.14 \\
21139.09 \\
21144.19\end{array}$ & $\begin{array}{l}-0.07 \\
-0.01 \\
-0.04 \\
+0.01 \\
+0.02\end{array}$ & $\begin{array}{l}45273_{3 \frac{1}{2}}^{\circ}-66395_{2 \frac{1}{2}} \\
34575_{\frac{1}{2}}^{\circ}-55702_{1 \frac{1}{2}} \\
45429_{3 \frac{1}{2}}^{\circ}-66558_{3 \frac{1}{2}} \\
44438_{3 \frac{1}{2}}^{\circ}-65577_{3 \frac{1}{2}}^{\circ} \\
58484_{4 \frac{1}{2}}^{\circ}-79628_{3 \frac{1}{2}}^{\circ}\end{array}$ & 6 & 1.43 & 1.69 & 0.40 & 1.56 \\
\hline $\begin{array}{l}4727.901 \\
4726.54 \\
4726.08 \\
4725.14 \\
4723.404\end{array}$ & 1000 & $\begin{array}{r}7 \\
1 \\
3000 \\
2 \\
4\end{array}$ & $\begin{array}{l}21145.12 \\
21151.21 \\
21153.27 \\
21157.48 \\
21165.25\end{array}$ & $\begin{array}{l}+0.01 \\
-0.02 \\
-0.10 \\
-0.08\end{array}$ & $\begin{array}{l}47005_{1 \frac{1}{2}}^{\circ}-68150_{1 \frac{1}{2}} \\
26759_{2}^{\circ}-47912_{3 \frac{1}{2}} \\
70858_{4 \frac{1}{2}}-92015_{4 \frac{1}{2}}^{\circ} \\
70136_{6 \frac{1}{2}}-91301_{6 \frac{1}{2}}^{\circ}\end{array}$ & 4 & 1.57 & 1.28 & 0.15 & 0.54 \\
\hline $\begin{array}{l}4720.390 \\
4715.672 \\
4712.78 \\
4705.40 \\
4705.04\end{array}$ & 40 & $\begin{array}{r}2 \\
3 \\
200 \\
4 \\
7\end{array}$ & $\begin{array}{l}21178.77 \\
21199.95 \\
21212.96 \\
21246.23 \\
21247.86\end{array}$ & $\begin{array}{r}-0.07 \\
-0.01 \\
+0.02 \\
-0.02 \\
0.00\end{array}$ & $\begin{array}{l}40035_{3 \frac{1}{2}}^{\circ}-61214_{4 \frac{1}{2}} \\
62163_{2 \frac{1}{2}}^{\circ}-83363_{3 \frac{1}{2}} \\
48923_{7 \frac{1}{2}}^{\circ}-70136_{6 \frac{1}{2}} \\
46169_{4 \frac{1}{2}}^{\circ}-67416_{4 \frac{1}{2}}^{\circ} \\
46902_{0 \frac{1}{2}}^{\circ}-68150_{1 \frac{1}{2}}\end{array}$ & 5 & $(1.20)$ & 1.16 & $w$ & 1.47 \\
\hline $\begin{array}{l}4704.23 \\
4703.012 \\
4699.045 \\
4698.62 \\
4697.688\end{array}$ & 10 & $\begin{array}{r}1 \\
4 \\
6 \\
40 \\
2\end{array}$ & $\begin{array}{l}21251.52 \\
21257.02 \\
21274.97 \\
21276.89 \\
21281.11\end{array}$ & $\begin{array}{l}\left\{\begin{array}{l}-0.01 \\
+0.04\end{array}\right. \\
-0.01 \\
-0.01 \\
+0.01\end{array}$ & $\begin{array}{l}69607_{2 \frac{1}{2}}-90859_{2 \frac{1}{2}}^{\circ} \\
72615_{5 \frac{1}{2}}^{\circ}-93867_{4 \frac{1}{2}}^{\circ} \\
53716_{2 \frac{1}{2}}^{\circ}-74973_{3 \frac{1}{2}}^{\circ} \\
53716_{2 \frac{1}{2}}^{\circ}-74991_{1 \frac{1}{2}} \\
38342_{4 \frac{1}{2}}^{\circ}-59618_{3 \frac{1}{2}}\end{array}$ & & & & & \\
\hline $\begin{array}{l}4697.34 \\
4696.842 \\
4692.41 \\
4691.982 \\
4690.810\end{array}$ & 20 & $\begin{array}{r}3 \\
15 \\
2 \\
4 \\
90\end{array}$ & $\begin{array}{l}21282.69 \\
21284.95 \\
21305.05 \\
21306.99 \\
21312.32\end{array}$ & $\begin{array}{r}-0.03 \\
-0.03 \\
-0.04 \\
0.00 \\
+0.01\end{array}$ & $\begin{array}{l}52987_{\frac{1}{2}}^{\circ}-74270_{2 \frac{1}{2}} \\
45273_{3 \frac{1}{2}}^{\circ}-66558_{3 \frac{1}{2}} \\
67416_{\frac{1}{2}}-88721_{3 \frac{1}{2}}^{\circ} \\
37516_{3 \frac{1}{2}}^{\circ}-58823_{2 \frac{1}{2}}^{\circ} \\
34389_{2 \frac{1}{2}}^{\circ}-55702_{1 \frac{1}{2}}\end{array}$ & & & & & \\
\hline $\begin{array}{l}4689.807 \\
4688.515 \\
4688.38 \\
4658.876 \\
4685.071\end{array}$ & $\begin{array}{r}1 \\
40 \\
5\end{array}$ & $\begin{array}{r}7 \\
100 \\
20 \\
6 \\
3\end{array}$ & $\begin{array}{l}21316.87 \\
21322.75 \\
21323.36 \\
21334.76 \\
21338.42\end{array}$ & $\begin{array}{r}-0.04 \\
+0.04 \\
-0.04 \\
-0.01 \\
0.00\end{array}$ & $\begin{array}{l}35059_{3 \frac{1}{2}}^{\circ}-56375_{2 \frac{1}{2}}^{\circ} \\
32981_{1 \frac{1}{2}}^{\circ}-54304_{0 \frac{1}{2}} \\
41688_{1 \frac{1}{2}}^{\circ}-63011_{1 \frac{1}{2}}^{\circ} \\
48272_{2 \frac{1}{2}}^{\circ}-69607_{2 \frac{1}{2}} \\
45012_{2 \frac{1}{2}}^{\circ}-66351_{2 \frac{1}{2}}^{\circ}\end{array}$ & 4 & 0.84 & 1.88 & 0.52 & 0.32 \\
\hline $\begin{array}{l}4683.810 \\
4681.088 \\
4678.171 \\
4675.66 \\
4674.644\end{array}$ & $\begin{array}{r}200 \\
2 \\
2\end{array}$ & $\begin{array}{r}1000 \\
3 \\
6 \\
4 \\
1\end{array}$ & $\begin{array}{l}21344.17 \\
21356.58 \\
21369.89 \\
21381.37 \\
21386.02\end{array}$ & $\begin{array}{r}+0.01 \\
0.00 \\
-0.04 \\
-0.03 \\
+0.03\end{array}$ & $\begin{array}{l}32371_{2 \frac{1}{2}}^{\circ}-53715_{3 \frac{1}{2}} \\
62861_{5 \frac{1}{2}}-84217_{4 \frac{1}{2}}^{\circ} \\
31568_{2 \frac{1}{2}}^{\circ}-52938_{1 \frac{1}{2}} \\
56500_{3 \frac{1}{2}}^{\circ}-77882_{3 \frac{1}{2}}^{\circ} \\
43075_{1 \frac{1}{2}}^{\circ}-64461_{1 \frac{1}{2}}\end{array}$ & 7 & (1.17) & 1.19 & 0 & 1.22 \\
\hline $\begin{array}{l}4674.493 \\
4671.386 \\
4670.586 \\
4669.280 \\
4667.05\end{array}$ & 50 & $\begin{array}{r}3 \\
3 \\
200 \\
4 \\
1\end{array}$ & $\begin{array}{l}21386.71 \\
21400.93 \\
21404.60 \\
21410.58 \\
21420.81\end{array}$ & $\begin{array}{r}+0.01 \\
+0.01 \\
0.00 \\
-0.02 \\
-0.03\end{array}$ & $\begin{array}{l}68756_{3 \frac{1}{2}}-90142_{3 \frac{1}{2}}^{\circ} \\
48556_{3 \frac{1}{2}}^{\circ}-69957_{4 \frac{1}{2}}^{\circ} \\
24332_{2 \frac{1}{2}}-45737_{1 \frac{1}{2}}^{\circ} \\
44940_{\frac{1}{2}}^{\circ}-66351_{2 \frac{1}{2}}^{\circ} \\
74270_{2 \frac{1}{2}}^{\circ}-95691_{3 \frac{1}{2}}^{\circ}\end{array}$ & 4 & $(1.20)$ & 1.30 & $w$ & 1.06 \\
\hline
\end{tabular}


TABlE 6. Yb II-Observed and classified lines-Continued

\begin{tabular}{|c|c|c|c|c|c|c|c|c|c|c|}
\hline 1 & 2 & 3 & 4 & 5 & 6 & 7 & 8 & 9 & 10 & 11 \\
\hline \multirow{2}{*}{$\begin{array}{l}\text { Wave- } \\
\text { length } \\
\text { air }\end{array}$} & \multicolumn{2}{|c|}{ Intensity } & \multirow{2}{*}{$\begin{array}{l}\text { Wave } \\
\text { number }\end{array}$} & \multirow{2}{*}{$o-c$} & \multirow{2}{*}{ Classification } & \multirow{2}{*}{$\begin{array}{l}\text { Zee- } \\
\text { man } \\
\text { type }\end{array}$} & \multirow{2}{*}{ lst $g$} & \multirow{2}{*}{ 2nd $g$} & \multicolumn{2}{|c|}{$\begin{array}{l}\text { Zeeman } \\
\text { components }\end{array}$} \\
\hline & $\begin{array}{l}\text { Meggers } \\
\text { lamp }\end{array}$ & $\begin{array}{c}\text { Thomson } \\
\text { lamp }\end{array}$ & & & & & & & Strong $p$ & Strong $n$ \\
\hline A & & & $K$ & K & & & & & & \\
\hline $\begin{array}{l}4666.145 \\
4660.906\end{array}$ & 2 & $\begin{array}{l}1 \\
4\end{array}$ & $\begin{array}{l}21424.97 \\
21449.05\end{array}$ & $\begin{array}{l}-0.09 \\
+0.01\end{array}$ & $\begin{array}{l}31979_{3 \frac{1}{2}}^{\circ}-53404_{2 \frac{1}{2}} \\
56621_{5 \frac{1}{2}}^{\circ}-78070_{4 \frac{1}{2}}^{\circ}\end{array}$ & & & & & \\
\hline 4659.910 & & 5 & 21453.64 & $\left\{\begin{array}{l}-0.03 \\
+0.01\end{array}\right.$ & $\begin{array}{l}48503_{5 \frac{1}{2}}^{\circ}-69957_{4 \frac{1}{2}} \\
68756_{31}-90209_{4 \frac{1}{\circ}}^{\circ}\end{array}$ & & & & & \\
\hline $\begin{array}{l}4659.60 \\
4658.746\end{array}$ & & $\begin{array}{l}1 \\
3\end{array}$ & $\begin{array}{l}21455.06 \\
21459.00\end{array}$ & -0.05 & $44940_{1 \frac{1}{2}}^{\circ}-66395_{2 \frac{1}{2}}^{\circ}$ & & & & & \\
\hline $\begin{array}{l}4658.443 \\
4657.43 \\
4654.070 \\
4652.667 \\
4652.279\end{array}$ & $\begin{array}{l}2 \\
1\end{array}$ & $\begin{array}{l}2 \\
1 \\
5 \\
5 \\
2\end{array}$ & $\begin{array}{l}21460.39 \\
21465.06 \\
21480.56 \\
21487.03 \\
21488.82\end{array}$ & $\begin{array}{l}+0.04 \\
-0.03 \\
-0.02 \\
-0.02\end{array}$ & $\begin{array}{l}68150_{1 \frac{1}{2}}-89615_{2 \frac{1}{2}}^{\circ} \\
68135_{2 \frac{1}{2}}^{\circ}-89615_{2 \frac{1}{2}}^{\circ} \\
56977_{0 \frac{1}{2}}^{\circ}-78464_{1 \frac{1}{2}} \\
60910_{1 \frac{1}{2}}^{\circ}-82398_{1 \frac{1}{2}}^{\circ}\end{array}$ & & & & & \\
\hline $\begin{array}{l}4649.756 \\
4647.076 \\
4645.05 \\
4643.14 \\
4640.11\end{array}$ & & $\begin{array}{r}2 \\
2 \\
10 \\
5 \\
15\end{array}$ & $\begin{array}{l}21500.48 \\
21512.88 \\
21522.27 \\
21531.12 \\
21545.18\end{array}$ & $\begin{array}{l}-0.04 \\
+0.02 \\
-0.01 \\
-0.03\end{array}$ & $\begin{array}{l}44438_{3 \frac{1}{2}}^{\circ}-65950_{4 \frac{1}{2}} \\
44940_{1 \frac{1}{2}}^{\circ}-66462_{0 \frac{1}{2}} \\
51248_{2 \frac{1}{2}}^{\circ}-72779_{3 \frac{1}{2}} \\
45012_{2 \frac{1}{2}}^{\circ}-66558_{3 \frac{1}{2}}\end{array}$ & & & & & \\
\hline $\begin{array}{l}4639.04 \\
4637.226 \\
4635.01 \\
4634.014\end{array}$ & 20 & $\begin{array}{r}1 \\
3 \\
1 \\
200\end{array}$ & $\begin{array}{l}21550.15 \\
21558.58 \\
21568.89 \\
21573.52\end{array}$ & $\begin{array}{l}-0.06 \\
-0.07 \\
-0.02\end{array}$ & $\begin{array}{l}68720_{6 \frac{1}{2}}-90279_{5 \frac{1}{2}}^{\circ} \\
52067_{0 \frac{1}{2}}^{\circ}-73636_{0 \frac{1}{2}} \\
37516_{3 \frac{1}{2}}^{\circ}-59090_{3 \frac{1}{2}}^{\circ}\end{array}$ & 7 & & & 0 & $1.05 ?$ \\
\hline 4630.614 & & 3 & 21589.36 & $\left\{\begin{array}{l}-0.06 \\
-0.06\end{array}\right.$ & $\begin{array}{l}52067_{0 \frac{1}{2}}^{\circ}-73657_{1 \frac{1}{2}}^{\circ} \\
56480_{4 \frac{1}{2}}^{\circ}-78070_{4 \frac{1}{2}}^{\circ}\end{array}$ & & & & & \\
\hline $\begin{array}{l}4629.75 \\
4624.93 \\
4623.91 \\
4623.060 \\
4621.83\end{array}$ & & $\begin{array}{l}2 \\
2 \\
1 \\
3 \\
2\end{array}$ & $\begin{array}{l}21593.39 \\
21615.89 \\
21620.66 \\
21624.64 \\
21630.39\end{array}$ & $\begin{array}{l} \\
-0.04 \\
-0.08 \\
-0.01\end{array}$ & $\begin{array}{l}43956_{2 \frac{1}{2}}^{\circ}-65577_{3 \frac{1}{2}}^{\circ} \\
56840_{\frac{1}{2}}^{\circ}-78464_{1 \frac{1}{2}}^{\circ} \\
69607_{\frac{21}{2}}-91237_{3 \frac{1}{2}}^{\circ}\end{array}$ & & & & & \\
\hline $\begin{array}{l}4620.51 \\
4615.740 \\
4614.020 \\
4611.90 \\
4610.70\end{array}$ & 1 & $\begin{array}{l}2 \\
2 \\
3 \\
1 \\
1\end{array}$ & $\begin{array}{l}21636.57 \\
21658.93 \\
21667.01 \\
21676.97 \\
21682.61\end{array}$ & $\begin{array}{l}-0.09 \\
-0.08 \\
-0.01 \\
+0.06 \\
-0.06\end{array}$ & $\begin{array}{l}47758_{3 \frac{1}{2}}^{\circ}-69395_{3 \frac{1}{2}} \\
56088_{4 \frac{1}{2}}^{\circ}-77747_{4 \frac{1}{2}} \\
777477_{4 \frac{1}{2}}-99414_{4 \frac{1}{2}}^{\circ} \\
67938_{3 \frac{1}{2}}-89615_{2 \frac{1}{2}}^{\circ} \\
54640_{4 \frac{1}{2}}^{\circ}-76323_{3 \frac{1}{2}}^{\circ}\end{array}$ & & & & & \\
\hline $\begin{array}{l}4609.600 \\
4608.131 \\
4600.300 \\
4598.85 \\
4598.361\end{array}$ & 300 & $\begin{array}{r}1 \\
15 \\
2 \\
2 \\
800\end{array}$ & $\begin{array}{l}21687.78 \\
21694.69 \\
21731.62 \\
21738.48 \\
21740.79\end{array}$ & $\begin{array}{l}-0.05 \\
-0.02 \\
-0.06 \\
-0.02\end{array}$ & $\begin{array}{l}52880_{4 \frac{1}{2}}^{\circ}-74568_{4 \frac{1}{2}} \\
49419_{0 \frac{1}{2}}^{\circ}-71113_{1 \frac{1}{2}} \\
47663_{4 \frac{1}{2}}^{\circ}-69395_{3 \frac{1}{2}}^{\circ} \\
31979_{3 \frac{1}{2}}^{\circ}-53720_{4 \frac{1}{2}}\end{array}$ & 5 & (1.33) & 1.29 & $w$ & 1.16 \\
\hline $\begin{array}{l}4597.980 \\
4597.279 \\
4597.087\end{array}$ & $\begin{array}{l}5 \\
2\end{array}$ & $\begin{array}{r}30 \\
30 \\
1\end{array}$ & $\begin{array}{l}21742.59 \\
21745.90 \\
21746.81\end{array}$ & $\begin{array}{l}-0.06 \\
-0.09\end{array}$ & $\begin{array}{l}37516_{3 \frac{1}{2}}^{\circ}-59259_{2 \frac{1}{2}} \\
37077_{2 \frac{1}{2}}^{\circ}-58823_{2 \frac{1}{2}}\end{array}$ & & & & & \\
\hline
\end{tabular}


TABLE 6. Yb II-Observed and classified lines-Continued

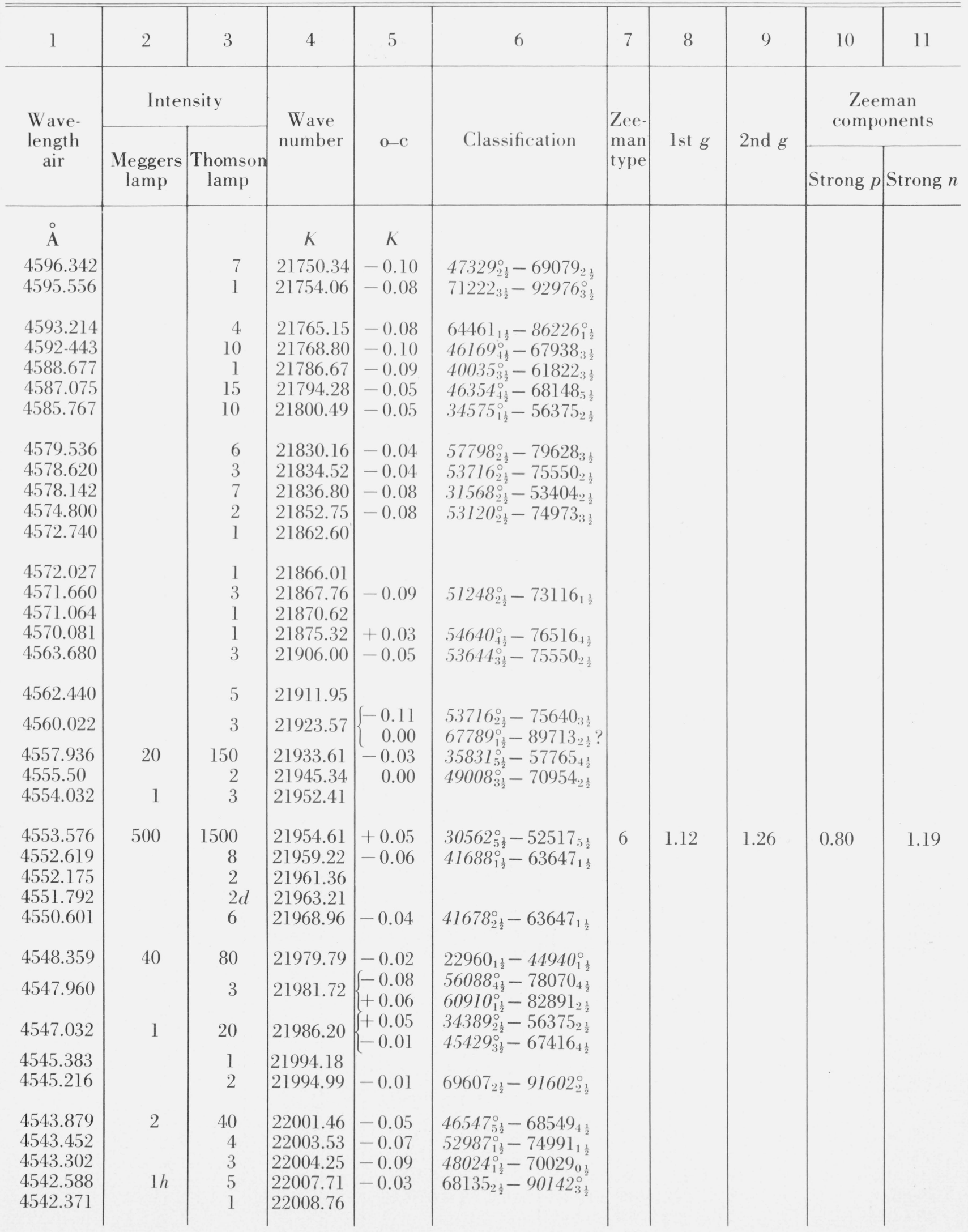


TABLE 6. Yb II-Observed and classified lines-Continued

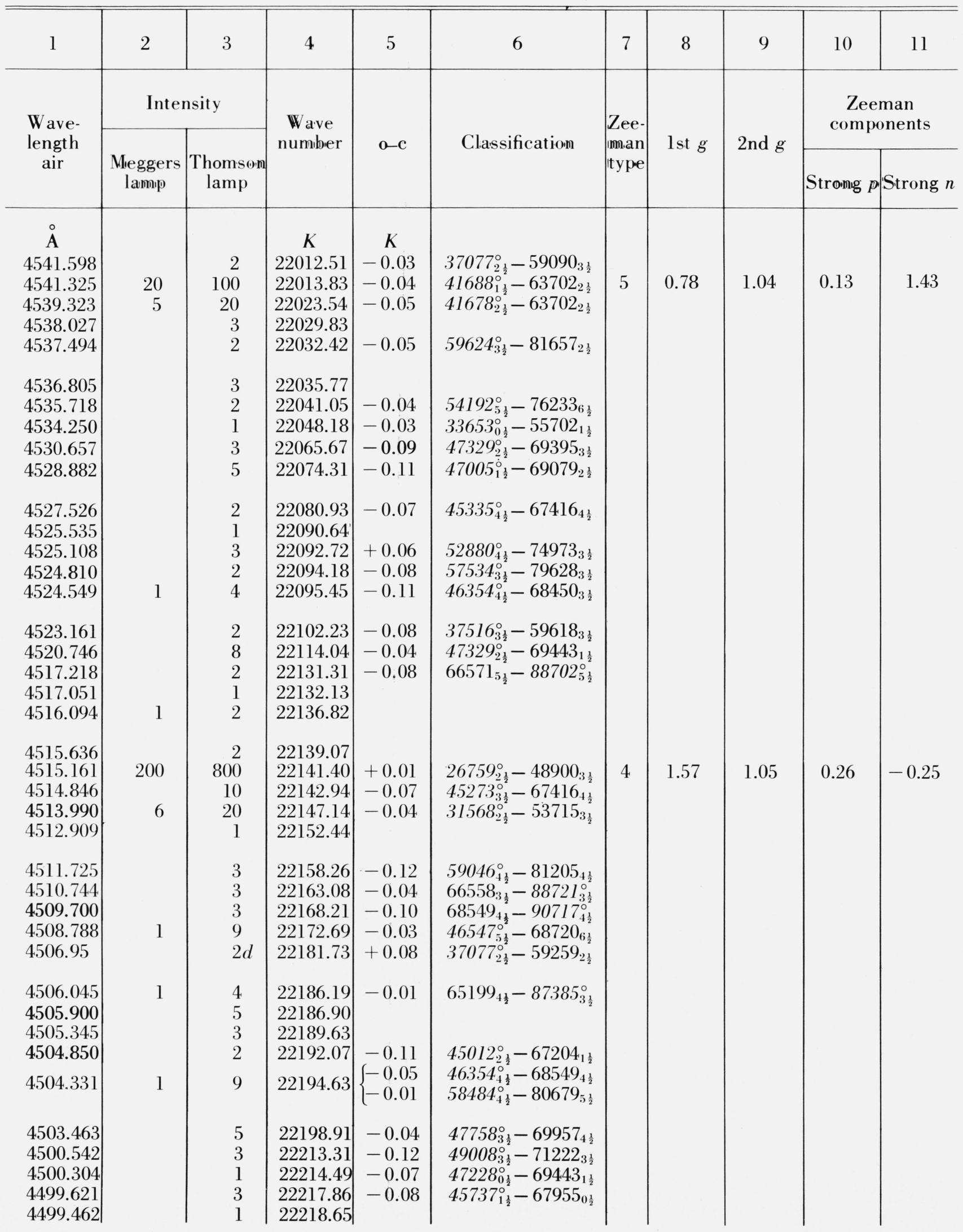


TABLE 6. Yb II-Observed and classified lines-Continued

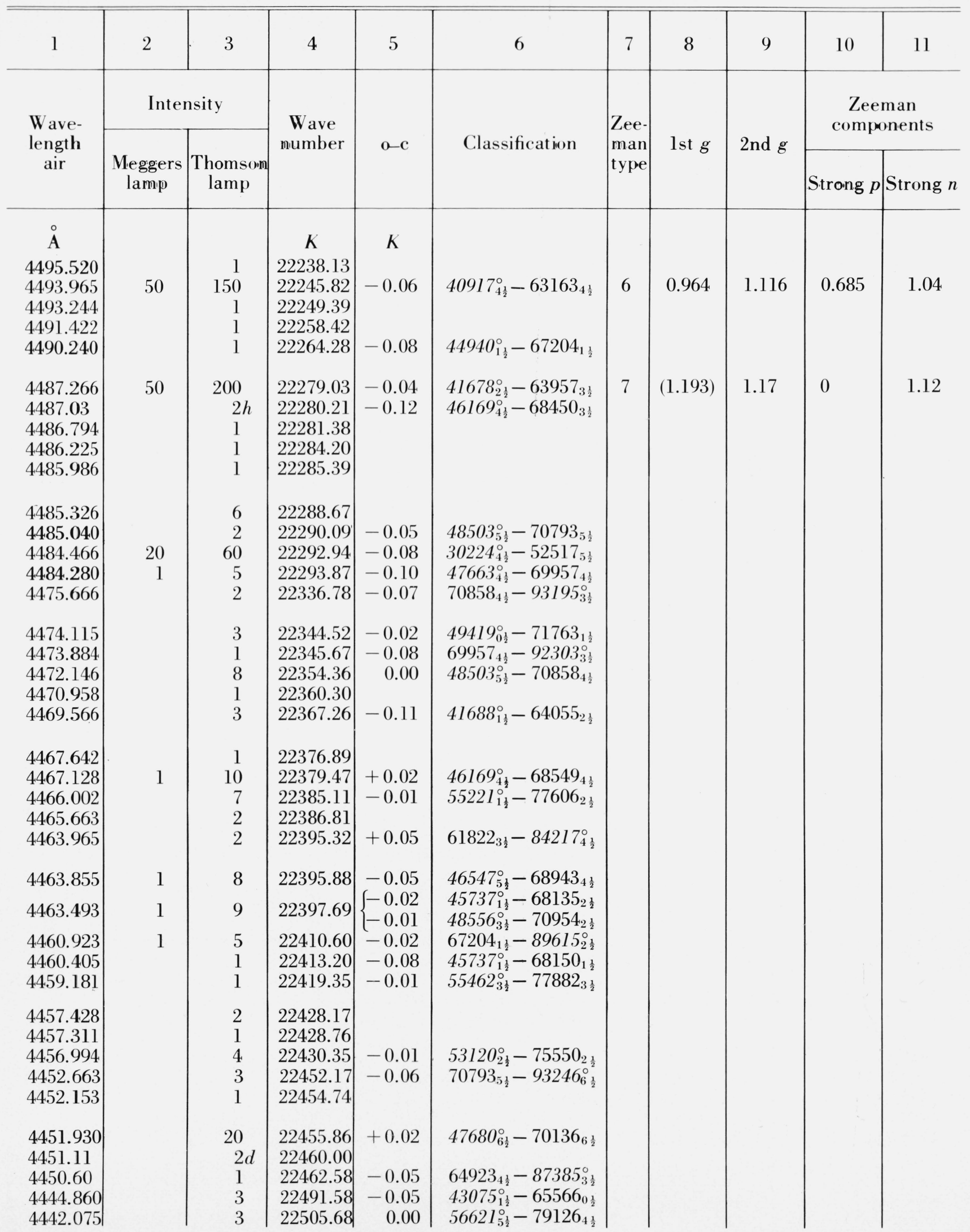


TABLE 6. Yb II-Observed and classified lines-Continued

\begin{tabular}{|c|c|c|c|c|c|c|c|c|c|c|}
\hline 1 & 2 & 3 & 4 & 5 & 6 & 7 & 8 & 9 & 10 & 11 \\
\hline \multirow{2}{*}{$\begin{array}{l}\text { Wave- } \\
\text { length } \\
\text { air }\end{array}$} & \multicolumn{2}{|c|}{ Intensity } & \multirow{2}{*}{$\begin{array}{l}\text { Wave } \\
\text { number }\end{array}$} & \multirow{2}{*}{$o-c$} & \multirow{2}{*}{ Classification } & \multirow{2}{*}{$\mid \begin{array}{l}\text { Zee- } \\
\text { man } \\
\text { type }\end{array}$} & \multirow{2}{*}{ lst $g$} & \multirow{2}{*}{ 2nd $g$} & \multicolumn{2}{|c|}{$\begin{array}{l}\text { Zeeman } \\
\text { components }\end{array}$} \\
\hline & $\begin{array}{l}\text { Meggers } \\
\text { lamp }\end{array}$ & $\begin{array}{c}\text { Thomson } \\
\text { lamp }\end{array}$ & & & & & & & Strong $p$ & Strong $n$ \\
\hline $\begin{array}{c}\AA \\
4441.484 \\
4438.142 \\
4435.150 \\
4434.178 \\
4431.595\end{array}$ & 40 & $\begin{array}{r}2 \\
7 \\
5 \\
100 \\
1\end{array}$ & \begin{tabular}{c|}
$K$ \\
22508.68 \\
22525.63 \\
22540.82 \\
22545.76 \\
22558.91
\end{tabular} & $\begin{array}{c}K \\
-0.06 \\
-0.06 \\
+0.01 \\
-0.02 \\
-0.02\end{array}$ & $\begin{array}{l}70954_{2 \frac{1}{2}}-93463_{3 \frac{1}{2}}^{\circ} \\
57103_{3 \frac{1}{2}}^{\circ}-79628_{3 \frac{1}{2}}^{\circ} \\
46902_{0 \frac{1}{2}}^{\circ}-69443_{1 \frac{1}{2}}^{\circ} \\
30392_{1 \frac{1}{2}}^{\circ}-52938_{1 \frac{1}{2}}^{\circ} \\
43007_{0 \frac{1}{2}}^{\circ}-65566_{0 \frac{1}{2}}^{\circ}\end{array}$ & & & & & \\
\hline $\begin{array}{l}4428.153 \\
4423.129 \\
4422.150 \\
4419.495 \\
4418.132\end{array}$ & & $\begin{array}{l}3 h \\
1 \\
3 \\
1 \\
3\end{array}$ & $\begin{array}{l}22576.44 \\
22602.08 \\
22607.09 \\
22620.67 \\
22627.65\end{array}$ & $\begin{array}{r}0.00 \\
-0.02 \\
-0.05 \\
-0.02\end{array}$ & $\begin{array}{l}47005_{1 \frac{1}{2}}^{\circ}-69607_{2 \frac{1}{2}} \\
53716_{2 \frac{1}{2}}^{\circ}-76323_{3 \frac{1}{2}} \\
66082_{5 \frac{1}{2}}-88702_{5 \frac{1}{2}}^{\circ} \\
58051_{5 \frac{1}{2}}^{\circ}-80679_{5 \frac{1}{2}}^{\circ}\end{array}$ & & & & & \\
\hline $\begin{array}{l}4417.814 \\
4417.080 \\
4414.548 \\
4410.740 \\
4409.685\end{array}$ & & $\begin{array}{l}1 \\
3 \\
1 \\
2 h \\
2 h\end{array}$ & $\begin{array}{l}22629.27 \\
22633.03 \\
22646.02 \\
22665.57 \\
22670.99\end{array}$ & $\begin{array}{l}-0.05 \\
-0.03 \\
-0.04 \\
-0.01 \\
-0.09\end{array}$ & $\begin{array}{l}52921_{3 \frac{1}{2}}^{\circ}-75550_{2 \frac{1}{2}} \\
37077_{2 \frac{1}{2}}^{\circ}-59710_{1 \frac{1}{2}} \\
56480_{4 \frac{1}{2}}^{\circ}-79126_{4 \frac{1}{2}} \\
59046_{4 \frac{1}{2}}^{\circ}-81692_{3 \frac{1}{2}} \\
45273_{3 \frac{1}{2}}^{\circ}-67938_{3 \frac{1}{2}}\end{array}$ & & & & & \\
\hline $\begin{array}{l}4409.346 \\
4408.220 \\
4407.650 \\
4405.496 \\
4404.208\end{array}$ & $\begin{array}{r}200 \\
1\end{array}$ & $\begin{array}{r}300 \\
1 \\
4 \\
1 \\
1\end{array}$ & $\begin{array}{l}22672.73 \\
22678.52 \\
22681.46 \\
22692.55 \\
22699.18\end{array}$ & $\begin{array}{l}-0.04 \\
-0.08 \\
-0.04 \\
-0.03 \\
-0.06\end{array}$ & $\begin{array}{l}24332_{2 \frac{1}{2}}-47005_{\frac{1}{2}}^{\circ} \\
53644_{3 \frac{1}{2}}^{\circ}-76323_{3 \frac{1}{2}}^{\circ} \\
48272_{2 \frac{1}{2}}^{\circ}-70954_{2 \frac{1}{2}}^{\circ} \\
67204_{1 \frac{1}{2}}^{\circ}-89897_{\frac{1}{2}}^{\circ} \\
49916_{4 \frac{1}{2}}^{\circ}-72615_{5 \frac{1}{2}}^{\circ}\end{array}$ & 5 & 1.20 & 1.02 & 0.09 & 1.47 \\
\hline $\begin{array}{l}4403.037 \\
4402.824\end{array}$ & & $\begin{array}{l}1 \\
5\end{array}$ & $\begin{array}{l}22705.22 \\
22706.32\end{array}$ & $\begin{array}{r}+0.04 \\
0.00\end{array}$ & $\begin{array}{l}45429_{3 \frac{1}{2}}^{\circ}-68135_{2 \frac{1}{2}}^{\circ} \\
35059_{3 \frac{1}{2}}^{\circ}-57765_{4 \frac{1}{2}}^{\circ}\end{array}$ & & & & & \\
\hline $\begin{array}{l}4402.300 \\
4401.824 \\
4401.445\end{array}$ & $\begin{array}{c}40 \\
1\end{array}$ & $\begin{array}{r}200 \\
1 \\
6\end{array}$ & $\begin{array}{l}22709.02 \\
22711.48 \\
22713.43\end{array}$ & $\begin{array}{l}-0.02 \\
-0.01\end{array}$ & $\begin{array}{l}38342_{4 \frac{1}{2}}^{\circ}-61051_{3 \frac{1}{2}}^{\circ} \\
48556_{3 \frac{1}{2}}^{\circ}-71270_{2 \frac{1}{2}}^{\circ}\end{array}$ & 4 & (1.093) & 1.18 & $w$ & 0.80 \\
\hline $\begin{array}{l}4400.583 \\
4399.391\end{array}$ & & $\begin{array}{l}1 \\
1\end{array}$ & $\begin{array}{l}22717.88 \\
22724.04\end{array}$ & $\begin{array}{l}-0.07 \\
-0.03\end{array}$ & $\begin{array}{l}51248_{2 \frac{1}{2}}^{\circ}-73966_{3 \frac{1}{2}}^{\circ} \\
68135_{2 \frac{1}{2}}-90859_{2 \frac{1}{2}}^{\circ}\end{array}$ & & & & & \\
\hline 4396.499 & 10 & 40 & 22738.98 & $\left\{\begin{array}{l}-0.04 \\
+0.03\end{array}\right.$ & $\begin{array}{l}26759_{2 \frac{1}{2}}^{\circ}-49498_{2 \frac{1}{2}}^{\circ} \\
71270_{2 \frac{1}{2}}^{\circ}-94008_{3 \frac{1}{2}}^{\circ}\end{array}$ & & & & & \\
\hline $\begin{array}{l}4392.825 \\
4390.49\end{array}$ & 50 & $\begin{array}{r}100 \\
1\end{array}$ & $\begin{array}{l}22758.00 \\
22770.10\end{array}$ & $\left\{\begin{array}{l}-0.05 \\
+0.05 \\
-0.07\end{array}\right.$ & $\begin{array}{c}39378_{0 \frac{1}{2}}^{\circ}-62136_{0 \frac{1}{2}}^{\circ} \\
\left(56056_{2 \frac{1}{2}}^{\circ}-78814_{2 \frac{1}{2}}\right) \\
65950_{4 \frac{1}{2}}-88721_{3 \frac{1}{2}}^{\circ}\end{array}$ & 6 & 1.84 & 0.08 & 0.88 & 0.96 \\
\hline $\begin{array}{l}4390.43 \\
4389.764 \\
4388.528 \\
4387.46 \\
4387.144\end{array}$ & 50 & $\begin{array}{r}1 \\
200 \\
8 \\
1 \\
10\end{array}$ & $\begin{array}{l}22770.42 \\
22773.87 \\
22780.28 \\
22785.83 \\
22787.47\end{array}$ & $\begin{array}{r}0.00 \\
-0.08 \\
+0.01\end{array}$ & $\begin{array}{l}46169_{4 \frac{1}{2}}^{\circ}-68943_{4 \frac{1}{2}} \\
68756_{3 \frac{1}{2}}-91536_{3 \frac{1}{2}}^{\circ} \\
64598_{3 \frac{1}{2}}-87385_{3 \frac{1}{2}}^{\circ}\end{array}$ & & & & & \\
\hline $\begin{array}{l}4385.97 \\
4385.66\end{array}$ & $\begin{array}{l}2 \\
3\end{array}$ & $\begin{array}{l}20 \\
10\end{array}$ & $\begin{array}{l}22793.57 \\
22795.18\end{array}$ & $\begin{array}{l}-0.07 \\
-0.03\end{array}$ & $\begin{array}{l}67416_{4 \frac{1}{2}}-90209_{4 \frac{1}{2}}^{\circ} \\
39378_{0 \frac{1}{2}}^{\circ}-62174_{1 \frac{1}{2}}^{\circ}\end{array}$ & & & & & \\
\hline
\end{tabular}


TABLE 6. Yb II-Observed and classified lines-Continued

\begin{tabular}{|c|c|c|c|c|c|c|c|c|c|c|}
\hline 1 & 2 & 3 & 4 & 5 & 6 & 7 & 8 & 9 & 10 & 11 \\
\hline \multirow{2}{*}{$\begin{array}{l}\text { W ave- } \\
\text { length } \\
\text { air }\end{array}$} & \multicolumn{2}{|c|}{ Intensity } & \multirow{2}{*}{$\begin{array}{c}\text { Wave } \\
\text { number }\end{array}$} & \multirow{2}{*}{$o-c$} & \multirow{2}{*}{ Classification } & \multirow{2}{*}{$\left|\begin{array}{l}\text { Zee- } \\
\text { man } \\
\text { type }\end{array}\right|$} & \multirow{2}{*}{ lst $g$} & \multirow{2}{*}{ 2nd $g$} & \multicolumn{2}{|c|}{$\begin{array}{c}\text { Zeeman } \\
\text { components }\end{array}$} \\
\hline & $\begin{array}{l}\text { Meggers } \\
\text { lamp }\end{array}$ & $\begin{array}{c}\text { Thomson } \\
\text { lamp }\end{array}$ & & & & & & & Strong $p$ & Strong $n$ \\
\hline A & & & $K$ & $K$ & & & & & & \\
\hline 4384.72 & & 2 & 22800.07 & -0.01 & $47228_{0 \frac{1}{2}}^{\circ}-70029_{0 \frac{1}{2}}$ & & & & & \\
\hline $\begin{array}{l}4383.93 \\
4383.11\end{array}$ & & 10 & 22804.18 & $\begin{array}{r}0.00 \\
-0.06\end{array}$ & $50832_{1 \frac{1}{2}}^{\circ}-73636_{0 \frac{1}{2}}$ & & & & & \\
\hline 4383.11 & & & 22808.44 & -0.06 & $54640_{4 \frac{1}{2}}^{\circ}-77449_{3 \frac{1}{2}}^{\circ}$ & & & & & \\
\hline 4383.02 & & 2 & 22808.91 & -0.01 & $40917_{+\frac{1}{2}}^{\circ}-63726_{4 \frac{1}{2}}$ & & & & & \\
\hline 4382.16 & & 1 & 22813.39 & +0.02 & $43075_{\frac{11}{2}}^{\circ}-65888_{\frac{1}{2}}^{\circ}$ & & & & & \\
\hline 4382.08 & & 2 & 22813.80 & -0.05 & $45335_{\frac{1}{2}}^{\circ}-68148_{5 \frac{1}{2}}^{2}$ & & & & & \\
\hline $\begin{array}{l}4381.80 \\
4379.50\end{array}$ & 20 & $\begin{array}{r}20 \\
300\end{array}$ & $\begin{array}{l}22815.26 \\
22827.24\end{array}$ & $\begin{array}{r}-0.04 \\
+0.04\end{array}$ & $\begin{array}{l}50468_{5 \frac{1}{2}}^{2}-73283_{\frac{1 \frac{1}{2}}{2}} \\
65875_{6 \frac{1}{2}}-88702^{\frac{1}{2}}\end{array}$ & & & & & \\
\hline 4377.80 & & 1 & 22836.11 & & 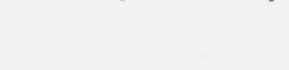 & & & & & \\
\hline 4376.851 & 1 & 40 & 22841.06 & -0.01 & $48272_{2 \frac{1}{2}}^{\circ}-71113_{1 \frac{1}{2}}$ & & & & & \\
\hline 4376.649 & & 2 & 22842.11 & & & & & & & \\
\hline 4374.861 & & 3 & 22851.45 & -0.04 & $58661_{2 \frac{1}{2}}^{\circ}-81512_{1 \frac{1}{2}}^{\circ}$ & & & & & \\
\hline 4372.854 & & 1 & 22861.94 & -0.04 & $45273_{3 \frac{1}{2}}^{\circ}-68135_{2 \frac{1}{2}}$ & & & & & \\
\hline 4372.65 & & 2 & 22863.00 & -0.05 & $67416_{4 \frac{1}{2}}-90279_{5 \frac{1}{2}}^{\circ}$ & & & & & \\
\hline 4372.560 & 2 & 30 & 22863.47 & -0.02 & $49916_{4 \frac{1}{2}}^{\circ}-72779_{3 \frac{1}{2}}^{\circ}$ & & & & & \\
\hline $\begin{array}{l}4371.083 \\
4371.05\end{array}$ & & $\begin{array}{l}3 \\
1\end{array}$ & $\begin{array}{l}22871.20 \\
22871.37\end{array}$ & -0.02 & $53644_{3 \frac{1}{2}}^{\circ}-76516_{4 \frac{1}{2}}$ & & & & & \\
\hline 4370.810 & 200 & 2000 & 22872.63 & -0.01 & $38342_{4 \frac{1}{2}}^{\circ}-61214_{4 \frac{1}{2}}$ & 6 & 1.10 & 1.07 & 0.15 & 1.08 \\
\hline 4368.89 & & 1 & 22882.68 & & & & & & & \\
\hline 4368.520 & & 1 & 22884.62 & -0.06 & $71113_{1 \frac{1}{2}}-9.3998_{1 \frac{1}{2}}^{\circ}$ & & & & & \\
\hline $\begin{array}{l}4365.948 \\
4364.056\end{array}$ & & $\begin{array}{l}2 \\
5\end{array}$ & $\begin{array}{l}22898.10 \\
22908.02\end{array}$ & -0.02 & $69395_{3 \frac{1}{2}}-92303_{3 \frac{1}{2}}^{\circ}$ & & & & & \\
\hline 4363.54 & & 1 & 22910.73 & +0.02 & $53.322_{7 \frac{1}{2}}^{\circ}-76233_{6 \frac{1}{2}}$ & & & & & \\
\hline 4363.280 & 6 & 90 & 22912.10 & -0.03 & $48.556_{3 \frac{1}{2}}^{\circ}-71468_{2 \frac{1}{2}}^{\circ}$ & & & & & \\
\hline 4362.333 & & 4 & 22917.07 & -0.07 & $66796_{3 \frac{1}{2}}^{\circ}-89713_{2 \frac{1}{2}}^{\circ}$ & & & & & \\
\hline 4362.156 & 1 & 6 & 22918.00 & -0.01 & $52987_{1 \frac{1}{2}}^{\circ}-75905_{0 \frac{1}{2}}^{\circ}$ & & & & & \\
\hline 4361.868 & & 2 & 22919.52 & -0.03 & $41678_{2 \frac{1}{2}}-64598_{3 \frac{1}{2}}$ & & & & & \\
\hline 4361.468 & 1 & 10 & 22921.62 & 0.00 & $52067_{0 \frac{1}{2}}^{-2}-74989_{0 \frac{1}{2}}^{2}$ & 7 & 0.50 & 0.50 & 0 & 0.500 \\
\hline $\begin{array}{l}4360.663 \\
4360.140\end{array}$ & 1 & $\begin{array}{l}4 \\
2\end{array}$ & $\begin{array}{l}22925.85 \\
22928.60\end{array}$ & -0.04 & $45012_{2 \frac{1}{2}}^{\circ}-67938_{3 \frac{1}{2}}$ & & & & & \\
\hline 4359.958 & & $\begin{array}{r}2 \\
20\end{array}$ & $\begin{array}{l}22928.00 \\
22929.56\end{array}$ & -0.01 & $48024_{1 \frac{1}{2}}^{\circ}-70954_{2 \frac{1}{2}}$ & & & & & \\
\hline $4359.165^{a}$ & 3 & 2 & 22933.73 & & $7001 \frac{1}{2}$ & & & & & \\
\hline 4356.160 & & 3 & 22949.55 & -0.04 & $48272_{2 \frac{1}{2}}^{\circ}-71222_{3 \frac{1}{2}}$ & & & & & \\
\hline 4355.58 & & 1 & 22952.60 & & & & & & & \\
\hline 4354.191 & 3 & 10 & 22959.93 & $\left\{\begin{array}{r}0.00 \\
+0.03\end{array}\right.$ & $\begin{array}{l}53716_{2 \frac{1}{2}}^{\circ} 76676_{2 \frac{1}{2}}^{\circ} \\
59439_{2 \frac{1}{2}}^{\circ}-82398_{1 \frac{1}{2}}^{\circ}\end{array}$ & & & & & \\
\hline $\begin{array}{l}4350.801 \\
4349.952\end{array}$ & & $\begin{array}{r}15 \\
2\end{array}$ & $\begin{array}{l}22977.81 \\
22982.30\end{array}$ & & $2 \frac{1}{2}-0201 \frac{1}{2}$ & & & & & \\
\hline 4348.087 & 3 & 15 & 22992.16 & -0.04 & $63234_{2 \frac{1}{2}}-86226_{1 \frac{1}{2}}^{\circ}$ & & & & & \\
\hline 4347.358 & & 3 & 22996.01 & +0.01 & $58661_{2 \frac{1}{2}}^{\circ}-81657_{2 \frac{1}{2}}$ & & & & & \\
\hline
\end{tabular}


TABLE 6. Yb II-Observed and classified lines-Continued

\begin{tabular}{|c|c|c|c|c|c|c|c|c|c|c|}
\hline 1 & 2 & 3 & 4 & 5 & 6 & 7 & 8 & 9 & 10 & 11 \\
\hline \multirow{2}{*}{$\begin{array}{l}\text { Wave- } \\
\text { length } \\
\text { air }\end{array}$} & \multicolumn{2}{|c|}{ Intensity } & \multirow{2}{*}{$\begin{array}{l}\text { Wave } \\
\text { number }\end{array}$} & \multirow[b]{2}{*}{$0-c$} & \multirow[b]{2}{*}{ Classification } & \multirow{2}{*}{ 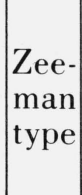 } & \multirow[b]{2}{*}{ lst $g$} & \multirow{2}{*}{2 nd $g$} & \multicolumn{2}{|c|}{$\begin{array}{c}\text { Zeeman } \\
\text { components }\end{array}$} \\
\hline & $\begin{array}{l}\text { Meggers } \\
\text { lamp }\end{array}$ & $\begin{array}{c}\text { Thomson } \\
\text { lamp }\end{array}$ & & & & & & & Strong $p$ & Strong $n$ \\
\hline A & & & K & $K$ & & & & & & \\
\hline $\begin{array}{l}4347.223 \\
4347.13 \\
4346.00 \\
4344.894\end{array}$ & 10 & $\begin{array}{r}20 \\
1 \\
1 \\
20\end{array}$ & $\begin{array}{l}22996.73 \\
22997.22 \\
23003.20 \\
23009.05\end{array}$ & $\begin{array}{l}-0.02 \\
-0.02 \\
+0.01 \\
+0.01\end{array}$ & $\begin{array}{l}24332_{2 \frac{1}{2}}-47329_{2 \frac{1}{2}}^{\circ} \\
48272_{2 \frac{1}{2}}^{\circ}-71270_{2 \frac{1}{2}}^{\circ} \\
61214_{4 \frac{1}{2}}-84217_{4 \frac{1}{2}}^{\circ} \\
70858_{4 \frac{1}{2}}-93867_{4 \frac{1}{2}}^{\circ}\end{array}$ & & & & & \\
\hline $\begin{array}{l}4344.204 \\
4343.845 \\
4342.753\end{array}$ & 60 & $\begin{array}{r}300 \\
5 \\
3\end{array}$ & $\begin{array}{l}23012.71 \\
23014.61 \\
23020.40\end{array}$ & $\begin{array}{l}-0.02 \\
-0.01 \\
+0.11\end{array}$ & $\begin{array}{l}30392_{1 \frac{1}{2}}^{\circ}-53404_{2 \frac{1}{2}} \\
44940_{1 \frac{1}{2}}^{\circ}-67955_{0 \frac{1}{2}} \\
45429_{3 \frac{1}{2}}^{\circ}-68450_{3 \frac{1}{2}}\end{array}$ & 4 & 1.320 & 1.034 & 0.143 & 0.605 \\
\hline $\begin{array}{l}4342.151 \\
4341.646\end{array}$ & 6 & $\begin{array}{l}80 \\
10\end{array}$ & $\begin{array}{l}23023.59 \\
23026.27\end{array}$ & $\begin{array}{r}+0.01 \\
0.00\end{array}$ & $\begin{array}{l}47005_{1 \frac{1}{2}}^{\circ}-70029_{0 \frac{1}{2}} \\
40917_{4 \frac{1}{2}}^{\circ}-63944_{5 \frac{1}{2}}\end{array}$ & 5 & 0.98 & 0.68 & 0.15 & 1.15 \\
\hline $\begin{array}{l}4340.673 \\
4340.269 \\
4340.147\end{array}$ & $\begin{array}{l}2 \\
1\end{array}$ & $\begin{array}{r}7 \\
1 \\
15\end{array}$ & $\begin{array}{l}23031.43 \\
23033.57 \\
23034.22\end{array}$ & $\begin{array}{r}+0.01 \\
0.00 \\
0.00\end{array}$ & $\begin{array}{l}53644_{3 \frac{1}{2}}^{\circ}-76676_{2 \frac{1}{2}} \\
71270_{2 \frac{1}{2}}-94303_{3}^{\circ} \\
66571_{5 \frac{1}{2}}-89605_{4 \frac{1}{2}}^{\circ}\end{array}$ & & & & & \\
\hline $\begin{array}{l}4339.082 \\
4337.235\end{array}$ & 150 & $\begin{array}{r}1000 \\
2\end{array}$ & $\begin{array}{l}23039.87 \\
23049.68\end{array}$ & $\begin{array}{l}-0.02 \\
+0.01\end{array}$ & $\begin{array}{l}40917_{4 \frac{1}{2}}^{\circ}-63957_{3 \frac{1}{2}}^{\circ} \\
53120_{2 \frac{1}{2}}^{\circ}-76170_{2 \frac{1}{2}}^{\circ}\end{array}$ & 4 & 0.977 & 1.147 & 0.085 & 0.383 \\
\hline $\begin{array}{l}4336.30 \\
4334.914 \\
4332.809 \\
4331.942 \\
4331.762\end{array}$ & 3 & $\begin{array}{l}1 \\
4 \\
4 \\
2 \\
3\end{array}$ & $\begin{array}{l}23054.65 \\
23062.02 \\
23073.23 \\
23077.85 \\
23078.81\end{array}$ & $\begin{array}{r}-0.04 \\
-0.03 \\
0.00\end{array}$ & $\begin{array}{l}70954_{2 \frac{1}{2}}-94008_{3 \frac{1}{2}}^{\circ} \\
70793_{5 \frac{1}{2}}-93867_{4 \frac{1}{2}}^{\circ} \\
69957_{4 \frac{1}{2}}-93036_{5 \frac{1}{2}}^{\circ}\end{array}$ & & & & & \\
\hline $\begin{array}{l}4331.318 \\
4331.032 \\
4329.818 \\
4329.654 \\
4327.853\end{array}$ & & $\begin{array}{r}1 \\
5 \\
15 \\
1 \\
9\end{array}$ & $\begin{array}{l}23081.17 \\
23082.69 \\
23 C^{\circ 9.17} \\
230 y 0.04 \\
23099.65\end{array}$ & $\begin{array}{r}-0.05 \\
0.00 \\
+0.03 \\
-0.07 \\
+0.01\end{array}$ & $\begin{array}{l}71222_{3 \frac{1}{2}}-94303_{3 \frac{1}{2}}^{\circ} \\
53716_{2 \frac{1}{2}}^{\circ}-76799_{1 \frac{1}{2}}^{\circ} \\
48024_{1 \frac{1}{2}}^{\circ}-71113_{1 \frac{1}{2}}^{\circ} \\
73291_{5 \frac{1}{2}}-96382_{5 \frac{1}{2}}^{\circ} \\
47758_{3 \frac{1}{2}}^{\circ}-70858_{4 \frac{1}{2}}^{\circ}\end{array}$ & & & & & \\
\hline $\begin{array}{l}4327.238 \\
4326.637 \\
4326.556 \\
4325.240 \\
4325.033\end{array}$ & 6 & $\begin{array}{r}2 \\
15 \\
9 \\
90 \\
2\end{array}$ & $\begin{array}{l}23102.93 \\
23106.14 \\
23106.57 \\
23113.60 \\
23114.71\end{array}$ & $\begin{array}{l}-0.01 \\
-0.01 \\
-0.01 \\
+0.04 \\
-0.02\end{array}$ & $\begin{array}{l}68135_{2 \frac{1}{2}}-91237_{3 \frac{1}{2}}^{\circ} \\
70136_{6 \frac{1}{2}}^{\circ}-93242_{5 \frac{1}{2}}^{\circ} \\
54640_{4 \frac{1}{2}}^{\circ}-77747_{4 \frac{1}{2}}^{\circ} \\
47680_{6 \frac{1}{2}}^{\circ}-70793_{5 \frac{1}{2}} \\
59777_{1 \frac{1}{2}}^{\circ}-82891_{2 \frac{1}{2}}\end{array}$ & & & & & \\
\hline $\begin{array}{l}4324.962 \\
4324.500 \\
4324.160 \\
4323.630 \\
4323.554\end{array}$ & 1 & $\begin{array}{l}1 \\
7 \\
1 \\
8 \\
5\end{array}$ & $\begin{array}{l}23115.09 \\
23117.56 \\
23119.38 \\
23122.21 \\
23122.62\end{array}$ & $\begin{array}{r}+0.01 \\
0.00 \\
-0.03 \\
0.00 \\
0.00\end{array}$ & $\begin{array}{l}45335_{4 \frac{1}{2}}^{\circ}-68450_{3 \frac{1}{2}} \\
57561_{6 \frac{1}{2}}^{\circ}-80679_{5 \frac{1}{2}}^{\circ} \\
45429_{3 \frac{1}{2}}^{\circ}-68549_{4 \frac{1}{2}}^{\circ} \\
45012_{2 \frac{1}{2}}^{\circ}-68135_{2 \frac{1}{2}}^{\circ} \\
53716_{2 \frac{1}{2}}^{\circ}-76839_{2 \frac{1}{2}}^{\circ}\end{array}$ & & & & & \\
\hline $\begin{array}{l}4322.851 \\
4322.56 \\
4322.53\end{array}$ & 2 & $\begin{array}{r}1 \\
30 \\
20\end{array}$ & $\begin{array}{l}23126.38 \\
23127.93 \\
23128.10\end{array}$ & $\begin{array}{l}+0.05 \\
-0.04 \\
+0.03\end{array}$ & $\begin{array}{l}46902_{0 \frac{1}{2}}^{\circ}-70029_{0 \frac{1}{2}} \\
40035_{3 \frac{1}{2}}^{\circ}-63163_{4 \frac{1}{2}} \\
56500^{\circ}-79628^{\circ}\end{array}$ & & & & & \\
\hline $\begin{array}{l}4322.230 \\
4320.723\end{array}$ & 200 & $\begin{array}{r}2000 \\
2\end{array}$ & $\begin{array}{l}23129.70 \\
23137.77\end{array}$ & $\begin{array}{l}+0.01 \\
-0.01\end{array}$ & $\begin{array}{l}35831_{5 \frac{1}{2}}^{\circ}-58961_{5 \frac{1}{2}}^{\circ} \\
45012_{2 \frac{1}{2}}^{\circ}-68150_{1 \frac{1}{2}}^{\circ}\end{array}$ & 6 & 1.22 & 0.98 & 1.30 & 1.11 \\
\hline 4319.556 & 1 & 9 & 23144.02 & +0.01 & $65577_{3 \frac{1}{2}}-88721_{3 \frac{1}{2}}^{\circ}$ & & & & & \\
\hline
\end{tabular}


TABLE 6. Yb II-Observed and classified lines-Continued

\begin{tabular}{|c|c|c|c|c|c|c|c|c|c|c|}
\hline 1 & 2 & 3 & 4 & 5 & 6 & 7 & 8 & 9 & 10 & 11 \\
\hline \multirow{2}{*}{$\begin{array}{l}\text { Wave- } \\
\text { length } \\
\text { air }\end{array}$} & \multicolumn{2}{|c|}{ Intensity } & \multirow{2}{*}{$\begin{array}{l}\text { Wave } \\
\text { number }\end{array}$} & \multirow{2}{*}{$o-c$} & \multirow{2}{*}{ Classification } & \multirow{2}{*}{ Zee- } & \multirow{2}{*}{ lst $g$} & \multirow{2}{*}{ 2nd $g$} & \multicolumn{2}{|c|}{$\begin{array}{c}\text { Zeeman } \\
\text { components }\end{array}$} \\
\hline & $\begin{array}{l}\text { Meggers } \\
\text { lamp }\end{array}$ & $\begin{array}{c}\text { Thomson } \\
\text { lamp }\end{array}$ & & & & & & & Strong $p$ & Strong $n$ \\
\hline $\begin{array}{c}\text { A } \\
4318.978 \\
4318.820 \\
4318.750 \\
4318.459\end{array}$ & 2 & $\begin{array}{r}1 \\
1 \\
2 \\
10\end{array}$ & $\begin{array}{c}K \\
23147.12 \\
23147.96 \\
23148.34 \\
23149.90\end{array}$ & $\begin{aligned} & K \\
&-0.01 \\
&+ 0.02 \\
& 0.00\end{aligned}$ & $\begin{array}{l}73750_{4 \frac{1}{2}}-96898_{5 \frac{1}{2}}^{\circ} \\
56480_{4 \frac{1}{2}}^{\circ}-79628_{3 \frac{1}{2}} \\
58162_{1 \frac{1}{2}}^{\circ}-81312_{2 \frac{1}{2}}^{\circ}\end{array}$ & & & & & \\
\hline $\begin{array}{l}4318.010 \\
4317.751 \\
4316.954 \\
4315.302 \\
4315.15\end{array}$ & $\begin{array}{r}1 \\
2000\end{array}$ & $\begin{array}{r}3 \\
15 \\
8000 \\
3 \\
1\end{array}$ & $\begin{array}{l}23152.31 \\
23153.69 \\
23157.97 \\
23166.83 \\
23167.65\end{array}$ & $\begin{array}{l}-0.07 \\
+0.05 \\
+0.04\end{array}$ & $\begin{array}{l}58051_{5 \frac{1}{2}}^{\circ}-81205_{4 \frac{1}{2}} \\
30562_{5 \frac{1}{2}}^{\circ}-53720_{4 \frac{1}{2}}^{\circ} \\
61051_{3 \frac{1}{2}}-84217_{4 \frac{1}{2}}^{\circ}\end{array}$ & 4 & 1.126 & 1.276 & 0.075 & 0.451 \\
\hline $\begin{array}{l}4310.230 \\
4310.124 \\
4309.886 \\
4309.308 \\
4308.586\end{array}$ & 1 & $\begin{array}{r}10 \\
20 \\
15 \\
4 \\
7\end{array}$ & $\begin{array}{l}23194.09 \\
23194.66 \\
23195.95 \\
23199.06 \\
23202.94\end{array}$ & $\begin{array}{r}-0.02 \\
0.00 \\
+0.02 \\
-0.03 \\
+0.03\end{array}$ & $\begin{array}{l}53644_{3 \frac{1}{2}}^{\circ}-76839_{2 \frac{1}{2}}^{\circ} \\
47663_{4 \frac{1}{2}}^{\circ}-70858_{4 \frac{1}{2}} \\
48272_{2 \frac{1}{2}}^{\circ}-71468_{2 \frac{1}{2}} \\
73283_{4 \frac{1}{2}}-96482_{4 \frac{1}{2}}^{\circ} \\
53120_{2 \frac{1}{2}}^{\circ}-76323_{3 \frac{1}{2}}^{\circ}\end{array}$ & & & & & \\
\hline $\begin{array}{l}4307.583 \\
4307.29 \\
4306.494 \\
4304.512 \\
4304.422\end{array}$ & 2 & $\begin{array}{r}4 \\
3 \\
80 \\
2 \\
2\end{array}$ & $\begin{array}{l}23208.35 \\
23209.93 \\
23214.22 \\
23224.90 \\
23225.39\end{array}$ & $\begin{array}{l}-0.03 \\
-0.03 \\
+0.02 \\
-0.01 \\
-0.03\end{array}$ & $\begin{array}{l}58484_{4 \frac{1}{2}}^{\circ}-81692_{3 \frac{1}{2}} \\
44940_{1 \frac{1}{2}}^{\circ}-68150_{1 \frac{1}{2}} \\
45335_{4 \frac{1}{2}}^{\circ}-68549_{4 \frac{1}{2}} \\
35059_{3 \frac{1}{2}}^{\circ}-58283_{2 \frac{1}{2}}^{\circ} \\
46169_{4 \frac{1}{2}}^{\circ}-69395_{3 \frac{1}{2}}^{\circ}\end{array}$ & & & & & \\
\hline $\begin{array}{l}4303.675 \\
4301.496 \\
4300.736 \\
4298.075 \\
4296.696\end{array}$ & 5 & $\begin{array}{r}2 \\
15 \\
4 \\
1 \\
3\end{array}$ & $\begin{array}{l}23229.42 \\
23241.19 \\
23245.30 \\
23259.69 \\
23267.15\end{array}$ & $\begin{array}{l}+0.01 \\
-0.03 \\
-0.01 \\
-0.03 \\
+0.01\end{array}$ & $\begin{array}{l}61214_{4 \frac{1}{2}}-84444_{3 \frac{1}{2}}^{\circ} \\
54640_{4 \frac{1}{2}}^{\circ}-77882_{3 \frac{1}{2}} \\
48024_{1 \frac{1}{2}}^{\circ}-71270_{2 \frac{1}{2}} \\
68756_{3 \frac{1}{2}}-92015_{4 \frac{1}{2}}^{\circ} \\
59624_{3 \frac{1}{2}}^{\circ}-82891_{2 \frac{1}{2}}\end{array}$ & & & & & \\
\hline $\begin{array}{l}4296.338 \\
4293.421 \\
4290.357 \\
4289.91 \\
4288.800\end{array}$ & & $\begin{array}{l}3 \\
8 \\
7 \\
1 \\
9\end{array}$ & $\begin{array}{l}23269.09 \\
23284.90 \\
23301.53 \\
23303.96 \\
23309.99\end{array}$ & $\begin{array}{r}0.00 \\
+0.02\end{array}$ & $\begin{array}{l}69957_{4 \frac{1}{2}}-93242_{5 \frac{1}{2}}^{\circ} \\
67416_{4 \frac{1}{2}}-90717_{4 \frac{1}{2}}^{\circ}\end{array}$ & & & & & \\
\hline $\begin{array}{l}4286.839 \\
4285.878 \\
4285.816 \\
4285.258 \\
4285.127\end{array}$ & $\begin{array}{l}1 \\
2\end{array}$ & $\begin{array}{r}7 \\
9 \\
1 \\
9 \\
15\end{array}$ & $\begin{array}{l}23320.65 \\
23325.88 \\
23326.22 \\
23329.25 \\
23329.97\end{array}$ & $\begin{array}{r}+0.02 \\
0.00 \\
+0.05\end{array}$ & $\begin{array}{l}43075_{1 \frac{1}{2}}^{\circ}-66395_{2 \frac{1}{2}} \\
45429_{3 \frac{1}{2}}^{\circ}-68756_{3 \frac{1}{2}}^{\circ} \\
64055_{2 \frac{1}{2}}-87385_{3 \frac{1}{2}}^{\circ}\end{array}$ & & & & & \\
\hline $\begin{array}{l}4284.942 \\
4283.453 \\
4282.808 \\
4281.972 \\
4281.584\end{array}$ & 20 & $\begin{array}{r}80 \\
1 \\
10 \\
50 \\
5\end{array}$ & $\begin{array}{l}23330.97 \\
23339.08 \\
23342.60 \\
23347.16 \\
23349.27\end{array}$ & $\begin{array}{r}0.00 \\
+0.01 \\
-0.04\end{array}$ & $\begin{array}{l}32371_{2 \frac{1}{3}}^{\circ}-55702_{1 \frac{1}{2}} \\
45737_{1 \frac{1}{2}}^{\circ}-69079_{2 \frac{1}{2}} \\
70954_{2 \frac{1}{2}}-94303_{3 \frac{1}{2}}^{\circ}\end{array}$ & & & & & - \\
\hline 4280.636 & & 2 & 23354.44 & -0.04 & $71468_{2 \frac{1}{2}}-94823_{1 \frac{1}{2}}^{\circ}$ & & & & & \\
\hline
\end{tabular}


TABLE 6. Yb II-Observed and classified lines-Continued

\begin{tabular}{|c|c|c|c|c|c|c|c|c|c|c|}
\hline 1 & 2 & 3 & 4 & 5 & 6 & 7 & 8 & 9 & 10 & 11 \\
\hline \multirow{2}{*}{$\begin{array}{l}\text { Wave- } \\
\text { length } \\
\text { air }\end{array}$} & \multicolumn{2}{|c|}{ Intensity } & \multirow{2}{*}{$\begin{array}{c}\text { Wave } \\
\text { number }\end{array}$} & \multirow{2}{*}{$\mathrm{o}-\mathrm{c}$} & \multirow{2}{*}{ Classification } & \multirow{2}{*}{ 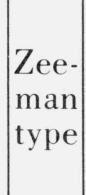 } & \multirow{2}{*}{ lst $g$} & \multirow{2}{*}{ 2nd $g$} & \multicolumn{2}{|c|}{$\begin{array}{c}\text { Zeeman } \\
\text { components }\end{array}$} \\
\hline & $\begin{array}{l}\text { Meggers } \\
\text { lamp }\end{array}$ & $\begin{array}{c}\text { Thomson } \\
\text { lamp }\end{array}$ & & & & & & & Strong $p$ & Strong $n$ \\
\hline$\AA$ & & & $K$ & $K$ & & & & & & \\
\hline 4280.594 & & 1 & 23354.67 & -0.02 & $57103_{3 \frac{1}{2}}^{\circ}-80457_{3 \frac{1}{2}}$ & & & & & \\
\hline 4277.092 & & 2 & 23373.79 & & & & & & & \\
\hline $\begin{array}{l}4276.78 \\
4273.340\end{array}$ & 20 & $\begin{array}{r}1 \\
150\end{array}$ & $\begin{array}{l}23375.50 \\
23394.32\end{array}$ & $\begin{array}{r}+0.05 \\
0.00\end{array}$ & $\begin{array}{l}49916_{1 \frac{1}{2}}^{\circ}-73291_{5 \frac{1}{2}} \\
32981_{1^{\frac{1}{2}}}-56375_{2 \frac{1}{2}}\end{array}$ & 5 & $(0.87)$ & 1.23 & 0.18 & $?$ \\
\hline 4271.963 & & 2 & 23401.86 & -0.01 & $52921_{3 \frac{1}{2}}^{\circ}-76323_{3 \frac{1}{2}}$ & & & & & \\
\hline 4270.515 & 4 & 60 & 23409.79 & +0.02 & $46547_{5 \frac{1}{2}}^{\circ}-69957_{4 \frac{1}{2}}^{\circ}$ & & & & & \\
\hline 4268.882 & & 1 & 23418.75 & -0.04 & $58672_{0 \frac{1}{2}}^{\circ}-82091_{0 \frac{1}{2}}^{\circ}$ & & & & & \\
\hline 4267.590 & 50 & 100 & 23425.84 & -0.01 & $24332_{2 \frac{1}{2}}-47758_{3 \frac{1}{2}}^{\circ}$ & & & & & \\
\hline 4266.952 & 10 & 100 & 23429.34 & -0.03 & $54640_{4 \frac{1}{2}}^{\circ}-78070_{4 \frac{1}{2}}^{\circ}$ & & & & & \\
\hline 4265.976 & & 2 & 23434.70 & +0.01 & $66462_{0 \frac{1}{2}}-89897_{1 \frac{1}{2}}^{\circ}$ & & & & & \\
\hline 4265.498 & & 1 & 23437.32 & 0.00 & $45012_{2 \frac{1}{2}}^{\sigma^{2}}-68450_{3 \frac{1}{2}}$ & & & & & \\
\hline 4264.802 & 1 & 30 & 23441.15 & -0.02 & $44497_{2 \frac{1}{2}}^{0^{2}}-67938_{3 \frac{1}{2}}$ & & & & & \\
\hline 4264.520 & & 1 & 23442.70 & -0.04 & $52880_{4 \frac{1}{2}}^{\circ}-76323_{3 \frac{1}{2}}^{\circ}$ & & & & & \\
\hline 4264.282 & & 5 & 23444.01 & +0.01 & $48024_{1 \frac{1}{2}}^{2}-71468_{2 \frac{1}{2}}$ & & & & & \\
\hline 4264.029 & & 6 & 23445.40 & 0.00 & $70858_{4 \frac{1}{2}}-94303_{3 \frac{1}{2}}^{\circ}$ & & & & & \\
\hline 4262.826 & 2 & 5 & 23452.02 & +0.05 & $68150_{1 \frac{1}{2}}-91602_{2 \frac{1}{2}}^{\circ}$ & & & & & \\
\hline 4262.691 & & 6 & 23452.76 & +0.04 & $59439_{2 \frac{1}{2}}^{\circ}-82891_{2 \frac{1}{2}}^{2}$ & & & & & \\
\hline 4262.273 & & 1 & 23455.06 & -0.01 & $43007_{0 \frac{1}{2}}^{0}-66462_{0 \frac{1}{2}}^{2}$ & & & & & \\
\hline 4260.678 & & 7 & 23463.84 & +0.02 & $47758_{3 \frac{1}{2}}^{\circ}-71222_{3 \frac{1}{2}}^{2}$ & & & & & \\
\hline 4259.17 & & 1 & 23472.15 & & & & & & & \\
\hline 4258.194 & 2 & 20 & 23477.53 & +0.01 & $70858_{4 \frac{1}{2}}-94335_{5 \frac{1}{2}}^{\circ}$ & & & & & \\
\hline $\begin{array}{l}4257.640 \\
4257.198\end{array}$ & 200 & $\begin{array}{r}2000 \\
6\end{array}$ & $\begin{array}{l}23480.58 \\
23483.02\end{array}$ & $\begin{array}{r}+0.02 \\
0.00\end{array}$ & $\begin{array}{l}38342_{4 \frac{1}{2}}^{\circ}-61822_{3 \frac{1}{2}}^{\circ} \\
45273^{\circ}-68756^{\circ}\end{array}$ & 4 & 1.10 & 1.34 & 0.12 & 0.26 \\
\hline 4255.765 & 500 & 2000 & 23490.93 & $\left\{\begin{array}{r}0.00 \\
+0.03\end{array}\right.$ & $\begin{array}{l}30224_{4 \frac{1}{2}}^{\circ}-53715_{3 \frac{1}{2}} \\
\left(48272_{2 \frac{1}{2}}^{\circ}-71763_{1 \frac{1}{2}}\right)\end{array}$ & 4 & $(0.94)$ & 1.19 & 0.125 & $?$ \\
\hline 4254.775 & 200 & 800 & 23496.39 & +0.01 & $30224_{4 \frac{1}{2}}^{\circ}-53720_{4 \frac{1}{2}}$ & 6 & 0.95 & 1.27 & 1.50 & 1.11 \\
\hline 4254.000 & & 9 & 23500.67 & +0.02 & $44438_{3 \frac{1}{2}}^{\circ}-67938_{3 \frac{1}{2}}^{2}$ & & & & & \\
\hline 4253.790 & & 1 & 23501.83 & $\left\{\begin{array}{r}0.00 \\
+0.01\end{array}\right.$ & $\begin{array}{l}66395_{\frac{1}{2}}-89897_{1 \frac{1}{2}}^{\circ} \\
70858_{4 \frac{1}{2}}-94360_{4 \frac{1}{2}}^{\circ}\end{array}$ & & & & & \\
\hline 4252.806 & & 3 & 23507.27 & -0.03 & $66205_{2 \frac{1}{2}}^{\circ}-89713_{2 \frac{1}{2}}$ & & & & & \\
\hline 4252.515 & 300 & 3000 & 23508.88 & +0.01 & $37077_{2 \frac{1}{2}}^{\circ}-60586_{1 \frac{1}{2}}$ & 4 & 1.113 & 1.326 & 0.107 & 0.794 \\
\hline 4252.040 & & 15 & 23511.50 & +0.03 & $47758_{3 \frac{1}{2}}^{\circ}-71270_{2 \frac{1}{2}}$ & & & & & \\
\hline 4250.21 & & 5 & 23521.63 & +0.05 & $65199_{4 \frac{1}{2}}-88721_{3 \frac{1}{2}}^{\circ}$ & & & & & \\
\hline 4249.861 & & 2 & 23523.56 & +0.01 & $66082_{\frac{1}{2}}^{2}-89605_{4 \frac{1}{2}}^{\circ}$ & & & & & \\
\hline 4249.81 & & 2 & 23523.84 & -0.06 & $69395_{3 \frac{1}{2}}-92919_{3 \frac{1}{2}}^{\circ}$ & & & & & \\
\hline 4249.748 & & 4 & 23524.18 & +0.01 & $66189_{1 \frac{1}{2}}^{\circ}-89713_{2 \frac{1}{2}}^{\circ}$ & & & & & \\
\hline 4248.12 & & 2 & 23533.20 & & & & & & & \\
\hline $\begin{array}{l}4247.885 \\
4247.04\end{array}$ & 40 & $\begin{array}{r}300 \\
2\end{array}$ & $\begin{array}{l}23534.50 \\
23539.18\end{array}$ & +0.03 & $37516_{3 \frac{1}{2}}^{\circ}-61051_{3 \frac{1}{2}}$ & 6 & 1.11 & 1.17 & 0.22 & 1.14 \\
\hline 4246.835 & & 10 & 23540.32 & 0.00 & $56088_{4 \frac{1}{2}}^{\circ}-79628_{3 \frac{1}{2}}$ & & & & & \\
\hline 4246.586 & 4 & 20 & 23541.70 & -0.04 & $70793_{5 \frac{1}{2}}-94335_{5 \frac{1}{2}}^{\circ}$ & & & & & \\
\hline
\end{tabular}


TABLE 6. Yb II-Observed and classified lines-Continued

\begin{tabular}{|c|c|c|c|c|c|c|c|c|c|c|}
\hline 1 & 2 & 3 & 4 & 5 & 6 & 7 & 8 & 9 & 10 & 11 \\
\hline \multirow{2}{*}{$\begin{array}{l}\text { Wave- } \\
\text { length } \\
\text { air }\end{array}$} & \multicolumn{2}{|c|}{ Intensity } & \multirow{2}{*}{$\begin{array}{c}\text { Wave } \\
\text { number }\end{array}$} & \multirow[b]{2}{*}{$\mathrm{o}-\mathrm{c}$} & \multirow[b]{2}{*}{ Classification } & \multirow{2}{*}{$\begin{array}{l}\text { Zee- } \\
\text { man } \\
\text { type }\end{array}$} & \multirow[b]{2}{*}{ lst $g$} & \multirow{2}{*}{ 2nd $g$} & \multicolumn{2}{|c|}{$\begin{array}{c}\text { Zeeman } \\
\text { components }\end{array}$} \\
\hline & $\begin{array}{l}\text { Meggers } \\
\text { lamp }\end{array}$ & $\begin{array}{c}\text { Thomson } \\
\text { lamp }\end{array}$ & & & & & & & Strong $p$ & Strong $n$ \\
\hline$\stackrel{\circ}{\AA}$ & \multirow{4}{*}{3} & & $K$ & $K$ & & \multirow{18}{*}{$5 ?$} & \multirow{18}{*}{1.11} & \multirow{18}{*}{ (1.07) } & \multirow{18}{*}{$w$} & \multirow{18}{*}{1.18} \\
\hline 4245.740 & & 15 & 23546.39 & $\left\{\begin{array}{l}+0.05 \\
-0.01\end{array}\right.$ & $\begin{array}{l}66351_{2 \frac{1}{2}}-89897_{1 \frac{1}{2}}^{\circ} \\
69957_{4 \frac{1}{2}}-93503_{4 \frac{1}{2}}^{\circ}\end{array}$ & & & & & \\
\hline $\begin{array}{l}4245.597 \\
4244.73\end{array}$ & & 2 & $\begin{array}{l}23547.18 \\
23551.99\end{array}$ & -0.02 & $68756_{3 \frac{1}{2}}-92303_{3 \frac{1}{2}}^{\circ}$ & & & & & \\
\hline 4244.51 & & $\begin{array}{l}1 \\
8\end{array}$ & $\begin{array}{l}25551.99 \\
23553.21\end{array}$ & +0.04 & $71270_{2 \frac{1}{2}}-94823_{1 \frac{1}{2}}^{\circ}$ & & & & & \\
\hline 4244.21 & 6 & 80 & 23554.88 & -0.01 & $54192_{5 \frac{1}{2}}^{\circ}-77747_{4 \frac{1}{2}}^{2}$ & & & & & \\
\hline 4244.054 & 6 & 15 & 23555.75 & +0.02 & $53120_{2 \frac{1}{2}}^{\circ}-76676_{2 \frac{1}{2}}$ & & & & & \\
\hline 4243.494 & & 9 & 23558.85 & +0.01 & $47663_{4 \frac{1}{2}}^{\circ}-71222_{3 \frac{1}{2}}$ & & & & & \\
\hline $\begin{array}{l}4242.425 \\
4242.299\end{array}$ & 4 & $\begin{array}{r}60 \\
4\end{array}$ & $\begin{array}{l}23564.79 \\
23565.49\end{array}$ & +0.03 & $49727_{6 \frac{1}{2}}^{\circ}-73291_{5 \frac{1}{2}}^{2}$ & & & & & \\
\hline 4241.67 & 2 & 6 & 23568.98 & & & & & & & \\
\hline 4241.24 & \multirow{4}{*}{1} & 2 & 23571.37 & & & & & & & \\
\hline 4241.159 & & 15 & 23571.82 & +0.02 & $56056_{2 \frac{1}{2}}^{\circ}-79628_{3 \frac{1}{2}}$ & & & & & \\
\hline 4241.116 & & $\begin{array}{l}5 \\
1\end{array}$ & $\begin{array}{l}23572.06 \\
23581.30\end{array}$ & 000 & $69395-09076^{\circ}$ & & & & & \\
\hline $\begin{array}{l}4239.455 \\
4238.84\end{array}$ & & $\begin{array}{l}1 \\
1\end{array}$ & $\begin{array}{l}23581.30 \\
23584.72\end{array}$ & $\begin{array}{r}0.00 \\
-0.02\end{array}$ & $\begin{array}{l}69393_{3 \frac{1}{2}}-92970_{3 \frac{1}{2}} \\
66558_{3 \frac{1}{2}}-90142_{3 \frac{1}{2}}^{\circ}\end{array}$ & & & & & \\
\hline 4238.345 & \multirow{3}{*}{2} & 1 & 23587.47 & -0.02 & $69607_{2 \frac{1}{2}}-93195_{3 \frac{1}{2}}^{\circ}$ & & & & & \\
\hline 4238.157 & & 30 & 23588.52 & 0.00 & $46547_{5 \frac{1}{2}}^{c^{2}}-70136_{6 \frac{1}{2}}$ & & & & & \\
\hline 4237.284 & & 3 & 23593.38 & -0.02 & $55221_{1 \frac{1}{2}}^{\circ}-78814_{2 \frac{1}{2}}^{\circ}$ & & & & & \\
\hline 4235.565 & 1 & 20 & 23602.95 & +0.01 & $46354_{4 \frac{1}{2}}^{\circ}-69957_{4 \frac{1}{2}}$ & & & & & \\
\hline 4235.012 & 1 & 20 & 23606.04 & -0.04 & $73291_{5 \frac{1}{2}}-96898_{5 \frac{1}{2}}^{\circ}$ & & & & & \\
\hline 4234.545 & 50 & 800 & 23608.64 & +0.02 & $45335_{4 \frac{1}{2}}^{\circ}-68943_{4 \frac{1}{2}}$ & \multirow[t]{3}{*}{6} & \multirow[t]{3}{*}{1.014} & \multirow[t]{3}{*}{0.870} & \multirow[t]{3}{*}{0.667} & \multirow[t]{3}{*}{0.942} \\
\hline 4232.427 & & 1 & 23620.45 & -0.03 & $49419_{0 \frac{1}{2}}^{\circ}-73039_{0 \frac{1}{2}}^{\circ}$ & & & & & \\
\hline 4231.648 & \multirow{3}{*}{200} & 6 & 23624.80 & -0.03 & $47329_{2 \frac{1}{2}}^{\circ}-70954_{2 \frac{1}{2}}^{2}$ & & & & & \\
\hline 4230.184 & & 400 & $\begin{array}{l}23632.98 \\
23625.70\end{array}$ & +0.02 & $39378_{0 \frac{1}{2}}^{\circ}-63011_{1 \frac{1}{2}}$ & \multirow[t]{2}{*}{4} & 1.865 & 0.775 & 0.545 & 0.230 \\
\hline & & & 25035. & & & & & & & \\
\hline 4229.271 & 3 & 30 & 23638.08 & -0.01 & $66571_{5 \frac{1}{2}}-90209_{4 \frac{1}{2}}^{\circ}$ & & & & & \\
\hline 4227.952 & 50 & 700 & 23645.45 & -0.01 & $48556_{3 \frac{1}{2}}^{\circ}-72202_{3 \frac{1}{2}}$ & 6 & 0.91 & 0.85 & 0.22 & 0.880 \\
\hline 4226.85 & & 2 & 23651.62 & -0.05 & $66558_{3 \frac{1}{2}}-90209_{4 \frac{1}{2}}^{\circ}$ & & & & & \\
\hline $\begin{array}{l}4226.595 \\
4226.273\end{array}$ & 1 & $\begin{array}{r}4 \\
20\end{array}$ & $\begin{array}{l}23653.05 \\
23654.85\end{array}$ & $\begin{array}{r}-0.01 \\
0.00\end{array}$ & $\begin{array}{l}44497_{2 \frac{1}{2}}^{\circ}-68150_{1 \frac{1}{2}}^{\circ} \\
65950_{4 \frac{1}{2}}-89605_{4 \frac{1}{2}}^{\circ}\end{array}$ & & & & & \\
\hline 4225.998 & & 8 & 23656.39 & -0.06 & $42915_{5}^{\circ}-66571_{5 \frac{1}{3}}$ & & & & & \\
\hline 4225.548 & 10 & 30 & 23658.91 & 0.00 & $63726_{4 \frac{1}{2}}-87385_{3 \frac{1}{2}}^{\circ}$ & & & & & \\
\hline 4224.196 & 20 & 150 & 23666.48 & -0.02 & $40035_{3 \frac{1}{2}}^{\circ}-63702_{2 \frac{1}{2}}^{2}$ & 4 & $(0.720)$ & 1.08 & 0.18 & \\
\hline 4224.064 & & 1 & 23667.22 & -0.07 & $62559_{2 \frac{1}{2}}-86226_{1 \frac{1}{2}}^{\circ}$ & & & & & \\
\hline 4223.466 & & 2 & 23670.57 & -0.06 & $45273_{3 \frac{1}{2}}^{\circ}-68943_{4 \frac{1}{2}}$ & & & & & \\
\hline 4222.486 & & 10 & 23676.06 & & & & & & & \\
\hline 4222.057 & & 4 & 23678.47 & -0.02 & $53120_{2 \frac{1}{2}}^{\circ}-76799_{1 \frac{1}{2}}^{\circ}$ & & & & & \\
\hline 4220.260 & & 1 & 23688.55 & 0.00 & $52987_{1 \frac{1}{2}}^{\circ}-76676_{2 \frac{1}{2}}$ & & & & & \\
\hline 4 i $\quad 646$ & & 10 & 23692.00 & -0.01 & $24332_{2 \frac{1}{2}}-48024_{1 \frac{1}{2}}^{\circ}$ & & & & & \\
\hline $42 l_{\text {e }} 565$ & 1000 & 5000 & 23698.07 & 0.00 & $37516_{3 \frac{1}{2}}^{\circ}-61214_{4 \frac{1}{2}}^{\circ}$ & 4 & 1.115 & 1.05 & $(0.035)$ & 0.80 \\
\hline
\end{tabular}


TABLE 6. Yb II-Observed and classified lines-Continued

\begin{tabular}{|c|c|c|c|c|c|c|c|c|c|c|}
\hline 1 & 2 & 3 & 4 & 5 & 6 & 7 & 8 & 9 & 10 & 11 \\
\hline \multirow{2}{*}{$\begin{array}{l}\text { Wave- } \\
\text { length } \\
\text { air }\end{array}$} & \multicolumn{2}{|c|}{ Intensity } & \multirow{2}{*}{$\begin{array}{c}\text { Wave } \\
\text { number }\end{array}$} & \multirow{2}{*}{$o-c$} & \multirow{2}{*}{ Classification } & \multirow{2}{*}{$\mid \begin{array}{l}\text { Zee- } \\
\text { man } \\
\text { type }\end{array}$} & \multirow{2}{*}{ lst $g$} & \multirow{2}{*}{ 2nd $g$} & \multicolumn{2}{|c|}{$\begin{array}{c}\text { Zeeman } \\
\text { components }\end{array}$} \\
\hline & $\begin{array}{c}\text { Meggers } \\
\text { lamp }\end{array}$ & $\begin{array}{c}\text { Thomson } \\
\text { lamp }\end{array}$ & & & & & & & Strong $p$ & Strong $n$ \\
\hline A & & & $K$ & $K$ & & & & & & \\
\hline 4217.79 & & 2 & 23702.42 & -0.03 & $72779_{3 \frac{1}{2}}-96482_{4 \frac{1}{3}}^{\circ}$ & & & & & \\
\hline 4217.69 & & 2 & 23702.98 & & 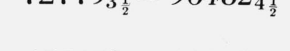 & & & & & \\
\hline 4217.110 & & 4 & 23706.24 & +0.01 & $45737_{1 \frac{1}{2}}^{\circ}-69443_{1 \frac{1}{2}}$ & & & & & \\
\hline 4216.885 & 6 & 60 & 23707.51 & +0.01 & $66571_{5 \frac{1}{2}}-90279_{5 \frac{1}{2}}^{\circ}$ & & & & & \\
\hline 4216.704 & 60 & 300 & 23708.53 & -0.01 & $34575_{1 \frac{1}{2}}^{\circ}-58283_{2 \frac{1}{2}}^{\circ}$ & 4 & 1.432 & 0.766 & 0.331 & -0.233 \\
\hline 4216.569 & & 2 & 23709.29 & -0.05 & $71113_{1 \frac{1}{2}}-94823_{1 \frac{1}{2}}^{\circ}$ & & & & & \\
\hline 4216.417 & & 8 & 23710.14 & -0.02 & $47758_{3 \frac{1}{2}}^{\circ}-71468_{2 \frac{1}{2}}^{\circ}$ & & & & & \\
\hline 4215.519 & 6 & 20 & 23715.19 & & & & & & & \\
\hline 4214.942 & 2 & 15 & 23718.44 & +0.02 & $53120_{2 \frac{1}{2}}^{\circ}-76839_{2 \frac{1}{2}}$ & & & & & \\
\hline 4213.856 & & 2 & 23724.55 & 0.00 & $51248_{2 \frac{1}{2}}^{\circ}-74973_{3 \frac{1}{2}}^{\circ}$ & & & & & \\
\hline 4213.407 & & 3 & 23727.08 & -0.05 & $65888_{1 \frac{1}{2}}-89615_{2 \frac{1}{2}}^{\circ}$ & & & & & \\
\hline 4212.361 & & 6 & 23732.97 & +0.03 & $53716_{2 \frac{1}{2}}^{\circ}-77449_{3 \frac{1}{2}}^{-2}$ & & & & & \\
\hline 4211.957 & & 1 & 23735.25 & & & & & & & \\
\hline 4211.502 & & 2 & 23737.81 & -0.04 & $58661_{2 \frac{1}{2}}^{\circ}-82398_{1 \frac{1}{2}}$ & & & & & \\
\hline 4211.298 & & 4 & 23738.96 & -0.01 & $48024_{1 \frac{1}{2}}^{\circ}-71763_{1 \frac{1}{2}}$ & & & & & \\
\hline 4210.664 & 4 & 40 & 23742.54 & +0.04 & $51248_{2 \frac{1}{2}}^{\circ}-74991_{1_{\frac{1}{2}}}$ & 7 & (1.034) & 1.05 & 0 & 1.01 \\
\hline 4209.870 & & 5 & 23747.01 & -0.01 & $66395_{2 \frac{1}{2}}^{2}-90142_{3 \frac{1}{2}}^{\circ \frac{1}{2}}$ & & & & & \\
\hline 4208.625 & & 4 & 23754.04 & +0.03 & $685499_{4 \frac{1}{2}}-92303_{3 \frac{1}{2}}^{\circ 0^{2}}$ & & & & & \\
\hline $\begin{array}{l}4208.519 \\
4207.65\end{array}$ & & 1 & 23754.64 & -0.05 & $52921_{3 \frac{1}{2}}^{\circ}-76676_{2 \frac{1}{2}}^{\circ}$ & & & & & \\
\hline & & 1 & 2569.54 & & & & & & & \\
\hline 4205.608 & & 4 & 23771.08 & +0.02 & $49008_{3 \frac{1}{2}}^{\circ}-72779_{3 \frac{1}{2}}^{\circ}$ & & & & & \\
\hline 4204.058 & & 5 & 23779.84 & -0.02 & $64923_{4 \frac{1}{2}}-88702_{5 \frac{1}{2}}^{\circ \frac{1}{2}}$ & & & & & \\
\hline 4203.254 & & 2 & 23784.39 & -0.01 & $47329_{2 \frac{1}{2}}^{\circ}-71113_{1 \frac{1}{2}}^{\circ}$ & & & & & \\
\hline 4204.666 & & 2 & 23787.72 & +0.01 & $46169_{4 \frac{1}{2}}^{\circ}-69957_{4 \frac{1}{2}}^{2}$ & & & & & \\
\hline 4202.44 & 15 & $30 h l$ & 23789.00 & & & & & & & \\
\hline 4201.991 & & 1 & 237.91 .54 & +0.01 & $66351_{2 \frac{1}{2}}-90142_{3 \frac{1}{2}}^{\circ}$ & & & & & \\
\hline 4201.460 & 2 & 20 & 23794.55 & -0.01 & $73291_{5 \frac{1}{2}}-97086_{4 \frac{1}{2}}^{\circ}$ & & & & & \\
\hline 4200.841 & & 5 & 23798.05 & +0.04 & $64923_{4 \frac{1}{2}}-88721_{3 \frac{1}{2}}^{\circ}$ & & & & & \\
\hline 4199.939 & & 4 & 23803.16 & 0.00 & $73283_{4 \frac{1}{2}}-97086_{4 \frac{1}{2}}^{\circ}$ & & & & & \\
\hline 4199.713 & & 10 & 23804.44 & +0.01 & $53644_{3 \frac{1}{2}}^{\circ}-77449_{3 \frac{1}{2}}^{\circ}$ & & & & & \\
\hline 4198.863 & & 1 & 23809.26 & +0.01 & $51248_{2 \frac{1}{2}}^{\circ}-75058_{2 \frac{1}{2}}$ & & & & & \\
\hline 4196.632 & 1 & 9 & 23821.92 & +0.01 & $67416_{4 \frac{1}{2}}^{2}-91237_{3 \frac{1}{2}}^{\circ}$ & & & & & \\
\hline 4193.807 & 2 & 10 & 23837.97 & +0.07 & $52067_{0 \frac{1}{2}}^{\circ}-75905_{0 \frac{1}{2}}^{\circ}$ & 6 & $(0.54)$ & 1.30 & 0.38 & \\
\hline 4193.533 & & 4 & 23839.52 & & & & & & & \\
\hline 4192.725 & & 3 & 23844.12 & & & & & & & \\
\hline 4191.473 & & 2 & 23851.24 & 0.00 & $52987_{1 \frac{1}{2}}^{\circ}-76839_{2 \frac{1}{2}}$ & & & & & \\
\hline 4190.297 & 200 & 2000 & 23857.93 & +0.03 & $37516_{3 \frac{1}{2}}^{\circ}-61374_{2 \frac{1}{2}}^{\circ}$ & 4 & 1.13 & 1.30 & 0.085 & 0.73 \\
\hline 4188.723 & & 7 & 23866.90 & +0.02 & $68148_{1 \frac{1}{2}}-92015_{4 \frac{1}{2}}^{\circ \frac{1}{2}}$ & & & & & \\
\hline 4188.371 & & 2 & 23868.90 & -0.01 & $70954_{2 \frac{1}{2}}^{2}-94823_{1 \frac{1}{2}}^{\circ \frac{2}{\circ}}$ & & & & & \\
\hline 4188.130 & 1 & 20 & 23870.28 & +0.03 & $45737_{1 \frac{1}{2}}^{\mathrm{o}^{2}}-69607_{\frac{1}{2}}^{2}$ & & & & & \\
\hline
\end{tabular}


TABLE 6. Yb II-Observed and classified lines-Continued

\begin{tabular}{|c|c|c|c|c|c|c|c|c|c|c|}
\hline 1 & 2 & 3 & 4 & 5 & 6 & 7 & 8 & 9 & 10 & 11 \\
\hline \multirow{2}{*}{$\begin{array}{l}\text { Wave- } \\
\text { length } \\
\text { air }\end{array}$} & \multicolumn{2}{|c|}{ Intensity } & \multirow{2}{*}{$\begin{array}{c}\text { Wave } \\
\text { number }\end{array}$} & \multirow[b]{2}{*}{$o-c$} & \multirow{2}{*}{ Classification } & \multirow{2}{*}{$\begin{array}{l}\text { Zee- } \\
\text { man } \\
\text { type }\end{array}$} & \multirow{2}{*}{ lst $g$} & \multirow{2}{*}{ 2nd $g$} & \multicolumn{2}{|c|}{$\begin{array}{c}\text { Zeeman } \\
\text { components }\end{array}$} \\
\hline & $\begin{array}{l}\text { Meggers } \\
\text { lamp }\end{array}$ & $\begin{array}{c}\text { Thomson } \\
\text { lamp }\end{array}$ & & & & & & & Strong $p$ & Strong $n$ \\
\hline A & & & $K$ & $K$ & & & & & & \\
\hline $\begin{array}{l}4186.83 \\
4186.027\end{array}$ & 15 & $\begin{array}{r}300 \\
2\end{array}$ & $\begin{array}{l}23877.69 \\
23882.27\end{array}$ & +0.01 & $54192_{5 \frac{1}{2}}^{\circ}-78070_{4 \frac{1}{2}}$ & 7 & $(1.11)$ & 1.11 & 0 & 1.11 \\
\hline 4185.565 & 2 & 30 & 23884.91 & +0.03 & $47228_{0 \frac{1}{2}}^{\circ}-71113_{1 \frac{1}{2}}$ & 5 & 0.74 & 1.00 & 0.13 & 1.13 \\
\hline $\begin{array}{l}4184.635 \\
4184.164\end{array}$ & 1 & $\begin{array}{r}20 \\
3\end{array}$ & $\begin{array}{l}23890.21 \\
23892.90\end{array}$ & $\begin{array}{l}+0.01 \\
-0.02\end{array}$ & $\begin{array}{l}53716_{2 \frac{1}{2}}^{\circ}-77606_{2 \frac{1}{2}}^{\circ} \\
47329_{2 \frac{1}{2}}^{\circ}-71222_{3 \frac{1}{2}}^{\circ}\end{array}$ & 7 & (1.183) & 1.12 & 0 & 1.15 \\
\hline $\begin{array}{l}4183.905 \\
4182.553\end{array}$ & & $\begin{array}{l}1 \\
2\end{array}$ & $\begin{array}{l}23894.38 \\
23902.11\end{array}$ & -0.03 & $57798_{2 \frac{1}{2}}^{\circ}-81692_{3 \frac{1}{2}}^{\circ}$ & & & & & \\
\hline 4180.809 & 10000 & 20000 & 23912.08 & +0.01 & $30392_{1 \frac{1}{2}}^{\circ}-54304_{0 \frac{1}{2}}$ & 4 & 1.33 & 2.01 & 0.34 & 0.99 \\
\hline 4179.084 & 10 & 80 & 23921.95 & -0.03 & $40035_{3 \frac{1}{2}}^{\circ}-63957_{3 \frac{1}{2}}^{2}$ & & & & & \\
\hline 4178.585 & 4 & 6 & 23924.80 & 0.00 & $59439_{2 \frac{1}{2}}^{\circ}-83363_{3 \frac{1}{2}}^{\circ}$ & & & & & \\
\hline 4178.225 & & 8 & 23926.86 & -0.04 & $68720_{6 \frac{1}{2}}-92647_{5 \frac{1}{2}}^{\circ}$ & & & & & \\
\hline 4177.81 & 4 & 80 & 23929.24 & -0.02 & $48272_{2 \frac{1}{2}}^{\circ}-72202_{3 \frac{1}{2}}^{\circ}$ & 4 & 1.14 & 0.86 & 0.144 & 0.149 \\
\hline $\begin{array}{l}4177.40 \\
4176204\end{array}$ & 2 & $\begin{array}{r}50 \\
3\end{array}$ & 23931.59 & & & & & & & \\
\hline $\begin{array}{l}4176.204 \\
4175.928\end{array}$ & 10 & $\begin{array}{r}3 \\
90\end{array}$ & $\begin{array}{l}23938.44 \\
23940.03\end{array}$ & $\begin{array}{l}-0.02 \\
-0.05\end{array}$ & $\begin{array}{l}69607_{2 \frac{1}{2}}-93546_{2 \frac{1}{2}}^{\circ} \\
24332_{2 \frac{1}{2}}^{\circ}-48272_{2 \frac{1}{2}}^{\circ}\end{array}$ & & & & & \\
\hline 4175.835 & & 30 & 23940.56 & -0.01 & $47329_{2 \frac{1}{2}}^{\circ}-71270_{2 \frac{1}{2}}$ & & & & & \\
\hline 4175.602 & 2 & 30 & 23941.89 & -0.02 & $22960_{1 \frac{1}{2}}-46902_{0 \frac{1}{2}}^{\circ}$ & 4 & 0.80 & 1.32 & 0.26 & 0.54 \\
\hline 4175.545 & 1 & 20 & 23942.22 & -0.02 & $35019_{4 \frac{1}{2}}^{\circ}-58961_{5 \frac{1}{2}}^{2}$ & & & & & \\
\hline $\begin{array}{l}4174.399 \\
4172.15\end{array}$ & & $\begin{array}{r}6 \\
20\end{array}$ & $\begin{array}{l}23948.79 \\
23961.70\end{array}$ & $\begin{array}{l}-0.02 \\
+0.01\end{array}$ & $\begin{array}{l}47005_{1 \frac{1}{2}}^{\circ}-70954_{2 \frac{1}{2}} \\
53644_{3 \frac{1}{2}}^{\circ}-77606_{2 \frac{1}{2}}^{\circ}\end{array}$ & 7 & (1.18) & 1.16 & 0 & 1.23 \\
\hline 4170.106 & 200 & 2000 & 23973.45 & -0.02 & $37077_{2 \frac{1}{3}}^{\circ}-61051_{3 \frac{1}{2}}$ & 5 & 1.09 & 1.15 & $(0.03)$ & 1.30 \\
\hline 4169.499 & 2 & 8 & 23976.94 & 0.00 & $56480_{4 \frac{1}{2}}^{\frac{2}{2}}-80457_{3 \frac{1}{2}}^{\frac{1}{2}}$ & & & & & \\
\hline 4165.692 & & 2 & 23998.85 & & & & & & & \\
\hline 4165.143 & & 6 & 24002.01 & & & & & & & \\
\hline 4164.660 & 5 & 50 & 24004.80 & -0.01 & $32371_{2 \frac{1}{2}}^{\circ}-56375_{2 \frac{1}{2}}$ & & & & & \\
\hline 4164.587 & & 15 & 24005.22 & +0.02 & $40917_{4 \frac{1}{2}}^{\circ}-64923_{4 \frac{1}{2}}$ & & & & & \\
\hline 4162.023 & & 5 & 24020.01 & +0.01 & $40035_{3 \frac{1}{2}}^{\circ}-64055_{\frac{21}{2}}^{\circ}$ & & & & & \\
\hline 4161.572 & & 1 & 24022.61 & & & & & & & \\
\hline 4160.299 & & 2 & 24029.96 & & & & & & & \\
\hline 4160.090 & 3 & 40 & 24031.17 & +0.04 & $35059_{3 \frac{1}{2}}^{\circ}-59090_{3 \frac{1}{2}}^{\circ}$ & & & & & \\
\hline 4157.755 & 100 & 200 & 24044.66 & 0.00 & $22960_{1 \frac{1}{2}}-47005_{1 \frac{1}{2}}^{\circ}$ & & & & & \\
\hline 4156.575 & 1 & 4 & 24051.49 & +0.02 & $69957_{4 \frac{1}{2}}-94008_{3 \frac{1}{2}}^{\circ}$ & & & & & \\
\hline 4156.444 & & 1 & 24052.25 & +0.04 & $62174_{1 \frac{1}{2}}-86226_{1 \frac{1}{2}}^{\circ \frac{1}{2}}$ & & & & & \\
\hline $\begin{array}{l}4153.88 \\
4153.758\end{array}$ & & $\begin{array}{l}6 \\
4\end{array}$ & $\begin{array}{l}24067.09 \\
24067.80\end{array}$ & $\begin{array}{r}0.00 \\
-0.01\end{array}$ & $\begin{array}{l}45012_{2 \frac{1}{2}}^{\circ}-69079 \cdot 9_{2 \frac{1}{2}} \\
69395^{\circ}-93463^{\circ}\end{array}$ & & & & & \\
\hline & & & 24006.00 & -0.01 & $09393_{3 \frac{1}{2}}-93403_{3 \frac{1}{2}}$ & & & & & \\
\hline $\begin{array}{l}4153.197 \\
415279\end{array}$ & & 7 & 24071.05 & +0.05 & $35019_{4 \frac{1}{2}}^{\circ}-59090_{3 \frac{1}{2}}^{\circ}$ & & & & & \\
\hline $\begin{array}{l}4152.19 \\
4148.440\end{array}$ & & $\begin{array}{l}3 \\
8\end{array}$ & $\begin{array}{l}24073.41 \\
24098.65\end{array}$ & & & & & & & \\
\hline 4147.774 & 1 & 30 & 24102.52 & $\left\{\begin{array}{l}+0.01 \\
+0.04\end{array}\right.$ & $53644_{3 \frac{1}{2}}^{\circ}-77747_{4 \frac{1}{2}}$ & & & & & \\
\hline 4146.984 & & 2 & 24107.11 & & $69443_{1 \frac{1}{2}}-93546_{2 \frac{1}{2}}^{\circ}$ & & & & & \\
\hline
\end{tabular}


TABLE 6. Yb II-Observed and classified lines-Continued

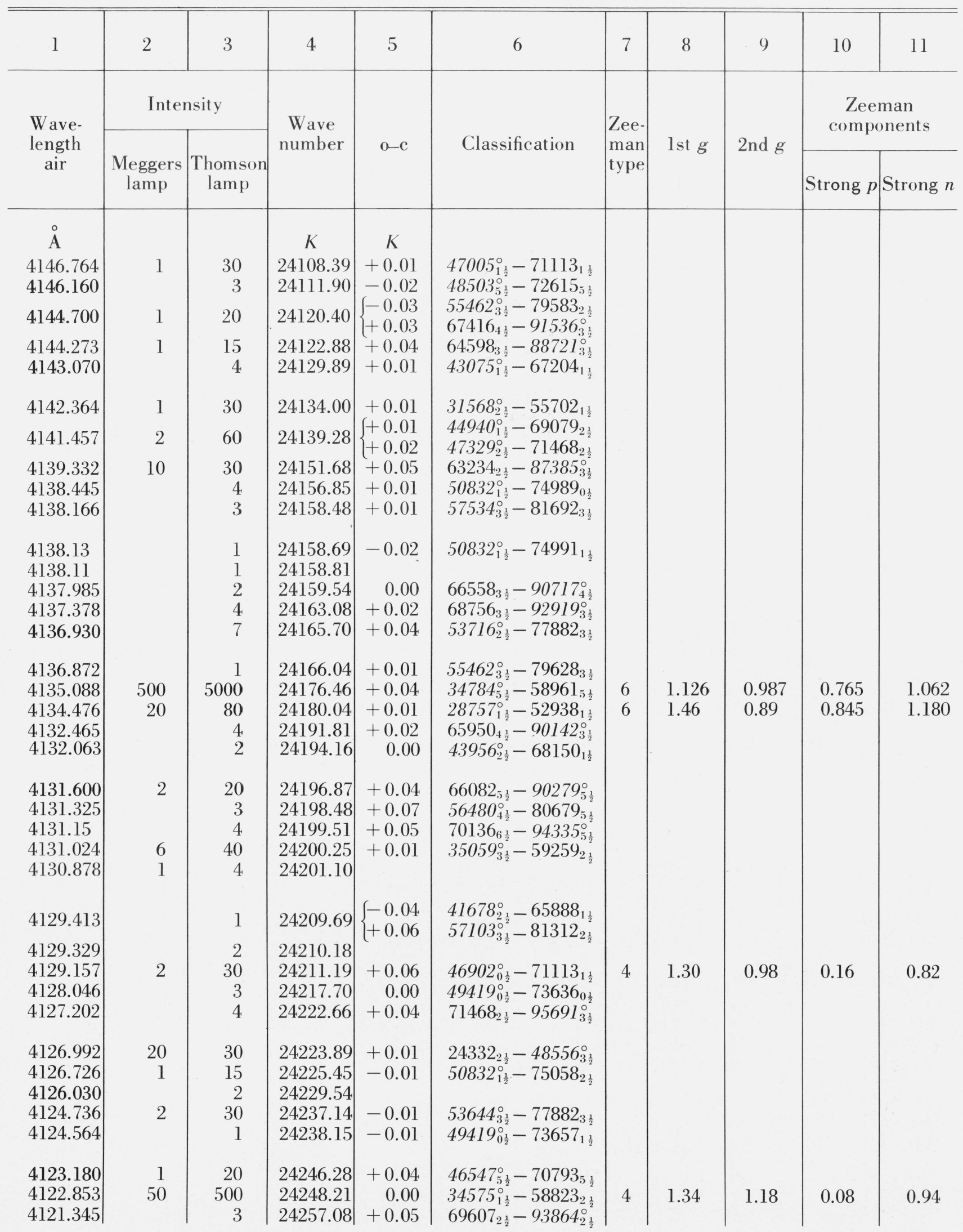


TABLE 6. Yb II-Observed and classified lines-Continued

\begin{tabular}{|c|c|c|c|c|c|c|c|c|c|c|}
\hline 1 & 2 & 3 & 4 & 5 & 6 & 7 & 8 & 9 & 10 & 11 \\
\hline \multirow{2}{*}{$\begin{array}{l}\text { Wave- } \\
\text { length } \\
\text { air }\end{array}$} & \multicolumn{2}{|c|}{ Intensity } & \multirow{2}{*}{$\begin{array}{l}\text { Wave } \\
\text { number }\end{array}$} & \multirow{2}{*}{$\mathrm{o}-\mathrm{c}$} & \multirow{2}{*}{ Classification } & \multirow{2}{*}{$\mid \begin{array}{l}\text { Zee- } \\
\text { man } \\
\text { type }\end{array}$} & \multirow{2}{*}{ lst $g$} & \multirow{2}{*}{2 nd $g$} & \multicolumn{2}{|c|}{$\begin{array}{c}\text { Zeeman } \\
\text { components }\end{array}$} \\
\hline & $\begin{array}{l}\text { Meggers } \\
\text { lamp }\end{array}$ & $\begin{array}{c}\text { Thomson } \\
\text { lamp }\end{array}$ & & & & & & & Strong $p$ & Strong $n$ \\
\hline A & & & $K$ & $K$ & & & & & & \\
\hline $\begin{array}{l}4120.071 \\
4119.812\end{array}$ & 1 & $\begin{array}{r}40 \\
2\end{array}$ & $\begin{array}{l}24264.58 \\
24266.11\end{array}$ & +0.03 & $47005_{1 \frac{1}{2}}^{\circ}-71270_{2 \frac{1}{2}}$ & & & & & \\
\hline $\begin{array}{l}4119.464 \\
4119.25 \\
4119.046 \\
4118.395 \\
4117.177\end{array}$ & $\begin{array}{r}200 \\
20 \\
3\end{array}$ & $\begin{array}{r}1500 \\
30 \\
60 \\
3 \\
30\end{array}$ & $\begin{array}{l}24268.16 \\
24269.42 \\
24270.62 \\
24274.46 \\
24281.64\end{array}$ & $\begin{array}{r}0.00 \\
-0.02 \\
+0.04 \\
+0.01\end{array}$ & $\begin{array}{l}22960_{1 \frac{1}{2}}-47228_{0 \frac{1}{2}}^{\circ} \\
33494_{3 \frac{1}{2}}^{\circ}-57765_{4 \frac{1}{2}} \\
49008_{3 \frac{1}{2}}^{\circ}-73283_{4 \frac{1}{2}} \\
40917_{4 \frac{1}{2}}^{\circ}-65199_{4 \frac{1}{2}}\end{array}$ & 5 & $0.76 ?$ & $0.56 ?$ & $0.10 w ?$ & 0.86 \\
\hline $\begin{array}{l}4116.654 \\
4115.454 \\
4114.591 \\
4113.879 \\
4113.707\end{array}$ & $\begin{array}{l}2 \\
1\end{array}$ & $\begin{array}{r}4 \\
9 \\
30 \\
20 \\
5\end{array}$ & $\begin{array}{l}24284.72 \\
24291.80 \\
24296.90 \\
24301.10 \\
24302.12\end{array}$ & $\begin{array}{r}+0.05 \\
0.00 \\
+0.03 \\
+0.04\end{array}$ & $\begin{array}{l}45737_{1 \frac{1}{2}}^{\circ}-70029_{0 \frac{1}{2}} \\
37077_{2 \frac{1}{2}}^{\circ}-61374_{2 \frac{1}{2}}^{\circ} \\
66558_{3 \frac{1}{2}}-90859_{2 \frac{1}{2}}^{\circ} \\
51248_{2 \frac{1}{2}}^{\circ}-75550_{2 \frac{1}{2}}^{\circ}\end{array}$ & & & & & \\
\hline $\begin{array}{l}4113.046 \\
4111.381 \\
4110.448 \\
4110.168 \\
4109.654\end{array}$ & 40 & $\begin{array}{r}400 \\
6 \\
2 \\
10 \\
400\end{array}$ & $\begin{array}{l}24306.02 \\
24315.87 \\
24321.39 \\
24323.04 \\
24326.08\end{array}$ & $\begin{array}{r}+0.03 \\
+0.01 \\
\\
0.00 \\
-0.01\end{array}$ & $\begin{array}{l}37516_{3 \frac{1}{2}}^{\circ}-61822_{3 \frac{1}{2}}^{\circ} \\
68720_{6 \frac{1}{2}}-93036_{5 \frac{1}{2}}^{\circ} \\
67955_{0 \frac{1}{2}}-92278_{1 \frac{1}{2}}^{\circ} \\
61822_{3 \frac{1}{2}}^{\circ}-86148_{2 \frac{1}{2}}^{\circ}\end{array}$ & 4 & 1.33 & 1.57 & 0.12 & 0.73 \\
\hline $\begin{array}{l}4109.306 \\
4108.845 \\
4106.284 \\
4106.070 \\
4103.416\end{array}$ & $\begin{array}{l}2 \\
5\end{array}$ & $\begin{array}{r}40 \\
15 \\
3 \\
5 \\
10\end{array}$ & $\begin{array}{l}24328.14 \\
24330.87 \\
24346.05 \\
24347.32 \\
24363.06\end{array}$ & $\begin{array}{l}\left\{\begin{array}{l}+0.01 \\
-0.05\end{array}\right. \\
+0.04 \\
-0.04\end{array}$ & $\begin{array}{l}65950_{4 \frac{1}{2}}-90279_{5 \frac{1}{2}}^{\circ} \\
71222_{3 \frac{1}{2}}-95550_{4 \frac{1}{2}}^{\circ} \\
65566_{0 \frac{1}{2}}-89897_{1 \frac{1}{2}}^{\circ} \\
69957_{4 \frac{1}{2}}-94303_{3 \frac{1}{2}}^{\circ}\end{array}$ & & & & & \\
\hline $\begin{array}{l}4103.113 \\
4102.478 \\
4102.264 \\
4100.877 \\
4100.160\end{array}$ & 20 & $\begin{array}{r}2 \\
20 \\
5 \\
1 \\
8\end{array}$ & $\begin{array}{l}24364.86 \\
24368.64 \\
24369.91 \\
24378.15 \\
24382.41\end{array}$ & $\begin{array}{r}-0.06 \\
0.00 \\
+0.04 \\
-0.06 \\
0.00\end{array}$ & $\begin{array}{l}68549_{4 \frac{1}{2}}-92914_{4 \frac{1}{2}}^{\circ} \\
22960_{\frac{1}{2}}^{\circ}-47329_{2 \frac{1}{2}}^{\circ} \\
68549_{\frac{1}{2}}-92919_{3 \frac{1}{2}}^{\circ}-94335_{5 \frac{1}{2}}^{\circ} \\
69957_{4 \frac{1}{2}}-9435_{3 \frac{1}{2}}^{\circ} \\
45012_{2 \frac{1}{2}}^{\circ}-6939\end{array}$ & & & & & \\
\hline $\begin{array}{l}4099.820 \\
4099.482 \\
4098.848 \\
4098.096 \\
4097.885\end{array}$ & 100 & $\begin{array}{r}2 \\
3 \\
2 \\
2 \\
600\end{array}$ & $\begin{array}{l}24384.43 \\
24386.44 \\
24390.22 \\
24394.69 \\
24395.95\end{array}$ & $\begin{array}{l}-0.06 \\
-0.06\end{array}$ & $55221_{1 \frac{1}{2}}^{\circ}-79605_{1 \frac{1}{2}}^{\circ}$ & 5 & 1.34 & 1.24 & 0.05 & 1.59 \\
\hline $\begin{array}{l}4096.968 \\
4096.785 \\
4096.644 \\
4096.156 \\
4094.351\end{array}$ & $\begin{array}{l}2 \\
1\end{array}$ & $\begin{array}{c}1 \\
3 \\
20 \\
15 \\
2\end{array}$ & $\begin{array}{l}24401.41 \\
24402.50 \\
24403.34 \\
24406.24 \\
24417.00\end{array}$ & $\begin{array}{r}-0.01 \\
-0.01 \\
+0.03 \\
-0.02\end{array}$ & $\begin{array}{l}69607_{2 \frac{1}{2}}-94008_{3 \frac{1}{2}}^{\circ} \\
69957_{4 \frac{1}{2}}-94360_{4 \frac{1}{2}}^{\circ} \\
65875_{6 \frac{1}{2}}-90279_{\frac{51}{2}}^{\circ} \\
65199_{4 \frac{1}{2}}-89605_{4 \frac{1}{2}}^{\circ}\end{array}$ & & & & & \\
\hline 4093.634 & 2 & 20 & 24421.28 & -0.03 & $71270_{2 \frac{1}{2}}-95691_{3 \frac{1}{2}}^{\circ}$ & & & & & \\
\hline
\end{tabular}


TABLE 6. Yb II-Observed and classified lines-Continued

\begin{tabular}{|c|c|c|c|c|c|c|c|c|c|c|}
\hline 1 & 2 & 3 & 4 & 5 & 6 & 7 & 8 & 9 & 10 & 11 \\
\hline \multirow{2}{*}{$\begin{array}{l}\text { Wave- } \\
\text { length } \\
\text { air }\end{array}$} & \multicolumn{2}{|c|}{ Intensity } & \multirow{2}{*}{$\begin{array}{l}\text { Wave } \\
\text { number }\end{array}$} & \multirow[b]{2}{*}{$\mathrm{o}-\mathrm{c}$} & \multirow{2}{*}{ Classification } & \multirow{2}{*}{$\begin{array}{l}\text { Zee- } \\
\text { man } \\
\text { type }\end{array}$} & \multirow{2}{*}{ lst $g$} & \multirow{2}{*}{2 nd $g$} & \multicolumn{2}{|c|}{$\begin{array}{c}\text { Zeeman } \\
\text { components }\end{array}$} \\
\hline & $\begin{array}{l}\text { Meggers } \\
\text { lamp }\end{array}$ & $\begin{array}{c}\text { Thomson } \\
\text { lamp }\end{array}$ & & & & & & & Strong $p$ & Strong $n$ \\
\hline A & & & $K$ & $K$ & & & & & & \\
\hline 4092.959 & & 10 & 24425.31 & +0.01 & $53644_{3 \frac{1}{2}}^{\circ}-78070_{4 \frac{1}{2}}$ & & & & & \\
\hline 4092.052 & 1 & 4 & 24430.72 & -0.01 & $45012_{2 \frac{1}{2}}^{\circ}-69443_{1 \frac{1}{2}}$ & & & & & \\
\hline 4091.53 & 6 & 100 & 24433.84 & +0.02 & $34389_{2 \frac{1}{2}}^{\circ}-58823_{2 \frac{1}{2}}$ & & & & & \\
\hline 4091.466 & 2 & 40 & 24434.22 & -0.01 & $47329_{2 \frac{1}{2}}^{\circ}-71763_{1 \frac{1}{2}}$ & 4 & 1.01 & 1.12 & 0.053 & 0.850 \\
\hline 4090.594 & & $\begin{array}{l}3 \\
2\end{array}$ & 24439.43 & +0.02 & $46354_{4 \frac{1}{2}}^{\circ}-70793_{5 \frac{1}{2}}$ & & & & & \\
\hline $\begin{array}{l}4088.633 \\
4088.528\end{array}$ & & $\begin{array}{l}2 \\
1\end{array}$ & $\begin{array}{l}24451.15 \\
24451.78\end{array}$ & & & & & & & \\
\hline 4086.61 & 7 & 100 & 24463.25 & +0.01 & $47005_{\frac{1}{2}}^{\circ}-71468_{2 \frac{1}{2}}$ & 7 & $(0.990)$ & 1.01 & 0 & 1.03 \\
\hline 4085.66 & 1 & 8 & 24468.94 & $\left\{\begin{array}{l}-0.05 \\
-0.02\end{array}\right.$ & $\begin{array}{l}68450_{3 \frac{1}{2}}-92919_{3 \frac{1}{2}}^{\circ} \\
71222_{3 \frac{1}{2}}-95691_{3 \frac{1}{2}}^{\circ}\end{array}$ & & & & & \\
\hline 4085.05 & & 3 & 24472.60 & -0.01 & $56840_{1 \frac{1}{2}}^{\circ}-81312_{2 \frac{1}{2}}$ & & & & & \\
\hline 4084.845 & & 3 & 24473.82 & & & & & & & \\
\hline 4084.515 & & 1 & 24475.80 & & & & & & & \\
\hline 4083.870 & & 1 & 24479.67 & & $53120^{\circ}-77606$ & & & & & \\
\hline 4082.814 & & 4 & 24486.00 & $\left\{\begin{array}{r}0.00 \\
-0.01\end{array}\right.$ & $\begin{array}{l}53120_{2 \frac{1}{2}}^{\circ}-77606_{2 \frac{1}{2}} \\
54640_{4 \frac{1}{2}}^{\circ}-79126_{4 \frac{1}{2}}\end{array}$ & & & & & \\
\hline 4082.64 & & 1 & 24487.04 & -0.03 & $68549_{4 \frac{1}{2}}-93036_{5 \frac{1}{2}}^{\circ}$ & & & & & \\
\hline $\begin{array}{l}4082.529 \\
408105\end{array}$ & & 1 & $\begin{array}{l}24487.71 \\
24496.58\end{array}$ & & & & & & & \\
\hline $\begin{array}{l}4081.05 \\
4079.991\end{array}$ & 2 & $\begin{array}{r}1 \\
40\end{array}$ & $\begin{array}{l}24496.58 \\
24502.94\end{array}$ & +0.03 & $44940_{1^{\frac{1}{2}}}^{\circ}-69443_{1 \frac{1}{2}}$ & 6 & 1.24 & 1.14 & 0.14 & 1.19 \\
\hline 4079.88 & & 6 & 24503.61 & -0.02 & $46354_{4 \frac{1}{2}}^{\circ}-70858_{4 \frac{1}{2}}$ & & & & & \\
\hline 4079.542 & & 3 & 24505.64 & +0.02 & $44438_{3 \frac{1}{2}}^{\circ}-68943_{4 \frac{1}{2}}$ & & & & & \\
\hline 4079.164 & 2 & 20 & 24507.91 & +0.05 & $66351_{2 \frac{1}{2}}-90859_{2 \frac{1}{2}}^{\circ}$ & & & & & \\
\hline 4078.36 & 7 & 70 & 24512.74 & +0.08 & $65093_{3 \frac{1}{2}}-89605_{4 \frac{1}{2}}^{\circ-2}$ & 4 & $(0.973)$ & 0.94 & $w$ & 0.830 \\
\hline 4077.713 & 10 & 20 & 24516.63 & & & & & & & \\
\hline 4077.276 & 200 & 2000 & 24519.26 & $\left\{\begin{array}{l}+0.01 \\
-0.10\end{array}\right.$ & $\begin{array}{c}38342_{4 \frac{1}{2}}^{\circ}-62861_{5 \frac{1}{2}}^{\circ} \\
\left(68943_{4 \frac{1}{2}}-93463_{3 \frac{1}{2}}^{\circ}\right)\end{array}$ & 5 & 1.06 & 1.10 & $(0.022)$ & 1.30 \\
\hline 4076.82 & & 1 & 24522.00 & +0.05 & $68720_{6 \frac{1}{2}}-93242_{5 \frac{1}{2}}^{\circ}$ & & & & & \\
\hline 4076.198 & & 3 & 24525.74 & -0.01 & $68720_{6 \frac{1}{2}}-93246_{6 \frac{1}{2}}^{\circ}$ & & & & & \\
\hline 4076.09 & & 3 & 24526.39 & 0.00 & $68450_{3 \frac{1}{2}}-92976_{3 \frac{1}{2}}^{\circ}$ & & & & & \\
\hline 4075.873 & & 3 & 24527.70 & $\left\{\begin{array}{r}+0.03 \\
0.00\end{array}\right.$ & $\begin{array}{l}45429_{3 \frac{1}{2}}^{\circ}-69957_{4 \frac{1}{2}} \\
52921_{3 \frac{1}{2}}^{\circ}-77449_{31}\end{array}$ & & & & & \\
\hline 4074.705 & 8 & 60 & 24534.73 & +0.02 & $47228_{0 \frac{1}{2}}^{\circ}-71763_{1 \frac{1}{2}}$ & 5 & 0.698 & 1.098 & 0.200 & 1.298 \\
\hline 4074.074 & & 1 & 24538.53 & +0.02 & $47663_{4 \frac{1}{2}}^{\circ}-72202_{3 \frac{1}{2}}$ & & & & & \\
\hline 4071.442 & & 1 & 24554.39 & & & & & & & \\
\hline 4071.334 & & 3 & 24555.04 & +0.004 & $69443_{1 \frac{1}{2}}-93998_{1 \frac{1}{2}}^{\circ}$ & & & & & \\
\hline 4070.527 & 8 & 50 & 24559.91 & +0.01 & $35059_{3 \frac{1}{2}}^{\circ}-59618_{3 \frac{1}{2}}^{\circ}$ & & & & & \\
\hline 4069.013 & & 4 & 24569.05 & & & & & & & \\
\hline 4068.50 & & 2 & 24572.14 & & & & & & & \\
\hline 4068.229 & & 2 & 24573.78 & & & & & & & \\
\hline 4067.031 & & 2 & 24581.02 & & & & & & & \\
\hline
\end{tabular}


TABLE 6. Yb II-Observed and classified lines-Continued

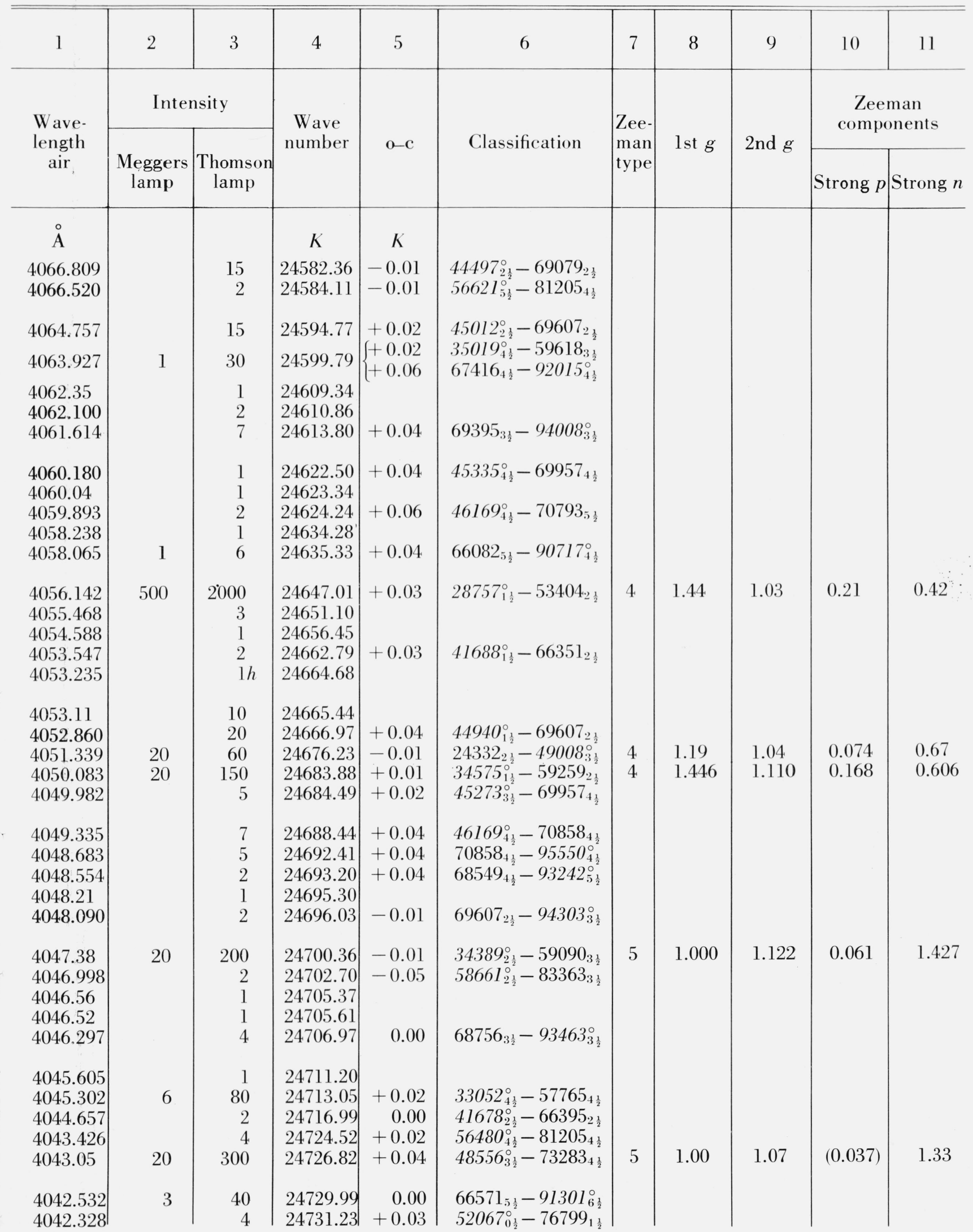


TABLE 6. Yb II-Observed and classified lines-Continued

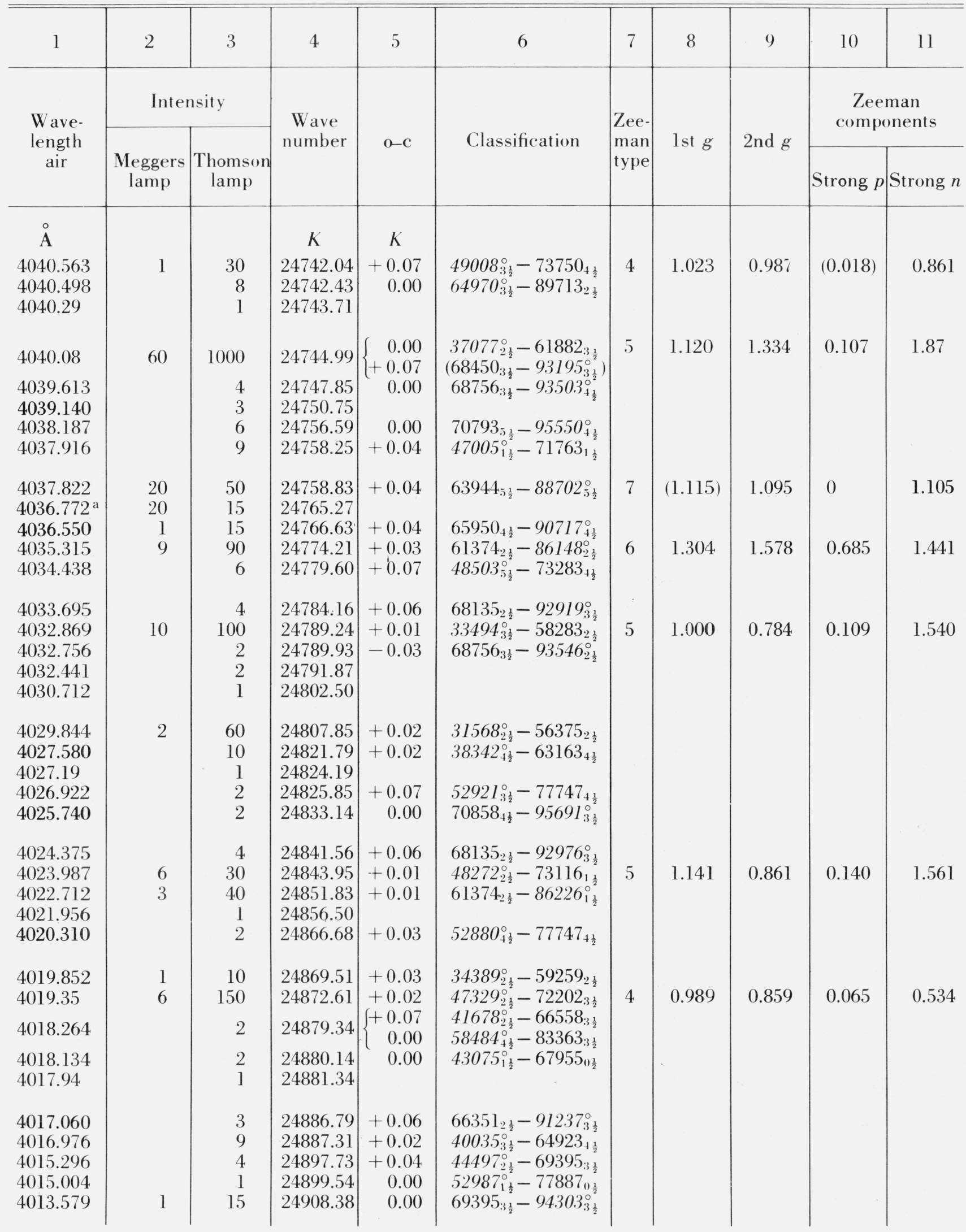


TABLE 6. Yb II-Observed and classified lines-Continued

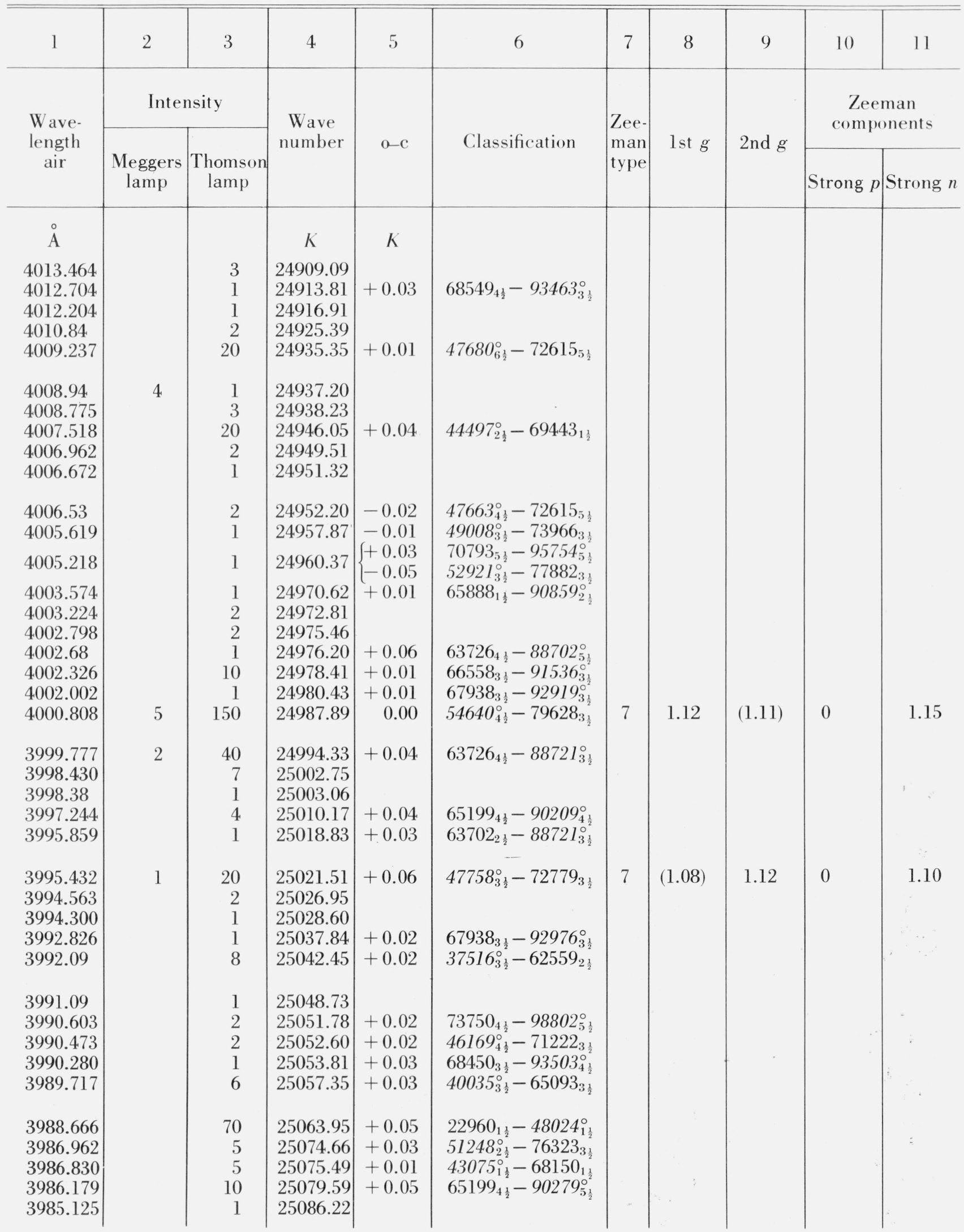


TABLE 6. Yb II-Observed and classified lines-Continued

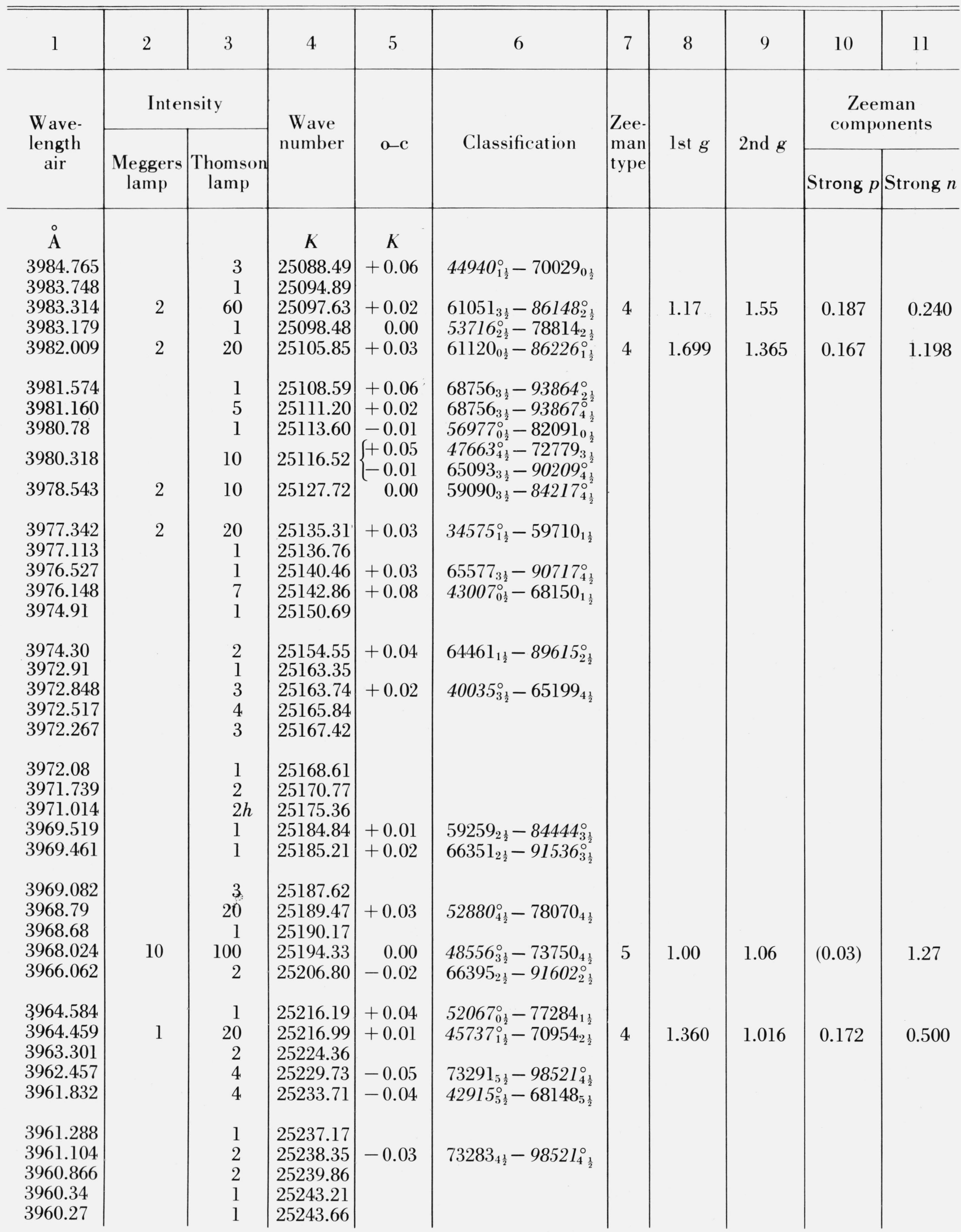


TABLE 6. Yb II-Observed and classified lines-Continued

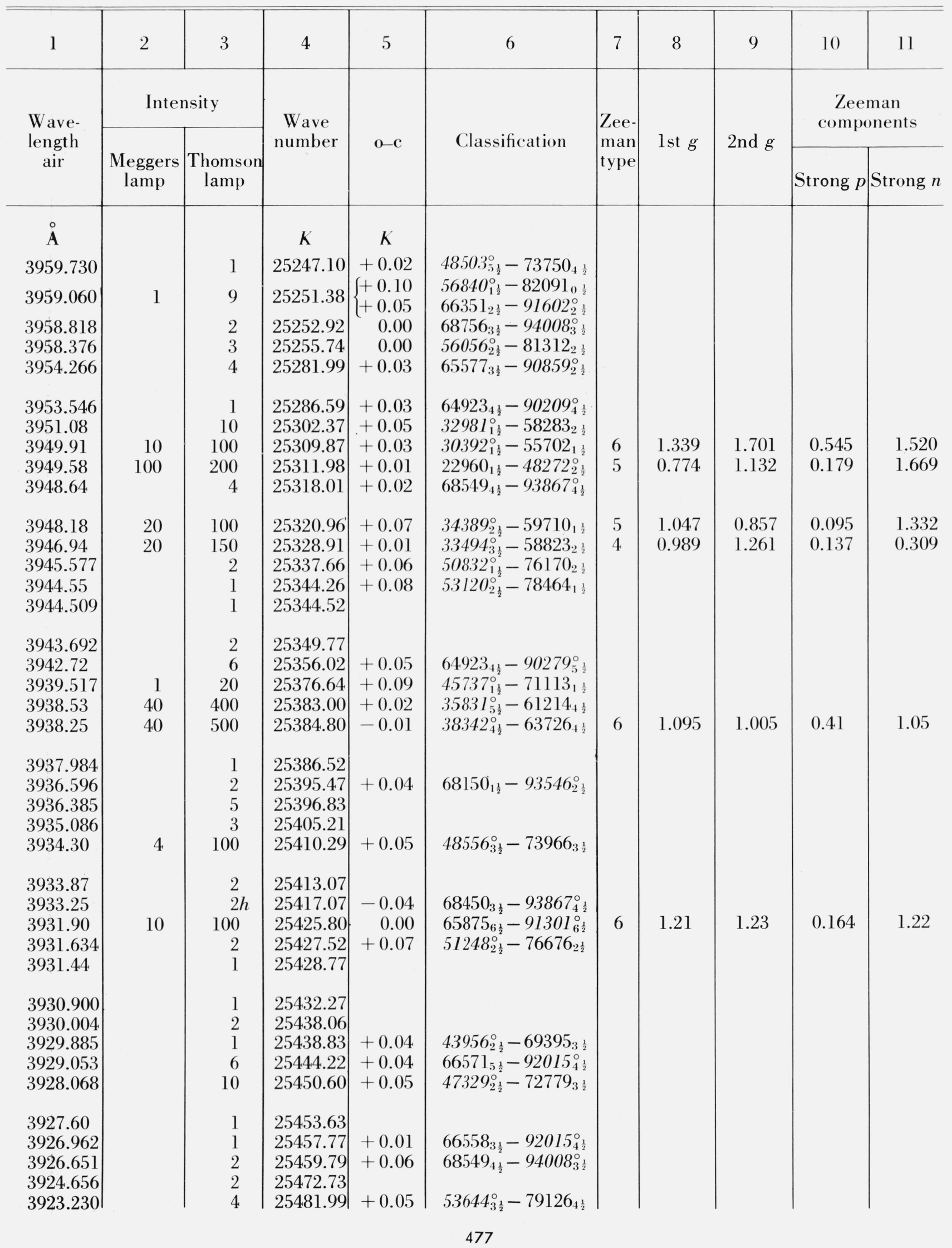


TABLE 6. Yb II-Observed and classified lines-Continued

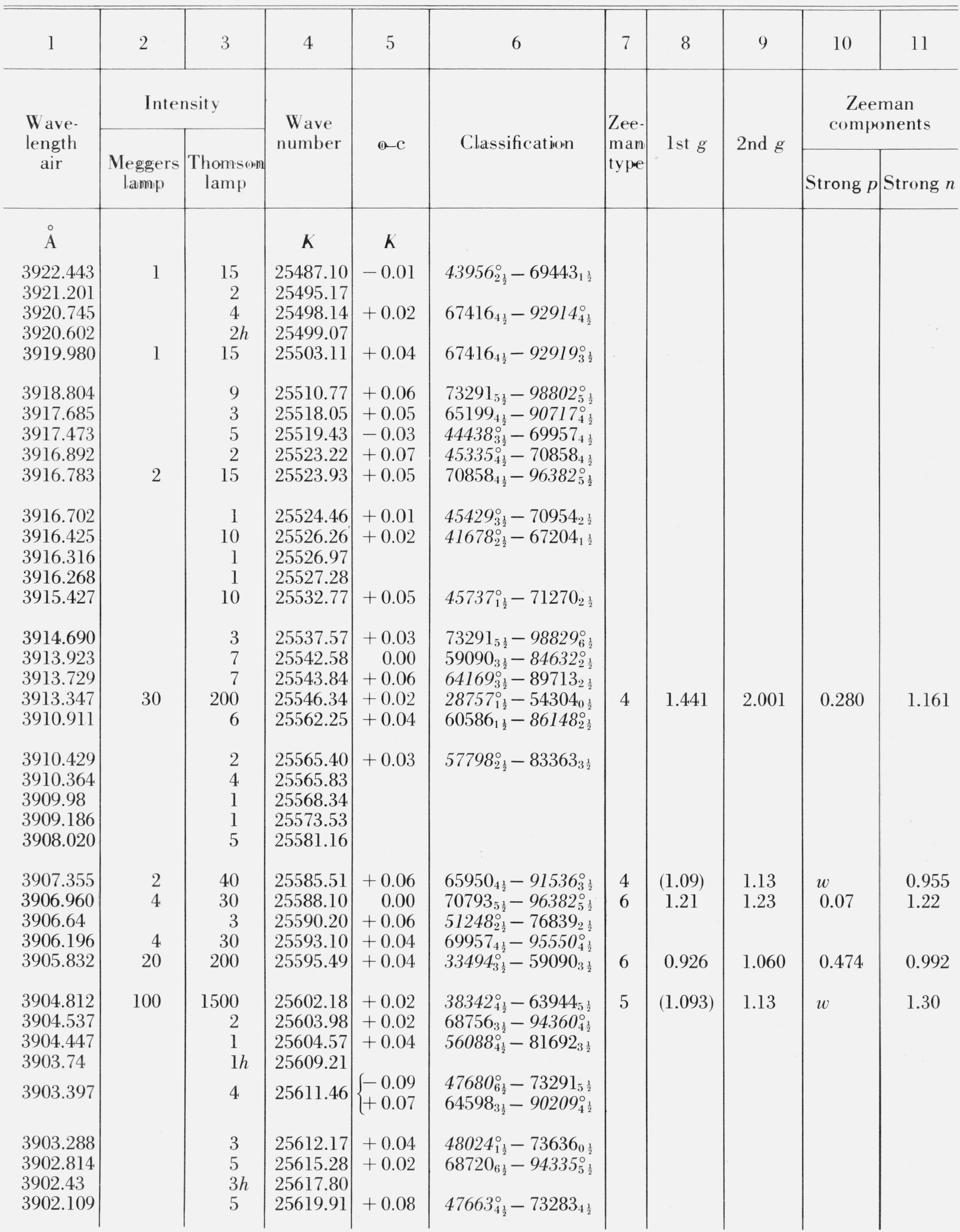


TABle 6. Yb II-Observed and classified lines-Continued

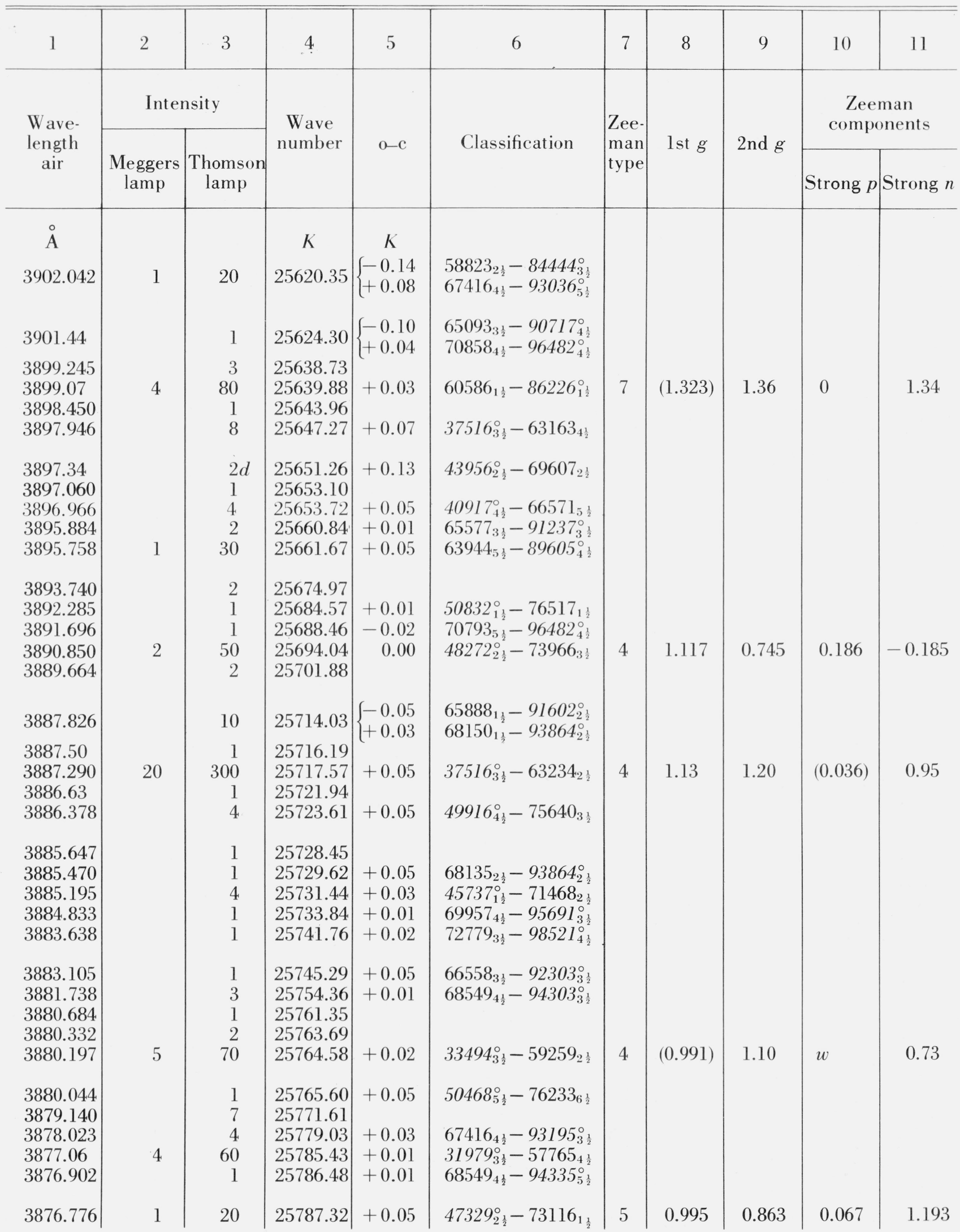


TABLE 6. Yb II-Observed and classified lines-Continued

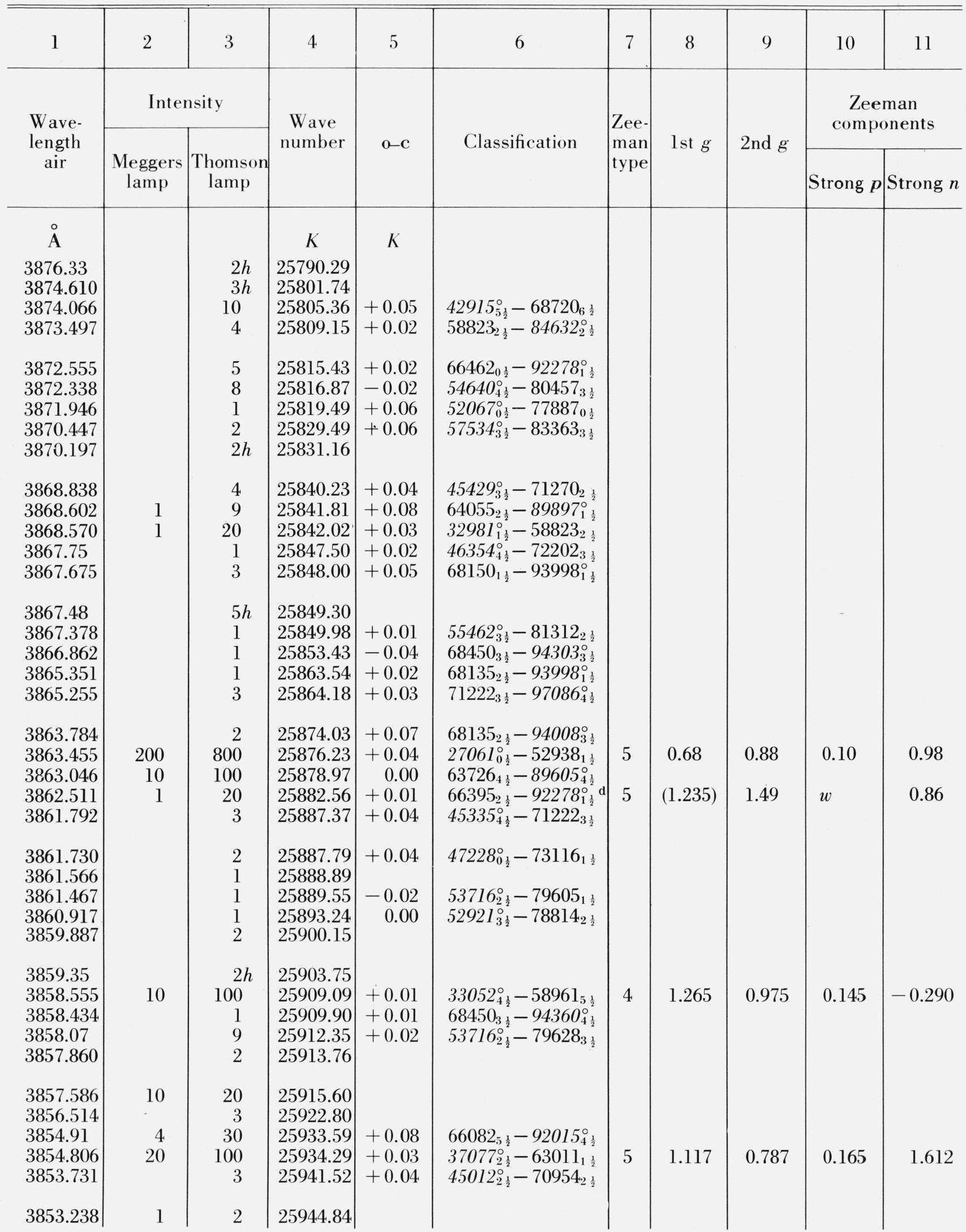


TABLE 6. Yb II-Observed and classified lines-Continued

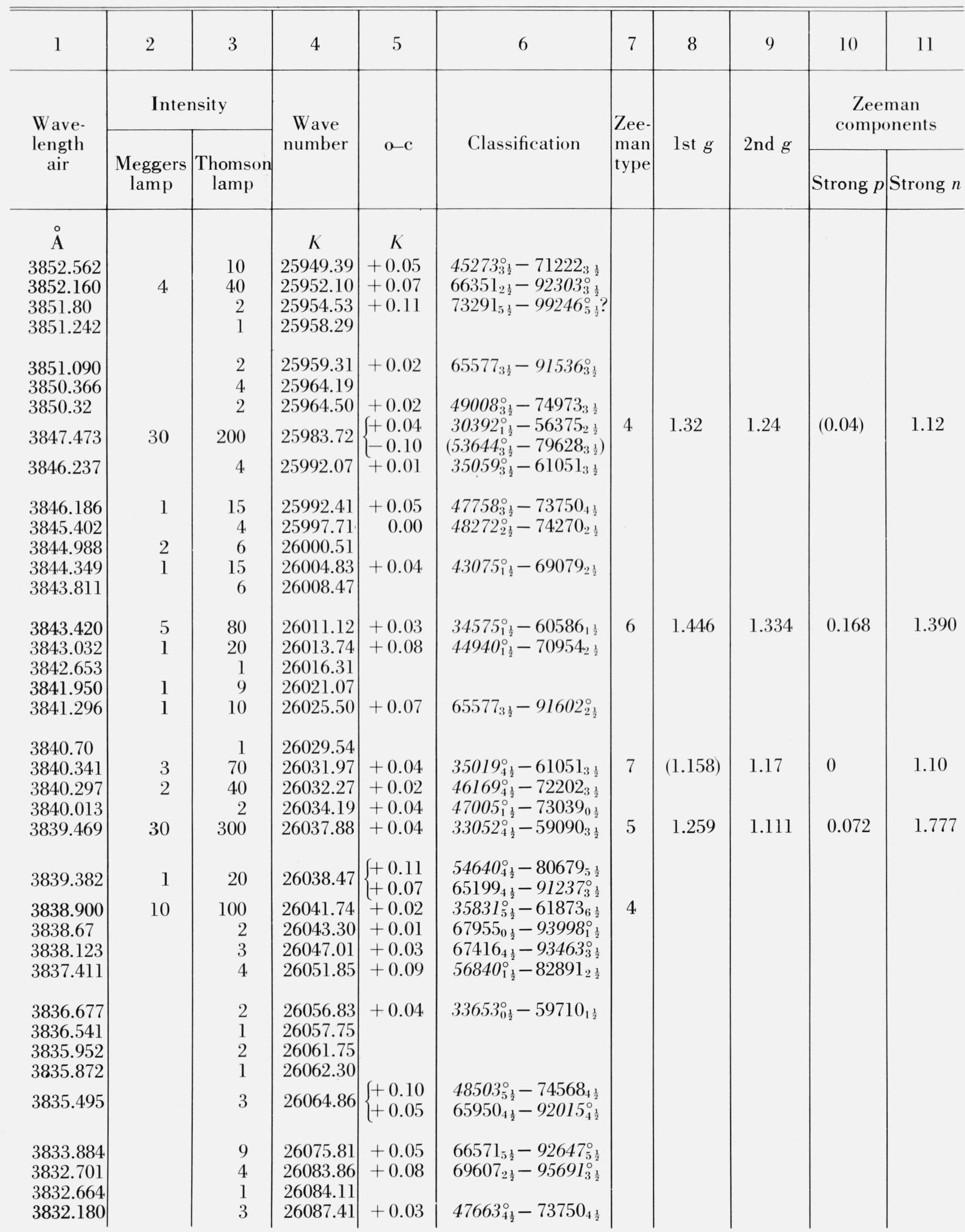


TABLE 6. Yb II-Observed and classified lines-Continued

\begin{tabular}{|c|c|c|c|c|c|c|c|c|c|c|}
\hline 1 & 2 & 3 & 4 & 5 & 6 & 7 & 8 & 9 & 10 & 11 \\
\hline \multirow{2}{*}{$\begin{array}{l}\text { Wave- } \\
\text { length } \\
\text { air }\end{array}$} & \multicolumn{2}{|c|}{ Intensity } & \multirow{2}{*}{$\begin{array}{l}\text { Wave } \\
\text { number }\end{array}$} & \multirow{2}{*}{$\mathrm{o}-\mathrm{c}$} & \multirow{2}{*}{ Classification } & \multirow{2}{*}{$\begin{array}{l}\text { Zee- } \\
\text { man } \\
\text { type }\end{array}$} & \multirow{2}{*}{ lst $g$} & \multirow{2}{*}{ 2nd $g$} & \multicolumn{2}{|c|}{$\begin{array}{c}\text { Zeeman } \\
\text { components }\end{array}$} \\
\hline & $\begin{array}{c}\text { Meggers } \\
\text { lamp }\end{array}$ & $\begin{array}{c}\text { Thomson } \\
\text { lamp }\end{array}$ & & & & & & & Strong $p$ & Strong $n$ \\
\hline $\begin{array}{c}\stackrel{\circ}{\text { A }} \\
3832.108\end{array}$ & & 3 & $\begin{array}{c}K \\
26087.90\end{array}$ & $\begin{array}{c}K \\
+0.04\end{array}$ & $67416_{4 \frac{1}{2}}-93503_{4 \frac{1}{2}}^{\circ}$ & & & & & \\
\hline $\begin{array}{l}3831.610 \\
3831.288 \\
3831.13 \\
3830.178 \\
3829.767\end{array}$ & 3 & $\begin{array}{r}20 \\
1 \\
1 \\
2 \\
2\end{array}$ & $\begin{array}{l}26091.29 \\
26093.48 \\
26094.56 \\
26101.04 \\
26103.84\end{array}$ & $\begin{array}{l}+0.10 \\
-0.01\end{array}$ & $\begin{array}{l}55221_{1 \frac{1}{2}}^{\circ}-81312_{2 \frac{1}{2}} \\
45012_{2 \frac{1}{2}}^{\circ}-71113_{1 \frac{1}{2}}\end{array}$ & 7 & (1.11) & 1.11 & 0 & 1.12 \\
\hline $\begin{array}{l}3829.736 \\
3827.494 \\
3827.030 \\
3826.768 \\
3826.51\end{array}$ & & $\begin{array}{l}1 \\
4 \\
2 \\
8 \\
1\end{array}$ & $\begin{array}{l}26104.05 \\
26119.34 \\
26122.51 \\
26124.30 \\
26126.06\end{array}$ & $\begin{array}{l}-0.02 \\
+0.08 \\
+0.04 \\
+0.08\end{array}$ & $\begin{array}{l}70793_{5 \frac{1}{2}}-96898_{5 \frac{1}{2}}^{\circ} \\
64598_{3 \frac{1}{2}}-90717_{4 \frac{1}{2}}^{\circ} \\
73291_{5 \frac{1}{2}}-99414_{4 \frac{1}{2}}^{\circ} \\
33494_{3 \frac{1}{2}}^{\circ}-59618_{3 \frac{1}{2}}^{\circ}\end{array}$ & & & & & \\
\hline $\begin{array}{l}3822.216 \\
3822.17 \\
3821.526 \\
3821.224 \\
3820.825\end{array}$ & & $\begin{array}{l}1 \\
1 \\
1 \\
1 \\
8\end{array}$ & $\begin{array}{l}26155.41 \\
26155.73 \\
26160.13 \\
26162.20 \\
26164.93\end{array}$ & $\begin{array}{l}+0.06 \\
+0.07 \\
-0.03 \\
+0.10\end{array}$ & $\begin{array}{l}69395_{3 \frac{1}{2}}-95550_{4 \frac{1}{2}}^{\circ} \\
35059_{3 \frac{1}{2}}^{\circ}-61214_{4 \frac{1}{2}}^{\circ} \\
58283_{2 \frac{1}{2}}-84444_{3 \frac{1}{2}}^{\circ} \\
62559_{2 \frac{1}{2}}-88721_{3 \frac{1}{2}}^{\circ}\end{array}$ & & & & & \\
\hline $\begin{array}{l}3820.288 \\
3819.916 \\
3818.365 \\
3817.777\end{array}$ & 1 & $\begin{array}{r}5 \\
15 \\
1 \\
20\end{array}$ & $\begin{array}{l}26168.61 \\
26171.16 \\
26181.79 \\
26185.82\end{array}$ & $\begin{array}{l}+0.03 \\
+0.08 \\
+0.04 \\
+0.09\end{array}$ & $\begin{array}{l}68135_{2 \frac{1}{2}}-94303_{3 \frac{1}{2}}^{\circ} \\
61214_{4 \frac{1}{2}}-87385_{3 \frac{1}{2}}^{\circ} \\
50468_{5 \frac{1}{2}}^{\circ}-76649_{5 \frac{1}{2}}^{\circ} \\
37516_{3 \frac{1}{2}}^{\circ}-63702^{\circ \frac{1}{3}}\end{array}$ & & & & & \\
\hline $\begin{array}{l}3817.777 \\
3817.624\end{array}$ & 3 & $\begin{array}{r}20 \\
7\end{array}$ & $\begin{array}{l}26185.82 \\
26186.87\end{array}$ & $\left\{\begin{array}{l}+0.09 \\
+0.05 \\
-0.05\end{array}\right.$ & $\begin{array}{l}37516_{3 \frac{1}{2}}^{\circ}-63702_{2 \frac{1}{2}} \\
68148_{5 \frac{1}{2}}-94335_{5 \frac{1}{2}}^{\circ} \\
72615_{5 \frac{1}{2}}-98802_{5 \frac{1}{2}}^{\circ}\end{array}$ & 5 & (1.12) & 1.05 & $w$ & 1.30 \\
\hline 3816.345 & 15 & 150 & 26195.65 & $\left\{\begin{array}{l}+0.12 \\
-0.03\end{array}\right.$ & $\begin{array}{l}35019_{4 \frac{1}{2}}^{\circ}-61214_{4 \frac{1}{2}} \\
\left(45273_{3 \frac{1}{2}}^{\circ}-71468_{2 \frac{1}{2}}\right)\end{array}$ & 6 & & & & \\
\hline $\begin{array}{l}3816.189 \\
3815.640 \\
3814.310\end{array}$ & 30 & $\begin{array}{r}300 \\
1 \\
10\end{array}$ & $\begin{array}{l}26196.72 \\
26200.49 \\
26209.62\end{array}$ & $\begin{array}{l}+0.02 \\
+0.03 \\
+0.05\end{array}$ & $\begin{array}{l}34389_{2 \frac{1}{2}}^{\circ}-60586_{1 \frac{1}{2}}^{\circ} \\
51248_{2 \frac{1}{2}}^{\circ}-77449_{3 \frac{1}{2}} \\
45012_{2 \frac{1}{2}}^{\circ}-71222_{3 \frac{1}{2}}^{\circ}\end{array}$ & 4 & 1.007 & 1.327 & 0.160 & 1.166 \\
\hline 3814.215 & 15 & 200 & 26210.28 & +0.04 & $37516_{3 \frac{1}{2}}^{\circ}-63726_{4 \frac{1}{2}}^{\circ}$ & 4 & 1.12 & 1.00 & 0.06 & 0.57 \\
\hline $\begin{array}{l}3814.084 \\
3813.665 \\
3811.584\end{array}$ & & $\begin{array}{l}4 \\
3 \\
2\end{array}$ & $\begin{array}{l}26211.18 \\
26214.06 \\
26228.37\end{array}$ & $\begin{array}{l}+0.06 \\
+0.06 \\
+0.04\end{array}$ & $\begin{array}{l}68148_{5_{\frac{1}{2}}}-94360_{4 \frac{1}{2}}^{\circ} \\
46902_{0 \frac{1}{2}}^{\circ}-73116_{1 \frac{1}{2}}^{\circ} \\
70858_{4 \frac{1}{2}}^{\circ}-97086_{4 \frac{1}{2}}^{\circ}\end{array}$ & & & & & \\
\hline $\begin{array}{l}3809.04 \\
3808.544\end{array}$ & 5 & $\begin{array}{r}30 \\
1\end{array}$ & $\begin{array}{l}26245.88 \\
26249.30\end{array}$ & $\left\{\begin{array}{l}+0.10 \\
+0.06 \\
-0.02\end{array}\right.$ & $\begin{array}{l}48024_{1 \frac{1}{2}}^{\circ}-74270_{2 \frac{1}{2}} \\
70136_{6 \frac{1}{2}}-96382_{5 \frac{1}{2}}^{\circ} \\
70793_{5 \frac{1}{2}}-97043_{6 \frac{1}{2}}^{\circ}\end{array}$ & & & & & \\
\hline $\begin{array}{l}3808.460 \\
3808.35 \\
3808.315 \\
3807.574\end{array}$ & 50 & $\begin{array}{r}2 \\
2 \\
2 \\
500\end{array}$ & $\begin{array}{l}26249.88 \\
26250.64 \\
26250.88 \\
26255.99\end{array}$ & +0.06 & $63647_{1 \frac{1}{2}}-89897_{1 \frac{1}{2}}^{\circ}$ & & & & & \\
\hline 3807.545 & 100 & 1000 & 26256.19 & -0.07 & $38342_{4 \frac{1}{2}}^{\circ}-64598_{3 \frac{1}{2}}$ & 4 & 1.08 & 1.23 & 0.076 & 0.55 \\
\hline 3806.996 & & 3 & $26259: 98$ & +0.03 & $41678_{2 \frac{1}{2}}^{\circ}-67938_{3 \frac{1}{2}}$ & & & & & \\
\hline
\end{tabular}


TABLE 6. Yb II-Observed and classified lines-Continued

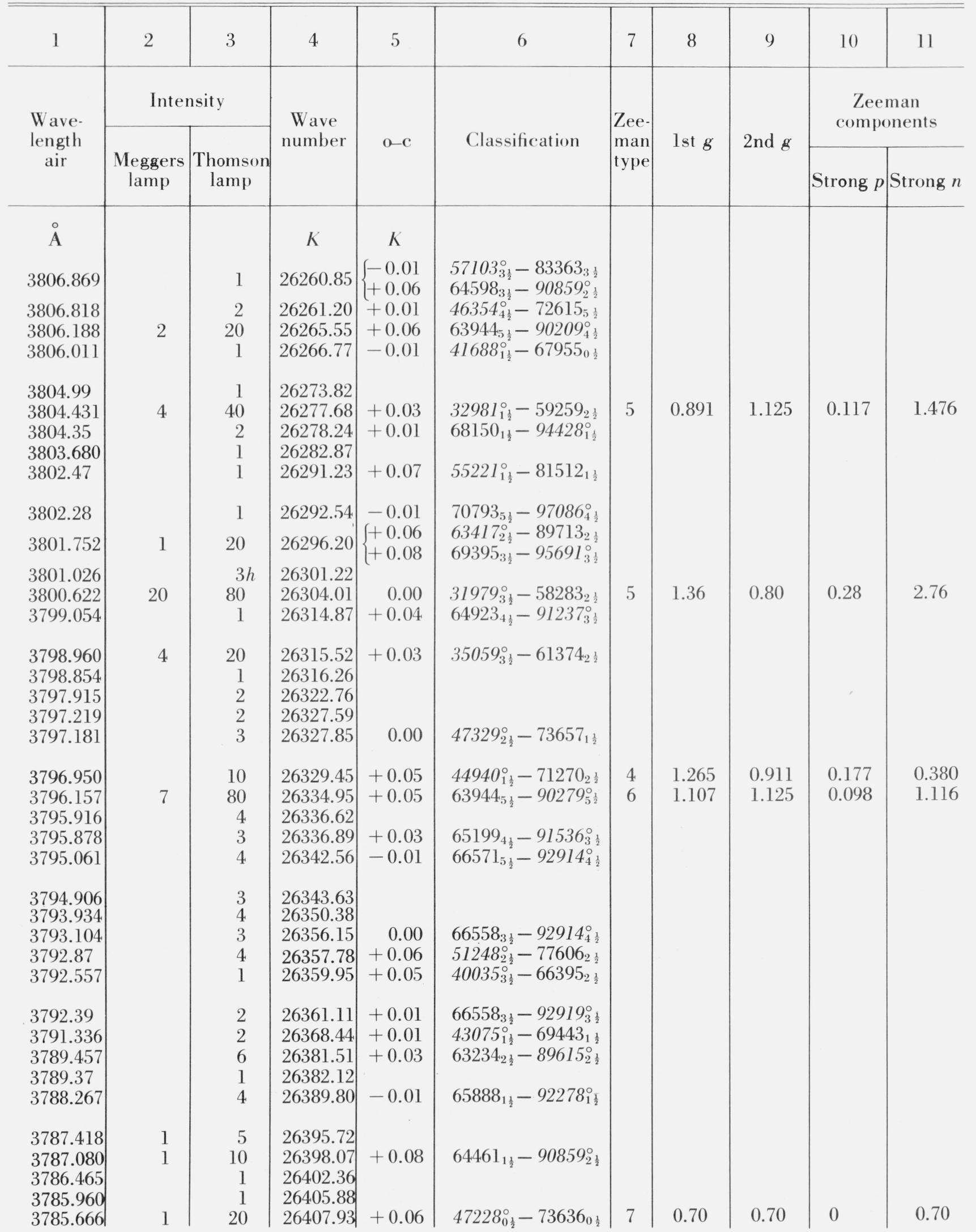


TABLE 6. Yb II-Observed and classified lines-Continued

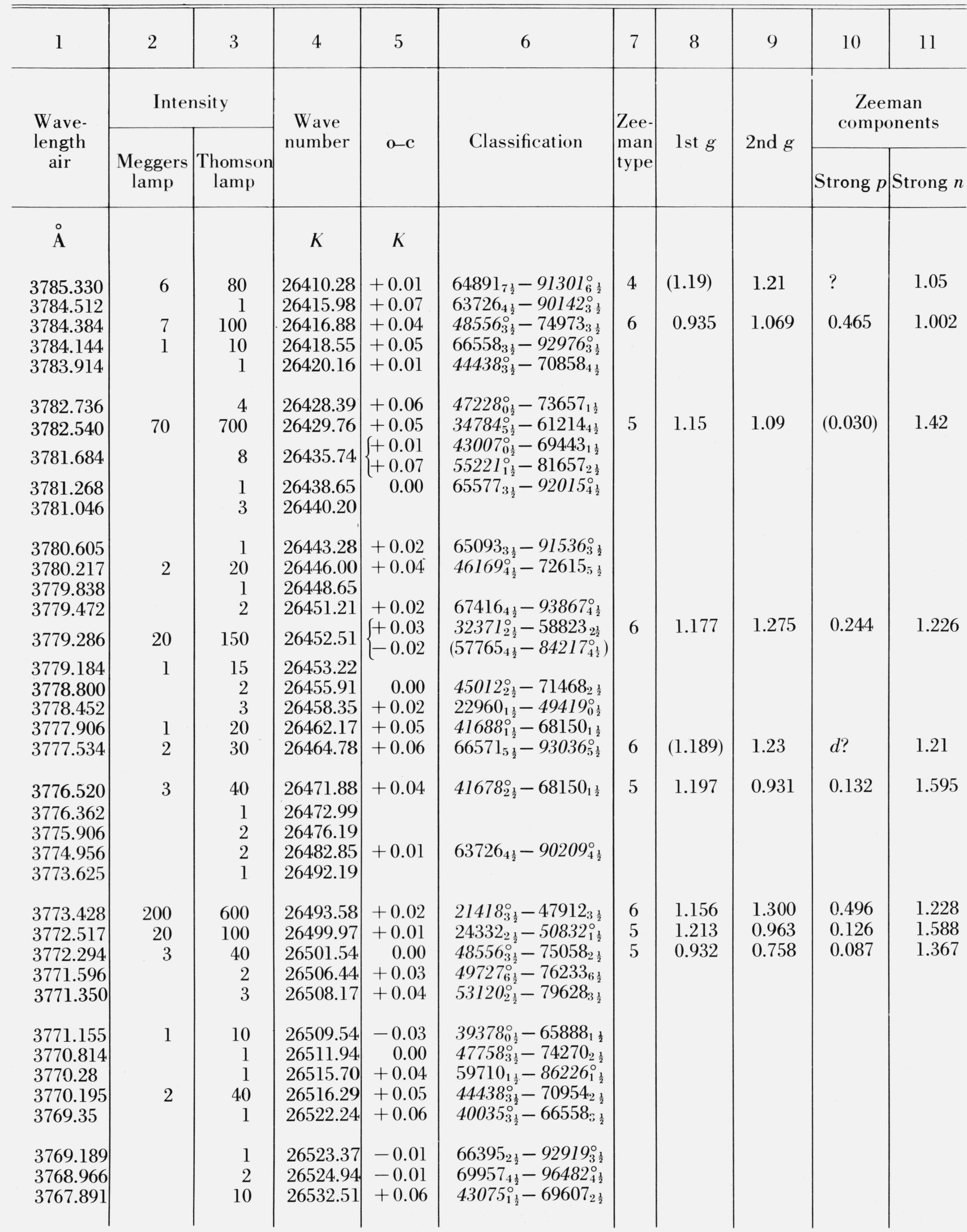


TABLE 6. Yb II-Observed and classified lines-Continued

\begin{tabular}{|c|c|c|c|c|c|c|c|c|c|c|}
\hline 1 & 2 & 3 & 4 & 5 & 6 & 7 & 8 & 9 & 10 & 11 \\
\hline \multirow{2}{*}{$\begin{array}{l}\text { W ave- } \\
\text { length } \\
\text { air }\end{array}$} & \multicolumn{2}{|c|}{ Intensity } & \multirow{2}{*}{$\begin{array}{l}\text { Wave } \\
\text { number }\end{array}$} & \multirow{2}{*}{$\mathrm{o}-\mathrm{c}$} & \multirow{2}{*}{ Classification } & \multirow{2}{*}{ Zee- } & \multirow{2}{*}{ lst $g$} & \multirow{2}{*}{ 2nd $g$} & \multicolumn{2}{|c|}{$\begin{array}{c}\text { Zeeman } \\
\text { components }\end{array}$} \\
\hline & $\begin{array}{l}\text { Meggers } \\
\text { lamp }\end{array}$ & $\begin{array}{c}\text { Thomson } \\
\text { lamp }\end{array}$ & & & & & & & Strong $p$ & Strong $n$ \\
\hline$\AA$ & & & $K$ & K & & & & & & \\
\hline $\begin{array}{l}3767.175 \\
3766.925\end{array}$ & 2 & $\begin{array}{r}1 \\
20\end{array}$ & $\begin{array}{l}26537.55 \\
26539.31\end{array}$ & +0.08 & $37516_{3 \frac{1}{2}}^{\circ}-64055_{2 \frac{1}{2}}$ & 5 & (1.12) & 1.11 & 0 & 1.15 \\
\hline $\begin{array}{l}3766.736 \\
3766.527\end{array}$ & & $\begin{array}{l}8 h \\
4\end{array}$ & $\begin{array}{l}26540.64 \\
26542.12\end{array}$ & +0.11 & $49008_{3 \frac{1}{2}}^{\circ}-75550_{2 \frac{1}{2}}$ & & & & & \\
\hline $\begin{array}{l}3766.101 \\
3765.080 \\
3764.56\end{array}$ & $\begin{array}{r}20 \\
1\end{array}$ & $\begin{array}{c}200 \\
8 \\
3 h\end{array}$ & $\begin{array}{l}26545.12 \\
26552.32 \\
26555.98\end{array}$ & $\begin{array}{r}0.00 \\
+0.07\end{array}$ & $\begin{array}{l}34575_{\frac{1}{2}}^{\circ}-61120_{0 \frac{1}{2}}^{\circ} \\
63726_{4 \frac{1}{2}}^{\circ}-90279_{5 \frac{1}{2}}^{\circ}\end{array}$ & 4 & 1.438 & 1.666 & 0.112 & 1.327 \\
\hline $\begin{array}{l}3764.03 \\
3763.35 \\
3763.27 \\
3763.045 \\
3762.98\end{array}$ & 1 & $\begin{array}{l}1 \\
1 \\
1 \\
6 \\
1\end{array}$ & $\begin{array}{l}26559.72 \\
26564.52 \\
26565.09 \\
26566.67 \\
26567.13\end{array}$ & $\begin{array}{r}+0.07 \\
0.00 \\
+0.06\end{array}$ & $\begin{array}{l}54640_{4 \frac{1}{2}}^{\circ}-81205_{4 \frac{1}{2}}^{\circ} \\
66082_{5 \frac{1}{2}}-92647_{5 \frac{1}{2}}^{\circ} \\
33052_{4 \frac{1}{2}}^{\circ}-59618_{3 \frac{1}{2}}^{\circ}\end{array}$ & & & & & \\
\hline $\begin{array}{l}3762.550 \\
3762.12 \\
3761.31\end{array}$ & 40 & $\begin{array}{r}400 \\
1 \\
1\end{array}$ & $\begin{array}{l}26570.17 \\
26573.21 \\
26578.93\end{array}$ & +0.03 & $37077_{2 \frac{1}{2}}^{\circ}-63647_{1 \frac{1}{2}}$ & 4 & (1.113) & 1.14 & $w$ & 1.07 \\
\hline $\begin{array}{l}3760.999 \\
3760.23\end{array}$ & 10 & $\begin{array}{l}200 \\
2 d\end{array}$ & $\begin{array}{l}26581.13 \\
26586.56\end{array}$ & +0.04 & $38342_{4 \frac{1}{2}}^{\circ}-64923_{4 \frac{1}{2}}$ & 6 & 1.103 & 1.089 & 0.06 & 1.096 \\
\hline $\begin{array}{l}3757.355 \\
3756.888 \\
3756.445 \\
3755.762 \\
3755.32\end{array}$ & & $\begin{array}{l}1 \\
2 \\
6 \\
2 \\
1\end{array}$ & $\begin{array}{l}26606.91 \\
26610.21 \\
26613.35 \\
26618.19 \\
26621.32\end{array}$ & $\begin{array}{r}+0.01 \\
0.00 \\
+0.06 \\
0.00\end{array}$ & $\begin{array}{l}68943_{4 \frac{1}{2}}-95550_{4 \frac{1}{2}}^{\circ} \\
46169_{4 \frac{1}{2}}^{\circ}-72779_{3 \frac{1}{2}}^{\circ} \\
64923_{4 \frac{1}{2}}^{\circ}-91536_{3 \frac{1}{2}}^{\circ} \\
52987_{1 \frac{1}{2}}^{\circ}-79605_{1 \frac{1}{2}}^{\circ}\end{array}$ & & & & & \\
\hline $\begin{array}{l}3754.83 \\
3754.74 \\
3754.606\end{array}$ & 2 & $\begin{array}{l}2 \\
1 \\
6\end{array}$ & $\begin{array}{l}26624.80 \\
26625.44 \\
26626.39\end{array}$ & +0.07 & $37077_{2 \frac{1}{2}}^{\circ}-63702_{2 \frac{1}{2}}$ & & & & & \\
\hline $\begin{array}{l}3753.897 \\
3753.65\end{array}$ & 8 & $\begin{array}{r}100 \\
1\end{array}$ & $\begin{array}{l}26631.41 \\
26633.17\end{array}$ & $\begin{array}{l}+0.04 \\
-0.01\end{array}$ & $\begin{array}{l}47005_{1 \frac{1}{2}}^{\circ}-73636_{0 \frac{1}{2}} \\
51248_{2 \frac{1}{2}}^{\circ}-77882_{3 \frac{1}{2}}\end{array}$ & 5 & 0.990 & 0.690 & 0.15 & 1.15 \\
\hline $\begin{array}{l}3753.49 \\
3753.053 \\
3752.730 \\
3752.56\end{array}$ & 4 & $\begin{array}{r}2 \\
60 \\
4 \\
1\end{array}$ & $\begin{array}{l}26634.30 \\
26637.40 \\
26639.70 \\
26640.90\end{array}$ & $\begin{array}{l}+0.03 \\
+0.04\end{array}$ & $\begin{array}{l}47329_{2 \frac{1}{2}}^{\circ}-73966_{3 \frac{1}{2}}^{\circ} \\
64598_{3 \frac{1}{2}}-91237_{3 \frac{1}{2}}^{\circ}\end{array}$ & & & & & \\
\hline 3751.847 & 10 & 40 & 26645.97 & +0.03 & $26759_{2 \frac{1}{2}}^{\circ}-53404_{2 \frac{1}{2}}$ & 6 & 1.58 & 1.04 & 1.36 & 1.31 \\
\hline $\begin{array}{l}3751.206 \\
3751.030 \\
3750.045\end{array}$ & 1 & $\begin{array}{l}2 \\
1 \\
8\end{array}$ & $\begin{array}{l}26650.52 \\
26651.77 \\
26658.77\end{array}$ & -0.06 & $47005_{1 \frac{1}{2}}^{\circ}-73657_{1 \frac{1}{2}}^{\circ}$ & & & & & \\
\hline $\begin{array}{l}3749.688 \\
3749.388\end{array}$ & $\begin{array}{r}40 \\
1\end{array}$ & $\begin{array}{r}400 \\
6\end{array}$ & $\begin{array}{l}26661.31 \\
26663.44\end{array}$ & $\begin{array}{r}+0.01 \\
0.00\end{array}$ & $\begin{array}{l}34389_{2 \frac{1}{2}}^{\circ}-61051_{3 \frac{1}{2}}^{\circ} \\
63234_{\frac{21}{2}}^{\circ}-89897_{1 \frac{1}{2}}^{\circ}\end{array}$ & 5 & 1.015 & 1.196 & 0.452 & 1.650 \\
\hline $\begin{array}{l}3748.345 \\
3748.204 \\
3748.102\end{array}$ & 3 & $\begin{array}{r}10 \\
2 \\
1\end{array}$ & $\begin{array}{l}26670.86 \\
26671.86 \\
26672.59\end{array}$ & $\begin{array}{l}+0.05 \\
-0.02\end{array}$ & $\begin{array}{l}66571_{5 \frac{1}{2}}-93242_{5 \frac{1}{2}}^{\circ} \\
68150_{1 \frac{1}{2}}-94823_{1 \frac{1}{2}}^{\circ}\end{array}$ & & & & & \\
\hline
\end{tabular}


TABLE 6. Yb II-Observed and classified lines-Continued

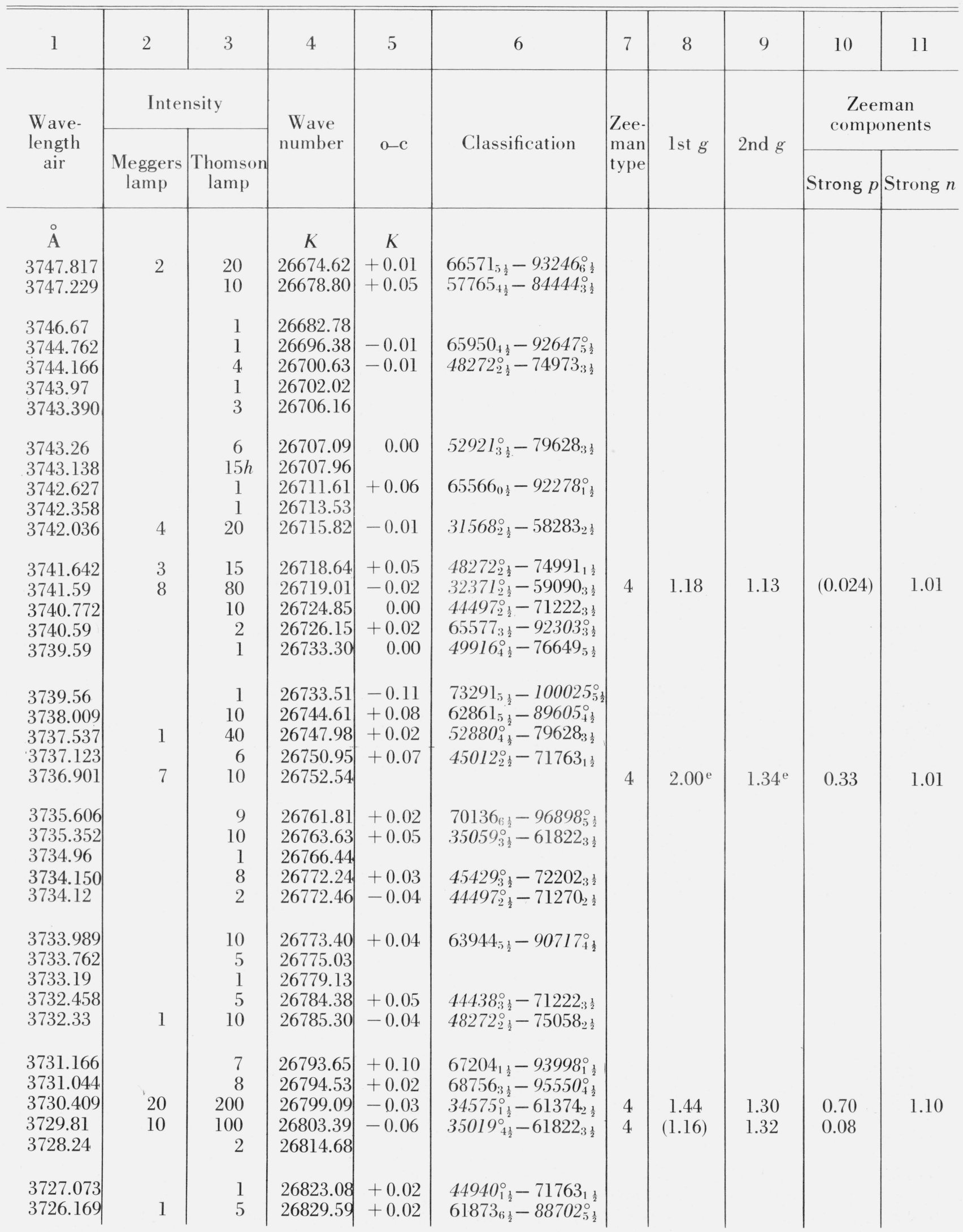


TABLE 6. Yb II-Observed and classified lines-Continued

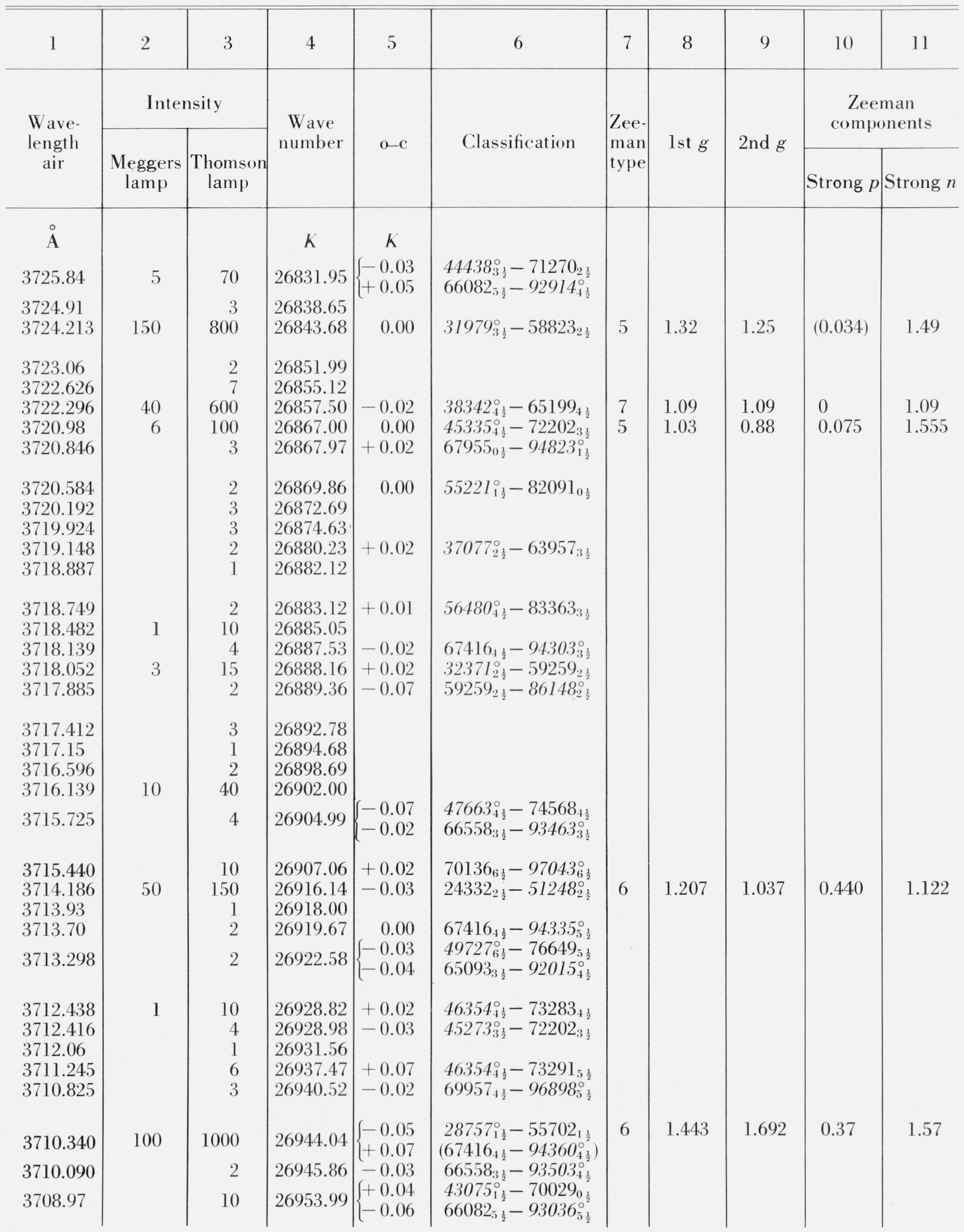


TABLE 6. Yb II-Observed and classified lines-Continued

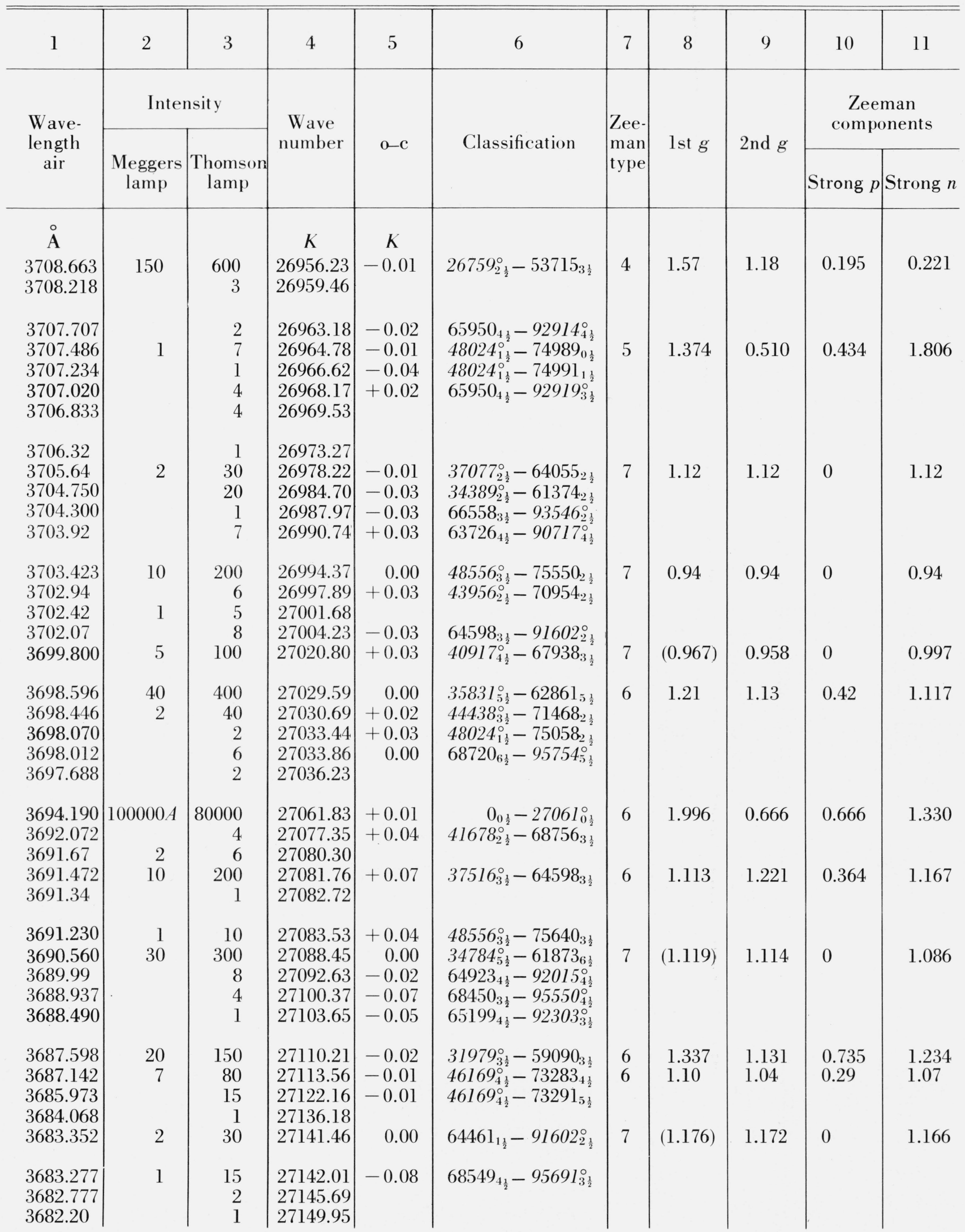


TABLE 6. Yb II-Observed and classified lines-Continued

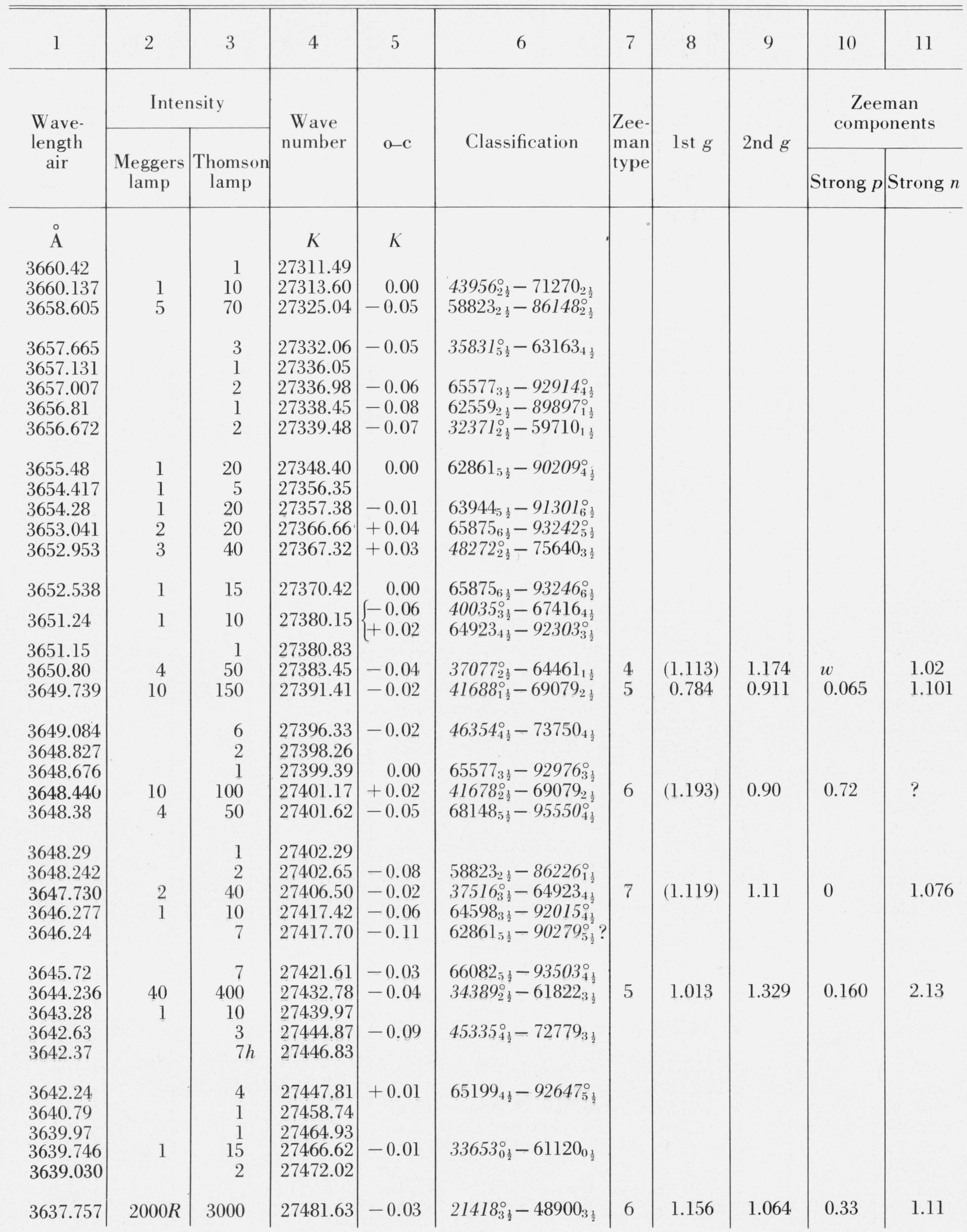


TABLE 6. Yb II-Observed and classified lines - Continued

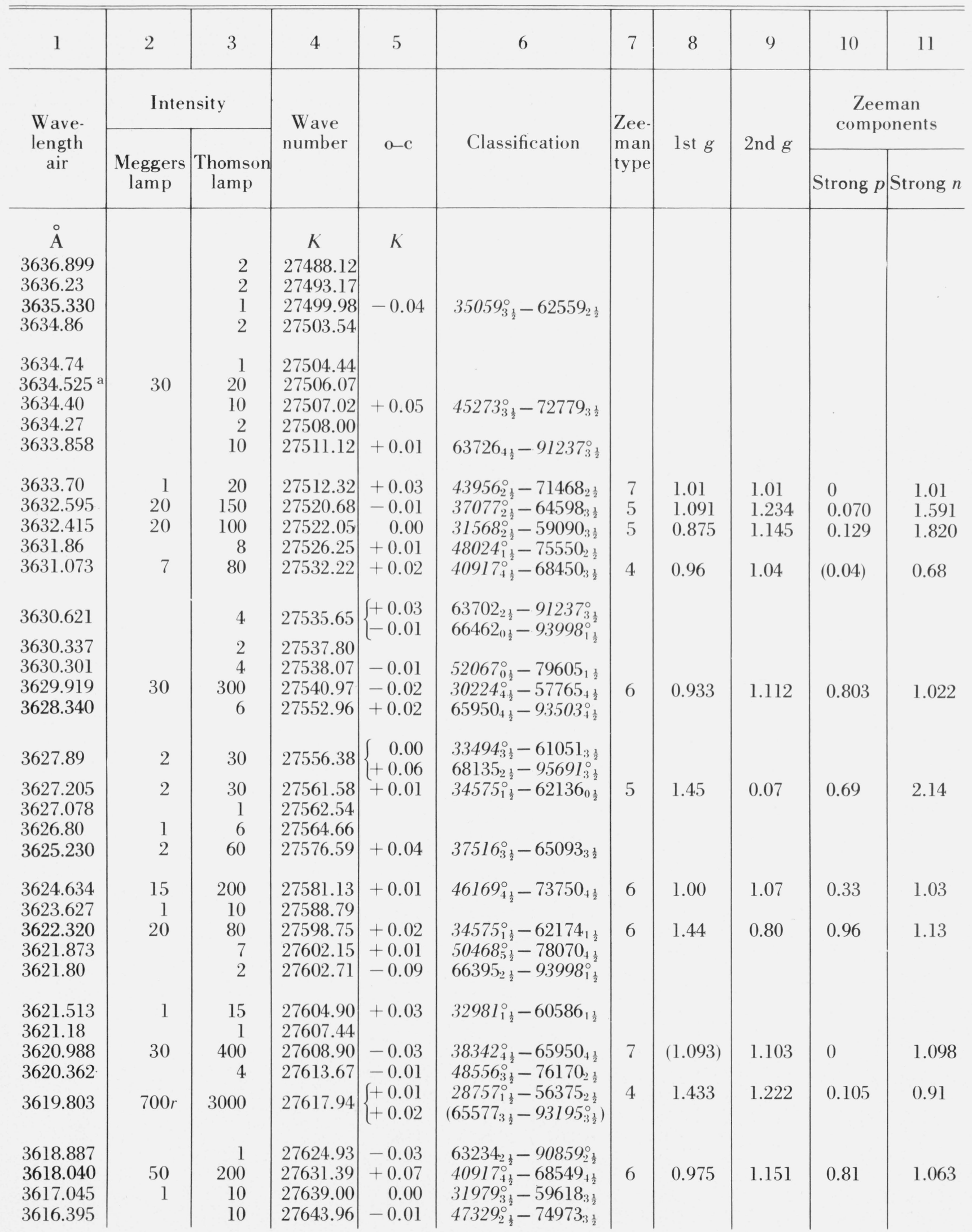


TABLE 6. Yb II-Observed and classified lines-Continued

\begin{tabular}{|c|c|c|c|c|c|c|c|c|c|c|}
\hline 1 & 2 & 3 & 4 & 5 & 6 & 7 & 8 & 9 & 10 & 11 \\
\hline \multirow{2}{*}{$\begin{array}{l}\text { Wave- } \\
\text { length } \\
\text { air }\end{array}$} & \multicolumn{2}{|c|}{ Intensity } & \multirow{2}{*}{$\begin{array}{c}\text { Wave } \\
\text { number }\end{array}$} & \multirow[b]{2}{*}{$\mathrm{o}-\mathrm{c}$} & \multirow{2}{*}{ Classification } & \multirow{2}{*}{$\begin{array}{l}\text { Zee- } \\
\text { man } \\
\text { type }\end{array}$} & \multirow{2}{*}{ lst $g$} & \multirow{2}{*}{ 2nd $g$} & \multicolumn{2}{|c|}{$\begin{array}{l}\text { Zeeman } \\
\text { components }\end{array}$} \\
\hline & $\begin{array}{l}\text { Meggers } \\
\text { lamp }\end{array}$ & $\begin{array}{c}\text { Thomson } \\
\text { lamp }\end{array}$ & & & & & & & Strong $p \mid$ & Strong $n$ \\
\hline$\stackrel{\circ}{\AA}$ & \multirow{10}{*}{5} & & $K$ & $K$ & & \multirow{10}{*}{4} & \multirow{10}{*}{$(0.990)$} & \multirow{10}{*}{1.04} & \multirow{10}{*}{$w$} & \multirow{10}{*}{0.91} \\
\hline 3616.031 & & 3 & 27646.75 & & & & & & & \\
\hline 3615.957 & & 2 & 27647.31 & 0.00 & $66351_{2 \frac{1}{2}}-93998_{1 \frac{1}{2}}^{\circ}$ & & & & & \\
\hline 3614.63 & & 1 & 27657.46 & -0.08 & $65888_{1 \frac{1}{2}}-93546_{2 \frac{1}{2}}^{\circ}$ & & & & & \\
\hline 3614.593 & & 6 & 27657.74 & -0.01 & $66351_{2 \frac{1}{2}}-94008_{3 \frac{1}{2}}$ & & & & & \\
\hline 3614.048 & & 100 & 27661.92 & 0.00 & $47329_{2 \frac{1}{2}}^{0}-74991_{1 \frac{1}{2}}^{2}$ & & & & & \\
\hline 3613.38 & & 3 & 27667.03 & -0.07 & $62046_{3 \frac{1}{2}}^{\circ}-89713_{2 \frac{1}{2}}$ & & & & & \\
\hline 3613.340 & & 3 & 27667.33 & -0.05 & $49008_{3 \frac{1}{2}}^{\circ}-76676_{2 \frac{1}{2}}$ & & & & & \\
\hline 3613.295 & & 2 & 27667.68 & -0.08 & $53644_{3 \frac{1}{2}}^{\circ}-81312_{2 \frac{1}{2}}^{\circ}$ & & & & & \\
\hline 3612.950 & & 4 & 27670.32 & -0.02 & $55221_{1 \frac{1}{2}}^{\circ}-82891_{2 \frac{1}{2}}$ & & & & & \\
\hline 3611.300 & 60 & 800 & 27682.96 & +0.01 & $37516_{3 \frac{1}{2}}^{\circ}-65199_{4 \frac{1}{2}}$ & 4 & 1.14 & 1.10 & $(0.02)$ & 0.96 \\
\hline 3610.233 & 100 & 700 & 27691.15 & -0.01 & $31568_{2 \frac{1}{2}}^{\circ}-59259_{2 \frac{1}{2}}^{\circ}$ & 6 & 0.862 & 1.105 & 0.613 & 0.99 \\
\hline 3609.24 & \multirow[b]{3}{*}{10} & i & 27698.76 & & & \multirow[b]{3}{*}{5} & \multirow[b]{3}{*}{0.711} & \multirow[b]{3}{*}{0.835} & \multirow[b]{3}{*}{0.06} & \multirow[b]{3}{*}{1.145} \\
\hline 3608.936 & & 3 & 27701.10 & & & & & & & \\
\hline $\begin{array}{l}3608.488 \\
3607.186\end{array}$ & & $\begin{array}{r}150 \\
2\end{array}$ & $\begin{array}{l}27704.54 \\
27714.54\end{array}$ & $\begin{array}{l}+0.02 \\
-0.07\end{array}$ & $\begin{array}{l}44497_{2 \frac{1}{2}}^{\circ}-72202_{3 \frac{1}{2}} \\
65199^{2}-92914^{\circ}\end{array}$ & & & & & \\
\hline 3606.478 & 300 & 2000 & $\begin{array}{l}2714.54 \\
27719.98\end{array}$ & $\begin{array}{r}-0.06 \\
0.00\end{array}$ & $\begin{array}{l}\text { 0.519994 } \\
33494_{3 \frac{1}{2}}^{\circ}-61214_{4 \frac{1}{2}}\end{array}$ & 5 & 0.98 & 1.04 & $(0.03)$ & 1.24 \\
\hline $\begin{array}{l}3605.347 \\
3605.208\end{array}$ & \multirow[b]{2}{*}{20} & 20 & $\begin{array}{l}27728.67 \\
27729.74\end{array}$ & $\begin{array}{r}0.00 \\
-0.04\end{array}$ & $\begin{array}{l}47329_{2 \frac{1}{2}}^{\circ}-75058_{2 \frac{1}{2}} \\
48503^{\circ}-76233^{2}\end{array}$ & 6 & 1.00 & 0.78 & 0.60 & 0.88 \\
\hline 3603.848 & & 300 & 27740.20 & -0.03 & $38342_{4 \frac{1}{2}}^{\circ \frac{2}{2}}-66082_{5 \frac{1}{2}}$ & 5 & 1.10 & 1.14 & $(0.019)$ & 1.31 \\
\hline 3602.237 & & 1 & 27752.61 & -0.03 & $67938_{3 \frac{1}{2}}-95691_{3 \frac{1}{2}}^{\circ}$ & & & & & \\
\hline 3601.917 & 2 & 20 & 27755.08 & +0.01 & $41688_{1 \frac{1}{2}}^{\circ}-69443_{1 \frac{1}{2}}^{\frac{1}{2}}$ & 6 & 0.77 & 1.15 & 0.58 & 0.96 \\
\hline 3601.209 & 2 & 15 & 27760.53 & $\left\{\begin{array}{r}0.00 \\
-0.08\end{array}\right.$ & $\begin{array}{l}47228_{0 \frac{1}{2}}^{\circ}-74989_{0 \frac{1}{2}} \\
62136_{01}-89897^{\circ}\end{array}$ & & & & & \\
\hline 3600.966 & 10 & 100 & 27762.41 & +0.01 & $47228_{0 \frac{1}{2}}^{\circ}-74991_{1 \frac{1}{2}}^{1 \frac{1}{2}}$ & & & & & \\
\hline 3600.763 & 20 & 300 & 27763.97 & -0.03 & $44438_{3 \frac{1}{2}}^{\circ 2}-72202_{3 \frac{1}{2}}^{\circ}$ & & & & & \\
\hline 3600.663 & 2 & 30 & 27764.74 & -0.05 & $41678_{2 \frac{1}{2}}^{\circ}-69443_{1 \frac{1}{2}}$ & & & & & \\
\hline 3600.383 & 10 & 150 & 27766.90 & $\left\{\begin{array}{l}-0.02 \\
+0.06\end{array}\right.$ & $\begin{array}{r}48556_{3 \frac{1}{2}}^{\circ}-76323_{3 \frac{1}{2}}^{\circ} \\
\left(59618_{3 \frac{1}{2}}^{\circ}-87385_{3 \frac{1}{2}}^{\circ}\right)\end{array}$ & 6 & 0.957 & 1.003 & 0.16 & 0.98 \\
\hline 3598.87 & & $2 h$ & 27778.57 & & & & & & & \\
\hline 3598.043 & 2 & 20 & 27784.96 & -0.01 & $66082_{5 \frac{1}{2}}-93867_{4 \frac{1}{2}}^{\circ}$ & & & & & \\
\hline 3597.60 & & 1 & 27788.38 & -0.04 & $66571_{5 \frac{1}{2}}-94360_{4 \frac{1}{2}}^{\circ}$ & & & & & \\
\hline 3597.078 & & 10 & 27792.41 & +0.01 & $47758_{3 \frac{1}{2}}^{\circ}-75550_{2 \frac{1}{2}}^{\circ}$ & & & & & \\
\hline 3596.488 & 1 & 30 & 27796.97 & -0.06 & $46169_{4 \frac{1}{2}}^{\circ}-73966_{3 \frac{1}{2}}$ & & & & & \\
\hline 3596.300 & & 4 & 27798.42 & -0.01 & $52880_{4 \frac{1}{2}}^{\circ}-80679_{5 \frac{1}{2}}$ & & & & & \\
\hline $\begin{array}{l}3595.844 \\
3595.649\end{array}$ & & $\begin{array}{l}5 \\
2\end{array}$ & $\begin{array}{l}27801.95 \\
27803.46\end{array}$ & -0.05 & $66558_{3 \frac{1}{2}}-94360_{4 \frac{1}{2}}^{\circ}$ & & & & & \\
\hline 3595.162 & & 9 & $\begin{array}{l}27803.40 \\
27807.22\end{array}$ & -0.04 & $43956_{2 \frac{1}{2}}^{\circ}-71763_{1 \frac{1}{2}}$ & & & & & \\
\hline 3594.864 & 1 & 10 & 27809.53 & -0.04 & $63726_{4 \frac{1}{2}}^{-2}-91536_{3 \frac{1}{2}}^{\circ}$ & & & & & \\
\hline $\begin{array}{l}3593.882 \\
3593.728\end{array}$ & & $\begin{array}{l}1 \\
2\end{array}$ & $\begin{array}{l}27817.13 \\
27818.32\end{array}$ & -0.06 & $64461_{1 \frac{1}{2}}-92278_{1 \frac{1}{2}}^{\circ}$ & & & & & \\
\hline
\end{tabular}


TABLE 6. Yb II-Observed and classified lines-Continued

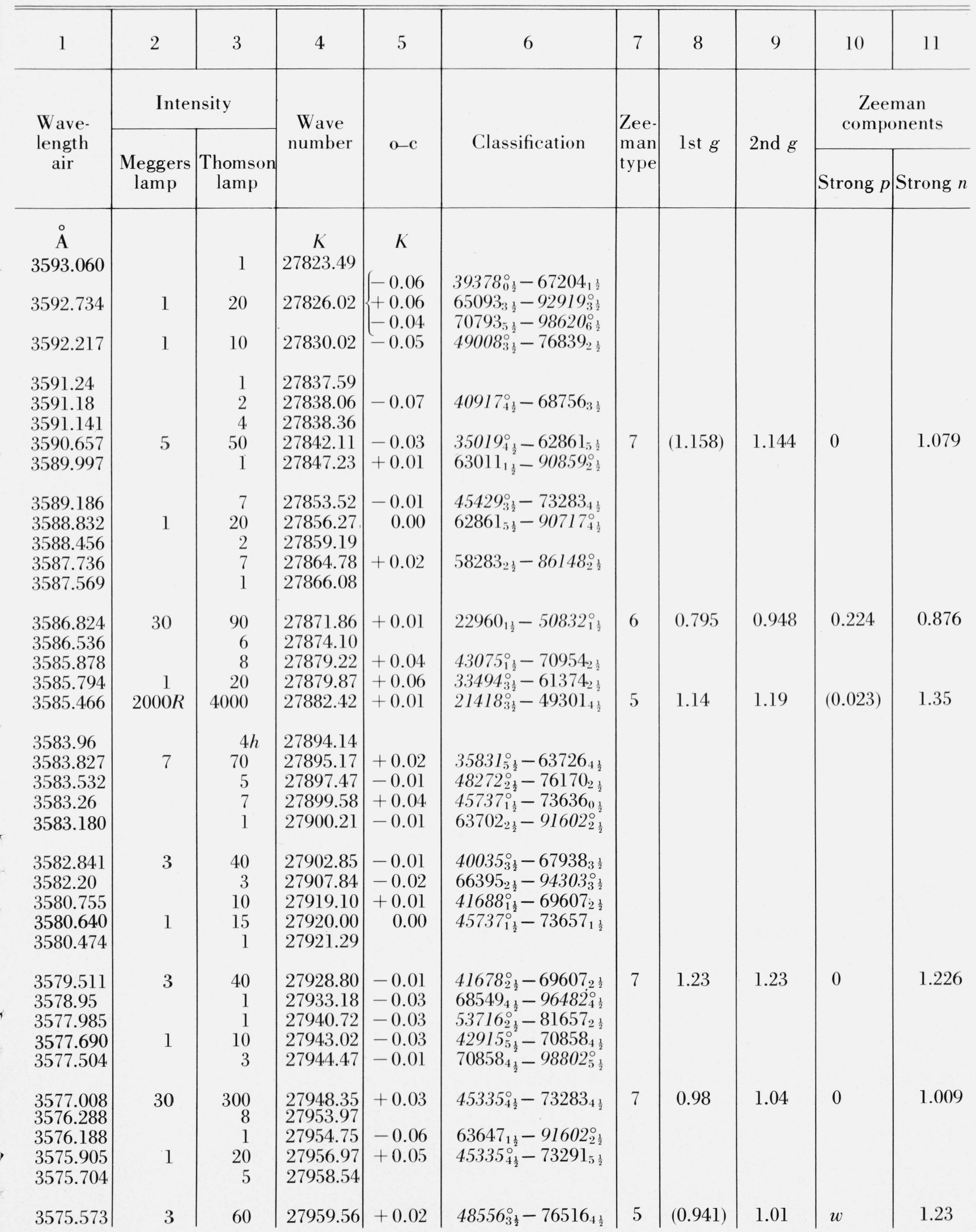


TABLE 6. Yb II-Observed and classified lines-Continued

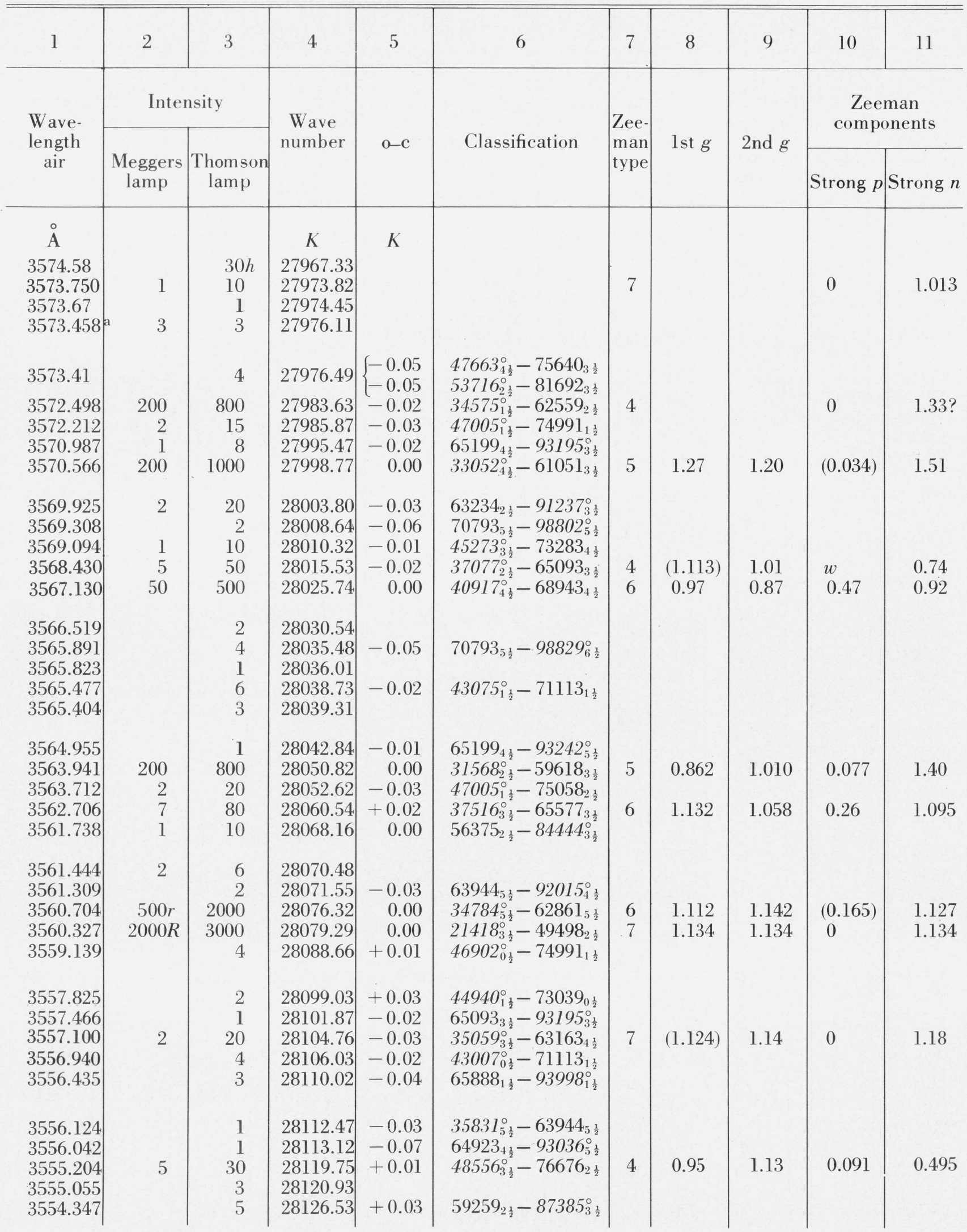


TABLE 6. Yb II-Observed and classified lines-Continued

\begin{tabular}{|c|c|c|c|c|c|c|c|c|c|c|}
\hline 1 & 2 & 3 & 4 & 5 & 6 & 7 & 8 & 9 & 10 & 11 \\
\hline \multirow{2}{*}{$\begin{array}{l}\text { Wave- } \\
\text { length } \\
\text { air }\end{array}$} & \multicolumn{2}{|c|}{ Intensity } & \multirow{2}{*}{$\begin{array}{c}\text { Wave } \\
\text { number }\end{array}$} & \multirow[b]{2}{*}{$\mathrm{o}-\mathrm{c}$} & \multirow{2}{*}{ Classification } & \multirow{2}{*}{$\mid \begin{array}{l}\text { Zee- } \\
\text { man } \\
\text { type }\end{array}$} & \multirow{2}{*}{ lst $g$} & \multirow{2}{*}{2 nd $g$} & \multicolumn{2}{|c|}{$\begin{array}{c}\text { Zeeman } \\
\text { components }\end{array}$} \\
\hline & $\begin{array}{l}\text { Meggers } \\
\text { lamp }\end{array}$ & $\begin{array}{c}\text { Thomson } \\
\text { lamp }\end{array}$ & & & & & & & Strong $p$ & Strong $n$ \\
\hline \begin{tabular}{c|} 
A \\
3553.82 \\
3553.340 \\
3553.192 \\
3553.153 \\
3552.324
\end{tabular} & 100 & $\begin{array}{r}1 \\
7 \\
3 \\
3 \\
1000\end{array}$ & \begin{tabular}{c|}
$K$ \\
28130.70 \\
28134.50 \\
28135.67 \\
28135.98 \\
28142.55
\end{tabular} & $\begin{array}{c}K \\
-0.02 \\
-0.02\end{array}$ & $\begin{array}{l}67416_{4 \frac{1}{2}}-95550_{4 \frac{1}{2}}^{\circ} \\
31568_{2 \frac{1}{2}}^{\circ}-59710_{1 \frac{1}{2}}^{\circ}\end{array}$ & 5 & 0.854 & 0.810 & $(0.022)$ & 0.92 \\
\hline $\begin{array}{l}3549.822 \\
3548.470 \\
3548.096 \\
3546.085 \\
3545.725\end{array}$ & 200 & $\begin{array}{r}2000 \\
2 \\
7 \\
1 \\
40 h\end{array}$ & $\begin{array}{l}28162.38 \\
28173.11 \\
28176.08 \\
28192.06 \\
28194.92\end{array}$ & $\begin{array}{r}+0.01 \\
-0.02 \\
\left\{\begin{array}{r}-0.01 \\
0.00 \\
0.00\end{array}\right.\end{array}$ & $\begin{array}{l}33052_{4 \frac{1}{2}}^{\circ}-61214_{4 \frac{1}{2}} \\
44940_{1 \frac{1}{2}}^{\circ}-73116_{1 \frac{1}{2}} \\
53120_{2 \frac{1}{2}}^{\circ}-81312_{2 \frac{1}{2}} \\
71222_{3 \frac{1}{2}}^{\circ}-99414_{4 \frac{1}{2}}^{\circ} \\
43075_{1 \frac{1}{2}}^{\circ}-71270_{2 \frac{1}{2}}\end{array}$ & 6 & 1.264 & 1.050 & 0.980 & 1.167 \\
\hline $\begin{array}{l}3543.890 \\
3543.324 \\
3543.159 \\
3542.385 \\
3542.272\end{array}$ & $\begin{array}{r}1 \\
30 \\
7 \\
1\end{array}$ & $\begin{array}{r}1 \\
10 \\
200 \\
100 \\
6\end{array}$ & $\begin{array}{l}28209.52 \\
28214.03 \\
28215.34 \\
28221.50 \\
28222.40\end{array}$ & $\begin{array}{r}0.00 \\
-0.02 \\
-0.05\end{array}$ & $\begin{array}{l}46354_{4 \frac{1}{2}}^{\circ}-74568_{4 \frac{1}{2}} \\
32371_{2 \frac{1}{2}}^{\circ}-60586_{1 \frac{1}{2}} \\
47329_{2 \frac{1}{2}}^{\circ}-75550_{2 \frac{1}{2}}^{\circ} \\
64055_{2 \frac{1}{2}}-92278_{1_{\frac{1}{2}}^{2}}^{\circ}\end{array}$ & $\begin{array}{l}4 \\
6\end{array}$ & $\begin{array}{l}1.202 \\
0.990\end{array}$ & $\begin{array}{l}1.370 \\
0.902\end{array}$ & $\begin{array}{l}0.084 \\
0.22\end{array}$ & $\begin{array}{l}0.960 \\
0.946\end{array}$ \\
\hline $\begin{array}{l}3541.375 \\
3540.926 \\
3540.61 \\
3540.21 \\
3540.09\end{array}$ & 7 & $\begin{array}{r}80 \\
3 \\
1 \\
2 \\
1\end{array}$ & $\begin{array}{l}28229.55 \\
28233.13 \\
28235.65 \\
28238.84 \\
28239.80\end{array}$ & $\begin{array}{l}-0.01 \\
-0.05 \\
\end{array}$ & $\begin{array}{l}38342_{4 \frac{1}{2}}^{\circ}-66571_{5 \frac{1}{2}} \\
68148_{5 \frac{1}{2}}-96382_{5 \frac{1}{2}}^{\circ}\end{array}$ & 5 & (1.093) & 1.18 & $w$ & 1.58 \\
\hline $\begin{array}{l}3540.02 \\
3539.932 \\
3539.362 \\
3538.369 \\
3537.964\end{array}$ & 20 & $\begin{array}{r}1 \\
3 \\
200 \\
2 \\
1\end{array}$ & $\begin{array}{l}28240.36 \\
28241.06 \\
28245.61 \\
28253.53 \\
28256.77\end{array}$ & $\begin{array}{l}-0.04 \\
-0.01 \\
+0.08 \\
-0.03\end{array}$ & $\begin{array}{l}61374_{2 \frac{1}{2}}-89615_{2 \frac{1}{2}}^{\circ} \\
43956_{2 \frac{1}{2}}^{\circ}-72202_{3 \frac{1}{2}}^{\circ} \\
66082_{5 \frac{1}{2}}-94335_{5 \frac{1}{2}}^{\circ} \\
56375_{2 \frac{1}{2}}-84632_{2 \frac{1}{2}}^{\circ}\end{array}$ & 4 & 0.990 & 0.865 & 0.067 & 0.530 \\
\hline $\begin{array}{l}3537.127 \\
3536.068\end{array}$ & 1 & $\begin{array}{l}6 \\
8\end{array}$ & $\begin{array}{l}28263.45 \\
28271.92\end{array}$ & $\begin{array}{r}-0.02 \\
0.00\end{array}$ & $\begin{array}{l}65199_{4 \frac{1}{2}}-93463_{3 \frac{1}{2}}^{\circ} \\
64923_{4 \frac{1}{2}}-93195_{3 \frac{1}{2}}^{\circ}\end{array}$ & & & & & \\
\hline 3534.752 & 4 & 20 & 28282.44 & $\left\{\begin{array}{l}-0.04 \\
+0.01\end{array}\right.$ & $\begin{array}{l}44497_{2 \frac{1}{2}}^{\circ}-72779_{3 \frac{1}{2}}^{\circ} \\
48556_{3 \frac{1}{2}}^{\circ}-76839_{2 \frac{1}{2}}\end{array}$ & & & & & \\
\hline $\begin{array}{l}3534.13 \\
3534.05\end{array}$ & 50 & $\begin{array}{r}2 \\
100\end{array}$ & $\begin{array}{l}28287.42 \\
28288.06\end{array}$ & $\begin{array}{r}-0.04 \\
0.00\end{array}$ & $\begin{array}{l}65577_{3 \frac{1}{2}}-93864_{2 \frac{1}{2}}^{\circ} \\
22960_{1 \frac{1}{2}}-51248_{2 \frac{1}{2}}^{\circ}\end{array}$ & 5 & 0.815 & 1.041 & 0.113 & 1.380 \\
\hline $\begin{array}{l}3533.80 \\
3533.107\end{array}$ & 20 & $\begin{array}{r}3 \\
60\end{array}$ & $\begin{array}{l}28290.06 \\
28295.61\end{array}$ & $\begin{array}{r}-0.05 \\
0.00\end{array}$ & $\begin{array}{l}65577_{3 \frac{1}{2}}-93867_{4 \frac{1}{2}}^{\circ} \\
59090_{3 \frac{1}{2}}-87385_{3 \frac{1}{2}}^{\circ}\end{array}$ & 6 & 1.12 & 1.14 & $d$ & 1.13 \\
\hline $\begin{array}{l}3531.24 \\
3530.58 \\
3530.16\end{array}$ & 5 & $\begin{array}{r}100 \\
1 \\
3\end{array}$ & $\begin{array}{l}28310.57 \\
28315.86 \\
28319.23\end{array}$ & $\begin{array}{l}-0.05 \\
-0.01 \\
-0.05\end{array}$ & $\begin{array}{l}47329_{2 \frac{1}{2}}^{\circ}-75640_{3 \frac{1}{2}}^{\circ} \\
64598_{3 \frac{1}{2}}-92914_{4}^{\circ} \\
64923_{4 \frac{1}{2}}-93242_{5 \frac{1}{2}}^{\circ}\end{array}$ & 4 & 0.995 & 0.813 & 0.091 & 0.358 \\
\hline $\begin{array}{l}3530.05 \\
3529.715 \\
3529.458 \\
3529.085\end{array}$ & 8 & $\begin{array}{r}1 \\
20 \\
2 \\
100\end{array}$ & $\begin{array}{l}28320.12 \\
28322.80 \\
28324.87 \\
28327.86\end{array}$ & $\begin{array}{l}-0.04 \\
-0.04 \\
-0.02 \\
-0.04\end{array}$ & $\begin{array}{l}61822_{3 \frac{1}{2}}-90142_{3 \frac{1}{2}}^{\circ} \\
68720_{6 \frac{1}{2}}^{\circ}-97043_{6 \frac{1}{2}}^{\circ} \\
52987_{1 \frac{1}{2}}^{\circ}-81312_{2 \frac{1}{2}} \\
33494_{3 \frac{1}{2}}^{\circ}-61822_{3 \frac{1}{2}}\end{array}$ & 6 & 1.001 & 1.340 & 1.185 & 1.171 \\
\hline
\end{tabular}


TABLE 6. Y $\mathrm{b}$ II-Observed and classified lines-Continued

\begin{tabular}{|c|c|c|c|c|c|c|c|c|c|c|}
\hline 1 & 2 & 3 & 4 & 5 & 6 & 7 & 8 & 9 & 10 & 11 \\
\hline \multirow{2}{*}{$\begin{array}{l}\text { Wave- } \\
\text { length } \\
\text { air }\end{array}$} & \multicolumn{2}{|c|}{ Intensity } & \multirow{2}{*}{$\begin{array}{l}\text { Wave } \\
\text { number }\end{array}$} & \multirow{2}{*}{$0-c$} & \multirow{2}{*}{ Classification } & \multirow{2}{*}{ Zee- } & \multirow{2}{*}{ lst $g$} & \multirow{2}{*}{ 2nd $g$} & \multicolumn{2}{|c|}{$\begin{array}{l}\text { Zeeman } \\
\text { components }\end{array}$} \\
\hline & $\begin{array}{c}\text { Meggers } \\
\text { lamp }\end{array}$ & $\begin{array}{c}\text { Thomson } \\
\text { lamp }\end{array}$ & & & & & & & Strong $p$ & Strong $n$ \\
\hline $\begin{array}{c}\text { A } \\
3528.771\end{array}$ & & 2 & $\begin{array}{c}K \\
28330.38\end{array}$ & $\begin{array}{c}K \\
-0.09\end{array}$ & $68756_{3 \frac{1}{2}}-97086_{4 \frac{1}{2}}^{\circ}$ & & & & & \\
\hline $\begin{array}{l}3527.501 \\
3527.332 \\
3526.477 \\
3525.722 \\
3525.456\end{array}$ & $\begin{array}{l}1 \\
2\end{array}$ & $\begin{array}{r}15 \\
20 \\
1 \\
50 \\
3\end{array}$ & $\begin{array}{l}28340.58 \\
28341.94 \\
28348.81 \\
28354.88 \\
28357.02\end{array}$ & $\begin{array}{l}-0.01 \\
-0.02 \\
+0.01 \\
-0.01 \\
-0.07\end{array}$ & $\begin{array}{l}41688_{1 \frac{1}{2}}^{\circ}-70029_{0 \frac{1}{2}} \\
44438_{3 \frac{1}{2}}^{\circ}-72779_{3 \frac{1}{2}}^{\circ} \\
68549_{4 \frac{1}{2}}-96898_{5 \frac{1}{2}}^{\circ} \\
64891_{7 \frac{1}{2}}-93246_{6 \frac{1}{2}}^{\circ} \\
51248_{2 \frac{1}{2}}^{\circ}-79605_{1 \frac{1}{2}}^{\circ}\end{array}$ & $\begin{array}{l}7 \\
7 \\
7 ?\end{array}$ & $\begin{array}{l}(0.784) \\
1.10 \\
(1.19)\end{array}$ & $\begin{array}{l}0.736 \\
1.10 \\
1.21\end{array}$ & $\begin{array}{l}0 \\
0 \\
w\end{array}$ & $\begin{array}{l}0.808 \\
1.105 \\
1.08\end{array}$ \\
\hline $\begin{array}{l}3525.171 \\
3525.05 \\
3524.82 \\
3524.044 \\
3523.734\end{array}$ & & $\begin{array}{l}2 \\
1 \\
1 \\
1 \\
3 h\end{array}$ & $\begin{array}{l}28359.31 \\
28360.28 \\
28362.13 \\
28368.38 \\
28370.88\end{array}$ & $\begin{array}{l}-0.04 \\
-0.05\end{array}$ & $\begin{array}{l}66462_{0 \frac{1}{2}}-94823_{1 \frac{1}{2}}^{\circ} \\
63234_{2 \frac{1}{2}}-91602_{2 \frac{1}{2}}^{\circ}\end{array}$ & & & & & \\
\hline $\begin{array}{l}3523.148^{\mathrm{a}} \\
3522.826 \\
3522.747 \\
3522.021 \\
3521.727\end{array}$ & 1 & $\begin{array}{r}2 \\
3 \\
10 \\
1 \\
3\end{array}$ & $\begin{array}{l}28375.59 \\
28378.19 \\
28378.82 \\
28384.67 \\
28387.04\end{array}$ & $\begin{array}{l}-0.03 \\
-0.02 \\
-0.08 \\
-0.05\end{array}$ & $\begin{array}{l}64598_{3 \frac{1}{2}}-92976_{3 \frac{1}{2}}^{\circ} \\
34784_{5 \frac{1}{2}}^{\circ}-63163_{4 \frac{1}{2}} \\
65950_{4 \frac{1}{2}}-94335_{5 \frac{1}{2}}^{\circ} \\
61822_{3 \frac{1}{2}}-90209_{4 \frac{1}{2}}^{\circ}\end{array}$ & & & & & \\
\hline $\begin{array}{l}3521.664 \\
3521.585 \\
3521.224 \\
3521.007 \\
3520.917\end{array}$ & 5 & $\begin{array}{r}2 \\
7 \\
7 \\
20 \\
10\end{array}$ & $\begin{array}{l}28387.55 \\
28388.19 \\
29391.10 \\
28392.85 \\
28393.57\end{array}$ & $\left\{\begin{array}{r}0.00 \\
+0.07 \\
-0.04 \\
-0.05 \\
-0.04\end{array}\right.$ & $\begin{array}{l}70858_{4 \frac{1}{2}}-99246_{5 \frac{1}{2}}^{\circ} \\
52921_{3 \frac{1}{2}}^{\circ}-81312_{2 \frac{1}{2}}^{\circ} \\
61214_{4 \frac{1}{2}}-89605_{4 \frac{1}{2}}^{\circ} \\
32981_{1 \frac{1}{2}}^{\circ}-61374_{2 \frac{1}{2}} \\
43075_{1 \frac{1}{2}}^{\circ}-71468_{2 \frac{1}{2}}^{\circ}\end{array}$ & & & & & \\
\hline $\begin{array}{l}3520.293 \\
3519.690 \\
3519.417 \\
3519.10 \\
3518.89\end{array}$ & $\begin{array}{c}600 r \\
5\end{array}$ & $\begin{array}{r}3000 \\
2 \\
50 \\
1 \\
1\end{array}$ & $\begin{array}{l}28398.61 \\
28403.47 \\
28405.67 \\
28408.23 \\
28409.93\end{array}$ & $\begin{array}{l}+0.03 \\
-0.07 \\
-0.01\end{array}$ & $\begin{array}{l}30562_{5 \frac{1}{2}}^{\circ}-58961_{5 \frac{1}{2}} \\
48272_{2 \frac{1}{2}}^{\circ}-76676_{2 \frac{1}{2}} \\
61873_{6 \frac{1}{2}}-90279_{5 \frac{1}{2}}^{\circ}\end{array}$ & 7 & $\begin{array}{l}1.111 \\
(1.11)\end{array}$ & $\begin{array}{l}0.972 \\
1.11\end{array}$ & $\begin{array}{l}0.770 \\
0\end{array}$ & $\begin{array}{l}1.043 \\
1.096\end{array}$ \\
\hline $\begin{array}{l}3518.795 \\
3518.674 \\
3518.353 \\
3518.156 \\
3516.60\end{array}$ & $\begin{array}{r}1 \\
20\end{array}$ & $\begin{array}{r}3 \\
1 \\
10 \\
300 \\
1\end{array}$ & $\begin{array}{l}28410.70 \\
28411.67 \\
28414.26 \\
28415.86 \\
28428.43\end{array}$ & $\begin{array}{l}-0.05 \\
-0.04 \\
-0.03 \\
-0.01\end{array}$ & $\begin{array}{l}65093_{3 \frac{1}{2}}-93503_{4 \frac{1}{2}}^{\circ} \\
47758_{3 \frac{1}{2}}^{\circ}-76170_{2 \frac{1}{2}} \\
40035_{3 \frac{1}{2}}^{\circ}-68450_{3 \frac{1}{2}} \\
45335_{4 \frac{1}{2}}^{\circ}-73750_{4 \frac{1}{2}}^{\circ}\end{array}$ & 7 & 1.00 & 1.00 & 0 & 1.00 \\
\hline 3516.180 & & 2 & 28431.82 & $\left\{\begin{array}{l}+0.02 \\
-0.03\end{array}\right.$ & $\begin{array}{l}65566_{0 \frac{1}{2}}-93998_{1 \frac{1}{2}}^{\circ} \\
65577_{3 \frac{1}{2}}-94008_{3 \frac{1}{2}}^{\circ}\end{array}$ & & & & & \\
\hline $\begin{array}{l}3515.865 \\
3515.610 \\
3515.138 \\
3513.640\end{array}$ & $\begin{array}{r}30 \\
10 \\
1\end{array}$ & $\begin{array}{r}300 \\
50 \\
15 \\
4\end{array}$ & $\begin{array}{l}28434.37 \\
28436.43 \\
28440.25 \\
28452.38\end{array}$ & $\begin{array}{l}+0.01 \\
-0.05 \\
-0.05 \\
-0.03\end{array}$ & $\begin{array}{l}37516_{3 \frac{1}{2}}^{\circ}-65950_{4 \frac{1}{2}}^{\circ} \\
34575_{\frac{1}{2}}^{\circ}-63011_{1 \frac{1}{2}}^{\circ} \\
62861_{5 \frac{1}{2}}-91301_{6 \frac{1}{2}}^{\circ}-9246_{5 \frac{1}{2}}^{\circ} \\
70793_{5 \frac{1}{2}}^{\circ}-99\end{array}$ & $\begin{array}{l}4 \\
6\end{array}$ & $\begin{array}{l}(1.119) \\
1.450\end{array}$ & $\begin{array}{l}1.09 \\
0.780\end{array}$ & $\begin{array}{l}w \\
0.90\end{array}$ & $\begin{array}{l}0.99 \\
1.120\end{array}$ \\
\hline $\begin{array}{l}3513.412 \\
3512.708\end{array}$ & 2 & $\begin{array}{r}2 \\
20\end{array}$ & $\begin{array}{l}28454.22 \\
28459.93\end{array}$ & 0.00 & $65875_{6 \frac{1}{2}}-94335_{5 \frac{1}{2}}^{\circ}$ & & & & & \\
\hline
\end{tabular}


TABLE 6. Yb II-Observed and classified limes-Continued

\begin{tabular}{|c|c|c|c|c|c|c|c|c|c|c|}
\hline 1 & 2 & 3 & 4 & 5 & 6 & 7 & 8 & 9 & 10 & 11 \\
\hline \multirow{2}{*}{$\begin{array}{l}\text { Wave- } \\
\text { leength } \\
\text { air }\end{array}$} & \multicolumn{2}{|c|}{ Intensity } & \multirow{2}{*}{$\begin{array}{l}\text { Wave } \\
\text { number }\end{array}$} & \multirow{2}{*}{$a-c$} & \multirow{2}{*}{ Classiffucation } & \multirow{2}{*}{ Zee- } & \multirow{2}{*}{ lst $g$} & \multirow{2}{*}{2 nd $g$} & \multicolumn{2}{|c|}{$\begin{array}{c}\text { Zeeman } \\
\text { components }\end{array}$} \\
\hline & $\begin{array}{l}\text { Meggers } \\
\text { lamp }\end{array}$ & $\begin{array}{c}\text { Thomsom } \\
\text { lamp }\end{array}$ & & & & & & & Strong $p$ & Strong $n$ \\
\hline $\begin{array}{c}\AA \\
3512.441 \\
3511.283 \\
3510.942\end{array}$ & & $\begin{array}{l}6 \\
8 \\
3\end{array}$ & \begin{tabular}{c|}
$K$ \\
28462.09 \\
28471.48 \\
28474.24
\end{tabular} & $K$ & & & & & & \\
\hline $\begin{array}{l}3510.500 \\
3509.86 \\
3509.776 \\
3508.95 \\
3507.830\end{array}$ & 200 & $\begin{array}{r}15 \\
10 \\
3 \\
1 \\
2000\end{array}$ & $\begin{array}{l}28477.83 \\
28483,02 \\
28483.70 \\
28490.40 \\
28499.50\end{array}$ & $\begin{array}{l}-0.05 \\
-0.06 \\
-0.08 \\
-0.02\end{array}$ & $\begin{array}{l}45273_{3 \frac{1}{2}}^{\circ}-73750_{4 \frac{1}{2}} \\
33653_{0 \frac{1}{2}}^{\circ}-62136_{0 \frac{1}{2}}^{\circ} \\
70136_{6 \frac{1}{2}}-98620_{6 \frac{1}{2}}^{\circ} \\
37077_{2 \frac{1}{2}}^{\circ}-65577_{3 \frac{1}{2}}^{\circ}\end{array}$ & 4 & 1.12 & 1.06 & $(0.028)$ & 0.92 \\
\hline $\begin{array}{l}3506.125 \\
3505.282 \\
3504.936 \\
3504.542 \\
3503.690\end{array}$ & $\begin{array}{r}8 \\
50 \\
3 \\
1 \\
4\end{array}$ & $\begin{array}{r}200 \\
150 \\
15 \\
15 \\
40\end{array}$ & $\begin{array}{l}28513.36 \\
28520.22 \\
28523.03 \\
28526.24 \\
28533.18\end{array}$ & $\begin{array}{l}-0.05 \\
-0.02 \\
-0.03 \\
-0.06 \\
-0.01\end{array}$ & $\begin{array}{l}40035_{3 \frac{1}{2}}^{\circ}-68549_{4 \frac{1}{2}} \\
33653_{0 \frac{1}{2}}^{\circ}-62174_{1 \frac{1}{2}}^{\circ} \\
61374_{2 \frac{1}{2}}-89897_{1 \frac{1}{2}}^{\circ} \\
48272_{2 \frac{1}{2}}^{\circ}-76799_{1 \frac{1}{2}} \\
45737^{\circ}\end{array}$ & $\begin{array}{l}5 \\
4\end{array}$ & $\begin{array}{l}0.714 \\
1.30\end{array}$ & $\begin{array}{l}1.160 \\
0.80\end{array}$ & $\begin{array}{l}0.223 \\
0.26\end{array}$ & $\begin{array}{l}2.72 \\
0.54\end{array}$ \\
\hline $\begin{array}{l}3503.277 \\
3503.190 \\
3503.011 \\
3502.572 \\
3502.180\end{array}$ & 10 & $\begin{array}{c}2 \\
3 \\
1 \\
4 h \\
100\end{array}$ & $\begin{array}{l}28536.54 \\
28537.25 \\
28538.71 \\
28542.28 \\
28545.48\end{array}$ & $\begin{array}{r}-0.01 \\
-0.03 \\
0.00\end{array}$ & $\begin{array}{l}53120_{2 \frac{1}{2}}^{\circ}-81657_{2 \frac{1}{2}}^{\circ} \\
68549_{4 \frac{1}{2}}-97086_{4 \frac{1}{2}}^{\circ} \\
47005_{1 \frac{1}{2}}^{\circ}-75550_{2 \frac{1}{2}}^{\circ}\end{array}$ & 4 & 1.00 & 0.92 & $(0.04)$ & 0.80 \\
\hline $\begin{array}{l}3500.863 \\
3500.131 \\
3499.886 \\
3499.85 \\
3499.791\end{array}$ & 10 & $\begin{array}{r}5 \\
30 \\
3 \\
1 \\
2\end{array}$ & $\begin{array}{l}28556.22 \\
28562.19 \\
28564.19 \\
28564.48 \\
28564.96\end{array}$ & $\begin{array}{l}-0.02 \\
+0.03 \\
-0.05 \\
-0.05 \\
+0.01\end{array}$ & $\begin{array}{l}70858_{4 \frac{1}{2}}-99414_{4 \frac{1}{2}}^{\circ} \\
58823_{2 \frac{1}{2}}-87385_{3 \frac{1}{2}}^{\circ} \\
69957_{4 \frac{1}{2}}-98521_{4 \frac{1}{2}}^{\circ} \\
61051_{3 \frac{1}{2}}-89615_{2 \frac{1}{2}}^{\circ} \\
47758_{3 \frac{1}{2}}^{\circ}-76323_{3 \frac{1}{2}}^{\circ}\end{array}$ & & & & & \\
\hline $\begin{array}{l}3499,636 \\
3499.419\end{array}$ & $\begin{array}{l}1 \\
5\end{array}$ & $\begin{array}{r}9 \\
10\end{array}$ & $\begin{array}{l}28566.23 \\
28568.00\end{array}$ & 0.00 & $48272_{2 \frac{1}{2}}^{\circ}-76839_{2 \frac{1}{2}}^{\circ}$ & & & & & \\
\hline $\begin{array}{l}3498.392 \\
3497.86 \\
3496.858\end{array}$ & 5 & $\begin{array}{r}40 \\
2 \\
20\end{array}$ & $\begin{array}{l}28576.38 \\
28580.73 \\
28588.92\end{array}$ & $\begin{array}{l}\left\{\begin{array}{l}+0.04 \\
-0.03\end{array}\right. \\
-0.05 \\
-0.01\end{array}$ & $\begin{array}{l}39378_{0 \frac{1}{2}}^{\circ}-67955_{0 \frac{1}{2}} \\
63726_{4 \frac{1}{2}}-92303_{3 \frac{1}{2}}^{\circ} \\
64923_{4 \frac{1}{2}}-93503_{4 \frac{1}{2}}^{\circ} \\
24332_{2 \frac{1}{2}}-52921_{3 \frac{1}{2}}^{\circ}\end{array}$ & & & & & \\
\hline $\begin{array}{l}3495.90 \\
3495.780 \\
3493.154 \\
3492.807 \\
3491.624\end{array}$ & $\begin{array}{l}1 \\
5 \\
6\end{array}$ & $\begin{array}{r}50 \\
3 \\
15 \\
30 \\
100\end{array}$ & $\begin{array}{l}28596.76 \\
28597.74 \\
28619.23 \\
28622.08 \\
28631.77\end{array}$ & $\begin{array}{l}+0.01 \\
+0.09 \\
+0.03 \\
-0.01 \\
-0.01\end{array}$ & $\begin{array}{l}64598_{3 \frac{1}{2}}-93195_{3 \frac{1}{2}}^{\circ} \\
49008_{3 \frac{1}{2}}^{\circ}-77606_{2 \frac{1}{2}}^{\circ} \\
44497_{2 \frac{1}{2}}^{\circ}-73116_{1 \frac{1}{2}}^{\circ} \\
34389_{2 \frac{1}{2}}^{\circ}-63011_{1 \frac{1}{2}}^{\circ} \\
45335_{4 \frac{1}{2}}^{\circ}-73966_{3 \frac{1}{2}}^{\circ}\end{array}$ & & & & & \\
\hline $\begin{array}{l}3491.063 \\
3490.880 \\
3490.58 \\
3490.213\end{array}$ & 1 & $\begin{array}{l}3 \\
2 h \\
1 \\
20\end{array}$ & $\begin{array}{l}28636.38 \\
28637.88 \\
28640.34 \\
28643.35\end{array}$ & $\begin{array}{l}-0.02 \\
+0.09 \\
+0.03\end{array}$ & $\begin{array}{l}68450_{3 \frac{1}{2}}-97086_{4 \frac{1}{2}}^{\circ} \\
27061_{0 \frac{1}{2}}^{\circ}-55702_{1 \frac{1}{2}}^{\circ} \\
35059_{3 \frac{1}{2}}^{\circ}-63702_{2 \frac{1}{2}}\end{array}$ & & & & & \\
\hline $\begin{array}{l}3488.786 \\
3488.43\end{array}$ & 200 & $\begin{array}{l}700 \\
10 h\end{array}$ & $\begin{array}{l}28655.06 \\
28657.99\end{array}$ & -0.01 & $24332_{2 \frac{1}{2}}-52987_{1 \frac{1}{2}}^{-2}$ & 5 & 1.18 & 1.12 & $(0.03)$ & 1.27 \\
\hline
\end{tabular}


TABLE 6. Yb II-Observed and classified lines-Continued

\begin{tabular}{|c|c|c|c|c|c|c|c|c|c|c|}
\hline 1 & 2 & 3 & 4 & 5 & 6 & 7 & 8 & 9 & 10 & 11 \\
\hline \multirow{2}{*}{$\begin{array}{l}\text { Wave- } \\
\text { length } \\
\text { air }\end{array}$} & \multicolumn{2}{|c|}{ Intensity } & \multirow{2}{*}{$\begin{array}{l}\text { Wave } \\
\text { number }\end{array}$} & \multirow[b]{2}{*}{$\mathrm{o}-\mathrm{c}$} & \multirow{2}{*}{ Classification } & \multirow{2}{*}{$\begin{array}{l}\text { Zee- } \\
\text { man } \\
\text { type }\end{array}$} & \multirow{2}{*}{ lst $g$} & \multirow{2}{*}{ 2nd $g$} & \multicolumn{2}{|c|}{$\begin{array}{l}\text { Zeeman } \\
\text { components }\end{array}$} \\
\hline & $\begin{array}{l}\text { Meggers } \\
\text { lamp }\end{array}$ & $\begin{array}{c}\text { Thomson } \\
\text { lamp }\end{array}$ & & & & & & & Strong $p$ & Strong $n$ \\
\hline$\AA$ & & & $K$ & $K$ & & & & & & \\
\hline 3488.34 & 6 & 50 & 28658.73 & $\left\{\begin{array}{l}-0.01 \\
-0.05\end{array}\right.$ & $\begin{array}{l}34575_{1 \frac{1}{2}}^{\circ}-63234_{2 \frac{1}{2}} \\
50468^{\circ}-79126^{2}\end{array}$ & & & & & \\
\hline 3487.402 & & 3 & 28666.44 & +0.02 & $70136_{6 \frac{1}{2}}-98802_{5 \frac{1}{2}}^{\frac{1}{2}}$ & & & & & \\
\hline 3487.233 & 6 & 100 & 28667.83 & 0.00 & $35059_{3 \frac{1}{2}}^{\circ}-63726_{4 \frac{1}{2}}$ & & & & & \\
\hline 3487.041 & & 2 & 28669.40 & +0.03 & $52987_{1 \frac{1}{2}}^{\circ}-81657_{2 \frac{1}{2}}^{\circ}$ & & & & & \\
\hline 3486.137 & 1 & 8 & 28676.84 & +0.03 & $47228_{0 \frac{1}{2}}^{\circ}-75905_{0 \frac{1}{2}}$ & & & & & \\
\hline 3485.757 & 200 & 1500 & 28679.96 & 0.00 & $32371_{2 \frac{1}{2}}^{\circ}-61051_{3 \frac{1}{2}}^{\circ}$ & 7 & (1.170) & 1.18 & 0 & 1.21 \\
\hline 3484.656 & & 1 & 28689.03 & & & & & & & \\
\hline $\begin{array}{l}3484.38 \\
3484.143\end{array}$ & 1 & $\begin{array}{r}2 \\
15\end{array}$ & $\begin{array}{l}28691.30 \\
28693.25\end{array}$ & 0.00 & $70136_{6 \frac{1}{2}}-98829_{6 \frac{1}{2}}^{\circ}$ & & & & & \\
\hline $\begin{array}{l}3484.09 \\
3484.06\end{array}$ & & $\begin{array}{l}2 \\
2\end{array}$ & $\begin{array}{l}28693.69 \\
28693.93\end{array}$ & -0.10 & $45273_{3 \frac{1}{2}}^{\circ}-73966_{3 \frac{1}{2}}$ & & & & & \\
\hline $\begin{array}{l}3484.06 \\
3483.782\end{array}$ & 1 & $\begin{array}{r}2 \\
10\end{array}$ & $\begin{array}{l}28693.93 \\
28696.22\end{array}$ & 0.00 & $44940_{1 \frac{1}{1}}^{\circ}-73636_{01}$ & & & & & \\
\hline 3482.942 & 1 & 10 & 28703.14 & -0.02 & $63944_{5 \frac{1}{2}}-92647_{5 \frac{1}{2}}^{0}$ & & & & & \\
\hline 3482.564 & 5 & $30 h$ & 28706.26 & & & & & & & \\
\hline 3482.390 & & 1 & 28707.69 & -0.01 & $35019_{4 \frac{1}{2}}^{\circ}-63726_{4 \frac{1}{2}}$ & & & & & \\
\hline 3481.706 & & 2 & 28713.33 & & & & & & & \\
\hline $\begin{array}{l}3481.302 \\
3479.912\end{array}$ & 1 & $\begin{array}{r}20 \\
2\end{array}$ & $\begin{array}{l}28716.66 \\
28728.14\end{array}$ & -0.02 & $44940_{1 \frac{1}{2}}^{\circ}-73657_{1 \frac{1}{2}}$ & & & & & \\
\hline 3478.835 & $3000 r$ & 10000 & 28737.03 & -0.01 & $30224_{4 \frac{1}{2}}^{\circ}-58961_{5 \frac{1}{2}}$ & 5 & 0.93 & 0.97 & $(0.022)$ & 1.17 \\
\hline 3477.022 & 20 & 30 & 28752.01 & & & & & & & \\
\hline 3476.303 & $10000 \mathrm{~A}$ & 8000 & 28757.96 & -0.02 & $0_{0 \frac{1}{2}}-28757_{1 \frac{1}{2}}^{\circ}$ & 4 & 1.999 & 1.437 & 0.281 & 1.160 \\
\hline $\begin{array}{l}3474.82 \\
3474.512\end{array}$ & 60 & 400 & 28770.23 & -0.06 & $33052_{4 \frac{1}{2}}^{\circ}-61822_{3 \frac{1}{2}}^{\circ \frac{1}{2}}$ & 4 & 1.28 & 1.33 & $(0.026)$ & 1.10 \\
\hline 3473.996 & & 5 & 28777.06 & 0.00 & $61120_{0 \frac{1}{2}}-89897_{1 \frac{1}{2}}^{\circ}$ & & & & & \\
\hline 3473.75 & & 3 & 28779.09 & & & & & & & \\
\hline 3473.01 & & 2 & 28785.23 & & & & & & & \\
\hline 3472.58 & & 1 & 28788.79 & & & & & & & \\
\hline $\begin{array}{l}3472.324 \\
3471.891\end{array}$ & 5 & $\begin{array}{r}20 \\
2\end{array}$ & $\begin{array}{l}28790.91 \\
28794.50\end{array}$ & & & & & & & \\
\hline 3471.46 & & 1 & 28798.08 & & & & & & & \\
\hline 3470.79 & 8 & 80 & 28803.64 & +0.01 & $46169_{4 \frac{1}{2}}^{\circ}-74973_{3 \frac{1}{2}}$ & 5 & (1.10) & 1.09 & $w$ & 1.13 \\
\hline $\begin{array}{l}3469.925 \\
3460.252\end{array}$ & $\begin{array}{l}5 \\
3\end{array}$ & $\begin{array}{r}70 \\
5\end{array}$ & 28810.82 & -0.05 & $37077_{2 \frac{1}{2}}^{0}-65888_{1 \frac{1}{2}}^{2}$ & 4 & 1.117 & 1.209 & 0.046 & 0.979 \\
\hline $\begin{array}{l}3409.253 \\
3468.395\end{array}$ & $\begin{array}{l}3 \\
1\end{array}$ & $\begin{array}{r}5 \\
20\end{array}$ & $\begin{array}{l}28816.40 \\
28823.53\end{array}$ & -0.05 & $43956_{2 \frac{1}{2}}^{\circ}-72779_{3 \frac{1}{2}}$ & & & & & \\
\hline 3468.00 & & $3 h$ & 28826.81 & & & & & & & \\
\hline 3467.066 & 10 & 200 & 28834.57 & -0.05 & $37516_{3 \frac{1}{2}}^{\circ}-66351_{2 \frac{1}{2}}$ & 7 & (1.119) & 1.115 & 0 & 1.128 \\
\hline 3466.346 & & 10 & 28840.56 & -0.10 & $45429_{3 \frac{1}{2}}^{\circ}-74270_{2 \frac{1}{2}}^{\circ}$ & & & & & \\
\hline 3465.898 & 3 & 40 & 28844.29 & -0.06 & $34389_{2 \frac{1}{2}}^{\circ}-63234_{2 \frac{1}{2}}$ & 6 & 1.00 & 1.19 & 0.48 & 1.090 \\
\hline 3465.792 & & 3 & 28845.17 & 0.00 & $69957_{4 \frac{1}{2}}-98802_{5 \frac{1}{2}}^{\circ}$ & & & & & \\
\hline
\end{tabular}


TABLE 6. Yb II-Observed and classified lines-Continued

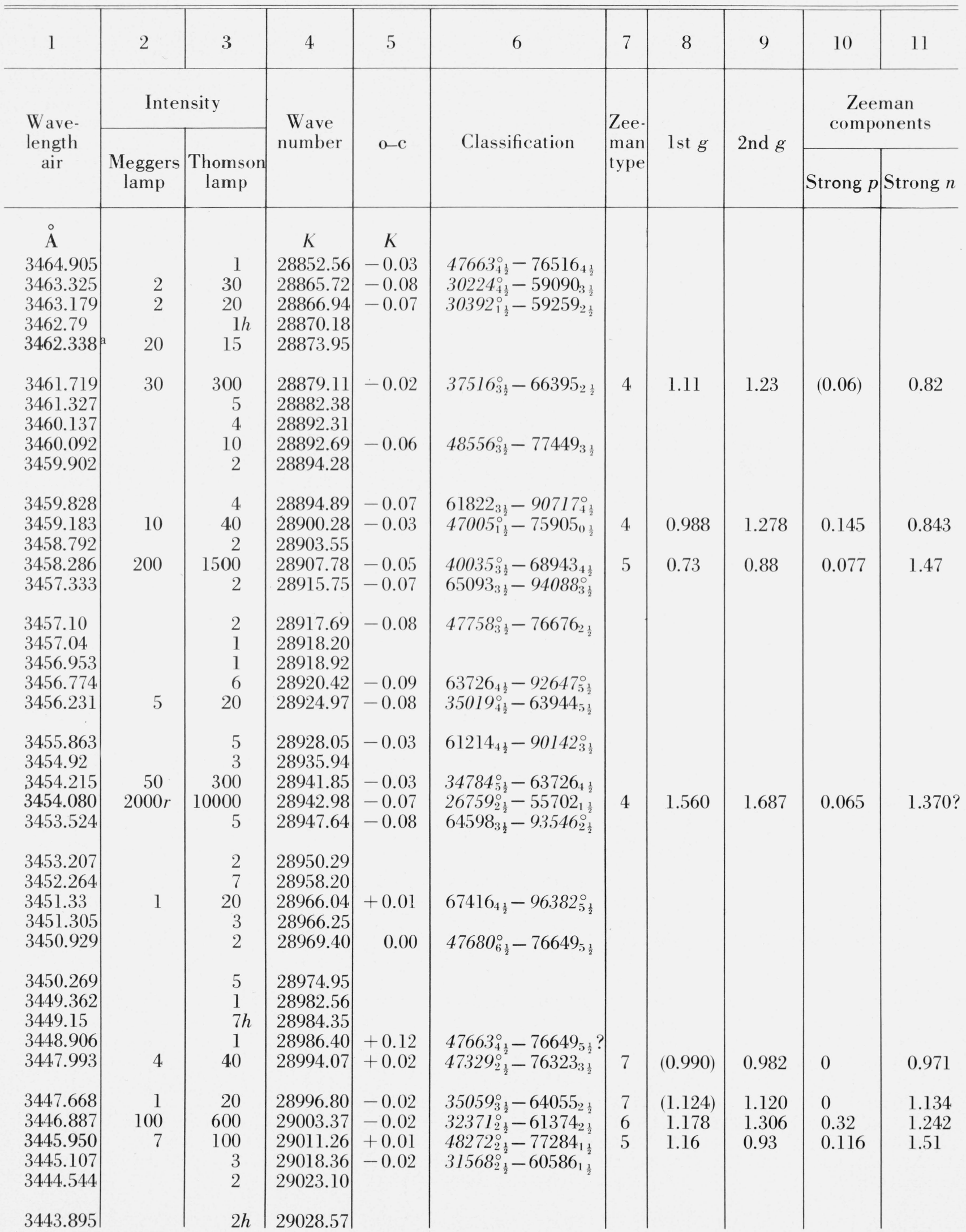


TABLE 6. Yb II-Observed and classified lines-Continued

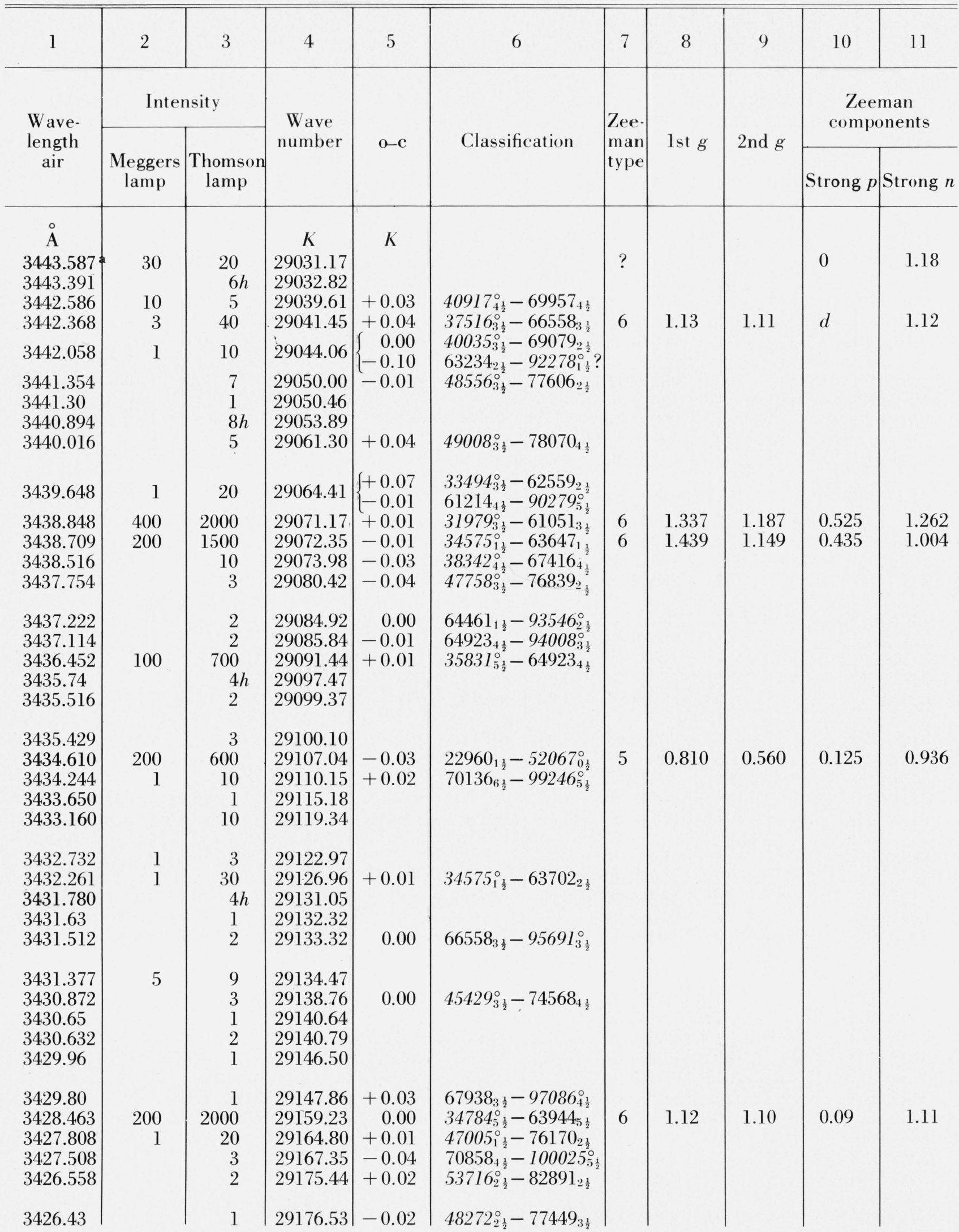


TABLE 6. Yb II-Observed and classified lines-Continued

\begin{tabular}{|c|c|c|c|c|c|c|c|c|c|c|}
\hline 1 & 2 & 3 & 4 & 5 & 6 & 7 & 8 & 9 & 10 & 11 \\
\hline \multirow{2}{*}{$\begin{array}{l}\text { Wave- } \\
\text { length } \\
\text { air }\end{array}$} & \multicolumn{2}{|c|}{ Intensity } & \multirow{2}{*}{$\begin{array}{c}\text { Wave } \\
\text { number }\end{array}$} & \multirow{2}{*}{$\mathrm{o}-\mathrm{c}$} & \multirow{2}{*}{ Classification } & \multirow{2}{*}{$\left|\begin{array}{l}\text { Zee- } \\
\text { man } \\
\text { type }\end{array}\right|$} & \multirow{2}{*}{ lst $g$} & \multirow{2}{*}{2 nd $g$} & \multicolumn{2}{|c|}{$\begin{array}{c}\text { Zeeman } \\
\text { components }\end{array}$} \\
\hline & $\begin{array}{l}\text { Meggers } \\
\text { lamp }\end{array}$ & $\begin{array}{c}\text { Thomson } \\
\text { lamp }\end{array}$ & & & & & & & Strong $p$ & Strong $n$ \\
\hline \begin{tabular}{c}
\multicolumn{1}{c}{ A } \\
3425.71 \\
3424.750 \\
3424.554 \\
3424.182
\end{tabular} & 3 & $\begin{array}{r}1 \\
5 \\
15 \\
1\end{array}$ & $\begin{array}{c}K \\
29182.66 \\
29190.84 \\
29192.51 \\
29195.68\end{array}$ & $\begin{aligned} & K \\
&- 0.06 \\
&+ 0.01 \\
& 0.00\end{aligned}$ & $\begin{array}{l}66571_{5 \frac{1}{2}}-95754_{5 \frac{1}{2}}^{\circ} \\
48556_{3 \frac{1}{2}}^{\circ}-77747_{4 \frac{1}{2}}^{\circ} \\
32981_{1 \frac{1}{2}}^{\circ}-62174_{1 \frac{1}{2}}^{\circ}\end{array}$ & & & & & \\
\hline 3422.454 & & 6 & 29210.42 & $\left\{\begin{array}{l}-0.02 \\
+0.09\end{array}\right.$ & $\begin{array}{l}65093_{3 \frac{1}{2}}-94303_{3 \frac{1}{2}}^{\circ} \\
49916_{4 \frac{1}{2}}^{\circ}-79126_{4 \frac{1}{2}}\end{array}$ & & & & & \\
\hline $\begin{array}{l}3421.409 \\
3419.742\end{array}$ & 2 & $\begin{array}{r}1 \\
20\end{array}$ & $\begin{array}{l}29219.34 \\
29233.59\end{array}$ & +0.04 & $45335_{4 \frac{1}{3}}^{\circ}-74568_{4 \frac{1}{2}}$ & 7 & 1.01 & (1.04) & 0 & 1.023 \\
\hline $\begin{array}{l}3419.606 \\
3418.571\end{array}$ & 20 & $\begin{array}{r}20 \\
1\end{array}$ & $\begin{array}{l}29234.75 \\
29243.60\end{array}$ & $\begin{array}{l}-0.07 \\
-0.01 \\
+0.02\end{array}$ & $\begin{array}{l}31979_{3 \frac{1}{2}}^{\circ}-61214_{4 \frac{1}{2}} \\
485033_{\frac{1}{2}}^{\circ}-77747_{4 \frac{1}{2}}\end{array}$ & 4 & 1.33 & 1.07 & 0.13 & 0.16 \\
\hline 3417.565 & 3 & 30 & 29252.21 & +0.01 & $45737_{1 \frac{1}{2}}^{\circ}-74989_{0 \frac{1}{2}}$ & 5 & 1.36 & 0.54 & 0.41 & 1.77 \\
\hline 3417.345 & 1 & 10 & 29254.09 & +0.02 & $45737_{1 \frac{1}{2}}^{\circ}-74991_{1 \frac{1}{2}}$ & & & & & \\
\hline $\begin{array}{l}3416.894 \\
3416.314\end{array}$ & 40 & $\begin{array}{r}200 \\
1\end{array}$ & $\begin{array}{l}29257.95 \\
29262.92\end{array}$ & -0.02 & $34389_{2 \frac{1}{2}}^{\circ}-63647_{1 \frac{1}{2}}$ & 4 & 1.00 & 1.14 & 0.070 & 0.79 \\
\hline 3415.974 & 10 & 100 & 29265.83 & +0.01 & $41688_{1 \frac{1}{2}}^{\circ}-70954_{2 \frac{1}{2}}^{\circ}$ & 5 & 0.800 & 1.028 & 0.114 & 1.370 \\
\hline 3415.853 & & 2 & 29266.87 & +0.01 & $65093_{3 \frac{1}{2}}-94360_{4 \frac{1}{2}}^{\circ}$ & & & & & \\
\hline $\begin{array}{l}3415.610 \\
3415.062\end{array}$ & 10 & $\begin{array}{r}6 \\
80\end{array}$ & $\begin{array}{l}29268.95 \\
29273.65\end{array}$ & $\begin{array}{l}+0.01 \\
+0.03\end{array}$ & $64598_{3 \frac{1}{2}}-93867_{4 \frac{1}{2}}^{\circ}$ & 7 & 1125 & 1125 & 0 & 125 \\
\hline $\begin{array}{l}3415.002 \\
3414.838\end{array}$ & 20 & 200 & 29275.57 & $\begin{array}{l}+0.03 \\
+0.03\end{array}$ & $\begin{array}{l}31078_{2 \frac{1}{2}}^{\circ}-00351_{2}^{\frac{1}{2}} \\
40954_{2 \frac{1}{2}}\end{array}$ & 6 & $\begin{array}{l}1.125 \\
1.191\end{array}$ & 1.008 & 0.454 & 1.099 \\
\hline 3413.357 & 5 & 15 & 29288.27 & +0.02 & $47228_{0 \frac{1}{2}}^{\circ}-76517_{1 \frac{1}{2}}^{2}$ & 5 & 0.77 & 0.87 & 0.05 & 0.92 \\
\hline 3412.507 & & 5 & 29295.57 & $\left\{\begin{array}{l}+0.01 \\
-0.03\end{array}\right.$ & $\begin{array}{l}45273_{3 \frac{1}{2}}^{\circ}-74568_{4 \frac{1}{2}} \\
663955_{2 \frac{1}{2}}-9591_{3}^{\circ}\end{array}$ & & & & & \\
\hline $3412.453^{\mathrm{a}}$ & 80 & 40 & 29296.03 & & & 7 & & & 0 & 1.13 \\
\hline & 10 & $\begin{array}{l}6 \\
8\end{array}$ & $\begin{array}{l}29302.07 \\
29306.45\end{array}$ & +0.06 & $63944_{5 \frac{1}{2}}-93246_{5_{\frac{1}{2}}}^{\circ}$ & & & & & \\
\hline 3410.880 & & 4 & 29309.54 & +0.07 & $63726_{4 \frac{1}{2}}-93036_{5 \frac{1}{2}}^{\circ}$ & & & & & \\
\hline 3410.689 & 1 & 6 & 29311.18 & +0.09 & $60586_{1 \frac{1}{2}}-89897_{1 \frac{1}{2}}^{\circ}$ & & & & & \\
\hline 3410.566 & 50 & 60 & 29312.24 & +0.04 & $24332_{2 \frac{1}{2}}-53644_{3 \frac{1}{2}}^{\circ}$ & 7 & (1.202) & 1.198 & 0 & 1.188 \\
\hline 3410.487 & 1 & 10 & 29312.92 & +0.05 & $44438_{3 \frac{1}{2}}^{\circ}-73750_{4 \frac{1}{2}}^{\frac{2}{2}}$ & & & & & \\
\hline 3409.875 & 6 & 70 & 29318.18 & +0.05 & $37077_{2 \frac{1}{2}}^{\circ}-66395_{\frac{1}{2}}^{\frac{2}{2}}$ & & & & & \\
\hline 3409.568 & 1 & 10 & 29320.82 & 0.00 & $45737_{1 \frac{1}{2}}^{\circ}-75058_{\frac{1}{2}}^{2}$ & & & & & \\
\hline 3409.022 & & 3 & 29325.51 & +0.04 & $48556_{3 \frac{1}{2}}^{\circ}-77882_{3 \frac{1}{2}}$ & & & & & \\
\hline & 10 & $\begin{array}{r}100 \\
3\end{array}$ & $\begin{array}{l}29329.87 \\
29332.59\end{array}$ & 0.00 & $44940_{1 \frac{1}{2}}^{\circ}-74270_{2 \frac{1}{2}}^{\circ}$ & 4 & 1.234 & 0.920 & 0.157 & 0.449 \\
\hline 3408.058 & & 7 & 29333.81 & 0.00 & $48272_{2 \frac{1}{2}}^{\circ}-77606_{2 \frac{1}{2}}$ & & & & & \\
\hline 3406.536 & 1 & 6 & 29346.91 & +0.04 & $47329_{2 \frac{1}{2}}^{\circ}-76676_{2 \frac{1}{2}}^{0.2}$ & & & & & \\
\hline 3405.79 & & 1 & 29353.34 & & & & & & & \\
\hline $\begin{array}{l}3405.249 \\
3405.093\end{array}$ & 5 & 30 & 29358.00 & +0.01 & $33653_{0 \frac{1}{2}}^{\circ}-63011_{1 \frac{1}{2}}$ & & & & & \\
\hline $\begin{array}{l}3405.093 \\
3405.03\end{array}$ & & $\begin{array}{l}2 \\
1\end{array}$ & $\begin{array}{l}29359.35 \\
29359.89\end{array}$ & -0.03 & $40035_{3 \frac{1}{2}}^{\circ}-69395_{3 \frac{1}{2}}$ & & & & & \\
\hline 3404.103 & 150 & 800 & 29367.89 & +0.03 & $35831_{5 \frac{1}{2}}^{\circ}-65199_{4 \frac{1}{2}}$ & 5 & 1.23 & 1.10 & $(0.065)$ & 1.815 \\
\hline
\end{tabular}


TABLE 6. Yb II-Observed and classified lines-Continued

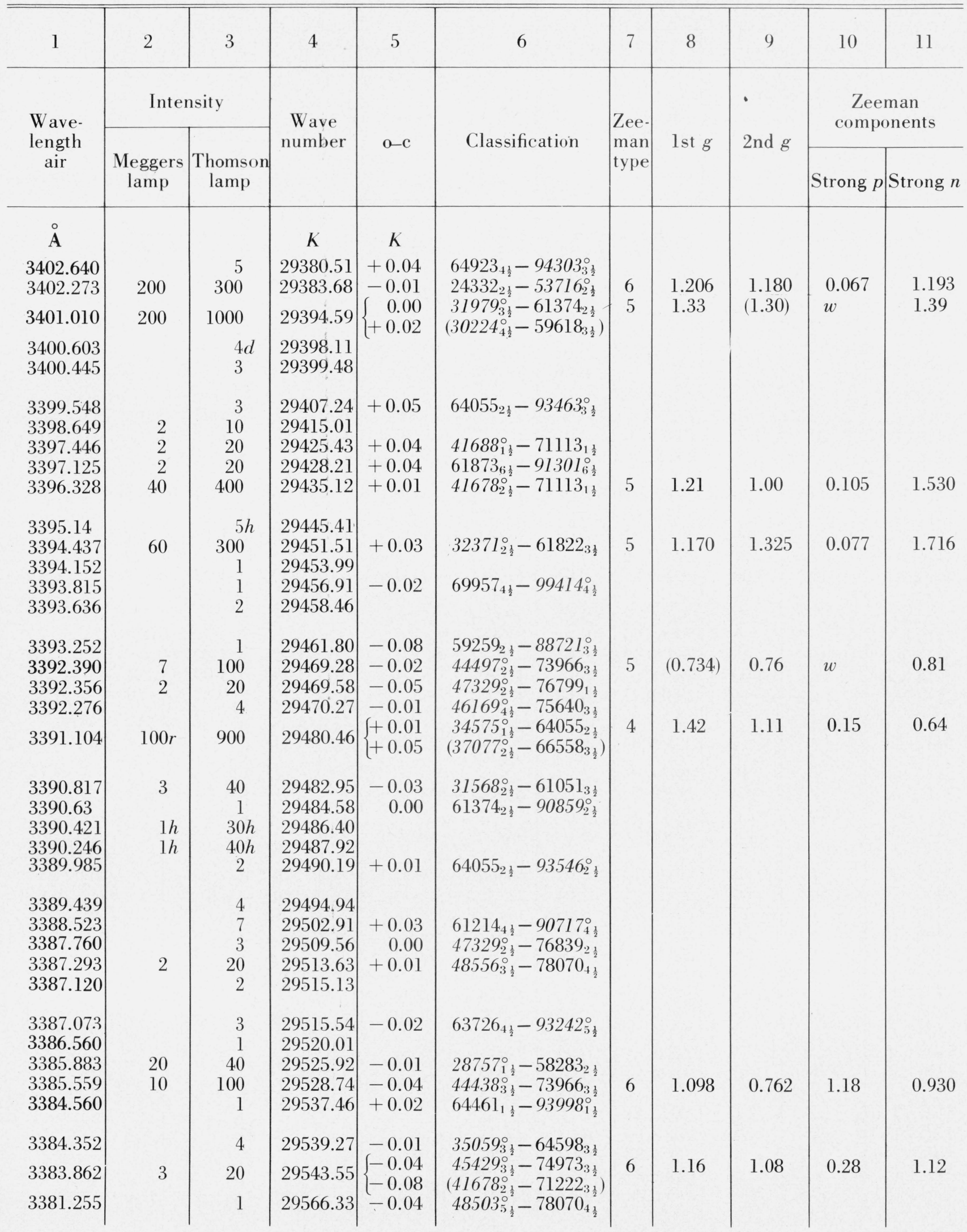


TABLE 6. Yb II-Observed and classified lines-Continued

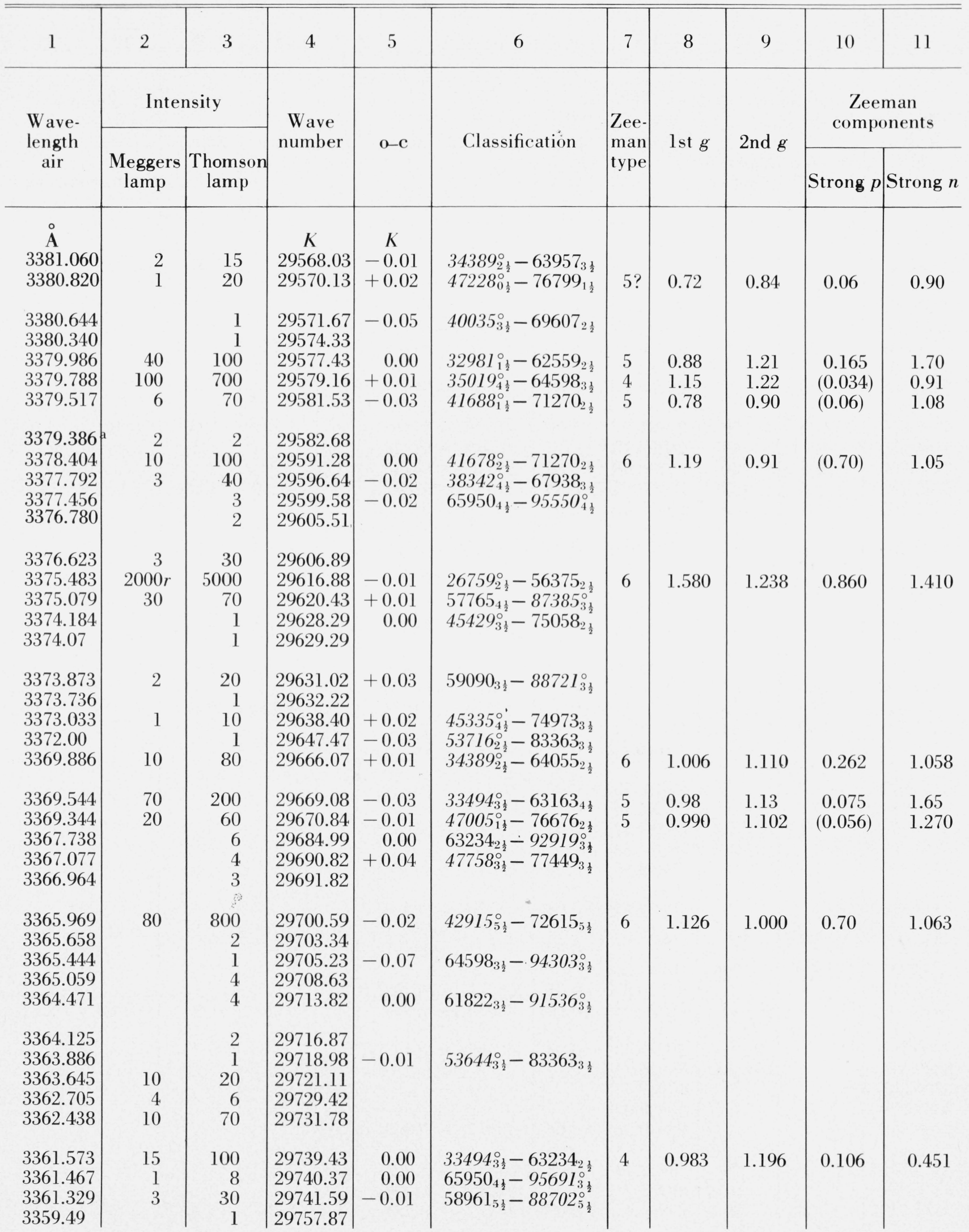


TABLE 6. Yb II-Observed and classified lines-Continued

\begin{tabular}{|c|c|c|c|c|c|c|c|c|c|c|}
\hline 1 & 2 & 3 & 4 & 5 & 6 & 7 & 8 & 9 & 10 & 11 \\
\hline \multirow{2}{*}{$\begin{array}{l}\text { Wave- } \\
\text { length } \\
\text { air }\end{array}$} & \multicolumn{2}{|c|}{ Intensity } & \multirow{2}{*}{$\begin{array}{c}\text { Wave } \\
\text { number }\end{array}$} & \multirow{2}{*}{$o-c$} & \multirow{2}{*}{ Classification } & \multirow{2}{*}{$\begin{array}{l}\text { Zee- } \\
\text { man } \\
\text { type }\end{array}$} & \multirow{2}{*}{ lst $g$} & \multirow{2}{*}{ 2nd $g$} & \multicolumn{2}{|c|}{$\begin{array}{c}\text { Zeeman } \\
\text { components }\end{array}$} \\
\hline & $\begin{array}{c}\text { Meggers } \\
\text { lamp }\end{array}$ & $\begin{array}{c}\text { Thomson } \\
\text { lamp }\end{array}$ & & & & & & & Strong $p$ & Strong $n$ \\
\hline$\AA$ & & & $K$ & $K$ & & & & & & \\
\hline 3359.193 & & $3 h$ & 29760.50 & & & & & & & \\
\hline $\begin{array}{l}3358.612 \\
3357809\end{array}$ & & 2 & 29765.65 & -0.04 & $68756_{3 \frac{1}{2}}-98521_{4 \frac{1}{2}}^{\circ}$ & & & & & \\
\hline $\begin{array}{l}3357.802 \\
3356.966\end{array}$ & $\begin{array}{r}5 \\
20\end{array}$ & $\begin{array}{r}40 \\
200\end{array}$ & $\begin{array}{l}29772.83 \\
29780.25\end{array}$ & $\begin{array}{r}+0.07 \\
0.00\end{array}$ & $\begin{array}{l}56375_{2 \frac{1}{2}}-86148^{\circ} \\
41688_{1}^{\circ}-71468^{2}\end{array}$ & 5 & 0.790 & 1.027 & 0.118 & 1.381 \\
\hline 3356.683 & & 1 & 29782.76 & & & 3 & 0.630 & 1.026 & 0.110 & 1.081 \\
\hline 3356.305 & 6 & 40 & 29786.11 & +0.04 & $62861_{5 \frac{1}{2}}-92647_{5 \frac{1}{2}}^{\circ}$ & & & & & \\
\hline 3355.870 & 20 & 200 & 29789.97 & 0.00 & $41678_{2 \frac{1}{2}}^{\circ}-71468_{2 \frac{1}{2}}$ & 6 & 1.189 & 1.007 & 0.463 & 1.098 \\
\hline 3354.020 & & 3 & 29806.40 & -0.01 & $31568_{2 \frac{1}{2}}^{\circ}-613744_{\frac{1}{2}}^{2}$ & & & & & \\
\hline 3353.966 & 2 & 30 & 29806.88 & +0.02 & $38342_{4 \frac{1}{2}}^{\circ}-68148_{5 \frac{1}{2}}$ & & & & & \\
\hline 3353.733 & 20 & 150 & 29808.95 & -0.03 & $33052_{4 \frac{1}{2}}^{\circ}-62861_{5 \frac{1}{2}}^{2}$ & 4 & 1.260 & 1.138 & 0.061 & 0.589 \\
\hline 3353.563 & 1 & 6 & 29810.46 & -0.02 & $66571_{5 \frac{1}{2}}-96382_{5 \frac{1}{2}}^{\circ}$ & & & & & \\
\hline 3353.200 & 3 & 40 & 29813.69 & +0.04 & $45737_{1 \frac{1}{2}}^{\circ}-75550_{2 \frac{1}{2}}^{\circ}$ & 4 & 1.334 & 0.908 & 0.213 & 0.269 \\
\hline 3352.491 & 10 & 30 & 29820.00 & & & & & & & \\
\hline $3351.265^{\mathrm{a}}$ & 20 & 20 & 29830.90 & & & & & & & \\
\hline 3351.087 & 6 & 60 & 29832.49 & +0.04 & $44438_{3 \frac{1}{2}}^{\circ}-74270_{2 \frac{1}{2}}$ & 5 & 1.103 & 0.921 & 0.091 & 1.558 \\
\hline 3350.967 & 5 & 40 & 29833.56 & +0.02 & $47005_{\frac{1}{2}}^{\circ}-76839_{2 \frac{1}{2}}^{\circ}$ & 5 & 0.996 & 1.116 & 0.06 & 1.296 \\
\hline 3349.942 & 20 & 150 & 29842.69 & +0.01 & $31979_{3 \frac{1}{2}}^{\circ}-61822_{3 \frac{1}{2}}$ & 7 & 1.333 & 1.333 & 0 & 1.333 \\
\hline 3349.074 & 10 & 60 & 29850.42 & +0.02 & $56375_{2 \frac{1}{2}}-86226_{1 \frac{1}{2}}^{\circ}$ & 4 & (1.226) & 1.314 & $w$ & 1.094 \\
\hline 3347.537 & 150 & 1000 & 29864.12 & +0.01 & $35059_{\frac{1}{2}}^{2}-64923_{\frac{1}{2}}^{2}$ & 7 & (.1.124) & 1.117 & 0 & 1.092 \\
\hline $3346.504^{\mathrm{a}}$ & 30 & 30 & 29873.34 & & 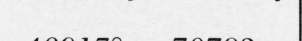 & & & & & \\
\hline 3346.192 & & 7 & 29876.13 & +0.08 & $40917_{4 \frac{1}{2}}^{\circ}-70793_{5 \frac{1}{2}}$ & & & & & \\
\hline 3345.921 & & 7 & 29878.55 & +0.02 & $65875_{6 \frac{1}{2}}-95754_{5 \frac{1}{2}}^{\circ}$ & & & & & \\
\hline 3345.187 & & 9 & 29885.10 & & & & & & & \\
\hline $\begin{array}{l}3344.715 \\
334278\end{array}$ & & 3 & $\begin{array}{l}29889.32 \\
20907.68\end{array}$ & -0.01 & $70136_{6 \frac{1}{2}}-100025_{5 \frac{1}{2}}^{\circ}$ & & & & & \\
\hline $\begin{array}{l}3343.78 \\
3343.705\end{array}$ & & $\begin{array}{l}1 \\
2\end{array}$ & $\begin{array}{l}29897.68 \\
29898.35\end{array}$ & +0.08 & $63647_{1 \frac{1}{2}}-93546_{2 \frac{1}{2}}^{\circ}$ & & & & & \\
\hline 3343.566 & & 2 & 29899.59 & +0.01 & $68720_{6 \frac{1}{2}}-98620_{6 \frac{1}{2}}^{\circ}$ & & & & & \\
\hline 3343.071 & 80 & 400 & 29904.02 & $\left\{\begin{array}{l}+0.04 \\
-0.02\end{array}\right.$ & $35019_{4 \frac{1}{2}}^{\circ}-64923_{4 \frac{1}{2}}$ & 6 & 1.16 & 1.11 & 0.21 & 1.14 \\
\hline 3342.93 & $20 h$ & $100 h$ & 29905.28 & & & & & & & \\
\hline 3342.310 & & 1 & 29910.83 & -0.03 & $66571_{5 \frac{1}{2}}-96482_{4 \frac{1}{2}}^{\circ}$ & & & & & \\
\hline 3341.097 & 3 & 40 & 29921.69 & +0.02 & $40035_{3 \frac{1}{2}}^{\circ}-69957_{\frac{1}{2}}^{2}$ & 5 & 0.66 & 1.08 & 0.208 & 2.54 \\
\hline 3340.791 & & 1 & 29924.43 & -0.01 & $66558_{3 \frac{1}{2}}-96482_{+\frac{1}{2}}^{\circ}$ & & & & & \\
\hline 3339.476 & & 3 & 29936.21 & & & & & & & \\
\hline $\begin{array}{l}3339.305 \\
3339.274\end{array}$ & & $\begin{array}{l}3 \\
1\end{array}$ & $\begin{array}{l}29937.74 \\
29938.02\end{array}$ & & & & & & & \\
\hline 3339.022 & & 1 & 29940.28 & +0.01 & $40917_{4 \frac{1}{2}}^{\circ}-70858_{\frac{1}{2}}$ & & & & & \\
\hline 3338.749 & 1 & 8 & 29942.73 & +0.03 & $64055_{2 \frac{1}{2}}-93998_{1 \frac{1}{2}}^{\circ}$ & & & & & \\
\hline 3337.581 & & 1 & 29953.21 & +0.07 & $64055_{2 \frac{1}{2}}^{2}-94008_{3 \frac{1}{2}}^{\circ}$ & & & & & \\
\hline 3337.420 & 5 & 20 & 29954.65 & +0.07 & $47329_{2 \frac{1}{2}}^{-2}-77284_{1 \frac{1}{2}}^{2}$ & 5 & 1.00 & $(0.90)$ & $w$ & 1.14 \\
\hline
\end{tabular}


TABLE 6. Yb II-Observed and classified lines-Continued

\begin{tabular}{|c|c|c|c|c|c|c|c|c|c|c|}
\hline 1 & 2 & 3 & 4 & 5 & 6 & 7 & 8 & 9 & 10 & 11 \\
\hline \multirow{2}{*}{$\begin{array}{l}\text { Wave- } \\
\text { length } \\
\text { air }\end{array}$} & \multicolumn{2}{|c|}{ Intensity } & \multirow{2}{*}{$\begin{array}{l}\text { Wave } \\
\text { number }\end{array}$} & \multirow[b]{2}{*}{$0-\mathrm{c}$} & \multirow{2}{*}{ Classification } & \multirow{2}{*}{$\begin{array}{l}\text { Zee- } \\
\text { man } \\
\text { type }\end{array}$} & \multirow{2}{*}{ lst $g$} & \multirow{2}{*}{ 2nd $g$} & \multicolumn{2}{|c|}{$\begin{array}{c}\text { Zeeman } \\
\text { components }\end{array}$} \\
\hline & $\begin{array}{l}\text { Meggers } \\
\text { lamp }\end{array}$ & $\begin{array}{c}\text { Thomson } \\
\text { iamp }\end{array}$ & & & & & & & Strong $p$ & Strong $n$ \\
\hline ‡ & & & $K$ & $K$ & & & & & & \\
\hline $\begin{array}{l}3337.171 \\
3337.005\end{array}$ & 30 & $\begin{array}{l}100 \\
5 h\end{array}$ & $\begin{array}{l}29956.89 \\
29958.38\end{array}$ & & & 7 & & & 0 & 1.019 \\
\hline $\begin{array}{l}3336.88 \\
3336.718 \\
3335.826 \\
3335.435 \\
3335.327\end{array}$ & 1 & $\begin{array}{r}1 \\
4 \\
10 \\
2 \\
3\end{array}$ & $\begin{array}{l}29959.50 \\
29960.95 \\
29968.96 \\
29972.48 \\
29973.45\end{array}$ & $\begin{array}{l}+0.03 \\
+0.02 \\
-0.02 \\
+0.01\end{array}$ & $\begin{array}{l}63234_{-\frac{1}{2}}-93195_{3 \frac{1}{2}}^{\circ} \\
463.54_{\frac{1}{2}}^{\circ}-76323_{3 \frac{1}{2}}^{\circ} \\
68549_{4 \frac{1}{2}}-98521_{\frac{1}{2}}^{\circ} \\
65577_{3 \frac{1}{2}}-95550_{4 \frac{1}{2}}^{\circ}\end{array}$ & & & & & \\
\hline $\begin{array}{l}3334.321 \\
3333.800\end{array}$ & 1 & $\begin{array}{l}3 \\
4\end{array}$ & $\begin{array}{l}29982.49 \\
29987.18\end{array}$ & & & & & & & \\
\hline $\begin{array}{l}3333.057 \\
3332.774\end{array}$ & 200 & $\begin{array}{r}1000 \\
7\end{array}$ & $\begin{array}{l}29993.86 \\
29996.41\end{array}$ & -0.01 & $33653_{0 \frac{1}{2}}^{\circ}-63647_{1 \frac{1}{2}}$ & 4 & 1.329 & 1.157 & 0.086 & 1.071 \\
\hline 3331.220 & 5 & 50 & 30010.40 & 0.00 & $43956_{2 \frac{1}{2}}^{\circ}-73966_{3 \frac{1}{2}}$ & 4 & 0.991 & 0.765 & 0.113 & 0.200 \\
\hline 3330.238 & & 1 & 30019.25 & +0.03 & $69395_{3 \frac{1}{2}}-99414_{4 \frac{1}{2}}^{\circ}$ & & & & & \\
\hline 3329.50 & 3 & 5 & 30025.90 & & & & & & & \\
\hline $\begin{array}{l}3329.382 \\
3329.087\end{array}$ & 50 & $\begin{array}{r}100 \\
4\end{array}$ & $\begin{array}{l}30026.97 \\
30029.63\end{array}$ & +0.01 & $22960_{1 \frac{1}{2}}-52987_{1 \frac{1}{2}}^{\circ}$ & 6 & 0.806 & 1.134 & 0.500 & 0.970 \\
\hline 3329.017 & 3 & 10 & 30030.26 & 0.00 & $32981_{1 \frac{1}{2}}^{\circ}-63011_{1 \frac{1}{2}}$ & & & & & \\
\hline 3328.584 & & 8 & 30034.17 & +0.03 & $35059_{3 \frac{1}{2}}^{\circ}-65093_{3 \frac{1}{2}}$ & & & & & \\
\hline $\begin{array}{l}3328.39 \\
3227756\end{array}$ & & 1 & 30035.92 & +0.02 & $43075_{11}^{\circ}-73116_{1 \frac{1}{3}}$ & 6 & 0960 & 0870 & 0,135 & 0915 \\
\hline $\begin{array}{l}3327.756 \\
3327.485\end{array}$ & $\begin{array}{l}8 \\
1\end{array}$ & $\begin{array}{l}80 \\
15\end{array}$ & $\begin{array}{l}30041.64 \\
30044.08\end{array}$ & $\begin{array}{l}+0.02 \\
-0.01\end{array}$ & $35831_{5 \frac{1}{2}}^{\frac{1}{2}}-65875_{6 \frac{1}{2}}^{\frac{1}{2}}$ & & 0.900 & 0.060 & 0.155 & 0.915 \\
\hline $3327.195^{\mathrm{a}}$ & 3 & 3 & 30046.70 & & & & & & & \\
\hline 3326.949 & 2 & 20 & 30048.93 & +0.05 & $44940_{1 \frac{1}{2}}^{\circ}-74989_{0 \frac{1}{2}}$ & 5 & 1.252 & 0.512 & 0.370 & 1.622 \\
\hline 3326.745 & & 4 & 30050.77 & +0.02 & $44940_{1 \frac{1}{2}}^{\circ}-74991_{1 \frac{1}{2}}$ & & & & & \\
\hline 3326.266 & 40 & 200 & 30055.09 & +0.03 & $47228_{0 \frac{1}{2}}^{\circ}-77284_{1 \frac{1}{2}}^{2}$ & 5 & 0.711 & 0.894 & 0.091 & 0.985 \\
\hline $\begin{array}{l}3325.104 \\
3324.80\end{array}$ & 9 & $\begin{array}{r}50 \\
2\end{array}$ & $\begin{array}{l}30065.60 \\
30068.35\end{array}$ & 0.00 & $28757_{\frac{1}{2}}^{\circ}-58823_{2 \frac{1}{2}}$ & 4 & 1.440 & 1.256 & 0.092 & 0.980 \\
\hline 3324.470 & 10 & 70 & 30071.33 & +0.01 & $34389_{2 \frac{1}{2}}^{\circ}-64461_{1 \frac{1}{2}}$ & 4 & 1.001 & 1.171 & 0.085 & 0.746 \\
\hline 3324.172 & 40 & 300 & 30074.03 & +0.02 & $35019_{4 \frac{1}{2}}^{0}-65093_{3 \frac{1}{2}}^{2}$ & 5 & 1.16 & 0.99 & 0.083 & 1.74 \\
\hline $\begin{array}{l}3324.043 \\
3323.46\end{array}$ & 2 & $\begin{array}{l}12 \\
3 h\end{array}$ & 30075.19 & -0.03 & $41688_{\frac{1}{2}}^{\circ}-71763_{1 \frac{1}{2}}^{2}$ & & & & & \\
\hline 3322.963 & 20 & $250^{\circ}$ & 30084.97 & +0.03 & $41678_{2 \frac{1}{2}}^{\circ}-71763_{1 \frac{1}{2}}$ & 5 & (1.193) & 1.10 & $w$ & $1.33 ?$ \\
\hline 3322.87 & 1 & 2 & 30085.81 & & & & & & & \\
\hline $\begin{array}{l}3322.58 \\
3320.802\end{array}$ & & 1 & 30088.44 & -0.07 & $59624_{3 \frac{1}{2}}^{\circ}-89713_{2 \frac{1}{2}}$ & & & & & \\
\hline 3320.642 & & 2 & 30106.00 & & & & & & & \\
\hline 3320.314 & 10 & 200 & 30108.97 & $\left\{\begin{array}{l}+0.05 \\
-0.08\end{array}\right.$ & $\begin{array}{l}43007_{0 \frac{1}{2}}^{\circ}-73116_{1 \frac{1}{2}} \\
\left(68720_{6 \frac{1}{2}}-98829_{6 \frac{1}{2}}^{\circ}\right)\end{array}$ & 5 & 0.158 & 0.862 & 0.352 & 1.213 \\
\hline 3320.032 & 5 & 30 & 30111.53 & +0.03 & $33052_{4 \frac{1}{2}}^{\circ}-63163_{4 \frac{1}{2}}$ & & & & & \\
\hline 3319.737 & & 2 & 30114.20 & -0.01 & $65577_{3 \frac{1}{2}}-95691_{3 \frac{1}{2}}^{\circ}$ & & & & & \\
\hline
\end{tabular}


TABLE 6. Yb II-Observed and classified lines-Continued

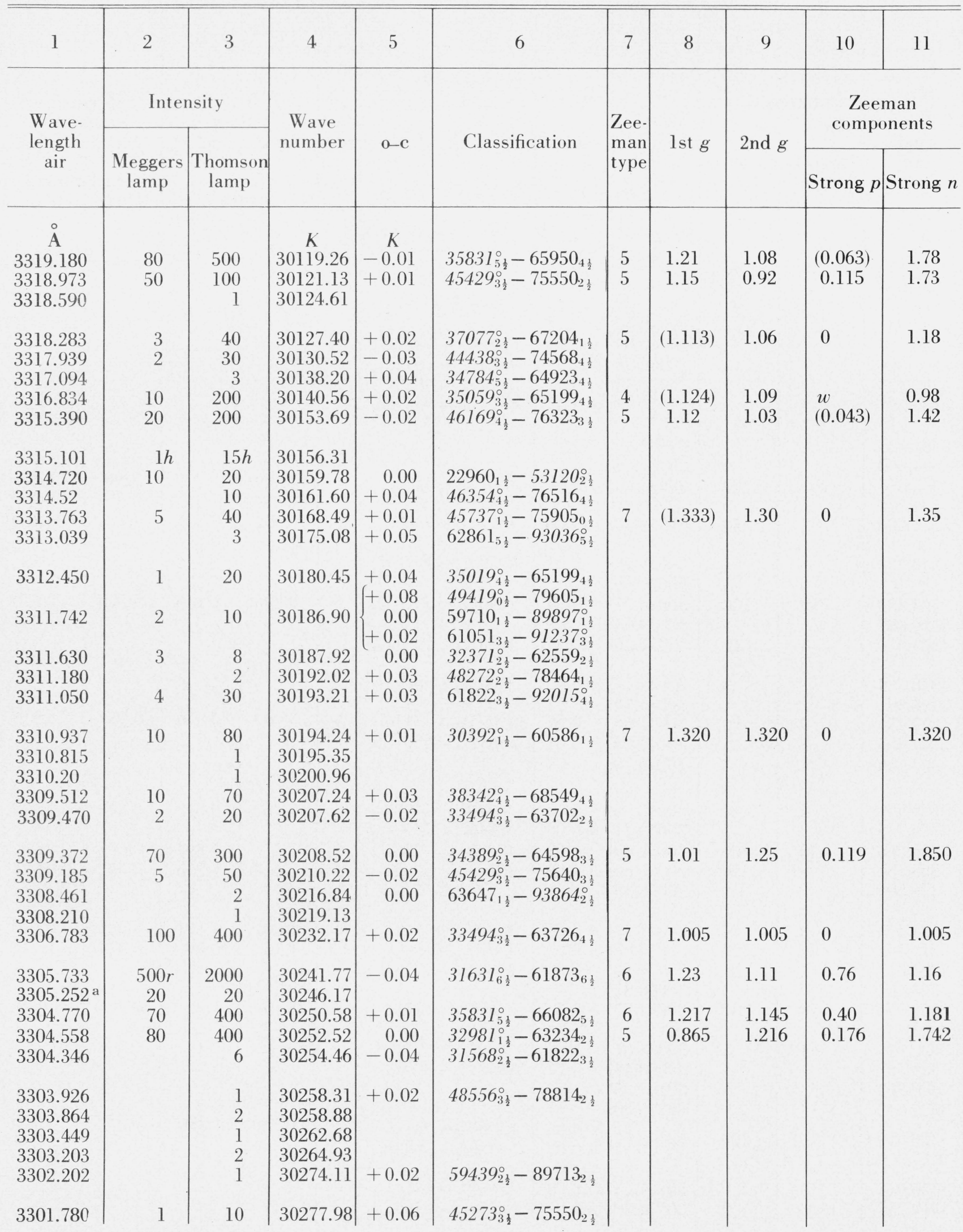


TABLE 6. Yb II-Observed and classified lines-Continued

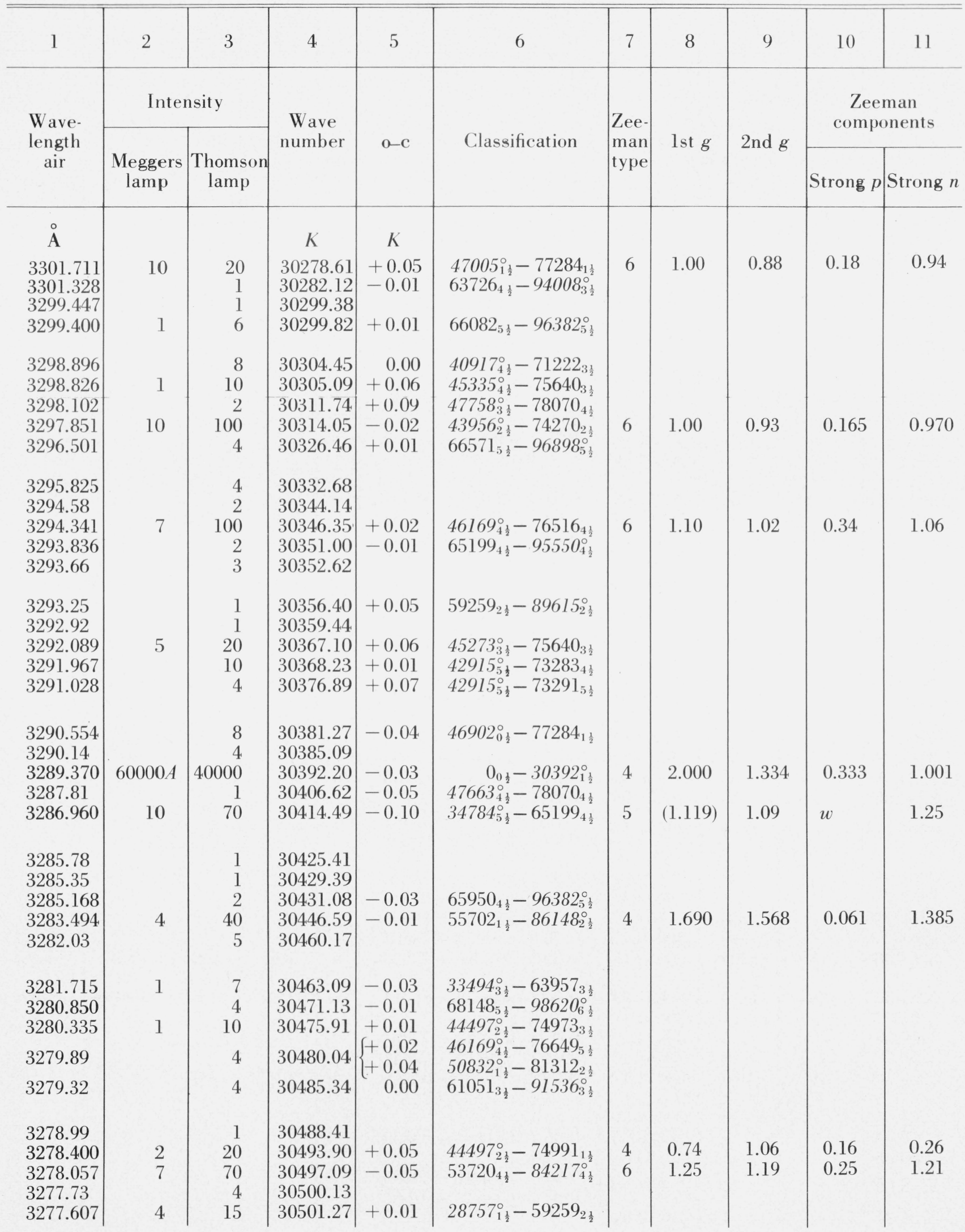


TABlE 6. Yb II-Observed and classified lines-Continued

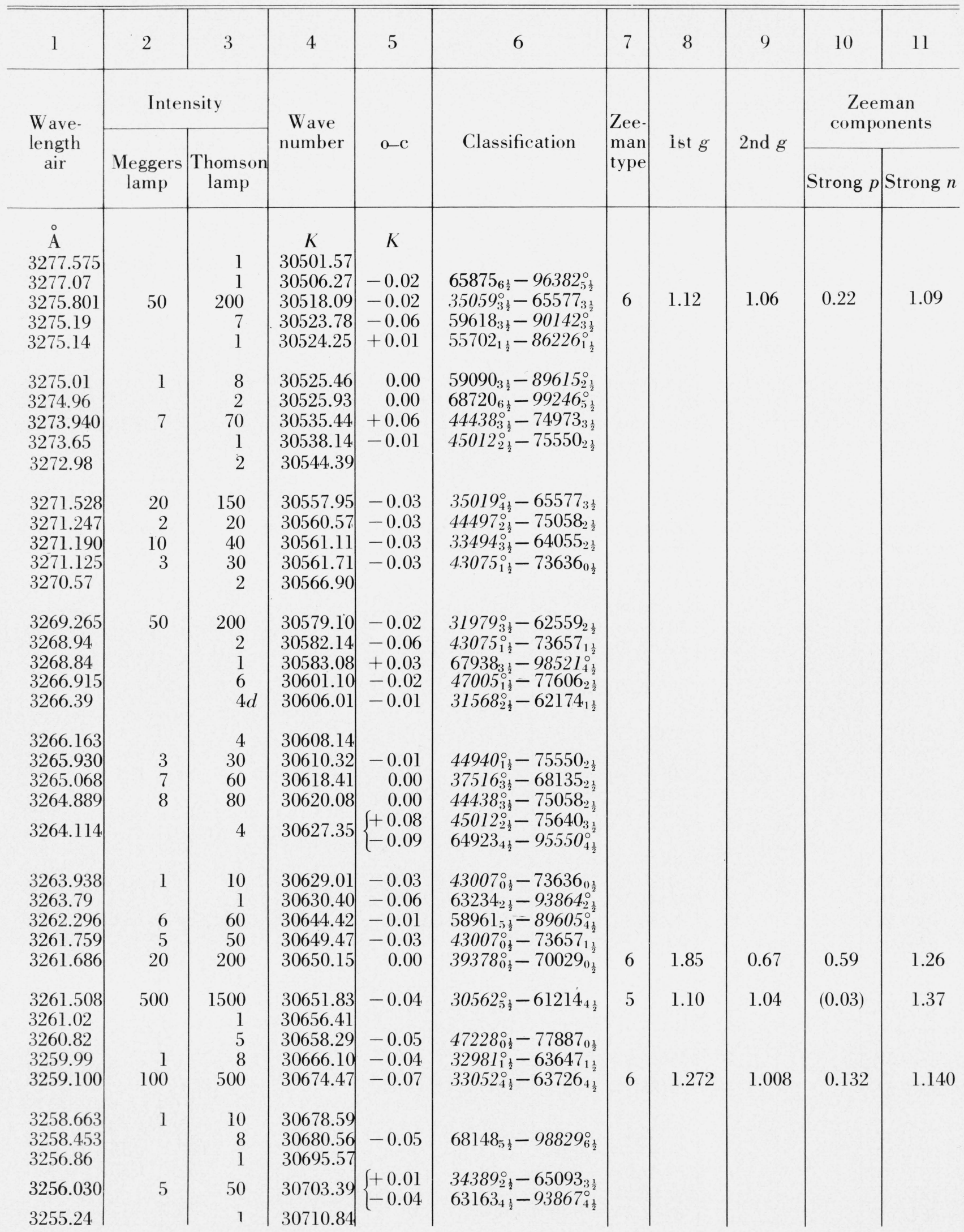


TABLE 6. Yb II-Observed and classified lines - Continued

\begin{tabular}{|c|c|c|c|c|c|c|c|c|c|c|}
\hline 1 & 2 & 3 & 4 & 5 & 6 & 7 & 8 & 9 & 10 & 11 \\
\hline \multirow{2}{*}{$\begin{array}{l}\text { Wave- } \\
\text { length } \\
\text { air }\end{array}$} & \multicolumn{2}{|c|}{ Intensity } & \multirow{2}{*}{$\begin{array}{c}\text { Wave } \\
\text { number }\end{array}$} & \multirow[b]{2}{*}{$\mathrm{o}-\mathrm{c}$} & \multirow{2}{*}{ Classification } & \multirow{2}{*}{$\begin{array}{l}\text { Zee- } \\
\text { man } \\
\text { type }\end{array}$} & \multirow{2}{*}{ lst $g$} & \multirow{2}{*}{2 nd $g$} & \multicolumn{2}{|c|}{$\begin{array}{c}\text { Zeeman } \\
\text { components }\end{array}$} \\
\hline & $\begin{array}{l}\text { Meggers } \\
\text { lamp }\end{array}$ & $\begin{array}{c}\text { Thomson } \\
\text { lamp }\end{array}$ & & & & & & & Strong $p$ & Strong $n$ \\
\hline A & & & $K$ & $K$ & & & & & & \\
\hline 3254.62 & & 2 & 30716.69 & & & & & & & \\
\hline 3254.197 & 100 & 300 & 30720.69 & -0.04 & $32981_{1 \frac{1}{2}}^{\circ}-63702_{2 \frac{1}{2}}$ & & & & & \\
\hline 3253.911 & 10 & 100 & 30723.39 & +0.03 & $53720_{4 \frac{1}{2}}-84444_{3 \frac{1}{2}}^{\circ}$ & & & & & \\
\hline 3253.340 & 20 & 120 & 30728.78 & -0.03 & $53715_{3 \frac{1}{2}}^{2}-84444_{3 \frac{1}{2}}^{\circ}$ & & & & & \\
\hline 3252.106 & 1 & 10 & 30740.44 & +0.01 & $45429_{3 \frac{1}{2}}^{\circ}-76170_{2 \frac{1}{2}}^{\circ}$ & & & & & \\
\hline 3250.505 & 1 & 5 & 30755.58 & 0.00 & $22960_{1 \frac{1}{2}}-53716_{2 \frac{1}{2}}^{\circ}$ & & & & & \\
\hline 3249.80 & & 1 & 30762.25 & +009 & $62224-02000^{\circ}$ & & & & & \\
\hline 3249.57 & & 1 & 30764.43 & +0.02 & $63234_{2 \frac{1}{2}}-93998_{\frac{1}{2}}^{\circ}$ & & & & & \\
\hline 3249.17 & & 4 & 30768.22 & +0.01 & $64923_{4 \frac{1}{2}}-95691_{3 \frac{1}{2}}^{\circ}$ & & & & & \\
\hline 3248.446 & 2 & 10 & 30775.07 & & & & & & & \\
\hline 3247.932 & 1 & 5 & 30779.94 & +0.02 & $45737_{1 \frac{1}{2}}^{\circ}-76517_{1 \frac{1}{2}}$ & & & & & \\
\hline 3246.85 & & 1 & 30790.20 & +0.04 & $48024_{1 \frac{1}{2}}^{\circ}-78814_{2 \frac{1}{2}}^{2}$ & & & & & \\
\hline 3246.657 & 1 & 7 & 30792.03 & +0.02 & $58823_{2 \frac{1}{2}}-89615_{2 \frac{1}{2}}^{0}$ & & & & & \\
\hline $\begin{array}{l}3246.28 \\
3246.060\end{array}$ & & $\begin{array}{r}1 \\
10\end{array}$ & $\begin{array}{l}30795.61 \\
30797.69\end{array}$ & & & & & & & \\
\hline 3245.70 & & 5 & 30801.11 & +0.01 & $61214_{41}-92015^{\circ}$ & & & & & \\
\hline 3245.05 & & 6 & 30807.28 & $\begin{array}{r}0.01 \\
+0.06\end{array}$ & & & & & & \\
\hline 3243.46 & & 5 & 30822.38 & +0.02 & $40035_{3 \frac{1}{2}}^{\circ}-70858_{4 \frac{1}{2}}^{\frac{1}{2}}$ & & & & & \\
\hline 3243.00 & 4 & 30 & 30826.75 & +0.02 & $30224_{4 \frac{1}{2}}^{\frac{3}{2}}-61051_{3 \frac{1}{2}}$ & & & & & \\
\hline 3242.12 & 1 & 10 & 30835.12 & & & & & & & \\
\hline 3242.048 & 4 & 50 & 30835.80 & +0.03 & $42915_{5 \frac{1}{2}}^{\circ}-73750_{4 \frac{1}{2}}$ & & & & & \\
\hline 3239.39 & 1 & 8 & 30861.10 & +0.01 & $37077_{2 \frac{1}{2}}^{\circ}-67938_{3 \frac{1}{3}}$ & & & & & \\
\hline 3239.195 & 50 & 200 & 30862.96 & -0.05 & $32371_{2 \frac{1}{2}}^{\circ}-63234_{2 \frac{1}{2}}^{2}$ & 6 & 1.18 & 1.22 & 0.09 & 1.20 \\
\hline 3237.215 & & 5 & 30881.84 & 0.00 & $47005_{\frac{1}{2}}^{\circ}-77887_{0 \frac{1}{2}}$ & & & & & \\
\hline 3236.488 & 20 & 60 & 30888.77 & 0.00 & $24332_{2 \frac{1}{2}}-55221_{1 \frac{1}{2}}^{\circ \frac{1}{2}}$ & & & & & \\
\hline 3236.160 & 40 & 300 & 30891.91 & $\left\{\begin{array}{l}-0.04 \\
+0.02\end{array}\right.$ & $35059_{3 \frac{1}{2}}^{\circ}-65950_{4 \frac{1}{2}}$ & 4 & (1.124) & 1.10 & $w$ & 1.03 \\
\hline 3235.975 & 3 & 30 & 30893.67 & $\begin{array}{r}(+0.02 \\
0.00\end{array}$ & $\begin{array}{r}\left(35052_{4 \frac{1}{2}}-039444_{5 \frac{1}{2}}\right) \\
45429_{3 \frac{1}{3}}^{\circ}-76323_{3 \frac{1}{2}}\end{array}$ & & & & & \\
\hline 3235.858 & & 6 & 30894.79 & & & & & & & \\
\hline 3235.61 & & 8 & 30897.16 & -0.07 & $45273_{3 \frac{1}{2}}^{\circ}-76170_{2 \frac{1}{2}}$ & & & & & \\
\hline 3234.740 & 2 & 10 & 30905.47 & -0.04 & $33052_{4 \frac{1}{2}}^{\circ}-63957_{3 \frac{1}{2}}^{2}$ & & & & & \\
\hline 3233.487 & 3 & 40 & 30917.44 & -0.01 & $53715_{3 \frac{1}{2}}-84632_{2 \frac{1}{2}}^{\circ}$ & & & & & \\
\hline 3233.38 & & 1 & 30918.46 & +0.01 & $40035_{3 \frac{1}{2}}^{\circ}-70954_{2 \frac{1}{2}}^{2 \frac{1}{2}}$ & & & & & \\
\hline 3232.29 & & 4 & 30928.89 & & & & & & & \\
\hline $\begin{array}{l}3231.986 \\
3231.90\end{array}$ & 50 & $\begin{array}{r}300 \\
10\end{array}$ & 30931.80 & -0.02 & $35019_{4 \frac{1}{2}}^{\circ}-65950_{4 \frac{1}{2}}$ & 6 & 1.15 & 1.07 & 0.36 & 1.11 \\
\hline 3231.90 & & 10 & 30932.62 & & & & & & & \\
\hline 3231.81 & & 10 & 30933.48 & -0.04 & $37516_{3 \frac{1}{2}}^{\circ}-68450_{3 \frac{1}{2}}$ & & & & & \\
\hline 3231.376 & 10 & 50 & 30937.64 & -0.01 & $57765_{4 \frac{1}{2}}-88702^{\circ} \frac{1}{2}$ & & & & & \\
\hline 3231.228 & & 2 & 30939.06 & +0.04 & $45737_{1 \frac{1}{2}}^{\circ}-76676_{2 \frac{1}{2}}$ & & & & & 12 \\
\hline 3229.814 & 50 & 150 & 30952.60 & -0.07 & $28757_{1 \frac{1}{2}}^{\circ}-59710_{1 \frac{1}{2}}$ & 6 & 1.44 & 0.80 & 0.96 & 1.12 \\
\hline 3229.475 & 2 & 20 & 30955.85 & +0.05 & $57765_{4 \frac{1}{2}}-88721_{3 \frac{1}{2}}^{\circ}$ & & & & & \\
\hline
\end{tabular}


TABLE 6. Y Y II-Observed and classified lines-Continued

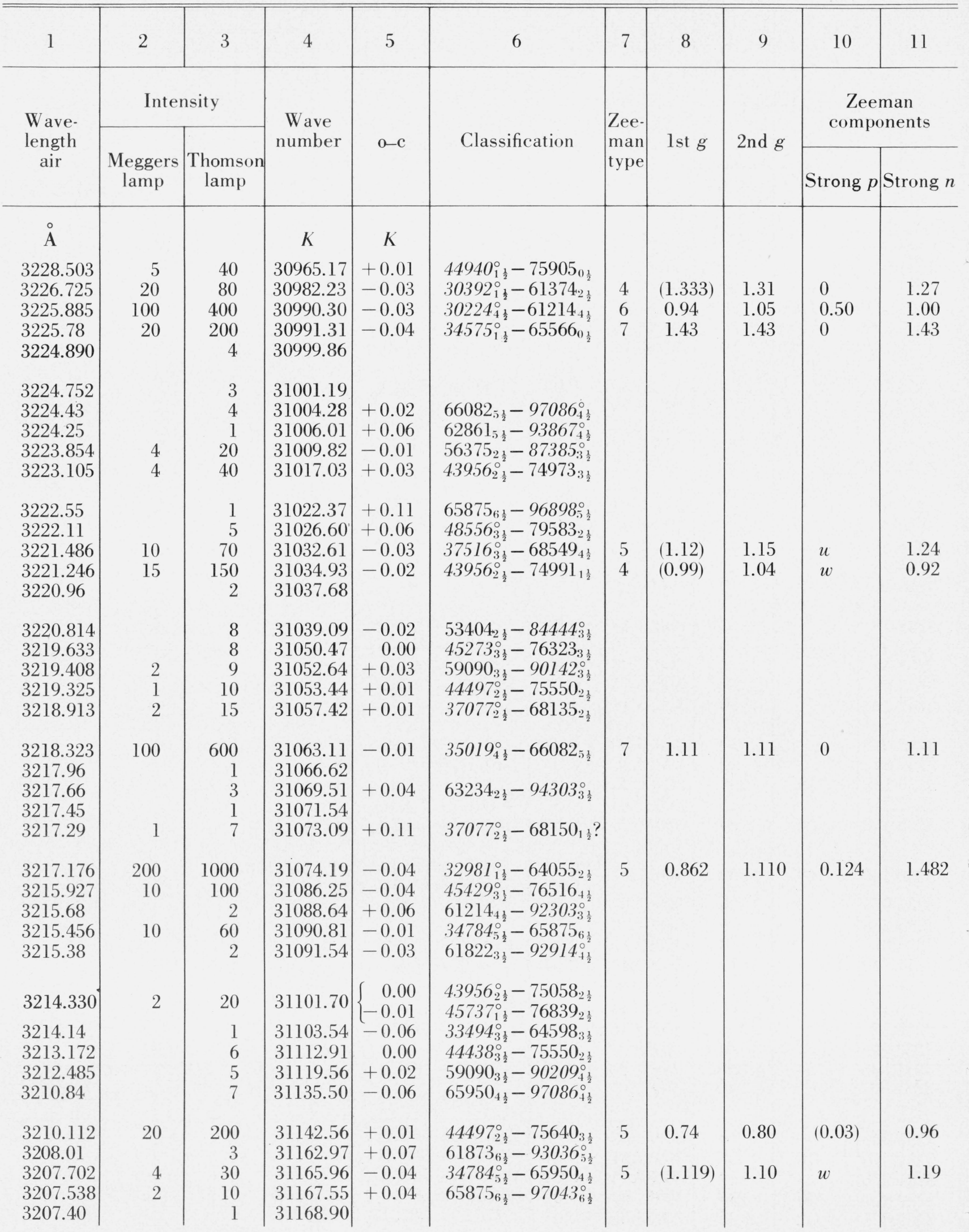


TABLE 6. Yb II-Observed and classified lines-Continued

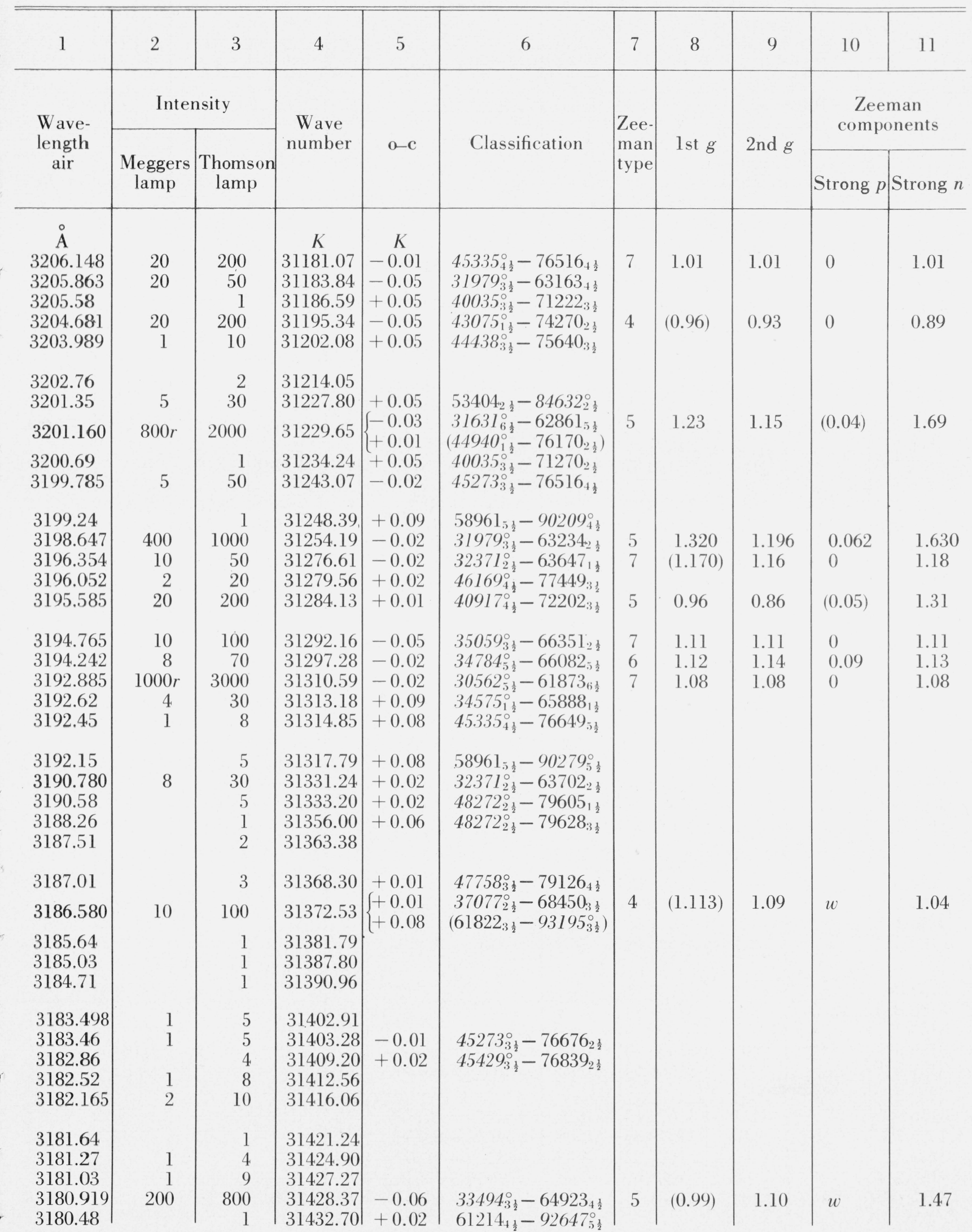


TABLE 6. Yb II-Observed and classified lines-Continued

\begin{tabular}{|c|c|c|c|c|c|c|c|c|c|c|}
\hline 1 & 2 & 3 & 4 & 5 & 6 & 7 & 8 & 9 & 10 & 11 \\
\hline \multirow{2}{*}{$\begin{array}{l}\text { Wave- } \\
\text { length } \\
\text { air }\end{array}$} & \multicolumn{2}{|c|}{ Intensity } & \multirow{2}{*}{$\begin{array}{c}\text { Wave } \\
\text { number }\end{array}$} & \multirow{2}{*}{$\mathbf{o}-\mathbf{c}$} & \multirow{2}{*}{ Classification } & \multirow{2}{*}{$\begin{array}{l}\text { Zee- } \\
\text { man } \\
\text { type }\end{array}$} & \multirow{2}{*}{ lst $g$} & \multirow{2}{*}{ 2nd $g$} & \multicolumn{2}{|c|}{$\begin{array}{c}\text { Zeeman } \\
\text { components }\end{array}$} \\
\hline & $\begin{array}{c}\text { Meggers } \\
\text { lamp }\end{array}$ & $\begin{array}{c}\text { Thomson } \\
\text { lamp }\end{array}$ & & & & & & & Strong $p$ & Strong $n$ \\
\hline$\AA$ & & & $K$ & $K$ & & & & & & \\
\hline 3179.94 & 1 & 10 & 31438.04 & +0.06 & $41678_{2 \frac{1}{2}}^{\circ}-73116_{1 \frac{1}{2}}$ & & & & & \\
\hline 3179.344 & 30 & 70 & 31443.93 & & & & & & & \\
\hline $\begin{array}{l}3178.85 \\
3177815\end{array}$ & & 1 & $\begin{array}{l}31448.82 \\
3145906\end{array}$ & +0.04 & $49008_{3 \frac{1}{2}}^{\circ}-80457_{3 \frac{1}{2}}$ & & & & & \\
\hline $\begin{array}{l}317.813 \\
3177.78\end{array}$ & & $\begin{array}{l}1 \\
2\end{array}$ & $\begin{array}{l}31459.00 \\
31459.41\end{array}$ & +0.11 & $47005_{1 \frac{1}{2}}^{\circ}-78464_{1 \frac{1}{2}} ?$ & & & & & \\
\hline 3176.26 & & 3 & 31474.46 & +0.03 & $62861_{5 \frac{1}{2}}-94335_{5 \frac{1}{2}}^{\circ}$ & & & & & \\
\hline 3176.06 & & 1 & 31476.45 & +0.11 & $68549_{4 \frac{1}{2}}^{2}-100025_{5 \frac{1}{2}}^{2}$ & $?$ & & & & \\
\hline 3175.759 & 25 & 150 & 31479.43 & -0.06 & $32981_{1 \frac{1}{2}}^{\circ}-64461_{1 \frac{1}{2}}$ & 6 & 0.873 & 1.177 & 0.455 & 1.025 \\
\hline \begin{tabular}{l|}
3174.22 \\
3173.797
\end{tabular} & 20 & $\begin{array}{r}2 \\
150\end{array}$ & $\begin{array}{l}31494.69 \\
31498.89\end{array}$ & -0.11 & $35059_{3 \frac{1}{2}}^{\circ}-66558_{3 \frac{1}{2}} ?$ & 7 & (1.124) & 1.10 & 0 & 1.11 \\
\hline 3171.176 & 100 & 300 & 31524.92 & +0.03 & $26759_{2 \frac{1}{2}}^{\circ}-58283_{2 \frac{1}{2}}^{\circ}$ & 6 & 1.58 & 0.78 & 2.00 & 1.18 \\
\hline 3169.056 & 200 & 1200 & 31546.01 & +0.02 & $33052_{4 \frac{1}{2}}^{\circ}-64598_{3 \frac{1}{2}}^{2}$ & 5 & $(1.264)$ & 1.244 & $w$ & 1.334 \\
\hline 3168.985 & 3 & 10 & 31546.72 & -0.01 & $45737_{1 \frac{1}{2}}^{\circ}-77284_{1 \frac{1}{2}}^{\circ}$ & & & & & \\
\hline 3168.405 & 8 & 60 & 31552.49 & +0.04 & $35019_{4 \frac{1}{2}}^{\circ}-66571_{5 \frac{1}{2}}^{\circ}$ & 5 & (1.158) & 1.18 & $w$ & 1.27 \\
\hline 3167.72 & & 1 & 31559.31 & -0.02 & $64923_{+\frac{1}{2}}-96482_{\frac{1}{2}}^{\circ}$ & & & & & \\
\hline 3167.044 & 1 & 8 & 31566.05 & +0.07 & $45273_{3 \frac{1}{2}}^{\circ}-76839_{2 \frac{1}{2}}$ & & & & & \\
\hline 3166.727 & 2 & 20 & 31569.21 & & & & & & & \\
\hline 3165.981 & 1 & 5 & 31576.65 & +0.05 & $44940_{1 \frac{1}{2}}^{\circ}-76517_{1 \frac{1}{2}}^{\circ}$ & & & & & \\
\hline 3165.875 & & 7 & 31577.70 & +0.08 & $46169_{4 \frac{1}{2}}^{\circ}-77747_{1 \frac{1}{2}}$ & & & & & \\
\hline 3165.206 & 60 & 500 & 31584.38 & +0.03 & $35831_{\frac{1}{2}}^{\circ}-67416_{4 \frac{1}{2}}$ & 4 & (1.214) & 1.25 & $w$ & 1.065 \\
\hline 3164.973 & 6 & 15 & 31586.70 & 0.00 & $32.371_{2 \frac{1}{2}}^{\circ}-63957_{3 \frac{1}{2}}$ & & & & & \\
\hline 3163.796 & 100 & 600 & 31598.45 & -0.01 & $33494_{3 \frac{1}{2}}^{\circ}-65093_{3 \frac{1}{2}}$ & 7 & 0.987 & 0.987 & 0 & 0.987 \\
\hline 3161.80 & & 2 & 31618.40 & & & & & & & \\
\hline 3160.894 & 1 & 8 & 31627.46 & +0.05 & $59090_{3 \frac{1}{2}}-90717_{4 \frac{1}{2}}^{\circ}$ & & & & & \\
\hline 3160.12 & & 1 & 31635.21 & & & & & & & \\
\hline 3160.01 & & 3 & 31636.31 & & & & & & & \\
\hline 3159.79 & & 2 & 31638.51 & & & & & & & \\
\hline 3159.59 & & 1 & 31640.52 & +0.09 & $61822_{3 \frac{1}{2}}-9.346 .3_{3 \frac{1}{2}}^{\circ}$ & & & & & \\
\hline 3159.49 & & 2 & 31641.52 & & & & & & & \\
\hline 3159.34 & & 1 & 31643.02 & +0.08 & $51248_{: \frac{1}{2}}^{\circ}-82891_{2 \frac{1}{2}}$ & & & & & \\
\hline 3159.04 & & 2 & 31646.03 & & & & & & & \\
\hline 3158.869 & 5 & 10 & 31647.74 & & & & & & & \\
\hline 3158.43 & 1 & 7 & 31652.14 & & & & & & & \\
\hline 3158.300 & 40 & 300 & 31653.44 & -0.01 & $42915_{\frac{5}{2} \frac{1}{2}}^{\circ}-74568_{4 \frac{1}{2}}$ & 5 & 1.12 & 1.04 & $(0.04)$ & 1.48 \\
\hline 3157.043 & & 7 & 31666.04 & +0.01 & $31568_{2 \frac{1}{2}}^{\circ}-63234 \cdot \frac{1}{2}$ & & & & & \\
\hline 3156.365 & & 4 & 31672.84 & +0.10 & $44497_{2 \frac{1}{2}}^{\circ}-76170_{2 \frac{1}{2}}$ & & & & & \\
\hline 3155.798 & 30 & 40 & 31678.54 & +0.09 & $37077_{2 \frac{1}{2}}^{-0}-68756_{3 \frac{1}{2}}^{2}$ & & & & & \\
\hline 3155.52 & & 2 & 31681.33 & +0.02 & $61822_{3 \frac{1}{2}}^{-2}-93503_{4 \frac{1}{2}}^{\circ}$ & & & & & \\
\hline 3155.284 & 5 & 50 & 31683.69 & +0.04 & $43956_{2 \frac{1}{2}}^{\circ}-75640_{3 \frac{1}{2}}^{\circ}$ & & & & & \\
\hline 3155.183 & 40 & 200 & 31684.71 & -0.01 & $32371_{2 \frac{1}{2}}^{0^{2}}-64055_{2 \frac{1}{2}}$ & 6 & 1.160 & 1.110 & 0.125 & 1.135 \\
\hline
\end{tabular}


TABLE 6. Yb II-Observed and classified lines-Continued

\begin{tabular}{|c|c|c|c|c|c|c|c|c|c|c|}
\hline 1 & 2 & 3 & 4 & 5 & 6 & 7 & 8 & 9 & 10 & 11 \\
\hline \multirow{2}{*}{$\begin{array}{l}\text { Wave- } \\
\text { length } \\
\text { air }\end{array}$} & \multicolumn{2}{|c|}{ Intensity } & \multirow{2}{*}{$\begin{array}{c}\text { Wave } \\
\text { number }\end{array}$} & \multirow[b]{2}{*}{$\mathrm{o}-\mathrm{c}$} & \multirow[b]{2}{*}{ Classification } & \multirow{2}{*}{$\begin{array}{l}\text { Zee- } \\
\text { man } \\
\text { type }\end{array}$} & \multirow[b]{2}{*}{ lst $g$} & \multirow[b]{2}{*}{ 2nd $g$} & \multicolumn{2}{|c|}{$\begin{array}{c}\text { Zeeman } \\
\text { components }\end{array}$} \\
\hline & $\begin{array}{c}\text { Meggers } \\
\text { lamp }\end{array}$ & $\begin{array}{c}\text { Thomson } \\
\text { lamp }\end{array}$ & & & & & & & Strong $p$ & Strong $n$ \\
\hline A & & & $K$ & $K$ & & & & & & \\
\hline 3154.194 & 5 & 40 & 31694.64 & -0.06 & $52938_{1 \frac{1}{2}}-84632_{2 \frac{1}{2}}^{\circ}$ & & & & & \\
\hline 3153.880 & 100 & 700 & 31697.80 & -0.03 & $40917_{4 \frac{1}{2}}^{\circ}-72615_{5 \frac{1}{2}}^{\circ}$ & 7 & $(0.967)$ & 0.978 & 0 & 1.028 \\
\hline 3153.616 & 20 & 100 & 31700.45 & -0.05 & $52517_{5 \frac{1}{2}}-84217_{4 \frac{1}{2}}^{\circ}$ & & & & & \\
\hline 3153.180 & 40 & 300 & 31704.84 & -0.02 & $33494_{3 \frac{1}{2}}^{\circ}-65199_{4 \frac{1}{2}}$ & 5 & 0.99 & 1.08 & $(0.044)$ & 1.38 \\
\hline 3152.44 & & 40 & 31712.28 & +0.02 & $46169_{4 \frac{1}{2}}^{\circ}-77882_{3 \frac{1}{2}}$ & & & & & \\
\hline $\begin{array}{l}3151.90 \\
3151.436\end{array}$ & 15 & $\begin{array}{r}1 \\
100\end{array}$ & $\begin{array}{l}31717.71 \\
31722.38\end{array}$ & -0.04 & & & & & & \\
\hline 3151.255 & $\begin{array}{r}10 \\
3\end{array}$ & $\begin{array}{r}100 \\
8\end{array}$ & $\begin{array}{l}31722.38 \\
31724.20\end{array}$ & $\begin{array}{l}-0.04 \\
-0.02\end{array}$ & $\begin{array}{l}31979_{3 \frac{1}{2}}-63702_{2 \frac{1}{2}} \\
24332_{2 \frac{1}{2}}-56056_{2 \frac{1}{2}}^{\circ}\end{array}$ & & & & & \\
\hline 3150.46 & 3 & 30 & 31732.21 & -0.01 & $44438_{3 \frac{1}{2}}^{\circ}-76170_{2 \frac{1}{2}}^{\circ}$ & & & & & \\
\hline 3150.19 & & 6 & 31734.93 & -0.02 & $39378_{0 \frac{1}{2}}^{\circ}-71113_{\frac{1}{2}}$ & & & & & \\
\hline 3150.12 & & 2 & 31735.63 & -0.07 & $44940_{1 \frac{1}{2}}^{\circ}-76676_{2 \frac{1}{2}}$ & & & & & \\
\hline $\begin{array}{l}3148.998 \\
3148.43\end{array}$ & 30 & $\begin{array}{r}200 \\
2\end{array}$ & $\begin{array}{l}31746.94 \\
31752.67\end{array}$ & +0.01 & $31979_{3 \frac{1}{2}}^{\circ}-63726_{4 \frac{1}{2}}$ & & & & & \\
\hline 3148.08 & & 1 & 31756.20 & +0.03 & $58961_{5 \frac{1}{2}}-90717_{4 \frac{1}{2}}^{\circ}$ & & & & & \\
\hline 3146.134 & 8 & 60 & 31775.84 & 0.00 & $34575_{1 \frac{1}{2}}^{\circ}-66351_{2 \frac{1}{2}}^{\circ}$ & & & & & \\
\hline 3145.540 & 150 & 500 & 31781.84 & -0.03 & $30392_{1 \frac{1}{2}}^{\circ}-62174_{1 \frac{1}{2}}$ & & & & & \\
\hline 3145.063 & 80 & 600 & 31786.66 & +0.03 & $34784_{5 \frac{1}{2}}^{\circ}-66571_{5 \frac{1}{2}}^{\circ}$ & 6 & 1.120 & 1.188 & 0.36 & 1.154 \\
\hline 3142.813 & 1 & 7 & 31809.41 & +0.01 & $47005_{\frac{1}{2}}^{\circ}-78814_{2 \frac{1}{2}}$ & & & & & \\
\hline 3142.74 & & 2 & 31810.15 & +0.03 & $63944_{5 \frac{1}{2}}-95754_{5 \frac{1}{2}}^{\circ}$ & & & & & \\
\hline 3141.733 & 60 & 400 & 31820.35 & 0.00 & $34575_{1 \frac{1}{2}}^{\circ}-66395_{2 \frac{1}{2}}$ & 4 & 1.420 & 1.234 & 0.093 & 0.954 \\
\hline $\begin{array}{l}3141.17 \\
3140.936\end{array}$ & $1000 r$ & $\begin{array}{r}3 \\
2000\end{array}$ & $\begin{array}{l}31826.05 \\
3182849\end{array}$ & $\begin{array}{l}+0.07 \\
-0.06\end{array}$ & $44497_{2 \frac{1}{2}}^{\circ}-76323_{3 \frac{1}{2}}$ & & & & & \\
\hline $\begin{array}{l}3140.930 \\
3140.28\end{array}$ & $1000 r$ & $\begin{array}{r}2000 \\
2\end{array}$ & $\begin{array}{l}31828.42 \\
31835.07\end{array}$ & -0.06 & $28757_{1 \frac{1}{2}}^{\circ}-60586_{1 \frac{1}{2}}^{\circ}$ & 6 & 1.440 & 1.320 & 0.180 & 1.380 \\
\hline $\begin{array}{l}3139.75 \\
3139.27\end{array}$ & 1 & $\begin{array}{l}6 \\
2\end{array}$ & $\begin{array}{l}31840.44 \\
31845.31\end{array}$ & -0.04 & $57765_{4 \frac{1}{2}}-89605_{4 \frac{1}{2}}^{\circ}$ & & & & & \\
\hline 3138.276 & & 5 & 31855.40 & & & & & & & \\
\hline 3138.07 & & 2 & 31857.49 & & & & & & & \\
\hline 3137.622 & & 10 & 31862.04 & -0.04 & $40917_{4 \frac{1}{2}}^{\circ}-72779_{3 \frac{1}{2}}$ & & & & & \\
\hline 3137.51 & & 1 & 31863.18 & +0.09 & $61051_{3 \frac{1}{2}}-92914_{4 \frac{1}{2}}^{\circ}$ & & & & & \\
\hline 3136.760 & 80 & 400 & 31870.79 & -0.03 & $33052_{4 \frac{1}{2}}^{\circ}-64923_{4 \frac{1}{2}}$ & 6 & 1.265 & 1.105 & 0.72 & 1.185 \\
\hline 3136.67 & 1 & 10 & 31871.71 & & & & & & & \\
\hline 3136.18 & & 2 & 31876.69 & 0.00 & $68148_{5 \frac{1}{2}}-100025_{5 \frac{1}{2}}^{\circ}$ & & & & & \\
\hline 3135.989 & & 4 & 31878.63 & +0.02 & $37516_{3 \frac{1}{2}}^{\circ}-69395_{3 \frac{1}{2}}^{\circ}$ & & & & & \\
\hline $\begin{array}{l}3135.93 \\
3135.43\end{array}$ & & $\begin{array}{l}1 \\
1\end{array}$ & $\begin{array}{l}31879.23 \\
31884.31\end{array}$ & & & & & & & \\
\hline 3135.316 & 1 & 10 & 31885.47 & +0.01 & $44438_{3 \frac{1}{3}}^{\circ}-76323_{3 \frac{1}{2}}$ & & & & & \\
\hline 3135.12 & 4 & 30 & 31887.47 & -0.02 & $34575_{1 \frac{1}{2}}^{\circ}-66462_{0 \frac{1}{2}}^{\circ}$ & & & & & \\
\hline 3133.96 & & 1 & 31899.27 & & $5 \operatorname{sen}-40-5$ & & & & & \\
\hline 3133.845 & & $\begin{array}{l}3 \\
3\end{array}$ & 31900.44 & +0.03 & $46169_{4 \frac{1}{2}}^{\circ}-78070_{4 \frac{1}{2}}$ & & & & & \\
\hline 3133.778 & & 3 & 31901.12 & -0.02 & $48556_{3 \frac{1}{2}}^{\circ}-80457_{3 \frac{1}{2}}$ & & & & & \\
\hline
\end{tabular}


TABLE 6. Yb II-Observed and classified lines-Continued

\begin{tabular}{|c|c|c|c|c|c|c|c|c|c|c|}
\hline 1 & 2 & 3 & 4 & 5 & 6 & 7 & 8 & 9 & 10 & 11 \\
\hline \multirow{2}{*}{$\begin{array}{l}\text { Wave- } \\
\text { length } \\
\text { air }\end{array}$} & \multicolumn{2}{|c|}{ Intensity } & \multirow{2}{*}{$\begin{array}{l}\text { Wave } \\
\text { number }\end{array}$} & \multirow{2}{*}{$\mathrm{o}-\mathrm{c}$} & \multirow{2}{*}{ Classification } & \multirow{2}{*}{$\begin{array}{l}\text { Zee- } \\
\text { man } \\
\text { type }\end{array}$} & \multirow{2}{*}{ lst $g$} & \multirow{2}{*}{ 2nd $g$} & \multicolumn{2}{|c|}{$\begin{array}{c}\text { Zeeman } \\
\text { components }\end{array}$} \\
\hline & $\begin{array}{c}\text { Meggers } \\
\text { lamp }\end{array}$ & $\begin{array}{c}\text { Thomson } \\
\text { lamp }\end{array}$ & & & & & & & Strong $p$ & Strong $n$ \\
\hline A & & & $K$ & $K$ & & & & & & \\
\hline $\begin{array}{l}3132.628 \\
3132.478 \\
3132.294 \\
3131.397 \\
3131.38\end{array}$ & $\begin{array}{r}15 \\
2 \\
1\end{array}$ & $\begin{array}{r}150 \\
15 \\
8 \\
1 \\
3\end{array}$ & $\begin{array}{l}31912.83 \\
31914.36 \\
31916.23 \\
31925.38 \\
31925.55\end{array}$ & $\begin{array}{l}-0.03 \\
-0.04 \\
-0.04 \\
-0.06\end{array}$ & $\begin{array}{l}33653_{0 \frac{1}{2}}^{\circ}-65566_{0 \frac{1}{2}} \\
43075_{1 \frac{1}{2}}^{\circ}-74989_{0 \frac{1}{2}} \\
43075_{1 \frac{1}{2}}^{\circ}-74991_{1 \frac{1}{2}}^{\circ} \\
61051_{3 \frac{1}{2}}-92976_{3 \frac{1}{2}}^{\circ}\end{array}$ & 6 & 1.32 & 1.42 & $(0.05)$ & 1.37 \\
\hline $\begin{array}{l}3129.145 \\
3128.60\end{array}$ & 8 & $\begin{array}{r}80 \\
1\end{array}$ & $\begin{array}{l}31948.35 \\
31953.92\end{array}$ & -0.03 & $41688_{1 \frac{1}{2}}^{\circ}-73636_{0 \frac{1}{2}}$ & 5 & 0.78 & 0.70 & $(0.04)$ & 0.82 \\
\hline $\begin{array}{l}3127.866 \\
3127.64 \\
3127.49\end{array}$ & 20 & $\begin{array}{r}120 \\
2 \\
2\end{array}$ & $\begin{array}{l}31961.41 \\
31963.72 \\
31965.26\end{array}$ & $\begin{array}{l}-0.04 \\
-0.01 \\
+0.07\end{array}$ & $\begin{array}{l}34389_{2 \frac{1}{2}}^{\circ}-66351_{2 \frac{1}{2}}^{\circ} \\
66558_{3 \frac{1}{2}}-98521_{4 \frac{1}{2}}^{\circ} \\
47663_{4 \frac{1}{2}}^{\circ}-79628_{3 \frac{1}{2}}^{\circ}\end{array}$ & 6 & $(1.01)$ & 1.12 & 0.27 & $?$ \\
\hline $\begin{array}{l}3127.31 \\
3127.138 \\
3126.25 \\
3126.192 \\
3125.885\end{array}$ & $\begin{array}{l}4 \\
1 \\
4 \\
1\end{array}$ & $\begin{array}{c}2 h \\
40 \\
5 \\
20 \\
10\end{array}$ & $\begin{array}{l}31967.10 \\
31968.86 \\
31977.94 \\
31978.53 \\
31981.67\end{array}$ & $\begin{array}{l}+0.02 \\
+0.04 \\
-0.03 \\
-0.03\end{array}$ & $\begin{array}{l}41688_{1 \frac{1}{2}}^{\circ}-73657_{1 \frac{1}{2}} \\
31979_{3 \frac{1}{2}}^{\circ}-63957_{3 \frac{1}{2}} \\
41678_{2 \frac{1}{2}}^{\circ}-73657_{1 \frac{1}{2}} \\
43007_{0 \frac{1}{2}}^{\circ}-74989_{0 \frac{1}{2}}^{\circ}\end{array}$ & & & & & \\
\hline 3125.700 & 5 & 20 & 31983.56 & $\left\{\begin{array}{l}-0.01 \\
-0.08\end{array}\right.$ & $\begin{array}{l}43007_{0 \frac{1}{2}}^{\circ}-74991_{1 \frac{1}{2}} \\
59618_{31}-91602^{\circ}{ }^{\circ}\end{array}$ & & & & & \\
\hline $\begin{array}{l}3125.443 \\
3124.25 \\
3123.870 \\
3123.510\end{array}$ & 50 & $\begin{array}{r}100 \\
2 \\
6 \\
60\end{array}$ & $\begin{array}{l}31986.19 \\
31998.41 \\
32002.30 \\
32005.99\end{array}$ & $\begin{array}{r}-0.02 \\
+0.02 \\
+0.01 \\
+0.03\end{array}$ & $\begin{array}{l}21418_{3 \frac{1}{2}}^{\circ \frac{1}{2}}-53404_{2 \frac{1}{2}}^{2 \frac{1}{2}} \\
67416_{4 \frac{1}{2}}^{\circ}-99414_{4 \frac{1}{2}}^{\circ} \\
37077_{2 \frac{1}{2}}^{\circ}-690799_{2 \frac{1}{2}}^{\circ} \\
34389_{2 \frac{1}{2}}^{\circ}-66395_{2 \frac{1}{2}}^{\circ}\end{array}$ & & & & & \\
\hline $\begin{array}{l}3122.74 \\
3122.48 \\
3122.185 \\
3121.38 \\
3119.73\end{array}$ & 2 & $\begin{array}{r}1 \\
3 \\
20 \\
2 \\
1\end{array}$ & $\begin{array}{l}32013.88 \\
32016.54 \\
32019.57 \\
32027.83 \\
32044.76\end{array}$ & $\begin{array}{l}+0.07 \\
+0.10 \\
+0.12\end{array}$ & $\begin{array}{l}45429_{3 \frac{1}{2}}^{\circ}-77449_{3 \frac{1}{2}}^{\circ} \\
61214_{4 \frac{1}{2}}-93242_{5 \frac{1}{2}}^{\circ} \\
61822_{3 \frac{1}{2}}-93867_{4 \frac{1}{2}}^{\circ}\end{array}$ & & & & & \\
\hline $\begin{array}{l}3119.37 \\
3119.10\end{array}$ & & $\begin{array}{l}2 \\
1\end{array}$ & $\begin{array}{l}32048.46 \\
32051.24\end{array}$ & +0.02 & $66571_{5 \frac{1}{2}}-98620_{6 \frac{1}{2}}^{\circ}$ & & & & & \\
\hline 3117.806 & $1000 r$ & 3000 & 32064.54 & -0.02 & $26759_{2 \frac{1}{2}}^{\circ}-58823_{2 \frac{1}{2}}$ & 6 & 1.568 & 1.260 & 0.770 & 1.414 \\
\hline $\begin{array}{l}3116.702 \\
3116.487\end{array}$ & $\begin{array}{l}60 \\
10\end{array}$ & $\begin{array}{r}300 \\
70\end{array}$ & $\begin{array}{l}32075.90 \\
32078.11\end{array}$ & $\begin{array}{r}-0.02 \\
+0.03\end{array}$ & $\begin{array}{l}31979_{3 \frac{1}{2}}^{\circ}-64055_{2 \frac{1}{2}}^{\circ} \\
44438^{\circ}-76516^{\frac{1}{2}}\end{array}$ & 5 & 1.333 & 1.111 & 0.110 & 1.888 \\
\hline 3116.342 & 10 & 40 & 32079.60 & -0.05 & $31568^{\circ}{ }_{13}-63647_{1 \frac{1}{3}}$ & & & & & \\
\hline 3116.070 & 15 & 70 & 32082.40 & -0.03 & $33494_{3 \frac{1}{2}}^{\circ-2}-65577_{3 \frac{1}{2}}^{12}$ & 6 & 1.00 & 1.05 & 0.18 & 1.02 \\
\hline 3115.340 & 100 & 400 & 32089.92 & -0.06 & $32371_{2 \frac{1}{2}}^{0}-64461_{1 \frac{1}{2}}^{0}$ & 7 & 1.162 & 1.162 & 0 & 1.162 \\
\hline $\begin{array}{l}3115.25 \\
3114.905\end{array}$ & $\begin{array}{l}1 \\
3\end{array}$ & $\begin{array}{r}8 \\
20\end{array}$ & $\begin{array}{l}32090.85 \\
32094.40\end{array}$ & -0.10 & $37516_{3 \frac{1}{2}}^{0^{2}}-69607_{2 \frac{1}{2}}$ & & & & & \\
\hline $\begin{array}{l}3114.69 \\
3114.505\end{array}$ & & $\begin{array}{l}1 \\
4\end{array}$ & $\begin{array}{l}32096.62 \\
32098.52\end{array}$ & & & & & & & \\
\hline 3112.980 & 1 & 10 & 32114.25 & -0.04 & $45335_{4 \frac{1}{2}}^{\circ}-77449_{3 \frac{1}{2}}$ & & & & & \\
\hline 3112.91 & & 4 & 32114.97 & -0.05 & $51248_{2 \frac{1}{2}}^{\circ}-83363_{3 \frac{1}{2}}$ & & & & & \\
\hline 3111.047 & 1 & 6 & 32134.20 & -0.04 & $31568_{2 \frac{1}{2}}^{\circ}-63702_{2 \frac{1}{2}}$ & & & & & \\
\hline
\end{tabular}


TABLE 6. Yb II-Observed and classified lines-Continued

\begin{tabular}{|c|c|c|c|c|c|c|c|c|c|c|}
\hline 1 & 2 & 3 & 4 & 5 & 6 & 7 & 8 & 9 & 10 & 11 \\
\hline \multirow{2}{*}{$\begin{array}{l}\text { Wave- } \\
\text { length } \\
\text { air }\end{array}$} & \multicolumn{2}{|c|}{ Intensity } & \multirow{2}{*}{$\begin{array}{c}\text { Wave } \\
\text { number }\end{array}$} & \multirow{2}{*}{$\mathrm{o}-\mathrm{c}$} & \multirow{2}{*}{ Classification } & \multirow{2}{*}{$\begin{array}{l}\text { Zee- } \\
\text { man } \\
\text { type }\end{array}$} & \multirow{2}{*}{ lst $g$} & \multirow{2}{*}{ 2nd $g$} & \multicolumn{2}{|c|}{$\begin{array}{c}\text { Zeeman } \\
\text { components }\end{array}$} \\
\hline & $\begin{array}{c}\text { Meggers } \\
\text { lamp }\end{array}$ & $\begin{array}{c}\text { Thomson } \\
\text { lamp }\end{array}$ & & & & & & & Strong $p$ & Strong $n$ \\
\hline$\AA$ & \multirow{3}{*}{5} & & $K$ & $K$ & \multirow{3}{*}{$\begin{array}{l}33052_{4 \frac{1}{2}}^{\circ}-65199_{4 \frac{1}{2}} \\
45737_{1 \frac{1}{2}}^{\circ}-77887_{0 \frac{1}{2}}\end{array}$} & \multirow{6}{*}{$\begin{array}{l}4 \\
5\end{array}$} & \multirow{6}{*}{$\begin{array}{l}(1.333) \\
(1.007)\end{array}$} & \multirow{6}{*}{$\begin{array}{l}1.25 \\
1.08\end{array}$} & \multirow{6}{*}{$\begin{array}{l}w \\
w\end{array}$} & \multirow{6}{*}{$\begin{array}{l}1.14 \\
1.26\end{array}$} \\
\hline 3109.786 & & 40 & 32147.23 & -0.02 & & & & & & \\
\hline 3109.52 & & 2 & 32149.98 & -0.03 & & & & & & \\
\hline 3109.10 & 1 & 5 & 32154.32 & & & & & & & \\
\hline 3107.902 & $1000 r$ & 3000 & 32166.72 & -0.07 & $30392_{1 \frac{1}{2}}^{\circ}-62559_{2 \frac{1}{2}}$ & & & & & \\
\hline 3107.760 & 100 & 500 & 32168.18 & -0.06 & $34389_{2 \frac{1}{2}}^{\circ}-66558_{3 \frac{1}{2}}^{\circ}$ & & & & & \\
\hline $\begin{array}{l}3106.97 \\
3106.94\end{array}$ & \multirow{5}{*}{2} & $\begin{array}{l}2 \\
6\end{array}$ & $\begin{array}{l}32176.36 \\
32176.67\end{array}$ & $\begin{array}{l}+0.06 \\
-0.09\end{array}$ & $\begin{array}{l}45273_{3 \frac{1}{2}}^{\circ}-77449_{3 \frac{1}{2}} \\
454290^{\circ}\end{array}$ & \multirow[b]{10}{*}{5} & \multirow[b]{10}{*}{ (1.170) } & \multirow[b]{10}{*}{1.23} & \multirow[b]{10}{*}{$w$} & \\
\hline & & & $\begin{array}{l}32160.07 \\
2917875\end{array}$ & $\int-0.05$ & $44497_{\frac{21}{2}}^{0}-76676_{2 \frac{1}{2}}^{0}$ & & & & & \\
\hline 3106.740 & & 10 & 32178.65 & $\{+0.03$ & $57534_{3 \frac{1}{2}}^{\circ}-89713_{2 \frac{1}{2}}^{2}$ & & & & & \\
\hline 3106.133 & & 3 & 32185.03 & +0.09 & $48272_{2 \frac{1}{2}}-80457_{3 \frac{1}{2}}$ & & & & & \\
\hline 3106.007 & & 4 & 32186.34 & -0.04 & $61822_{3 \frac{1}{2}}-94008_{3 \frac{1}{2}}^{\circ}$ & & & & & \\
\hline 3105.37 & & 1 & 32192.94 & & & & & & & \\
\hline 3103.81 & & 4 & 32209.12 & & & & & & & \\
\hline 3103.36 & & 1 & 32213.79 & -0.05 & $43956_{.2 \frac{1}{2}}^{\circ}-76170_{2 \frac{1}{2}}$ & & & & & \\
\hline 3102.71 & & $\begin{array}{r}2 \\
150\end{array}$ & 32220.54 & & & & & & & \\
\hline 3102.074 & 30 & 150 & 32227.15 & -0.03 & $32.371_{2 \frac{1}{2}}^{\circ}-64598_{3 \frac{1}{2}}^{\circ}$ & & & & & 1.37 \\
\hline 3101.688 & & 5 & 32231.16 & +0.08 & $66571_{5 \frac{1}{2}}-98802_{5 \frac{1}{2}}^{\circ}$ & & & & & \\
\hline 3101.361 & 50 & 250 & 32234.55 & -0.05 & $33653_{0 \frac{1}{2}}^{\circ}-65888_{1 \frac{1}{2}}^{\circ}$ & 4 & 1.32 & 1.22 & $(0.05)$ & 1.17 \\
\hline 3101.004 & 15 & 100 & 32238.26 & -0.02 & $44438_{3 \frac{1}{2}}^{\circ}-76676_{2 \frac{1}{2}}$ & & & & & \\
\hline $\begin{array}{l}3100.25 \\
3100.03\end{array}$ & & $\begin{array}{l}4 \\
2\end{array}$ & $\begin{array}{l}32246.11 \\
32248.39\end{array}$ & +0.04 & $61214_{4 \frac{1}{2}}-93463_{3 \frac{1}{2}}^{\circ}$ & & & & & \\
\hline 3099.114 & 1 & 10 & 32257.93 & +0.02 & $66571_{5 \frac{1}{2}}-98829_{6 \frac{1}{3}}^{\circ}$ & & & & & \\
\hline 3098.850 & 4 & 10 & 32260.67 & +0.01 & $22960_{1 \frac{1}{2}}-55221_{1 \frac{1}{2}}^{\circ}$ & & & & & \\
\hline 3098.384 & & 3 & 32265.52 & & & & & & & \\
\hline 3097.331 & 1 & 8 & 32276.49 & -0.02 & $47329_{2 \frac{1}{2}}^{\circ}-79605_{1 \frac{1}{2}}$ & & & & & \\
\hline 3096.278 & & 4 & 32287.47 & & & & & & & \\
\hline 3096.10 & & 1 & 32289.33 & +0.10 & $61214_{4 \frac{1}{2}}-93503_{4 \frac{1}{2}}^{\circ}$ & & & & & \\
\hline 3095.415 & 30 & 60 & 32296.47 & -0.04 & $21418_{3 \frac{1}{2}}^{\circ}-53715_{3 \frac{1}{2}}^{\circ}$ & & & & & \\
\hline 3095.221 & 10 & 60 & 32298.50 & +0.02 & $30562_{5 \frac{1}{2}}^{\circ}-62861_{5 \frac{1}{2}}$ & 6 & (1.112) & 1.15 & $w$ & 1.13 \\
\hline 3094.894 & 100 & 200 & 32301.91 & -0.05 & $21418_{3 \frac{1}{2}}-53720_{4 \frac{1}{2}}$ & & & & & \\
\hline 3093.870 & $200 r$ & 1000 & 32312.60 & +0.01 & $31631_{6 \frac{1}{2}}^{\circ}-63944_{5 \frac{1}{2}}^{\circ}$ & 5 & 1.24 & 1.13 & $(0.056)$ & 1.86 \\
\hline 3093.423 & 3 & 30 & 32317.27 & +0.07 & $35831_{5 \frac{1}{2}}^{\circ}-68148_{5 \frac{1}{2}}$ & 6 & (1.214) & 1.23 & $w$ & 1.22 \\
\hline 3093.40 & 1 & 10 & 32317.51 & $\left\{\begin{array}{l}-0.10 \\
-0.07\end{array}\right.$ & $\begin{array}{l}37077_{2 \frac{1}{2}}^{\circ}-69395_{3 \frac{1}{2}} \\
45429^{\circ}-77747^{\circ}\end{array}$ & & & & & \\
\hline 3092.10 & 1 & 8 & 32331.10 & -0.01 & $26759_{\frac{21}{2}}^{\circ}-59090_{3 \frac{1}{2}}^{\circ}$ & & & & & \\
\hline 3091.10 & 1 & 6 & 32341.55 & +0.06 & $44497_{2 \frac{1}{2}}^{0}-76839_{2 \frac{1}{2}}$ & & & & & \\
\hline 3090.924 & 6 & 15 & 32343.40 & -0.01 & $44940_{1 \frac{1}{2}}^{\circ}-77284_{1 \frac{1}{2}}$ & & & & & \\
\hline 3090.747 & 1 & 10 & 32345.25 & +0.04 & $56375_{2 \frac{1}{3}}-88721_{3 \frac{1}{3}}^{\circ}$ & & & & & \\
\hline 3089.61 & 3 & 20 & 32357.15 & +0.12 & $35059_{3 \frac{1}{2}}^{\circ}-67416_{4 \frac{1}{2}}$ & & & & & \\
\hline 3089.102 & $150 r$ & 500 & 32362.47 & -0.04 & $28757_{1 \frac{1}{2}}^{\circ}-61120_{0 \frac{1}{2}}$ & 4 & 1.437 & 1.658 & 0.110 & 1.325 \\
\hline
\end{tabular}


TABLE 6. Yb II-Observed and classified lines-Continued

\begin{tabular}{|c|c|c|c|c|c|c|c|c|c|c|}
\hline 1 & 2 & 3 & 4 & 5 & 6 & 7 & 8 & 9 & 10 & 11 \\
\hline \multirow{2}{*}{$\begin{array}{l}\text { Wave- } \\
\text { length } \\
\text { air }\end{array}$} & \multicolumn{2}{|c|}{ Intensity } & \multirow{2}{*}{$\begin{array}{c}\text { Wave } \\
\text { number }\end{array}$} & \multirow{2}{*}{$o-c$} & \multirow{2}{*}{ Classification } & \multirow{2}{*}{$\begin{array}{l}\text { Zee- } \\
\text { man } \\
\text { type }\end{array}$} & \multirow{2}{*}{ lst $g$} & \multirow{2}{*}{ 2nd $g$} & \multicolumn{2}{|c|}{$\begin{array}{c}\text { Zeeman } \\
\text { components }\end{array}$} \\
\hline & $\begin{array}{l}\text { Meggers } \\
\text { lamp }\end{array}$ & $\begin{array}{c}\text { Thomson } \\
\text { lamp }\end{array}$ & & & & & & & Strong $p$ & Strong $n$ \\
\hline A & & & $K$ & $K$ & & & & & & \\
\hline 3088.823 & 3 & 30 & 32365.39 & -0.05 & $40917_{4 \frac{1}{2}}^{\circ}-73283_{4 \frac{1}{2}}$ & & & & & \\
\hline 3088.78 & 1 & 9 & 32365.84 & -0.09 & $37077_{2 \frac{1}{2}}^{\circ}-69443_{1 \frac{1}{2}}$ & & & & & \\
\hline 3088.66 & & 3 & 32367.10 & +0.02 & $43956_{2 \frac{1}{2}}^{\circ}-76323_{3 \frac{1}{2}}$ & & & & & \\
\hline 3087.998 & 1 & 15 & 32374.04 & 0.00 & $40917_{4 \frac{1}{2}}^{-0^{2}}-73291_{5 \frac{1}{2}}$ & & & & & \\
\hline 3087.720 & & 6 & 32376.96 & -0.03 & $47228_{0 \frac{1}{2}}^{\circ}-79605_{1 \frac{1}{2}}$ & & & & & \\
\hline $\begin{array}{l}3087.46 \\
3086.971\end{array}$ & 10 & $\begin{array}{r}2 \\
100\end{array}$ & $\begin{array}{l}32379.68 \\
32384.81\end{array}$ & +0.03 & $39378_{0 \frac{1}{2}}^{\circ}-71763_{1 \frac{1}{2}}$ & 4 & 1.84 & 1.10 & 0.37 & 0.72 \\
\hline 3086.508 & 8 & 40 & 32389.67 & -0.05 & $31568_{2 \frac{1}{2}}^{\circ}-63957_{3 \frac{1}{2}}$ & & & & & \\
\hline 3085.817 & 10 & 70 & 32396.92 & +0.02 & $35019_{4 \frac{1}{2}}^{-2}-67416_{4 \frac{1}{2}}$ & 6 & 1.16 & 1.26 & 0.44 & 1.21 \\
\hline 3085.434 & 9 & 60 & 32400.94 & -0.03 & $44438_{3 \frac{1}{2}}^{\circ}-76839_{2 \frac{1}{2}}$ & & & 1 & & \\
\hline 3084.347 & 7 & 70 & 32412.36 & -0.01 & $45335_{4 \frac{1}{2}}^{\circ}-77747_{4 \frac{1}{2}}^{\circ}$ & 6 & 1.00 & 1.07 & 0.30 & $1.04 ?$ \\
\hline 3083.809 & & 3 & 32418.02 & & & & & & & \\
\hline 3083.44 & & 1 & 32421.89 & & & & & & & \\
\hline 3082.34 & & 1 & 32433.46 & +0.05 & $53715_{3 \frac{1}{2}}-86148_{2 \frac{1}{2}}^{\circ}$ & & & & & \\
\hline 3082.264 & & 6 & 32434.26 & & & & & & & \\
\hline 3082.05 & & 1 & 32436.52 & -0.01 & $45012_{2 \frac{1}{2}}^{\circ}-77449_{3 \frac{1}{2}}$ & & & & & \\
\hline 3081.625 & & 8 & 32440.99 & +0.09 & $37516_{3 \frac{1}{2}}^{0}-69957_{4 \frac{1}{2}}^{\circ}$ & & & & & \\
\hline $\begin{array}{l}3081.18 \\
3080552\end{array}$ & & $\begin{array}{r}1 \\
30\end{array}$ & $\begin{array}{l}32445.67 \\
3245299\end{array}$ & & & & & & & \\
\hline $\begin{array}{l}3080.552 \\
3080.49\end{array}$ & 3 & $\begin{array}{r}30 \\
3\end{array}$ & $\begin{array}{l}32452.29 \\
32452.94\end{array}$ & $\begin{array}{l}+0.07 \\
+0.11\end{array}$ & $\begin{array}{l}45429_{3 \frac{1}{2}}^{1}-77882_{3 \frac{1}{2}} \\
61051_{3 \frac{1}{2}}-93503^{\circ} \frac{1 \frac{1}{2}}{?}\end{array}$ & 6 & 1.15 & 1.13 & $(0.07)$ & 1.14 \\
\hline $\begin{array}{l}3080.17 \\
3070502\end{array}$ & 3 & 20 & 32456.31 & +0.04 & $33494_{3 \frac{1}{2}}^{2}-65950_{4 \frac{1}{2}}$ & & & & & \\
\hline 3079.593 & & 7 & 32462.39 & +0.09 & $61873_{6 \frac{1}{2}}-94335_{\frac{5}{2}}^{\circ}$ & & & & & \\
\hline 3078.450 & 1 & 10 & 32474.45 & +0.07 & $45273_{3 \frac{1}{2}}^{\circ}-77747_{+\frac{1}{2}}$ & & & & & \\
\hline 3078.31 & 1 & 10 & 32475.92 & +0.07 & $43075_{1 \frac{1}{2}}^{\circ}-75550_{2 \frac{1}{2}}$ & & & & & \\
\hline $\begin{array}{l}3077.82 \\
3077.34\end{array}$ & & $\begin{array}{l}2 \\
7\end{array}$ & $\begin{array}{l}32481.09 \\
32486.16\end{array}$ & +0.09 & $61822_{3 \frac{1}{2}}-94303_{3 \frac{1}{2}}^{\circ}$ & & & & & \\
\hline 3077.18 & & 8 & 32487.85 & +0.11 & $31568_{2 \frac{1}{2}}^{\circ}-64055_{2 \frac{1}{2}} ?$ & & & & & \\
\hline 3077.14 & & 6 & 32488.27 & +0.04 & $64598_{3 \frac{1}{2}}-97086_{4 \frac{1}{\circ}}^{\circ}$ & & & & & \\
\hline 3076.50 & & 1 & 32495.03 & +0.09 & $61051_{3 \frac{1}{2}}-93546_{2 \frac{1}{2}}^{\circ \frac{2}{2}}$ & & & & & \\
\hline $\begin{array}{l}3076.013 \\
3075.10\end{array}$ & 30 & $\begin{array}{r}150 \\
2\end{array}$ & $\begin{array}{l}32500.17 \\
32509.82\end{array}$ & -0.05 & $26759_{2 \frac{1}{2}}^{\circ}-59259_{2 \frac{1}{2}}^{\circ}$ & 6 & 1.572 & 1.104 & 1.170 & 1.338 \\
\hline 3074.92 & & 2 & $\begin{array}{l}32509.02 \\
32511.73\end{array}$ & & & & & & & \\
\hline 3074.84 & & 3 & 32512.57 & & & & & & & \\
\hline $\begin{array}{l}3074.50 \\
3073.89\end{array}$ & 1 & $\begin{array}{r}10 \\
3\end{array}$ & $\begin{array}{l}32516.17 \\
32522.62\end{array}$ & +0.01 & $38342_{4 \frac{1}{2}}^{\circ}-70858_{4 \frac{1}{2}}$ & & & & & \\
\hline 3073.680 & 20 & 100 & 32524.84 & +0.02 & $33052_{4 \frac{1}{2}}^{\circ}-65577_{3 \frac{1}{2}}$ & & & & & \\
\hline 3073.19 & & 3 & 32530.03 & +0.08 & $37077_{2 \frac{1}{2}}^{\circ}-69607_{2 \frac{1}{2}}^{3 \frac{1}{2}}$ & & & & & \\
\hline 3072.486 & 6 & 60 & 32537.48 & +0.06 & $61822_{3 \frac{1}{2}}-94360_{4 \frac{1}{2}}^{\circ}$ & & & & & \\
\hline $\begin{array}{l}3072.41 \\
3071.583\end{array}$ & & $\begin{array}{r}4 \\
50\end{array}$ & $\begin{array}{l}32538.29 \\
3254705\end{array}$ & +0.03 & $63944_{5 \frac{1}{2}}-96482_{4 \frac{1}{2}}^{\circ}$ & & & & & \\
\hline 3071.26 & 5 & $\begin{array}{r}50 \\
1\end{array}$ & $\begin{array}{l}32547.05 \\
32550.47\end{array}$ & +0.04 & $45335_{4 \frac{1}{2}}^{\circ}-77882_{3 \frac{1}{2}}$ & & & & & \\
\hline
\end{tabular}


TABLE 6. Yb II-Observed and classified lines-Continued

\begin{tabular}{|c|c|c|c|c|c|c|c|c|c|c|}
\hline 1 & 2 & 3 & 4 & 5 & 6 & 7 & 8 & 9 & 10 & 11 \\
\hline \multirow{2}{*}{$\begin{array}{l}\text { Wave- } \\
\text { length } \\
\text { air }\end{array}$} & \multicolumn{2}{|c|}{ Intensity } & \multirow{2}{*}{$\begin{array}{c}\text { Wave } \\
\text { number }\end{array}$} & \multirow{2}{*}{$o-c$} & \multirow{2}{*}{ Classification } & \multirow{2}{*}{$\begin{array}{l}\text { Zee- } \\
\text { man } \\
\text { type }\end{array}$} & \multirow{2}{*}{ lst $g$} & \multirow{2}{*}{ 2nd $g$} & \multicolumn{2}{|c|}{$\begin{array}{c}\text { Zeeman } \\
\text { components }\end{array}$} \\
\hline & $\begin{array}{l}\text { Meggers } \\
\text { lamp }\end{array}$ & $\begin{array}{c}\text { Thomson } \\
\text { lamp }\end{array}$ & & & & & & & Strong $p$ & Strong $n$ \\
\hline$\stackrel{\circ}{A}$ & & & $K$ & $K$ & & & & & & \\
\hline 3070.28 & 2 & 7 & 32560.86 & +0.06 & $43956^{\circ \frac{1}{2}}-76517_{1 \frac{1}{2}}^{\circ}$ & & & & & \\
\hline 3069.34 & & 2 & 32570.83 & +0.05 & $65950_{4 \frac{1}{2}}-98521_{4 \frac{1}{2}}^{\circ}$ & & & & & \\
\hline 3068.69 & 1 & 10 & 32577.73 & +0.08 & $47005_{1 \frac{1}{2}}^{\circ}-79583_{\frac{21}{2}}^{\circ}$ & & & & & \\
\hline 3068.284 & 7 & 60 & 32582.04 & +0.01 & $41688_{1 \frac{1}{2}}^{\circ}-74270_{2 \frac{1}{2}}^{2}$ & & & & & \\
\hline 3067.986 & 2 & 10 & 32585.20 & +0.07 & $32981_{1 \frac{1}{2}}^{\circ}-65566_{0 \frac{1}{2}}$ & & & & & \\
\hline 3067.365 & 10 & 70 & 32591.80 & +0.05 & $41678_{2 \frac{1}{2}}^{\circ}-74270_{2 \frac{1}{2}}^{\circ}$ & 6 & (1.19) & 0.93 & 0.65 & $?$ \\
\hline 3066.54 & & 2 & 32600.57 & +0.08 & $47005_{1 \frac{1}{2}}^{\circ}-79605_{1 \frac{1}{2}}$ & & & & & \\
\hline 3066.487 & 2 & 6 & 32601.13 & +0.13 & $30562_{5 \frac{1}{2}}^{\circ}-63163_{4 \frac{1}{2}} ?$ & & & & & \\
\hline 3065.74 & & 7 & 32609.07 & +0.05 & $45273_{3 \frac{1}{2}}^{\circ}-77882_{3 \frac{1}{2}}^{2}$ & & & & & \\
\hline 3065.52 & & 2 & 32611.41 & & & & & & & \\
\hline 3065.48 & & 1 & 32611.84 & & & & & & & \\
\hline 3065.040 & 300 & 1000 & 32616.52 & +0.01 & $28757_{1 \frac{1}{2}}^{\circ}-61374_{2 \frac{1}{2}}$ & 4 & 1.45 & 1.30 & 0.075 & 1.08 \\
\hline 3064.868 & 30 & 150 & 32618.35 & -0.03 & $31979_{3 \frac{1}{2}}^{\circ}-64598_{3 \frac{1}{2}}^{-2}$ & & & & & \\
\hline 3064.75 & & 2 & 32619.61 & -0.01 & $30392_{\frac{1}{2}}^{\circ}-63011_{1 \frac{1}{2}}$ & & & & & \\
\hline $\begin{array}{l}3063.80 \\
3063.671\end{array}$ & 20 & $\begin{array}{r}4 \\
100\end{array}$ & $\begin{array}{l}32629.72 \\
32631.10\end{array}$ & $\begin{array}{l}+0.12 \\
+0.02\end{array}$ & $\begin{array}{l}34575_{\frac{1}{2}}^{\circ}-67204_{1 \frac{1}{2}}^{2} \\
34784_{5 \frac{1}{3}}^{\circ}-67416_{4 \frac{1}{3}}^{\circ}\end{array}$ & & & & & \\
\hline 3063.350 & 2 & 10 & 32634.51 & +0.04 & $61374_{2 \frac{1}{2}}-94008_{3 \frac{1}{2}}^{\circ}$ & & & & & \\
\hline 3063.125 & 40 & 200 & 32636.91 & -0.03 & $30224_{4 \frac{1}{2}}^{\circ}-62861_{5 \frac{1}{2}}$ & 5 & 0.935 & 1.137 & 0.101 & 2.047 \\
\hline 3062.524 & & 4 & 32643.32 & & & & & & & \\
\hline 3062.06 & & 1 & 32648.26 & +0.06 & $49008_{3 \frac{1}{2}}^{\circ}-81657_{2_{\frac{1}{2}}}$ & & & & & \\
\hline 3061.646 & & 6 & 32652.68 & +0.12 & $61214_{4 \frac{1}{2}}-93867_{4 \frac{1}{2}}^{\mathrm{o}^{2}} ?$ & & & & & \\
\hline 3060.394 & & 7 & 32666.03 & +0.06 & $44940_{1 \frac{1}{2}}^{\circ}-77606_{2 \frac{1}{2}}^{\circ}$ & & & & & \\
\hline 3059.568 & & 7 & 32674.85 & +0.06 & $66571_{5 \frac{1}{2}}^{1 \frac{1}{2}}-99246_{5 \frac{1}{2}}^{\circ \frac{1}{2}}$ & & & & & \\
\hline 3059.362 & & 4 & 32677.05 & & & & & & & \\
\hline 3058.71 & & 2 & 32684.02 & +0.03 & $49008_{3 \frac{1}{2}}^{\circ}-81692_{3 \frac{1}{2}}$ & & & & & \\
\hline 3058.66 & & 5 & 32684.55 & & & & & & & \\
\hline 3058.288 & & 4 & 32688.53 & & & & & & & \\
\hline 3058.20 & & 1 & 32689.47 & & & & & & & \\
\hline 3057.29 & & 1 & 32699.20 & +0.03 & $47758_{3 \frac{1}{2}}^{\circ}-80457_{3 \frac{1}{2}}$ & & & & & \\
\hline 3056.01 & & 2 & 32712.89 & +0.07 & $58823_{2 \frac{1}{2}}-91536_{3 \frac{1}{2}}^{\circ \frac{1}{2}}$ & & & & & \\
\hline 3055.60 & & 1 & 32717.28 & & & & & & & \\
\hline 3055.46 & & 2 & 32718.78 & & & & & & & \\
\hline 3055.350 & 5 & 15 & 32719.96 & +0.06 & $43956_{2 \frac{1}{2}}^{\circ}-76676_{2 \frac{1}{2}}$ & & & & & \\
\hline 3055.157 & 10 & 60 & 32722.03 & -0.01 & $32371_{2 \frac{1}{2}}^{0}-65093_{3 \frac{1}{2}}^{-2}$ & 4 & 1.168 & 0.978 & 0.095 & 0.503 \\
\hline 3054.649 & 1 & 10 & 32727.47 & 0.00 & $45737_{1 \frac{1}{2}}^{0}-78464_{1 \frac{1}{2}}$ & & & & & \\
\hline 3053.928 & 2 & 20 & 32735.20 & +0.04 & $45335_{4 \frac{1}{2}}^{\circ}-78070_{4 \frac{1}{2}}^{2}$ & & & & & \\
\hline 3053.452 & & 8 & 32740.30 & & & & & & & \\
\hline 3053.084 & 1 & 10 & 32744.24 & $\left\{\begin{array}{l}+0.07 \\
-0.01\end{array}\right.$ & $40035_{3 \frac{1}{2}}^{\circ}-72779_{3 \frac{1}{2}}$ & & & & & \\
\hline 3052.20 & & 2 & 32753.73 & & & & & & & \\
\hline
\end{tabular}


TABlE 6. Yb II-Observed and classified lines-Continued

\begin{tabular}{|c|c|c|c|c|c|c|c|c|c|c|}
\hline 1 & 2 & 3 & 4 & 5 & 6 & 7 & 8 & 9 & 10 & 11 \\
\hline \multirow{2}{*}{$\begin{array}{l}\text { Wave- } \\
\text { length } \\
\text { air }\end{array}$} & \multicolumn{2}{|c|}{ Intensity } & \multirow{2}{*}{$\begin{array}{l}\text { Wave } \\
\text { number }\end{array}$} & \multirow{2}{*}{$0-c$} & \multirow{2}{*}{ Classification } & \multirow{2}{*}{$\begin{array}{l}\text { Zee- } \\
\text { man } \\
\text { type }\end{array}$} & \multirow{2}{*}{ lst $g$} & \multirow{2}{*}{ 2nd $g$} & \multicolumn{2}{|c|}{$\begin{array}{l}\text { Zeeman } \\
\text { components }\end{array}$} \\
\hline & $\begin{array}{l}\text { Meggers } \\
\text { lamp }\end{array}$ & $\begin{array}{c}\text { Thomson } \\
\text { lamp }\end{array}$ & & & & & & & Strong $p$ & Strong $n$ \\
\hline А & & & $K$ & K & & & & & & \\
\hline $\begin{array}{l}3052.021 \\
3051.98\end{array}$ & 1 & $\begin{array}{l}7 \\
2\end{array}$ & $\begin{array}{l}32755.65 \\
32756.09\end{array}$ & $\begin{array}{l}+0.04 \\
+0.01\end{array}$ & $\begin{array}{l}63726_{4 \frac{1}{2}}-96482_{4 \frac{1}{2}}^{\circ} \\
48556_{3 \frac{1}{2}}^{\circ}-81312_{2 \frac{1}{2}}^{\circ}\end{array}$ & & & & & \\
\hline $\begin{array}{l}3051.11 \\
3050.64 \\
3050.50 \\
3049.84 \\
3049.14\end{array}$ & & $\begin{array}{l}2 \\
1 \\
6 \\
2 \\
7\end{array}$ & $\begin{array}{l}32765.43 \\
32770.48 \\
32771.98 \\
32779.07 \\
32786.60\end{array}$ & $\begin{array}{l}+0.11 \\
+0.09\end{array}$ & $\begin{array}{l}58823_{2 \frac{1}{2}}-91602_{2 \frac{1}{2}}^{\circ} \\
44497_{2 \frac{1}{2}}^{\circ}-77284_{1 \frac{1}{2}}^{\circ}\end{array}$ & & & & & \\
\hline $\begin{array}{l}3048.82 \\
3048.41 \\
3048.15 \\
3047.052 \\
3046.482\end{array}$ & $\begin{array}{l}20 \\
30\end{array}$ & $\begin{array}{r}2 \\
6 \\
2 \\
100 \\
200\end{array}$ & $\begin{array}{l}32790.04 \\
32794.45 \\
32797.24 \\
32809.06 \\
32815.20\end{array}$ & $\begin{array}{l}+0.15 \\
+0.07 \\
+0.06 \\
-0.01\end{array}$ & $\begin{array}{l}61214_{4 \frac{1}{2}}-94008_{3 \frac{1}{2}}^{\circ} \\
45273_{3 \frac{1}{2}}^{\circ}-78070_{4 \frac{1}{2}}^{\circ} \\
33653_{0 \frac{1}{2}}^{\circ}-66462_{0 \frac{1}{2}}^{\circ} \\
34389_{2 \frac{1}{2}}^{\circ}-67204_{1 \frac{1}{2}}^{\circ}\end{array}$ & $\begin{array}{l}6 \\
4\end{array}$ & $\begin{array}{l}1.315 \\
(1.007)\end{array}$ & $\begin{array}{l}1.107 \\
1.03\end{array}$ & $\begin{array}{l}0.104 \\
w\end{array}$ & $\begin{array}{l}1.211 \\
0.97\end{array}$ \\
\hline $\begin{array}{l}3046.27 \\
3045.91 \\
3045.84 \\
3045.46 \\
3045.044\end{array}$ & 1 & $\begin{array}{l}3 \\
2 \\
1 \\
4 \\
7\end{array}$ & $\begin{array}{l}32817.48 \\
32821.36 \\
32822.12 \\
32826.21 \\
32830.70\end{array}$ & +0.02 & $\begin{array}{l}53404_{2 \frac{1}{2}}-86226_{1 \frac{1}{2}}^{\circ} \\
43075_{1 \frac{1}{2}}^{\circ}-75905_{0 \frac{1}{2}}^{\circ}\end{array}$ & & & & & \\
\hline \begin{tabular}{l|}
3044.83 \\
3044.004 \\
3043.93 \\
3043.07 \\
3042.78
\end{tabular} & $\begin{array}{l}10 \\
20\end{array}$ & $\begin{array}{r}60 \\
100 \\
4 \\
1 \\
3\end{array}$ & $\begin{array}{l}32833.00 \\
32841.91 \\
32842.71 \\
32851.99 \\
32855.12\end{array}$ & $\begin{array}{l}+0.01 \\
+0.03 \\
+0.05\end{array}$ & $\begin{array}{l}40917_{4 \frac{1}{2}}^{\circ}-73750_{4 \frac{1}{2}} \\
30392_{1 \frac{1}{2}}^{\circ}-63234_{2 \frac{1}{2}} \\
43956_{2 \frac{1}{2}}^{\circ}-76799_{\frac{1}{2}}\end{array}$ & $\begin{array}{l}6 \\
4\end{array}$ & $\begin{array}{l}0.97 \\
1.335\end{array}$ & $\begin{array}{l}0.99 \\
1.205\end{array}$ & $\begin{array}{l}0.07 ? \\
(0.065)\end{array}$ & $\begin{array}{l}0.98 ? \\
1.01\end{array}$ \\
\hline $\begin{array}{l}3042.650 \\
3042.34 \\
3040.83 \\
3040.504 \\
3040.444\end{array}$ & $\begin{array}{l}5 \\
2\end{array}$ & $\begin{array}{r}300 \\
2 \\
2 \\
30 \\
15\end{array}$ & $\begin{array}{l}32856.53 \\
32859.87 \\
32876.19 \\
32879.72 \\
32880.37\end{array}$ & $\begin{array}{r}0.00 \\
-0.01 \\
+0.04 \\
+0.03\end{array}$ & $\begin{array}{l}33494_{3 \frac{1}{2}}^{\circ}-66351_{2 \frac{1}{2}} \\
26759_{2 \frac{1}{2}}^{\circ}-59618_{3 \frac{1}{2}} \\
35059_{3 \frac{1}{2}}^{\circ}-67938_{3 \frac{1}{2}} \\
38342_{4 \frac{1}{2}}^{\circ}-71222_{3 \frac{1}{2}}\end{array}$ & 4 & 0.990 & 1.122 & $(0.066)$ & 0.66 \\
\hline $\begin{array}{l}3040.235 \\
3039.665\end{array}$ & $\begin{array}{r}5 \\
40\end{array}$ & $\begin{array}{r}15 \\
300\end{array}$ & $\begin{array}{l}32882.63 \\
32888.79\end{array}$ & $\begin{array}{l}+0.04 \\
+0.03\end{array}$ & $\begin{array}{l}43956_{2 \frac{1}{2}}^{\circ}-76839_{2 \frac{1}{2}} \\
35831_{5 \frac{1}{2}}^{\circ}-68720_{6 \frac{1}{2}}\end{array}$ & 4 & 1.211 & 1.159 & $(0.026)$ & 0.871 \\
\hline $\begin{array}{l}3039.276 \\
3038.81 \\
3038.75\end{array}$ & 2 & $\begin{array}{r}6 \\
2 \\
10\end{array}$ & $\begin{array}{l}32893.00 \\
32898.04 \\
32898.69\end{array}$ & $\left\{\begin{array}{r}0.00 \\
-0.03 \\
+0.06 \\
+0.03\end{array}\right.$ & $\begin{array}{l}31568_{2 \frac{1}{2}}^{\circ}-64461_{1 \frac{1}{2}}^{\circ} \\
62861_{5 \frac{1}{2}}^{\circ}-95754_{5 \frac{1}{2}}^{\circ} \\
43007_{0 \frac{1}{2}}^{\circ}-75905_{0 \frac{1}{2}}^{\circ} \\
33052_{\frac{1}{2}}^{\circ}-65950_{4 \frac{1}{2}}^{\circ}\end{array}$ & & & & & \\
\hline $\begin{array}{l}3038.531 \\
3037.992 \\
3036.820 \\
3036.13 \\
3035.945\end{array}$ & $\begin{array}{r}5 \\
15 \\
10 \\
1\end{array}$ & $\begin{array}{r}30 \\
90 \\
60 \\
1 \\
7\end{array}$ & $\begin{array}{l}32901.07 \\
32906.90 \\
32919.60 \\
32927.08 \\
32929.09\end{array}$ & $\begin{array}{r}+0.03 \\
+0.03 \\
+0.05 \\
\\
0.00\end{array}$ & $\begin{array}{l}33494_{3 \frac{1}{2}}^{\circ}-66395_{2 \frac{1}{2}} \\
32981_{1 \frac{1}{2}}^{\circ}-65888_{1 \frac{1}{2}} \\
35019_{4 \frac{1}{2}}^{\circ}-67938_{3 \frac{1}{2}}^{\circ} \\
61374_{2 \frac{1}{2}}-94303_{3 \frac{1}{2}}^{\circ}\end{array}$ & 6 & 0.865 & 1.204 & 0.508 & 1.035 \\
\hline $\begin{array}{l}3034.99 \\
3034.642\end{array}$ & $\begin{array}{r}1 \\
40\end{array}$ & $\begin{array}{r}5 \\
200\end{array}$ & \begin{tabular}{|l|}
32939.45 \\
32943.23
\end{tabular} & $\begin{array}{l}-0.01 \\
+0.02\end{array}$ & $\begin{array}{l}30224_{4 \frac{1}{2}}^{\circ}-63163_{4 \frac{1}{2}} \\
31979_{3 \frac{1}{2}}^{\circ}-64923_{4 \frac{1}{2}}\end{array}$ & 4 & 1.336 & 1.113 & 0.111 & 0.330 \\
\hline
\end{tabular}


TABLE 6. Yb II-Observed and classified lines-Continued

\begin{tabular}{|c|c|c|c|c|c|c|c|c|c|c|}
\hline 1 & 2 & 3 & 4 & 5 & 6 & 7 & 8 & 9 & 10 & 11 \\
\hline \multirow{2}{*}{$\begin{array}{l}\text { Wave- } \\
\text { length } \\
\text { air }\end{array}$} & \multicolumn{2}{|c|}{ Intensity } & \multirow{2}{*}{$\begin{array}{c}\text { Wave } \\
\text { number }\end{array}$} & \multirow{2}{*}{$\mathrm{o}-\mathrm{c}$} & \multirow{2}{*}{ Classification } & \multirow{2}{*}{$\begin{array}{l}\text { Zee- } \\
\text { man } \\
\text { type }\end{array}$} & \multirow{2}{*}{ lst $g$} & \multirow{2}{*}{ 2nd $g$} & \multicolumn{2}{|c|}{$\begin{array}{c}\text { Zeeman } \\
\text { components }\end{array}$} \\
\hline & $\begin{array}{l}\text { Meggers } \\
\text { lamp }\end{array}$ & $\begin{array}{c}\text { Thomson } \\
\text { lamp }\end{array}$ & & & & & & & Strong $p$ & Strong $n$ \\
\hline A & & & K & $K$ & & & & & & \\
\hline $\begin{array}{l}3034.52 \\
3034.32\end{array}$ & & $\begin{array}{l}2 \\
1\end{array}$ & $\begin{array}{l}32944.55 \\
32946.72\end{array}$ & $\begin{array}{r}-0.07 \\
+0.03\end{array}$ & $\begin{array}{l}65577_{3 \frac{1}{2}}-98521_{4 \frac{1}{2}}^{\circ} \\
44940_{1 \frac{1}{2}}^{\circ}-77887_{0 \frac{1}{2}}^{\circ}\end{array}$ & & & & & \\
\hline 3033.860 & 30 & 100 & 32951.72 & $\left\{\begin{array}{l}+0.09 \\
-0.09\end{array}\right.$ & $\begin{array}{l}26759_{2 \frac{1}{2}}^{\circ}-59710_{1 \frac{1}{2}}^{2} \\
44497_{2 \frac{1}{2}}^{\circ}-77449_{3 \frac{1}{2}}^{\circ}\end{array}$ & & & & & \\
\hline 3033.664 & 2 & 10 & 32953.85 & 0.00 & $63944_{5 \frac{1}{2}}-96898_{5 \frac{1}{2}}^{\circ}$ & & & & & \\
\hline $\begin{array}{l}3033.40 \\
3033.28\end{array}$ & & $\begin{array}{l}2 \\
1\end{array}$ & $\begin{array}{l}32956.72 \\
32958.02\end{array}$ & +0.12 & $6105]_{31}-94008^{\circ}$ & & & & & \\
\hline 3033.14 & & 1 & 32959.54 & 0.00 & $60586_{1 \frac{1}{2}}-93546_{2 \frac{1}{2}}^{\circ}$ & & & & & \\
\hline 3031.110 & $5000 A$ & 3000 & 32981.61 & +0.02 & $0_{0 \frac{1}{2}}-32981_{1 \frac{1}{2}}^{\circ}$ & 4 & 1.996 & 0.864 & 0.566 & 0.298 \\
\hline 3028.38 & 2 & 20 & 33011.34 & +0.05 & $44438_{3 \frac{1}{2}}^{\circ}-77449_{3 \frac{1}{2}}$ & & & & & \\
\hline 3026.669 & 100 & 700 & 33030.00 & +0.04 & $33052_{4 \frac{1}{2}}^{\circ}-66082_{5 \frac{1}{2}}^{2}$ & 4 & 1.266 & 1.140 & $(0.063)$ & 0.570 \\
\hline $\begin{array}{l}3025.76 \\
3025.38\end{array}$ & & $\begin{array}{l}2 \\
6\end{array}$ & $\begin{array}{l}33039.93 \\
33044.08\end{array}$ & $\begin{array}{l}+0.05 \\
+0.08\end{array}$ & $\begin{array}{l}48272_{2 \frac{1}{2}}^{\circ}-81312_{2 \frac{1}{2}}^{2} \\
59259^{2}-92303^{\circ}\end{array}$ & & & & & \\
\hline 3024.935 & 2 & 15 & 33048.94 & +0.04 & $40917_{\frac{1}{2}}^{\circ}-73966_{3 \frac{1}{2}}^{\circ}$ & & & & & \\
\hline $\begin{array}{l}3023.614 \\
3022.58\end{array}$ & 5 & $\begin{array}{r}30 \\
1\end{array}$ & $\begin{array}{l}33063.38 \\
33074.69\end{array}$ & +0.06 & $33494_{3 \frac{1}{2}}^{\circ}-66558_{3 \frac{1}{2}}$ & 6 & 0.99 & 1.09 & 0.35 & 1.04 \\
\hline 3022.454 & 10 & 60 & 33076.07 & +0.07 & $35059_{3 \frac{1}{2}}^{\circ}-68135_{2 \frac{1}{2}}$ & 7 & 1.11 & (1.087) & $w$ & 1.17 \\
\hline $\begin{array}{l}3021.27 \\
3020.99\end{array}$ & & $\begin{array}{l}1 \\
4\end{array}$ & $\begin{array}{l}33089.03 \\
33092.09\end{array}$ & +0.11 & $61214_{4 \frac{1}{2}}-94303_{3 \frac{1}{2}}^{\circ}$ & & & & & \\
\hline 3020.703 & 20 & 100 & 33095.24 & +0.08 & $43075_{1 \frac{1}{2}}^{\circ}-76170_{2 \frac{1}{2}}$ & 5 & & & & \\
\hline 3020.345 & 2 & 10 & 33099.16 & +0.06 & $63944_{5 \frac{1}{2}}-97043_{6 \frac{1}{2}}^{\circ \frac{2}{2}}$ & & & & & \\
\hline 3020.211 & & 5 & 33100.63 & +0.07 & $48556_{3 \frac{1}{2}}^{\circ}-81657_{2 \frac{1}{2}}^{\circ}$ & & & & & \\
\hline 3019.440 & 2 & 15 & 33109.08 & +0.01 & $44497_{2 \frac{1}{2}}^{\circ}-77606_{2 \frac{1}{2}}^{2}$ & & & & & \\
\hline 3019.17 & & 2 & 33112.04 & +0.07 & $35831_{5 \frac{1}{2}}^{\circ}-68943_{4 \frac{1}{2}}$ & & & & & \\
\hline 3019.058 & 8 & 40 & 33113.27 & +0.03 & $31979_{3 \frac{1}{2}}^{\circ}-65093_{3 \frac{1}{2}}$ & & & & & \\
\hline 3017.560 & 100 & 800 & 33129.71 & -0.04 & $35019_{4 \frac{1}{2}}^{\circ}-68148_{5 \frac{1}{2}}^{\circ}$ & 5 & (1.158) & 1.20 & $w$ & 1.40 \\
\hline $\begin{array}{l}3016.95 \\
3016.40\end{array}$ & & $\begin{array}{l}6 \\
1\end{array}$ & $\begin{array}{l}33136.41 \\
33142.45\end{array}$ & +0.06 & $48556_{3 \frac{1}{2}}^{\circ}-81692_{3 \frac{1}{2}}$ & & & & & \\
\hline 3014.97 & & 2 & 33158.17 & & & & & & & \\
\hline 3014.526 & 10 & 60 & 33163,05 & & & 7 & & & 0 & 1.12 \\
\hline 3014.427 & 40 & 200 & 33164.14 & $\left\{\begin{array}{l}+0.10 \\
+0.02\end{array}\right.$ & $\begin{array}{l}30562_{5 \frac{1}{2}}^{\circ}-63726_{4 \frac{1}{2}}^{\circ} \\
\left(66082_{5 \frac{1}{2}}-99246_{5 \frac{1}{2}}^{\circ}\right)\end{array}$ & 5 & 1.11 & 1.01 & $(0.05)$ & 1.56 \\
\hline $\begin{array}{l}3014.02 \\
3013.08 \\
3012.80\end{array}$ & & $\begin{array}{l}8 \\
2 \\
3\end{array}$ & $\begin{array}{l}33168.62 \\
33178.96 \\
33182.05\end{array}$ & +0.07 & $44438_{3 \frac{1}{2}}^{\circ}-77606_{2 \frac{1}{2}}$ & & & & & \\
\hline 3012.28 & & 1 & 33187.77 & & & & & & & \\
\hline 3011.842 & & 4 & 33192.60 & & & & & & & \\
\hline $\begin{array}{l}3011.00 \\
3010.625\end{array}$ & 50 & $\begin{array}{r}1 \\
300\end{array}$ & $\begin{array}{l}33201.88 \\
33206.02\end{array}$ & $\begin{array}{l}+0.12 \\
+0.01\end{array}$ & $\begin{array}{l}24332_{2 \frac{1}{2}}-56534_{3 \frac{1}{2}}^{2} \\
32371_{2 \frac{1}{2}}^{\circ}-65577_{3 \frac{1}{2}}^{\circ}\end{array}$ & 4 & 1.17 & 1.05 & 0.06 & 0.75 \\
\hline 3009.392 & 100 & 500 & 33219.62 & -0.02 & $31979_{3 \frac{1}{2}}^{\circ}-65199_{4 \frac{1}{2}}^{\circ}$ & 4 & 1.328 & 1.076 & 0.126 & 0.195 \\
\hline
\end{tabular}


TABLE 6. Yb II-Observed and classified lines-Continued

\begin{tabular}{|c|c|c|c|c|c|c|c|c|c|c|}
\hline 1 & 2 & 3 & 4 & 5 & 6 & 7 & 8 & 9 & 10 & 11 \\
\hline \multirow{2}{*}{$\begin{array}{l}\text { Wave- } \\
\text { length } \\
\text { air }\end{array}$} & \multicolumn{2}{|c|}{ Intensity } & \multirow{2}{*}{$\begin{array}{c}\text { Wave } \\
\text { number }\end{array}$} & \multirow{2}{*}{$\mathrm{o}-\mathrm{c}$} & \multirow{2}{*}{ Classification } & \multirow{2}{*}{$\begin{array}{l}\text { Zee- } \\
\text { man } \\
\text { type }\end{array}$} & \multirow{2}{*}{ lst $g$} & \multirow{2}{*}{2 nd $g$} & \multicolumn{2}{|c|}{$\begin{array}{c}\text { Zeeman } \\
\text { components }\end{array}$} \\
\hline & $\begin{array}{c}\text { Meggers } \\
\text { lamp }\end{array}$ & $\begin{array}{c}\text { Thomson } \\
\text { lamp }\end{array}$ & & & & & & & Strong $p$ & Strong $n$ \\
\hline A & & & $K$ & $K$ & & & & & & \\
\hline 3007.923 & 5 & 30 & 33235.85 & & & & & & & \\
\hline 3007.56 & & 3 & 33239.86 & +0.01 & $48272_{2 \frac{1}{2}}^{\circ}-81512_{1 \frac{1}{2}}$ & & & & & \\
\hline $\begin{array}{l}3006.863 \\
3006.30\end{array}$ & 10 & $\begin{array}{r}70 \\
3\end{array}$ & $\begin{array}{l}33247.56 \\
33253.79\end{array}$ & +0.03 & $40035_{3 \frac{1}{2}}^{\circ}-73283_{4 \frac{1}{2}}^{2}$ & 5 & 0.720 & 1.020 & 0.150 & 2.070 \\
\hline 3006.14 & 5 & 40 & 33255.56 & +0.06 & $30392_{1 \frac{1}{2}}^{\circ}-63647_{1 \frac{1}{2}}$ & & & & & \\
\hline 3005.766 & $800 r$ & 3000 & 33259.70 & -0.01 & $31631_{6 \frac{1}{2}}^{\circ}-64891_{7 \frac{1}{2}}$ & 4 & (1.230) & 1.20 & $w$ & 1.02 \\
\hline $\begin{array}{l}3004.642 \\
3004.46\end{array}$ & $\begin{array}{l}1 \\
3\end{array}$ & $\begin{array}{l}15 \\
10 h\end{array}$ & $\begin{array}{l}33272.14 \\
33274.15\end{array}$ & -0.01 & $46354_{4 \frac{1}{1}}^{\circ}-79628_{31}$ & & & & & \\
\hline $\begin{array}{l}3004.097 \\
3003.60\end{array}$ & 1 & $\begin{array}{l}5 \\
2\end{array}$ & $\begin{array}{l}33278.17 \\
33283.68\end{array}$ & +0.06 & $60586_{1 \frac{1}{2}}-93864_{2 \frac{1}{2}}^{\circ \frac{1}{2}}$ & & & & & \\
\hline 3002.608 & 30 & 200 & 33294.68 & 0.00 & $41678_{2 \frac{1}{2}}^{\circ}-74973_{3 \frac{1}{2}}$ & & & & & \\
\hline 3002.47 & & 2 & 33296.21 & & & & & & & \\
\hline 3002.035 & 5 & 30 & 33301.03 & -0.01 & $41688_{1 \frac{1}{2}}^{\circ}-74989_{0 \frac{1}{2}}$ & 5 & 0.80 & 0.52 & 0.14 & 0.94 \\
\hline 3001.864 & 2 & 10 & 33302.93 & +0.02 & $41688_{1 \frac{1}{2}}^{\circ}-74991_{1 \frac{1}{2}}^{\circ}$ & & & & & \\
\hline 3001.283 & 4 & 40 & 33309.37 & 0.00 & $44438_{3 \frac{1}{2}}^{\circ}-77747_{+\frac{1}{2}}^{2}$ & 4 & $(1.10)$ & 1.10 & $w$ & 1.09 \\
\hline 3000.987 & 2 & 15 & 33312.66 & +0.03 & $41678_{2 \frac{1}{2}}^{\circ}-74991_{1 \frac{1}{2}}$ & & & & & \\
\hline 3000.465 & 100 & 300 & 33318.45 & -0.02 & $42915_{5 \frac{1}{2}}^{\circ}-76233_{6 \frac{1}{2}}^{2}$ & 4 & (1.115) & 1.10 & $w$ & 1.05 \\
\hline 3000.13 & & 2 & 33322.17 & -0.02 & $65199_{4 \frac{1}{2}}-98521_{4 \frac{1}{2}}^{\circ 2}$ & & & & & \\
\hline $\begin{array}{l}2999.642 \\
2998.377\end{array}$ & $\begin{array}{l}5 \\
4\end{array}$ & $\begin{array}{l}10 \\
30\end{array}$ & $\begin{array}{l}33327.59 \\
33341.66\end{array}$ & $\begin{array}{l}-0.02 \\
+0.07\end{array}$ & $\begin{array}{l}43956_{2 \frac{1}{2}}^{\circ}-77284_{1 \frac{1}{2}} \\
37516_{3 \frac{1}{2}}^{\circ}-70858_{4 \frac{1}{2}}\end{array}$ & & & & & \\
\hline $\begin{array}{l}2996.75 \\
2996.373\end{array}$ & & $\begin{array}{l}2 \\
5\end{array}$ & $\begin{array}{l}33359.76 \\
33363.95\end{array}$ & $\begin{array}{l}+0.08 \\
+0.02\end{array}$ & $\begin{array}{l}63726_{4 \frac{1}{2}}-97086_{\frac{1}{2}}^{\circ} \\
34784_{5 \frac{1}{2}}^{\circ}-68148_{5 \frac{1}{2}}^{\circ}\end{array}$ & & & & & \\
\hline 2995.862 & 20 & 100 & 33369.64 & $\left\{\begin{array}{l}+0.02 \\
-0.02\end{array}\right.$ & $\begin{array}{c}32981_{1 \frac{1}{2}}^{\circ}-66351_{2 \frac{1}{2}} \\
\left(4688^{\circ}-75058\right.\end{array}$ & 5 & 0.880 & 1.128 & 0.124 & 1.500 \\
\hline $\begin{array}{l}2995.03 \\
2994.94\end{array}$ & $\begin{array}{l}6 \\
2\end{array}$ & $\begin{array}{l}30 \\
20\end{array}$ & $\begin{array}{l}33378.91 \\
33379.92\end{array}$ & $\begin{array}{r}-0.05 \\
+0.06\end{array}$ & $\begin{array}{l}28757_{1 \frac{1}{2}}^{\circ}-62136_{0 \frac{1}{2}}^{\circ} \\
34575_{\frac{1}{2}}^{\circ}-67955_{\left(\frac{1}{2}\right.}^{\circ}\end{array}$ & & & & & \\
\hline 2994.805 & 150 & 600 & 33381.42 & +0.03 & $30562_{5 \frac{1}{3}}^{\circ}-63944_{5 \frac{1}{1}}$ & 6 & 1.101 & 1.119 & 0.10 & 1.110 \\
\hline 2994.53 & 2 & 20 & 33384.49 & -0.04 & $44497_{2 \frac{1}{2}}^{\circ}-77882_{3 \frac{1}{2}}$ & & & & & \\
\hline 2994.48 & 1 & 10 & 33385.04 & 0.00 & $45429_{3 \frac{1}{2}}^{\circ}-78814_{2 \frac{1}{2}}^{\circ}$ & & & & & \\
\hline $\begin{array}{l}2993.939 \\
2992.28\end{array}$ & 10 & $\begin{array}{r}70 \\
5\end{array}$ & $\begin{array}{l}33391.08 \\
33409.59\end{array}$ & -0.03 & $35059_{3 \frac{1}{2}}^{\circ}-68450_{3 \frac{1}{3}}^{\circ}$ & 6 & 1.12 & 1.08 & 0.14 & 1.10 \\
\hline 2991.872 & 40 & 200 & 33414.14 & +0.01 & $32981_{1 \frac{1}{2}}^{\circ}-66395_{2 \frac{1}{2}}$ & 5 & 0.867 & 1.248 & 0.189 & 1.813 \\
\hline 2991.70 & 5 & 9 & 33416.07 & -0.05 & $28757_{1 \frac{1}{2}}^{\circ}-62174_{1 \frac{1}{2}}^{2}$ & & & & & \\
\hline 2991.33 & & 1 & 33420.20 & +0.05 & $48272_{2 \frac{1}{2}}^{\circ}-81692_{3 \frac{1}{2}}^{2}$ & & & & & \\
\hline 2991.240 & 1 & 6 & 33421.20 & & & & & & & \\
\hline 2990.366 & 30 & 200 & 33430.97 & -0.01 & $35019_{4 \frac{1}{2}}^{\circ}-68450_{3 \frac{1}{2}}$ & 5 & (1.158) & 1.09 & $w$ & 1.38 \\
\hline 2989.768 & 2 & 15 & 33437.66 & -0.02 & $37516_{3 \frac{1}{2}}^{\circ}-70954_{2 \frac{1}{2}}$ & & & & & \\
\hline 2989.376 & 6 & 10 & 33442.04 & -0.08 & $43075_{1 \frac{1}{2}}^{\circ}-76517_{1 \frac{1}{2}}^{\circ}$ & & & & & \\
\hline $\begin{array}{l}2989.20 \\
2988.95\end{array}$ & 2 & 20 & $\begin{array}{l}33444.01 \\
3344681\end{array}$ & $\begin{array}{r}0.00 \\
+0.08\end{array}$ & $44438_{3 \frac{1}{2}}^{\circ}-77882_{3 \frac{1}{2}}$ & 6 & 1.09 & 1.12 & 0.12 & 1.106 \\
\hline 2988.56 & & $\begin{array}{l}5 n \\
1\end{array}$ & $\begin{array}{l}33440.81 \\
33451.17\end{array}$ & +0.08 & $47758_{3 \frac{1}{2}}-81205_{+\frac{1}{2}}$ & & & & & \\
\hline
\end{tabular}


TABLE 6. Yb II-Observed and classified lines-Continued

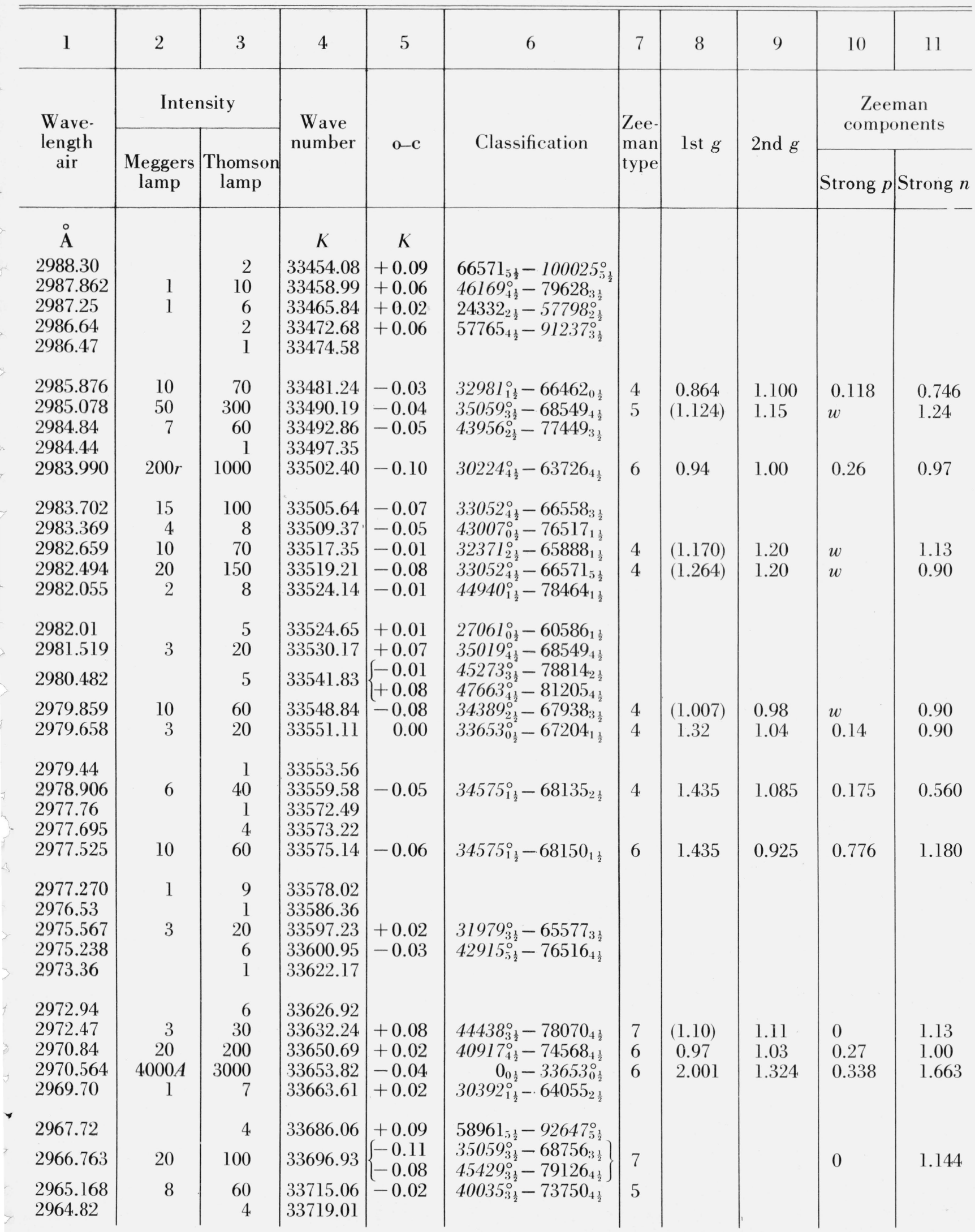


TABLE 6. Yb II-Observed and classified lines-Continued

\begin{tabular}{|c|c|c|c|c|c|c|c|c|c|c|}
\hline 1 & 2 & 3 & 4 & 5 & 6 & 7 & 8 & 9 & 10 & 11 \\
\hline \multirow{2}{*}{$\begin{array}{l}\text { Wave- } \\
\text { length } \\
\text { air }\end{array}$} & \multicolumn{2}{|c|}{ Intensity } & \multirow{2}{*}{$\begin{array}{c}\text { Wave } \\
\text { number }\end{array}$} & \multirow{2}{*}{$o-c$} & \multirow{2}{*}{ Classification } & \multirow{2}{*}{$\begin{array}{l}\text { Zee- } \\
\text { man } \\
\text { type }\end{array}$} & \multirow{2}{*}{ lst $g$} & \multirow{2}{*}{2 nd $g$} & \multicolumn{2}{|c|}{$\begin{array}{c}\text { Zeeman } \\
\text { components }\end{array}$} \\
\hline & $\begin{array}{c}\text { Meggers } \\
\text { lamp }\end{array}$ & $\begin{array}{c}\text { Thomson } \\
\text { lamp }\end{array}$ & & & & & & & Strong $p$ & Strong $n$ \\
\hline А & & & $K$ & $K$ & & & & & & \\
\hline 2964.755 & $100 r$ & 500 & 33719.75 & -0.10 & $30224_{4 \frac{1}{2}}^{\circ}-63944_{5 \frac{1}{2}}$ & 5 & 0.944 & 1.110 & 0.083 & 1.852 \\
\hline $\begin{array}{l}2964.39 \\
2963.96\end{array}$ & 15 & $\begin{array}{r}100 \\
4\end{array}$ & $\begin{array}{l}33723.90 \\
33728.80\end{array}$ & $\begin{array}{l}-0.08 \\
+0.08\end{array}$ & $\begin{array}{l}43075_{1 \frac{1}{2}}^{\circ}-76799_{1 \frac{1}{2}} \\
64891_{7 \frac{1}{2}}-98620_{6 \frac{1}{2}}^{\circ}\end{array}$ & 6 & 0.97 & 0.85 & 0.17 & 0.91 \\
\hline 2963.46 & 40 & 300 & 33734.49 & & & 6 & & & 0.16 & 1.08 \\
\hline 2963.255 & 10 & 70 & 33736.82 & -0.09 & $35019_{4 \frac{1}{2}}^{\circ}-68756_{3 \frac{1}{2}}$ & & & & & \\
\hline 2963.17 & 8 & 50 & 33737.79 & -0.03 & $39378_{0 \frac{1}{2}}^{\circ}-73116_{1 \frac{1}{2}}$ & & & & & \\
\hline 2962.522 & 40 & 250 & 33745.17 & -0.07 & $34389_{2 \frac{1}{2}}^{\circ}-68135_{2 \frac{1}{2}}$ & 6 & 1.00 & 1.08 & 0.19 & 1.04 \\
\hline 2961.801 & 4 & 30 & 33753.38 & -0.04 & $37516_{3 \frac{1}{2}}^{\circ}-71270_{2 \frac{1}{2}}^{\circ}$ & & & & & \\
\hline 2961.150 & 1 & 6 & 33760.80 & -0.01 & $34389_{2 \frac{1}{2}}^{\circ}-68150_{1 \frac{1}{2}}^{\circ}$ & & & & & \\
\hline $\begin{array}{l}2960.850 \\
2958.425\end{array}$ & 10 & $\begin{array}{r}60 \\
3\end{array}$ & $\begin{array}{l}33764.22 \\
33791.90\end{array}$ & $\begin{array}{l}-0.06 \\
+0.10\end{array}$ & $\begin{array}{l}34784_{5 \frac{1}{2}}^{\circ}-68549_{4 \frac{1}{2}} \\
45335_{\frac{1}{2}}^{\circ}-79126_{4 \frac{1}{2}}^{\circ}\end{array}$ & 4 & 1.13 & (1.157) & $w$ & 1.01 \\
\hline 2957.632 & 40 & 150 & 33800.96 & -0.08 & $28757_{1 \frac{1}{2}}^{\circ}-62559_{2 \frac{1}{2}}$ & & & & & \\
\hline 2957.05 & & 3 & 33807.61 & & & & & & & \\
\hline 2955.61 & & 2 & 33824.08 & +0.06 & $59090_{3 \frac{1}{2}}-92914_{4 \frac{1}{2}}^{\circ}$ & & & & & \\
\hline 2955.318 & 40 & 200 & 33827.42 & -0.02 & $26759_{2 \frac{1}{2}}^{\circ}-60586_{1 \frac{1}{2}}^{\circ}$ & 5 & 1.576 & 1.334 & 0.121 & 1.940 \\
\hline 2955.09 & 1 & 4 & 33830.03 & -0.03 & $24332_{2 \frac{1}{2}}-58162_{1 \frac{1}{2}}^{\circ}$ & & & & & \\
\hline 2954.45 & & 1 & 33837.36 & +0.05 & $65577_{3 \frac{1}{2}}-99414_{4 \frac{1}{2}}^{\circ}$ & & & & & \\
\hline 2953.016 & 5 & 40 & 33853.79 & -0.02 & $45273_{3 \frac{1}{2}}^{\circ}-79126_{4 \frac{1}{2}}$ & 4 & (1.18) & 1.15 & $w$ & 1.03 \\
\hline $\begin{array}{l}2952.475 \\
2952.253\end{array}$ & 1 & $\begin{array}{r}4 \\
10\end{array}$ & $\begin{array}{l}33859.99 \\
33862.54\end{array}$ & $\begin{array}{l}-0.02 \\
+0.05\end{array}$ & $\begin{array}{l}38342_{4 \frac{1}{2}}^{\circ}-72202_{3 \frac{1}{2}} \\
41688^{\circ}-755500^{2}\end{array}$ & & & & & \\
\hline $\begin{array}{l}2952.253 \\
2951.716\end{array}$ & 1 & 8 & $\begin{array}{l}33862.54 \\
33868.70\end{array}$ & $\left\{\begin{array}{l}+0.05 \\
+0.04 \\
-0.04\end{array}\right.$ & $\begin{array}{l}41688_{1 \frac{1}{2}}-75550_{2 \frac{1}{2}} \\
45737_{1 \frac{1}{2}}^{\circ}-79605_{1 \frac{1}{2}} \\
618222_{3 \frac{1}{2}}-95691_{3 \frac{1}{2}}^{\circ}\end{array}$ & & & & & \\
\hline 2951.406 & 5 & 40 & 33872.26 & +0.05 & $41678_{2 \frac{1}{2}}^{\circ}-75550_{2 \frac{1}{2}}$ & & & & & \\
\hline 2951.022 & 8 & 50 & 33876.67 & -0.01 & $37077_{2 \frac{1}{2}}^{\circ}-70954_{2 \frac{1}{2}}$ & & & & & \\
\hline $\begin{array}{l}2950.80 \\
2950.64\end{array}$ & 2 & $\begin{array}{l}8 \\
1\end{array}$ & $\begin{array}{l}33879.21 \\
33881.05\end{array}$ & -0.03 & $22960_{1 \frac{1}{2}}-56840_{1 \frac{1}{2}}^{\mathrm{o}^{2}}$ & & & & & \\
\hline 2950.52 & 2 & 20 & 33882.43 & & & & & & & \\
\hline 2950.326 & 20 & 150 & 33884.66 & +0.01 & $35059_{3 \frac{1}{2}}^{a}-68943_{4 \frac{1}{2}}$ & 4 & 1.143 & 0.889 & 0.127 & 0 \\
\hline 2949.840 & & 5 & 33890.24 & & & & & & & \\
\hline $\begin{array}{l}2948.65 \\
2947.76\end{array}$ & & $\begin{array}{l}2 \\
3\end{array}$ & 33903.92 & & & & & & & \\
\hline $\begin{array}{l}2947.60 \\
2947.131\end{array}$ & 4 & $\begin{array}{r}3 \\
30\end{array}$ & $\begin{array}{l}33914.15 \\
33921.39\end{array}$ & +0.04 & $33494_{3 \frac{1}{2}}^{\circ}-67416_{4 \frac{1}{2}}$ & & & & & \\
\hline 2946.95 & & 5 & 33923.47 & +0.02 & $64598_{3 \frac{1}{2}}-98521_{4 \frac{1}{2}}^{\circ}$ & & & & & \\
\hline 2946.852 & 2 & 20 & 33924.60 & +0.08 & $35019_{4 \frac{1}{2}}^{\circ}-68943_{4 \frac{1}{2}}$ & & & & & \\
\hline 2946.765 & 10 & 70 & 33925.60 & -0.03 & $43956_{2 \frac{1}{2}}^{\circ}-77882_{3 \frac{1}{2}}$ & 5 & $(0.99)$ & 1.15 & $w$ & 1.55 \\
\hline 2946.305 & 30 & 200 & 33930.90 & -0.09 & $40035_{3 \frac{1}{2}}^{\circ}-73966_{3 \frac{1}{2}}^{2}$ & 6 & 0.71 & 0.76 & 0.17 & 0.733 \\
\hline 2945.907 & $100 r$ & 600 & 33935.48 & -0.01 & $34784_{5 \frac{1}{2}}^{0}-68720_{6 \frac{1}{2}}^{\circ}$ & 5 & (1.119) & 1.14 & $w$ & 1.28 \\
\hline 2945.22 & & 3 & 33943.40 & +0.08 & $66082_{\frac{5}{2}}-100025_{5 \frac{1}{2}}^{\circ}$ & & & & & \\
\hline 2944.465 & 5 & 30 & 33952.10 & -0.01 & $37516_{3 \frac{1}{2}}^{\circ}-71468_{2 \frac{1}{2}}^{\circ}$ & & & & & \\
\hline 2944.19 & & 2 & 33955.27 & & & & & & & \\
\hline
\end{tabular}


TABLE 6. Yb II-Observed and classified lines-Continued

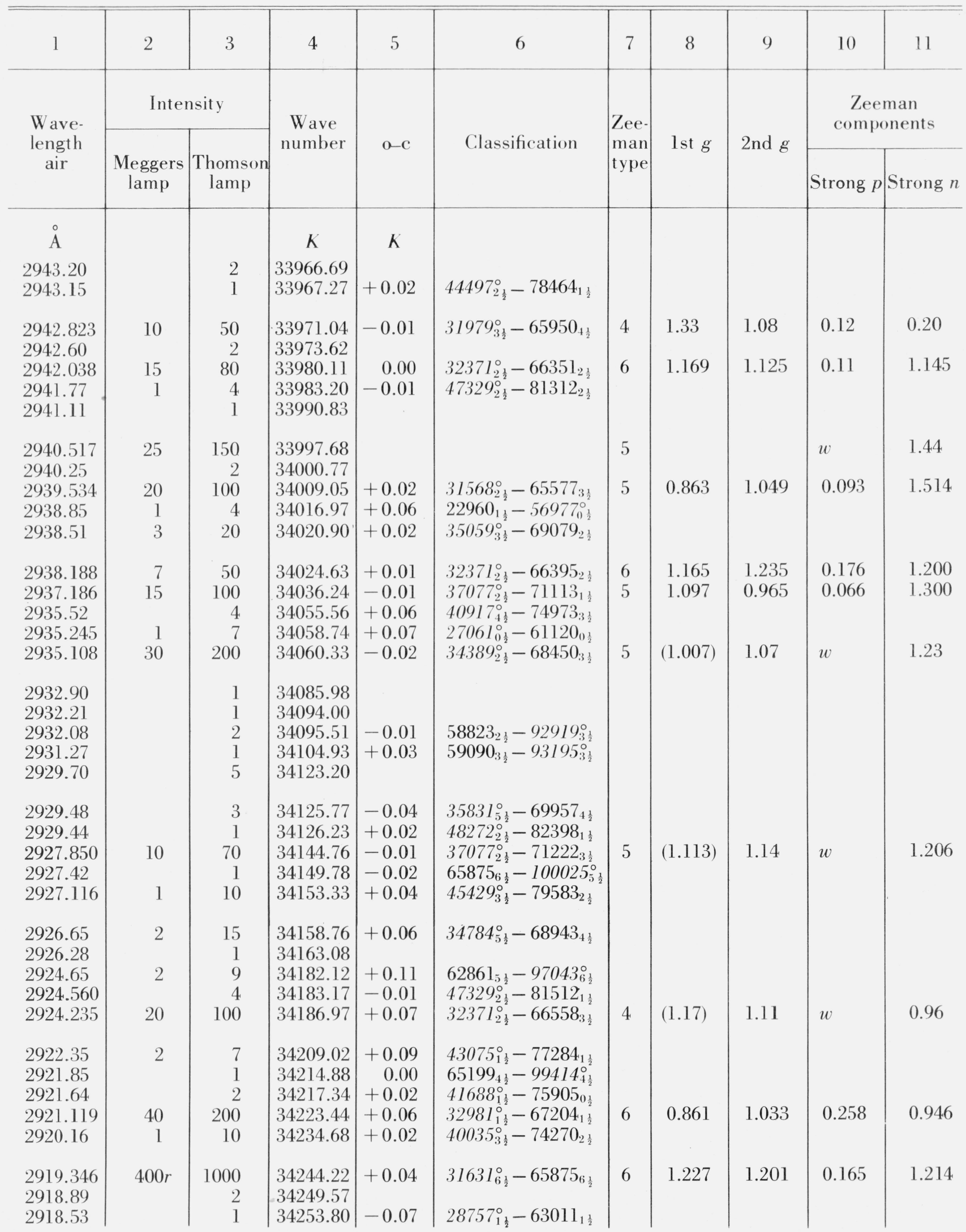


TABLE 6. Yb II-Observed and classified lines-Continued

\begin{tabular}{|c|c|c|c|c|c|c|c|c|c|c|}
\hline 1 & 2 & 3 & 4 & 5 & 6 & 7 & 8 & 9 & 10 & 11 \\
\hline \multirow{2}{*}{$\begin{array}{l}\text { Wave- } \\
\text { length } \\
\text { air }\end{array}$} & \multicolumn{2}{|c|}{ Intensity } & \multirow{2}{*}{$\begin{array}{c}\text { Wave } \\
\text { number }\end{array}$} & \multirow[b]{2}{*}{$\mathrm{o}-\mathrm{c}$} & \multirow[b]{2}{*}{ Classification } & \multirow{2}{*}{$\begin{array}{l}\text { Zee- } \\
\text { man } \\
\text { type }\end{array}$} & \multirow[b]{2}{*}{ lst $g$} & \multirow{2}{*}{ 2nd $g$} & \multicolumn{2}{|c|}{$\begin{array}{c}\text { Zeeman } \\
\text { components }\end{array}$} \\
\hline & $\begin{array}{c}\text { Meggers } \\
\text { lamp }\end{array}$ & $\begin{array}{c}\text { Thomson } \\
\text { lamp }\end{array}$ & & & & & & & Strong $p$ & Strong $n$ \\
\hline$\AA$ & & & $K$ & $K$ & & & & & & \\
\hline $\begin{array}{l}2916.83 \\
2916.61\end{array}$ & $\begin{array}{l}2 \\
7\end{array}$ & $\begin{array}{l}10 \\
30\end{array}$ & $\begin{array}{l}34273.76 \\
34276.35\end{array}$ & $\begin{array}{l}+0.04 \\
+0.12\end{array}$ & $\begin{array}{l}38342_{4 \frac{1}{2}}^{\circ}-72615_{5 \frac{1}{2}} \\
43007_{0 \frac{1}{2}}^{\circ}-77284_{1 \frac{1}{2}}\end{array}$ & & & & & \\
\hline $\begin{array}{l}2916.431 \\
2915.99\end{array}$ & 10 & $\begin{array}{r}70 \\
4\end{array}$ & $\begin{array}{l}34278.45 \\
34283.63\end{array}$ & $\begin{array}{l}+0.05 \\
-0.03\end{array}$ & $\begin{array}{l}39378_{0 \frac{1}{2}}^{\circ}-73657_{1 \frac{1}{2}} \\
47228_{0 \frac{1}{2}}^{\circ}-81512_{1 \frac{1}{3}}\end{array}$ & 4 & 1.86 & 1.05 & 0.40 & 0.64 \\
\hline 2915.275 & $100 r$ & 300 & 34292.04 & 0.00 & $26759_{2 \frac{1}{2}}^{\circ}-61051_{3 \frac{1}{2}}^{\circ}$ & 4 & 1.56 & 1.18 & 0.19 & 0.22 \\
\hline $\begin{array}{l}2915.14 \\
2914.84\end{array}$ & & $\begin{array}{l}4 \\
8\end{array}$ & $\begin{array}{l}34293.63 \\
34297.16\end{array}$ & -0.05 & $45335_{4 \frac{1}{2}}^{\circ}-79628_{3 \frac{1}{2}}$ & & & & & \\
\hline 2914.48 & 7 & 40 & 34301.39 & +0.02 & $33653_{0 \frac{1}{2}}^{\circ}-67955_{0 \frac{1}{2}}$ & 6 & 1.310 & 0.900 & 0.205 & 1.105 \\
\hline 2914.210 & $100 r$ & 600 & 34304.57 & +0.01 & $35831_{5 \frac{1}{2}}^{\circ}-70136_{6 \frac{1}{2}}^{\circ}$ & 4 & 1.22 & 1.15 & $(0.034)$ & 0.78 \\
\hline 2913.75 & & 3 & 34309.99 & -0.10 & $45273_{3 \frac{1}{2}}^{\circ}-79583_{2 \frac{1}{2}}^{\circ}$ & & & & & \\
\hline 2913.12 & 1 & 10 & 34317.41 & +0.06 & $44497_{2 \frac{1}{2}}^{\circ}-78814_{2 \frac{1}{2}}$ & & & & & \\
\hline 2912.865 & 10 & 50 & 34320.41 & +0.03 & $31568_{\frac{1}{2}}^{\circ}-65888_{1 \frac{1}{2}}^{\circ}$ & 4 & 0.86 & 1.20 & 0.17 & 0.36 \\
\hline 2912.51 & & 5 & 34324.59 & -0.04 & $46354_{4 \frac{1}{2}}^{\circ}-80679_{5 \frac{1}{2}}$ & & & & & \\
\hline 2912.25 & & 2 & 34327.66 & -0.03 & $47329_{2 \frac{1}{2}}^{\circ}-81657_{2 \frac{1}{2}}^{2}$ & & & & & \\
\hline 2911.61 & & 3 & 34335.20 & -0.03 & $48556_{3 \frac{1}{2}}^{\circ}-82891_{2 \frac{1}{2}}^{\circ}$ & & & & & \\
\hline 2911.523 & 40 & 200 & 34336.23 & +0.03 & $35059_{3 \frac{1}{2}}^{\circ}-69395_{3 \frac{1}{2}}^{\circ}$ & 6 & 1.12 & 0.94 & 0.63 & 1.05 \\
\hline 2910.43 & & 2 & 34349.12 & & & & & & & \\
\hline 2909.88 & & 4 & 34355.62 & -0.07 & $45273_{3 \frac{1}{2}}^{\circ}-79628_{3 \frac{1}{2}}$ & & & & & \\
\hline 2909.48 & 30 & 150 & 34360.34 & +0.02 & $30562_{5 \frac{1}{2}}^{\circ}-64923_{4 \frac{1}{2}}$ & 5 & (1.112) & 1.10 & 0 & 1.153 \\
\hline 2909.19 & 20 & 100 & 34363.76 & +0.02 & $33052_{4 \frac{1}{2}}^{\circ}-67416_{4 \frac{1}{2}}^{\circ}$ & 7 & 1.26 & 1.26 & 0 & 1.266 \\
\hline 2908.55 & 1 & 9 & 34371.33 & +0.02 & $31979_{3 \frac{1}{2}}^{\circ}-66351_{2 \frac{1}{2}}^{\circ}$ & & & & & \\
\hline 2908.33 & 10 & 50 & 34373.93 & -0.02 & $30224_{4 \frac{1}{2}}^{\mathrm{o}^{2}}-64598_{3 \frac{1}{2}}^{2}$ & 4 & 0.93 & 1.23 & 0.150 & -0.12 \\
\hline 2908.15 & & 4 & 34376.05 & -0.02 & $35019_{4 \frac{1}{2}}^{\circ}-69395_{3 \frac{1}{2}}$ & & & & & \\
\hline 2908.09 & 5 & 40 & 34376.76 & -0.07 & $44438_{3 \frac{1}{2}}^{\circ}-78814_{2 \frac{1}{2}}^{\circ}$ & & & & & \\
\hline 2906.88 & 7 & 60 & 34391.07 & -0.04 & $37077_{2 \frac{1}{2}}^{\circ}-71468_{2 \frac{1}{2}}^{2}$ & 6 & 1.110 & 1.018 & 0.23 & 1.064 \\
\hline $\begin{array}{l}2905.61 \\
2904.97\end{array}$ & & $\begin{array}{l}3 \\
3\end{array}$ & $\begin{array}{l}34406.10 \\
34413.68\end{array}$ & -0.08 & $59090_{3 \frac{1}{2}}-93503_{4 \frac{1}{2}}^{\circ}$ & & & & & \\
\hline 2904.79 & 1 & 7 & 34415.81 & -0.01 & $31979_{3 \frac{1}{2}}^{\circ}-66395_{2 \frac{1}{2}}$ & & & & & \\
\hline 2904.20 & & 1 & 34422.81 & & & & & & & \\
\hline 2902.924 & 8 & 80 & 34437.94 & -0.03 & $38342_{4 \frac{1}{2}}^{\circ}-72779_{3 \frac{1}{2}}$ & 5 & (1.093) & 1.064 & $w$ & 1.065 \\
\hline 2902.41 & 6 & 40 & 34444.03 & +0.03 & $33494_{3 \frac{1}{2}}^{\circ}-67938_{3 \frac{1}{2}}$ & 6 & 0.99 & 0.97 & 0.06 & 0.98 \\
\hline 2901.86 & & 1 & 34450.56 & -0.10 & $31631_{6 \frac{1}{2}}^{\circ}-66082_{5 \frac{1}{2}}$ & & & & & \\
\hline 2901.60 & & 1 & 34453.65 & & & & & & & \\
\hline 2900.39 & & 2 & 34468.02 & & & & & & & \\
\hline 2899.705 & 40 & 200 & 34476.16 & +0.03 & $28757_{1 \frac{1}{2}}^{\circ}-63234_{2 \frac{1}{2}}$ & 4 & 1.44 & 1.21 & 0.115 & 0.86 \\
\hline 2899.236 & 5 & 40 & 34481.74 & -0.06 & $41688_{1 \frac{1}{2}}^{\circ}-76170_{2 \frac{1}{2}}$ & 5 & 0.77 & 1.12 & $0.175 u s$ & $1.646 u s$ \\
\hline 2897.98 & & 2 & 34496.68 & -0.03 & $33653_{0 \frac{1}{2}}^{\circ}-68150_{1 \frac{1}{2}}^{\circ}$ & & & & & \\
\hline 2897.32 & & 4 & 34504.54 & +0.03 & $34575_{1 \frac{1}{2}}^{\circ}-69079_{2 \frac{1}{2}}$ & & & & & \\
\hline 2896.902 & 10 & 100 & 34509.52 & +0.12 & $46169_{4 \frac{1}{2}}^{\circ}-80679_{5 \frac{1}{2}}$ & 4 & 1.06 & 1.00 & $(0.03 ?)$ & 0.73 \\
\hline 2895.06 & 2 & 15 & 34531.48 & -0.01 & $43075_{1 \frac{1}{2}}^{\circ}-77606_{2 \frac{1}{2}}$ & & & & & \\
\hline
\end{tabular}


TABLE 6. Yb II-Observed and classified lines-Continued

\begin{tabular}{|c|c|c|c|c|c|c|c|c|c|c|}
\hline 1 & 2 & 3 & 4 & 5 & 6 & 7 & 8 & 9 & 10 & 11 \\
\hline \multirow{2}{*}{$\begin{array}{l}\text { Wave- } \\
\text { length } \\
\text { air }\end{array}$} & \multicolumn{2}{|c|}{ Intensity } & \multirow{2}{*}{$\begin{array}{c}\text { Wave } \\
\text { number }\end{array}$} & \multirow{2}{*}{$a-c$} & \multirow{2}{*}{ Classification } & \multirow{2}{*}{ Zee- } & \multirow{2}{*}{ lst $g$} & \multirow{2}{*}{ 2nd $g$} & \multicolumn{2}{|c|}{$\begin{array}{c}\text { Zeeman } \\
\text { components }\end{array}$} \\
\hline & $\begin{array}{c}\text { Meggers } \\
\text { lamp }\end{array}$ & $\begin{array}{c}\text { Thomson } \\
\text { lamp }\end{array}$ & & & & & & & Strong $p$ & Strong $n$ \\
\hline A & & & $K$ & $K$ & & & & & & \\
\hline 2894.95 & 3 & 30 & 34532.79 & +0.03 & $40035_{3 \frac{1}{2}}^{\circ}-74568_{4 \frac{1}{2}}$ & 5 & & & & \\
\hline 2893.620 & 10 & 100 & 34548.66 & +0.12 & $35059_{3 \frac{1}{2}}^{\circ}-69607_{2 \frac{1}{2}}^{2}$ & 4 & 1.12 & 1.21 & $(0.044)$ & 0.90 \\
\hline 2891.384 & $5000 \mathrm{~A}$ & 2000 & 34575.38 & +0.01 & $0_{0 \frac{1}{2}}-34575_{1 \frac{1}{2}}^{\circ}$ & 4 & 1.999 & 1.430 & 0.285 & 1.145 \\
\hline 2891.16 & 4 & 40 & 34578.06 & -0.04 & $31979_{3 \frac{1}{2}}^{\circ}-66558_{3 \frac{1}{2}}^{\circ}$ & & & & & \\
\hline $\begin{array}{l}2890.28 \\
288804\end{array}$ & $\begin{array}{c}1 \\
100 r\end{array}$ & $\begin{array}{r}8 \\
300\end{array}$ & 34588.58 & & $267500^{\circ}$ & & & & & \\
\hline $\begin{array}{l}2888.04 \\
2887.74\end{array}$ & $\begin{array}{c}100 r \\
2\end{array}$ & $\begin{array}{r}300 \\
7\end{array}$ & $\begin{array}{l}34615.41 \\
34619.00\end{array}$ & $\begin{array}{l}-0.06 \\
-0.03\end{array}$ & $\begin{array}{l}26759_{2 \frac{1}{2}}^{\circ}-61374_{2 \frac{1}{2}} \\
48272_{2 \frac{1}{2}}^{\circ}-82891_{2 \frac{1}{2}}\end{array}$ & 6 & 1.567 & 1.292 & 0.692 & 1.430 \\
\hline 2886.263 & 30 & 200 & 34636.72 & -0.03 & $30562_{5 \frac{1}{2}}-65199_{4 \frac{1}{2}}$ & 5 & 1.10 & 1.06 & $(0.02)$ & 1.28 \\
\hline 2885.97 & 10 & 70 & 34640.24 & $\left\{\begin{array}{l}-0.08 \\
-0.02\end{array}\right.$ & $\begin{array}{c}33494_{3 \frac{1}{2}}^{\circ}-68135_{2 \frac{1}{2}} \\
\left(61051_{3}-9569 l^{\circ}\right)\end{array}$ & 4 & 1.00 & 1.09 & $(0.044)$ & 0.78 \\
\hline $\begin{array}{l}2885.594 \\
2885.02 \\
2884.51\end{array}$ & 1 & $\begin{array}{l}7 \\
8 \\
2\end{array}$ & $\begin{array}{l}34644.75 \\
34651.64 \\
34657.77\end{array}$ & $\begin{array}{l}-0.02 \\
-0.01 \\
-0.03\end{array}$ & $\begin{array}{l}41678_{2 \frac{1}{2}}^{\circ}-76323_{3 \frac{1}{2}} \\
47005_{1 \frac{1}{2}}^{\circ}-81657_{2 \frac{1}{2}}\end{array}$ & & & & & \\
\hline $\begin{array}{l}2883.87 \\
2883.01\end{array}$ & 1 & $\begin{array}{r}10 \\
6\end{array}$ & $\begin{array}{l}34665.46 \\
34675.80\end{array}$ & $\begin{array}{l}+0.12 \\
-0.04\end{array}$ & $\begin{array}{l}44940_{1 \frac{1}{2}}^{\circ}-79605_{1 \frac{1}{2}} \\
63944_{5 \frac{1}{3}}-98620_{6 \frac{1}{\circ}}^{\circ}\end{array}$ & 6 & 1.236 & 1.152 & 0.125 & 1.194 \\
\hline 2882.155 & 7 & 50 & 34686.09 & +0.01 & $37077_{2 \frac{1}{2}}^{\circ}-71763_{1 \frac{1}{2}}^{\circ}$ & 7 & 1.12 & 1.12 & 0 & 1.122 \\
\hline 2882.01 & & 1 & 34687.83 & & & & & & & \\
\hline 2881.93 & 7 & 60 & 34688.79 & & & 5 & & & $w$ & 1.24 \\
\hline $\begin{array}{l}2881.10 \\
2879.83\end{array}$ & & $\begin{array}{l}6 \\
1\end{array}$ & $\begin{array}{l}34698.79 \\
34714.09\end{array}$ & +0.01 & $30224_{4 \frac{1}{2}}^{\circ}-64923_{4 \frac{1}{2}}$ & & & & & \\
\hline 2879.159 & 10 & 70 & 34722.18 & +0.03 & $40917_{4 \frac{1}{2}}^{\circ}-75640_{3 \frac{1}{2}}$ & 5 & 0.976 & 0.820 & 0.078 & 1.522 \\
\hline $\begin{array}{l}2876.45 \\
2874.83\end{array}$ & & $\begin{array}{l}1 \\
1\end{array}$ & $\begin{array}{l}34754.88 \\
34774.46\end{array}$ & & $59090_{3 \frac{1}{2}}-93864_{2 \frac{1}{2}}^{\circ}$ & & & & & \\
\hline 2874.11 & & 6 & 34783.17 & +0.04 & $31568_{2 \frac{1}{2}}^{\circ}-66351_{2 \frac{1}{2}}$ & & & & & \\
\hline 2873.14 & & 3 & 34794.91 & +0.01 & $63726_{4 \frac{1}{2}}-98521_{4 \frac{1}{2}}^{\circ \frac{1}{2}}$ & & & & & \\
\hline 2872.82 & & 1 & 34798.79 & & & & & & & \\
\hline 2872.11 & 2 & 10 & 34807.39 & +0.08 & $48556_{3 \frac{1}{2}}^{\circ}-83363_{3 \frac{1}{2}}$ & & & & & \\
\hline 2871.712 & 2 & 10 & 34812.22 & +0.01 & $43075_{1 \frac{1}{2}}^{\circ}-77887_{0 \frac{1}{2}}^{2}$ & & & & & \\
\hline 2870.57 & & 3 & 34826.06 & +0.03 & $65199_{4 \frac{1}{2}}-100025_{5 \frac{1}{2}}^{\circ}$ & & & & & \\
\hline 2870.061 & 20 & $\begin{array}{r}3 \\
100\end{array}$ & $\begin{array}{l}34826.79 \\
34832.24\end{array}$ & -0.03 & $42915_{5 \frac{1}{2}}^{\circ}-77747_{4 \frac{1}{2}}$ & 5 & 1.10 & 1.07 & $(0.017)$ & 1.25 \\
\hline 2869.61 & & 1 & 34837.71 & 0.00 & $22960_{1 \frac{1}{2}}-57798_{2 \frac{1}{2}}^{\circ}$ & & & & & \\
\hline 2869.546 & 1 & 15 & 34838.49 & +0.01 & $41678_{2 \frac{1}{2}}^{\circ}-76517_{1 \frac{1}{2}}^{\circ}$ & & & & & \\
\hline 2868.536 & & 5 & 34850.76 & +0.04 & $46354_{4 \frac{1}{2}}^{\circ}-81205_{4 \frac{1}{2}}$ & & & & & \\
\hline 2867.90 & & 7 & 34858.49 & $\left\{\begin{array}{l}+0.01 \\
+0.04\end{array}\right.$ & $63944_{5 \frac{1}{2}}-98802_{5 \frac{1}{2}}^{\circ}$ & & & & & \\
\hline 2867.58 & 5 & 15 & 34862.38 & $\begin{array}{l}+0.04 \\
+0.02\end{array}$ & $\begin{array}{l}43950_{2 \frac{1}{2}}-8814_{2 \frac{1}{2}} \\
472228_{0 \frac{1}{2}}^{\circ}-82091_{0 \frac{1}{2}}\end{array}$ & & & & & \\
\hline 2867.20 & 1 & 7 & 34867.00 & -0.10 & $48024_{1 \frac{1}{2}}^{\circ}-82891_{2 \frac{1}{2}}^{\circ}$ & & & & & \\
\hline 2867.06 & $100 r$ & 500 & 34868.70 & -0.11 & $30224_{4 \frac{1}{2}}^{\circ}-65093_{3 \frac{1}{2}}^{2}$ & 4 & 0.94 & 1.00 & $(0.03)$ & 0.72 \\
\hline 2866.179 & 4 & 30 & 34879.42 & -0.09 & $43007_{0 \frac{1}{2}}^{\circ}-77887_{0 \frac{1}{2}}$ & 6 & 0.14 & 2.18 & 1.02 & 1.16 \\
\hline
\end{tabular}


TABLE 6. Yb II-Observed and classified lines-Continued

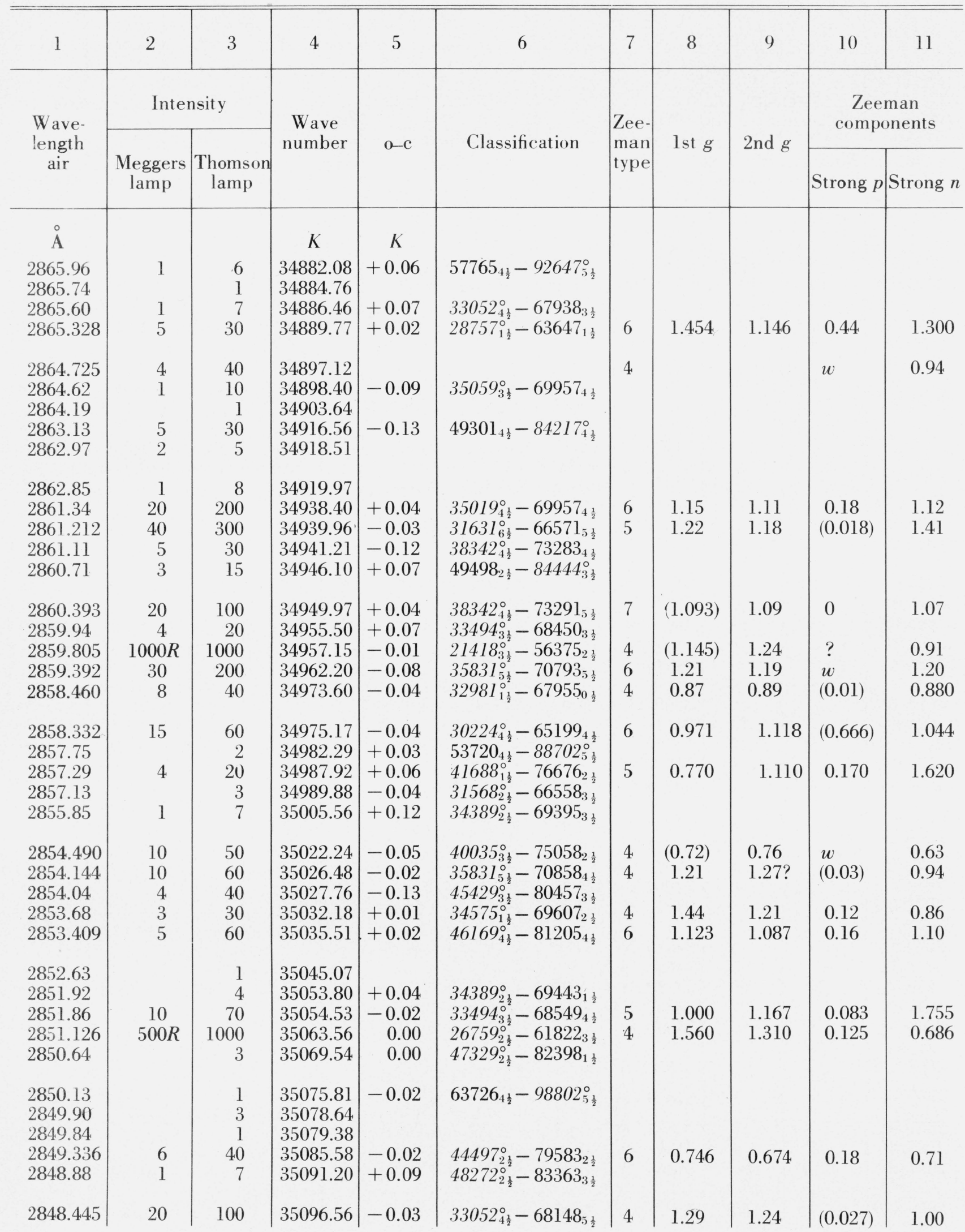


TABLE 6. Yb II-Observed and classified lines-Continued

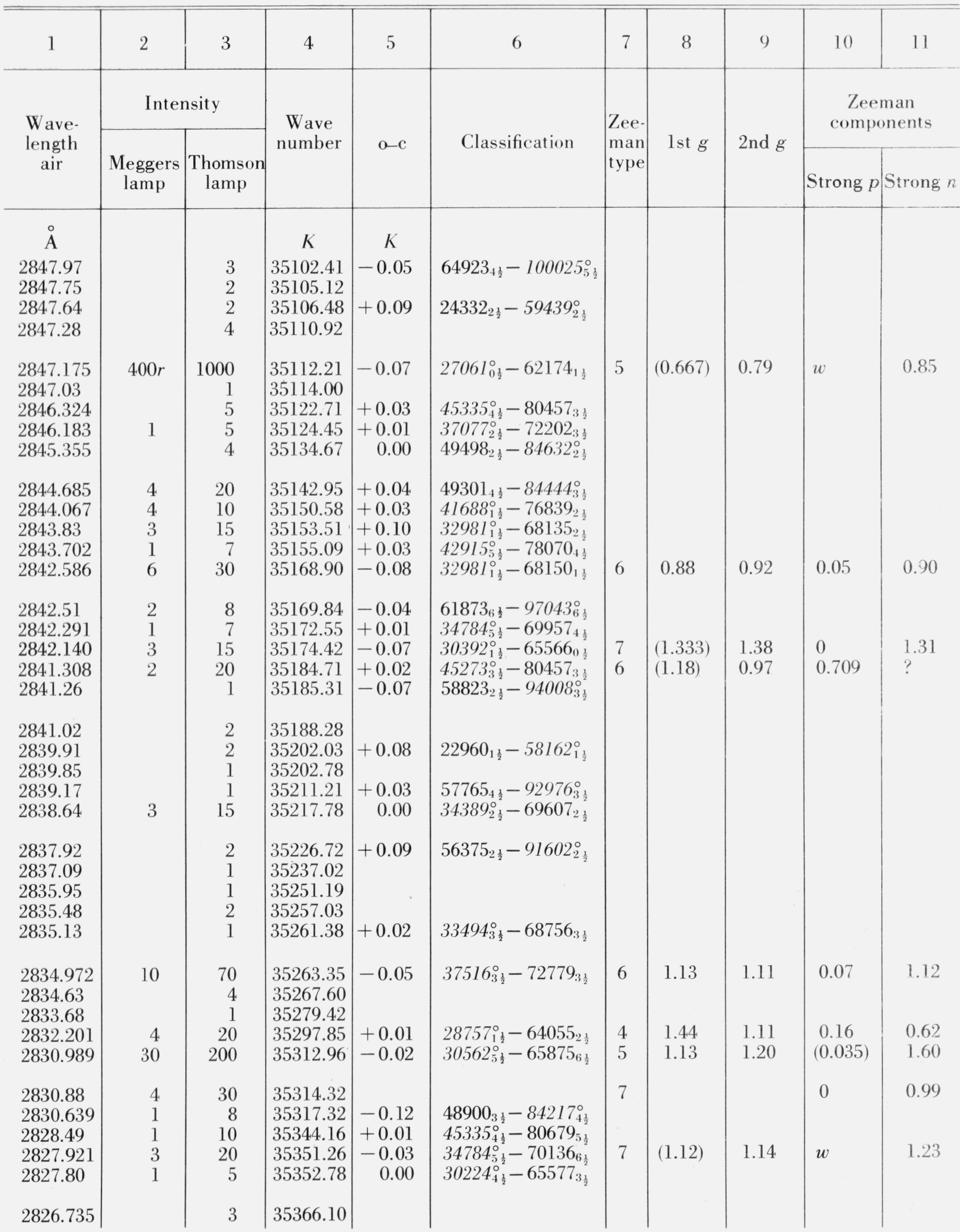


TABLE 6. Yb II-Observed and classified lines-Continued

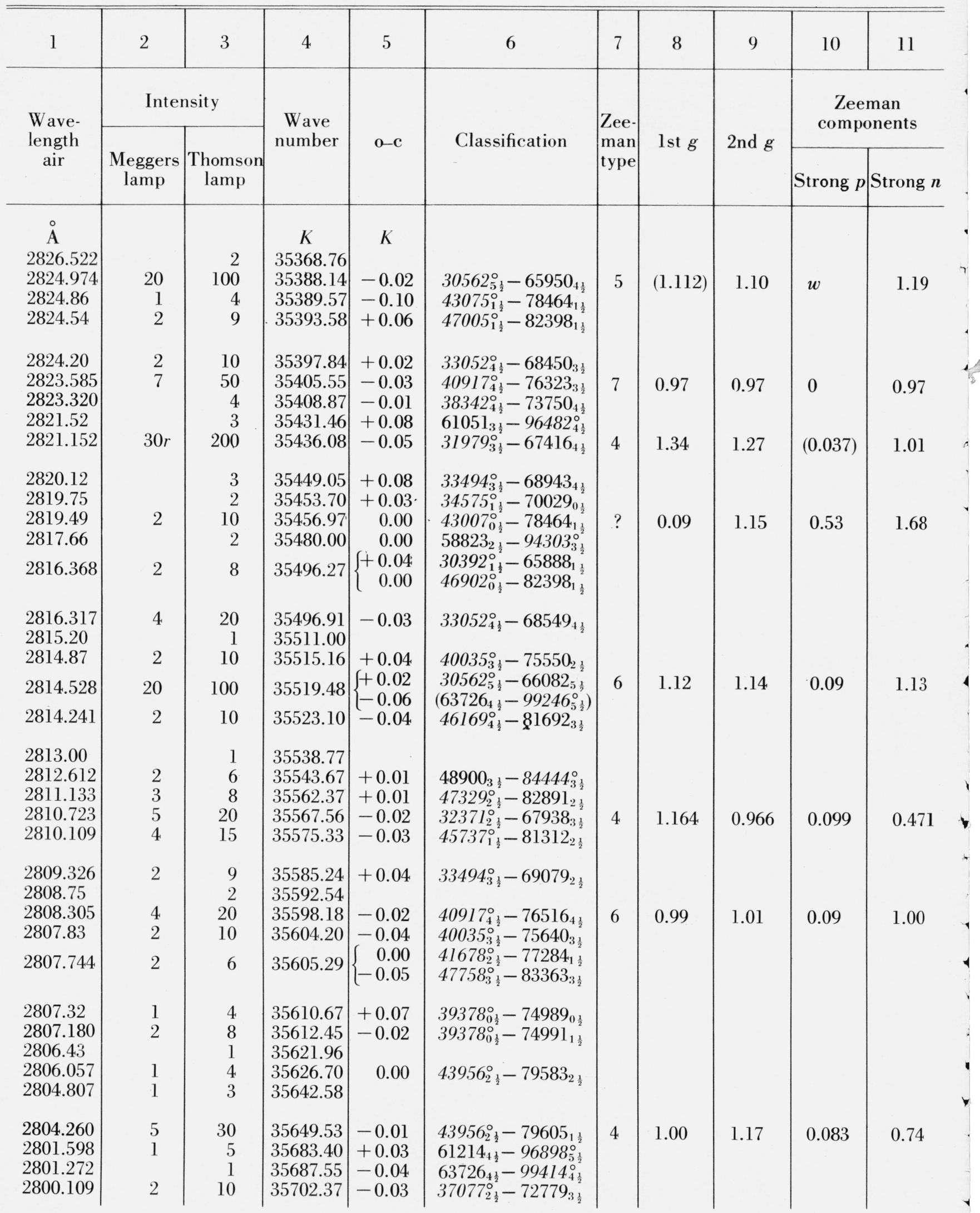


TABLE 6. Yb II-Observed and classified lines-Continued

\begin{tabular}{|c|c|c|c|c|c|c|c|c|c|c|}
\hline 1 & 2 & 3 & 4 & 5 & 6 & 7 & 8 & 9 & 10 & 11 \\
\hline \multirow{2}{*}{$\begin{array}{l}\text { Wave- } \\
\text { length } \\
\text { air }\end{array}$} & \multicolumn{2}{|c|}{ Intensity } & \multirow{2}{*}{$\begin{array}{c}\text { Wave } \\
\text { number }\end{array}$} & \multirow{2}{*}{$o-c$} & \multirow{2}{*}{ Classification } & \multirow{2}{*}{$\begin{array}{l}\text { Zee- } \\
\text { man } \\
\text { type }\end{array}$} & \multirow{2}{*}{ lst $g$} & \multirow{2}{*}{ 2nd $g$} & \multicolumn{2}{|c|}{$\begin{array}{c}\text { Zeeman } \\
\text { components }\end{array}$} \\
\hline & $\begin{array}{l}\text { Meggers } \\
\text { lamp }\end{array}$ & $\begin{array}{c}\text { Thomson } \\
\text { lamp }\end{array}$ & & & & & & & Strong $p$ & Strong $n$ \\
\hline A & & & $K$ & $K$ & & & & & & \\
\hline 2800.060 & 6 & 30 & 35703.00 & -0.10 & $28757_{1 \frac{1}{2}}^{\circ}-6446 l_{1 \frac{1}{2}}$ & & & & & \\
\hline 2800.006 & 8 & 50 & 35703.69 & -0.06 & $33052_{4 \frac{1}{2}}^{\circ}-68756_{3 \frac{1}{2}}$ & & & & & \\
\hline 2799.376 & 30 & 40 & 35711.72 & -0.01 & $22960_{1 \frac{1}{2}}-58672_{0 \frac{1}{2}}^{\circ}$ & 5 & 0.807 & 0.541 & 0.133 & 0.940 \\
\hline 2798.211 & 20 & 100 & 35726.59 & -0.03 & $30224_{4 \frac{1}{2}}^{\circ}-65950_{\frac{1}{2}}$ & 6 & 0.931 & 1.091 & 0.720 & 1.10 \\
\hline $\begin{array}{l}2797.796 \\
279728\end{array}$ & 10 & 80 & 35731.89 & $\begin{array}{r}0.00 \\
-0.09\end{array}$ & $40917_{4 \frac{1}{2}}^{\circ}-76649_{5 \frac{1}{2}} \mathrm{~d}$ & 5 & & & $0.43 w$ & 1.58 \\
\hline 2797.28 & & 2 & 35738.48 & -0.09 & $57765_{4 \frac{1}{2}}-93503_{4 \frac{1}{2}}^{\circ}$ & & & & & \\
\hline $\begin{array}{l}2796.764 \\
2795.292\end{array}$ & 6 & $\begin{array}{r}2 \\
20\end{array}$ & $\begin{array}{l}35745.07 \\
35763.89\end{array}$ & -0.01 & $32371_{2 \frac{1}{2}}^{\circ}-68135_{2 \frac{1}{2}}$ & 6 & 1.17 & 1.09 & 0.20 & 1.13 \\
\hline 2795.074 & 8 & 40 & 35766.68 & -0.08 & $37516_{3 \frac{1}{2}}^{\circ}-73283_{4 \frac{1}{2}}^{\circ} d$ & 4 & 1.16 & 1.09 & $(0.04)$ & 0.63 \\
\hline 2794.774 & 7 & 40 & 35770.52 & -0.07 & $41678_{2 \frac{1}{2}}^{\circ}-77449_{3 \frac{1}{2}}$ & 7 & (1.193) & 1.18 & 0 & 1.14 \\
\hline 2794.441 & 10 & 70 & 35774.79 & -0.04 & $35019_{4 \frac{1}{2}}^{\circ}-70793_{5 \frac{1}{2}}^{\circ}$ & 5 & 1.15 & 1.20 & $(0.025)$ & 1.43 \\
\hline 2794.08 & 2 & 10 & 35779.41 & -0.06 & $32371_{2 \frac{1}{2}}^{\circ}-68150_{1 \frac{1}{2}}$ & & & & & \\
\hline 2793.280 & 10 & 60 & 35789.65 & -0.01 & $33653_{0 \frac{1}{2}}^{\circ}-69443_{1 \frac{1}{2}}^{2}$ & 4 & 1.32 & 1.15 & 0.09 & 1.06 \\
\hline $\begin{array}{l}2792.54 \\
2789.431\end{array}$ & 3 & $\begin{array}{r}3 \\
15\end{array}$ & $\begin{array}{l}35799.14 \\
35839.04\end{array}$ & $\begin{array}{l}-0.04 \\
-0.01\end{array}$ & $\begin{array}{l}35059_{3 \frac{1}{2}}^{\circ}-70858_{4 \frac{1}{2}} \\
35019_{41}^{\circ}-70858_{4}\end{array}$ & & & & & \\
\hline 2787.965 & 7 & 30 & 35857.88 & -0.04 & $30224_{4 \frac{1}{2}}^{\circ}-66082_{5 \frac{1}{2}}^{\circ}$ & 5 & $(0.935)$ & 1.15 & 0.107 & $?$ \\
\hline 2787.010 & 1 & 7 & 35870.17 & -0.07 & $45335_{4 \frac{1}{2}}^{\circ}-81205_{4 \frac{1}{2}}$ & & & & & \\
\hline 2786.025 & 1 & 5 & 35882.85 & +0.02 & $45429_{3 \frac{1}{2}}^{\circ}-81312_{2 \frac{1}{2}}^{\circ}$ & & & & & \\
\hline 2785.750 & 8 & 30 & 35886.39 & +0.05 & $47005_{\frac{1}{2}}^{\circ}-82891_{2 \frac{1}{2}}^{2}$ & 5 & $(0.99)$ & 1.03 & $w$ & 1.09 \\
\hline 2785.066 & & 2 & 35895.20 & $\begin{array}{l}-0.07 \\
-0.03\end{array}$ & $\begin{array}{l}35059_{3 \frac{1}{2}}^{\circ}-70954_{2 \frac{1}{2}} \\
33494_{31}^{\circ}-69395_{312}^{2}\end{array}$ & & & & & \\
\hline 2784.656 & 30 & 150 & 35900.49 & $\left\{\begin{array}{l}-0.03 \\
+0.02\end{array}\right.$ & $\begin{array}{l}334944_{3 \frac{1}{2}}-09595_{3 \frac{1}{2}} \\
\left(55702_{1 \frac{1}{2}}-91602_{2 \frac{1}{2}}^{\circ}\right)\end{array}$ & 0 & 0.99 & 0.95 & 0.15 & 0.91 \\
\hline 2783.16 & & 2 & 35919.78 & -0.06 & $45737_{1 \frac{1}{2}}^{\circ}-81657_{2 \frac{1}{2}}$ & & & & & \\
\hline 2782.58 & & 3 & 35927.27 & -0.06 & $56375_{2 \frac{1}{2}}-92303_{3 \frac{1}{2}}^{\circ}$ & & & & & \\
\hline 2782.54 & 1 & 5 & 35927.79 & -0.06 & $41678_{2 \frac{1}{2}}^{\circ}-77606_{2 \frac{1}{2}}$ & & & & & \\
\hline $\begin{array}{l}2782.203 \\
2782.102\end{array}$ & $\begin{array}{l}1 \\
1\end{array}$ & $\begin{array}{l}5 \\
2\end{array}$ & $\begin{array}{l}35932.14 \\
35933.44\end{array}$ & -0.11 & $45273_{3 \frac{1}{2}}^{\circ}-81205_{4 \frac{1}{2}}$ & & & & & \\
\hline 2781.493 & & 2 & 35941.31 & -0.08 & $62861_{5 \frac{1}{2}}-98802_{5 \frac{1}{2}}^{\circ}$ & & & & & \\
\hline $2780.820^{\circ}$ & 1 & 3 & 35950.01 & -0.02 & $27061_{0 \frac{1}{2}}^{\circ}-63011_{1 \frac{1}{2}}^{\circ}$ & & & & & \\
\hline 2780.125 & & 3 & 35958.99 & +0.01 & $30392_{1 \frac{1}{2}}^{\circ}-66351_{2 \frac{1}{2}}^{2}$ & & & & & \\
\hline 2780.032 & 2 & 15 & 35960.20 & 0.00 & $44497_{2 \frac{1}{2}}^{0}-80457_{3 \frac{1}{2}}^{2}$ & & & & & \\
\hline 2776.688 & 1 & 5 & 36003.50 & +0.01 & $30392_{\frac{1}{2}}^{\circ}-66395_{2 \frac{1}{2}}$ & & & & & \\
\hline 2776.280 & $60 r$ & 200 & 36008.79 & 0.00 & $30562_{5 \frac{1}{2}}^{\circ}-66571_{5 \frac{1}{2}}$ & 6 & 1.12 & 1.19 & 0.36 & 1.16 \\
\hline 2775.440 & 2 & 10 & 36019.69 & $\left\{\begin{array}{l}+0.01 \\
+0.02\end{array}\right.$ & $\begin{array}{c}44438_{3 \frac{1}{2}}^{\circ}-80457_{3 \frac{1}{2}}^{\circ} \\
\left(58283_{\frac{21}{2}}^{\circ}-94303_{3 \frac{1}{2}}^{\circ}\right)\end{array}$ & 6 & (1.10) & $0.99 ?$ & 0.38 & \\
\hline $\begin{array}{l}2774.304 \\
2773.91 \\
2771.86\end{array}$ & 5 & $\begin{array}{r}20 \\
2 \\
2\end{array}$ & $\begin{array}{l}36034.44 \\
36039.56 \\
36066.21\end{array}$ & $\begin{array}{r}0.00 \\
-0.07\end{array}$ & $\begin{array}{l}47329_{\frac{21}{2}}^{\mathrm{o}}-83363_{3 \frac{1}{2}} \\
45273_{3 \frac{1}{2}}^{\mathrm{O}}-81312_{2 \frac{1}{2}}\end{array}$ & 7 & $(0.990)$ & 0.97 & 0 & 0.911 \\
\hline $\begin{array}{l}2771.324 \\
2770.405\end{array}$ & 20 & $\begin{array}{r}100 \\
2\end{array}$ & $\begin{array}{l}36073.18 \\
36085.15\end{array}$ & -0.05 & $34784_{5 \frac{1}{2}}^{\circ}-70858_{4 !}$ & 4 & 1.12 & 1.28 & 0.080 & 0.40 \\
\hline
\end{tabular}


TABle 6. Yb II-Observed and classified lines-Continued

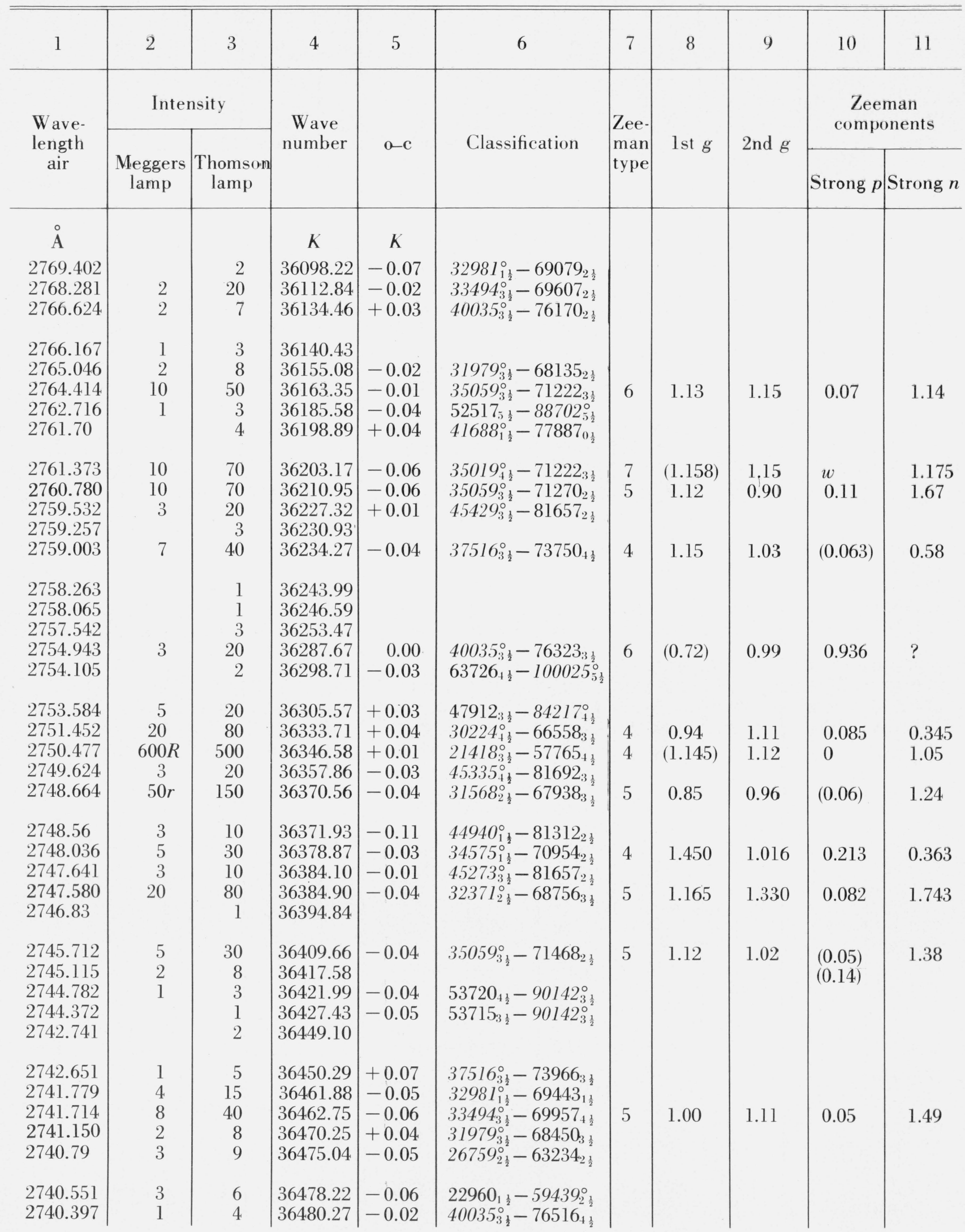


TABLE 6. Yb II-Observed and classified lines-Continued

\begin{tabular}{|c|c|c|c|c|c|c|c|c|c|c|}
\hline 1 & 2 & 3 & 4 & 5 & 6 & 7 & 8 & 9 & 10 & 11 \\
\hline \multirow{2}{*}{$\begin{array}{l}\text { W ave- } \\
\text { length } \\
\text { air }\end{array}$} & \multicolumn{2}{|c|}{ Intensity } & \multirow{2}{*}{$\begin{array}{c}\text { Wave } \\
\text { number }\end{array}$} & \multirow[b]{2}{*}{$o-c$} & \multirow[b]{2}{*}{ Classification } & \multirow{2}{*}{$\begin{array}{l}\text { Zee- } \\
\text { man } \\
\text { type }\end{array}$} & \multirow[b]{2}{*}{ lst $g$} & \multirow[b]{2}{*}{ 2nd $g$} & \multicolumn{2}{|c|}{$\begin{array}{c}\text { Zeeman } \\
\text { components }\end{array}$} \\
\hline & $\begin{array}{c}\text { Meggers } \\
\text { lamp }\end{array}$ & $\begin{array}{c}\text { Thomson } \\
\text { lamp }\end{array}$ & & & & & & & Strong $p$ & Strong $n$ \\
\hline A & & & K & $K$ & & & & & & \\
\hline 2740.215 & 2 & 6 & 36482.69 & & & & & & & \\
\hline 2739.475 & 1 & 2 & 36492.55 & -0.04 & $53404_{2 \frac{1}{2}}-89897_{1 \frac{1}{2}}^{\circ}$ & & & & & \\
\hline 2738.818 & 2 & 8 & 36501.30 & 0.00 & $43956_{2 \frac{1}{2}}^{\circ}-80457_{3 \frac{1}{2}}^{2}$ & 7 & $(0.99)$ & 0.99 & 0 & 0.99 \\
\hline & & 1 & 36508.07 & +0.05 & $43075_{1 \frac{1}{2}}^{\circ}-79583_{2 \frac{1}{2}}$ & & & & & \\
\hline 2737.61 & 3 & 15 & 36517.41 & +0.12 & $31631_{6 \frac{1}{2}}^{\circ}-68148_{5 \frac{1}{2}}^{2}$ & 5 & $(1.230)$ & 1.22 & 0 & 1.28 \\
\hline $\begin{array}{l}2736.553 \\
2736.050\end{array}$ & 4 & $\begin{array}{r}20 \\
2\end{array}$ & $\begin{array}{l}36531.51 \\
36538.23\end{array}$ & $\begin{array}{l}+0.10 \\
-0.03\end{array}$ & $\begin{array}{l}40917_{4 \frac{1}{2}}^{\circ}-77449_{3 \frac{1}{2}} \\
57765^{\frac{1}{2}}-94303^{\circ}\end{array}$ & & & & & \\
\hline 2735.673 & 2 & $\begin{array}{l}2 \\
6\end{array}$ & $\begin{array}{l}30350.20 \\
36543.26\end{array}$ & $\begin{array}{l}-0.03 \\
+0.07\end{array}$ & $\begin{array}{l}56375_{2 \frac{1}{2}}-943013_{3 \frac{1}{2}}^{\circ} \\
0 . \frac{1}{3}\end{array}$ & & & & & \\
\hline $\begin{array}{l}2734.928 \\
2734.413\end{array}$ & & $\begin{array}{l}2 \\
1\end{array}$ & $\begin{array}{l}36553.22 \\
36560.10\end{array}$ & +0.07 & $62861_{5 \frac{1}{2}}-99414_{4 \frac{1}{2}}^{\circ}$ & & & & & \\
\hline 2734.087 & 5 & 30 & 36564.46 & -0.05 & $34389_{2 \frac{1}{2}}^{\circ}-70954_{2_{1}}$ & 7 & 1.00 & 1.00 & 0 & 1.00 \\
\hline 2733.724 & & 4 & 36569.31 & -0.02 & $31979_{3 \frac{1}{2}}^{\circ}-68549_{4 \frac{1}{2}}^{\circ}$ & & & & & \\
\hline 2733.65 & & 2 & 36570.30 & -0.08 & $57765_{4 \frac{1}{2}}-94335_{5 \frac{1}{2}}^{\circ}$ & & & & & \\
\hline 2733.112 & 5 & 10 & 36577.50 & +0.05 & $24332_{2 \frac{1}{2}}-60910_{1 \frac{1}{2}}^{\circ}$ & 5 & (1.202) & 1.12 & $w$ & 1.32 \\
\hline 2732.942 & 1 & 4 & 36579.78 & +0.08 & $37077_{2 \frac{1}{2}}^{\circ}-73657_{1 \frac{1}{2}}$ & & & & & \\
\hline $\begin{array}{l}2732.742 \\
2732.50 \\
2732.033\end{array}$ & $30 r$ & $\begin{array}{r}150 \\
1 \\
1\end{array}$ & $\begin{array}{l}36582.45 \\
36585.69 \\
36591.95\end{array}$ & -0.04 & $31568_{2 \frac{1}{2}}^{\circ}-68150_{1 \frac{1}{2}}^{\circ}$ & 4 & $(0.862)$ & 0.90 & $d$ & 0.81 \\
\hline 2731.83 & & 1 & 36594.67 & -0.01 & $57765_{4 \frac{1}{2}}-94360_{4 \frac{1}{2}}^{\circ}$ & & & & & \\
\hline 2731.573 & & 3 & 36598.11 & -0.05 & $43007_{0 \frac{1}{2}}^{\circ}-79605_{1 \frac{1}{2}}^{\frac{1}{2}}$ & & & & & \\
\hline $\begin{array}{l}2731.517 \\
2730.62\end{array}$ & & $\begin{array}{l}3 \\
1\end{array}$ & $\begin{array}{l}36598.86 \\
36610.88\end{array}$ & & & & & & & \\
\hline 2729.50 & 2 & 8 & 36625.90 & -0.05 & $32981_{1 \frac{1}{2}}^{\circ}-69607_{2 \frac{1}{2}}$ & 5 & $(0.866)$ & 1.21 & 0.17 & \\
\hline $\begin{array}{l}2729.093 \\
2728.47\end{array}$ & 1 & $\begin{array}{l}5 \\
1\end{array}$ & $\begin{array}{l}36631.36 \\
36639.73\end{array}$ & -0.03 & $38342_{4 \frac{1}{2}}^{\circ}-74973_{3 \frac{1}{2}}$ & & & & & \\
\hline 2728.420 & 6 & 20 & 36640.40 & -0.09 & $40035_{3 \frac{1}{2}}^{\circ}-76676_{2 \frac{1}{2}}$ & 4 & 0.726 & 1.112 & 0.193 & 0.240 \\
\hline $\begin{array}{l}2727.66 \\
2727.22\end{array}$ & & $\begin{array}{l}1 \\
1\end{array}$ & $\begin{array}{l}36650.61 \\
36656.52\end{array}$ & -0.02 & $49498_{2 \frac{1}{2}}-86148_{2 \frac{1}{2}}^{2}$ & & & & & \\
\hline 2724.65 & 1 & 3 & 36691.09 & & & & & & & \\
\hline 2724.41 & & 2 & 36694.33 & & & & & & & \\
\hline 2723.338 & & 3 & 36708.77 & -0.01 & $32371_{2 \frac{1}{2}}^{\circ}-69079_{2 \frac{1}{2}}$ & & & & & \\
\hline 2722.759 & & 2 & 36716.58 & +0.06 & $44940_{1 \frac{1}{2}}^{\circ}-81657_{2 \frac{1}{2}}^{\circ}$ & & & & & \\
\hline 2722.475 & 1 & 5 & 36720.41 & +0.01 & $47912_{3 \frac{1}{2}}-84632_{2 \frac{1}{2}}^{\circ}$ & & & & & \\
\hline 2722.205 & 8 & 40 & 36724.05 & -0.03 & $34389_{2 \frac{1}{2}}^{\circ}-71113_{1 \frac{1}{2}}$ & 7 & 0.99 & $(0.967)$ & 0 & 1.03 \\
\hline 2721.895 & & 3 & 36728.23 & -0.04 & $49498_{2 \frac{1}{2}}-86226_{1 \frac{1}{2}}^{\circ}$ & & & & & \\
\hline 2721.183 & & 3 & 36737.84 & +0.06 & $53404_{2 \frac{1}{2}}-90142_{3 \frac{1}{2}}^{\circ}$ & & & & & \\
\hline $\begin{array}{l}2720.522 \\
2719.996\end{array}$ & 1 & $\begin{array}{l}5 \\
2\end{array}$ & $\begin{array}{l}36746.77 \\
36753.87\end{array}$ & -0.02 & $37516_{31}^{\circ}-74270_{2}$ & & & & & \\
\hline 2719.005 & 3 & 15 & 36767.27 & +0.03 & $44438_{3 \frac{1}{2}}^{\circ}-81205_{4 \frac{1}{3}}$ & 7 & $(1.10)$ & 1.11 & 0 & 1.157 \\
\hline 2718.349 & $40 r$ & 200 & 36776.14 & 0.00 & $31979_{3 \frac{1}{2}}^{\circ}-68756_{3 \frac{1}{2}}^{\circ}$ & 7 & 1.328 & 1.328 & 0 & 1.328 \\
\hline
\end{tabular}


TABlE 6. Yb II-Observed and classified lines-Continued

\begin{tabular}{|c|c|c|c|c|c|c|c|c|c|c|}
\hline 1 & 2 & 3 & 4 & 5 & 6 & 7 & 8 & 9 & 10 & 11 \\
\hline \multirow{2}{*}{$\begin{array}{l}\text { Wave- } \\
\text { length } \\
\text { air }\end{array}$} & \multicolumn{2}{|c|}{ Intensity } & \multirow{2}{*}{$\begin{array}{l}\text { Wave } \\
\text { number }\end{array}$} & \multirow{2}{*}{$\mathrm{o}-\mathrm{c}$} & \multirow{2}{*}{ Classification } & \multirow{2}{*}{$\left|\begin{array}{l}\text { Zee- } \\
\text { man } \\
\text { type }\end{array}\right|$} & \multirow{2}{*}{ lst $g$} & \multirow{2}{*}{2 nd $g$} & \multicolumn{2}{|c|}{$\begin{array}{l}\text { Zeeman } \\
\text { components }\end{array}$} \\
\hline & $\begin{array}{l}\text { Meggers } \\
\text { lamp }\end{array}$ & $\begin{array}{c}\text { Thomson } \\
\text { lamp }\end{array}$ & & & & & & & Strong $p$ & Strong $n$ \\
\hline A & & & $K$ & K & & & & & & \\
\hline $\begin{array}{l}2717.76 \\
2717.661 \\
2717.617\end{array}$ & $\begin{array}{l}3 \\
1\end{array}$ & $\begin{array}{r}2 \\
10 \\
4\end{array}$ & $\begin{array}{l}36784.11 \\
36785.45 \\
36786.04\end{array}$ & $\begin{array}{l}+0.05 \\
+0.01\end{array}$ & $\begin{array}{l}35831_{5 \frac{1}{2}}^{\circ}-72615_{5 \frac{1}{2}}^{\circ} \\
41678_{2 \frac{1}{2}}^{\circ}-78464_{1 \frac{1}{2}}\end{array}$ & & & & & \\
\hline $\begin{array}{l}2717.106 \\
2716.354 \\
2716.177\end{array}$ & $\begin{array}{l}4 \\
3\end{array}$ & $\begin{array}{r}20 \\
5 \\
3\end{array}$ & $\begin{array}{l}36792.96 \\
36803.15 \\
36805.55\end{array}$ & $\begin{array}{l}+0.03 \\
-0.03\end{array}$ & $\begin{array}{l}58961_{5 \frac{1}{2}}-95754_{5 \frac{1}{2}}^{\circ} \\
40035_{3 \frac{1}{2}}^{\circ}-76839_{2 \frac{1}{2}}^{\circ}\end{array}$ & 7 & 0.89 & 0.89 & 0 & 0.89 \\
\hline $\begin{array}{l}2715.94 \\
2715.640\end{array}$ & $\begin{array}{l}3 \\
1\end{array}$ & $\begin{array}{r}15 \\
4\end{array}$ & $\begin{array}{l}36808.76 \\
36812.82\end{array}$ & $\begin{array}{l}+0.02 \\
+0.08\end{array}$ & $\begin{array}{l}28757_{1 \frac{1}{2}}^{\circ}-65566_{0 \frac{1}{2}} \\
30392_{1^{\frac{1}{2}}}^{\circ}-67204_{1 \frac{1}{2}}\end{array}$ & 7 & 1.45 & 1.45 & 0 & 1.45 \\
\hline $\begin{array}{l}2715.383 \\
2715.17 \\
2714.408 \\
2714.18 \\
2713.718\end{array}$ & $\begin{array}{l}4 \\
4\end{array}$ & $\begin{array}{r}7 \\
1 \\
30 \\
2 \\
2\end{array}$ & $\begin{array}{l}36816.31 \\
36819.19 \\
36829.53 \\
36832.62 \\
36838.89\end{array}$ & $\begin{array}{l}+0.04 \\
+0.07 \\
+0.04 \\
+0.02\end{array}$ & $\begin{array}{l}22960_{1 \frac{1}{2}}-59777_{1 \frac{1}{2}}^{\circ} \\
56375_{2 \frac{1}{2}}^{\circ}-93195_{3 \frac{1}{2}}^{\circ} \\
40917_{4 \frac{1}{2}}^{\circ}-77747_{4 \frac{1}{2}} \\
34389_{2 \frac{1}{2}}^{\circ}-71222_{3 \frac{1}{2}}^{\circ}\end{array}$ & 6 & 0.965 & 1.073 & 0.49 & 1.02 \\
\hline $\begin{array}{l}2712.658 \\
2711.785 \\
2711.09 \\
2710.674 \\
2710.544\end{array}$ & $\begin{array}{r}15 \\
20 \\
\\
6 \\
20\end{array}$ & $\begin{array}{r}80 \\
30 \\
1 \\
30 \\
80\end{array}$ & $\begin{array}{l}36853.29 \\
36865.15 \\
36874.60 \\
36880.26 \\
36882.03\end{array}$ & $\begin{array}{r}+0.05 \\
-0.01 \\
-0.02 \\
+0.01 \\
0.00\end{array}$ & $\begin{array}{l}30562_{5 \frac{1}{2}}^{\circ}-67416_{4 \frac{1}{2}} \\
21418_{3 \frac{1}{2}}^{\circ}-58283_{2 \frac{1}{2}} \\
44438_{3 \frac{1}{2}}^{\circ}-81312_{2 \frac{1}{2}}^{\circ} \\
34389_{2 \frac{1}{2}}^{\circ}-71270_{2 \frac{1}{2}}^{\circ} \\
31568_{2 \frac{1}{2}}^{\circ}-68450_{\frac{1}{2}}^{\circ}\end{array}$ & $\begin{array}{l}4 \\
5 \\
6 \\
5\end{array}$ & $\begin{array}{l}(1.112) \\
1.141 \\
\\
1.02 \\
0.87\end{array}$ & $\begin{array}{l}1.23 \\
0.770 \\
\\
0.92 \\
1.09\end{array}$ & $\begin{array}{l}? \\
0.190 \\
0.26 \\
0.111\end{array}$ & $\begin{array}{l}0.52 \\
2.071 \\
\\
0.97 \\
1.651\end{array}$ \\
\hline $\begin{array}{l}2710.046 \\
2709.715 \\
2708.842 \\
2708.46 \\
2705.108\end{array}$ & $\begin{array}{l}2 \\
4 \\
5\end{array}$ & $\begin{array}{r}5 \\
20 \\
30 \\
2 \\
3\end{array}$ & $\begin{array}{l}36888.81 \\
36893.31 \\
36905.20 \\
36910.41 \\
36956.14\end{array}$ & $\begin{array}{r}+0.10 \\
-0.02 \\
0.00 \\
+0.05\end{array}$ & $\begin{array}{l}26759_{2 \frac{1}{2}}^{\circ}-63647_{1 \frac{1}{2}} \\
34575_{\frac{1}{2}}^{\circ}-71468_{2 \frac{1}{2}} \\
33052_{4 \frac{1}{2}}^{\circ}-69957_{+\frac{1}{2}} \\
61873_{6 \frac{1}{2}}-98829_{6 \frac{1}{\frac{1}{2}}}^{\circ}\end{array}$ & $\begin{array}{l}4 \\
6\end{array}$ & $\begin{array}{l}1.46 \\
1.27\end{array}$ & $\begin{array}{l}1.02 \\
1.13\end{array}$ & $\begin{array}{l}0.22 \\
0.65\end{array}$ & $\begin{array}{l}0.36 \\
1.20\end{array}$ \\
\hline $\begin{array}{l}2704.75 \\
2704.53 \\
2702.133 \\
2701.733 \\
2701.130\end{array}$ & $\begin{array}{l}3 \\
1 \\
1 \\
3\end{array}$ & $\begin{array}{r}2 \\
15 \\
4 \\
4 \\
5\end{array}$ & $\begin{array}{l}36961.03 \\
36964.04 \\
36996.83 \\
37002.30 \\
37010.56\end{array}$ & $\begin{array}{r}-0.09 \\
0.00 \\
+0.02\end{array}$ & $\begin{array}{l}40917_{4 \frac{1}{2}}^{\circ}-77882_{3 \frac{1}{2}} \\
53720_{4 \frac{1}{2}}-90717_{4 \frac{1}{2}}^{\circ}-90717_{4 \frac{1}{2}}^{\circ} \\
53715_{3 \frac{1}{2}}-900\end{array}$ & & & & & \\
\hline $\begin{array}{l}2700.797 \\
2698.617 \\
2698.447\end{array}$ & $\begin{array}{l}6 \\
2\end{array}$ & $\begin{array}{r}40 \\
8 \\
1\end{array}$ & $\begin{array}{l}37015.13 \\
37045.03 \\
37047.36\end{array}$ & $\begin{array}{l}+0.02 \\
-0.09\end{array}$ & $\begin{array}{l}44497_{2 \frac{1}{2}}^{\circ}-81512_{1 \frac{1}{2}} \\
32981_{1 \frac{1}{2}}^{\circ}-70029_{0 \frac{1}{2}}\end{array}$ & 5 & 0.74 & 0.48 & 0.130 & 1.14 \\
\hline $\begin{array}{l}2696.625 \\
2696.150\end{array}$ & 4 & $\begin{array}{r}20 \\
2\end{array}$ & $\begin{array}{l}37072.39 \\
37078.92\end{array}$ & $\begin{array}{l}-0.03 \\
-0.02\end{array}$ & $\begin{array}{l}32371_{2 \frac{1}{2}}^{\circ}-69443_{1 \frac{1}{2}} \\
34389_{2 \frac{1}{2}}^{\circ}-71468_{2 \frac{1}{2}}\end{array}$ & 7 & (1.170) & 1.16 & 0 & 1.19 \\
\hline $\begin{array}{l}2695.556 \\
2695.427 \\
2694.622 \\
2692.703 \\
2692.408\end{array}$ & $\begin{array}{l}8 \\
1 \\
2 \\
3\end{array}$ & $\begin{array}{r}2 \\
60 \\
4 \\
10 \\
15\end{array}$ & $\begin{array}{l}37087.09 \\
37088.87 \\
37099.95 \\
37126.38 \\
37130.45\end{array}$ & $\begin{array}{l}-0.01 \\
+0.02 \\
-0.03 \\
-0.03 \\
-0.03\end{array}$ & $\begin{array}{l}56375_{2 \frac{1}{2}}-93463_{3 \frac{1}{2}}^{\circ} \\
31631_{6 \frac{1}{2}}^{\circ}-68720_{6 \frac{1}{2}}^{\circ} \\
31979_{3 \frac{1}{2}}^{\circ}-69079_{2 \frac{1}{2}}^{\circ} \\
41688_{1 \frac{1}{2}}^{\circ}-78814_{2 \frac{1}{2}}^{\circ} \\
28757_{1 \frac{1}{2}}^{\circ}-65888_{1 \frac{1}{2}}^{\circ}\end{array}$ & 6 & (1.230) & 1.16 & 0.44 & $?$ \\
\hline $\begin{array}{l}2691.996 \\
2691.49\end{array}$ & 5 & $\begin{array}{r}30 \\
3\end{array}$ & $\begin{array}{l}37136.14 \\
37143.12\end{array}$ & $\begin{array}{l}+0.01 \\
+0.09\end{array}$ & $\begin{array}{l}41678_{2 \frac{1}{2}}^{\circ}-78814_{2 \frac{1}{2}} \\
35059_{3 \frac{1}{2}}^{\circ}-72202_{3 \frac{1}{2}}\end{array}$ & 6 & (1.193) & 1.26 & $d$ & 1.225 \\
\hline
\end{tabular}


TABLE 6. Yb II-Observed and classified lines-Continued

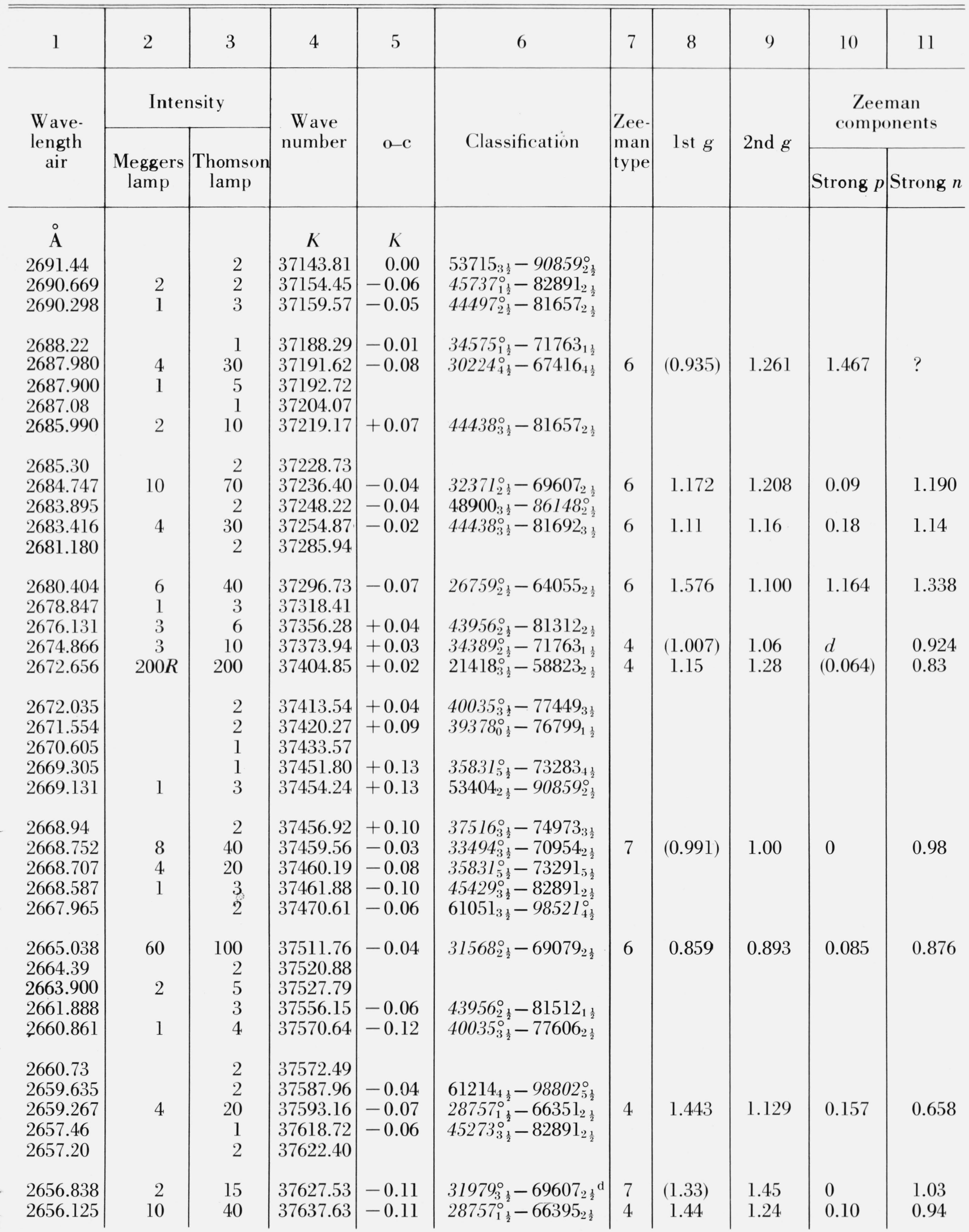


TABLE 6. Yb II-Observed and classified lines-Continued

\begin{tabular}{|c|c|c|c|c|c|c|c|c|c|c|}
\hline 1 & 2 & 3 & 4 & 5 & 6 & 7 & 8 & 9 & 10 & 11 \\
\hline \multirow{2}{*}{$\begin{array}{l}\text { Wave- } \\
\text { length } \\
\text { air }\end{array}$} & \multicolumn{2}{|c|}{ Intensity } & \multirow{2}{*}{$\begin{array}{l}\text { Wave } \\
\text { number }\end{array}$} & \multirow{2}{*}{$0-c$} & \multirow{2}{*}{ Classification } & \multirow{2}{*}{ Zee- } & \multirow{2}{*}{ lst $g$} & \multirow{2}{*}{ 2nd $g$} & \multicolumn{2}{|c|}{$\begin{array}{l}\text { Zeeman } \\
\text { components }\end{array}$} \\
\hline & $\begin{array}{c}\text { Meggers } \\
\text { lamp }\end{array}$ & $\begin{array}{c}\text { Thomson } \\
\text { lamp }\end{array}$ & & & & & & & Strong $p$ & Strong $n$ \\
\hline A & & & $K$ & $K$ & & & & & & \\
\hline $\begin{array}{l}2653.75 \\
2650.795\end{array}$ & $\begin{array}{c}1000 R \\
2\end{array}$ & $\begin{array}{r}1000 \\
10\end{array}$ & $\begin{array}{l}37671.31 \\
37713.30\end{array}$ & $\begin{array}{l}-0.07 \\
-0.08\end{array}$ & $\begin{array}{l}21418_{3 \frac{1}{2}}^{\circ}-59090_{3 \frac{1}{2}}^{\circ} \\
24332_{2 \frac{1}{2}}^{\circ}-62046_{3 \frac{1}{2}}^{\circ}\end{array}$ & 7 & $(1.145)$ & 1.13 & 0 & 1.136 \\
\hline 2650.728 & 9 & 30 & 37714.26 & -0.09 & $30224_{4 \frac{1}{2}}^{\circ}-67938_{3 \frac{1}{2}}^{\circ}$ & 4 & $(0.935)$ & 0.96 & & 0.863 \\
\hline $\begin{array}{l}2649.787 \\
2649.165\end{array}$ & 10 & $\begin{array}{r}40 \\
3\end{array}$ & $\begin{array}{l}37727.65 \\
37736.51\end{array}$ & $\begin{array}{r}-0.03 \\
0.00\end{array}$ & $\begin{array}{l}33494_{3 \frac{1}{2}}^{\circ}-71222_{3 \frac{1}{2}} \\
43956_{2 \frac{1}{2}}^{\circ}-81692_{3 \frac{1}{2}}\end{array}$ & 6 & 0.98 & 1.14 & 0.54 & 1.06 \\
\hline 2648.803 & 6 & 30 & 37741.66 & -0.01 & $33052_{4 \frac{1}{2}}^{\circ}-70793_{5 \frac{1}{2}}^{\circ}$ & 4 & 1.24 & 1.20 & $(0.017)$ & 1.05 \\
\hline 2647.455 & 8 & 40 & 37760.88 & +0.02 & $35019_{4 \frac{1}{2}}^{\circ}-72779_{3 \frac{1}{2}}^{\circ}$ & 5 & 1.16 & 1.12 & $(0.02)$ & 1.30 \\
\hline 2647.233 & 2 & 10 & 37764.05 & 0.00 & $42915_{5 \frac{1}{2}}^{\circ}-80679_{5 \frac{1}{2}}^{\circ \frac{2}{2}}$ & & & & & \\
\hline $\begin{array}{l}2646.442 \\
2645.691\end{array}$ & 6 & $\begin{array}{r}30 \\
2\end{array}$ & $\begin{array}{l}37775.33 \\
37786.06\end{array}$ & 0.00 & $33494_{3 \frac{1}{2}}^{\circ}-71270_{2 \frac{1}{2}}$ & 5 & 0.98 & 0.90 & $(0.04)$ & 1.18 \\
\hline 2644.306 & $20 r$ & 80 & 37805.85 & -0.04 & $33052_{4 \frac{1}{2}}^{\circ}-70858_{4 \frac{1}{2}}$ & 6 & 1.265 & 1.279 & $(0.06)$ & 1.272 \\
\hline 2643.237 & & 3 & 37821.13 & -0.01 & $53715_{3 \frac{1}{2}}-91536_{3 \frac{1}{2}}^{\circ}$ & & & & & \\
\hline 2642.815 & & 5 & 37827.17 & +0.05 & $31568_{2 \frac{1}{2}}^{\circ}-69395_{3 \frac{1}{2}}^{\circ}$ & & & & & \\
\hline 2642.558 & 10 & 1000 & 37830.85 & +0.06 & $34784_{5 \frac{1}{2}}^{\circ}-72615_{5 \frac{1}{2}}$ & 6 & 1.20 & 1.07 & 0.73 & 1.132 \\
\hline 2641.97 & 2 & 10 & 37839.27 & +0.01 & $26759_{2 \frac{1}{2}}^{\circ}-64598_{3 \frac{1}{2}}^{\circ}$ & & & & & \\
\hline 2641.886 & 50 & 50 & 37840.47 & -0.02 & $21418_{3 \frac{1}{2}}^{\circ}-59259_{2 \frac{1}{2}}^{2}$ & 5 & (1.145) & 1.12 & $w$ & 1.20 \\
\hline $\begin{array}{l}2641.485 \\
2639448\end{array}$ & & 2 & 37846.22 & 0.00 & $40035_{3 \frac{1}{2}}^{\circ}-77882_{3 \frac{1}{2}}^{2}$ & & & & & \\
\hline 2639.448 & 10 & 40 & 37875.42 & -0.02 & $31568_{2 \frac{1}{2}}^{\circ}-69443_{1 \frac{1}{2}}$ & 4 & 0.86 & 1.14 & 0.14 & 0.42 \\
\hline 2638.596 & 4 & 5 & 37887.65 & -0.05 & $49498_{2 \frac{1}{2}}-87385_{3 \frac{1}{2}}^{\circ}$ & & & & & \\
\hline 2637.637 & & 2 & 37901.43 & -0.04 & $44497_{2 \frac{1}{2}}^{\circ}-82398_{1 \frac{1}{2}}$ & & & & & \\
\hline $\begin{array}{l}2637.378 \\
2636.519\end{array}$ & 2 & $\begin{array}{l}2 \\
6\end{array}$ & $\begin{array}{l}37905.15 \\
37917.50\end{array}$ & $\begin{array}{r}+0.02 \\
0.00\end{array}$ & $\begin{array}{l}39378_{0 \frac{1}{2}}^{\circ}-77284_{1_{\frac{1}{2}}} \\
\left.4680^{\circ}-7960\right)^{2}\end{array}$ & & & & & \\
\hline $\begin{array}{l}2030.319 \\
2636.399\end{array}$ & & $\begin{array}{l}0 \\
3\end{array}$ & $\begin{array}{l}37917.50 \\
37919.22\end{array}$ & 0.00 & $\begin{array}{l}41688_{1 \frac{1}{2}}-79605_{1 \frac{1}{2}} \\
35831_{5 \frac{1}{2}}^{\circ}-73750_{4 \frac{1}{2}}\end{array}$ & 6 & $(0.784)$ & 1.16 & 0.58 & \\
\hline 2636.275 & 2 & 8 & 37921.01 & -0.05 & $52938_{1 \frac{1}{2}}-90859_{2 \frac{1}{2}}^{\circ}$ & & & & & \\
\hline 2635.197 & 1 & 4 & 37936.52 & -0.14 & $58961_{5 \frac{1}{2}}-96898_{5 \frac{1}{2}}^{\circ}$ & & & & & \\
\hline 2634.306 & 30 & 40 & 37949.35 & +0.01 & $22960_{1 \frac{1}{2}}-60910_{1 \frac{1}{2}}^{\circ}$ & 6 & 0.806 & 1.000 & 0.29 & 0.903 \\
\hline 2634.183 & & 3 & 37951.12 & -0.07 & $44940_{1 \frac{1}{2}}^{\circ}-82891_{2 \frac{1}{2}}^{\circ}$ & & & & & \\
\hline 2632.69 & 2 & 8 & 37972.64 & -0.04 & $32981_{1 \frac{1}{2}}^{\circ}-70954_{2 \frac{1}{2}}$ & & & & & \\
\hline 2631.73 & 3 & 15 & 37986.49 & +0.05 & $30562_{5 \frac{1}{2}}^{\circ}-68549_{4 \frac{1}{2}}$ & 4 & (1.112) & 1.14 & $w$ & 1.00 \\
\hline 2628.80 & 2 & 7 & 38028.83 & -0.02 & $45335_{4 \frac{1}{2}}^{\circ}-83363_{3 \frac{1}{2}}$ & & & & & \\
\hline 2628.605 & 1 & 4 & 38031.65 & -0.06 & $61214_{4 \frac{1}{2}}-99246_{5 \frac{1}{2}}^{\circ}$ & & & & & \\
\hline 2628.424 & & 2 & 38034.27 & $\left\{\begin{array}{l}-0.08 \\
-0.10\end{array}\right.$ & $\begin{array}{l}37516_{3 \frac{1}{2}}^{\circ}-75550_{2 \frac{1}{2}} \\
40035_{3 \frac{1}{2}}^{\circ}-78070_{4 \frac{1}{2}}\end{array}$ & & & & & \\
\hline 2628.07 & 3 & 20 & 38039.39 & -0.07 & $31568_{2 \frac{1}{2}}^{\circ}-69607_{2 \frac{1}{2}}$ & 6 & $(0.862)$ & 1.22 & 0.88 & \\
\hline 2624.949 & 3 & 5 & 38084.62 & +0.04 & $49301_{4 \frac{1}{2}}-87385_{3 \frac{1}{2}}^{\circ}$ & & & & & \\
\hline 2624.52 & & 2 & 38090.84 & -0.02 & $45273_{3 \frac{1}{2}}^{\circ}-83363_{3 \frac{1}{2}}^{\circ}$ & & & & & \\
\hline 2623.22 & 2 & $\begin{array}{r}9 \\
20\end{array}$ & 38109.72 & -0.09 & $33653_{0 \frac{1}{2}}^{\circ}-71763_{\frac{1}{2}}^{2}$ & 4 & 1.32 & 1.10 & 0.11 & 0.99 \\
\hline $\begin{array}{l}2621.67 \\
2619.93\end{array}$ & $\begin{array}{l}4 \\
3\end{array}$ & $\begin{array}{l}20 \\
15\end{array}$ & 38132.25 & 0.00 & $32981_{1 \frac{1}{2}}^{\circ}-71113_{1 \frac{1}{2}}$ & & & & & \\
\hline 2619.93 & 3 & 15 & 38157.57 & -0.08 & $30562_{5 \frac{1}{2}}^{\circ}-68720_{6 \frac{1}{2}}$ & 5 & $(1.112)$ & 1.13 & $d$ & 1.25 \\
\hline 2619.074 & 4 & 20 & 38170.04 & -0.03 & $33052_{4 \frac{1}{2}}^{\circ}-71222_{3 \frac{1}{2}}$ & & & & & \\
\hline
\end{tabular}


TABLE 6. Yb II-Observed and classified lines - Continued

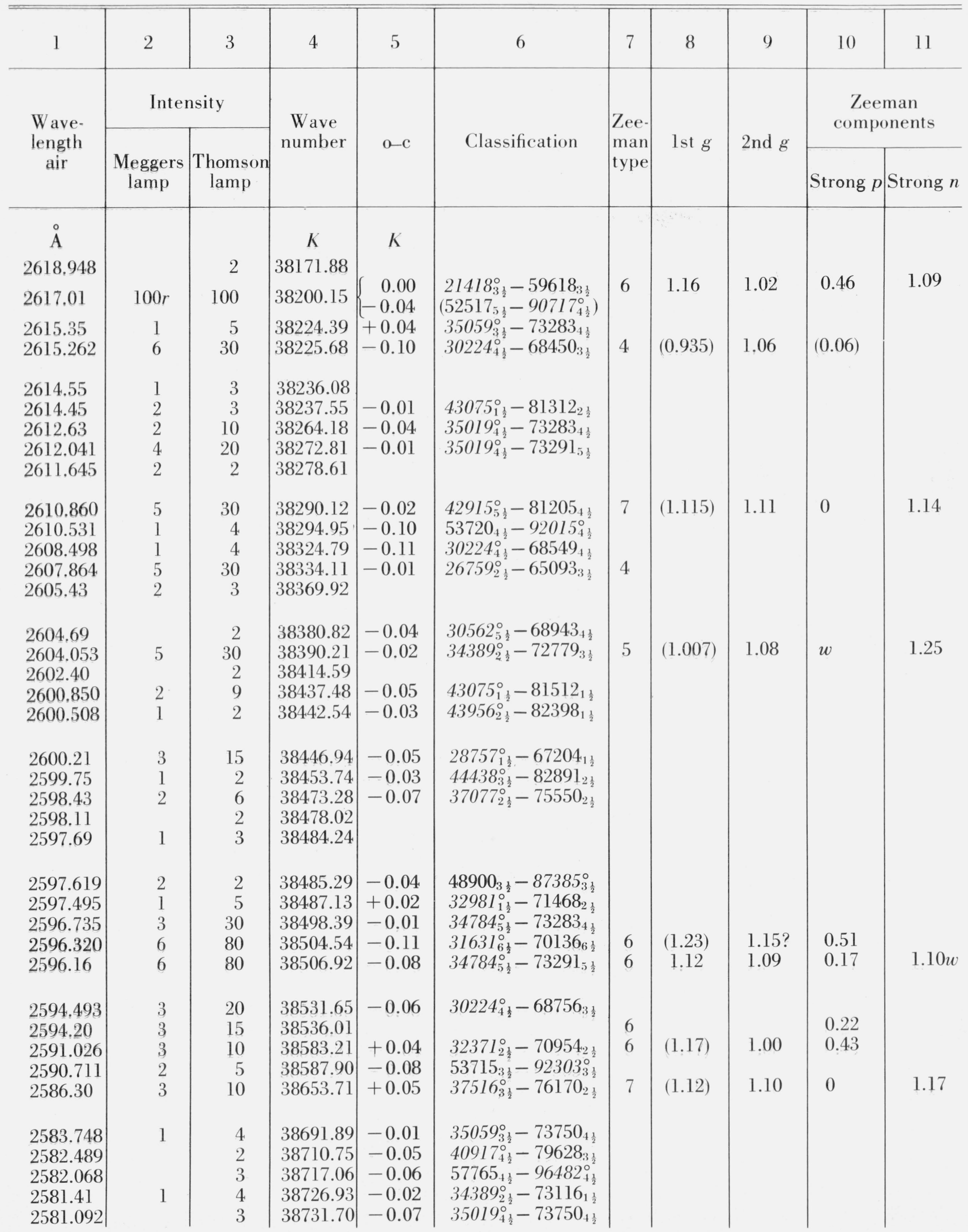


TABLE 6. Yb II-Observed and classified lines-Continued

\begin{tabular}{|c|c|c|c|c|c|c|c|c|c|c|}
\hline 1 & 2 & 3 & 4 & 5 & 6 & 7 & 8 & 9 & 10 & 11 \\
\hline \multirow{2}{*}{$\begin{array}{l}\text { Wave- } \\
\text { length } \\
\text { air }\end{array}$} & \multicolumn{2}{|c|}{ Intensity } & \multirow{2}{*}{$\begin{array}{c}\text { Wave } \\
\text { number }\end{array}$} & \multirow{2}{*}{$\mathrm{o}-\mathrm{c}$} & \multirow{2}{*}{ Classification } & \multirow{2}{*}{ 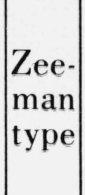 } & \multirow{2}{*}{ lst $g$} & \multirow{2}{*}{ 2nd $g$} & \multicolumn{2}{|c|}{$\begin{array}{c}\text { Zeeman } \\
\text { components }\end{array}$} \\
\hline & $\begin{array}{c}\text { Meggers } \\
\text { lamp }\end{array}$ & $\begin{array}{c}\text { Thomson } \\
\text { lamp }\end{array}$ & & & & & & & Strong $p$ & Strong $n$ \\
\hline$\stackrel{\circ}{\AA}$ & & & $K$ & $K$ & & & & & & \\
\hline 2577.74 & 3 & 10 & 38782.06 & -0.02 & $32981_{1 \frac{1}{2}}^{\circ}-71763_{1 \frac{1}{2}}$ & & & & & \\
\hline 2577.605 & 5 & 20 & 38784.09 & -0.13 & $52517_{5 \frac{1}{2}}-91301_{6 \frac{1}{3}}^{\circ}$ & & & & & \\
\hline 2576.09 & & 2 & 38806.90 & 0.00 & $37516_{3 \frac{1}{2}}^{\circ}-76323_{3 \frac{1}{2}}$ & & & & & \\
\hline $\begin{array}{l}2574.78 \\
0573147\end{array}$ & 2 & 10 & 38826.64 & $\begin{array}{r}0.00 \\
+0.02\end{array}$ & $27061_{0 \frac{1}{2}}^{\circ}-65888_{1 \frac{1}{2}}$ & & & & & \\
\hline & & & 38851.28 & +0.02 & $32371_{2 \frac{1}{3}}^{\circ}-71222_{3 \frac{1}{3}}$ & 7 & (1.17) & 1.16 & 0 & 1.12 \\
\hline 2572.15 & 1 & 4 & 38866.34 & -0.03 & $44497_{2 \frac{1}{2}}^{\circ}-83363_{3 \frac{1}{2}}$ & & & & & \\
\hline 2571.69 & 1 & 2 & 38873.29 & -0.02 & $53404_{2 \frac{1}{2}}^{2}-92278_{1 \frac{1}{2}}^{\circ}$ & & & & & \\
\hline 2571.36 & 7 & 50 & 38878.28 & 0.00 & $31979_{3 \frac{1}{2}}^{\circ}-70858_{4 \frac{1}{2}}^{\circ}$ & 4 & (1.331) & 1.30 & $w$ & 1.18 \\
\hline $\begin{array}{l}2569.61 \\
2568.60\end{array}$ & 1 & $\begin{array}{l}2 \\
2\end{array}$ & $\begin{array}{l}38904.76 \\
38920.05\end{array}$ & & & & & & & \\
\hline 2568.17 & 3 & 10 & 38926.57 & -0.06 & $53720_{4 \frac{1}{2}}-92647_{5 \frac{1}{3}}^{\circ}$ & & & & & \\
\hline 2565.573 & 10 & 40 & 38965.97 & +0.02 & $34784_{5 \frac{1}{2}}^{\circ}-73750_{4 \frac{1}{2}}$ & & & & & \\
\hline 2563.92 & 2 & 2 & 38991.09 & & & & & & & \\
\hline 2559,972 & 2 & 5 & 39051,22 & -0.07 & $30392_{1 \frac{1}{2}}^{\circ}-69443_{1 \frac{1}{2}}$ & & & & & \\
\hline 2558,40 & 3 & 2 & 39075.21 & & & & & & & \\
\hline 2557.804 & 4 & 5 & 39084.32 & -0.02 & $24332_{21}-63417_{2 \frac{1}{3}}^{\circ}$ & & & & & \\
\hline 2557.703 & 1 & 5 & 39085.86 & -0.01 & $39378_{0 \frac{1}{2}}^{\circ}-78464_{1 \frac{1}{3}}^{\circ}$ & & & & & \\
\hline 2557,259 & 4 & 20 & 39092,64 & -0.02 & $37077_{\frac{1}{2}}^{\circ}-76170_{2 \frac{1}{2}}$ & 7 & (1.113) & 1.13 & 0 & 1.12 \\
\hline 2556.30 & & 3 & 39107,31 & +0.01 & $38342_{41}^{\circ}-77449_{3}$ & & & & & \\
\hline 2554.641 & & 2 & 39132.70 & $=0.01$ & $57765_{4 \frac{1}{3}}-96898^{0}$ & & & & & \\
\hline 2552,88 & 2 & 2 & 39159.70 & -0.02 & $37516_{3 \frac{1}{2}}^{\circ}-76676_{2 \frac{1}{2}}$ & & & & & \\
\hline 2552.70 & 10 & 60 & 39162.46 & +0.09 & $31631_{6 \frac{1}{2}}^{\circ}-70793_{5 \frac{1}{2}}^{\circ}$ & 7 & $(1.230)$ & 1.224 & 0 & 1.264 \\
\hline 2552.147 & 15 & 80 & 39170.94 & +0.07 & $30224_{4 \frac{1}{2}}^{\circ}-69395_{3 \frac{1}{2}}^{2}$ & 7 & $(0.935)$ & 0.942 & 0 & 0.912 \\
\hline 2550.786 & & 1 & 39191.84 & & & & & & & \\
\hline 2550.68 & 3 & 10 & 39193.47 & +0.03 & $53720_{4 \frac{1}{9}}-92914_{4 \frac{1}{3}}$ & & & & & \\
\hline 2550,060 & 10 & 20 & 39203,00 & $-0,12$ & $22960_{1 \frac{1}{3}}-62163_{2 \frac{1}{3}}^{\circ}$ & & & & & \\
\hline 2550.023 & & 3 & 39203.57 & & & & & & & \\
\hline $\begin{array}{l}2548.756 \\
254800\end{array}$ & 3 & 15 & 39223,05 & -0.03 & $49498_{2 \frac{1}{2}}-88721_{3 \frac{1}{2}}^{\circ}$ & 7 & $(1,145)$ & 1.156 & 0 & 1.182 \\
\hline $\begin{array}{l}2548.00 \\
2547.498\end{array}$ & 2 & $\begin{array}{l}3 \\
8 \\
8\end{array}$ & $\begin{array}{l}39234.69 \\
39242.42\end{array}$ & $=0.04$ & $31979_{3 \frac{1}{2}}^{\circ}-71222_{3 \frac{1}{3}}$ & & & & & \\
\hline 2547.23 & 2 & 5 & 39246.55 & & & & & & & \\
\hline 2547.20 & & $\stackrel{4}{2}$ & 39247.01 & & & & & & & \\
\hline 2546.886 & 1 & 2 & 39251.85 & & & & & & & \\
\hline 2546.28 & 2 & 5 & 39261.19 & -0.05 & $53715_{3 \frac{1}{2}}-92976_{3 \frac{1}{2}}^{\circ}$ & & & & & \\
\hline 2545.871 & 2 & 4 & 39267.50 & -0.03 & $34389^{\circ}-73657_{1 \frac{1}{2}}$ & & & & & \\
\hline 2545.545 & & 3 & 39272.53 & & & & & & & \\
\hline 2544.72 & & 2 & 39285.26 & -0.05 & $33494_{3 \frac{1}{2}}^{\circ}-72779_{3 \frac{1}{2}}$ & & & & & \\
\hline 2542.76 & 3 & 10 & 39315.54 & $\left\{\begin{array}{l}-0.05 \\
+0.13\end{array}\right.$ & $\begin{array}{l}53720_{4 \frac{1}{2}}-93036_{5^{\frac{1}{2}}}^{\circ} \\
56375^{2}-95691^{\circ}\end{array}$ & & & & & \\
\hline $\begin{array}{l}2542.318 \\
2541.82\end{array}$ & 4 & $\begin{array}{r}10 \\
1\end{array}$ & $\begin{array}{l}39322.37 \\
39330.08\end{array}$ & -0.04 & $37516_{3 \frac{1}{2}}^{\circ \frac{1}{2}}-76839_{2 \frac{1}{2}}^{3}$ & & & & & \\
\hline
\end{tabular}


TABLE 6. Yb II-Observed and classified lines-Continued

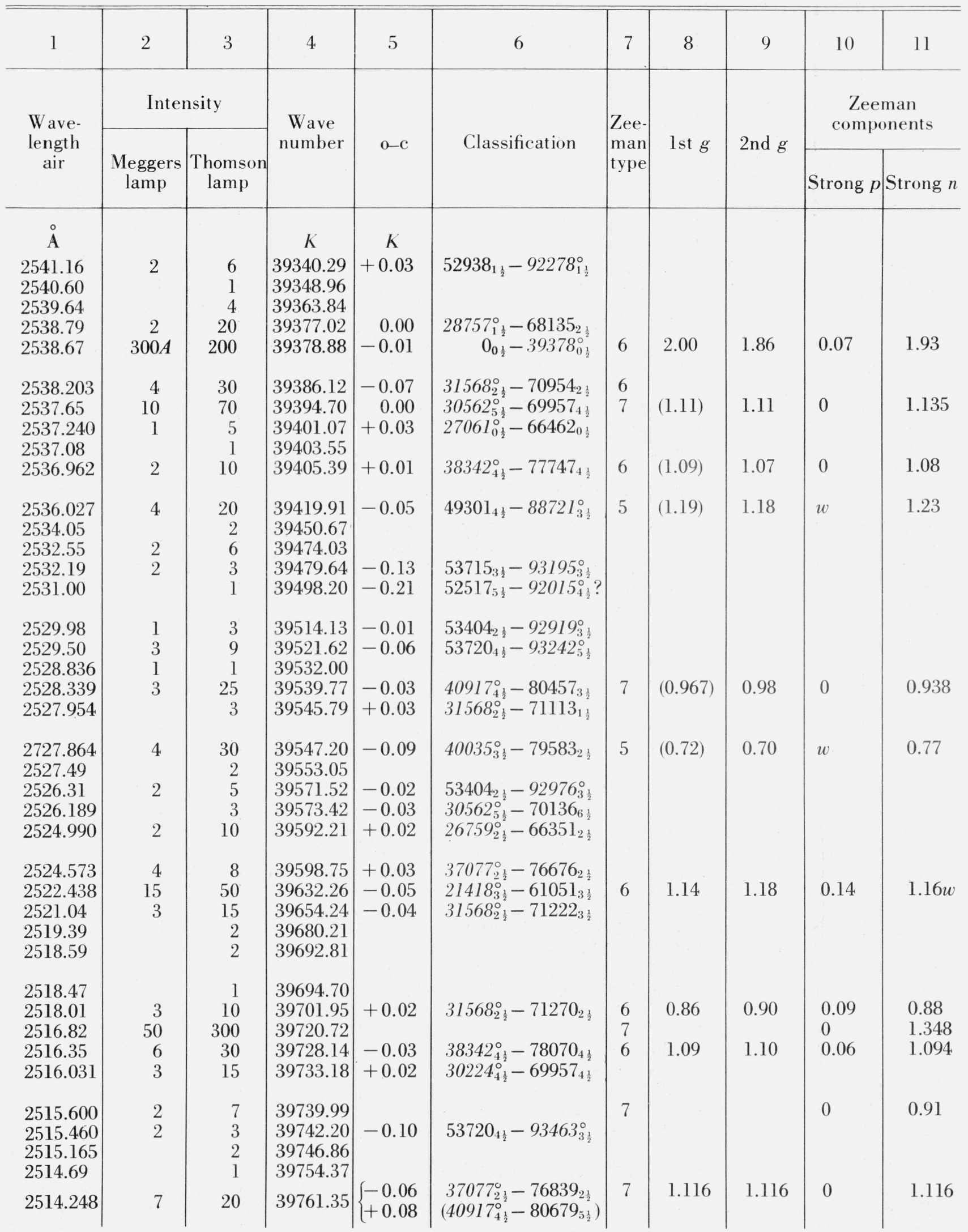


TABLE 6. Yb II-Observed and classified lines-Continued

\begin{tabular}{|c|c|c|c|c|c|c|c|c|c|c|}
\hline 1 & 2 & 3 & 4 & 5 & 6 & 7 & 8 & 9 & 10 & 11 \\
\hline \multirow{2}{*}{$\begin{array}{l}\text { Wave- } \\
\text { length } \\
\text { air }\end{array}$} & \multicolumn{2}{|c|}{ Intensity } & \multirow{2}{*}{$\begin{array}{l}\text { Wave } \\
\text { number }\end{array}$} & \multirow[b]{2}{*}{$\mathrm{o}-\mathrm{c}$} & \multirow{2}{*}{ Classification } & \multirow{2}{*}{ 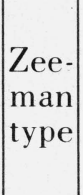 } & \multirow[b]{2}{*}{ lst $g$} & \multirow[b]{2}{*}{ 2nd $g$} & \multicolumn{2}{|c|}{$\begin{array}{c}\text { Zeeman } \\
\text { components }\end{array}$} \\
\hline & $\begin{array}{l}\text { Meggers } \\
\text { lamp }\end{array}$ & $\begin{array}{c}\text { Thomson } \\
\text { lamp }\end{array}$ & & & & & & & Strong $p \mid$ & Strong $n$ \\
\hline$\AA$ & & & $K$ & $K$ & & & & & & \\
\hline $\begin{array}{l}2513.64 \\
2512.87\end{array}$ & & $\begin{array}{l}1 \\
2\end{array}$ & $\begin{array}{l}39770.97 \\
39783.16\end{array}$ & -0.02 & $53720_{4 \frac{1}{2}}-93503_{4 \frac{1}{2}}^{\circ}$ & & & & & \\
\hline 2512.524 & 5 & 20 & 39788.64 & $\left\{\begin{array}{l}-0.03 \\
+0.01\end{array}\right.$ & $\begin{array}{c}33494_{3 \frac{1}{2}}^{\circ}-73283_{\frac{1}{2}}^{\circ} \\
\left(53715_{3 \frac{1}{2}}-93503_{4 \frac{1}{2}}^{\circ}\right)\end{array}$ & 5 & $(0.99)$ & 1.02 & $w$ & 1.11 \\
\hline $\begin{array}{l}2512.061 \\
2511.868\end{array}$ & $\begin{array}{c}50 r \\
4\end{array}$ & $\begin{array}{r}100 \\
20\end{array}$ & $\begin{array}{l}39795.97 \\
39799.03\end{array}$ & $\begin{array}{l}+0.06 \\
+0.05\end{array}$ & $\begin{array}{l}21418_{\frac{1}{2}}^{\circ}-61214_{4 \frac{1}{2}}^{\circ} \\
26759_{2 \frac{1}{2}}^{\circ}-66558_{3 \frac{1}{2}}^{\circ}\end{array}$ & 4 & 1.12 & 1.05 & $(0.036)$ & 0.80 \\
\hline $\begin{array}{l}2510.50 \\
2510.29 \\
2510.03 \\
2509.86 \\
2508.073\end{array}$ & $\begin{array}{r}2 \\
1 \\
1 \\
2 \\
10\end{array}$ & $\begin{array}{r}7 \\
2 \\
1 \\
5 \\
20\end{array}$ & $\begin{array}{l}39820.71 \\
39824.04 \\
39828.17 \\
39830.86 \\
39859.24\end{array}$ & $\begin{array}{r}0.00 \\
-0.13 \\
-0.07 \\
-0.04\end{array}$ & $\begin{array}{l}48900_{3 \frac{1}{2}}-88721_{3 \frac{1}{2}}^{\circ} \\
41688_{1 \frac{1}{2}}^{\circ}-81512_{1 \frac{1}{2}}^{\circ} \\
32371_{2 \frac{1}{2}}^{\circ}-72202_{3 \frac{1}{2}}^{\circ} \\
24332_{2 \frac{1}{2}}^{\circ}-64191_{1 \frac{1}{2}}^{\circ}\end{array}$ & & & & & \\
\hline $\begin{array}{l}2505.477 \\
2503.46 \\
2502.02 \\
2501.21 \\
2500.568\end{array}$ & $\begin{array}{r}6 \\
30 \\
1 \\
2\end{array}$ & $\begin{array}{r}30 \\
1 \\
70 \\
3 \\
6\end{array}$ & $\begin{array}{l}39900.54 \\
39932.68 \\
39955.66 \\
39968.60 \\
39978.86\end{array}$ & $\begin{array}{l}-0.08 \\
-0.05 \\
-0.08 \\
-0.08\end{array}$ & $\begin{array}{l}31568_{2 \frac{1}{2}}^{\circ} 71468_{2 \frac{1}{2}} \\
37516_{3 \frac{1}{2}}^{\circ}-77449_{3 \frac{1}{2}} \\
21418_{3 \frac{1}{2}}^{\circ}-61374_{2 \frac{1}{2}} \\
41688_{1 \frac{1}{2}}^{\circ}-81657_{2 \frac{1}{2}}\end{array}$ & 4 & $\begin{array}{l}0.86 \\
1.14\end{array}$ & $\begin{array}{l}1.00 \\
1.29\end{array}$ & $\begin{array}{l}0.36 ? \\
(0.07)\end{array}$ & $\begin{array}{l}0.93 \\
0.77\end{array}$ \\
\hline $\begin{array}{l}2498.73 \\
2498.36 \\
2496.85\end{array}$ & 2 & $\begin{array}{l}2 \\
5 \\
2\end{array}$ & $\begin{array}{l}40008.27 \\
40014.19 \\
40038.39\end{array}$ & 0.00 & $41678_{2 \frac{1}{2}}^{\circ}-81692_{3 \frac{1}{2}}$ & & & & 0 & \\
\hline 2495.63 & 5 & 20 & 40057.96 & $\left\{\begin{array}{l}-0.06 \\
-0.09\end{array}\right.$ & $\begin{array}{l}32981_{1 \frac{1}{2}}^{\circ}-73039_{0 \frac{1}{2}}^{\circ} \\
53404_{2 \frac{1}{2}}-93463_{3 \frac{1}{2}}^{\circ}\end{array}$ & & & & & \\
\hline 2495.05 & 4 & 3 & 40067.27 & & & & & & & \\
\hline $\begin{array}{l}2493.64 \\
2491.15 \\
2488.92 \\
2487.08\end{array}$ & $\begin{array}{l}3 \\
3\end{array}$ & $\begin{array}{r}10 \\
5 \\
3 \\
3\end{array}$ & $\begin{array}{l}40089.93 \\
40130.00 \\
40165.95 \\
40195.66\end{array}$ & $\begin{array}{l}-0.06 \\
+0.01 \\
+0.07\end{array}$ & $\begin{array}{l}37516_{3 \frac{1}{2}}^{\circ}-77606_{2 \frac{1}{2}}^{\circ} \\
52517_{\frac{1}{2}}^{\circ}-92647_{\frac{1}{2}}^{\circ} \\
31568_{2 \frac{1}{2}}^{\circ}-71763_{\frac{1}{2}}^{\circ}\end{array}$ & & & & 0 & \\
\hline 2484.89 & 5 & 20 & 40231.09 & $\left\{\begin{array}{l}-0.08 \\
+0.03\end{array}\right.$ & $\begin{array}{c}30562_{5 \frac{1}{2}}^{0^{2}}-70793_{5 \frac{1}{2}} \\
\left(33052_{4 \frac{1}{2}}^{\circ}-73283_{4 \frac{1}{2}}\right)\end{array}$ & 6 & (1.112) & 1.20 & 0.49 & \\
\hline $\begin{array}{l}2481.42 \\
2481.36 \\
2481.03 \\
2479.29 \\
2479.15\end{array}$ & $\begin{array}{l}1 \\
1\end{array}$ & $\begin{array}{l}3 \\
2 \\
2 \\
2 \\
5\end{array}$ & $\begin{array}{l}40287.34 \\
40288.32 \\
40293.67 \\
40321.95 \\
40324.23\end{array}$ & $\begin{array}{l}-0.02 \\
+0.07 \\
-0.03 \\
+0.05 \\
-0.06\end{array}$ & $\begin{array}{l}40917_{4 \frac{1}{2}}^{\circ}-81205_{4 \frac{1}{2}} \\
53720_{4 \frac{1}{2}}-94008_{3 \frac{1}{2}}^{\circ} \\
53715_{3 \frac{1}{2}}-94008_{3 \frac{1}{2}}^{\circ} \\
28757_{1 \frac{1}{2}}^{\circ}-69079_{2 \frac{1}{2}}^{\circ} \\
59090_{3 \frac{1}{2}}-99414_{4 \frac{1}{2}}^{\circ}\end{array}$ & & & & & \\
\hline $\begin{array}{l}2477.76 \\
2476.62 \\
2475.92 \\
2474.52 \\
2474.27\end{array}$ & $\begin{array}{l}2 \\
1\end{array}$ & $\begin{array}{l}1 \\
2 \\
2 \\
1 \\
3\end{array}$ & $\begin{array}{l}40346.85 \\
40365.42 \\
40376.83 \\
40399.67 \\
40403.75\end{array}$ & $\begin{array}{l}-0.03 \\
+0.16 \\
-0.08\end{array}$ & $\begin{array}{l}37516_{3 \frac{1}{2}}^{\circ}-77882_{3 \frac{1}{2}} \\
49498_{2 \frac{1}{2}}-89897_{1 \frac{1}{2}}^{\circ} \\
21418_{3 \frac{1}{2}}^{\circ}-61822_{3 \frac{1}{2}}\end{array}$ & & & & & \\
\hline $\begin{array}{l}2473.95 \\
2473.16 \\
2471.06\end{array}$ & 2 & $\begin{array}{l}1 \\
2 \\
3\end{array}$ & $\begin{array}{l}40408.98 \\
40421.89 \\
40456.24\end{array}$ & $\begin{array}{r}+0.09 \\
0.00 \\
+0.01\end{array}$ & $\begin{array}{l}32371_{2 \frac{1}{2}}^{\circ}-72779_{3 \frac{1}{2}} \\
40035_{3 \frac{1}{2}}^{\circ}-80457_{3 \frac{1}{2}} \\
22960_{\frac{1}{2}}-63417_{2 \frac{1}{2}}^{\circ}\end{array}$ & & & & & \\
\hline
\end{tabular}


TABLE 6. Yb II-Observed and classified lines-Continued

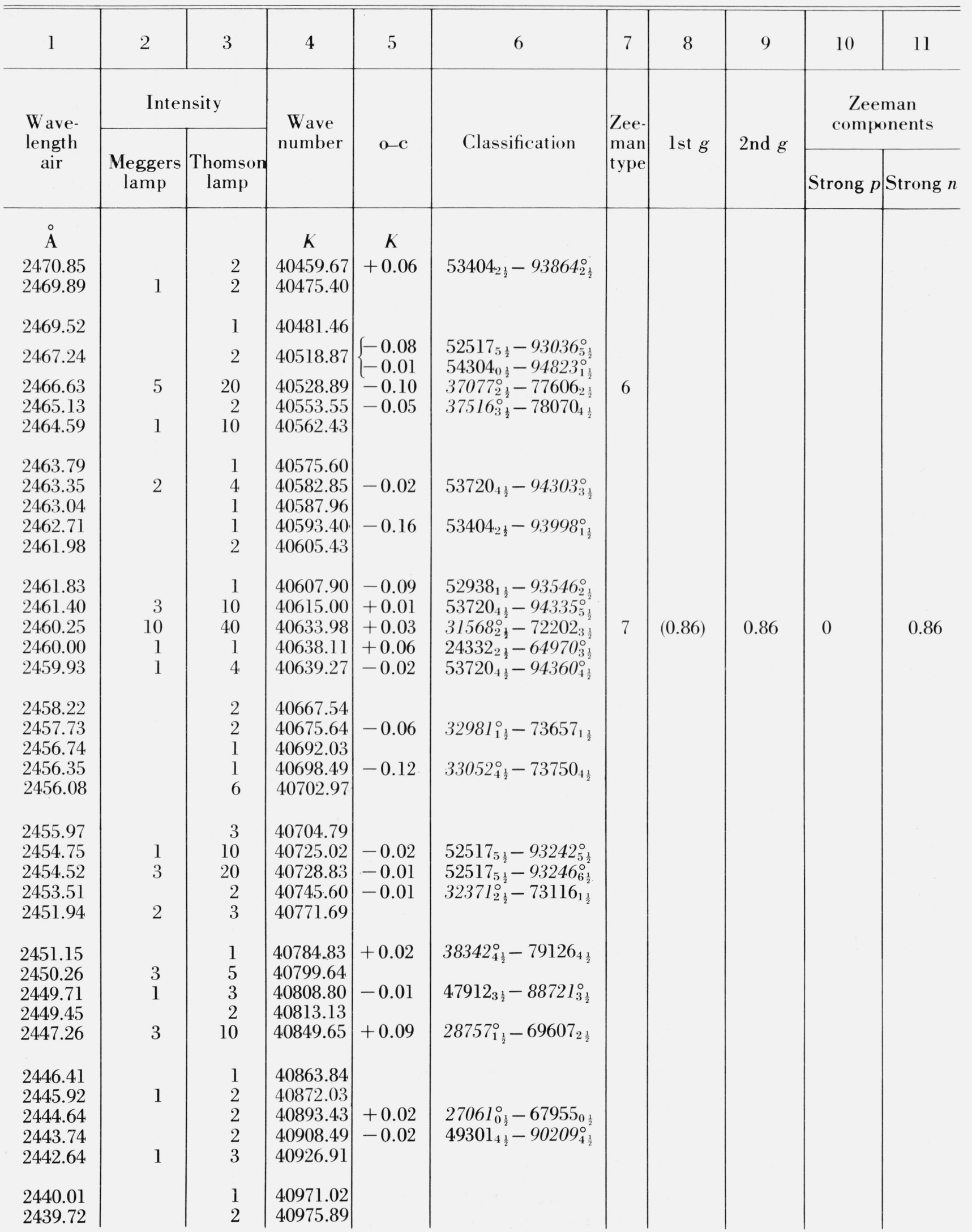


TABLE 6. Yb II-Observed and classified lines-Continued

\begin{tabular}{|c|c|c|c|c|c|c|c|c|c|c|}
\hline 1 & 2 & 3 & 4 & 5 & 6 & 7 & 8 & 9 & 10 & 11 \\
\hline \multirow{2}{*}{$\begin{array}{l}\text { Wave- } \\
\text { length } \\
\text { air }\end{array}$} & \multicolumn{2}{|c|}{ Intensity } & \multirow{2}{*}{$\begin{array}{c}\text { Wave } \\
\text { number }\end{array}$} & \multirow{2}{*}{$\mathrm{o}-\mathrm{c}$} & \multirow{2}{*}{ Classification } & \multirow{2}{*}{$\begin{array}{l}\text { Zee- } \\
\text { man } \\
\text { type }\end{array}$} & \multirow{2}{*}{ lst $g$} & \multirow{2}{*}{ 2nd $g$} & \multicolumn{2}{|c|}{$\begin{array}{c}\text { Zeeman } \\
\text { components }\end{array}$} \\
\hline & $\begin{array}{c}\text { Meggers } \\
\text { lamp }\end{array}$ & $\begin{array}{c}\text { Thomson } \\
\text { lamp }\end{array}$ & & & & & & & Strong $p$ & Strong $n$ \\
\hline$\AA$ & & & $K$ & $K$ & & & & & & \\
\hline 2439.59 & 3 & 5 & 40978.07 & +0.15 & $49301_{4 \frac{1}{2}}-90279_{5 \frac{1}{2}}^{\circ}$ & & & & & \\
\hline 2437.24 & 4 & 7 & 41017.58 & & & & & & & \\
\hline 2436.87 & 1 & 2 & 41023.81 & -0.03 & $53404_{2 \frac{1}{2}}-94428_{1 \frac{1}{2}}^{\circ}$ & & & & & \\
\hline 2436.06 & & 5 & 41037.45 & +0.11 & $57765_{4 \frac{1}{2}}-98802_{5 \frac{1}{2}}^{\circ}$ & & & & & \\
\hline 2434.02 & & 2 & 41071.84 & +0.09 & $30392^{\circ}-71468$ & & & & & \\
\hline $\begin{array}{l}2433.74 \\
2433.62\end{array}$ & 1 & $\begin{array}{l}1 \\
3\end{array}$ & $\begin{array}{l}41076.56 \\
41078.59\end{array}$ & +0.09 & $30392_{1 \frac{1}{2}}^{1}-71468_{2 \frac{1}{2}}$ & & & & & \\
\hline 2433.16 & 1 & 2 & 41086.35 & & & & & & & \\
\hline 2433.05 & & 1 & 41088.21 & & & & & & & \\
\hline 2432.62 & 2 & 3 & 41095.47 & & & & & & & \\
\hline 2432.01 & & 2 & 41105.78 & & & & & & & \\
\hline 2431.69 & & 1 & 41111.19 & $\begin{array}{l}-0.06 \\
-0.05\end{array}$ & $35059_{3 \frac{1}{2}}^{\circ}-76170_{2 \frac{1}{2}}$ & & & & & \\
\hline 2429.01 & 1 & 10 & 41156.55 & -0.05 & $48556_{3 \frac{1}{2}}^{\circ}-89713_{2 \frac{1}{2}}$ & & & & & \\
\hline $\begin{array}{l}2428.74 \\
2428.30\end{array}$ & & 1 & $\begin{array}{l}41161.12 \\
41168.58\end{array}$ & -0.06 & $34389_{2 \frac{1}{2}}^{\circ}-75550_{2 \frac{1}{2}}$ & & & & & \\
\hline $\begin{array}{l}2428.30 \\
2428.12\end{array}$ & & $\begin{array}{l}1 \\
6\end{array}$ & $\begin{array}{l}41108.58 \\
41171.63\end{array}$ & & & & & & & \\
\hline 2427.66 & & 1 & 41179.43 & & & & & & & \\
\hline 2427.20 & & 1 & 41187.23 & & & & & & & \\
\hline 2426.19 & & 1 & 41204.38 & & & & & & & \\
\hline 2425.68 & & 1 & 41213.04 & -0.03 & $41678_{2 \frac{1}{2}}^{\circ}-82891_{2 \frac{1}{2}}$ & & & & & \\
\hline 2424.61 & 4 & 6 & 41231.23 & +0.06 & $22960_{1 \frac{1}{2}}-64191_{1 \frac{1}{2}}^{\circ \frac{2}{2}}$ & & & & & \\
\hline 2423.96 & & 1 & 41242.28 & -0.05 & $48900_{3 \frac{1}{2}}-90142_{3 \frac{1}{2}}^{\circ}$ & & & & & \\
\hline 2422.84 & 10 & 20 & 41261.35 & -0.02 & $24332_{2 \frac{1}{2}}^{2}-65594_{2 \frac{1}{2}}^{\circ}$ & & & & & \\
\hline 2421.35 & 10 & 50 & 41286.74 & +0.05 & $38342_{4 \frac{1}{2}}^{\circ}-79628_{3 \frac{1}{2}}$ & 4 & (1.09) & 1.12 & $w$ & 1.00 \\
\hline 2420.03 & 1 & 4 & 41309.25 & -0.01 & $48900_{3 \frac{1}{2}}-90209_{4 \frac{1}{2}}^{\circ}$ & & & & & \\
\hline 2419.41 & & 1 & 41319.84 & & & & & & & \\
\hline $\begin{array}{l}2419.20 \\
2418.38\end{array}$ & & $\begin{array}{l}2 \\
2\end{array}$ & $\begin{array}{l}41323.42 \\
41337.44\end{array}$ & -0.06 & $33653_{01}^{\circ}-74991_{11}$ & & & & & \\
\hline & & & 41006 & 0.00 & $50000 \frac{1}{2}-14791 \frac{1}{2}$ & & & & & \\
\hline 2417.01 & & 3 & 41360.86 & -0.17 & $49498_{2 \frac{1}{2}}-90859_{2 \frac{1}{2}}^{\circ}$ & & & & & \\
\hline 2415.39 & & 2 & 41388.60 & & $24332_{01}-65739^{\circ}$ & & & & & \\
\hline $\begin{array}{l}2414.33 \\
2413.77\end{array}$ & 2 & $\begin{array}{l}6 \\
2\end{array}$ & $\begin{array}{l}41406.77 \\
41416.38\end{array}$ & $\begin{array}{r}-0.01 \\
0.00\end{array}$ & $\begin{array}{l}24332_{2 \frac{1}{2}}-65739_{2 \frac{1}{2}}^{\circ} \\
49301_{4 \frac{1}{2}}-90717^{\circ}\end{array}$ & & & & & \\
\hline 2413.66 & 1 & 5 & 41418.27 & +0.05 & $53404_{2 \frac{1}{2}}-94823_{\frac{1}{2}}^{\circ \frac{1}{2}}$ & & & & & \\
\hline 2409.44 & & 1 & 41490.80 & +0.01 & $52938_{1 \frac{1}{2}}-94428_{1 \frac{1}{2}}^{\circ}$ & & & & & \\
\hline 2409.06 & & 5 & 41497.35 & & & & & & & \\
\hline 2407.98 & & 1 & 41515.96 & & & & & & & \\
\hline $\begin{array}{l}2407.76 \\
240679\end{array}$ & & 1 & $\begin{array}{l}41519.75 \\
4153648\end{array}$ & & & & & & & \\
\hline & & 1 & 41500.40 & & & & & & & \\
\hline 2406.09 & 1 & 10 & 41548.57 & -0.06 & $31568_{2 \frac{1}{2}}^{\circ}-73116_{1 \frac{1}{2}}$ & & & & & \\
\hline 2403.41 & & 4 & 41594.89 & +0.01 & $34575_{1 \frac{1}{2}}^{\circ}-76170_{2 \frac{1}{2}}^{\circ}$ & & & & & \\
\hline
\end{tabular}


TABLE 6. Yb II-Observed and classified lines-Continued

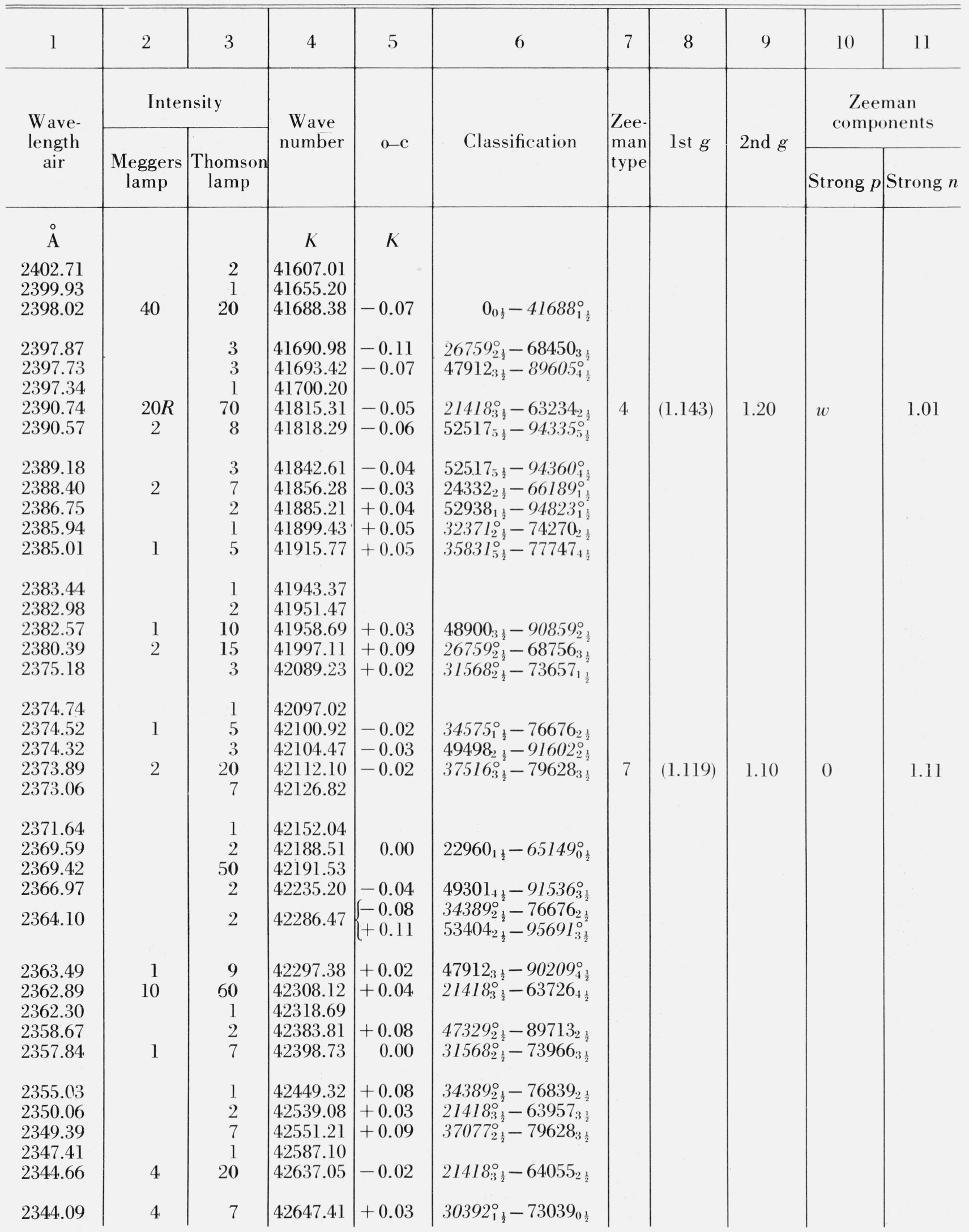


TABLE 6. Yb II-Observed and classified lines-Continued

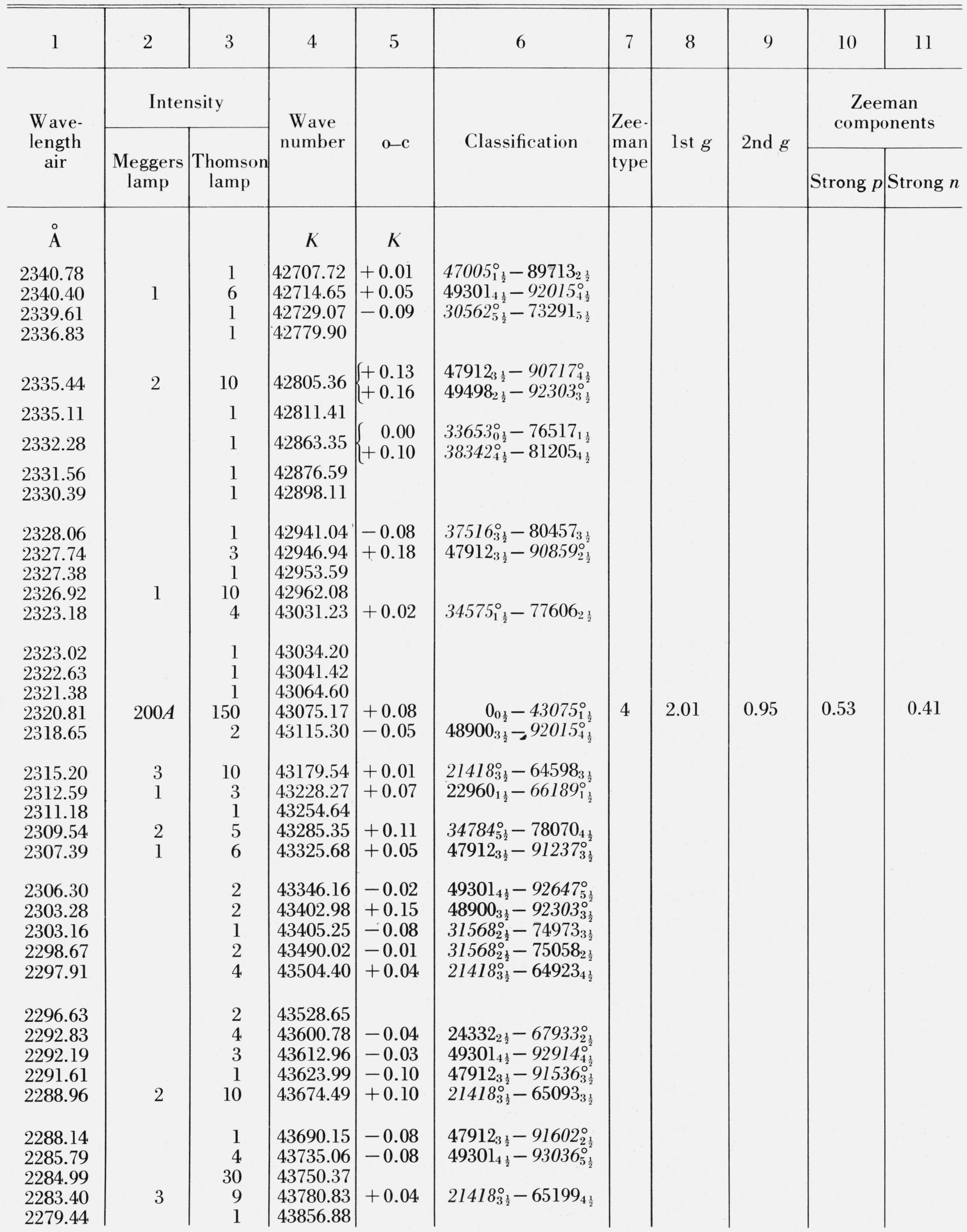


TABLE 6. Yb II-Observed and classified lines-Continued

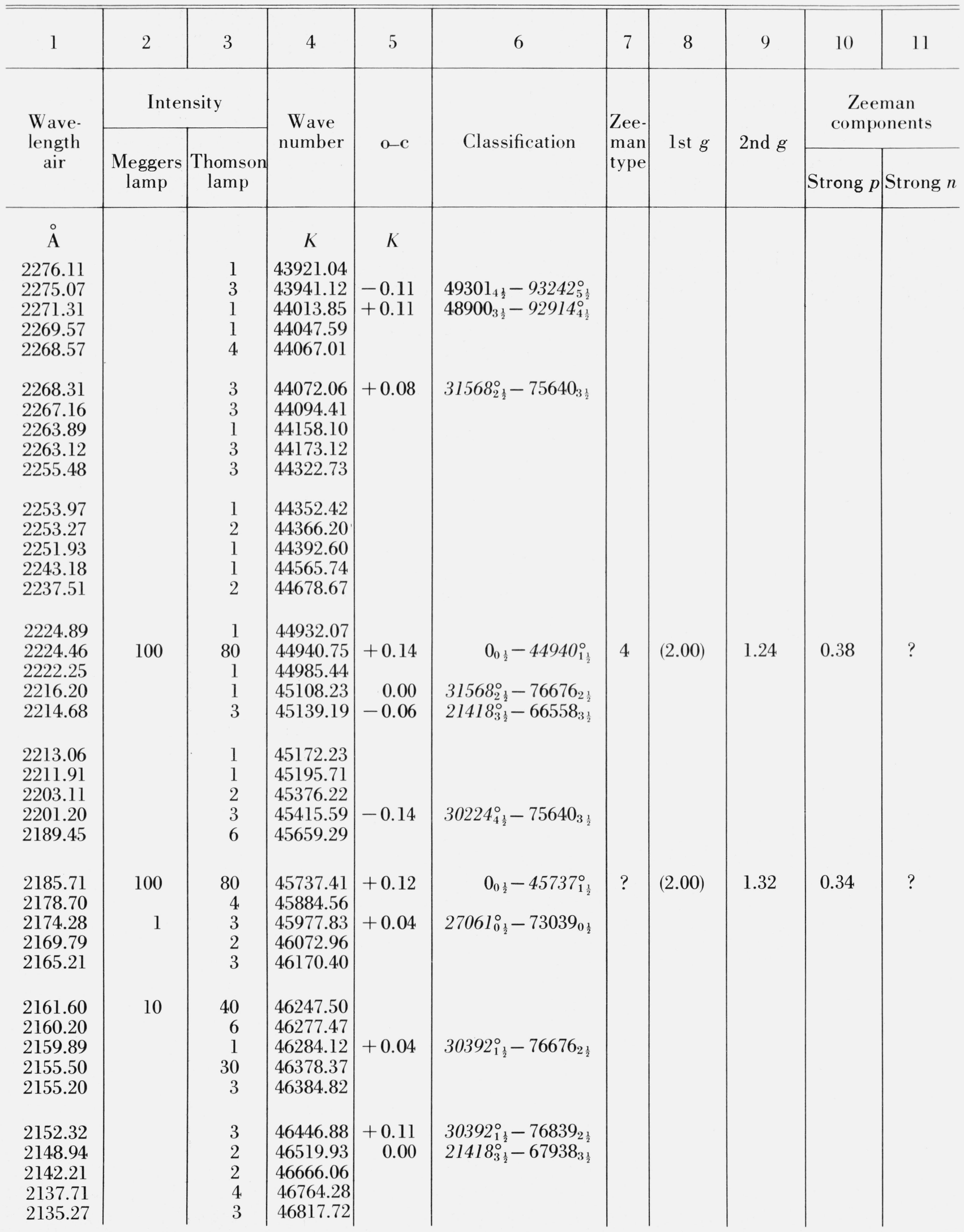


TABLE 6. Yb II-Observed and classified lines-Continued

\begin{tabular}{|c|c|c|c|c|c|c|c|c|c|c|}
\hline 1 & 2 & 3 & 4 & 5 & 6 & 7 & 8 & 9 & 10 & 11 \\
\hline \multirow{2}{*}{$\begin{array}{l}\text { Wave- } \\
\text { length } \\
\text { air }\end{array}$} & \multicolumn{2}{|c|}{ Intensity } & \multirow{2}{*}{$\begin{array}{c}\text { Wave } \\
\text { number }\end{array}$} & \multirow{2}{*}{$\mathrm{o}-\mathrm{c}$} & \multirow{2}{*}{ Classification } & \multirow{2}{*}{$\left|\begin{array}{c}\text { Zee- } \\
\text { man } \\
\text { type }\end{array}\right|$} & \multirow{2}{*}{ lst $g$} & \multirow{2}{*}{ 2nd $g$} & \multicolumn{2}{|c|}{$\begin{array}{c}\text { Zeeman } \\
\text { components }\end{array}$} \\
\hline & $\begin{array}{c}\text { Meqqers } \\
\text { lamp }\end{array}$ & $\begin{array}{c}\text { Thomson } \\
\text { lamp }\end{array}$ & & & & & & & Strong $p$ & Strong $n$ \\
\hline$\stackrel{\circ}{\AA}$ & & & K & $K$ & & & & & & \\
\hline 2131.40 & 6 & 5 & 46902.72 & +0.01 & $0_{0 \frac{1}{2}}-46902_{0 \frac{1}{2}}^{\circ}$ & & & & & \\
\hline 2126.74 & 100 & 80 & 47005.47 & +0.01 & $0_{0 \frac{1}{2}}-47005_{1 \frac{1}{2}}^{\circ}$ & & & & & \\
\hline 2125.57 & & 4 & 47031.34 & -0.02 & $21418_{3 \frac{1}{2}}^{\circ}-68450_{3 \frac{1}{2}}^{\circ}$ & & & & & \\
\hline 2120.36 & & 3 & 47146.89 & -0.04 & $31979_{3 \frac{1}{2}}^{\circ}-79126_{4 \frac{1}{2}}$ & & & & & \\
\hline 2117.62 & & 6 & 47207.89 & +0.10 & $26759_{2 \frac{1}{2}}^{\circ}-73966_{3 \frac{1}{2}}^{\circ}$ & & & & & \\
\hline 2116.67 & 100 & 80 & 47229.07 & +0.11 & $0_{0 \frac{1}{2}}-47228_{0 \frac{1}{2}}^{\circ}$ & & & & & \\
\hline 2102.73 & 5 & 20 & 47542.14 & & & & & & & \\
\hline 2022.03 & & 2 & 49439.31 & -0.12 & $21418_{3 \frac{1}{2}}^{\circ}-70858_{+\frac{1}{2}}$ & & & & & \\
\hline 2021.36 & & 2 & 49455.70 & & & & & & & \\
\hline 2018.08 & & 2 & 49536.06 & & & & & & & \\
\hline
\end{tabular}

${ }^{a}$ Line is a blend of $\mathrm{Yb} \mathrm{I}$ and $\mathrm{Yb}$ II.

b) Parentheses in column 6 denote masked lines.

' $J=01 / 2$ assumed in deriving $g$-values for $\lambda$ 8093.030.

d Observed Zeeman Effect and classification are discordant.

e For $\lambda 7362.920 J=1 \frac{11}{2}$, lst $g$; $01 / 2$ 2nd $g$.

For $\lambda 3736.901 J=01 / 2$, lst $g$; $1 \frac{1 / 2}{2}$ 2nd $g$. 


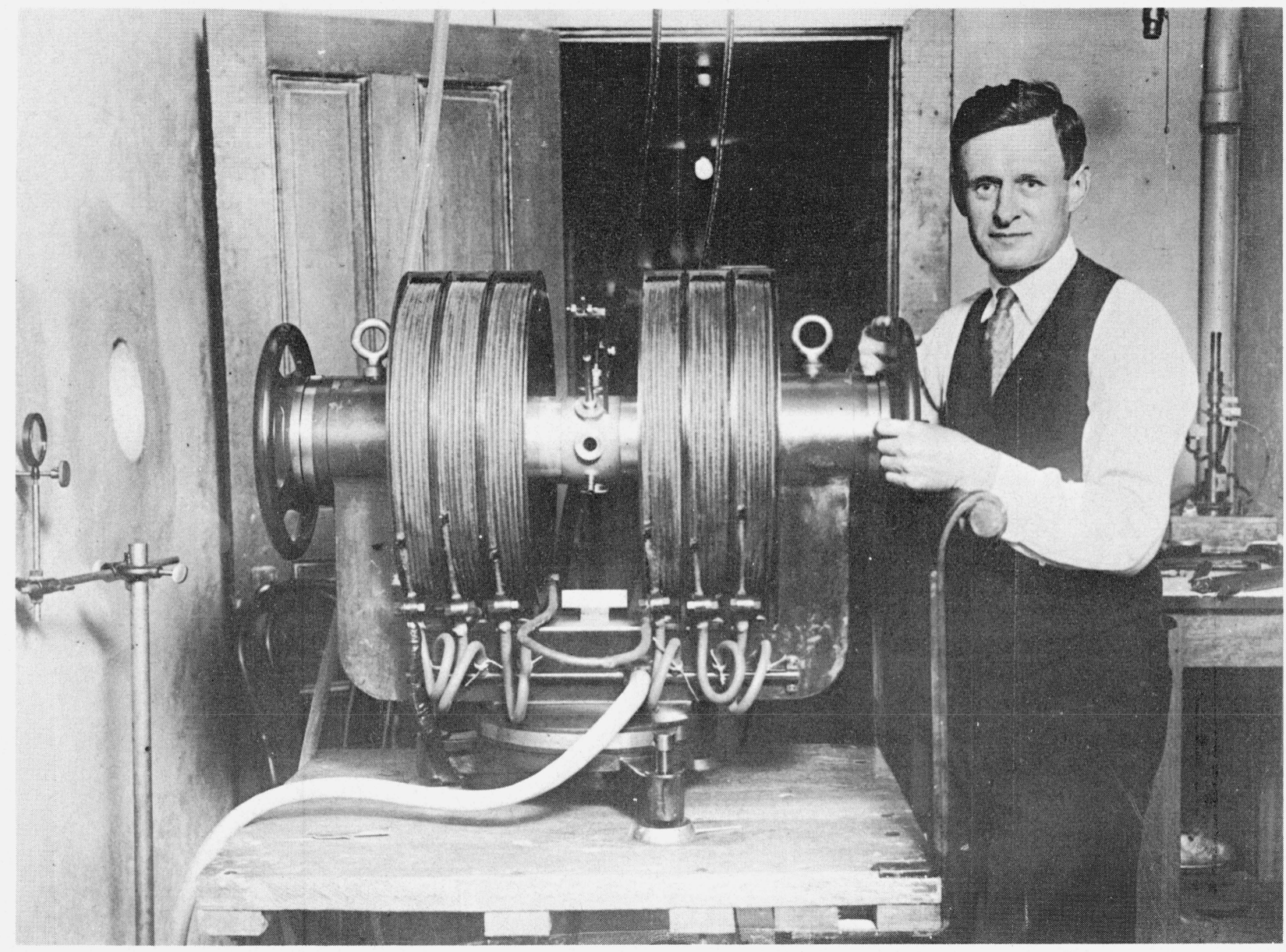

W. F. Meggers shown in 1925 with the electromagnet used by him in his pioneering application of the Zeeman effect to the interpretation of atomic spectra. 
